RUNNING HEAD: WISDOM, BIAS, AND BALANCE

Wisdom, bias, and balance: Toward a process-sensitive measurement of wisdomrelated cognition

\author{
Justin P. Brienza* $\quad$ Franki Y. H. Kung \\ Henri C. Santos $\quad$ D. Ramona Bobocel \\ Igor Grossmann
}

University of Waterloo, Canada

Author Note

*Corresponding authors:

Justin P. Brienza, 200 University Avenue West, Waterloo, Ontario, Canada N21 3G1, e-mail:

jbrienza@uwaterloo.ca

Igor Grossmann, 200 University Avenue West, Waterloo, Ontario, Canada N21 3G1, e-mail:

igrossma@uwaterloo.ca

The present research was funded by the Social Sciences and Humanities Research Council of Canada Insight Grants 435-2012-0306 (to D.R.B.) and 435-2014-0685 (to I.G.). 


\begin{abstract}
Philosophers and behavioral scientists refer to wisdom as unbiased reasoning that guides one toward balance of interests and promotes a good life. However, major instruments developed to test wisdom appear biased, and it is unclear whether they capture balance-related tendencies. We examined whether shifting from global, de-contextualized reports to state-level reports about concrete situations provides a less biased method to assess wise reasoning (e.g., intellectual humility, recognition of uncertainty and change, consideration of the broader context at hand and perspectives of others, integration of these perspectives/compromise), which may be aligned with the notion of balancing interests. Results of a large-scale psychometric investigation $(N=$ 4,463 ) revealed that the novel situated wise reasoning scale (SWIS) is reliable and appears independent of psychological biases (attribution bias, bias blind spot, self-deception, impression management), whereas global wisdom reports are subject to such biases. Moreover, SWIS scores were positively related to indices of living well (e.g., adaptive emotion regulation, mindfulness), and balancing of cooperative and self-protective interests, goals (influence-vs.-adjustment) and causal inferences about conflict (attribution to the self-vs.-other party). In contrast, global wisdom reports were unrelated or negatively related to balance-related measures. Notably, people showed modest within-person consistency in wise reasoning across situations/over time, suggesting that a single-shot measurement may be insufficient for whole understanding of traitlevel wisdom. We discuss theoretical and practical implications for research on wisdom, judgment and decision making, well-being, and prosociality.
\end{abstract}

Keywords: reasoning, goal conflict, conflict resolution, prosociality, person-situation 


\section{Wisdom, bias, and balance: Toward a process-sensitive measurement of wisdom- related cognition}

Throughout human history, people from different philosophies, cultures, and religions have considered wisdom as a supreme and valuable concept (Assmann, 1994; Birren \& Svensson, 2005). Wisdom has been linked to pragmaticism (Baltes \& Smith, 2008), reduced bias (McKee \& Barber, 1999), bigger-picture, prosocial orientation (Baltes \& Smith, 2008;

Staudinger \& Glück, 2011; Vervaeke \& Ferraro, 2013) and is often used in reference to historical exemplars who have remarkable acumen into matters of social life (e.g., Buddha, Gandhi, or Martin Luther King, Jr.; Weststrate, Ferrari, \& Ardelt, 2016).

Contemporary behavioral scientists characterize wisdom through unbiased thought, which is conducive to working through challenging life situations (Baltes \& Staudinger, 2000; Grossmann, Na, Varnum, Kitayama, \& Nisbett, 2013; McKee \& Barber, 1999; Staudinger \& Glück, 2011; Sternberg, 1998). Examples of such thought involve intellectual humility, recognition of world in flux and change, and consideration of the bigger picture beyond immediate self-interest (Basseches, 1984; Clayton, 1982; Grossmann, 2017; Staudinger \& Glück, 2011). Quantitative information about wisdom-related thought can be useful for a wide range of fields, including decision making, conflict management and negotiation, counseling, living well, and would be beneficial to anyone who wishes to work through a difficult challenge and improve their well-being (Grossmann, Na, Varnum, Kitayama, \& Nisbett, 2013). Indeed, contemporary scholars and practitioners have called for wisdom in many challenging social domains, including education, conflict resolution, leadership, and business (Baltes \& Smith, 2008b; Goold \& Campbell, 1998; Haque, 2010; Nonaka \& Takeuchi, 2011; Rooney \& McKenna, 2008; Staudinger \& Glück, 2011; Sternberg, 2010). 
Despite broad interest in wisdom, empirical inquiry into this topic has been constrained by methodological limitations and a non-alignment between dominant theoretical propositions about wisdom and their measurement. The methodological limitation chiefly concerns the lack of consensus about how to measure wisdom. Some scholars advocate measuring wisdom-related characteristics via intensive observer-based evaluations of people's narratives (Baltes \& Smith, 2008; Baltes \& Staudinger, 2000; Bluck \& Glück, 2004; Grossmann et al., 2010; König \& Glück, 2014; Kunzmann \& Baltes, 2003; Mickler \& Staudinger, 2008). However, such observerbased evaluations require costly narrative analysis techniques and therefore remain limited in their ecological application for large-scale investigations. Conversely, other scholars (e.g., Ardelt, 2003; Levenson, Jennings, Aldwin, \& Shiraishi, 2005; Park \& Peterson, 2008; Webster, 2003) advocate using single-shot self-report questionnaires assessing wisdom-related characteristics that are socially desirable. In these tests, people are instructed to report on their global tendencies to be reflective, to show benevolence towards others, to perspective-take, or to report on other desirable characteristics. This approach, while easier to administer than observerbased methods, does not factor in the dynamic nature of wisdom-related characteristics (Staudinger \& Glück, 2011), precluding context-sensitive assessment within individuals and across specific states (Dunlop, 2015; Fleeson \& Jayawickreme, 2015; Fleeson \& Noftle, 2008b, 2012).

The theory-measurement non-alignment concerns the question of a criterion against which wisdom-related characteristics should be evaluated. Numerous theorists have proposed that wisdom-related thought is critical for balancing various interests and trade-offs in the process of making important life decisions. The notion of balancing various interests is central to Sternberg's balance theory of wisdom (1998), Baltes and colleagues' notion of managing 
uncertainties (Baltes \& Smith, 2008), Ardelt's multi-dimensional wisdom theory (1997), Grossmann's work on person-context interaction in expression of wise thought (2017), and earlier works by Clayton (1975) and Kitchener and Brenner (1990). Despite the centrality of the notion of balancing interests to the wisdom construct, this link has remained theoretical (e.g., Clayton, 1975; Grossmann, 2017; Sternberg, 1998) or implicit (e.g., Ardelt, 1997; Baltes \& Smith, 2008; Kitchener \& Brenner, 1990). In particular, there has been little effort to explicitly use balance-related criteria to evaluate the hypothesized effectiveness of wisdom-related cognitive processes. To an extent, this void in empirical research on the relationship between wisdom and balancing-related criterion may be attributed to methodology: Dominant global measures of wisdom conceptualize wisdom-related characteristics as an outcome of being balanced without ever testing this quality, and they lack precision in measurement to evaluate the actual state of balancing interests.

Building on advances in survey methodology and the psychology of wisdom, we introduce a novel, hybrid method for assessing aspects of thought that philosophers and behavioral scholars have associated with wisdom (henceforth "wise reasoning;" Grossmann, 2017). The new method aims to minimize the social desirability biases associated with global self-reports by grounding the assessment in the context of respondents' concrete experiences, versus focusing on global, decontextualized characteristics. To optimize the method, we incorporated the efficiency of self-report scales with the potential for ecological and construct validity that can be achieved with performance assessments and experience sampling (Kahneman, Krueger, Schkade, Schwarz, \& Stone, 2004; Schwarz, Kahneman, \& Xu, 2009). The new hybrid method enabled us to conduct the first large-scale evaluation of state-level wise reasoning, focusing on social challenges (work-related challenges and interpersonal conflicts) 
people encounter in their lives. We evaluate the utility of this method (vis-à-vis global wisdom measures) against markers of social cognitive bias as well as the process of balancing goals and causal judgments.

\section{Defining Wise Reasoning}

In lay terms, wisdom can mean many things, ranging from rationality and intellect to leadership qualities, to knowledge drawn from traumatic life experiences (Grossmann, 2017; Staudinger \& Glück, 2011). Notably, philosophers and psychological scientists view general knowledge or intelligence as insufficient for wisdom (Ardelt, 2004; Baltes \& Kunzmann, 2004; Baltes \& Smith, 2008; Baltes \& Staudinger, 2000; Jeste et al., 2010; Kekes, 1983; McKee \& Barber, 1999; Sternberg, 1998; Vervaeke \& Ferraro, 2013). Rather, psychological scientists interested in wisdom have proposed to examine specific processes involved in understanding and navigating one's social world - aspects of reasoning involved in the context-sensitive processing of knowledge (Baltes \& Smith, 2008; Baltes \& Staudinger, 1993; Grossmann, 2017; Vervaeke \& Ferraro, 2013). As Sternberg (1998, p. 353) pointed out, "information processing in and of itself is not wise or unwise. Its degree of wisdom depends on the fit of a wise solution to its context." Dynamic processing of knowledge is particularly important for flexibly navigating life's uncertainties (Baltes \& Kunzmann, 2004; Baltes \& Smith, 2008; Baltes \& Staudinger, 2000; Grossmann, Na, Varnum, et al., 2013).

The study of cognitions involved in a wise judgment started with Clayton $(1975,1982)$, who defined wisdom as a tendency to understand and accept paradoxes and contradictions that mark social situations, guided by the principle of dialectical thinking (i.e., the recognition that the world is in flux and therefore changes). Clayton (1982) proposed that dialectical thinking can enable individuals to identify relationships and even commonalities between seemingly 
conflicting interests. Invoking dialecticism allowed Clayton to distinguish between wisdom and domain-general cognitive abilities characterizing rational/analytical thought (e.g., intelligence). Specifically, domain-general abilities draw on symbolic rules and procedures such as propositional logic (Peng \& Nisbett, 1999; Piaget, Inhelder, \& Piaget, 2013) which are (better) suited for solving well-structured problems (e.g., Haugeland, 1989). In contrast, ill-structured problems (e.g., those involving value trade-offs, incomplete information for a decision, unclear means or end-goals or problems with missing information about initial- or goal-states, or means to a solution; cf. Jonassen, 1997; Simon, 1973) are more complex and dynamic, and therefore they are harder to resolve via processes like symbolic and propositional logic. Rather, they require open, nuanced, and dynamic processing of information (Clayton, 1982; Hieronymi, 2013; Sinnott, 1984; 1989). Importantly, the problems encountered in everyday social life (even the relatively simple ones) can often be characterized as ill-structured (Allaire \& Marsiske, 2002; Frensch \& Funke, 2014; Mienaltowski, 2011). Later empirical work has built on the claim of relative insufficiency for domain-general abilities for resolving social challenges, demonstrating that abstract cognitive abilities, executive functioning, and rationality are not sufficient for wise reasoning (Grossmann, Na, Varnum, et al., 2013; Grossmann, Sahdra, \& Ciarrochi, 2016; Staudinger, Lopez, \& Baltes, 1997; Sternberg, 1998).

Building on the earlier work by neo-Piagetian developmental psychologists (Basseches, 1984; Clayton, 1982; Kitchner, 1983; for a review, see Kallio, 2015), Baltes and colleagues defined wisdom as "excellence in mind and virtue ... an expert knowledge system [for] dealing with the conduct and understanding of life" (Baltes, 2004; Baltes \& Smith, 2008). In their view, “an expert knowledge system" concerns an individual's ability to understand and manage challenging life situations. This ability draws from characteristics of reasoning such as 
consideration of the relationship between varied contexts of life and how they change over time, the recognition that values and life goals differ between individuals and groups, and acknowledgment of the uncertainties of life, together with ways to manage those uncertainties. Other scholars (Grossmann et al., 2010; Kramer, 2002) have specified aspects of wisdom-related cognition involved in dialectical, self-transcendent reflection on ill-structured problems: intellectual humility, acknowledgment of different points of view, appreciation of the context within which the issue unfolds, sensitivity to the possibility of change in social relations, acknowledgment of the likelihood of multiple outcomes of a conflict, and preference for compromise in resolving opposing viewpoints. Recent reviews suggest that these aspects of reasoning appear across a wide range of definitions of wisdom in behavioral sciences (Bangen, Meeks, \& Jeste, 2013; Grossmann \& Kung, in press).

\section{Balancing Interests as a Criterion of Wise Reasoning}

The notion of balancing interests unites different theoretical models of wisdom in general, and wise reasoning in particular. For instance, Sternberg's (1998) influential balance theory of wisdom has pointed out to various intrapersonal, interpersonal, and extrapersonal goals, long- and short-term plans, and goals of adjusting to versus influencing one's environment (Sternberg, 1998, 2003). The centrality of balancing to wisdom goes beyond Sternberg's conceptualization. Indeed, when summarizing the last few decades of psychological wisdom research, Staudinger and Glück (2011) concluded that:

Wisdom concerns mastering the basic dialectics shaping human existence, such as the dialectic between good and bad, positivity and negativity, dependency and independence, certainty and doubt, control and lack of control, finiteness and eternity, strength and weakness, and selfishness and altruism. (p. 217) 
Such dialectics, or trade-offs, are especially pronounced in ill-structured situations, with wise reasoning conceptualized as a process promoting balance between these conflicting interests (Achenbaum \& Orwoll, 1991; Grossmann, 2017).

Parallel ideas about the centrality of balancing as an outcome of managing ill-structured complexities of human life have also been discussed in the adult developmental literature on identity development. For instance, Erikson ( 1984) conceptualized wisdom as a form of personal maturation promoting mastery of uncertainties involved in the later-life crisis of integrity versus despair. Like other psychoanalytic theorists (e.g., Jung, 1965), Erikson suggested that such self-development is oriented towards balancing seemingly opposed desires and transcending the limitations of the egoistic self. Subsequent empirical scholars have expanded on Erikson's ideas when measuring developmental maturation (e.g., Ryff \& Heincke, 1983; Ryff \& Keyes, 1995) and wisdom-related personality characteristics (e.g., Helson \& Wink, 1987; Wink \& Helson, 1997).

It appears that despite different theoretical assumptions, various perspectives on wisdom share a great deal in common in their focus on balancing interests (for a review, see Grossmann, 2017). Psychometrically speaking, balancing of interests can be considered as a criterion to evaluate the incremental validity of existing measures of wisdom. ${ }^{1}$

\section{Measuring Wise Reasoning: Extant Views and Challenges}

Observer-based evaluations of narratives. To assess wisdom-related cognition, Baltes and colleagues (Baltes \& Kunzmann, 2004; Baltes \& Smith, 2008; Baltes \& Staudinger, 1993,

\footnotetext{
${ }^{1}$ Theoretically, it is also possible that balance is a central indicator of wisdom writ large. Thus, when testing whether the hypothesized wisdom-related characteristics reflect wisdom broadly defined, balance-related indicators represent the critical feature of the nomological network. Irrespective of one's theoretical position (balance as a criterion or as an outcome), a critical empirical step concerns evaluating the relations between a hypothesized wisdom-related characteristic and markers of balance. We aim to test these relations in the present work.
} 
2000; Kunzmann \& Baltes, 2003; Staudinger \& Glück, 2011; Staudinger et al., 1997; Staudinger, Smith, \& Baltes, 1994) have proposed to examine how people reflect on difficult social situations. In their paradigm, participants are instructed to provide "stream-of-thought" reflections on what should be done in response to hypothetical life situations (e.g., a dilemma between family and job). Subsequently, trained coders perform narrative analyses of participants' responses, scoring responses regarding application of certain aspects of wisdomrelated cognition described above. Similarly, in a paradigm developed by Grossmann and colleagues (Grossmann et al., 2012; Grossmann, Na, Varnum, et al., 2013; Kross \& Grossmann, 2012), participants verbally reflect on interpersonal or intergroup conflict scenarios, responding to a set of prompts (e.g., What do you think will happen next? Why will it happen in that way? What do you think should be done in the situation?). Again, trained coders rate participants' narratives across various aspects of wise reasoning, including intellectual humility, recognition of uncertainty and change, consideration of multiple ways a situation could unfold, appreciation of others' perspectives, consideration of/search for compromise, and acknowledgment of the importance of conflict resolution.

The strengths of observer-based evaluations center around their potential to focus on wisdom-related cognitions in situ—studying how people reason in the context of concrete situations (Grossmann \& Kross, 2014; Kross \& Grossmann, 2012; Kunzmann \& Thomas, 2014; Staudinger \& Baltes, 1996) — understanding of which is essential for gaining insights into the dynamic nature of wisdom (Staudinger \& Glück, 2011). At the same time, this approach to assessing wise reasoning has several drawbacks. Recording of stream-of-thought reflections is not viable in the context of acute social challenges, limiting the utility of observer-based evaluations for ecological assessment of wise reasoning across a range of situations. Further, it 
can be impractical due to the costs and high levels of researcher burden when evaluating respondents' narratives (Glück et al., 2013), with a substantial time investment into the training of raters to establish inter-rater reliability and to score the narratives. Indeed, observer-based evaluations of narratives are typically constrained to small-moderate sample sizes $(N<150)$. Finally, observer-based evaluations of written or transcribed narratives often involve grounding of the coding categories in the nuances of specific scenarios, resulting in distinct coding systems for different situations. For instance, distinct codebooks are available for analyses of intrapersonal reflections (Mickler \& Staudinger, 2008), reflections on prescriptive actions regarding interpersonal dilemmas (Baltes \& Staudinger, 2000), reflections on interpersonal and intergroup conflicts (Grossmann et al., 2010), and reflections on political election outcomes (Kross \& Grossmann, 2012). Though these codebooks overlap in their overarching content, the specific categories are somewhat idiosyncratic, which presents a challenge for direct comparability across content-analytic methods assessing wisdom. Thus, observer-based evaluations of narratives, while invaluable, have led to relatively underpowered studies, and preclude direct comparability and efficient use by the vast majority of researchers.

Global self-report assessments. Given the methodological difficulties in conducting observer-based evaluations of wisdom, some scholars have proposed to assess wisdom by using global self-report questionnaires (e.g., Ardelt, 2003; Glück et al., 2013; Levenson et al., 2005; Webster, 2003), similar to those used when assessing personality. In these global evaluations, participants respond to items capturing personal wisdom (Glück et al., 2013). Depending on the scale, participants indicate their overall ability to reflect, to see different perspectives, to tolerate ambiguity, to be concerned with amicable conflict resolution, to be accepting of contradictions 
and irony of life, or ability to (self-) transcend immediate concerns and recognize that the world is in flux (Ardelt, 2003; Glück et al., 2013; Levenson et al., 2005; Webster, 2003).

This global self-report method for assessing wisdom suffers from many drawbacks. First, relying on participants' global, de-contextualized self-evaluations, existing self-reports of wisdom do not reveal information about how people navigate challenges in their lives, thereby providing no insight into how wisdom may vary as a function of the situation. This is noteworthy, because contemporary standards in research on personality and individual differences suggest that the consideration of situation- (or state-) specific responses is essential for understanding the nature of personality constructs as a whole (e.g., Fleeson \& Jayawickreme, 2015; Fleeson \& Noftle, 2008b, 2012; Funder, 2009; McLean, Pasupathi, Greenhoot, \& Fivush, 2016; Mischel, 2004; Mischel, Shoda, \& Mendoza-Denton, 2002). For instance, Fleeson (2001) proposed a density-distribution account of personality—specifically, that traits should be conceived as frequency distributions of their corresponding states. Accumulating over time and across situations, a person's distribution of states indicates the typical frequency with which the individual is at each level of the state. Modern personality psychologists (Fleeson \& Noftle, 2008b, 2012; Mischel, 2004; Mischel \& Shoda, 1995; Mischel et al., 2002) recommend examining people in concrete situations, preferably several times, to draw inferences about the reliability of general tendencies (i.e., traits).

Second, global self-evaluations require participants to filter through and condense years of experience to derive an overall portrait of the self, resulting in bias toward casting the self in a positive light (Dunning, Heath, \& Suls, 2004; Kihlstrom, Eich, Sandbrand, \& Tobias, 2000; Wilson \& Bar-Anan, 2008). Indeed, when self-assessing highly desirable qualities such as wisdom (Assmann, 1994) in a de-contextualized fashion, participants may be more tempted to 
respond in a socially desirable fashion by exaggerating, faking, and lying, in part driven by involuntary self-deception (Paulhus \& Vazire, 2007; Vazire \& Carlson, 2010). Self-biased responding and memory distortions are of particular relevance when measuring wisdom, because the central pillars of wisdom concern intellectual humility and the absence of bias (Glück et al., 2013; Staudinger \& Glück, 2011). Ironically, it appears that the global self-report approach to wisdom is most likely to be contaminated by psychological biases (Taylor, Bates, \& Webster, 2011; Zacher, McKenna, \& Rooney, 2013), the absence of which the scales aim to explore (Glück et al., 2013; Staudinger \& Glück, 2011).

\section{Current Research: A State-Level Hybrid Method of Wise Reasoning}

In the present paper, we introduce a state-level method that integrates the in situ advantages of observer-based evaluations with the convenience of self-report assessments, enabling a high-powered exploration of state-level variability in wise reasoning and its relationship to balancing of interests and desires. To avoid potential biases associated with global self-reports, we build on recent advances in survey methodology, concerning the eventreconstruction of specific experiences (Kahneman et al., 2004; Schwarz et al., 2009).

Event-reconstruction protocol. The chief methodological challenge with self-report assessments concerns difficulty with gaining reliable self-insight when assessing global tendencies, which leads to memory bias and desirability-related distortions in responding (Kahneman et al., 2004; Schwarz et al., 2009). One way to facilitate accurate responding is to provide greater access to episodic memory by illuminating details with the help of recall cues concerning the "what," "where," "when," and "how" of the recalled experience (Robinson \& Clore, 2002; Wagenaar, 1986). For instance, describing how the event has unfolded (rather than why it happened) can facilitate concrete reliving and re-experiencing of the episode (Kross \& 
Ayduk, 2011; Trope \& Liberman, 2010). Indeed, autobiographic self-reports adopting the eventreconstruction method-instructions to reconstruct the details of a specific episode prior to answering self-report questions about their experience - can approximate responses observed via ambulatory monitoring (e.g., experience-sampling; Grube, Schroer, Hentzschel, \& Hertel, 2008; Kahneman et al., 2004; Schwarz et al., 2009; Stone \& Litcher-Kelly, 2006). Notably, the eventreconstruction method appears well-suited for assessing thought-based components of a specific life experience (White \& Dolan, 2009). On the basis of these insights, we propose a hybrid method for assessing wisdom-related thought about concrete situations, combining a self-report approach with the recently developed event-reconstruction technique to reinstate the social experiences (Schwarz et al., 2009).

State-level method to assess wise reasoning. The new hybrid method cues participants to recall an interpersonal conflict that they experienced. To maximize precision in recall, we guide participants to recall a recent episode (Schwarz \& Oyserman, 2001). Respondents are then guided to reconstruct features of the conflict experience by answering questions about the what, where, when, and how of the situation, including the thoughts and feelings they experienced. This reconstruction process aims to increase accuracy and reduce bias in the recall of the experience. Finally, respondents answer questions designed to assess wise reasoning, tapping into intellectual humility, recognition of a world in flux and change, appreciation of different perspectives, application of an outsider's vantage point, consideration of and search for compromise and conflict resolution.

\section{Research Overview}

We conducted a large-scale study exploring the utility of the state-focused method (vis-àvis global scales) for assessing wisdom-related cognition in an unbiased fashion. Study flow is 
described in Figure 1. In the first step, we examined the internal reliability of the state-level measure of wise reasoning. In the second step, we examined intra-individual stability (vs. variability) in wise reasoning across several situations encountered by the same person. We also examined its construct validity: we assessed convergence of hybrid method scores with scores obtained from the existing global wisdom scales, observer-based performance scores of wise reasoning, and reasoning about an intergroup conflict. Further, we examined the relative associations between the state-level method (vs. global wisdom scales) and markers of psychological bias. To expand the nomological network (Cronbach \& Meehl, 1955) of wise reasoning, we further assessed how wise reasoning is related to markers of adaptive emotion regulation, mindfulness, and prosocial orientation (e.g., agreeableness, communal relationship orientation).

In the third step, we tested the relation between wise reasoning scores and various indicators of balance, including balancing cooperative and self-protective intentions (Kelley \& Stahelski, 1970), balancing influence versus adjustment goals (Sternberg, 1998; also see Heckhausen, Wrosch, \& Schulz, 2010), balancing various social inferences about the world (Nisbett \& Ross, 1985; Gilovich \& Ross, 2015), and balancing various conflicts in one's life (Carlson, Kacmar, \& Williams, 2000). Finally, we tested the role of demographic and situationspecific contexts for wise reasoning.

\section{-- Insert Figure 1 about here --}

\section{General Procedure and Samples}

We recruited participants from a diverse demographic background and different conflict situations (i.e., workplace conflicts, conflicts with a friend) to increase the generalizability of the results. Table 1 includes demographic information for each sample included in the current study. 
Some participants (Samples A-F, and I) were recruited via Amazon Mechanical Turk (MTurk) platform. For MTurk samples $(N=3,195)$, we restricted recruitment to native English-speaking adults (age of majority $>17$ years in the US) to reduce the impact of potential confounds related to life experience, language comprehension, and cultural differences. Our research tested wise reasoning in different social contexts. To examine wise reasoning in the workplace, the first two samples comprised full-time employees from a wide range of occupations (e.g., business, administration, construction, food services). To examine wise reasoning in non-work contexts, we instructed subsequent samples to reflect on recent interpersonal conflicts with a friend. In one sample (Sample E), in addition to the state-level measure of wise reasoning, participants completed a version of the state-level measure adapted to assess wise reasoning about an intergroup conflict (the 2014 political revolution in Ukraine) and provided their thoughts about the same conflict via open-text responses. Participants from these six samples were compensated $\$ 0.50$ for their participation.

To replicate the psychometric structure of state-level wise reasoning beyond MTurk, we also sampled undergraduate students at the University of Waterloo, Canada (Samples G and $\mathrm{H} ; N$ =968). A sub-group of these students completed an alternate, global (i.e., decontextualized) version of the wise reasoning scale items, to compare its relative susceptibility to biased responding. Students received course credit for their participation. Finally, to generalize our findings to a broader population, we recruited a non-student community sample (Sample $\mathrm{J} ; N=$ 300) on Prolific Academic (ProA), a recently established UK-based crowdsourcing platform, developed at Oxford University specifically for accommodating researcher needs. ProA matches researchers with a community sample of individuals willing to complete studies for reimbursement. We selected ProA because it retains a more diverse pool of participants of non- 
student adults from Europe as well as North America, shown to be more naïve and conscientious compared to MTurk workers (Peer, Brandimarte, Samat, \& Acquisti, 2017). These participants were compensated $2.5 £$ for $30 \mathrm{~min}$ of their time.

-- Insert Table 1 about here --

\section{Study 1: Scale Development}

The purpose of Study 1 was to develop a Situated WIse reasoning Scale (SWIS). First, we designed a set of 46 items - each assessing one of the pre-existing theoretical aspects of wise reasoning - and conducted principal components analyses and principal axis factoring to trim the scale. Second, we tested the hypothesis that each aspect of wise reasoning would covary with the others, and would relate to a latent wise reasoning construct. Finally, we tested alternative factor structures of wise reasoning.

\section{Ethics Review Board Statement}

This study was approved by a University of Waterloo Research Ethics Committee (Title of study: Test of materials for future study; Protocol \#18966). Informed consent was obtained from all participants. This research was carried out following the recommendations of the Human Research Ethics Committee at the University of Waterloo, Canada, with written informed consent following the Declaration of Helsinki.

\section{Method}

\section{Wise Reasoning Assessment}

Event reconstruction. We asked participants to reconstruct a specific, recent life experience before responding to the scale items. Our instructions mirrored existing eventreconstruction methods that facilitate accurate recall (Kahneman et al., 2004; Schwarz et al., 2009). First, participants recalled a difficult situation — a single conflict situation or disagreement 
instead of a recurring problem — that happened between them and a workmate (Samples A and B) or with a friend (Samples C-G) in the past few months. We selected work conflicts for our initial samples because social conflicts are frequent in the workplace (Andersson \& Pearson, 1999; Estes \& Wang, 2008). Participants then reflected on what they thought and felt during that difficult situation. To increase the accuracy of recall, participants were guided by questions that helped them reconstruct the context of their experience (e.g., "Where were you at the time?"; Table S14).

Situated wise reasoning scale (SWIS). After the event reconstruction task (and after being reminded of anonymity and confidentiality, to minimize social desirability influence; Paulhus \& Vazire, 2007; Podsakoff, MacKenzie, \& Podsakoff, 2012), participants reported the extent to which they used different reasoning strategies during the event. In the item selection phase, these strategies represented a set of 46 items ( 9 reverse coded) concerning wise reasoning (Baltes \& Smith, 2008; Basseches, 1984; Grossmann et al., 2010, 2013; Grossmann \& Kross, 2014; Kross \& Grossmann, 2012; Kramer, 1990; Labouvie-Vief, 1982; Riegel, 1973; Staudinger \& Glück, 2011; Sternberg \& Jordan, 2005). To generate items, we built on prior work (Grossmann, 2017; also see Bangen, Meeks, \& Jeste, 2013) characterizing wise reasoning through a set of interrelated facets that provide a base for a single, second-order factor: i) intellectual humility/recognition of the limits of one's knowledge (e.g., "Looked for any extraordinary circumstances before forming my opinion"; 10 items), ii) consideration of change (e.g., "Looked for different solutions as the situation evolved"; 8 items), iii) consideration of multiple ways a situation may unfold (e.g., "Believed the situation could lead to a number of different outcomes"; 6 items), iv) recognition of others' perspectives (e.g., "Made an effort to take the other person's perspective"; 6 items), v) consideration of/search for compromise (e.g., 
"Tried my best to find a way to accommodate both of us"; 6 items), vi) recognition of importance of conflict resolution (e.g., "Tried to anticipate how the conflict might be resolved"; 5 items), and vii) application of an outsider's viewpoint (e.g., "Tried to see the conflict from the point of view of an uninvolved person"; 5 items). A complete list of original items is presented in Table S1 in the supplementary online materials. Participants answered the following question, "While this situation was unfolding, I did the following ..." by rating items on a 5-point scale (1 $=$ Not at all, $3=$ Somewhat, $5=$ Very much $)$.

Sample A completed the original set of 46 items. Sample B completed the reduced set of wise reasoning items and 7 new items that seemed plausible in a conflict situation yet were theoretically unrelated to wisdom-related cognition (see Table S10), to test whether their inclusion altered the responses to original items. Samples C-G, I, and J completed the final 21 items (see Appendix). Depending on the sample, participants also completed a battery of other measures, described fully in subsequent studies. To minimize responder burden, each participant completed only a subset of the measures, such that the total completion time would not extend over 30 minutes.

\section{Results}

\section{Item Reduction}

We aimed to select 2-4 items for each aspect of wise reasoning. Together, these aspects would capture the hypothesized single, second-order latent wise reasoning construct. To this end, we analyzed Sample A responses. Our plan for item-selection integrated theoretical and empirical insights. First, we designed a set of items capturing the most frequently mentioned cognitive aspects of wisdom (e.g., Bangen et al., 2013; Grossmann et al., 2010). To maximize the coverage and utility of the SWIS measure, our goal was to select the items that apply to most 
situations. In the subsequent empirical step, we selected items based on factor-analytic methods, to reveal the items that covaried best with each other and the first- and second-order wise reasoning constructs. This empirical step was imposed to avoid researcher bias (e.g., tendency to select subpar items, based on personal speculation). At the same time, we remained mindful of theory and therefore eliminated items if they adhered together empirically, for non-theoretical reasons (e.g., negatively worded items). Thus, through this combination of a priori theorizing and empirical procedures, we were able to retain the items that reflect the most common ecologically-sensitive expression of wise thought, though not every unique and possible way wise reasoning could be expressed.

The complete analytic protocol, including each step of the item reduction procedure, is presented in the online supplement (Tables S2-S6). First, we conducted a preliminary principal component analysis on all items, using eigenvalues $>1$ and Promax rotation, to determine the presence of any problematic items or conceptually unrelated components (e.g., results of psychometric artifacts rather than aspects of wise reasoning). This process revealed eight components, two of which identified reverse coded items, and not any particular aspect of wise reasoning. The presence of components that consisted solely of reverse-coded items suggested that participants responded similarly to these items due to their negative wording. Therefore, we removed reverse-coded items from further analyses.

Next, we conducted iterated principal axis factoring analyses, to determine the 2-4 items that best represented just one of seven aspects (Child, 2006; Floyd \& Widaman, 1995; Furr \& Bacharach, 2014) of wise reasoning. Our a priori prediction was that each aspect of wise reasoning would covary and load onto a single, second-order latent factor. Thus, we imposed a 7factor solution and utilized Promax rotation to allow the factors to correlate. At each iteration, 
we removed items that did not load strongly onto a single factor (i.e., coefficients < .4; e.g., Cohen, Wolf, Panter, \& Insko, 2011), or that cross-loaded substantially on more than one factor (<.2 difference between loadings on different factors). We repeated this process until only the highest-ranking 2-4 items in each of the seven wise reasoning factors remained (i.e., to allow approximately equal weighting per aspect/factor). The item-reduction process resulted in 27 items explaining $62.02 \%$ of the total variance (Table S6).

To further reduce the initial set of items to approximately equal item-weighting between aspects of wise reasoning (i.e., 2-4 items per aspect), we conducted structural equation modeling, using AMOS 22.0.0 for SPSS. We used standard criteria to assess the 7-factor model, including standardized root-mean-square residual $(\mathrm{RMSR})<.10$, root-mean-square error of approximation $($ RMSEA $)<.08$, comparative fit index $(\mathrm{CFI})>.95$, and probability of close fit (PCLOSE) $>.05$ (Hu \& Bentler, 1999; Meyers, Gamst, \& Guarino, 2006). According to these guidelines, results of the confirmatory factor analysis (CFA) suggested that the initial 7-factor model could be improved (i.e., PCLOSE <.01). We eliminated items that did not fall in the factor expected by the theoretical model of wise reasoning, or that exhibited the greatest frequency of standardized residual covariance $>.4$. This process resulted in items $4,6,8,14,24$, and 42 being removed from the model. We also covaried individual-item error terms with high modification indices (Kenny, 2016). The 21-item model indicated good fit, $\mathrm{RMSR}=.046, \mathrm{RMSEA}=.036, \mathrm{CFI}=$ .983 , and PCLOSE $=.997$. Item loadings for the final 21 items in Sample A are presented in Table 2.

-- Insert Table 2 about here -- 


\section{Replicating the Internal Structure of Wise Reasoning in Followup Samples}

In Sample B we included seven distractor items (Table S10), designed to be high in face validity but without theoretical relation to wisdom, and interspersed them with the reduced set of 21 wise reasoning items. We tested whether including such items would disrupt the original dimension loadings found in our initial model. Using principal component analysis on the responses from Sample B, we found that each of the distractor items either 1) clustered only with other distractor items, or 2) cross-loaded sufficiently onto multiple components to warrant discarding them from the analysis (Table S11). Importantly, in subsequent principal component analysis with distractor items removed, factor structure found in Sample A was maintained in Sample B (Table S12). Most importantly, results of a follow-up CFA indicated that responses from Sample B converged well with those from Sample A. Specifically, we tested the identical 7-factor model as revealed from item-reduction in Sample A, finding a good model fit, RMSR = $.045, \mathrm{RMSEA}=.037, \mathrm{CFI}=.978$, and PCLOSE $=.998$.

Next, we present a large-scale CFA on responses from Samples C-F, as they were independent of the responses used for item-reduction and selection and initial confirmatory tests (Samples A and B). First, we tested the 7-to-1 factor model (i.e., seven first-order factors, one second-order factor) and found a good fit to the data. Next, we tested whether alternative models also exhibited good or better fit (Figures S4-S9). To this end, we systematically combined factors that had most conceptual overlap (e.g., multiple outcomes and change, compromise and resolution), to form the 5-to-1 and 6-to-1 factor models; these models were tested because they are similar to the initial 7-to-1 factor model, but with increased parsimony. We also examined a single-factor model, in which all items would load onto a single-order factor of wise reasoning. We tested this model on the notion that all aspects of wise reasoning together represented a 
unitary reasoning style. Furthermore, we included a two-factor model of social and cognitive wisdom, consistent with some prior theorizing (e.g., Meeks \& Jeste, 2009; Takahashi \& Bordia, 2000). In the latter model, intellectual humility, change, multiple outcomes, and outsider's vantage point comprised the cognitive factor and recognition of others' perspectives and search for a compromise and conflict resolution comprised the social factor.

All models exhibited acceptable fit. Because the different models are not nested and include structural differences in parameters (e.g., different latent factors), we examined the Akaike Information Criterion (AIC) measure of model fit. This index allows for comparison between the relative fit of different models (Kline, 1998), where lower AIC values represent better fit. As seen in Table 3, according to AIC, the 5-factor model exhibited the best relative fit and was therefore accepted as our present model of wise reasoning in interpersonal conflicts. Figure 2 shows the final model, in which a single world in flux/change dimension subsumes the dimensions of change and multiple outcomes, and in which a single search for compromise/resolution dimension subsumes the dimensions of compromise and importance of resolution. Factor loadings are presented in Table 4.

-- Insert Figure 2 about here --

In the present study, we focus on wise reasoning as a single, second-order index. Our method to calculate scores ensures that each theoretical aspect of wise reasoning receives appropriate weighting based on data-reduction. Specifically, we compute wise reasoning scores by calculating the average of items from each of the five aspects of wise reasoning and conduct a principal component analysis on all five indexes, saving the resulting single component as a new 
index of wise reasoning. ${ }^{2}$ Additionally, in several studies we present exploratory tests on individual aspects of wise reasoning.

-- Insert Tables 3-4 about here --

\section{Inter-individual Variability}

We examined the distribution of wise reasoning across different samples. ${ }^{3} \mathrm{We}$ found that, in each case, wise reasoning scores exhibited no skewness or kurtosis (both $<|1|$ ). As seen in Figure 3, we observed a rather symmetric distribution of responses across all samples, indicating substantial inter-individual variability and no skewness across both workplace conflicts and conflicts with friends. Consistent with prior research (Grossmann, Gerlach, \& Denissen, 2016), people report greater wise reasoning in reflections on interpersonal situations involving a friend compared to work-related disagreements, $t(1549.30)^{4}=5.89, p<.001$.

-- Insert Figure 3 about here --

\section{Study 1 Summary}

Overall, the SWIS measure showed a high internal consistency of items and good model fit. Notably, we observed no apparent skewness in the distribution of the wise reasoning scores, meaning that at least with respect to work-related and recent interpersonal conflicts with friends, expression of wise reasoning appears to be normally distributed, which provides initial evidence that the measure avoids biased responding (e.g., no floor or ceiling effects due to social

\footnotetext{
${ }^{2}$ Results from all main analyses in this study were replicated when calculating wise reasoning scores by averaging items (Cronbach's $\alpha>.90$ in all samples). Separate PCA scores were computed for Sample A and Sample B individually since they were included in larger scales with other items used for scale creation.

${ }^{3}$ Since different PCA scores were computed in Samples A and B compared to the other samples, we examined average scores of means across items on each of the five aspects of wise reasoning to compare the distribution of wise reasoning across samples.

${ }^{4}$ All degrees of freedom in linear regression analyses are based on Welch-version of the t-tests, correcting for potential unequal variances.
} 
desirability biases). Additionally, we observed substantial cross-situational variability, with greater reports of wisdom in reflections on conflicts involving a friend vs. a workmate or a boss.

\section{Study 2: Intra-Individual Stability vs. Variability}

Building on the emerging scholarship in personality research on the importance of measuring intra-individual differences, Study 2 aimed to explore intra-individual variability in wise reasoning across multiple sampling points, to estimate trait-level stability and variability in the construct (e.g., Fleeson \& Jayawickreme, 2014). In this study, trait-level individual differences are conceptualized as an average across multiple sampling points. Of particular importance here, recent diary work has indicated that the intra-individual variability in wise reasoning is comparable in magnitude to the variability of established personality constructs (Grossmann et al., 2016). This work also suggested that some aspects of wise reasoning (i.e., "self-transcendence") may be more stable across situations than others (i.e., perspective taking). In light of these previous findings, we examined the intra-individual variability in each of the five aspects of wise reasoning utilizing the hybrid method.

We recruited participants $(n=293)$ from the prior studies to fill out a follow-up wise reasoning survey at least two years upon completion of the initial survey. We expected that wise reasoning scores would exhibit significant convergence across points in time. However, on the notion that wise reasoning is context-dependent (Grossmann, 2017), we expected the relations between wise reasoning at Time 1 and Time 2 would be small-to-medium in magnitude. 


\section{Method}

\section{Participants, Procedure, and Measures}

Participants were drawn from Samples A-F, instructed to complete the wise reasoning measure, and several additional indices (see Study 6). Sample characteristics are presented in Table S13.

\section{Results}

We explored the intra-individual stability of wise reasoning and each of its five aspects. We did so by estimating correlations, based on the variance explained between Wave 1 and Wave 2 data points. Similar to past research (Grossmann et al., 2016), we found moderate positive associations between Wave 1 and Wave $2, r_{\text {SWIS }}(n=290)=.48, r_{\text {Perspectives }}(n=290)=$ $.56, r_{\text {Multiple Outcomes }}(n=290)=.48, r_{\text {Limits }}(n=290)=.56, r_{\text {Compromise/Resolution }}(n=290)=.47$, rOutsider's Viewpoint $(n=290)=.66$. We also estimated intra-individual correlations for global wisdom scores, across Wave 1 and Wave 2. Here, we found positive associations between waves, $r_{\text {SAWS }}(n=37)=.53, r_{3 \mathrm{D}-\mathrm{WS}}(n=41)=.42$, and $r_{\text {ASTI }}(n=40)=.54$, comparable to those observed with SWIS. Notably, the latter estimates are based on underpowered sub-samples and should be interpreted with caution.

\section{Study 2 Summary}

Study 2 showed initial evidence for the intra-individual stability in wise reasoning. Conceptualizing trait-level individual differences as an average across multiple sampling points, we found positive associations between Wave 1 and Wave 2 wise reasoning and each of its five aspects. The results indicated a somewhat greater association between different measurement points in wise reasoning as compared to that observed in prior work (Grossmann et al., 2016), likely due to a larger number of items and greater internal reliability of the present instrument, 
compared to the one used in prior research. Given that Wave 1 and Wave 2 assessments were separated by at least two years, these findings present a conservative test of the variationconvergence of wise reasoning across different points in time.

\section{Study 3: Initial Validity Tests}

In Study 3, we report the initial validity tests, specifically aiming to establish preliminary convergent and discriminant validity of the SWIS. First, we established the convergent validity of the state-level measure by comparing state-level wise reasoning scores to scores obtained from global, decontextualized measures and observer-ratings of wise reasoning. We compared people's wise reasoning scores to scores on existing global measures of wisdom: Self-Assessed Wisdom Scale (Webster, 2003), Three-Dimensional Wisdom Scale (Ardelt, 2003), Adult SelfTranscendence Inventory (Levenson et al., 2005). Further, we explored participants' expression of wise reasoning when reflecting on their challenges and when reflecting on a societal conflict that was heightened at the time (March $\left.20^{\text {th }}-24^{\text {th }}, 2014\right)$ - the Crimean referendum in Ukraine. Additionally, we examined whether state-level wise reasoning about the societal conflict would correspond to participants' wise reasoning scores as rated by outside observers (Grossmann et al., 2010; Grossmann, Na, Varnum, et al., 2013). To examine this question, we asked participants to respond to several questions about the societal conflict in Crimea, and their responses were later assessed by trained coders for expression of wise reasoning and compared to the scale responses.

Next, we established discriminant validity by comparing the relations between SWIS scores and measures of bias, in juxtaposition with global wisdom measures. Specifically, we examined whether the SWIS measure would be less susceptible to biased responding as compared to global self-report measures of wisdom (Glück et al., 2013; Taylor, et al., 2011). To 
distinguish the new measure from extant measures of wisdom, we assessed Sample D participants for biased responding (i.e., impression management and self-deception; Paulhus, 1984, 1988), and compared these responses to scores on wise reasoning and global wisdom measures. Further, because at the conceptual level wisdom promotes unbiased thinking and judgment, we included measures of socio-cognitive bias, namely bias blind spot (i.e., the tendency to attribute greater psychological bias to others than to the self; Pronin, 2008) and dispositional and situational bias in attributions of others' behavior (Grossmann \& Varnum, 2011; Kitayama, Ishii, Imada, Takemura, \& Ramaswamy, 2006). Finally, to control for the possibility that the bias-related differences between global and state-level measures are due to different item content, we created a global, decontextualized version of the SWIS, adapting items from the state-level measure to refer to general tendencies, testing its relation with biased responding.

We expected small-to-moderate relations between scores from SWIS and global wisdom scales. Further, to the extent that the hybrid method captures variance of wisdom-related cognition more accurately, we expected that its scores would be less prone to (i.e., less related to) psychological bias compared to scores obtained from the global wisdom scales.

\section{Method}

\section{Samples}

Participants in Study 3 were drawn from Samples D, E, and H. In Samples D and E, participants completed the state-level wise reasoning measure and a subset of the validation measures (see Table 5). Sample H participants completed the alternative, global (i.e., decontextualized) measure of wise reasoning. Reliability indices and descriptive information for each measure are presented in Table 5. 
-- Insert Table 5 about here --

\section{Measures}

Convergent validity. For tests of convergent validity, we included 3 global measures of wisdom. We also included an adapted SWIS measure that focused on intergroup conflicts, and individuals' scores of observer-rated wise reasoning performance in terms of text responses to the same intergroup conflict.

Self-Assessed Wisdom Scale (SAWS). The SAWS (Webster, 2003) is a 40-item measure assessing multiple wisdom dimensions: Experience (e.g., "I have overcome many painful events in my life"), Emotional Regulation (e.g., "I am “tuned” into my own emotions"), Reminiscence/Reflection (e.g., Recalling my earlier days helps me gain insight into important life matters"), Humor (e.g., "Now I find that I can really appreciate life's little ironies"), and Openness (e.g., "I like being around persons whose views are strongly different from mine”). Statements are assessed on 6-point scales $(1=$ Strongly disagree to $6=$ Strongly agree $)$. Scores are determined by summing all items to form a total wisdom score.

Three-Dimensional Wisdom Scale (3D-WS). The 3D-WS (Ardelt, 2003) is a 39-item measure that assesses wisdom as a composite of cognitive (e.g., "I always try to look at all sides of a problem"), reflective (e.g., "When I look back on what's happened to me, I feel cheated"), and affective dimensions (e.g., "I either get very angry or depressed if things go wrong”). Statements are assessed on 5-point scales $(1=$ Strongly agree to $5=$ Strongly disagree or $1=$ Definitely true of myself to $5=$ Not true of myself). Scores are determined by computing the mean of each of the three dimensions and taking the mean of these three-dimensional scores.

Adult Self-Transcendence Inventory (ASTI). The ASTI (Levenson et al., 2005) assesses wisdom as the development of self-transcendence, using ten items. This scale asks participants to 
rate themselves as they are now, compared to five years ago (e.g., "I am more likely to engage in quiet contemplation"). Statements are assessed on 4-point scales $(1=$ Disagree strongly to $4=$ Agree strongly). Scores are determined by summing items.

Observer-rated wise reasoning performance. To evaluate the accuracy of self-reports in the new state-level method, Sample E $(N=240)$ participants provided written reflections on a recent societal conflict, which were rated by independent observers (as in Grossmann et al., 2010). Participants were surveyed following the Crimea referendum in Eastern Europe (March $\left.20^{\text {th }}-24^{\text {th }}, 2014\right)$. We compiled a summary of the ongoing socio-political conflict in the Ukraine (see Table S15 for exact wording). After reading the summary, participants were asked to provide their thoughts about the conflict, guided by three questions in the following order: "How do you think the situation in Ukraine might unfold?", "Why do you think the issue in Ukraine might unfold in the way you just wrote?", and "What do you think should be done in the situation in Ukraine?" (Grossmann et al., 2010; Grossmann, Na, Varnum, et al., 2013).

Following established procedures, two trained, hypothesis-blind raters content-analyzed participants' narrative reflections on 5 aspects of wisdom-related thought: recognition of the limits of one's own knowledge, recognition of uncertainty and change, recognition of others' perspectives, consideration of/search for compromise, and importance of conflict resolution (Grossmann et al., 2010; Grossmann \& Kross, 2014; Grossmann, Na, Varnum, et al., 2013; Kross \& Grossmann, 2012). In line with how observer ratings were conducted in prior scholarship (Grossmann et al., 2010), compromise and resolution were coded separately. Recognition of multiple ways a situation may unfold and recognition of change, previously coded as two separate components (Grossmann et al., 2010; 2012; 2013), were collapsed into one dimension of recognition of uncertainty and change, as done in subsequent research 
(Grossmann et al., 2016; Kross \& Grossmann, 2012). Further, given that participants were not involved in the conflict (i.e., default $3^{\text {rd }}$ person perspective), we did not code responses for the application of an outsider's viewpoint. Raters used a scale from 0 (Not at all) to 2 (A great deal). Inter-rater reliabilities for each aspect were in the medium-high range (Table 5). Following prior research (Grossmann et al., 2010), the aspects of wisdom-related thought were subjected to a principal component analysis, which yielded a single component solution, with the resulting score used as a metric of observer-rated scores of wise reasoning.

Wise reasoning about an intergroup conflict. Upon reading the Ukraine conflict summary and writing down their thoughts about the conflict, participants completed the set of 21 wise reasoning items. Instructions asked participants to indicate the extent to which they engaged in wise reasoning as they were thinking and writing down their open-text responses about the intergroup conflict, with all other aspects being identical to the measure for interpersonal conflicts (Table S16). Similar to the internal reliability of the interpersonal wise reasoning instrument, reliability of the adapted measure of wise reasoning about intergroup conflicts was very high.

Global wise reasoning scale. We adapted two items from each of the initial seven dimensions of the wise reasoning measure to refer to global self-ratings (e.g., "I keep an eye out for ways things might change over time"). Statements were assessed on 6-point scales ( $1=$ Strongly disagree to $6=$ Strongly agree). Principal component analysis, using Promax rotation and eigenvalues $>1$, on all 14 items indicated that one item (item 7), initially designed to be a reverse-coded item, did not covary well with the other items; further, scale reliability analysis indicated that for this item the corrected item-total $r=.17)$. Therefore, we discarded this item, which resulted in a highly reliable 13-item scale (Table S17). 
Discriminant validity. To distinguish the SWIS at the psychometric level, we tested its relation to measures of biased responding, in juxtaposition with extant self-report measures of wisdom. To distinguish wise reasoning from bias at the conceptual level, we tested its relations with social-cognitive biases — bias blind spot and attribution bias_again in juxtaposition with global wisdom scores.

Biased responding. We used the 20-item Self-Deception and 20-item Impression Management subscales of the BIDR (Paulhus, 1984, 1988). Self-Deception assesses overconfidence in oneself (e.g., "I never regret my decisions"), and Impression Management assesses the tendency to over-report desirable and under-report undesirable behavior (e.g., "I never cover up my mistakes"). Statements were assessed on 7-point scales ( 1 = Strongly disagree to $7=$ Strongly agree). A score of 1 is assigned for each item for which the participant scores an extreme score (i.e., 6 or 7), and a score of 0 for each item that is scored otherwise. Following Paulhus $(1984 ; 1988)$, item scores for each subscale were summed, resulting in a total range from 0 (low desirable responding) to 20 (high desirable responding). Sample D participants completed the full 20-item self-deceptive positivity and impression management items. Sample $\mathrm{H}$ participants completed a brief version of this measure, including five impression management items and five self-deception items. To form the measures used in Sample H, we selected items based on scale reliability tests for each subscale on Sample D responses (i.e., we took the five highest loading items from each dimension).

Bias blind spot. Using a paradigm developed by Pronin et al. (2002), participants read a description of the "self-serving bias" and were asked about their own susceptibility to this bias (i.e., "To what extent do you believe that you show this effect or tendency?") and about the susceptibility of the average American to this bias (i.e., "To what extent do you believe the 
average American shows this effect or tendency?"), on 9-point scales $(1=$ Not at all to $9=$ Strongly). Presentation order was counterbalanced. Scores were computed by calculating a difference score between participants' ratings of their susceptibility vs. others' susceptibility to the self-serving bias. Higher scores represent greater bias blind spot.

Biased (vs. balanced) attributions. Participants read four vignettes which depict an individual who performed either a desirable or an undesirable action under some extenuating context (Table S18; Grossmann \& Varnum, 2011; Kitayama, Ishii, Imada, Takemura, \& Ramaswamy, 2006). After reading each vignette, participants answered two questions indicating 1) the extent to which features of the individual, such as his/her character, attitude, or temperament, influenced the individuals' behavior (dispositional attribution), and 2) the extent to which features of the environment that surround the individual, such as atmosphere, social norms, or other contextual factors, influenced the individuals' behavior (situational attribution; 1 = Strongly disagree, 6 = Strongly agree). Vignette and question presentation order were counterbalanced. For each vignette, a "biased attribution" score was assigned a score of 1 if participants reported only dispositional or situational factors as influential to the individuals' behavior; otherwise, we assigned a score of 0 . We calculated a composite index of biased attribution by averaging the scores from the four vignettes.

\section{Results}

\section{Convergent Validity}

Global wisdom measures. Findings from Study 2 are presented in Table 6. We first examined the relationships between scores on the wise reasoning (interpersonal conflicts) measure and the three global measures of wisdom. We observed a small-to-medium positive 
association between SWIS scores and indices of global self-assessed wisdom, $.19 \leq r \mathrm{~s} \leq .39$, suggesting that the SWIS is related to but distinct from existing global measures of wisdom.

Wise reasoning performance. We also found that self-reported wise reasoning about the intergroup conflict was positively associated with observer-rated wise reasoning performance about the intergroup conflict, $B=.19, t(200)=2.67, p=.008$. This association is comparable in magnitude to the degree of convergence of self- and observer-ratings on other established individual difference constructs (Meyer et al., 2001). Moreover, participants reporting higher state-level wisdom in interpersonal conflicts were also more likely to report higher state-level wisdom in the domain of intergroup conflict, $r=.44, p<.001$. The convergence across methods of assessments and domains is noteworthy and suggests that people's wise reasoning strategies may generalize across domains.

\section{Discriminant Validity}

Biased responding. Next, we tested whether the state-level and global measures of wisdom predict biased responding. Replicating past findings (Glück et al., 2013; Taylor et al., 2011), global wisdom measures were associated with biased responding, self-deception: $.17 \leq r \mathrm{~s}$ $\leq .36, p \mathrm{~s}<.001$, impression management: $.22 \leq r \mathrm{~s} \leq .40$. In contrast, SWIS scores were negligibly related to biased responding, $-.05 \leq r \mathrm{~s} \leq .07$, establishing evidence that the state-level measure of wise reasoning is largely independent of biased responding. Similar to established global measures of wisdom, the global measure of wise reasoning (comprised of decontextualized items similar to those in the state-level measure) was subject to self-deception, $r=$ $.17, p<.001$, and impression management, $r=.30, p<.001$. The latter finding suggests that it is not the idiosyncratic nature of the items, but rather the focus on concrete situations and use of event reconstruction that contributes to less bias in the SWIS. 
Social biases. Finally, we compared the relationships between each of the SWIS and three global measures of wisdom with social biases. As Table 6 indicates, global scores of wisdom were associated with a greater bias blind spot, $.18 \leq r \mathrm{~s} \leq .25$, whereas state-level scores of wise reasoning were not, $r<.01$. Furthermore, participants scoring higher on the SWIS were less likely to make biased (vs. balanced) attributions, $r=-.11, p=.003$, as were participants scoring higher on one global wisdom scale, Self-Assessed Wisdom Scale (SAWS): $r=-.13, p=$ .04 , with other global scales showing no significant relationship to attributional judgments, $r$ s $<$ $|.05|$.

To investigate further, we compared the relations between the SWIS versus the SAWS to biased attributions. The size of the association between state-level wise reasoning and biased attribution was comparable when controlling for SAWS, $r=-.12, p=.08$ (note that the relation between wise reasoning and biased attributions controlling [vs. not controlling] for SAWS scores size remains the same but the $p$-value increases due to smaller sub-sample containing both SAWS and wise reasoning data). In contrast, the reverse was not true- the relationship between SAWS and biased attribution was no longer significant when controlling for SWIS, $r=-.08, p=$ .24. Also, state-level wise reasoning was associated with less biased attributions, even when controlling for individual differences included in Sample D, all of which speaks to the incremental validity of the SWIS over existing global self-report wisdom measures. ${ }^{5}$ Supplementary analyses of the relationships between each aspect of state-level wise reasoning

\footnotetext{
${ }^{5}$ We also tested the relationship between state-focused wise reasoning scores and individual attribution scores (situational vs. dispositional) for each vignette. Wise reasoning scores interacted with attribution score on vignette 1 (see Appendix $\mathrm{E}$ for vignette items), $B=0.23$, $t(1409)=3.16, p=.002$, and on vignette $3, B=0.14, t(1410)=1.93, p=.05$, such that people with higher wise reasoning scores tended to endorse situational attributions more than people with lower wise reasoning scores $\left(r_{\text {vignettel }}=.14, p_{\text {vignette } 1}<.001, r_{\text {vignette } 3}=.10, p_{\text {vignette } 3}<.006\right)$. This effect did not reach significance for vignette 2 or vignette 4 .
} 
and the outcomes from Study 3 are also presented in Table 6, and show that the relationship between wise reasoning and the outcomes was not exclusive to one particular aspect but is rather present across each aspect of wise reasoning.

-- Insert Table 6 about here --

\section{Study 3 Summary}

Altogether, Study 3 showed that SWIS scores showed good convergent validity, with consistent relations with extant global wisdom scores and observer-rated wisdom scores, as well as wise reasoning about intergroup conflicts. Further, Study 2 also showed that the SWIS showed excellent discriminant validity. In contrast to extant global wisdom measures, the new state-level measure was independent of biased responding and social-cognitive biases.

\section{Study 4: Extending the Nomological Network of State-Level Wise Reasoning}

According to psychological theorizing on wisdom (Baltes \& Smith, 2008; Erikson, 1984;

Grossmann et al., 2013; Kekes, 1995; Ryff \& Heincke, 1983; Ryff \& Keyes, 1995; Staudinger \& Glück, 2011; Sternberg, 1998; Tiberius, 2008; Weststrate \& Glück, in press), the wise individual ought to be balanced in thinking, judgment, and action, skilled at emotion regulation and intelligence, and oriented to collective well-being (vs. being predominantly self-serving).

Does wise reasoning relate to such tendencies? The purpose of Study 4 was to address this question by extending the nomological network (Cronbach \& Meehl, 1955) of the SWIS. We hypothesized that state-level wise reasoning would be positively associated with such constructs (Baltes \& Staudinger, 2000; Kunzmann \& Baltes, 2003; Tiberius, 2008), without fully overlapping with them (Grossmann, Na, Varnum, et al., 2013; Staudinger \& Glück, 2011; Staudinger et al., 1997). Mindfulness, openness, emotional intelligence, and attributional complexity have been shown to lead to positive social-cognitive outcomes (Fletcher, Danilovics, 
Fernandez, Peterson, \& Reeder, 1986; Goldstein \& Gigerenzer, 2002; Kabat-Zinn, 2000; Law, Wong, \& Song, 2004; Wong \& Law, 2002; see Table 6) that are also associated with wisdom (Baltes \& Smith, 2008; Dambrun \& Ricard, 2011; Garland, Farb, Goldin, \& Fredrickson, 2015; Grossmann, Na, Varnum, et al., 2013; Staudinger \& Glück, 2011; Sternberg, 1998). Therefore, we selected these measures as part of our nomological network assessment. Most psychological perspectives suggest that wisdom involves recognition and management of uncertainties in life (Baltes \& Smith, 2008b; Basseches, 1984; Grossmann et al., 2010; Staudinger \& Glück, 2011; for a review, see Grossmann, 2017), hence also we expected our wise reasoning scores to be associated with changeable (or incremental) beliefs about conflicts and social life in general. Measures such as emotion suppression, neuroticism, and rumination were selected because of the large body of research indicating that they lead to negative outcomes (Gross \& John, 2003; Treynor, Gonzalez, \& Nolen-Hoeksema, 2003). Therefore, we expected negative or null relationships between these constructs and wise reasoning. Finally, based on the notion that wisdom-related qualities are associated with an orientation to others' concerns, we included measures of social orientation, such as communal relationship orientation, dispositional perspective-taking, agreeableness, and attending to others' emotions, expecting that wise reasoning would relate to a greater orientation toward collective well-being. We had no a priori expectation for the relationship between wise reasoning, extraversion and conscientiousness, but assessed these along with the other Big Five constructs. 


\section{Method}

\section{Participants}

Participants in Samples A, B, D, and G completed the SWIS and a subset of individual difference measures.

\section{Measures}

Table 5 indicates general information regarding each measure (sample inclusion and number of items) and descriptive statistics.

Big five personality traits. We used John, Naumann, and Soto's (2008) Big Five Inventory to assess personality. Participants indicated the extent to which they agreed with 44 statements about themselves, assessing openness to experience (e.g., "Is curious about many different things"), Conscientiousness (e.g., "Does a thorough job”), Extraversion (e.g., "Has an assertive personality"), Agreeableness (e.g., "Is helpful and unselfish with others"), and Neuroticism (e.g., “Can be moody”) on 5-point scales (1 = Disagree strongly, $5=$ Agree strongly). Scores were calculated by averaging the items.

Intellect. We used Mussel's (2013) 24-item scale assessing two motivational dimensions of intellect: seek and conquer. The seek dimension includes 12 items referring to openness and positivity toward situations that are intellectually challenging (e.g., "I would like to learn new ways of doing things"). The conquer dimension includes 12 items assessing how one is motivated to resolve situational incongruities and master intellectual challenges, once they arise (e.g., "I am able to think about things in a lengthy, focused way"). All statements were assessed on 7-point scales $(1=$ Strongly disagree, $7=$ Strongly agree $)$. Scores were calculated by averaging respective items. 
Attributional complexity. Fletcher and colleagues' (1986) measure of attributional complexity assesses the degree to which individuals are motivated to uncover more or less indepth information about social events. Participants were asked to rate the extent to which they agreed with 28 statements (e.g., "I think very little about the different ways that people influence one another") on 7-point scales ( 1 = Disagree strongly, $7=$ Agree strongly $)$. Scores were calculated by averaging the items.

Perspective taking. We used the perspective taking dimension of Davis' (1983) Empathy Questionnaire. Participants rated the degree to which nine statements describe them (e.g., "I try to look at everybody's side of an agreement before I make a decision") on a 5-point Likert-type scale (1 = Does not describe me well, 5 = Describes me very well).

Emotional intelligence. We used Wong and Law's (2002) 16-item Emotional Intelligence Scale measuring 4 dimensions of emotional intelligence: self-emotions appraisal (e.g., "I really understand what I feel”), others-emotions appraisal (e.g., "I have good understanding of the emotions of people around me"), use of emotion (e.g., "I would always encourage myself to try my best"), and regulation of emotion (e.g., "I can always calm down quickly when I am very angry”). All statements were assessed on 7-point scales ( 1 = Totally disagree to $7=$ Totally agree . Scores were calculated by averaging the items for each dimension.

Mindfulness. We used Baer and colleagues' (2006) 39-item Five Factor Mindfulness Questionnaire, which measures non-reactivity to inner experience (e.g., "In difficult situations, I can pause without immediately reacting"), observing/attending (e.g., "I pay attention to how my emotions affect my thoughts and behavior"), acting with awareness (e.g., "I find myself doing things without paying attention"), describing/labeling with words (e.g., "I'm good at finding the 
words to describe my feelings"), and nonjudging of experience (e.g., "I criticize myself for having irrational or inappropriate emotions"). Participants responded to statements on 5-point scales $(1=$ Never or very rarely true, $5=$ Very often or always true $)$. Scores were calculated by averaging respective items.

Communal relationship orientation. Clark, Ouellette, Powell, and Milberg's (1987) scale was used. Participants responded to how characteristic of them each of 10 statements is (e.g., "I'm not especially sensitive to other people's feelings"), on a 5-point scale (1 = Extremely characteristic of me, $5=$ Extremely uncharacteristic of me). Communal relationship orientation scores were calculated by recoding (such that higher values mean greater communal orientation) and averaging the items.

Emotion regulation. Gross and John's (Gross \& John, 2003) 10-item Emotion Regulation Questionnaire assesses two dimensions of emotional regulation. The first dimension, reappraisal, includes 6 statements assessing the extent to which individuals control their emotions by changing the way they think about situations (e.g., "When I want to feel more positive emotion, I change the way I'm thinking about the situation”). The second dimension, suppression, includes 4 statements assessing how individuals withhold expressing their emotions as a way of dealing with them (e.g., "I control my emotions by not expressing them"). All statements were assessed on 7-point scales $(1=$ Strongly disagree, $7=$ Strongly agree $)$. Scores were calculated by averaging the items.

Rumination. We used Nolen-Hoeksema and Morrow's (1991) 10-item Ruminative Responses Scale that assesses two dimensions of rumination: reflection (e.g., "Go someplace alone to think about your feelings") and brooding (e.g., "Think: What am I doing to deserve 
this?"). Each dimension was assessed with 5 items on 4-point scales $(1=$ Almost never to $4=$ Almost always). Scores were calculated by averaging respective items.

Growth mindset about social relations. We measured peoples' growth (i.e., incremental) versus fixed (i.e., entity) mindset of social relations. We asked participants the extent to which they agree with 3 statements regarding a growth mindset of interpersonal relations (e.g., "People can always change their own interpersonal ability"; Hui, Bond, \& Molden, 2012). We adapted extant items to create the second measure that used 3 statements to measure a growth mindset of social conflict (e.g., "The degree of conflict between people can change over time"). Participants replied to these statements on 7 -point scales $(1=$ Strongly disagree, $7=$ Strongly agree $)$. Scores were calculated by averaging the items.

\section{Results}

Results are presented in Table 7. State-level wise reasoning was significantly associated with open-minded beliefs and cognitive styles, including growth mindset about social relations, intellect, attributional complexity, and openness. SWIS scores were further associated with greater social orientation, as measured by perspective-taking, agreeableness, extraversion, and communal relationship orientation. Finally, SWIS scores were related to individual differences related to aspects of emotion regulation, three sub-scales of mindfulness (non-reactivity, observing and attending, describing with words), and self-reported emotional intelligence (selfand others-emotions appraisal, use of emotions, regulation of emotions). Although maladaptive brooding was positively correlated to SWIS scores, brooding normally shares variance with adaptive reflection (Treynor et al., 2003). When controlling for reflection, the relationship of SWIS to brooding became negligible, $r=.06, p=.10$. In contrast, SWIS still showed a smallmoderate positive association to reflection scores when controlling for brooding, $r=.23, p<$ 
.001 . These results suggest that wise reasoning is more closely related to adaptive reflection rather than maladaptive brooding. Associations with other individual differences did not reach statistical significance. In each sample, all individual differences together accounted for less than $25 \%$ of the variance in wise reasoning, indicating that wise reasoning is not fully accounted for by existing individual difference measures. ${ }^{6}$ Some prior research has investigated perspectivetaking, and its positive relations to important psychological and social outcomes (e.g., prosociality; Underwood \& Moore, 1982). Therefore, we additionally explored the relations between each aspect of wise reasoning and individual differences, finding similar results across each aspect (Table 7). Finally, we conducted tests of the incremental validity of wise reasoning, controlling for the variance explained by perspective taking aspect of wise reasoning. Here, we found that the pattern of results was similar, albeit with a slightly smaller magnitude of association in a few cases (see SOM for full results).

-- Insert Table 7 about here --

\section{Study 4 Summary}

Taken together, Study 4 findings confirm theory arguing that wisdom is related to adaptive psycho-social functioning (cf. Wink \& Staudinger, 2015), dovetailing with burgeoning bodies of research on interpersonal relations (Van Lange et al., 2013), and well-being (Diener, Kanazawa, Suh, \& Oishi, 2015; Diener \& Tay, 2014; Fredrickson, 2006; Gross, 1998). Higher

\footnotetext{
${ }^{6}$ We examined the relationships between wise reasoning and individual differences, controlling for global trait-level wisdom measures (Sample D responses: emotion regulation and emotional intelligence). Each participant completed only one global wisdom measure, so we controlled for each separately. Controlling for the global wisdom scores, we found that wise reasoning was still positively related to adaptive reflection, $.15<r s<.31$. Controlling for SAWS, wise reasoning was not significantly related to emotional intelligence. Controlling for 3D-WS, wise reasoning was related to emotional intelligence, specifically the "use of emotion" dimension, $r=.15$. Controlling for ASTI, wise reasoning was related to emotional intelligence, specifically the "others" emotions" dimension, $r=21$.
} 
SWIS scores were related to balanced thinking and attention, adaptive emotional functioning, and a more social orientation.

\section{Study 5: Cooperative Balancing of Self- vs. Other-focused Intentions}

In Study 5, we further probed the relationship between wise reasoning and social orientation (Staudinger \& Glück, 2011; Sternberg, 1998), extending it beyond global individual differences in cooperation to concrete cooperative (vs. self-focused) behaviors and cooperative tendencies in social dilemma tasks that capture managing conflicting (self- vs. other-focused) interests. By examining these outcomes, we started addressing one of the key criteria postulated by various wisdom theorists - the notion of balance between different interpersonal interests (Grossmann, 2017; Staudinger \& Glück, 2011; Sternberg, 1998). The idea of balancing selfprotective and cooperative intentions is central to interpersonal conflicts and can be simulated in the lab via social dilemma games (e.g., prisoner's and commons dilemmas). In social dilemma games, losses are incurred upon cooperative players (those focused on mutual gains), when paired with competitive players (those focused on self-gains), who gain from defecting on cooperators. A choice to defect here reflects a myopic concern about maximizing benefits for self, which neglects benefits for the partner player(s). As such, even those with intuitively cooperative goals may cede their goals and orient themselves towards protecting their selfinterests (Kelley \& Stahelski, 1970). In contrast, take the benefits for the partner into consideration can help to sustain a balance between self- and other-interest, yielding mutually beneficial outcomes (also called joint gain) (Axelrod \& Hamilton, 1981). This insight is consistent with theorizing in wisdom scholarship, which suggests that the wise participant is better able to balance such dichotomies (e.g., self versus others; Staudinger \& Glück, 2011). 
To the extent that wise reasoning affords a bigger-picture, more integrative perspective, then we expected that wise reasoning would relate to increased prosociality (Grossmann, Brienza, \& Bobocel, 2017; Sternberg, 1998), both in classic economic dilemmas and in individuals' behavior in their own conflict experiences.

\section{Method}

\section{Participants and Procedure}

Participants from Sample C completed two of the most common social dilemma games used to study cooperative vs. self-protective responding (Axelrod, 2006; Rand \& Nowak, 2013; Van Lange, Joireman, Parks, \& Van Dijk, 2013): the prisoner's dilemma and the commons dilemma. Participants from Samples A-F reported the behaviors they engaged in within their conflicts.

Social dilemma tasks. Sample C participants first responded to the SWIS and a filler task, to minimize the potential influence of the wise reasoning measure on the responses in the social dilemma tasks. Next, a set of participants $(n=379)$ was randomly assigned to complete one of the social dilemmas-prisoner's dilemma (PD) or commons dilemma (CD). Another set of participants $(n=109)$ completed both the PD and CD. The tasks (PD, CD, both) were counterbalanced in presentation order for participants who completed both tasks. Given that participants did not receive a performance-contingent reward for their participation in these tasks, we characterize responses as generalized cooperative intentions (i.e., cooperative intentions that are not related specifically to kinship, reciprocity, reputation, or threat of punishment; for reviews, see Fehr \& Fischbacher, 2003; Jordan, Peysakhovich, \& Rand, 2014). Prosocial and self-protective behaviors. Next, we tested the relationship between wise reasoning and people's behavior within their social conflicts. Participants reported behaviors they 
enacted in response to the interpersonal conflict they reflected on in response to the wise reasoning measure. Some of these conflict-behaviors could help to resolve the situation to the benefit of both people in the conflict (e.g., trying to solve the problem together, seeking impartial mediator), whereas others could protect oneself at the expense of the other person (e.g., build alliances against the other person; Rahim \& Magner, 1995). Participants in Samples B-D and F completed the wise reasoning measure and a set of items capturing conflict-related behaviors. To ensure that the relationship between wise reasoning and reported conflict-related behaviors was not a result of having completed the wise reasoning measure before the conflict-related behavior items (e.g., artifacts, demand effects), presentation order was reversed in Sample F.

\section{Measures}

Social dilemma tasks. We implemented two social dilemma tasks: the fisherman's dilemma, which is a variant of the prisoner's dilemma that is less known among MTurk participants, and the commons dilemma. We sought to avoid competitive framing effects on game outcomes (Kay \& Ross, 2003) and to make the tasks more ecologically relevant by altering the tasks from their typical presentation (see below).

Prisoner's dilemma. To avoid potential negative effects of competitive framing and to make the task more ecologically relevant, we framed the prisoner's dilemma game as a fisherman's dilemma, maintaining the reward structure of the prisoner's dilemma (Rapoport \& Guyer, 1967). In the prisoner's dilemma, dyadic gains/interests are always maximized when both players cooperate. However, either player can capitalize on their partner's cooperation by defecting, which increases self-gains and partner's losses. This threat can evoke self-protective behavior - pre-emptively defecting in case the other player takes advantage of one's cooperation. Players were asked to envision themselves harvesting fish in collective waters with other players, 
and decide whether to capitalize on resources (i.e., to trawl/defect; coded $=0$ ), or to leave the collective resources intact for the group (i.e., to take a sustainable harvest/cooperate; coded =1).

Commons dilemma. In the commons dilemma (van Dijk \& De Cremer, 2006; Van Lange et al., 2013), group gains/interests are always maximized by leaving resources intact. However, any player can take resources for the self, leaving other group members without resources. This threat can evoke self-protective behavior-pre-emptively taking resources in case another player leaves nothing for the group. This task was altered to make it distinct from a typical group task (e.g., a public goods game). Participants were told that there are 400 chips in a collective pot and that they and other players will take turns choosing whether to take chips for themselves or leave chips for the collective. The chips that were left in the pot after each player had taken their turn would be doubled and split among the group members, thus furthering collective gains. Participants, who were always the first player, were requested to select a number of chips they would allocate to themselves (0-400).

Prosocial and anti-social behaviors. Based on research on conflict resolution (De Dreu, Evers, Beersma, Kluwer, \& Nauta, 2001; Deutsch, Coleman, \& Marcus, 2011; Rahim \& Magner, 1995) we created items assessing the conflict-related behaviors participants reported within their conflicts. Participants indicated whether they engaged (Yes/No) in one of the seven strategies concerning their thinking, feeling, and behaving during the conflict, including four prosocial strategies: I tried to find another person to hear both sides of the story; I tried to find somebody to give me impartial advice; I tried to communicate with the other person to try to solve the problem together; I forgave the other person, and three anti-social strategies: Tried to just disengage from the other person and/or the situation; Retaliated against the other person; Tried to find an ally against the other person, indicating whether they engaged the behavior. 


\section{Results}

First, we examined the relation between SWIS and relatively more cooperative (vs. selfprotective) intentions in social dilemma games. Results of a multi-level regression model with state-level wise reasoning as a predictor of standardized task scores nested within participants indicated that higher level of wise reasoning was positively associated with cooperative intentions across social dilemma tasks, $B=.13, S E=.05, t(394.60)=2.66, p=.008$. Participants reporting greater wisdom in reflections on personal conflicts were more likely to act cooperatively in the prisoner's dilemma, Spearman's $\rho=.17, p=.006, N=261$, and marginally more likely to do so in the commons dilemma, $r=.12, p=.065, N=225$.

Concerning the relationship between wise reasoning and conflict-related behaviors, we found that participants who reported greater wise reasoning were also more likely to report engaging in behaviors promoting balanced conflict resolution, $.07<$ Spearman's $\rho$ s $<.22$, ps $<$ .001 . Notably, wise reasoning was either unrelated, Spearman's $\rho \mathrm{s}=.01$, or negatively related to the engagement in behaviors that inhibit conflict resolution or undermine the other person, $-.13<$ Spearman's $\rho \mathrm{s}<-.11, p \mathrm{~s}<.001$ (see Table 8). This set of associations held across age and gender groups, and also when controlling for presentation order of the materials and socially desirable responding. These findings suggest that state-level wise reasoning is associated with endorsement of cooperative (vs. self-protective) conflict resolution strategies.

-- Insert Table 8 about here --

\section{Study 5 Summary}

Taken together, Study 5 showed that state-level wise reasoning scores captured with the SWIS related to a social orientation towards greater balance between cooperative and selffocused goals, as indicated by more cooperative intentions and behavior across two different 
domains. In social dilemmas, people with higher wise reasoning were more likely to make cooperative choices that balance communal with self-protective outcomes. Likewise, in their own conflicts, people with higher wise reasoning were more likely to report behaviors that could help to resolve the situation toward mutual benefit (e.g., try to resolve the situation together) and less likely to report behaviors that could benefit the self at the expense of the other person.

\section{Study 6: Balancing Goals, Causal Inferences, and Life Domain Conflicts}

Study 6 sought to further probe the relationship between wise reasoning and several aspects of balance, specifically, in the domains of goals, attribution formation, and evaluation of life domain conflicts. Balance is one of the key goals of wisdom (e.g., Kitchner \& Brenner, 1990; Kramer, 1990; Labouvie-Vief, 1990). According to Sternberg's (1998) balance theory, wisdom occurs as a process or interaction between individuals and their environment, specifically in adapting to different contexts-wise responding to contexts involves striking a balance between adjusting to the situation versus influencing it. This view of wisdom also requires balancing one's attention to both personal and contextual factors to arrive at wise decisions, something that people often fail to accomplish (e.g., Gilbert \& Malone, 1995). For example, when making attributions, people often overemphasize contextual factors for forming attributions about their roles and behaviors in negative situations (e.g., conflicts), and underemphasize contextual factors when forming attributions about others' roles and behaviors (Jones \& Nisbett, 1972). Wisdom involves forming accurate representations about the particular details about a problem (Sternberg, 1998), which requires balancing attributions about selfversus others' roles in conflict situations, and showing more situational sensitivity when forming judgments about others' behaviors. In line with these ideas, in Study 6 we assessed balance in influence versus adjustment goals, balancing of causal inferences about their own and others' 
role in the conflict, and increased situational sensitivity in attributions toward others' behavior in the conflict.

In addition to testing balancing at the level of the situation, we also wanted to examine assessments of balance between different types of conflict people may encounter in their own lives. Thus, in Study 6, we compared reports of work-related challenges due to family-related concerns as compared to family-related challenges due to work-related concerns (Carlson, Kacmar, \& Williams, 2000; Greenhaus \& Beutell, 1985). We chose the intersection of work and family life domains because work-life conflicts are multidimensional. That is, people tend to report work-life conflict in terms of direction-work interference with family, and family interference with work (Carlson, Kacmar, \& Williams, 2000). Using this structure, we could determine the extent to which people report global experiences in conflict for each of these two directions, and whether wise reasoning related to balance in reports of work $\rightarrow$ family and family $\rightarrow$ work conflict.

\section{Method}

\section{Participants and Procedure}

Participants in Study 6 were drawn from Sample I, which included some new participants as well as Wave 2 participants explored in Study 2. Participants completed the SWIS, two measures of balancing in goals and judgments about their conflict. Participants then completed three global wisdom measures, counterbalanced in presentation order. Then, they completed a measure of work and family conflicts.

\section{Measures}

Balancing influence and adjustment goals. Participants responded to items assessing the influence and adjustment goals they had when engaging in their conflict. We focused on 
influence and adjustment goals given their centrality to prior theoretical work on the notion of balance for wisdom (Sternberg, 1998). To this end, we modified several items from an existing trait-style measure of influence and adjustment goals (Tsai, Miao, Seppala, Fung, \& Yeung, 2007). Participants were asked how important it was for them to engage in nine different strategies, during their conflict. Example items assessing influence included, "Assert yourself," and "Have the other person listen to what you have to say." Example items assessing adjustment included, "Go along with what the other person wants," and "Make sure that the other person does not see you as getting in his/her way." Participants responded to these statements on a 6point scale $(0=$ Not at all important, to $5=$ Extremely important $)$. The full measure is presented in Table S19. Reliabilities for items reflecting influence and adjustment were good ( $\alpha \mathrm{s} \geq .79)$. Therefore, the items were averaged into respective indices of influence and adjustment goals $\left(M_{\text {Influence }}=4.10, S D=1.20 ; M_{\text {Adjustment }}=2.30, S D=1.10\right)$. To estimate the degree of balance between these goals, we calculated the relative strength and intensity of different goals using the similarity-intensity model (Thompson, Zanna, \& Griffin, 1995), with $S$ and $L$ representing different goals:

$$
(S+L) / 2-|S-L|
$$

Higher scores on this metric indicate a greater balance in the weighting of influencing and adjusting goals when navigating one's conflict.

Balancing causal inferences. Participants responded to six items assessing their causal inferences concerning their and the other person's role in the conflict, using a 5-point scale $(1=$ Not at all, to $5=$ Very much). Three items concerned self-focused inferences: "Do you think that you were primarily responsible for the incident?" "To what extent were you to blame" and "Could you have been more "wise" in the situation?" Three other items concerned other-focused 
inferences: "Do you think that the other person was primarily responsible for the incident?" "To what extent was the other person to blame?" and "Could this other person have been more "wise" in the situation?" Reliability for each type of causal inferences was good ( $\alpha \mathrm{s} \geq .76)$. Therefore, respective scores were averaged into indices of internal vs. external causal inferences $\left(M_{\text {Self }}=\right.$ $\left.2.40, S D=1.10 ; M_{\text {Other }}=3.80, S D=1.40\right)$. Similar to the balance in goals, we calculated balance in the weighting of internal and external causal inferences with the similarity-intensity formula depicted above.

Additionally, we assessed the degree of situational sensitivity when evaluating others' behavior by asking participants "Do you think the situation may have influenced this other person to say or do things that they otherwise would not have done?" using the same response scale as above $(M=3.80, S D=1.40)$. The full measure is presented in Table $S 20$.

Work-family conflict. Participants responded to six items from Carlson, Kacmar, and Williams' (2000) work-family conflict scale. This scale conceptualizes challenges at work and in family life in terms of two directions - work obligations interfering with family life (work $\rightarrow$ family) and family stress interfering with work (family $\rightarrow$ work). We used three items assessing each direction. Work $\rightarrow$ family items included "I have to miss family activities due to the amount of time I must spend on work responsibilities," "I am often so emotionally drained when I get home from work that it prevents me from contributing to my family," and "The behaviors I perform that make me effective at work do not help me to be a better parent and spouse." Family $\rightarrow$ work items included "I have to miss work activities due to the amount of time I must spend on family responsibilities," "Because I am often stressed from family responsibilities, I have a hard time concentrating on my work," and "Behavior that is effective and necessary for me at home would be counterproductive at work." Participants reported the 
extent to which they agreed with these statements on a 6-point scale $(1=$ Strongly disagree, to 6 $=$ Strongly agree $; \alpha=.78 ; M=2.68, S D=1.06)$. Items 3 and 6 assess behavioral specificity for work and family domains and did not refer to any challenges, and were therefore analyzed separately $(r=.46 ; M=2.95, S D=1.47)$. Correlations between the two items used for each type of challenge were strong $(r s \geq .63)$ and were averaged into indices of work $\rightarrow$ family $(M=3.04$, $S D=1.68)$ vs. family $\rightarrow$ work $(M=2.24, S D=1.37)$. Similar to the balance in goals and causal inferences, we calculated a measure of balance between severity of work $\rightarrow$ family and family $\rightarrow$ work challenges with the help of the similarity-intensity formula depicted above.

Global wisdom measures. Each participant completed three global trait-level wisdom measures, as described in Study 2, followed by demographics. To avoid carry-over effects, global wisdom scales were presented in a randomized order.

Self-Assessed Wisdom Scale (SAWS). Participants completed the 40-item SAWS (Webster, 2003), see Study 2 for further details on the method $(\alpha=.92 ; M=4.48, S D=0.62)$.

Three-Dimensional Wisdom Scale (3D-WS). Participants completed Thomas, Bangen, Ardelt, and Jeste's (2015) abbreviated three-dimensional wisdom scale, which uses 12 items from the original scale, described fully in Study $2(\alpha=.74 ; M=3.42, S D=0.63)$.

Adult Self-Transcendence Inventory (ASTI). Participants completed the 16-item ASTI (Levenson et al., 2005), see Study 2 for further details on the method $(\alpha=.82 ; M=3.61, S D=$ $0.71)$.

\section{Results}

First, we examined the relations between participants' scores on the SWIS, global wisdom measures, and balancing of influencing and adjustment goals within their conflict. We conducted multiple regressions predicting influencing and adjusting goals, the first with only wise 
reasoning as the predictor, and the second (to examine incremental validity of wise reasoning) with all three trait-level wisdom scores in the first step, and wise reasoning in the second step, as predictors. Results are presented in Table 9. In the first regression, we found that wise reasoning predicted greater balancing of influencing and adjusting goals within participants' own conflicts, $B=.41, S E=.06, t=7.00, p<.001$. In the second regression, in step one we found that the SAWS and 3D-WS negatively predicted balancing goals, whereas ASTI was not significantly associated with goal balance. In the second step, we found that adding wise reasoning to the model resulted in significant incremental variance explaining the balance of influencing and adjusting goals, $\Delta R^{2}=.123$ (see SOM for detailed analyses).

Next, we looked at the relations between participants' wise reasoning, global wisdom measures, and the index of balancing causal inferences about their conflict, using identical methods to the previous tests. In the first regression, we found that wise reasoning predicted greater balancing of causal inferences, $B=.14, S E=.06, t=2.40, p=.017$. In the second regression, in step one, global measures were unrelated to the balance of causal inference, though each measure was trending in a negative direction and SAWS negatively predicted the balance of causal inferences in step 2. Again, we found that adding wise reasoning to the model resulted in significant incremental variance explaining the balance of self- vs. other -focused inferences about the cause of conflict, $\Delta R^{2}=.018$ (SOM). Examining participants' degree of situational attributions when evaluating others' behavior (see SOM for full results), in the same manner as the previous two tests, we additionally found that wise reasoning predicted more situational attributions, $B=.27, S E=.07, t=3.72, p<.001$. In the second test, the SAWS also predicted more situational attributions. The 3D-WS and ASTI did not predict situational attributions, and 
the ASTI trended in the negative direction. Finally, adding wise reasoning to the model explained a significant additional variance in situational attributions, $\Delta R^{2}=.015$.

Last, we examined the relations between participants' wise reasoning, global wisdom measures, and balance of two different types of life challenges (work $\rightarrow$ family vs. family $\rightarrow$ work), with methods identical to those in the previous tests, focusing only on participants who were employed. In the first test, we found that wise reasoning predicted balanced reports of severity of different types of challenges, $B=.19, S E=.06, t=3.14, p=.002$. In the second test, the 3D-WS predicted less balance and the ASTI predicted marginally less balance; the SAWS did not predict balance. In the third test, adding wise reasoning to the model resulted in significant incremental variance explaining the balance between different challenges, $\Delta R^{2}=.024$. Notably, these effects of balance were not due to greater moderacy-bias among wise reasoners. As supplementary analyses indicated, higher SWIS scores were linked to greater focus on adjustment goals and consideration of personal responsibility for the conflict.

-- Insert Table 9 about here --

\section{Study 6 Summary}

Study 6 showed that state-level wise reasoning related to several indices of situational balancing within people's own conflicts. We found that wise reasoning predicted greater balance in influence and adjustment goals, greater balance in people's inferences about the causes of their conflicts, and more situational attributions of others' behaviors in the conflict. In supplementary analyses, we found that these effects were a result of participants' wise reasoning relating to increased adjustment goals (vs. influencing goals), and increased internal attributions (vs. external attributions) about their conflicts. In contrast, global wisdom scores were either unrelated or, more often, negatively related to these indices of balance. We also found parallel 
results in people's general ratings of balance in their own life, as shown in more balanced reporting of work $\rightarrow$ family compared to family $\rightarrow$ work conflict. In sum, Study 6 showed strong evidence that wise reasoning relates to balancing at both the state- and general-level.

\section{Study 7: Wise Reasoning across Distinct Conflicts}

Study 7 had three goals. First, we aimed to further probe the reliability and factor structure of the wise reasoning construct using a different, socioeconomically, and culturally heterogeneous sample of non-student community participants. Prior research suggests possible cross-cultural differences in the meaning of wisdom (Grossmann \& Kung, in press, for a review), as well as performance on wise reasoning tasks (Grossmann, Na et al., 2012). Given that Studies 1-6 in the present research employed North American participants, we explored whether the reliability and factor structure of situated wise reasoning and its relationship to balance generalizes beyond North American populations on MTurk. Second, we aimed to replicate and extend the results from Studies 2 and 6, evaluating the stability of wise reasoning (Study 2), and the intra-individual variability in the relationship of wise reasoning to balance indices (Study 6). In Study 2, participants were free to recall any conflict they wished. If participants selected a conflict for the second session that was qualitatively similar to the one they recalled in their first session, it could have artificially inflated the relations between Wave 1 and Wave 2 wise reasoning. To address this issue, we assessed participants' reasoning about two distinct conflict episodes in the same session. Third, Study 7 examined the relative contribution of state- and trait-level components of wise reasoning for balance indices across several distinct conflicts assessed on the same participants. 


\section{Method}

\section{Participants and Procedure}

We collected data from 300 non-college participants collected through Prolific Academic (ProA; Sample J), an organization that matches researchers with a community sample of individuals willing to complete studies for reimbursement. ProA retains a more culturally-diverse population of participants, who have been shown to be more naïve and conscientious than MTurk workers (Peer, Brandimarte, Samat, \& Acquisti, 2017). ProA participants came from different countries, $43 \%=$ North America $(4.3 \%=$ Canada, $0.7 \%=$ Mexico, \& $38.0 \%=$ United States), 51.2\% = UK/Ireland, 5.2\% = other (e.g., Australia, Germany, Israel, Spain, Hong Kong; see online supplement for full details). Full sample characteristics are in Table 1. Study 7 participants were compensated $2.5 £$ for $25 \mathrm{~min}$ of their time.

Participants completed a two-part study, separated by a standard filler task (a series of four simple anagrams). In the first half of the survey, participants completed the wise reasoning measure about a recent interpersonal conflict, and two indices of balancing goals and causal inferences within their conflict as in Study 6. In the second half, participants completed the same measures as in the first half but were asked to think of a different recent interpersonal conflict than that recalled in the first half. Specifically, participants first recorded the type of conflict in the first wise reasoning assessment: (1) with a friend, (2) a co-worker, (3) a romantic partner, or (4) a family member. Upon completion of the SWIS, goals, and inference-related measures for the first situation, the survey automatically instructed participants to reflect on a type of conflict that was distinct from the first one. Participants then completed another global wisdom measure (described below). 


\section{Measures}

Situated wise reasoning scale (SWIS). For each conflict, participants filled out the hybrid measure of wise reasoning. Reliabilities for each aspect of wise reasoning were good, Conflict 1: $\alpha_{\text {Perspective: }}=.82, \alpha_{\text {Change }}=.82, \alpha_{\text {Humility }}=.75, \alpha_{\text {Compromise }}=.84, \alpha_{\text {Outsider }}=.91 ;$ Conflict 2 : $\alpha_{\text {Perspective: }}=.87, \alpha_{\text {Change }}=.83, \alpha_{\text {Humility }}=.82, \alpha_{\text {Compromise }}=.88, \alpha_{\text {Outsider }}=.93$. To compare results across two conflicts, we averaged individual items into five first-order scores, which we subsequently averaged into a second-order score. This way, each aspect of wise reasoning remained equally weighted in the composite index.

Balancing influence and adjustment goals. Participants completed the identical measure to the one reported in Study 6. Reliabilities for influence and adjustment goals were good $(\alpha \mathrm{s}>$.78). Therefore, respective scores were averaged into indices of influence goals and adjustment goals, which were in turn subjected to a similarity-intensity formula to obtain a relative degree of balance in conflict-related goals. Central tendency for Study 7 variables presented in Table 10.

Balancing causal inferences. Participants completed the identical measure to the one reported in Study 6. Reliabilities for self-focused and others-focused causal inferences were good $(\alpha \mathrm{s}>.80)$. Therefore, respective scores were averaged into indices of self- and others-focused causal inferences, which were in turn subjected to a similarity-intensity formula to obtain a relative degree of balance in causal inferences. As in Study 6, additionally, we measured sensitivity to situational inferences when evaluating others' behavior.

Global wisdom measure. Participants completed Glück et al.’s (2013) Brief Wisdom Screening Scale (BWSS), which takes 20 items based on the longer-format scales - the SAWS, the 3D-WS, and the ASTI-on a 5-point scale (1 = Strongly disagree, to $5=$ Strongly agree $)$. 
This measure was chosen for pragmatic considerations of reducing time commitment of participants, and because it assesses the most central aspect across most common global wisdom scales (SAWS, 3D-WD, and ASTI), as identified by Glück and colleagues (2013) in their prior research. Reliability of this measure was good $(\alpha=.85)$. Therefore, the items were averaged into a single index of global wisdom.

\section{Results}

To this point, all of our tests have been conducted on responses from participants residing in North America. Thus, we wished to examine any cultural differences in the structure of wise reasoning as a function of the region from which responses were drawn. We first examined the factor structure and reliability of wise reasoning per region: North American participants, and non-North American participants. Given that a small portion of non-North American responses came from outside the UK, we also provide results of the same tests as above omitting non-UK responses in the online supplement, finding nearly identical results. As in previous studies, for both sampling points we conducted principal components analyses on five indices of wise reasoning (first conflict: North America: 55\% variance explained; non-North America: 63\% variance explained; second conflict: North America: 69\% variance explained; non-North America: $70 \%$ variance explained). To examine the factor structure across regions and conflicts, we then conducted region-specific (North American vs. non-North American) principal component analyses. As seen in Table 4, factor loadings across regions were very similar to one another, and to those found for MTurk and student samples, presented in Study 1 (see Table 4). For the first conflict that we assessed, Cronbach's $\alpha_{\text {North America }}=.91$, and $\alpha_{\text {non-North America }}=93$; for the second conflict that we assessed participants, Cronbach's $\alpha_{\text {North America }}=.91$, and $\alpha_{\text {non-North }}$ 
America $=94$. Given the similarities in factor structure and reliabilities between regions, in subsequent analyses, we collapsed regions and tested all participants as a single group.

Testing the convergence of SWIS across all participants, we found a small-moderate degree of intra-individual stability in wise reasoning across sampled conflicts, $r_{\mathrm{WR}}=.29$, suggesting substantial intra-individual variability in wise reasoning across different contexts. We found similar results with each individual aspect of wise reasoning, $r_{\text {Perspectives }}=.18, r_{\text {Multiple }}$ Outcomes $=.24, r_{\text {Limits }}=.24, r_{\text {Compromise/Resolution }}=.16, r_{\text {Outsider's Viewpoint }}=.45$. Further zero-order correlations of focal variables in Study 7 are presented in Table 10. SWIS scores were related to global wisdom scores and, in contrast to global wisdom scores, were also related to all balancerelated criteria, in both Conflict 1 and Conflict 2.

\section{-- Insert Table 10 about here --}

Next, we examined the contribution of trait-level (i.e., average across two state-level scores; Finnigan \& Vazire, 2017; Fleeson \& Jayawickreme, 2015) and state-level (i.e., statespecific deviation from the average) wise reasoning in predicting balance-related indices. Specifically, for each of the balance-related criterion variables, we conducted multi-level generalized linear model analyses, with balance scores nested within individuals, and global wisdom, trait-level wise reasoning, and state-specific wise reasoning as predictors. We conducted separate multi-level mixed effect models (with responses nested within participants), one with just wise reasoning indices as predictors, and another to examine incremental variance over global wisdom scores. Results presented in Table 11 indicate that both trait-level and statespecific wise reasoning predicted significantly greater balancing of influencing and adjusting goals and greater balancing of causal inferences. In contrast, global wisdom scores were negatively related to balance in causal inferences and trended in the negative direction for 
balanced influence and adjustment goals. This latter effect was largely driven by an effect of global wisdom scores on attributing less blame for the situation on the self (see SOM for complete Study 7 analyses).

-- Insert Table 11 about here -

\section{Study 7 Summary}

Study 7 presented further evidence for the generalizability of the factor structure and reliability of the SWIS. Reliability and internal structure of wise reasoning were nearly identical to those from our initial tests. Study 7 showed a small-moderate degree of stability in wise reasoning across two distinct conflicts, indicating substantial intra-personal variation in wise reasoning across situations (as in Grossmann et al., 2016, and Study 2 in the current research). State and trait wise-reasoning scores were consistently and positively related to balance, in terms of balancing one's influencing and adjustment goals, and in one's attributions toward others in one's conflicts. This was not the case for global wisdom scores, which showed either nonsignificant or significant negative relations to balance-related indicators. In summary, replicating findings from Studies 1, 5, and 6, SWIS scores showed strong reliability and related to balanced psychological functioning, over-and-above that of the most central items from three most widely used global wisdom measures (Glück et al., 2013).

\section{Study 8: Demographic and Social-Contextual Effects}

Finally, in Study 8, we conducted exploratory tests of the effects of demographics and social-contextual variables on wise reasoning across all responses to this point (Samples A-G, I, J), for which we assessed single-shot SWIS. Given much debate and research on wisdom as a function of age (e.g., Ardelt, 2011; Grossmann et al., 2010; Grossmann et al., 2012, Jordan, 2005; Staudinger, 1999), and theorizing about curvilinear effects of age on wisdom-related 
qualities (e.g., Labouvie-Vief, 1990), we examined wise reasoning as a function of age and age ${ }^{2}$. Similarly, there has been some recent attention to possible gender differences in wisdom-related qualities (e.g., Aldwin, 2009; Ardelt, 2009; Levenson, 2009), and therefore we examined wise reasoning as a function of participant gender. Given the situation-specific focus of the present method for assessing wisdom-related qualities, we were also able to evaluate how SWIS scores are impacted by gender of the other person in participants' conflicts, and the participant gender $\times$ gender of the other person interaction (i.e., "gender-context"). To generalize our results across other socio-demographic factors, in exploratory analyses, we also tested wise reasoning as a function of ethnicity, education, employment status, and participants' perceptions of the level of seriousness of their conflict. We first examined across MTurk and student North American participants (Samples A-G, and I)), and then examined ProA North American and non-North American participants (Sample J) separately, with an aim to exploring whether we would replicate the effects on the more regionally-heterogeneous sample.

\section{Results}

\section{North American Samples (MTurk and College Students)}

We first conducted a large-scale test of the relationships between demographic and social-contextual variables and wise reasoning on Samples A-G and I. First, we conducted a multiple regression with SWIS scores as the criterion and gender, age, and age ${ }^{2}$ (to explore possible curvilinear effects of age) as the predictors. We found that males reported lower wise reasoning than females, $t(3547)=-2.89, p=.004$ (see Figure 5). There were also significant linear and quadratic effects of age, age: $t(3547)=-7.22, p<.001$ age $^{2}: t(3547)=4.51, p<.001$. To get a fuller understanding of the nature of the curvilinear effect of age, we plotted the data and used the loess function to fit the trend (Figure 4), which indicated that wise reasoning 
decreased with age until approximately age 45 , and then appeared to increase. To further analyze the effect, we created two groups, one including younger participants (age $\leq 45$; Group 1), and one with older participants (age > 45; Group 2), analyzing them separately for the effect of age on wise reasoning. In line with the curvilinear findings, we found a negative effect of age on wise reasoning in Group 1, $t(3034)=-5.72, p<.001$, and a positive effect of age on wise reasoning in Group 2, $t(520)=2.08, p=.038$.

An omnibus ANOVA of ethnicity $($ Black $=1$, Asian $=2$, Latino $=3$, Other $=4$, White/European $=5$ ) on wise reasoning revealed a significant effect, $F(4,3096)=3.78, p=.005$. Exploratory post-hoc comparisons showed that, compared to Black participants, White participants reported engaging in wise reasoning more, $t(3096)=1.98, p=.048$, as did "Other" ethnicity, $t(3096)=2.50, p=.013$. Other comparisons were not significant. We also examined wise reasoning as a function of education (High school $=1$, Some college $=2$, College $=3$, Postgraduate $=4)$ and employment status $(\mathrm{No} /$ Yes $)$, finding no significant effects, $p s>.819$.

Finally, we examined wise reasoning as a function of social-contextual factors, including perceived conflict seriousness and gender of the other party. To fully understand the latter variable, we also added participants' gender, and their personal $\times$ other party gender interaction as predictors. Results indicated that greater perceived seriousness of conflicts predicted significantly higher wise reasoning, $t(2163)=5.13, p<.001$. We also found a significant effect of the gender of the other person in participants' conflicts, with higher wise reasoning when people were in a conflict with a female, $t(2163)=2.43, p<.015$, and a non-significant trend for the 2-way interaction between participant gender and gender of the other person in their conflict, $t(2163)=-1.60 p=.11$. To examine the interaction, we analyzed the effect of gender of the other person in participants' conflicts separately for female and male participants. Here, we found that 
male participants had significantly higher wise reasoning when the other person in their conflicts was a female versus a male, $t(1191)=-2.38, p=.017$, whereas we observed no significant effect for females, $t(1594)=-1.61, p=.11($ see Figure 5$)$.

\section{Prolific Academic Sample (Sample J)}

Next, we tested the contribution of demographic and social-contextual variables to wise reasoning in Sample $\mathrm{J}(n=300)$. It was important to examine this sample separately because it was a community sample consisting of participants living in different cultures. To explore whether contextual effects generalize beyond North American context (Henrich, Heine, \& Norenzayan, 2010), we included region as one of the contextual factors into multi-level analyses with wise reasoning for each of the two conflicts nested within participants. Other contextual variables included age, age $^{2}$, level of education, employment (No/Yes), perceived seriousness of the conflict, gender, and gender of the other person in the conflict. Given substantial heterogeneity in ethnic make-up and its meaning across countries in the non-North American sample, and given insufficient power for some of the ethnic groups in the Prolific Academic sample, we opted not to examine ethnicity-related relationships in the analyses involving Prolific Academic sample.

--Insert Figure 4 around here--

Replicating the MTurk/student results, analyses indicated higher wise reasoning when participants perceived their conflict to be more serious, $t(569.50)=2.59, p=.010 .{ }^{7}$ Further replicating MTurk results, we observed a linear and quadratic trends of age, age: $t(287.40)=-$

\footnotetext{
${ }^{7}$ Do wiser reasoners consider social conflicts as more serious or is the relationship between perceived conflict seriousness and wise reasoning rather reflect state-specific effects? To address this question, we examined whether trait vs. state-level wise reasoning contributes to perceived conflict seriousness. Results of a multi-level model with conflict-specific ratings of seriousness nested within participants indicated that state-level wise reasoning was significantly associated with seriousness, $B=.189, S E=.087, t(297.22)=2.17, p=.031$, whereas trait-level wise reasoning was not, $B=.097, S E=.080, t(296.94)=1.21, p=.228, \Delta \mathrm{AIC}=2.70$.
} 
$2.45, p=.015$, age $^{2}: t(286.70)=1.60, p=.111$ (see Figure 4$)$, as well as a significant effect of gender, $t(285.70)=1.96, p=.050$, and participant gender $\times$ other person's gender interaction, $t(535.60)=-1.71, p=.09$. Linear effects of age, as well as gender-specific interactions were moderated by region. Therefore we further analyzed North American and non-North American samples separately. Looking at North American sample, we found no significant effect of age, $t(122.67)=-0.75, p=.454$, and no gender-related effects, $p s>.172$. Looking at the non-North American sample, we found a negative effect of age, $t(169.80)=-2.80, p=.006$, as well as a gender $\times$ other's gender interaction, $t(318.00)=-2.87, p=.004$. Replicating the MTurk results on the non-North American sample, males showed significantly wiser reasoning in conflicts with females than with males, $t(129.74)=2.43, p=.017$ (see Figure 5). Notably, for females we observed a non-significant reversal, $t(189.90)=-1.93, p=.055$.

--Insert Figure 5 around here--

\section{Study 8 Summary}

Study 8 explored variability in wise reasoning as a function of demographic and socialcontextual characteristics. The results indicated that people are more likely to report wise reasoning as a function of perceived seriousness of the conflict. Further, we observed that wise reasoning scores decreased as a function of age until approximately 45 years of age, and increased after that. This finding appears to connect with Labouvie-Vief's (2003) theoretical position on lifespan development of socio-emotional processes and complex cognitions, which suggests the lower likelihood of mastering complex cognitive processes in middle adulthood as compared to younger and older adulthood. Notably, this effect was more prominent in the MTurk samples compromised of U.S. and Canadian participants. Though a similar curvilinear effect of age was also noticeable in the Prolific Academic sample, it was weaker and largely driven by 
North American sub-sample. In contrast, looking at the Non-North American ProA subsample (consisting predominantly of Western European participants), we mainly observed a linear negative effect of age on wise reasoning. We will return to the cross-sample difference in sociodemographic findings in the general discussion. Finally, we found several significant socialcontextual effects. North American MTurk and student participants (Samples A-G and I) were more likely to report higher wise reasoning when they were in a conflict with a woman, but this finding was qualified by an interaction showing that male participants were driving the effect: males reported lower wise reasoning when they were in a conflict with another male. Participants from the more culturally-diverse Prolific Academic sample (Sample J) were more likely to report wiser reasoning when they were in a conflict with someone of the opposite gender. The latter gender-specific interaction, along with a negative effect of age, was particularly pronounced among the non-North American sub-sample of Prolific Academic.

\section{General Summary and Discussion}

Despite many inspirational books ready to teach one how to become wiser, psychological scientists have so far been unable to provide practical and reliable methods to assess wisdom. Some scholars have employed global self-assessed measures of wisdom, which produce memory-related biases, and biases concerning bias blind spot (Pronin et al., 2002) and social desirability (Paulhus, 1988). Other scholars have employed narrative techniques to examine wisdom-related reflections in concrete difficult life situations (Baltes \& Smith, 2008; Grossmann et al., 2013; Staudinger et al., 1994). However, such narrative techniques are expensive, require situation-specific codebooks, often target hypothetical scenarios (vs. personally-relevant concerns), and can be inefficient for large-scale ecological assessments. In addition, past literature on wisdom is limited by a lack of clarity about criteria by which wisdom-related 
characteristics should be evaluated. In particular, although the concept of balance appears to be a central outcome in several popular theories on wisdom (e.g., Sternberg, 1998; Baltes \& Smith, 2008; Grossmann, 2017; Staudinger \& Glück, 2011), the link remains theoretical, with little empirical scholarship testing the relationship of characteristics attributed to wisdom against indices of balancing one's interests and inferences about the world.

Building on recent insights in psychology and survey methodology (Schwarz et al., 2009), we introduced a new hybrid method—Situated Wise Reasoning Scale (SWIS) — aiming to provide an efficient, yet reliable and accurate way to assess wisdom-related responses. This method operates on the level of a situation and by doing so allows for context-sensitive reconstruction of wise reasoning in a survey-based format (Schwarz et al., 2009). Examination of the psychometric properties of the SWIS indicates that it provides accurate responses that are either unrelated or negatively related to a host of bias-related tendencies, from social desirability — self-deception and impression management — to bias blind spot and attributional bias. In contrast, global methods to assess wisdom were vulnerable to these psychological biases. Further testing of SWIS indicates remarkably similar factor structure and reliability across regions compromised of socio-demographically diverse samples, speaking to the ecological generalizability of the method. The current methodological application extends prior research on advantages of event-reconstruction, compared to global assessments, for measuring everyday life experiences (Kahneman et al., 2004; Schwarz et al., 2009). Further, it contributes to an emerging scholarship on the relationship between wisdom and unbiased thought (e.g., Gilovich \& Ross, 2015; Grossmann et al., 2016) by proving a more precise tool for testing the effect of wisdom on bias. 
Moreover, the hybrid method allowed us to establish the connection between wisdomrelated cognition and balancing of interests, trade-offs, and inferences one makes about the social world. We found that wise reasoning was not only related to balance between self-focused and other-oriented intentions in classic decision-making tasks, but also to people's tendency to strike a balance between their influence and adjustment goals, and between their attributions to the self vs. another party in their conflicts. Conversely, global wisdom measures were either unrelated or more often inversely related these markers of balance. Thereby, the current research provides the first large-scale empirical support to the conceptual claims of numerous wisdom scholars (e.g., Grossmann, 2017; Sternberg, 1998; Staudinger \& Glück, 2011).

\section{Utility of Process-Oriented Measurement of Wisdom-Related Characteristics}

Recent insights from research on personality and social cognition both call for greater attention to state-level processes for a deeper understanding of psychological phenomena. Personality psychologists have pointed out that greater attention to the state (vs. global) levels of analysis can provide a more holistic perspective on how traits are represented through the profiles of density distributions respecting specific states (Fleeson, 2001; Mischel \& Shoda, 1995). According to this research, multi-state measurements provide a more accurate estimate of individual differences and allow for the detection of systematic patterns of responding to situational contingencies (Mischel \& Shoda, 1995). Simultaneously, social cognitive researchers have pointed out that cognitive processes are not isolated inner representations but rather interdependent with the current physical, social, and cultural environment, as demonstrated in the domains of knowledge accessibility (Yeh \& Barsalou, 2006), affect and judgment (Schwarz, 2011), cognitive style (Wegner \& Vallacher, 1986), and the theory of "situated social cognition" (Smith \& Semin, 2007). 
The present hybrid method of state-level wise reasoning integrates these insights, showing that people vary in their wise reasoning across situations and respond with more or less wise reasoning depending on the features of the situation. For instance, we found that wise reasoning was associated with situations that participants viewed as more serious. This observation is not trivial and may appear counterintuitive at first: serious conflicts can be more polarizing, with lower willingness to adopt others' perspectives. We also observed that male participants were more likely to reason wisely in conflict situations involving a female rather than a male counterpart (see Figure 5). Though further work is needed to replicate these observations and explore potential underlying influences from culture and gender norms (e.g., Eagly, 2009), these preliminary observations provide further evidence to the utility of the hybrid method. Revealing seriousness and gender-related findings would not have been possible without shifting the focus from traditional global reports to the current state-level of analysis.

By providing an opportunity for new insights concerning the role of situational contingencies for wisdom, the state-level measurement of wise reasoning contributes to an empirical foundation for nurturing new developments of wisdom-enhancing environments (Grossmann, 2017). In particular, the state-level measurement of wise reasoning (across multiple situations) can be a valuable tool for examining which components of wisdom are dispositional and which are state-specific (Fleeson \& Jayawickreme, 2015; Fleeson \& Noftle, 2008a, 2012; McCabe \& Fleeson, 2015; Mischel, Shoda, \& Mendoza-Denton, 2002b). By assessing wisdom via a global single-shot measurement of wisdom-related characteristics (Ardelt, 2003; Levenson et al., 2005; Webster, 2003, 2007), prior studies have made an implicit assumption that wisdomrelated qualities are rather stable individual differences. However, global single-shot measures make it impossible to determine whether responses reflect intra-individually stable differences in 
wisdom, differences in bias, or some features of the situation. In contrast, the hybrid method assesses wisdom-related characteristics on the level of the situation. As demonstrated in Study 7 , it is ideally suited for multi-situation assessment (e.g., examining interactions with parents vs. close friends), enabling researchers to differentiate situational contingencies from the trait-level tendencies by averaging responses across situations (Fleeson \& Jayawickreme, 2015;

Grossmann, Gerlach, \& Denissen, 2016; Mischel et al., 2002). Indeed, the simultaneous analyses of trait- and state-level components of wise reasoning on markers of balance indicated a unique contribution of each component, suggesting that both trait and state-specific factors contribute to balancing of interests and inferences that people make about the world around them. This finding is noteworthy, as it suggests that it is not only the case that wiser reasoners tend to be more balanced on average, but also that people are likely to be more balanced in situations that they express wise reasoning above their average level.

Moreover, separating trait from state-level components of wise reasoning enables a better understanding of the relationship between wise reasoning and seriousness. Specifically, we were able to evaluate whether the relationship between wise reasoning and seriousness means that wise reasoners are more aware of the seriousness of the situation (trait-level effects) or whether people are more motivated to reason wisely in the face of more challenging situations (i.e. statelevel effect). Our results indicated that this relationship was mainly present at the state level of analysis. Future work targeting these multi-level questions will benefit from the present hybrid method, especially when integrating it with experimental or longitudinal designs to unpack the complexity within these questions.

From a practical perspective, the ability to measure situational contingencies of wisdomrelated characteristics in an ecologically-sensitive manner can also contribute to greater 
knowledge about how wisdom may be practiced, developed, and enhanced across a variety of settings (e.g., health-promotion, education, and business). Given that desirable psychological tendencies and processes may be harmful in some situations (McNulty \& Fincham, 2012), the current method also makes it possible to determine conditions under which engaging in wise reasoning may, in fact, be maladaptive.

Finally, the state-level measurement of wise reasoning affords novel inquiries about the stability of wise reasoning across multiple situations and offers practical estimates of how many measurement units may be necessary for an accurate estimation of individuals' tendency to reason wisely. The present results in Studies 2 and 7 reveal that the extent to which individuals express wise reasoning varies substantially over time and across different situations, dovetailing with emerging evidence on day-to-day variability in wise reasoning (Grossmann et al., 2016). Nonetheless, we also observed a moderate degree of convergence (over time: $r=.48$ and across distinct situations: $r=.31$ ). Applying the Spearman-Brown Prophecy formula, it appears that to obtain average reliability of .70 , one would require at least two sampling units if the sampled episodes are similar (e.g., in type; in level of seriousness), or at least five sampling units if they are heterogeneous. One practical recommendation would be first to consider what degree of reliability one would deem acceptable, and second consider whether one wants to sample individuals in similar or diverse contexts. For a high degree of reliability, a few sampled episodes may not be sufficient when evaluating performance across diverse contexts. However, if a lower degree of reliability is acceptable, three sampling points may be enough to obtain a trait-level estimate. Based on the present insights, three measurement points with at least two distinct types of situation may be a good compromise between accuracy and ecological applicability across distinct situations. 


\section{Theoretical Implications}

Beyond providing empirical support for the notion of balance as a key criterion in numerous wisdom theories (e.g., Grossmann, 2017; Sternberg, 1998; Staudinger \& Glueck, 2011), the insights from the state-level hybrid method generate several new ideas for advancing theorizing about wisdom. First and foremost, state-level measurement provides a fruitful foundation for testing the process-oriented aspects of wisdom. The notion of process is evident across numerous theories (for reviews, see Grossmann, 2017; Staudinger \& Glück, 2011). For instance, Sternberg indicated that "information processing in and of itself is not wise or unwise. Its degree of wisdom depends on the fit of a wise solution to its context" (1998, p. 353). Similarly, scholars such as Baltes and Staudinger (1996) have theorized how the meaning and function of wisdom operates within the larger socio-cultural context. The hybrid method for assessing wise reasoning advances these insights empirically, providing an efficient platform for evaluating the role of contextual factors in understanding how wisdom-related qualities manifest and develop. It does so by conceptualizing individual differences in wise reasoning as a density distribution of specific states.

Notably, the notion of a situation-sensitive distribution of how wisdom-related characteristics are expressed by a given individual has two critical implications. First, it suggests that any theoretical claims about the relationship between wisdom and various well-being enhancing or prosocial tendencies ought to consider the level of analysis. For instance, consider the debate about the role of wisdom-related characteristics for various markers of subjective well-being (for review, see Grossmann, 2017): Whereas some scholars suggest that wisdom may promote more positive emotions and greater life satisfaction (e.g., Bergsma \& Ardelt, 2012), other scholars proposed that wisdom is linked with a deeper understanding of twists and turns of 
one's life, and therefore may not necessary contribute to one's subjective feeling of happiness (Staudinger \& Kunzmann, 2005). Importantly, in most of these cases wisdom-related characteristics were measured only once, thus it is unclear whether effects concerned trait- or state-level associations between wisdom and happiness/mood, or could even be accounted by various additional situational contingencies (e.g., desire to present oneself in a positive light; Zacher et al., 2013). The present observation of separate trait- and state-level effects of wise reasoning suggest that through paying attention to state- vs. trait-level effects and measuring wisdom-related characteristics several times, one can obtain a more precise estimate. Indeed, using this approach, recent work began to advocate that wise reasoning is a more potent correlate of well-being on a state- as compared to trait-levels of analysis (Grossmann et al., 2016). Whereas wise reasoners may not necessarily be happier people (consistent with Staudinger \& Kunzmann, 2005), wiser reflection on a concrete situation at hand may in fact be aligned with psychological benefits of more adaptive emotion regulation and greater satisfaction with one's life (Gross \& Thompson, 2007; Kekes, 1983, 1995; Kross \& Ayduk, 2011). In a given situation, focusing on sustaining one's well-being may be a healthy tendency. However, if this principle is used rigidly across all contexts, it may likely backfire (e.g., Ford et al., 2015; Grant \& Schwartz, 2011).

In a similar vein, attention to context is important when evaluating claims about the relationship between wisdom-related characteristics and prosociality (cf. Sternberg, 1998). Past research has indicated that though prosociality can be of universal advantage for individual and group survival, prosociality is expressed to different degrees across various contexts, especially when comparing (post-) industrial vs. small-scale societies (e.g., Henrich et al., 2010). In particular, the meaning of prosocial acts tends to vary as a function of expectations of trust 
towards ingroup vs. strangers, such that differences in degree of trust towards ingroup vs. strangers lead to different patterns of prosociality, as demonstrated in numerous comparisons of East Asian vs. North American contexts (Huff \& Kelley, 2003; Yamagishi, 1988; Yamagishi, Jin, \& Miller, 1998; c.f. Buchan \& Croson, 2004; Yuki, Maddux, Brewer, \& Takemura, 2005). The relationship between wise reasoning and prosocial behavior is sensitive to situational context as well, as demonstrated in a recent experimental study on the role of deliberation for a robust association between these characteristics (Grossmann, Brienza, \& Bobocel, 2017). Second, when building a developmental theory of wisdom (Erikson, 1984; Loevinger, 1976), the notion of traits as a distribution of numerous states allows for a more fine-grained understanding of how hypothesized antecedents of wisdom interact in fostering this quality. For instance, instead of conceptualizing ideas of mastering the developmental dialectics of integrity vs. despair (Erikson, 198) as a developmental stage of a person, the current approach allows probabilistic modelling of the way personal characteristics interact with the ever-changing environment the person finds themselves in (for a similar interactionist argument, also see Loevinger, 1976). Thereby, the current approach enables a more accurate portrayal of the ways people, in fact, develop wisdom-related skills over time. In short, instead of merely speculating about the dynamic nature of wisdom as is the case to date, the current method allows for actual dynamic modeling of the relationship between the person and their situation.

\section{Limitations and Future Directions}

Future research using a reliable tool for assessing wise reasoning on the level of a situation can help to integrate an apparent paradox in the domains of social psychology and mental health. Is wisdom always characterized by unbiased thought or is it possible, as some have suggested (Taylor et al., 2011), that under certain circumstances a wise response could 
involve self-deception, positive illusions, or overconfidence? After all, past research indicates that such biases can be adaptive when used to regulate one's response to negative information in a way that makes the negative information appear less threatening (Taylor \& Brown, 1988). By using the situated wise reasoning scale (SWIS), it is now possible to identify conditions under which otherwise wise people would demonstrate psychological bias. It is further possible to explore whether such bias results from motivated reasoning (Kunda, 1990) or involuntary reactivity to concrete situational demands. Given the self-report nature of the current measure, the state-level method could be further bolstered with additional methods, such as utilizing informants' state-specific evaluations of whether a person demonstrates wisdom-related characteristics.

Beyond understanding wise reasoning in particular, the present hybrid method also has a potential to shed new light on the improvement of ecological methods for testing cognition and social phenomena by utilizing the event reconstruction method to improve the accuracy of recall. We suggest that such techniques could be used in future studies to improve the incremental validity of trait-level measurement of many individual differences for understanding and predicting behavior and other-reports (cf. Finnigan \& Vazire, 2017).

In MTurk and student samples (Samples A-G and I) we found consistent effects of perceived seriousness of a conflict, and curvilinear effect of participant age, on wise reasoning, effects that were replicated in our Prolific Academic (ProA) sample (Sample J). At the same time, when evaluating the role of socio-demographic factors such as age, ethnicity, or gender to wise reasoning, it is worth pointing out that some of the observed effects were small in magnitude, requiring adequately powered samples to detect and replicate the role of sociodemographic factors for wise reasoning. Indeed, in the present report, we opted not to evaluate 
the role of ethnicity in the ProA sample due to underpowered sub-samples from some ethnic subpopulations. Similar power considerations likely impacted the minor inconsistencies in sociodemographic effects of participants' age, gender, and gender of the other person involved in the conflict on wise reasoning when comparing the Mturk and (much smaller) ProA samples. Surmising that this lack of replication may have resulted from underpowered tests or sampling error due to the smaller ProA subsamples and greater power necessary to detect quadratic and interaction effects, we suggest that future research conduct more thorough and higher powered tests of the effects of age, gender, gender context, and ethnicity on wise reasoning, as these findings may be important for understanding when and why people increase their use of wise reasoning and how doing so impacts on the outcomes of interpersonal conflicts and relationships in general.

Going beyond the quantitative considerations of adequate power to detect the sociodemographic effects for wise reasoning, it is also worth considering whether the meaning of agerelated, gender, educational and ethnic factors across different cultural and situational contexts may moderate their effects on wise reasoning (see Grossmann \& Kung, in press, and Grossmann \& Huynh, 2012, for related arguments). The latter insight underscores the importance of examining the relationship between social-demographic factors and wisdom-related qualities in the context of how the social-demographic factors are construed by the specific cultural group in a specific situation (Grossmann, 2017). Mixed method approaches combining qualitative studies of intersectionality, and statistical methods quantifying the subjective meaning of relevant sociodemographic factors for wise reasoning may be a useful path forward when attempting to get a fuller understanding of social-demographic considerations for wise thought. 
The context-sensitive method is expandable to a range of social domains (e.g., workplace, interpersonal, or intergroup conflict). Moreover, it can be tailored to include a broad range of wisdom-related characteristics in addition to wisdom-related cognitions that we highlight here. On the premise that wisdom is inherently a social process (Baltes \& Smith, 2008; Sternberg, 1998), we focused on social conflict situations and the most commonly-tested aspects of wisdom-related thought. We found more robust relationships to other measures (nomological network and outcomes) with a single second-order wise reasoning construct (as opposed to any of the individual first-order aspects). However, it remains an open question when or under what contexts other, more unique aspects of wise reasoning or emotion regulation could be expressed and how they contribute to balance vs. bias in decision making and making sense of one's social environment. Another fruitful avenue for future research may be to examine other contexts (e.g., legal and political decision-making) and to examine the role of state-specific motivational and neurophysiological processes for wise judgment (also see Grossmann, 2017; Meeks \& Jeste, 2009; Staudinger \& Glück, 2011).

\section{Conclusion}

We introduced the Situated WIse reasoning Scale (SWIS) and revealed that the new measure is reliable and less susceptible to psychological biases (attribution bias, bias blind spot, self-deception, impression management), compared to global wisdom measures. State-level scores were positively related to indices of living well and to greater balancing of cooperative and self-protective interests, goals (influence vs. adjustment), and causal inferences (internal vs. external) about other people's behavior. In contrast, global wisdom reports were unrelated or negatively related to balance-related indices. We observed a weak-moderate level of withinperson consistency in wise reasoning across situations, recommending multi-state measurements 
for a complete understanding of trait-level wisdom. Future research should utilize the new method to examine the benefits of wise reasoning in a broad range of domains and venture beyond cognitive aspects, as the new method is easily modifiable to examine other aspects of wisdom or personality in general. 


\section{References}

Aldwin, C. M. (2009). Gender and wisdom: A brief overview. Research in Human Development, $6(1), 1-8$.

Allaire, J. C., \& Marsiske, M. (2002). Well- and ill-defined measures of everyday cognition: Relationship to older adults' intellectual ability and functional status. Psychology and Aging, 17(1), 101-115.

Andersson, L. M., \& Pearson, C. M. (1999). Tit for tat? The spiraling effect of incivility in the workplace. Academy of Management Review, 24(3), 452-471.

Andrew, W., \& Orwoll, L. (1991). Becoming wise: A psycho-gerontological interpretation of the Book of Job. The International Journal of Aging \& Human Development, 32(1), 21-39.

Ardelt, M. (2003). Empirical assessment of a three-dimensional wisdom scale. Research on Aging, 25(3), 275-324.

Ardelt, M. (2004). Where can wisdom be found? Human Development, 47(5), 304-307.

Ardelt, M. (2009). How similar are wise men and women? A comparison across two age cohorts. Research in Human Development, 6(1), 9-26.

Ardelt, M. (2011). Wisdom, age, and well-being. In Schaie, K. W., \& Willis, S. L. (Eds.), Handbook of the Psychology of Aging, (pp.279-291). Chicago, IL, US: Academic Press.

Assmann, A. (1994). Wholesome knowledge: Concepts of wisdom in a historical and crosscultural perspective. In D. L. Featherman, R. M. Lerner, \& M. Perlmutter (Eds.), Lifespan development and behavior, Vol. 12 (pp. 187-224). Hillsdale, NJ, US: Lawrence Erlbaum Associates, Inc.

Axelrod, R. M. (2006). The evolution of cooperation. Basic books.

Axelrod, R., \& Hamilton, W. D. (1981). The evolution of cooperation, Science, 211, 1390-1396. 
Baer, R. A., Smith, G. T., Hopkins, J., Krietemeyer, J., \& Toney, L. (2006). Using self-report assessment methods to explore facets of mindfulness. Assessment, 13(1), 27-45. https://doi.org/10.1177/1073191105283504

Balliet, D., \& Joireman, J. (2010). Ego depletion reduces proselfs' concern with the well-being of others. Group Processes \& Intergroup Relations, 13(2), 227-239.

Balliet, D., \& Lange, P. A. M. V. (2013). Trust, punishment, and cooperation across 18 societies A meta-analysis. Perspectives on Psychological Science, 8(4), 363-379.

Baltes, P. B. (2004). Wisdom as orchestration of mind and virtue. Unpublished Book. Retrievable at Mpibberlin.Mpg.de/en/institut/dok/full/baltes/orchestr/Wisdom_compl.Pdf.

Baltes, P. B., \& Kunzmann, U. (2004). The two faces of wisdom: Wisdom as a general theory of knowledge and judgment about excellence in mind and virtue vs. wisdom as everyday realization in people and products. Human Development, 47(5), 290-299.

Baltes, P. B., \& Smith, J. (2008). The fascination of wisdom: Its nature, ontogeny, and function. Perspectives on Psychological Science, 3(1), 56-64.

Baltes, P. B., \& Staudinger, U. M. (1993). The search for a psychology of wisdom. Current Directions in Psychological Science, 2(3), 75-80.

Baltes, P. B., \& Staudinger, U. M. (1996). Interactive minds: Life-span perspectives on the social foundation of cognition. New York, NY, US: Cambridge Univerisity Press.

Baltes, P. B., \& Staudinger, U. M. (2000). Wisdom: A metaheuristic (pragmatic) to orchestrate mind and virtue toward excellence. The American Psychologist, 55(1), 122-136.

Bangen, K. J., Meeks, T. W., \& Jeste, D. V. (2013). Defining and assessing wisdom: A review of the literature. The American Journal of Geriatric Psychiatry, 21(12), 1254-1266.

Basseches, M. (1980). Dialectical schemata. Human Development, 23(6), 400-421. 
Basseches, M. (1984). Dialectical thinking and adult development. New Jersey, NJ: Ablex.

Bergsma, A., \& Ardelt, M. (2012). Self-reported wisdom and happiness: An empirical investigation. Journal of Happiness Studies, 13(3), 481-499.

Birren, J. E., \& Svensson, C. M. (2005). Wisdom in History. In R. J. Sternberg \& J. Jordan (Eds.), A handbook of wisdom: Psychological perspectives (pp. 3-28). New York, NY, US: Cambridge University Press.

Bluck, S., \& Glück, J. (2004). Making Things Better and Learning a Lesson: Experiencing Wisdom Across the Lifespan. Journal of Personality, 72(3), 543-572.

Buchan, N., \& Croson, R. (2004). The boundaries of trust: Own and others' actions in the US and China. Journal of Economic Behavior \& Organization, 55(4), 485-504.

Carlson, D. S., Kacmar, K. M., \& Williams, L. J. (2000). Construction and initial validation of a multidimensional measure of work-family conflict. Journal of Vocational behavior, 56(2), 249-276. doi:10.1006/jvbe.1999.1713

Child, D. (2006). The essentials of factor analysis (3rd ed.). New York, NY: Continuum International Publishing Group.

Chiu, C. Y., Hong, Y. Y., \& Dweck, C. S. (1997). Lay dispositionism and implicit theories of personality. Journal of Personality and Social Psychology, 73, 19-30.

Clark, M. S., Oullette, R., Powell, M. C., \& Milberg, S. (1987). Recipient's mood, relationship type, and helping. Journal of Personality and Social Psychology, 53(1), 94-103.

Clayton, V. (1975). Erikson's theory of human development as it applies to the aged: Wisdom as contradictive cognition. Human Development, 18(1-2), 119-128.

Clayton, V. (1982). Wisdom and intelligence: The nature and function of knowledge in the later years. The International Journal of Aging \& Human Development, 15(4), 315-321. 
Cohen, T. R., Wolf, S. T., Panter, A. T., \& Insko, C. A. (2011). Introducing the GASP scale: a new measure of guilt and shame proneness. Journal of Personality and Social Psychology, 100(5), 947-966.

Cronbach, L. J., \& Meehl, P. E. (1955). Construct validity in psychological tests. Psychological Bulletin, 52(4), 281-302.

Dambrun, M., \& Ricard, M. (2011). Self-centeredness and selflessness: A theory of self-based psychological functioning and its consequences for happiness. Review of General Psychology, 15(2), 138-157.

Davis, M. H. (1983). Measuring individual differences in empathy: evidence for a multidimensional approach. Journal of Personality and Social Psychology, 44(1), 113126.

De Dreu, C. K., Evers, A., Beersma, B., Kluwer, E. S., \& Nauta, A. (2001). A theory-based measure of conflict management strategies in the workplace. Journal of Organizational Behavior, 22(6), 645-668.

Deutsch, M., Coleman, P. T., \& Marcus, E. C. (2011). The handbook of conflict resolution: Theory and practice. San Francisco, CA: John Wiley \& Sons.

Diener, E., Kanazawa, S., Suh, E. M., \& Oishi, S. (2015). Why people are in a generally good mood. Personality and Social Psychology Review, 19(3), 235-256.

Diener, E., \& Tay, L. (2014). Review of the day reconstruction method (DRM). Social Indicators Research, 116(1), 255-267.

Dunlop, W. L. (2015). Contextualized personality, beyond traits. European Journal of Personality, 29(3), 310-325. 
Dunning, D., Heath, C., \& Suls, J. M. (2004). Flawed self-assessment: Implications for health, education, and the workplace. Psychological Science in the Public Interest, 5(3), 69-106.

Eagly, A. H. (2009). The his and hers of prosocial behavior: an examination of the social psychology of gender. American Psychologist, 64(8), 644.

Erikson, E. H. (1984). Reflections on the last stage - and the first. Psychoanalytic Study of the Child, 39, 155-165.

Estes, B., \& Wang, J. (2008). Workplace incivility: Impacts on individual and organizational performance. Human Resource Development Review, 7(2), 218-240.

Fehr, E., \& Fischbacher, U. (2003). The nature of human altruism. Nature, 425(6960), 785-791.

Finnigan, K. M. \& Vazire, S. (2017). The incremental validity of average state self-reports over global self-reports of personality. Journal of Personality and Social Psychology, doi:10.1037/pspp0000136

Fleeson, W. (2001). Toward a structure- and process-integrated view of personality: Traits as density distributions of states. Journal of Personality and Social Psychology, 80(6), $1011-1027$.

Fleeson, W., \& Jayawickreme, E. (2015). Whole trait theory. Journal of Research in Personality, $56,82-92$.

Fleeson, W., \& Noftle, E. (2008a). The End of the Person-Situation Debate: An Emerging Synthesis in the Answer to the Consistency Question. Social and Personality Psychology Compass, 2(4), 1667-1684. https://doi.org/10.1111/j.1751-9004.2008.00122.x

Fleeson, W., \& Noftle, E. E. (2008b). Where does personality have its influence? A supermatrix of consistency concepts. Journal of Personality, 76(6), 1355-1386. 
Fleeson, W., \& Noftle, E. E. (2012). Personality research. In M. R. Mehl \& T. S. Conner (Eds.), Handbook of research methods for studying daily life (pp. 525-538). New York, NY, US: Guilford Press.

Fletcher, G. J. O., Danilovics, P., Fernandez, G., Peterson, D., \& Reeder, G. D. (1986). Attributional complexity: An individual differences measure. Journal of Personality and Social Psychology, 51(4), 875-884.

Floyd, F. J., \& Widaman, K. F. (1995). Factor analysis in the development and refinement of clinical assessment instruments. Psychological Assessment, 7(3), 286-299.

Fredrickson, B. L. (2006a). The broaden-and-build theory of positive emotions. In M. Csikszentmihalyi \& I. S. Csikszentmihalyi (Eds.), A life worth living: Contributions to positive psychology (pp. 85-103). New York, NY, US: Oxford University Press.

Fredrickson, B. L. (2006b). Unpacking positive emotions: Investigating the seeds of human flourishing. The Journal of Positive Psychology, 1(2), 57-59.

Frensch, P. A., \& Funke, J. (2014). Complex problem solving: The European perspective. Psychology Press.

Funder, D. C. (2009). Persons, behaviors and situations: An agenda for personality psychology in the postwar era. Journal of Research in Personality, 43(2), 120-126.

Furr, R. M., \& Bacharach, V. R. (2014). Psychometrics: An introduction. Thousand Oaks, CA: Sage Publications.

Garland, E. L., Farb, N. A., R. Goldin, P., \& Fredrickson, B. L. (2015). Mindfulness broadens awareness and builds eudaimonic meaning: a process model of mindful positive emotion regulation. Psychological Inquiry, 26(4), 293-314. 
Gilbert, D. T., \& Malone, P. S. (1995). The correspondence bias. Psychological Bulletin, 117(1), 21.

Gilovich, T., \& Ross, L. (2015). The Wisest One in the Room: How You Can Benefit from Social Psychology's Most Powerful Insights. Simon and Schuster.

Glück, J., König, S., Naschenweng, K., Redzanowski, U., Dorner, L., Straßer, I., \& Wiedermann, W. (2013). How to measure wisdom: content, reliability, and validity of five measures. Frontiers in Psychology, 4, 405.

Goldstein, D. G., \& Gigerenzer, G. (2002). Models of ecological rationality: The recognition heuristic. Psychological Review, 109(1), 75-90.

Goold, M., \& Campbell, A. (1998). Desperately seeking synergy. Harvard Business Review, 76(5), 131-143.

Grant, A. M., \& Schwartz, B. (2011). Too much of a good thing: The challenge and opportunity of the inverted U. Perspectives on Psychological Science, 6(1), 61-76.

Greenhaus, J. H., \& Beutell, N. J. (1985). Sources of conflict between work and family roles. Academy of Management Review, 10(1), 76-88.

Gross, J. J. (1998). The emerging field of emotion regulation: An integrative review. Review of General Psychology, 2(3), 271-299.

Gross, J. J., \& John, O. P. (2003). Individual differences in two emotion regulation processes: Implications for affect, relationships, and well-being. Journal of Personality and Social Psychology, 85(2), 348-362.

Gross, J. J. \& Thompson, R. A. (2007). Emotion Regulation: Conceptual Foundations. In J. J. Gross (Ed.) Handbook of emotion regulation, (pp. 3-24). New York, NY: Guilford Press. 
Grossmann, I., Gerlach, T. M., \& Denissen, J. J. A. (2016). Wise reasoning in the face of everyday life challenges. Social Psychological and Personality Science, 7(7), 611-622.

Grossmann, I., Karasawa, M., Izumi, S., Na, J., W, E., Kitayama, S., \& Nisbett, R. E. (2012). Aging and wisdom: Culture matters. Psychological Science, 23(10), 1059-1066.

Grossmann, I., \& Kross, E. (2014). Exploring Solomon's Paradox: Self-distancing eliminates the self-other asymmetry in wise reasoning about close relationships in younger and older adults. Psychological Science, 25(8), 1571-1580.

Grossmann, I., Na, J., Varnum, M. E. W., Kitayama, S., \& Nisbett, R. E. (2013). A route to wellbeing: Intelligence versus wise reasoning. Journal of Experimental Psychology: General, 142(3), 944-953.

Grossmann, I., Na, J., Varnum, M. E. W., Park, D. C., Kitayama, S., \& Nisbett, R. E. (2010). Reasoning about social conflicts improves into old age. Proceedings of the National Academy of Sciences of the United States of America, 107(16), 7246-7250.

Grossmann, I., Na, J., W, E., Kitayama, S., \& Nisbett, R. E. (2013). A route to well-being: Intelligence versus wise reasoning. Journal of Experimental Psychology: General, 142(3), 944-953.

Grossmann, I., Sahdra, B. K., \& Ciarrochi, J. (2016). A heart and a mind: Self-distancing facilitates the association between heart rate variability, and wise reasoning. Frontiers in Behavioral Neuroscience, 10, 68.

Grossmann, I., \& Varnum, M. E. W. (2011). Social class, culture, and cognition. Social Psychological and Personality Science, 2(1), 81-89. 
Grube, A., Schroer, J., Hentzschel, C., \& Hertel, G. (2008). The event reconstruction method: An efficient measure of experience-based job satisfaction. Journal of Occupational and Organizational Psychology, 81(4), 669-689.

Haque, U. (2010). The wisdom manifesto. Harvard Business Review. https://hbr.org/2010/02/the-wisdom-planifesto

Haugeland, J. (1989). Artificial intelligence: The very idea. MIT press.

Heckhausen, J., Wrosch, C., \& Schulz, R. (2010). A motivational theory of life-span development. Psychological Review, 117(1), 32.

Helson, R., \& Wink, P. (1987). Two conceptions of maturity examined in the findings of a longitudinal study. Journal of Personality and Social Psychology, 53(3), 531-541.

Henrich, J., Heine, S. J., \& Norenzayan, A. (2010). Most people are not WEIRD. Nature, 466(7302), 29-29.

Hieronymi, P. (2013). The use of reasons in thought (and the use of earmarks in arguments). Ethics, 124(1), 114-127.

Hu, L., \& Bentler, P. M. (1999). Cutoff criteria for fit indexes in covariance structure analysis: Conventional criteria versus new alternatives. Structural Equation Modeling: A Multidisciplinary Journal, 6(1), 1-55.

Huff, L., \& Kelley, L. (2003). Levels of organizational trust in individualist versus collectivist societies: A seven-nation study. Organization Science, 14(1), 81-90.

Hui, C. M., Bond, M. H., \& Molden, D. C. (2012). Why do(n't) your partner's efforts at selfimprovement make you happy? An implicit theories perspective. Personality and Social Psychology Bulletin, 38(1), 101-113. http://doi.org/10.1177/0146167211420734 
Jeste, D. V., Ardelt, M., Blazer, D., Kraemer, H. C., Vaillant, G., \& Meeks, T. W. (2010). Expert consensus on characteristics of wisdom: A Delphi method study. The Gerontologist, $50(5), 668-680$.

John, O. P., Naumann, L. P., \& Soto, C. J. (2008). Paradigm shift to the integrative big give trait taxonomy. In O. P. John, R. W. Robins, \& L. A. Pervin (Eds.), Handbook of personality: Theory and research (pp. 114-158). New York, NY: Guilford Press.

Jolliffe, I. (2002). Principal component analysis and factor analysis. Aberdeen, UK: Wiley Online Library.

Jonassen, D. H. (1997). Instructional design models for well-structured and III-structured problem-solving learning outcomes. Educational Technology Research and Development, 45(1), 65-94.

Jones, E. E., \& Nisbett, R. E. (1972). The actor and the observer: Divergent perceptions of the causes of behavior. In E. E. Jones et al. (Eds.), Attribution: Perceiving the causes of behavior. Morristown, NJ: General Learning Press.

Jordan, J. (2005). The quest for wisdom in adulthood: A psychological perspective. In R. J. Sternberg \& J. Jordan (Eds.), A handbook of wisdom: Psychological perspectives (pp. 160-188). New York, NY, US: Cambridge University Press.

Jordan, J. J., Peysakhovich, A., \& Rand, D. G. (2014). Why we cooperate. The Moral Brain: Multidisciplinary Perspectives. Cambridge, MA: MIT Press.

Jung, C. G. (1965). The relations between the ego and the unconscious. In H. Read, M. Fordham, \& G. Adler (Eds.), Collected works (pp. 173-241). Princeton, NJ: Princeton University Press. 
Kabat-Zinn, J. (2000). Indra's net at work: The mainstreaming of Dharma practice in society. In G. Watson, S. Batchelor, \& G. Clazton (Eds.), The psychology of awakening: Buddhism, science, and our day-to-day lives (pp. 225-249). York Beach, ME: Samuel Weiser.

Kahneman, D., Krueger, A. B., Schkade, D. A., Schwarz, N., \& Stone, A. A. (2004). A survey method for characterizing daily life experience: The day reconstruction method. Science, 306(5702), 1776-1780.

Kallio, E. (2015). From causal thinking to wisdom and spirituality: some perspectives on a growing research field in adult (cognitive) development. Approaching Religion, 5(2), $27-$ 41.

Kay, A. C., \& Ross, L. (2003). The perceptual push: The interplay of implicit cues and explicit situational construals on behavioral intentions in the Prisoner's Dilemma. Journal of Experimental Social Psychology, 39(6), 634-643.

Kekes, J. (1983). Wisdom. American Philosophical Quarterly, 20(3), 277-286.

Kekes, J. (1995). Moral wisdom and good lives (Vol. x). Ithaca, NY, US: Cornell University Press.

Kelley, H. H., \& Stahelski, A. J. (1970). Social interaction basis of cooperators' and competitors' beliefs about others. Journal of Personality and Social Psychology, 16(1), 66-91.

Keltner, D., Kogan, A., Piff, P. K., \& Saturn, S. R. (2014). The sociocultural appraisals, values, and emotions (SAVE) framework of prosociality: Core processes from gene to meme. Annual Review of Psychology, 65(1), 425-460.

Kihlstrom, J. F., Eich, E., Sandbrand, D., \& Tobias, B. A. (2000). Emotion and memory: Implications for self-report. In A. A. Stone, J. S. Turkkan, C. A. Bachrach, J. B. Jobe, H. 
S. Kurtzman, \& V. S. Cain (Eds.), The science of self-report: Implications for research and practice (pp. 81-99). Mahwah, NJ, US: Lawrence Erlbaum Associates Publishers.

Kitayama, S., Ishii, K., Imada, T., Takemura, K. K., \& Ramaswamy, J. (2006). Voluntary settlement and the spirit of independence: Evidence from Japan's "Northern Frontier." Journal of Personality and Social Psychology, 91(3), 369-384.

Kitchner, K. S. (1983). Cognition, metacognition, and epistemic cognition. Human Development, 26(4), 222-232.

Kitchener, K. S., \& Brenner, H. G. (1990). 10 Wisdom and Reflective Judgment: knowing in the face of uncertainty. In R. J. Sternberg (Ed.) Wisdom: Its nature, origins, and development (pp. 212-278). New York, NY: Cambridge University Press.

Kline, R. B. (1998). Software review: Software programs for structural equation modeling: Amos, EQS, and LISREL. Journal of psychoeducational assessment, 16(4), 343-364.

König, S., \& Glück, J. (2014). “Gratitude is with me all the time”: How gratitude relates to wisdom. The Journals of Gerontology Series B: Psychological Sciences and Social Sciences, 69(5), 655-666.

Kramer, D. A. (1990). 13 Conceptualizing wisdom: the primacy of affect-cognition relations. In R. J. Sternberg (Ed.) Wisdom: Its nature, origins, and development (pp. 279-313). New York, NY: Cambridge University Press.

Kramer, D. A. (2002). The ontogeny of wisdom in its variations. In Handbook of adult development (pp. 131-151). New York, NY: Springer.

Kross, E., \& Ayduk, O. (2011). Making meaning out of negative experiences by self-distancing. Current Directions in Psychological Science, 20(3), 187-191. 
Kross, E., \& Grossmann, I. (2012). Boosting wisdom: Distance from the self enhances wise reasoning, attitudes, and behavior. Journal of Experimental Psychology: General, 141(1), $43-8$.

Kunda, Z. (1990). The case for motivated reasoning. Psychological Bulletin, 108(3), 480-498.

Kunzmann, U., \& Baltes, P. B. (2003). Wisdom-related knowledge: Affective, motivational, and interpersonal correlates. Personality \& Social Psychology Bulletin, 29(9), 1104-1119.

Kunzmann, U., \& Thomas, S. (2014). Multidirectional age differences in anger and sadness. Psychology and Aging, 29(1), 16-27.

Labouvie-Vief, G. (1982). Dynamic development and mature autonomy. Human Development, 25(3), 161-191.

Labouvie-Vief, G. (1990). Wisdom as integrated thought: Historical and developmental perspectives. In R. J. Sternberg (Ed.) Wisdom: Its nature, origins, and development (pp. 52-83). New York, NY: Cambridge University Press.

Labouvie-Vief, G. (2003). Dynamic integration affect, cognition, and the self in adulthood. Current Directions in Psychological Science, 12(6), 201-206.

Law, K. S., Wong, C.-S., \& Song, L. J. (2004). The Construct and Criterion Validity of Emotional Intelligence and Its Potential Utility for Management Studies. Journal of Applied Psychology, 89(3), 483-496.

Levenson, M. R. (2009). Gender and wisdom: The roles of compassion and moral development. Research in Human Development, 6(1), 45-59.

Levenson, M. R., Jennings, P. A., Aldwin, C. M., \& Shiraishi, R. W. (2005). Self-transcendence: Conceptualization and measurement. The International Journal of Aging and Human Development, 60(2), 127-143. 
Loevinger, J., \& Blasi, A. (1976). Ego development. San Francisco, US. Jossey-Bass.

McCabe, K. O., \& Fleeson, W. (2015). Are traits useful? Explaining trait manifestations as tools in the pursuit of goals. Journal of Personality and Social Psychology.

McKee, P., \& Barber, C. (1999). On defining wisdom. The International Journal of Aging \& Human Development, 49(2), 149-164.

McLean, K. C., Pasupathi, M., Greenhoot, A. F., \& Fivush, R. (2016). Does intra-individual variability in narration matter and for what? Journal of Research in Personality. http://dx.doi.org/10.1016/j.jrp.2016.04.003

McNulty, J. K., \& Fincham, F. D. (2012). Beyond positive psychology? Toward a contextual view of psychological processes and well-being. American Psychologist, 67(2), 101-110.

Meeks, T. W., \& Jeste, D. V. (2009). Neurobiology of wisdom: A literature overview. Archives of General Psychiatry, 66(4), 355-365.

Meyer, G. J., Finn, S. E., Eyde, L. D., Kay, G. G., Moreland, K. L., Dies, R. R., .. Reed, G. M. (2001). Psychological testing and psychological assessment: A review of evidence and issues. American Psychologist, 56(2), 128-165.

Meyers, L. S., Gamst, G., \& Guarino, A. J. (2006). Applied multivariate research: Design and interpretation. Thousand Oaks, CA: Sage Publications.

Mickler, C., \& Staudinger, U. M. (2008). Personal wisdom: Validation and age-related differences of a performance measure. Psychology and Aging, 23(4), 787-799.

Mienaltowski, A. (2011). Everyday problem solving across the adult life span: Solution diversity and efficacy. Annals of the New York Academy of Sciences, 1235(1), 75-85.

Mischel, W. (2004). Toward an integrative science of the person. Annual Review of Psychology, $55,1-22$. 
Mischel, W., \& Shoda, Y. (1995). A cognitive-affective system theory of personality:

Reconceptualizing situations, dispositions, dynamics, and invariance in personality structure. Psychological Review, 102(2), 246-268.

Mischel, W., Shoda, Y., \& Mendoza-Denton, R. (2002). Situation-behavior profiles as a locus of consistency in personality. Current Directions in Psychological Science, 11(2), 50-54.

Mussel, P. (2013). Intellect: A theoretical framework for personality traits related to intellectual achievements. Journal of Personality and Social Psychology, 104(5), 885-906.

Nisbett, R. E., \& Ross, L. (1980). Human inference: Strategies and shortcomings of social judgment. Englewood Cliffs, NJ: PrenticeHall.

Nolen-Hoeksema, S., \& Morrow, J. (1991). A prospective study of depression and posttraumatic stress symptoms after a natural disaster: The 1989 Loma Prieta earthquake. Journal of Personality and Social Psychology, 61(1), 115-121.

Nonaka, I., \& Takeuchi, H. (2011). The Big Idea: The Wise Leader. Harvard Business Review. https://hbr.org/2011/05/the-big-idea-the-wise-leader.

Park, N., \& Peterson, C. (2008). The cultivation of character strengths. In M. Ferrari \& G. Potworowski (Eds.), Teaching for wisdom: Cross-cultural perspectives on fostering wisdom (pp. 59-77). New York, NY, US: Springer Science + Business Media.

Paulhus, D. L. (1984). Two-component models of socially desirable responding. Journal of Personality and Social Psychology, 46, 598-609.

Paulhus, D. L. (1988). Assessing self-deception and impression management in self-reports: The balanced inventory of desirable responding. Unpublished manuscript. Vancouver, BC: University of British Columbia. 
Paulhus, D. L., \& Vazire, S. (2007). The self-report method. In R. W. Robins, R. C. Fraley, \& R. F. Krueger (Eds.), Handbook of research methods in personality psychology (pp. 224239). New York, NY: Guilford Press.

Peer, E., Brandimarte, L., Samat, S., \& Acquisti, A. (2017). Beyond the Turk: Alternative platforms for crowdsourcing behavioral research. Journal of Experimental Social Psychology, 70, 153-163.

Peng, K., \& Nisbett, R. E. (1999). Culture, dialectics, and reasoning about contradiction. American Psychologist, 54(9), 741.

Piaget, J., Inhelder, B., \& Piaget, J. (2013). The growth of logical thinking from childhood to adolescence: An essay on the construction of formal operational structures (Vol. 84). Routledge.

Podsakoff, P. M., MacKenzie, S. B., \& Podsakoff, N. P. (2012). Sources of method bias in social science research and recommendations on how to control it. Annual Review of Psychology, 63, 539-569.

Pronin, E. (2008). How we see ourselves and how we see others. Science, 320(5880), 11771180.

Pronin, E., Lin, D. Y., \& Ross, L. (2002). The bias blind spot: Perceptions of bias in self versus others. Personality and Social Psychology Bulletin, 28(3), 369-381.

Rahim, M. A., \& Magner, N. R. (1995). Confirmatory factor analysis of the styles of handling interpersonal conflict: first-order factor model and its invariance across groups. Journal of Applied Psychology, 80(1), 122.

Rand, D. G., Greene, J. D., \& Nowak, M. A. (2012). Spontaneous giving and calculated greed. Nature, 489(7416), 427-430. 
Rand, D. G., \& Nowak, M. A. (2013). Human cooperation. Trends in Cognitive Sciences, 17(8), 413-425.

Rapoport, A., \& Guyer, M. (1967). A taxonomy of 2 × 2 games. Bobbs-Merrill.

Riegel, K. F. (1973). Dialectic operations: The final period of cognitive development. Human Development, 16(5), 346-370.

Robinson, M. D., \& Clore, G. L. (2002). Belief and feeling: evidence for an accessibility model of emotional self-report. Psychological Bulletin, 128(6), 934-960.

Rooney, D., \& McKenna, B. (2008). Wisdom in public administration: Looking for a sociology of wise practice. Public Administration Review, 68(4), 709-721. doi:10.1111/j.15406210.2008.00909.x

Ryff, C. D., \& Heincke, S. G. (1983). Subjective organization of personality in adulthood and aging. Journal of Personality and Social Psychology, 44(4), 807-816.

Ryff, C. D., \& Keyes, C. L. M. (1995). The structure of psychological well-being revisited. Journal of Personality and Social Psychology, 69(4), 719-727.

Schwarz, N. (2011). Feelings-as-Information Theory. In P. A. M. Van Lange, A. W. Kruglanski, \& E. T. Higgins (Eds.), Handbook of Theories of Social Psychology (pp. 289-308). Thousand Oaks, CA: SAGE.

Schwarz, N., Kahneman, D., \& Xu, J. (2009). Global and episodic reports of hedonic experience. In R. Belli, D. Alwin, \& F. Stafford (Eds.), Calendar and time diary: Methods in life course research (pp. 157-174). Newbury Park, CA: Sage Publications.

Schwarz, N., \& Oyserman, D. (2001). Asking questions about behavior: Cognition, communication, and questionnaire construction. American Journal of Evaluation, 22(2), 127-160. 
Simon, H. A. (1973). The structure of ill structured problems. Artificial Intelligence, 4(3-4), $181-201$.

Sinnott, J. D. (1984). Postformal reasoning: The relativistic stage. Beyond Formal Operations, 1, $298-325$.

Sinnott, J. D. (1989). Changing the known; knowing the changing: The general systems theory metatheory as a conceptual framework to study complex change and complex thoughts. In Transformation in clinical and developmental psychology (pp. 51-69). Springer.

Smith, E. R., \& Semin, G. R. (2007). Situated social cognition. Current Directions in Psychological Science, 16(3), 132-135.

Staudinger, U. M. (1999). Older and wiser? Integrating results on the relationship between age and wisdom-related performance. International Journal of Behavioral Development, 23(3), 641-664.

Staudinger, U. M., \& Baltes, P. B. (1996). Interactive minds: A facilitative setting for wisdomrelated performance? Journal of Personality and Social Psychology, 71(4), 746-762.

Staudinger, U. M., \& Glück, J. (2011). Psychological wisdom research. Annual Review of Psychology, 62(1), 215-241.

Staudinger, U. M., \& Kunzmann, U. (2005). Positive adult personality development: Adjustment and/or growth?. European Psychologist, 10(4), 320-329.

Staudinger, U. M., Lopez, D. F., \& Baltes, P. B. (1997). The Psychometric Location of WisdomRelated Performance: Intelligence, Personality, and more? Personality and Social Psychology Bulletin, 23(11), 1200-1214. 
Staudinger, U. M., Smith, J., \& Baltes, P. B. (1994). Manual for the assessment of wisdomrelated knowledge. Berlin, Germany: Max Planck Institute for Human Development and Education.

Sternberg, R. J. (1998). A balance theory of wisdom. Review of General Psychology, 2(4), 347365.

Sternberg, R. J. (2010). Why schools should teach for wisdom: The balance theory of wisdom in educational settings. International Psychologist, 36(4), 227-245. doi:10.1207/S15326985EP3604_2

Sternberg, R., \& Jordan, J. (Eds.). (2005). A handbook of wisdom: Psychological perspectives. New York, NY: Cambridge University Press.

Stone, A. A., \& Litcher-Kelly, L. (2006). Momentary Capture of Real-World Data. In M. Eid \& E. Diener (Eds.), Handbook of multimethod measurement in psychology (pp. 61-72). Washington, DC, US: American Psychological Association.

Takahashi, M., \& Bordia, P. (2000). The concept of wisdom: A cross-cultural comparison. International Journal of Psychology, 35(1), 1-9.

Taylor, M., Bates, G., \& Webster, J. D. (2011). Comparing the psychometric properties of two measures of wisdom: Predicting forgiveness and psychological well-being with the SelfAssessed Wisdom Scale (SAWS) and the Three-Dimensional Wisdom Scale (3D-WS). Experimental Aging Research, 37(2), 129-141.

Taylor, S. E., \& Brown, J. D. (1988). Illusion and well-being: a social psychological perspective on mental health. Psychological Bulletin, 103(2), 193. 
Thomas, M. L., Bangen, K. J., Ardelt, M., \& Jeste, D. V. (2017). Development of a 12-Item Abbreviated Three-Dimensional Wisdom Scale (3D-WS-12) Item Selection and Psychometric Properties. Assessment, 24(1), 71-82. doi:10.1177/1073191115595714

Thompson, M. M., Zanna, M. P., \& Griffin, D. W. (1995). Let's not be indifferent about (attitudinal) ambivalence. In R. E. Petty \& J. A. Krosnick (Eds.), Attitude strength: Antecedents and consequences (pp. 361-386). Hillsdale, NJ: Erlbaum

Tiberius, V. (2008). Reflective Wisdom: Living Wisely with Our Limits. Cambridge, UK: Oxford University Press.

Treynor, W., Gonzalez, R., \& Nolen-Hoeksema, S. (2003). Rumination reconsidered: A psychometric analysis. Cognitive Therapy and Research, 27(3), 247-259.

Trope, Y., \& Liberman, N. (2010). Construal-level theory of psychological distance. Psychological Review, 117(2), 440-463.

Tsai, J. L., Miao, F. F., Seppala, E., Fung, H. H., \& Yeung, D. Y. (2007). Influence and adjustment goals: sources of cultural differences in ideal affect. Journal of Personality and Social Psychology, 92(6), 1102.

van Dijk, E., \& De Cremer, D. (2006). Self-benefiting in the allocation of scarce resources: Leader-follower effects and the moderating effect of social value orientations. Personality and Social Psychology Bulletin, 32(10), 1352-1361.

Van Lange, P. A., Joireman, J., Parks, C. D., \& Van Dijk, E. (2013). The psychology of social dilemmas: A review. Organizational Behavior and Human Decision Processes, 120(2), $125-141$.

Vazire, S., \& Carlson, E. N. (2010). Self-knowledge of personality: Do people know themselves? Social and Personality Psychology Compass, 4(8), 605-620. 
Vervaeke, J., \& Ferraro, L. (2013). Relevance, meaning and the cognitive science of wisdom. In The scientific study of personal wisdom (pp. 21-51). The Netherlands: Springer.

Wagenaar, W. A. (1986). My memory: A study of autobiographical memory over six years. Cognitive Psychology, 18(2), 225-252.

Webster, J. D. (2003). An exploratory analysis of a self-assessed wisdom scale. Journal of Adult Development, 10(1), 13-22.

Webster, J. D. (2007). Measuring the character strength of wisdom. The International Journal of Aging and Human Development, 65(2), 163-183.

Wegner, D. M., Vallacher, R. R., Kiersted, G. W., \& Dizadji, D. (1986). Action identification in the emergence of social behavior. Social Cognition, 4(1), 18-38.

Weststrate, N. M., \& Glück, J. (in press). Wiser but not sadder, blissful but not ignorant: Exploring the co-development of wisdom and well-being over time. In M. Robinson \& Mi. Eid (Eds.), The happy mind: cognitive contributions to well-being. New York, NY: Springer.

Weststrate, N. M., Ferrari, M., \& Ardelt, M. (2016). The many faces of wisdom: An investigation of cultural-historical wisdom exemplars reveals practical, philosophical, and benevolent prototypes. Personality and Social Psychology Bulletin, 42(5), 662-676.

White, M. P., \& Dolan, P. (2009). Accounting for the richness of daily activities. Psychological Science, 20(8), 1000-1008.

Wilson, T. D., \& Bar-Anan, Y. (2008). The Unseen Mind. Science, 321(5892), 1046-1047.

Wink, P., \& Helson, R. (1997). Practical and transcendent wisdom: Their nature and some longitudinal findings. Journal of Adult Development, 4(1), 1-16. 
Wink, P., \& Staudinger, U. M. (2015). Wisdom and psychosocial functioning in later life. Journal of personality, 84(3), 306-318. doi: : 10.1111/jopy.12160

Wong, C., \& Law, K. S. (2002). The effects of leader and follower emotional intelligence on performance and attitude. The Leadership Quarterly, 13(3), 243-274.

Yamagishi, T. (1988). The provision of a sanctioning system in the United States and Japan. Social Psychology Quarterly, 51(3), 265-271.

Yamagishi, T., Jin, N., \& Miller, A. S. (1998). In-group bias and culture of collectivism. Asian Journal of Social Psychology, 1(3), 315-328.

Yeh, W., \& Barsalou, L. W. (2006). The situated nature of concepts. The American Journal of Psychology, 199(3) 349-384.

Yuki, M., Maddux, W. W., Brewer, M. B., \& Takemura, K. (2005). Cross-cultural differences in relationship-and group-based trust. Personality and Social Psychology Bulletin, 31(1), 48-62.

Chicago

Zacher, H., McKenna, B., \& Rooney, D. (2013). Effects of self-reported wisdom on happiness: Not much more than emotional intelligence?. Journal of Happiness Studies, 14(6), 16971716. 
Table 1

Sample Characteristics

\begin{tabular}{|c|c|c|c|c|c|c|c|c|c|c|}
\hline Context & Sample A & Workplace & Sample C & Sample D & $\begin{array}{l}\text { Sample E } \\
\text { Interpers. } \\
\& \\
\text { Intergroup }\end{array}$ & Sample F & $\begin{array}{l}\text { Sample G } \\
\text { Interpers. } \\
\text { (Students) }\end{array}$ & $\begin{array}{c}\text { Sample H } \\
\text { Global } \\
\text { (Students) }\end{array}$ & $\begin{array}{l}\text { Sample I } \\
\text { Interpers. } \\
\text { or } \\
\text { Workplace }\end{array}$ & $\begin{array}{c}\text { Sample J } \\
\text { Workplace } \\
\text { and } \\
\text { Interpers. }\end{array}$ \\
\hline Recruited $N$ & 653 & 629 & 398 & 773 & 278 & 340 & 532 & 501 & 619 & 300 \\
\hline Valid $N$ & 404 & 469 & 398 & 730 & 240 & 340 & 467 & 501 & 614 & 300 \\
\hline Age $M(S D)$ & $\begin{array}{c}32.25(10.2 \\
6)\end{array}$ & $\begin{array}{c}32.09(10.2 \\
0)\end{array}$ & $\begin{array}{c}31.51(10.1 \\
9)\end{array}$ & $\begin{array}{c}33.38(11.7 \\
3)\end{array}$ & $\begin{array}{c}29.90(7.81 \\
)\end{array}$ & $\begin{array}{c}34.35(12.4 \\
5)\end{array}$ & $\begin{array}{c}20.04(3.51 \\
)\end{array}$ & $\begin{array}{c}20.05(1.18 \\
)\end{array}$ & $\begin{array}{c}37.82(13.6 \\
0)\end{array}$ & $\begin{array}{c}37.60(12.5 \\
1)\end{array}$ \\
\hline $\begin{array}{c}\text { Gender }(\% \circ) \\
\text { Ethnicity }(\%) \\
\text { Asian- }\end{array}$ & 48.26 & 49.25 & 58.54 & 64.93 & 52.5 & 55.58 & 54.81 & 39.32 & 56.10 & 55.30 \\
\hline $\begin{array}{l}\text { American } \\
\text { African- }\end{array}$ & 5.69 & 6.60 & 5.52 & 4.10 & 8.89 & 5.29 & & & 6.40 & \\
\hline American & 9.65 & 9.38 & 8.04 & 6.98 & 6.68 & 7.05 & & & 9.20 & \\
\hline White & 73.76 & 72.92 & 78.39 & 76.02 & 74.72 & 80.00 & & & 77.80 & \\
\hline Latino & 4.45 & 7.03 & 4.77 & 4.38 & 6.03 & 5.88 & & & 6.60 & \\
\hline Other & 6.43 & 4.05 & 3.26 & 4.52 & 3.83 & 1.76 & & & - & \\
\hline $\begin{array}{l}\text { Median Income } \\
(\$)\end{array}$ & & $\begin{array}{c}35,001- \\
50,000\end{array}$ & $\begin{array}{c}35,001- \\
50,000\end{array}$ & $\begin{array}{c}35,001- \\
50,000\end{array}$ & $\begin{array}{c}25,001- \\
35,000\end{array}$ & $\begin{array}{c}35,001- \\
50,000\end{array}$ & & & $\begin{array}{c}50,001- \\
75,000\end{array}$ & \\
\hline Edu $(\%)$ & & & & & & & & & & \\
\hline High school & & 13.60 & 14.65 & 12.00 & 9.95 & 11.47 & & & 12.00 & 17.00 \\
\hline Some college & & 31.03 & 35.22 & 35.14 & 34.33 & 29.12 & & & 28.50 & 18.00 \\
\hline College & & 44.87 & 38.05 & 38.57 & 39.30 & 44.12 & & & 45.80 & 51.70 \\
\hline Post-grad & & 10.50 & 12.08 & 14.29 & 16.42 & 15.29 & & & 13.70 & 13.30 \\
\hline Med $t$ (Survey) & 15.36 & 20.07 & 11.51 & 24.67 & 20.43 & 7.27 & 23.26 & 16.00 & 17.00 & 20.00 \\
\hline
\end{tabular}

Note. Valid $N=$ participants who passed screening criteria and completed the study. Percentages by socio-demographic group reflect proportion of Valid $N$ in the respective sample. Due to administrative error, income and education questions were not included in Samples A, G, and H, and ethnicity questions were also not included in samples G and H. Sample J ethnicity and income not included due to cross-country differences in meaning of ethnic group, currency, average population income, and its utility. 
Table 2

Principle axis factoring on final 21 wise reasoning items (Sample A)

\begin{tabular}{|c|c|c|c|c|c|c|c|}
\hline \multirow[b]{2}{*}{ Item\# } & \multicolumn{7}{|c|}{ Factor } \\
\hline & 1 & 2 & 3 & 4 & 5 & 6 & 7 \\
\hline 1 & .59 & & & & & & \\
\hline 2 & .41 & & & & .35 & & \\
\hline 3 & .90 & & & & & & \\
\hline 4 & .68 & & & & & & \\
\hline 5 & & .94 & & & & & \\
\hline 6 & & .84 & & & & & \\
\hline 7 & & & .85 & & & & \\
\hline 8 & & & .94 & & & & \\
\hline 9 & & & & .81 & & & \\
\hline 10 & & & & .88 & & & \\
\hline 11 & & & & .51 & .23 & & \\
\hline 12 & & & & .38 & & & \\
\hline 13 & & & & & .64 & & \\
\hline 14 & & & & & .77 & & \\
\hline 15 & & & & & .82 & & \\
\hline 16 & & & & & & .69 & \\
\hline 17 & & & & & & .92 & \\
\hline 18 & & & & & & & .84 \\
\hline 19 & & & & & & & .81 \\
\hline 20 & & & & & & & .90 \\
\hline 21 & & & & & & & .84 \\
\hline
\end{tabular}


Table 3

Model Fit Indices for 1-, 2- 5-, 6-, and 7-factor Models of Wise Reasoning

\begin{tabular}{lcccccc}
\hline Model & AIC & CMIN/DF & CFI & RMSR & RMSEA & PCLOSE \\
\hline Single-factor model & 1144.13 & 6.704 & .972 & .052 & .047 & .974 \\
2-Factor model & 1334.85 & 7.165 & .965 & .061 & .049 & .822 \\
5-Factor model & 1087.21 & 6.617 & .969 & .057 & .046 & .990 \\
6 Factor model (v. A) & 1334.85 & 7.165 & .965 & .061 & .049 & .822 \\
6 factor model (v. B) & 1325.96 & 7.143 & .966 & .060 & .048 & .835 \\
7 factor model & 1409.90 & 7.072 & .966 & .061 & .048 & .876 \\
\hline
\end{tabular}

Note. We assessed model fit with Akaike Information Criterion (AIC), chi-square tests (CMIN/DF), comparative fit index (CFI), standardized root-mean-square residual (RMSR), rootmean-square error of approximation (RMSEA), and probability of close fit (PCLOSE). Model 6 (version A) combined multiple outcomes and change dimension items into a single factor. Model 6 (version B) combined compromise and resolution dimension items into a single factor. Model 5 combined multiple outcomes / change dimension items, and compromise / resolution items into two respective factors. 
Table 4

Loadings from a Principal Component Analysis (PCA) of the five components of wise reasoning on a second-order component

\begin{tabular}{|c|c|c|c|c|c|c|}
\hline & & Interpersonal & & Interpersonal & North America & UK/Europe/Other \\
\hline Dimension & Workplace $^{\mathrm{a}}$ & Online $\mathrm{e}^{\mathrm{b}}$ & Intergroup $^{c}$ & Student $^{\mathrm{d}}$ & $(\text { Conflict 1/Conflict 2) })^{\mathrm{e}}$ & $\left(\right.$ Conflict $1 /$ Conflict 2) ${ }^{\mathrm{e}}$ \\
\hline $\begin{array}{l}\text { Change/multiple } \\
\text { outcomes }\end{array}$ & .76 & .72 & .75 & .77 & $.78 / .77$ & $.78 / .84$ \\
\hline Intellectual humility & .80 & .81 & .79 & .77 & $.86 / .74$ & $.82 / .85$ \\
\hline $\begin{array}{l}\text { Search for } \\
\text { compromise/resolution }\end{array}$ & .84 & .82 & .86 & .80 & $.76 / .84$ & $.84 / .87$ \\
\hline
\end{tabular}

Note. ${ }^{\mathrm{a}}($ Samples A \& B $) ;{ }^{\mathrm{b}}\left(\right.$ Samples C-F); ${ }^{\mathrm{c}}\left(\right.$ Sample E); ${ }^{\mathrm{d}}\left(\right.$ Sample G); ${ }^{\mathrm{e}}$ (Sample J). Standardized coefficients are presented; a single component solution was used to obtain dimension loadings. 
Table 5

Nomological Network Measures, Sample Inclusion, and Number of Items per Measure

\begin{tabular}{|c|c|c|c|c|c|}
\hline Constructs & Sample $(n)$ & Items & $M$ & $S D$ & Reliability $(\alpha / \kappa)$ \\
\hline \multicolumn{6}{|l|}{ Global Self-Assessment of Wisdom } \\
\hline SAWS & $\mathrm{D}(238)$ & 40 & 4.58 & 0.61 & .91 \\
\hline 3DWS & $\mathrm{D}(240)$ & 39 & 3.28 & 0.47 & .87 \\
\hline ASTI & $\mathrm{D}(240)$ & 10 & 3.67 & 0.69 & .80 \\
\hline Global Wise Reasoning & $\mathrm{H}(501)$ & 13 & 4.36 & 0.68 & .89 \\
\hline Intergroup Wise Reasoning (z-scores) & $\mathrm{E}(240)$ & 21 & -- & -- & .93 \\
\hline Observer-rated Wise Reasoning & $E(202)$ & 3 & 0.07 & 1.08 & $.71-.79^{\kappa}$ \\
\hline \multicolumn{6}{|l|}{ Bias-Related Indicators } \\
\hline \multicolumn{6}{|l|}{ Socially Desirable Responding } \\
\hline \multirow[t]{2}{*}{ Self-Deception } & $\mathrm{D}(730)$ & 20 & 0.12 & 0.11 & .71 \\
\hline & $\mathrm{H}(497)$ & 5 & 0.19 & 0.26 & .75 \\
\hline \multirow[t]{2}{*}{ Impression Management } & $\mathrm{D}(698)$ & 20 & 0.12 & 0.11 & .76 \\
\hline & $\mathrm{H}(497)$ & $3^{\mathrm{a}}$ & 0.09 & 0.19 & .66 \\
\hline Bias Blindspot & $\mathrm{D}(705)$ & 1 & 1.42 & 1.81 & -- \\
\hline Biased (vs. Balanced) Attributions & $\mathrm{D}(708)$ & 8 & -0.57 & 0.33 & 60 \\
\hline \multicolumn{6}{|l|}{ Individual Differences } \\
\hline \multicolumn{6}{|l|}{ Big-Five Personality } \\
\hline Openness & B1 (220) & 8 & 3.72 & 0.64 & .82 \\
\hline Conscientiousness & B1 (220) & 8 & 3.88 & 0.69 & .85 \\
\hline Extraversion & B1 (220) & 8 & 3.14 & 0.91 & .83 \\
\hline Agreeableness & B1 (220) & 8 & 3.8 & 0.70 & .83 \\
\hline Neuroticism & B1 (220) & 8 & 2.63 & 0.83 & .85 \\
\hline Intellect & & 24 & & & \\
\hline Seek & B2 (220) & 12 & 5.39 & 1.06 & .95 \\
\hline Conquer & B2 (217) & 12 & 5.00 & 1.15 & .96 \\
\hline Attributional Complexity & B1 (218) & 28 & 4.69 & 0.87 & .92 \\
\hline Perspective taking & A (404) & 7 & 3.41 & 0.73 & .80 \\
\hline Emotional Intelligence & & 12 & & & \\
\hline Self-emotions appraisal & $\mathrm{D}(702)$ & 4 & 5.21 & 1.20 & .90 \\
\hline Others-emotions appraisal & $\mathrm{D}(701)$ & 4 & 5.2 & 1.10 & .89 \\
\hline Use of emotion & $\mathrm{D}(701)$ & 4 & 5.22 & 1.26 & .88 \\
\hline Regulation of emotion & $\mathrm{D}(701)$ & 4 & 4.83 & 1.38 & .90 \\
\hline Mindfulness & & 39 & & & \\
\hline Nonreactivity & B2 (223) & 7 & 3.27 & 0.75 & .87 \\
\hline Observing and attending & B2 (223) & 8 & 3.54 & 0.75 & .87 \\
\hline Acting with awareness & B2 (223) & 8 & 3.30 & 0.88 & .92 \\
\hline Describing with words & B2 (221) & 8 & 3.39 & 0.75 & .85 \\
\hline Non-judging of experience & B2 (220) & 8 & 3.04 & 0.96 & .93 \\
\hline Communal relationship orientation & A (404) & 14 & 2.52 & 0.79 & .88 \\
\hline Emotion Regulation & & 10 & & & \\
\hline Reappraisal & B2 (216) & 6 & 4.88 & 1.22 & .93 \\
\hline Suppression & B2 (216) & 4 & 4.29 & 1.38 & .85 \\
\hline Ruminative Response & & 10 & & & \\
\hline Reflection & $\mathrm{D}(701)$ & 5 & 2.59 & 0.68 & .77 \\
\hline Brooding & $\mathrm{D}(701)$ & 5 & 2.54 & 0.77 & .82 \\
\hline \multicolumn{6}{|l|}{ Social Relations Growth Mindset } \\
\hline Interpersonal relations are changeable & $\mathrm{G}(466)$ & 3 & 4.75 & 1.07 & .79 \\
\hline Social conflicts are changeable & $\mathrm{G}(466)$ & 3 & 5.28 & 0.92 & .85 \\
\hline
\end{tabular}

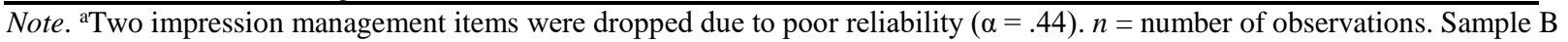
participants responded to one half of the individual differences measures (i.e., B1 or B2). No reliability is reported for Bias Blindspot, which is a single score. $\kappa=$ Cohen's kappa scores for coded dimensions. 
Table 6

Pearson's Correlation between Wise Reasoning (and Its Five Aspects), Global Measures of Wisdom, and Bias-Related Indicators

\begin{tabular}{|c|c|c|c|c|c|c|c|c|c|c|}
\hline Constructs & SWIS & Persp. & Change & Humility & Compr. & $\begin{array}{l}\text { Outsider } \\
\text { Viewpoint }\end{array}$ & SAWS & 3D-WS & ASTI & $\begin{array}{l}\text { Global Wise } \\
\text { Reasoning }\end{array}$ \\
\hline \multicolumn{11}{|l|}{ Global Self-rated Wisdom } \\
\hline SAWS & $.39 * * *$ & $.26 * * *$ & $.30 * * *$ & $.35 * * *$ & $.36 * * *$ & $.28 * * *$ & & & & \\
\hline 3D-WS & $.21 * * *$ & $.17 * *$ & $.14^{*}$ & $.15^{* *}$ & $.26 * * *$ & .01 & & & & \\
\hline ASTI & $.19 * *$ & $.22 * * *$ & $.17 * * *$ & .03 & $.16^{* *}$ & $.13^{*}$ & & & & \\
\hline Observer-rated Wisdom ${ }^{a}$ & $.19 * *$ & $.18^{*}$ & $.27 * *$ & .11 & $.29 * *$ & .05 & & & & \\
\hline \multicolumn{11}{|l|}{ Bias-Related Indicators } \\
\hline Social Desirability & & & & & & & & & & \\
\hline Self-Deception & -.05 & -.03 & .02 & $-.07 *$ & -.01 & $-.09 *$ & $.17 * *$ & $.36 * * *$ & $.23 * * *$ & $.17 * * *$ \\
\hline Imp. Management & $.07 *$ & -.03 & .05 & .04 & $.13 * * *$ & .02 & $.22 * * *$ & $.40 * * *$ & $.24 * * *$ & $.30 * * *$ \\
\hline $\begin{array}{l}\text { Bias Blindspot } \\
\text { Biased (vs. Balanced) }\end{array}$ & $<.01$ & .03 & -.01 & -.05 & .02 & -.01 & $.25 * * *$ & $.18^{* *}$ & $.19 * *$ & - \\
\hline Attributions & $-.11 * *$ & $-.09 *$ & $-.08 *$ & -.04 & $-.11 * *$ & $-.10 * *$ & $-.13^{*}$ & -.04 & -.02 & - \\
\hline
\end{tabular}

Note. SWIS = Situated Wise Reasoning scale; SAWS = Self-assessed wisdom scale. 3D-WS = Three-dimensional wisdom scale. ASTI = Adult self-transcendence inventory. The sample with the Global Measure of Wise Reasoning did not have items for Bias Blindspot and Biased Attributions.

${ }^{\text {a }}$ Unstandardized estimates from a linear regression with wise reasoning (and its five aspects) predicting observer-rated wisdom are reported.

$* p \leq .05, * * p \leq .01, * * * p \leq .001$. 
Table 7

Pearson's Correlations between Wise Reasoning (and Its Five Aspects) and Individual Difference Measures.

\begin{tabular}{|c|c|c|c|c|c|c|}
\hline \multirow[b]{2}{*}{ Constructs } & \multirow{2}{*}{$\begin{array}{l}\text { SWIS } \\
\text { Index }\end{array}$} & \multicolumn{5}{|c|}{ Component of Wise Reasoning } \\
\hline & & Persp. & Change & Humility & Compromise & Outsider \\
\hline \multicolumn{7}{|l|}{ Big-Five Personality } \\
\hline Openness & $.19 * *$ & .08 & $.20 * *$ & $.15^{*}$ & $.16^{*}$ & $.13 \dagger$ \\
\hline Conscientiousness & .02 & -.07 & .08 & .05 & $.12 \dagger$ & $-.13 \dagger$ \\
\hline Extraversion & $.24 * * *$ & $.14^{*}$ & $.21 * * *$ & $.19 * * *$ & $.14 *$ & $.22 * * *$ \\
\hline Agreeableness & $.12 \dagger$ & .04 & $.21 * * *$ & $<.01$ & $.19^{* *}$ & $<.01$ \\
\hline Neuroticism & -.10 & -.09 & -.10 & $-.12 \dagger$ & -.11 & .05 \\
\hline \multicolumn{7}{|l|}{ Intellect } \\
\hline Seek & $.23 * * *$ & $.17 * *$ & $.23 * * *$ & $.13^{*}$ & $.20 * *$ & $.16^{*}$ \\
\hline Conquer & $.24 * * *$ & $.22 * * *$ & $.22 * * *$ & $.16^{*}$ & $.16^{*}$ & $.20 * *$ \\
\hline Attributional Complexity & $.22 * * *$ & .10 & $.16^{*}$ & $.21 * * *$ & $.18 * *$ & $.18 * *$ \\
\hline Perspective taking & $.48 * * *$ & $.39 * * *$ & $.38 * * *$ & $.31 * * *$ & $.48 * * *$ & $.37 * * *$ \\
\hline \multicolumn{7}{|l|}{ Emotional Intelligence } \\
\hline Self-emotions appraisal & $.10 * *$ & $.07 \dagger$ & $.12 * *$ & .05 & $.11 * *$ & $<.01$ \\
\hline Others-emotions appraisal & $.21 * * *$ & $.19 * * *$ & $.13 * * *$ & $.09 *$ & $.23 * * *$ & $.13 * * *$ \\
\hline Use of emotion & $.11^{* *}$ & .04 & $.11 * *$ & .05 & $.13 * * *$ & $.07 \dagger$ \\
\hline Regulation of emotion & $.12 * * *$ & $.11 * *$ & $.09^{*}$ & $.08^{*}$ & $.11 * *$ & $.07 \dagger$ \\
\hline \multicolumn{7}{|l|}{ Mindfulness } \\
\hline Nonreactivity & $.19^{* *}$ & $.17 * *$ & $.16^{*}$ & $.16^{*}$ & $.18 * *$ & .07 \\
\hline Observing and attending & $.42 * * *$ & $.38 * * *$ & $.36^{* * *}$ & $.32 * * *$ & $.34 * * *$ & $.27 * * *$ \\
\hline Acting with awareness & $-.12 \dagger$ & $-.13 \dagger$ & -.03 & -.09 & -.04 & $-.20 * *$ \\
\hline Describing with words & $.17 * *$ & $.14 *$ & $.21 * * *$ & .11 & .11 & .08 \\
\hline Non-judging of experience & -.11 & -.10 & -.11 & -.07 & .01 & $-.18 * *$ \\
\hline Communal relationship orientation & $.24 * * *$ & $.20 * * *$ & $.22 * * *$ & $.18 * * *$ & $.23 * * *$ & $.14 * *$ \\
\hline \multicolumn{7}{|l|}{ Emotion Regulation } \\
\hline Reappraisal & $.23 * * *$ & $.20 * *$ & $.24 * * *$ & $.17 *$ & $.21 * * *$ & .10 \\
\hline Suppression & .05 & .11 & .02 & .11 & .02 & -.06 \\
\hline \multicolumn{7}{|l|}{ Ruminative Response } \\
\hline Reflection & $.26 * * *$ & $.21 * * *$ & $.15^{* * *}$ & $.21 * * *$ & $.19 * * *$ & $.22 * * *$ \\
\hline Brooding & $.13 * * *$ & $.08^{*}$ & $.07 *$ & $.12 * *$ & $.07 \dagger$ & $.19 * * *$ \\
\hline \multicolumn{7}{|l|}{ Social Relations Growth Mindset } \\
\hline Interpersonal rel. are changeable & $.16^{* * *}$ & $.11^{*}$ & $.15^{* * *}$ & $.19 * * *$ & $.11 *$ & .03 \\
\hline Social conflicts are changeable & $.15^{* * * *}$ & $.08 \dagger$ & $.20 * * *$ & $.16^{* * * *}$ & $.11 *$ & $<.01$ \\
\hline
\end{tabular}

Note. $\dagger p \leq .08,{ }^{*} p \leq .05,{ }^{* *} p \leq .01,{ }^{* * *} p \leq .001$. 


\section{Table 8}

Spearman's Correlations between the Wise Reasoning Index (and Its Five Aspects) and Conflict Resolution Behaviors

\begin{tabular}{lcccccc}
\hline Behavior & SWIS & & & & \\
& Index & Persp. & Change & Humility & Compr. & Outsider Viewpoint \\
\hline Find someone to hear both sides & $.15 * * *$ & $.07 * * *$ & $.09 * * *$ & $.12 * * *$ & $.08 * * *$ & $.17 * * *$ \\
Find someone to give advice & $.17 * * *$ & $.11 * * *$ & $.12 * * *$ & $.15 * * *$ & $.12 * * *$ & $.16^{* * *}$ \\
Solve problem together & $.24 * * *$ & $.21 * * *$ & $.13 * * *$ & $.14 * * *$ & $.29 * * *$ & $.05^{*}$ \\
Forgive & $.07 * * *$ & $.11^{* * *}$ & $.06 * *$ & $.04 \dagger$ & $.09 * * *$ & -.02 \\
Disengage & $-.11 * * *$ & $-.17 * * *$ & $-.08^{* * *}$ & $-.06 *$ & $-.13 * * *$ & .03 \\
Retaliate & $-.13 * * *$ & $-.14 * * *$ & $-.11 * * *$ & $-.11 * * *$ & $-.13 * * *$ & -.04 \\
Try to find ally & .01 & -.03 & .01 & $<.01$ & -.01 & $.06 *$ \\
\hline
\end{tabular}

Note. $\dagger p \leq .08, * p \leq .05, * * p \leq .01, * * * p \leq .001$. 
Table 9

Unstandardized Coefficients (Standard Error Estimates in Parentheses) from the Hierarchical Regression Analyses Predicting Balanced Influencing and Adjusting Goals, Causal Inferences, and Work/Family Challenges in Study 5.

\begin{tabular}{|c|c|c|c|c|c|c|}
\hline & \multicolumn{2}{|c|}{ Influencing and Adjusting Goals } & \multicolumn{2}{|c|}{ Causal Inferences } & \multicolumn{2}{|c|}{ Work-Family Conflict } \\
\hline Predictor & Step 1 & Step 2 & Step 1 & Step 2 & Step 1 & Step 2 \\
\hline Intercept & $3.95 * * *(.46)$ & $5.12 * * *(.45)$ & $2.66^{* * *}(.45)$ & $3.09 * * *(.46)$ & $1.99 * * *(.50)$ & $2.51 * * *(.52)$ \\
\hline SAWS & $-.40 * * *(.12)$ & $-.61 * * *(.11)$ & $-.19(.12)$ & $-.27 *(.12)$ & $-.10(.13)$ & $-.18(.13)$ \\
\hline 3D-WS & $-.36 * * *(.10)$ & $-.37 * * *(.10)$ & $-.14(.10)$ & $-.15(.10)$ & $-.23 *(.11)$ & $-.24 *(.11)$ \\
\hline ASTI & $.07(.10)$ & $0.03(.10)$ & $-.03(.10)$ & $-.05(.10)$ & $.18(.11)$ & $.15(.11)$ \\
\hline SWIS & & $.55 * * *(.06)$ & & $.20 * * *(.06)$ & & $.23^{* * *}(.06)$ \\
\hline$R^{2}$ & .06 & .19 & .02 & .04 & .01 & .04 \\
\hline$\Delta R^{2}$ & & $.12 * * *$ & & $.02 * * *$ & & $.02 * * *$ \\
\hline
\end{tabular}

Note : SWIS = Situated wise reasoning scale. SAWS = Self-assessed wisdom scale. $3 \mathrm{D}-\mathrm{WS}=$ Three dimensional wisdom scale. ASTI $=$ Adult self-transcendence inventory. $* p<.05, * * p<.01, * * * p<.001$ 
Table 10.

Means, Standard Deviations, and Intercorrelations of Variables in Study 7.

\begin{tabular}{|c|c|c|c|c|c|c|}
\hline \multirow[b]{2}{*}{ Variables } & \multirow[b]{2}{*}{$M$} & \multirow[b]{2}{*}{$S D$} & \multicolumn{4}{|c|}{ Correlations } \\
\hline & & & 1 & 2 & 3 & 4 \\
\hline \multicolumn{7}{|l|}{ Conflict 1} \\
\hline 1. BWSS & 3.48 & 0.49 & & & & \\
\hline 2. SWIS & 2.94 & 0.75 & $.18 * *$ & & & \\
\hline 3. Balanced Goals & 1.18 & 1.43 & -.02 & $.19 * *$ & & \\
\hline 4. Balanced Causal Inferences & 1.26 & 1.34 & -.06 & $.14^{*}$ & $.24 * *$ & \\
\hline 5. Situational Sensitivity & 3.17 & 1.22 & .09 & $.25^{* *}$ & .07 & $.18 * *$ \\
\hline \multicolumn{7}{|l|}{ Conflict 2} \\
\hline 1. BWSS & 3.48 & 0.49 & & & & \\
\hline 2. SWIS & 3.01 & 0.81 & $.19 * *$ & & & \\
\hline 3. Balanced Goals & 1.24 & 1.57 & -.02 & $.33 * *$ & & \\
\hline 4. Balanced Causal Inferences & 1.06 & 1.30 & $-.13 *$ & $.14^{*}$ & $.23 * *$ & \\
\hline 5. Situational Sensitivity & 3.13 & 1.27 & -.02 & $.13^{*}$ & .10 & $.14^{*}$ \\
\hline
\end{tabular}


Table 11.

Unstandardized Estimates (and Standard Errors) from Multi-Level Models Predicting Balanced Influencing and Adjusting Goals and Causal Inferences in Study 7.

\begin{tabular}{|c|c|c|c|c|}
\hline & \multicolumn{2}{|c|}{ Influencing and Adjusting Goals } & \multicolumn{2}{|c|}{ Causal Inferences } \\
\hline Predictor & Model 1 & Model 2 & Model 1 & Model 2 \\
\hline Intercept & $1.38 * * *(.53)$ & $.23 * * *(.55)$ & $2.16 * * *(.43)$ & $1.53 * * *(.47)$ \\
\hline BWSS & $-.05(.15)$ & $-.22(.15)$ & $-.27 * *(.12)$ & $-.36 * *(.12)$ \\
\hline Trait SWIS & & $.59 * * *(.12)$ & & $.32 * * *(.10)$ \\
\hline State SWIS & & $.45 * * *(.10)$ & & $.18 \dagger(.10)$ \\
\hline$A I C$ & 2132.10 & 2090.00 & 2016.90 & 2007.10 \\
\hline
\end{tabular}




\section{Wise Reasoning: Study Flow}

Scale Development

\section{Study 1}

Item generation, item

selection, reliability

and internal structure

Rellability, Validity, and

Nomological Network

\section{Study 2}

Intra-individual

reliability across two

points in time

Study 3

Initial convergent

and discriminant

validity tests
Study 4

Relations to

individual difference

variables
Implications

and Replications

\section{Study 6}

Balancing influence

vs. adjustment, internal vs, external causal inferences

\section{Study 7}

Intra-individual reliability and

balance across

distinct conflicts

\section{Study 8}

Demographic and social-contextual effects on wise reasoning

Figure 1. Study flow for the current research. 


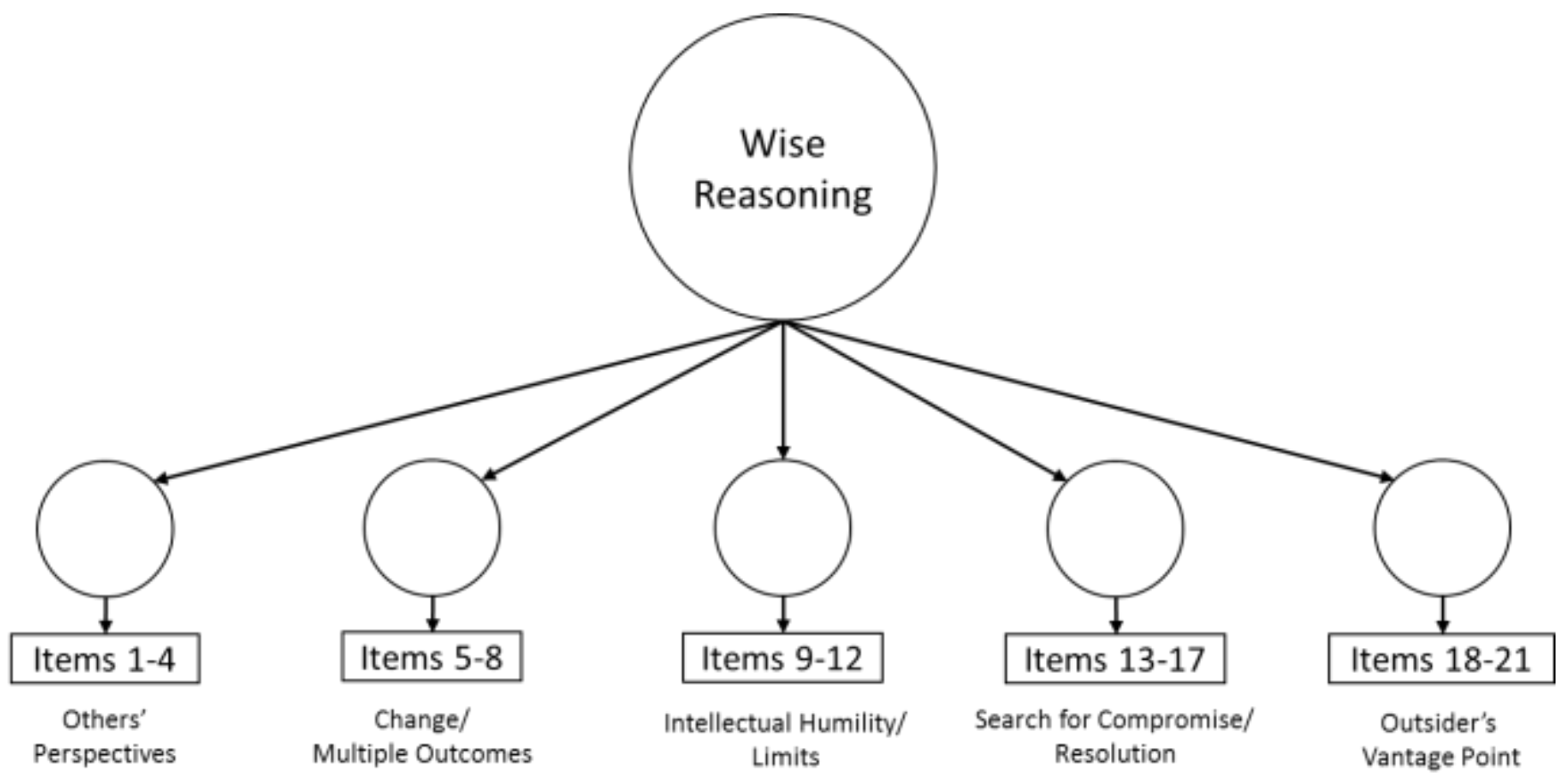

Figure 2. Final 5-factor model of the situated wise reasoning scale. 


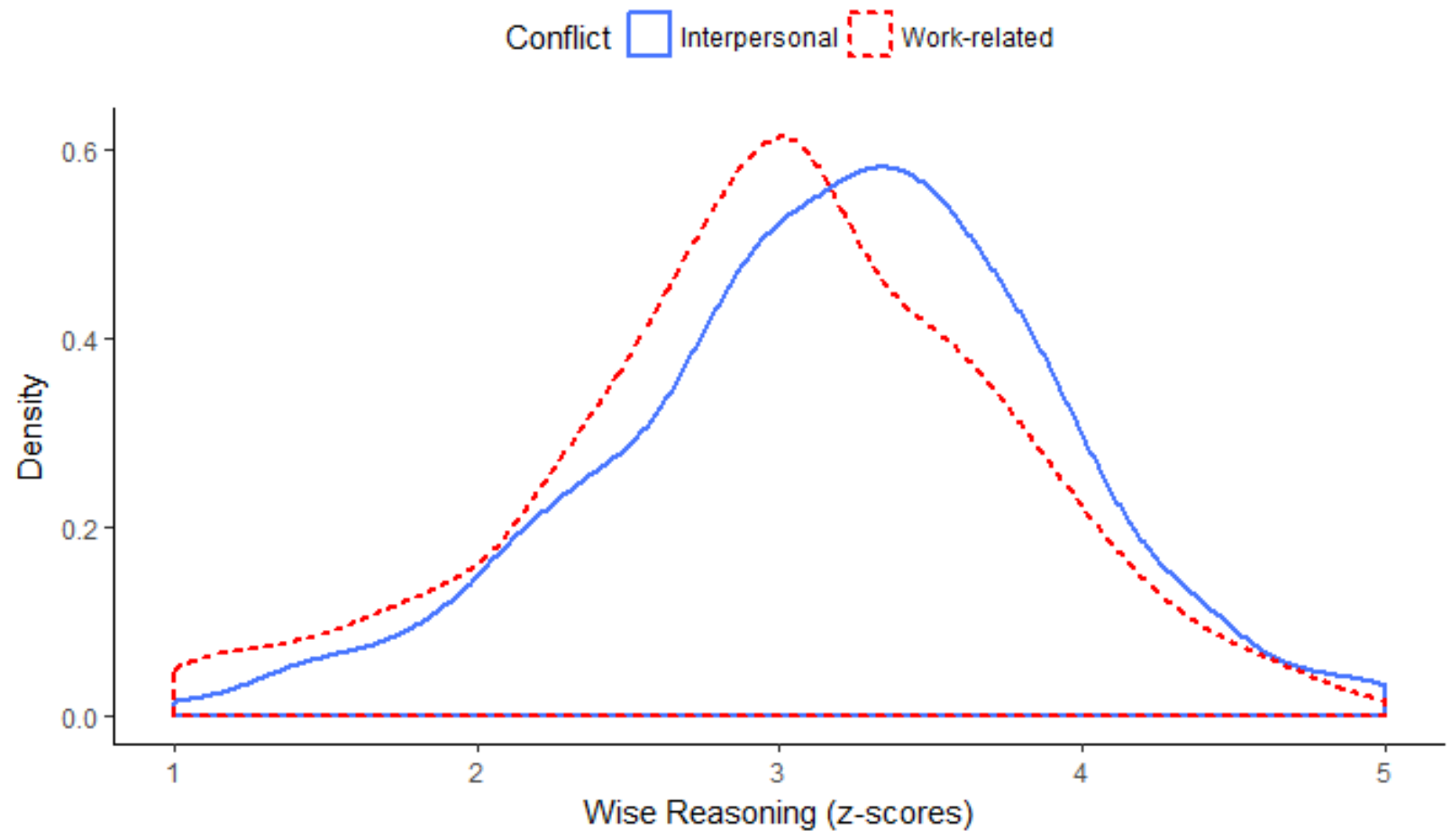

Figure 3. Distribution of wise reasoning across individuals reflecting on interpersonal conflicts involving a friend or in the workplace. 

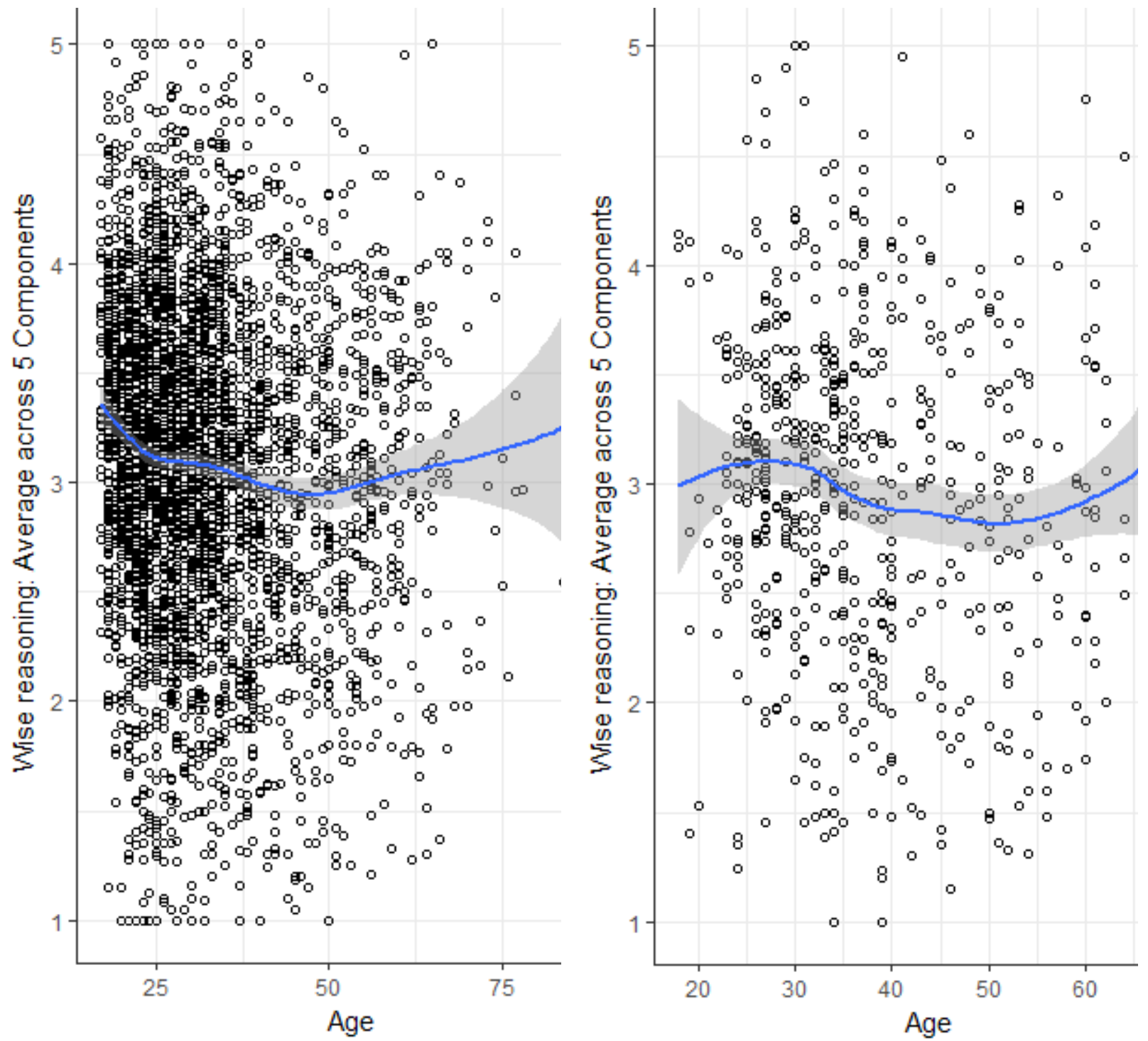

Figure 4. Curvilinear effects of participant age on state-level wise reasoning in conflicts. Panel A: Mturk \& Student Samples C-I, Panel B: Prolific Academic Sample J. 


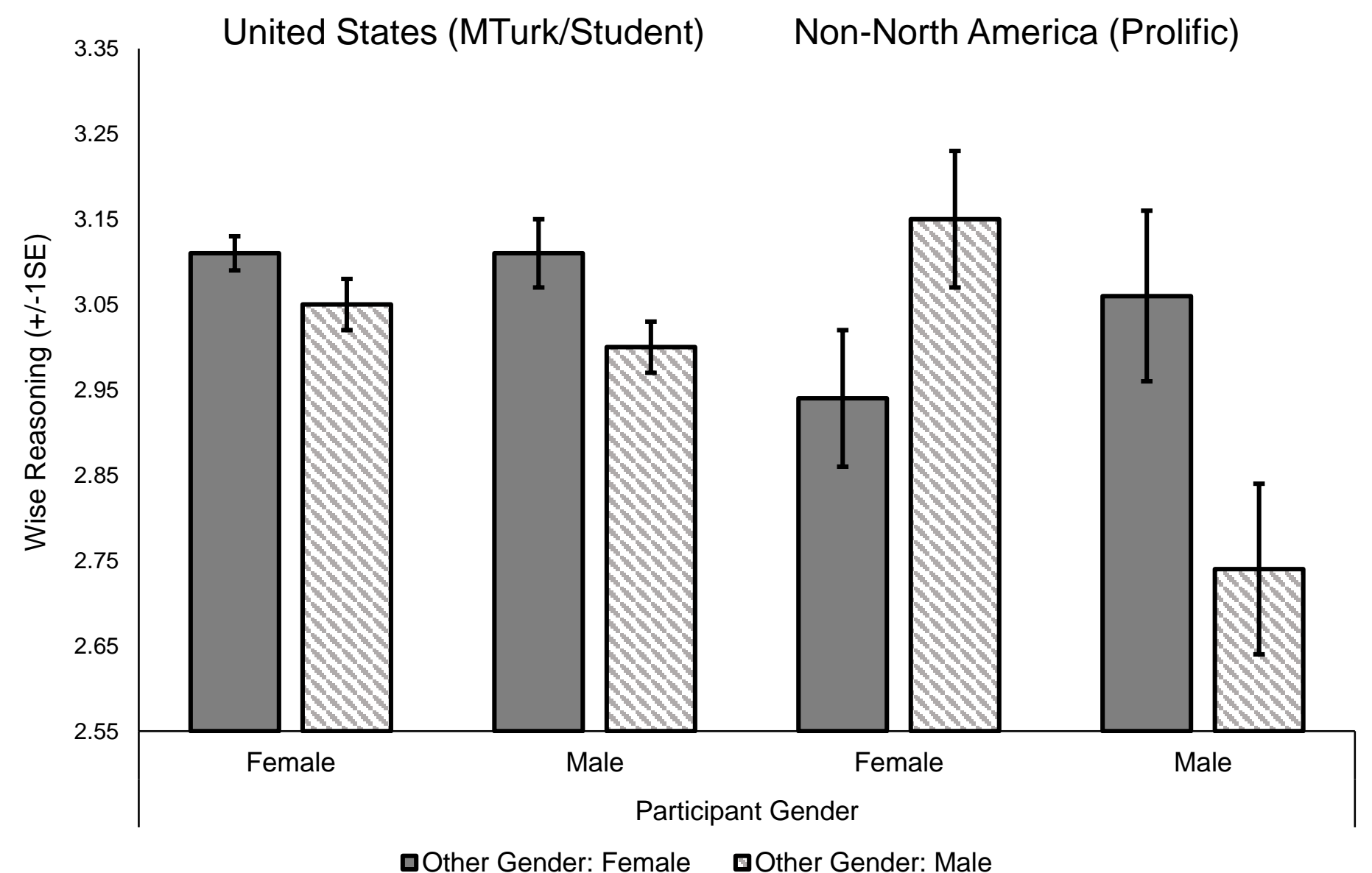

Figure 5. Gender-context (gender $\times$ other's gender interaction) effects in Study 8 . 


\section{Appendix}

\section{Situated WIse reasoning Scale (SWIS)}

1. Put myself in the other person's shoes

2. Tried to communicate with the other person what we might have in common

3. Made an effort to take the other person's perspective

4. Took time to get the other person's opinions on the matter before coming to a conclusion

5. Looked for different solutions as the situation evolved

6. Considered alternative solutions as the situation evolved

7. Believed the situation could lead to a number of different outcomes

8. Thought the situation could unfold in many different ways

9. Double-checked whether my opinion on the situation might be incorrect

10. Double-checked whether the other person's opinions might be correct

11. Looked for any extraordinary circumstances before forming my opinion

12. Behaved as if there may be some information to which I did not have access

13. Tried my best to find a way to accommodate both of us

14. Though it may not have been possible, I searched for a solution that could result in both of us being satisfied

15. Considered first whether a compromise was possible in resolving the situation

16. Viewed it as very important that we resolve the situation

17. Tried to anticipate how the conflict might be resolved

18. Wondered what I would think if I was somebody else watching the situation

19. Tried to see the conflict from the point of view of an uninvolved person

20. Asked myself what other people might think or feel if they were watching the conflict

21. Thought about whether an outside person might have a different opinion from mine about the situation

Note. 1-4: others' perspectives; 5-8: consideration of change and multiple ways situation may unfold; 9-12: intellectual humility/recognition of limits of knowledge; 13-17: search for a compromise / conflict resolution; 18-21: view of the event through the vantage point of an outsider. 
Wisdom, bias, and balance:

Toward a process-sensitive measurement of wisdom-related cognition

SUPPLEMENTARY MATERIALS 


\section{Table of Contents}

Theoretical Model of Wise Reasoning.............................. 3

Initial Scale Items........................................... 4

Scale Development........................................... 5

Phase 1: Principal Components Analysis....................... 5

Phase 2: Principal Axis Factor................................ 8

Phase 3: Confirmatory Factor Analysis...................... 12

Post-peer review Factor Analytic Tests...................... 28

Supplemental Analyses....................................... 31

Additional Materials.............................................. 366 


\section{Theoretical model of wise reasoning}

We used the below model to guide item-design and item-reduction procedures.

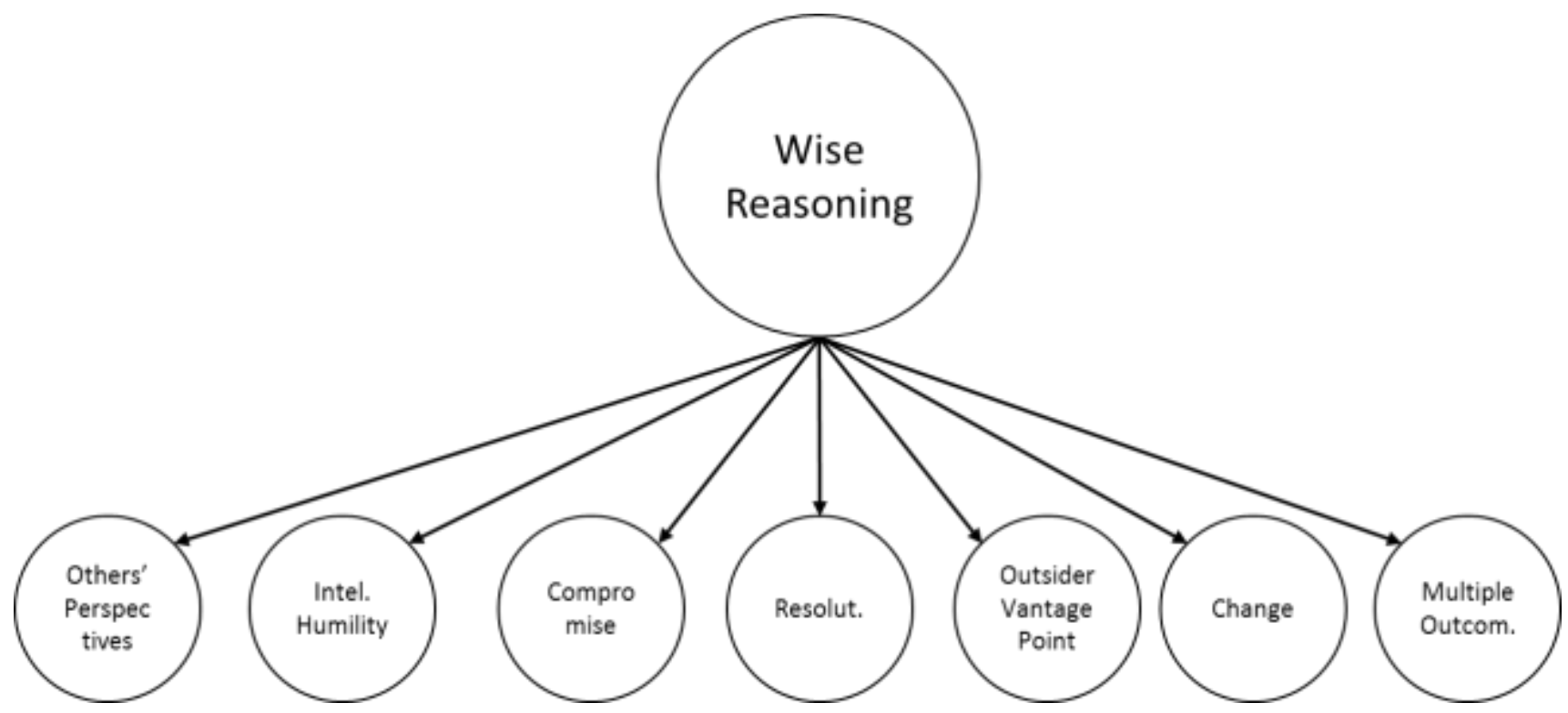

Figure S1. A priori theorized model of wise reasoning (drawing on Bangen et al., 2013; Grossmann et al., 2010; 2013). 


\section{Original scale items (final items are marked by an asterisk)}

\section{Table S1}

\section{Original set of 46 items before item reduction procedure}

\begin{tabular}{|c|c|}
\hline Item \# & Label \\
\hline $1 *$ & Put myself in the other person's shoes \\
\hline $2 * *$ & Tried to not waste time thinking about the other person's beliefs - they did something wrong, and that's that \\
\hline $3 * *$ & Told myself that the other person was wrong for what they were doing \\
\hline 4 & Wondered whether the other person might be right \\
\hline $5 *$ & Tried to communicate with the other person what we might have in common \\
\hline 6 & Wondered about the possibility that we could both be right and/or wrong \\
\hline $7 *$ & Made an effort to take the other person's perspective \\
\hline 8 & Spent time thinking about why the other person felt the way they did \\
\hline $9 *$ & Took time to get the other person's opinions on the matter before coming to a conclusion \\
\hline 10 & Considered how the situation might change through time \\
\hline $11^{* *}$ & Believed that how the situation would work out was set in stone right from the beginning \\
\hline 12 & Thought about how the other person's intentions and opinions might change as the situation evolves \\
\hline 13 & Wondered whether my own intentions and opinions might change as the situation evolves \\
\hline 14 & Approached the situation assuming there were two sides to the situation \\
\hline $15^{*}$ & Looked for different solutions as the situation evolved \\
\hline $16^{*}$ & Considered alternative solutions as the situation evolved \\
\hline 17 & Assumed that there could be multiple ways the situation might unfold \\
\hline $18 * *$ & Knew whose side to take as soon as the conflict began \\
\hline $19 * *$ & Knew exactly how the situation would end \\
\hline 20 & Did not form judgment until I saw the outcome of the situation \\
\hline $21 *$ & Believed the situation could lead to a number of different outcomes \\
\hline $22 *$ & Thought the situation could unfold in many different ways \\
\hline 23 & Wondered whether the situation seemed to be about one issue, but really was about something else \\
\hline 24 & Search for underlying reasons for the situation \\
\hline $25^{*}$ & Double-checked whether my opinion on the situation might be incorrect \\
\hline $26^{*}$ & Double-checked whether the other person's opinions might be correct \\
\hline 27 & Tried to establish whether both parties had common ideas about the situation \\
\hline $28 *$ & Looked for any extraordinary circumstances before forming my opinion \\
\hline $29 *$ & Behaved as if there may be some information to which I did not have access \\
\hline 30 & Tried to understand the context of the situation \\
\hline $31 * *$ & Told myself that I was in the right, and not to worry about extra details \\
\hline $32 * *$ & "Stuck to my guns" on the matter - I knew who was wrong and who was right \\
\hline $33 *$ & Tried my best to find a way to accommodate both of us \\
\hline 34 & Realized that it was not possible for a single involved party to come out as a sole winner from the situation \\
\hline $35^{*}$ & Though it may not have been possible, I searched for a solution that could result in both of us being satisfied \\
\hline $36^{*}$ & Considered first whether a compromise was possible in resolving the situation \\
\hline $37 * *$ & Considered a compromise to be a weak solution in this situation \\
\hline $38 * *$ & Avoided compromise, since it would make it impossible for me to come out satisfied \\
\hline $39^{*}$ & Viewed it as very important that we resolve the situation \\
\hline $40 *$ & Tried to anticipate how the conflict might be resolved \\
\hline 41 & Tried to "step outside myself" to gain perspective on the situation \\
\hline 42 & Attempted to view the situation from a 3rd person perspective \\
\hline $43^{*}$ & Wondered what I would think if I was somebody else watching the situation \\
\hline $44^{*}$ & Tried to see the conflict from the point of view of an uninvolved person \\
\hline $45^{*}$ & Asked myself what other people might think or feel if they were watching the conflict \\
\hline $46^{*}$ & Thought about whether an outside person might have a different opinion from mine about the situation \\
\hline
\end{tabular}

*Item included in final measure.

** Reverse-keyed item 


\section{Scale Development Phase 1: Principal Components Analysis (PCA)}

In the first step we aimed to identify potentially problematic items that might skew the subsequent item reduction procedure (presented in the next step below). To this end, we utilized a principal components analysis (PCA). PCA is well-suited for this purpose. It makes no assumptions about the underlying structure (e.g., latent construct model or a causal model), with a sole purpose of data reduction. Specifically, it reveals overall patterns in the data concerning main components (Jolliffe, 2002). We used PCA to determine the existence of anomalies in responses to the initial set of items (e.g., particularly bad items or components that represented constructs other than an aspect of wise reasoning). We left the PCA unconstrained by specifying eigenvalues $>1$, and requested promax rotation to allow components to correlate.

Through the resulting analysis, we identified that reverse-worded items (highlighted below) clustered together-Component 4: 2,3,11,18,19,31,32; Component 7: 37,38. These items were removed during the subsequent item-reduction procedure. 
Table S2: Principal components analysis on Sample A

\begin{tabular}{|c|c|c|c|c|c|c|c|c|}
\hline \multirow{2}{*}{ Item\# } & \multicolumn{8}{|c|}{ Component } \\
\hline & 1 & 2 & 3 & 4 & 5 & 6 & 7 & 8 \\
\hline 1 & .603 & & & & & & & \\
\hline 2 & & & & .564 & & & & \\
\hline 3 & -.344 & & & .555 & & & & \\
\hline 4 & 906 & & & & & -.380 & & \\
\hline 5 & .714 & & -.252 & & & & & \\
\hline 6 & .924 & & & & & -.241 & & \\
\hline 7 & 953 & & & & & & & \\
\hline 8 & .578 & & & & & & & .449 \\
\hline 9 & .774 & & & & & .258 & & \\
\hline 10 & .524 & & & & & & & \\
\hline 11 & .365 & & & .393 & -.282 & & & .352 \\
\hline 12 & .388 & & & & & & & \\
\hline 13 & .475 & & & & .245 & -.218 & & \\
\hline 14 & .652 & & & & & & & \\
\hline 15 & .232 & & & & & .248 & & .296 \\
\hline 16 & .254 & & & & & .223 & -.243 & .282 \\
\hline 17 & & & & & .460 & & & \\
\hline 18 & & & & .510 & & .220 & & \\
\hline 19 & & & & .529 & -.301 & & & \\
\hline 21 & & & & & .901 & & & \\
\hline 22 & & & & & .949 & & & \\
\hline 23 & & & 416 & & & & & .300 \\
\hline 24 & & & .868 & & & & & .285 \\
\hline 25 & & & .769 & & & & & \\
\hline 26 & .334 & & .700 & & & -.224 & & \\
\hline 27 & .475 & & .361 & & & & & \\
\hline 28 & & & .629 & & & & & \\
\hline 29 & & & .486 & & & & & \\
\hline 30 & & & .307 & & & .387 & & \\
\hline 31 & & & & .618 & & & & \\
\hline 32 & -.246 & & & .556 & & & & \\
\hline 33 & .376 & & & & & .385 & & -.210 \\
\hline 34 & .332 & & & & & & & \\
\hline 35 & .379 & & & & & .255 & -.280 & \\
\hline 36 & .319 & & & & & & -.239 & \\
\hline 37 & & & & & & .346 & .831 & \\
\hline 38 & .236 & & & .306 & & & 699 & \\
\hline 39 & & & & & & .795 & & \\
\hline 40 & & & & & & .894 & .211 & \\
\hline 41 & & .366 & & & & .400 & & \\
\hline 42 & & .622 & & & & .223 & & \\
\hline 43 & & .865 & & & & & & \\
\hline 44 & & .859 & & & & & & \\
\hline 45 & & .955 & & & & & & \\
\hline 46 & & .891 & & & & -.201 & & \\
\hline
\end{tabular}

Notes: Highlights indicate reverse-worded items. 


\section{Scale Development Phase 2, Round 1: Principal Axis Factor (PAF)}

Upon removing negative items that were having undue influence on the component structure in principle components analysis, we began our item-reduction procedure. Here we used principle axis factoring (PAF). We selected PAF because, unlike PCA, it assumes an underlying latent (psychological / causal) structure influencing similarities in responses to different factors of items (Jolliffe, 2002). Theory guiding construction of our items suggested that such latent variables (e.g., bigger-picture thinking) could drive individuals' behaviors when reasoning about a social conflict (e.g., taking an outsider's point of view). Therefore, PAF appeared well-suited for our goal of item reduction. We used this logic for each iteration of the item-reduction procedure, detailed below. Based on the theoretical rationale about seven aspects of wise reasoning employed in prior research (see main text), we requested a forced 7-factor solution and used promax rotation to allow factors to correlate. We looked for items which cross-loaded strongly onto more than one factor; we planned to eliminate any items with $<.4$ loading and with $>.2$ absolute difference between any cross-loading.

Round 1: The matrix in Table S3 identified six items with low factor loading $(12,13,30,41)$ and suboptimal cross-loading $(17,23)$. These items are highlighted in Table S3 and were removed before performing a subsequent item-reduction test.

Round 2: The matrix reported in Table S4 revealed six items with suboptimal cross-loadings (10, 27). These items are highlighted in Table $S 4$ and were removed before performing the subsequent iteration of the item-reduction procedure.

Round 3: The matrix in Table S5 revealed one item with suboptimal cross-loadings (20). This item is highlighted in Table S5 and was removed before performing the subsequent iteration of the item-reduction procedure.

Round 4: The matrix in Table S6 showed that all item loadings were above .4, with no crossloadings above .2. Thus, we stopped the process of item iteration. Item set was reduced to 27 items. The pattern matrix revealed the following items clustered in seven factors: Perspectives $(1,4,5,6,7,8,9,14)$, Change $(15,16)$, Outcomes $(21,22)$, Limits $(24,25,26,28,29)$, Compromise (33, 35, 36), Resolution (39, 40), Outsider (42, 43, 44, 45, 46). We subsequently continued to perform a confirmatory factor analysis (CFA) in IBM SPSS AMOS. 
Table S3

Principal axis factoring on Sample A, round 1

\begin{tabular}{|c|c|c|c|c|c|c|c|}
\hline \multirow[b]{2}{*}{ Item\# } & \multicolumn{7}{|c|}{ Factor } \\
\hline & 1 & 2 & 3 & 4 & 5 & 6 & 7 \\
\hline 7 & .913 & & & & & & \\
\hline 6 & .798 & & & & -.218 & & \\
\hline 9 & .750 & & & & .228 & & \\
\hline 4 & .737 & & & & -.302 & & \\
\hline 8 & .621 & & & & & -.355 & \\
\hline 1 & .585 & & & & & & \\
\hline 5 & .570 & & -.244 & & & .214 & \\
\hline 14 & .507 & & & & & .276 & \\
\hline 10 & .455 & & & & 217 & & \\
\hline 12 & .337 & & & & & & \\
\hline 13 & .326 & & & & -.232 & .250 & \\
\hline 45 & & .935 & & & & & \\
\hline 44 & & .886 & & & & & \\
\hline 46 & & .881 & & & & & \\
\hline 43 & & .881 & & & & & \\
\hline 42 & & .634 & & & & & \\
\hline 41 & & .362 & & & .323 & & \\
\hline 24 & & & . 772 & & & & \\
\hline 25 & & & .713 & & & & \\
\hline 26 & & & .609 & & & .276 & \\
\hline 28 & & & .533 & & & .311 & \\
\hline 29 & & & .451 & & & & \\
\hline 23 & & & .421 & .202 & & -.249 & \\
\hline 22 & & & & .932 & & & \\
\hline 21 & & & & .862 & & & \\
\hline 40 & & & & & .800 & & \\
\hline 39 & & & & & .708 & & \\
\hline 30 & & & .268 & & .279 & & \\
\hline 33 & & & & & & .636 & \\
\hline 36 & & & & & & .622 & \\
\hline 35 & & & & & & .518 & \\
\hline 27 & .272 & & .247 & & & .488 & \\
\hline 20 & & & & & & .437 & \\
\hline 16 & & & & & & & .892 \\
\hline 15 & & & & & & & .808 \\
\hline 17 & & & & .313 & & & .407 \\
\hline
\end{tabular}

Note. Highlights indicate items that were removed in the next round. 
Table S4

Principal axis factoring on Sample A, round 2

\begin{tabular}{|c|c|c|c|c|c|c|c|}
\hline \multirow[b]{2}{*}{ Item\# } & \multicolumn{7}{|c|}{ Factor } \\
\hline & 1 & 2 & 3 & 4 & 5 & 6 & 7 \\
\hline 7 & .893 & & & & & & \\
\hline 6 & .777 & & & & & -.209 & \\
\hline 9 & .725 & & & & & .201 & \\
\hline 4 & .691 & & & & & -.246 & \\
\hline 8 & .583 & & & -.282 & & & \\
\hline 5 & .539 & & -.234 & .289 & & & \\
\hline 1 & .536 & & & & & & \\
\hline 14 & .482 & & & .241 & & & \\
\hline 10 & .447 & & & & & .261 & \\
\hline 45 & & .911 & & & & & \\
\hline 43 & & .861 & & & & & \\
\hline 46 & & .855 & & & & & \\
\hline 44 & & .853 & & & & & \\
\hline 42 & & .608 & & & & & \\
\hline 25 & & & .755 & & & & \\
\hline 26 & & & .705 & & & & \\
\hline 24 & & & .700 & & & & \\
\hline 28 & & & .558 & .272 & & & \\
\hline 29 & & & .431 & & & & \\
\hline 36 & & & & .670 & & & \\
\hline 33 & & & & .654 & & & \\
\hline 35 & & & & .589 & & & \\
\hline 27 & .242 & & .301 & .445 & & & \\
\hline 20 & & & & .435 & & & \\
\hline 22 & & & & & .897 & & \\
\hline 21 & & & & & .864 & & \\
\hline 40 & & & & & & .816 & \\
\hline 39 & & & & & & .723 & \\
\hline 15 & & & & & & & .896 \\
\hline 16 & & & & & & & .821 \\
\hline
\end{tabular}

Note. Highlights indicate items that were removed in the next round. 


\section{Table S5}

Principal axis factoring on Sample A, round 3

\begin{tabular}{|c|c|c|c|c|c|c|c|}
\hline \multirow[b]{2}{*}{ Item\# } & \multicolumn{7}{|c|}{ Factor } \\
\hline & 1 & 2 & 3 & 4 & 5 & 6 & 7 \\
\hline 7 & .934 & & & & & & \\
\hline 9 & .745 & & & & & .233 & \\
\hline 6 & .722 & & & & & -.242 & \\
\hline 4 & .634 & & & & & -.288 & \\
\hline 1 & .568 & & & & & & \\
\hline 8 & .534 & & & & -.245 & & .205 \\
\hline 14 & .522 & & & & & & \\
\hline 5 & .515 & & -.219 & & .282 & & \\
\hline 45 & & .907 & & & & & \\
\hline 43 & & .862 & & & & & \\
\hline 46 & & .858 & & & & & \\
\hline 44 & & .854 & & & & & \\
\hline 42 & & .613 & & & & & \\
\hline 25 & & & .769 & & & & \\
\hline 26 & & & .695 & & & & \\
\hline 24 & & & .689 & & & & \\
\hline 28 & & & .547 & & .253 & & \\
\hline 29 & & & .423 & & & & \\
\hline 22 & & & & .906 & & & \\
\hline 21 & & & & .878 & & & \\
\hline 36 & & & & & .730 & & \\
\hline 33 & & & & & .613 & & \\
\hline 35 & & & & & .608 & & \\
\hline 20 & & & & & .327 & & \\
\hline 40 & & & & & & .784 & \\
\hline 39 & & & & & & .693 & \\
\hline 15 & & & & & & & .885 \\
\hline 16 & & & & & & & .838 \\
\hline
\end{tabular}

Note. Highlights indicate items that were removed in the next round. 
Table S6

Principal axis factoring on Sample A, round 4

\begin{tabular}{|c|c|c|c|c|c|c|c|}
\hline \multirow[b]{2}{*}{ Item\# } & \multicolumn{7}{|c|}{ Factor } \\
\hline & $\overline{1}$ & 2 & 3 & 4 & 5 & 6 & 7 \\
\hline 7 & .939 & & & & & & \\
\hline 9 & .765 & & & & .248 & & \\
\hline 6 & .699 & & & & -.293 & & \\
\hline 4 & .611 & & & & -.334 & & \\
\hline 1 & .583 & & & & & & \\
\hline 14 & .548 & & & & & & \\
\hline 5 & .523 & & -.230 & & & & .291 \\
\hline 8 & .509 & & & & & .203 & \\
\hline 45 & & .901 & & & & & \\
\hline 43 & & .861 & & & & & \\
\hline 46 & & .857 & & & & & \\
\hline 44 & & .849 & & & & & \\
\hline 42 & & .615 & & & & & \\
\hline 25 & & & .785 & & & & \\
\hline 26 & .201 & & .709 & & & & \\
\hline 24 & & & .689 & & & & \\
\hline 28 & & & .549 & & & & .231 \\
\hline 29 & & & .412 & & & & \\
\hline 22 & & & & .943 & & & \\
\hline 21 & & & & .847 & & & \\
\hline 40 & & & & & .750 & & \\
\hline 39 & & & & & .670 & & \\
\hline 15 & & & & & & .901 & \\
\hline 16 & & & & & & .837 & \\
\hline 36 & & & & & & & .691 \\
\hline 35 & & & & & & & .568 \\
\hline 33 & & & & & & & .532 \\
\hline
\end{tabular}




\section{Scale Development Phase 3, Step 1: Confirmatory factor analysis (CFA) on Sample A}

We conducted CFA to test the fit of our model. We assessed model fit with standard criteria using standardized root-mean-square residual (RMSR; $<.10$ ), root-mean-square error of approximation (RMSEA; < .08), comparative fit index (CFI; > .95), and probability of close fit (PCLOSE; > .05) (Hu \& Bentler, 1999; Meyers, Gamst, \& Guarino, 2006). According to these guidelines, CFA that the initial 7-factor model could be improved (PCLOSE < .01).

Additionally, we used the results of the CFA to further reduce the scale to approximately 20 items. Our goal was to keep 2-4 items for each factor, to allow for approximately equal weighting in the final measure. From the PAF, above, and as seen in the diagram below, we found that three factors remained with over four items, and needed to be reduced.

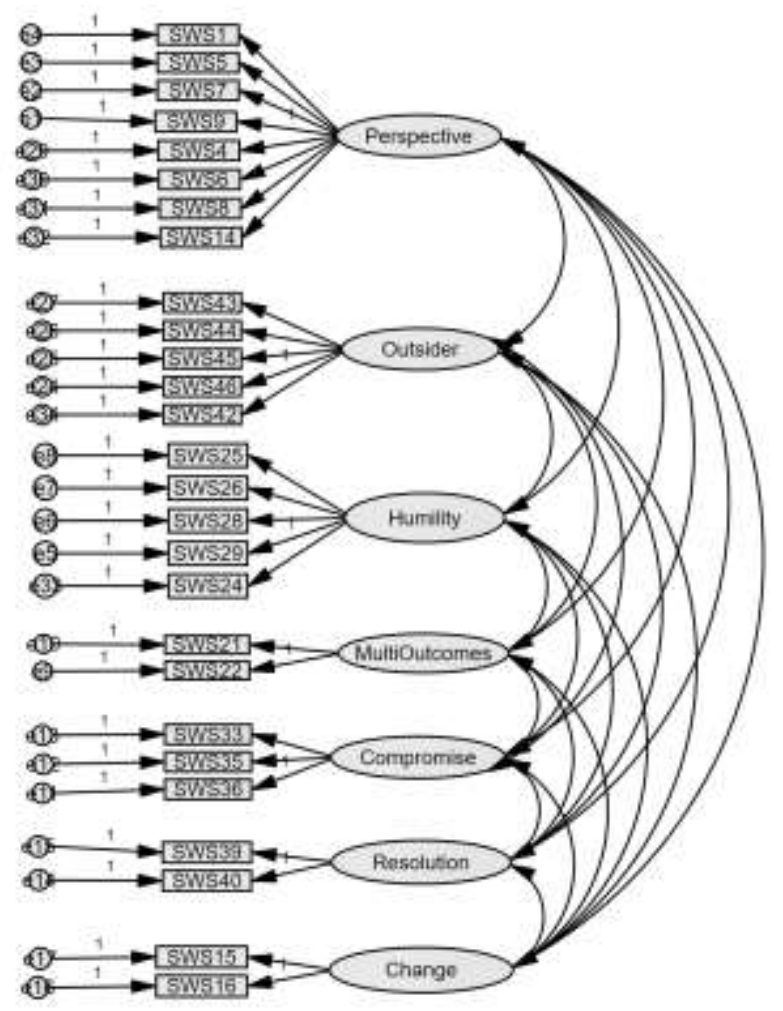

Figure S2. Confirmatory factor analysis on Sample A - 27 items.

We conducted a confirmatory factor analysis on the model depicted in Figure S2, examining model fit. Model fit was good, but showed PCLOSE <.05, indicating that the model could be improved. To examine potential adjustments, we next examined modification indices to further strengthen the model fit by allowing some error terms to covary.

$\begin{array}{ll}\text { CMIN/df } & 2.556 \\ \text { CFI } & .930 \\ \text { RMSR } & .073 \\ \text { RMSEA } & .062 \\ \text { PCLOSE } & .000\end{array}$


Modification indices in Table S7 indicates four pairs of error terms (highlighted in grey) with relatively high modification indices (> 20), suggesting that covarying these error term-pairs could improve the model fit.

Table S7

Modification indices for the 7-factor model of wise reasoning (Figure S2)

\begin{tabular}{|c|c|c|c|c|c|}
\hline Error terms & M.I. & Change & Error terms & M.I. & Change \\
\hline e31<-->e33 & 17.971 & 0.191 & e7<-->e29 & 13.241 & 0.137 \\
\hline e31<-->e32 & 6.136 & -0.1 & e7<-->e27 & 6.456 & -0.076 \\
\hline e30<-->e33 & 5.368 & -0.103 & e7<-->e24 & 4.458 & 0.071 \\
\hline e29<-->e33 & 4.829 & -0.093 & e7<-->e14 & 11.376 & -0.116 \\
\hline e29<-->e30 & 82.235 & 0.377 & e7<-->e8 & 47.697 & 0.257 \\
\hline e27<-->e33 & 8.346 & 0.098 & e6<-->e34 & 6.89 & 0.091 \\
\hline e27<-->e30 & 7.327 & -0.09 & e6<-->e24 & 9.714 & -0.102 \\
\hline e25<-->e34 & 9.002 & -0.096 & e6<-->e8 & 8.275 & -0.104 \\
\hline e25<-->e31 & 5.955 & 0.089 & e6<-->e7 & 5.548 & -0.079 \\
\hline e24<-->e29 & 4.093 & 0.072 & e5<-->e30 & 4.917 & 0.102 \\
\hline e24<-->e25 & 20.849 & 0.138 & e5<-->e26 & 6.81 & 0.098 \\
\hline e17<-->e33 & 4.659 & 0.07 & e5<-->e8 & 19.646 & -0.194 \\
\hline e17<-->e32 & 10.058 & 0.093 & e5<-->e7 & 8.474 & -0.119 \\
\hline e16<-->e32 & 5.32 & -0.067 & e5<-->e6 & 21.749 & 0.185 \\
\hline e14<-->e26 & 4.351 & 0.065 & e4<-->e34 & 8.177 & 0.104 \\
\hline e14<-->e24 & 9.143 & -0.099 & e4<-->e30 & 6.605 & -0.102 \\
\hline e13<-->e33 & 6.251 & -0.1 & e3<-->e33 & 5.638 & -0.115 \\
\hline e13<-->e29 & 5.531 & -0.088 & e2<-->e17 & 6.749 & -0.066 \\
\hline e12<-->e32 & 6.42 & -0.078 & e2<-->e9 & 6.51 & 0.062 \\
\hline e11<-->e32 & 6.356 & 0.086 & e2<-->e4 & 4.966 & 0.07 \\
\hline e11<-->e31 & 7.715 & -0.105 & e1<-->e31 & 5.939 & 0.093 \\
\hline e11<-->e29 & 5.432 & 0.083 & e1<-->e30 & 6.769 & -0.098 \\
\hline e9<-->e26 & 5.447 & -0.058 & e1<-->e29 & 8.617 & -0.106 \\
\hline e9<-->e24 & 4.25 & 0.054 & e1<-->e24 & 10.595 & -0.106 \\
\hline e8<-->e32 & 4.79 & -0.084 & e1<-->e17 & 4.368 & 0.058 \\
\hline e7<-->e33 & 7.685 & -0.11 & e1<-->e15 & 4.91 & 0.083 \\
\hline e7<-->e30 & 5.516 & 0.092 & $\mathrm{e} 1<-->\mathrm{e} 11$ & 7.78 & -0.09 \\
\hline
\end{tabular}

Note. Highlights indicate items with high modification indices.

After covarying error terms, we reexamined the model fit, observing an improved fit.

$\begin{array}{ll}\text { CMIN/df } & 1.972 \\ \text { CFI } & .957 \\ \text { RMSR } & .066 \\ \text { RMSEA } & .049 \\ \text { PCLOSE } & .592\end{array}$


Next, we turned to reducing potential excess items, following three criteria:

1. Keep 2-4 items per factor (perspectives/humility/compromise/resolution/outsider's vantage point/change/multiple ways a situation may unfold), for approximately equal weighting of each factor;

2. Remove items clustering in a factor that we did not a priori hypothesize (e.g., an item written for "outsider's vantage point clustering with "intellectual humility" items);

3. Remove items with greatest frequency of absolute standardized residual covariances $>.4$, indicating over- or under- estimating relations between variables

As indicated in Table S8 below, item 14 grouped into a different factor (other's perspective) than initially planned (recognition of limits of knowledge). Therefore, we removed this item for from the final item list. Next, we checked standardized residual covariances, including items from the dimensions "other's perspective," "outsider's vantage point," and "intellectual humility/limits of one's knowledge" that had the most frequent covariances $>.4$. As highlighted below, items 4, 6, 8,24 and 42 exhibited the most frequent covariances $>.4$. These items were therefore selected for removal from the final item list. These processes left us with a set of 21 final wise reasoning items. 
Table S8

Standardized residual covariances for the 7-factor model of wise reasoning (Figure S2) with those > .4 shaded in grey

\begin{tabular}{|c|c|c|c|c|c|c|c|c|c|c|c|c|c|c|c|c|c|c|c|c|c|c|c|c|c|c|c|}
\hline Item & 42 & 24 & 14 & 8 & 6 & 4 & 43 & 44 & 45 & 46 & 15 & 16 & 39 & 40 & 33 & 35 & 36 & 21 & 22 & 25 & 26 & 28 & 29 & 1 & 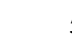 & 7 & 9 \\
\hline $42^{\mathrm{d}}$ & 0 & & & & & & & & & & & & & & & & & & & & & & & & & & \\
\hline $24^{\mathrm{c}}$ & 0.59 & 0 & & & & & & & & & & & & & & & & & & & & & & & & & \\
\hline $14^{\mathrm{a}}$ & 1.37 & -0.87 & 0 & & & & & & & & & & & & & & & & & & & & & & & & \\
\hline $8^{\mathrm{b}}$ & 2.44 & 1.85 & -1.19 & 0 & & & & & & & & & & & & & & & & & & & & & & & \\
\hline $6^{\mathrm{b}}$ & 0.46 & -1.39 & -0.20 & 0.11 & 0 & & & & & & & & & & & & & & & & & & & & & & \\
\hline $4^{\mathrm{b}}$ & 0.03 & -1.02 & -0.30 & 0.05 & 0 & 0 & & & & & & & & & & & & & & & & & & & & & \\
\hline $43^{\mathrm{d}}$ & -0.21 & 0.77 & -0.62 & 1.15 & -2.66 & -1.37 & 0 & & & & & & & & & & & & & & & & & & & & \\
\hline $44^{\mathrm{d}}$ & 0.08 & -0.56 & 0.36 & 0.70 & -1.47 & -0.80 & -0.07 & 0 & & & & & & & & & & & & & & & & & & & \\
\hline $45^{\mathrm{d}}$ & -0.56 & 0.14 & 0.82 & 2.16 & -1.80 & -1.11 & 0.29 & 0.20 & 0 & & & & & & & & & & & & & & & & & & \\
\hline $46^{\mathrm{d}}$ & -0.19 & 0.76 & 1.13 & 2.14 & -0.41 & 0.31 & 0.24 & -0.06 & 0 & 0 & & & & & & & & & & & & & & & & & \\
\hline 15 & 1.21 & 1.23 & 1.31 & 1.62 & -1.05 & -1.17 & -0.42 & 0.01 & -0.18 & -0.35 & 0 & & & & & & & & & & & & & & & & \\
\hline 16 & 0.99 & 0.72 & 0.10 & 1.61 & -0.61 & -0.75 & -0.08 & -0.42 & -0.31 & 0.22 & 0 & 0 & & & & & & & & & & & & & & & \\
\hline 39 & 0.93 & 0.38 & -0.01 & 1.01 & -2.28 & -2.67 & 0.14 & -0.33 & -0.67 & -0.27 & 0.31 & -0.00 & 0 & & & & & & & & & & & & & & \\
\hline 40 & 1.03 & 0.24 & -0.52 & 1.46 & -2.38 & -2.52 & 0.32 & 0.16 & -0.92 & -1.24 & -0.38 & 0.27 & 0 & 0 & & & & & & & & & & & & & \\
\hline 33 & 2.15 & -1.72 & 0.88 & 0.06 & -0.08 & -0.99 & 0.16 & -0.17 & -0.44 & 0.06 & 0.42 & 0.65 & 0.43 & 0.65 & 0 & & & & & & & & & & & & \\
\hline 35 & 2.11 & -0.41 & -0.26 & 0.26 & 0.05 & -0.08 & 0.07 & -0.77 & -0.32 & -0.46 & 0.04 & -0.11 & -0.03 & -0.29 & -0.13 & 0 & & & & & & & & & & & \\
\hline 36 & 1.34 & -0.38 & 0.97 & -1.07 & 0.21 & 0.70 & -0.50 & -0.72 & -0.89 & -0.36 & -0.61 & -0.06 & -0.44 & 0.00 & -0.06 & 0.13 & 0 & & & & & & & & & & \\
\hline 21 & 1.59 & 0.48 & 1.63 & 0.31 & -0.22 & -0.64 & 0.22 & -0.78 & 0.44 & 0.85 & 0.23 & 0.04 & -0.57 & -0.42 & 0.97 & -0.30 & 0.62 & 0 & & & & & & & & & \\
\hline 22 & 1.25 & 0.26 & 1.46 & 0.26 & -0.29 & -0.26 & -0.42 & -1.49 & 0.25 & 0.96 & 0.05 & -0.24 & 0.01 & 0.37 & 0.46 & -0.80 & 0.35 & 0 & 0 & & & & & & & & \\
\hline $25^{\mathrm{c}}$ & 0.64 & 1.09 & -0.08 & 0.76 & 0.49 & 1.41 & -1.24 & -1.02 & -0.69 & 0.28 & -0.31 & -0.39 & 0.33 & -0.14 & -0.57 & -0.05 & 0.54 & -0.96 & -0.88 & 0 & & & & & & & \\
\hline $26^{\mathrm{c}}$ & 0.04 & -0.51 & 1.78 & 0.62 & 2.04 & 2.99 & -1.61 & -1.13 & -0.52 & 0.18 & -0.50 & -0.82 & -0.89 & -1.92 & -0.08 & 0.69 & 0.80 & 0.34 & 0.18 & 0 & 0 & & & & & & \\
\hline $28^{\mathrm{c}}$ & 2.06 & 0.22 & 0.64 & -0.45 & 0.29 & 0.50 & 0.12 & -0.03 & -0.46 & -0.56 & -0.17 & $\begin{array}{l}-0.28 \\
\end{array}$ & 0.24 & 0.76 & -0.05 & 0.13 & 0.33 & 0.14 & 0.03 & -0.17 & 0.00 & 0 & & & & & \\
\hline $29^{\mathrm{c}}$ & 2.29 & 0.58 & 0.12 & 0.93 & 1.32 & 1.20 & 0.50 & 1.43 & 0.22 & 0.95 & 0.33 & 0.37 & 0.28 & 0.95 & -0.02 & -0.13 & -0.14 & 0.03 & -0.19 & -1.12 & -0.41 & 0 & 0 & & & & \\
\hline $1^{\mathrm{b}}$ & 3.12 & -0.18 & 0.10 & -0.69 & -0.86 & -0.58 & -0.15 & 0.92 & 0.30 & 0.65 & -0.88 & -1.33 & -0.78 & 0.11 & 0.33 & 0.71 & 0.08 & -1.89 & -1.74 & 1.29 & 1.04 & -0.02 & -0.2 & 0 & & & \\
\hline $5^{\mathrm{b}}$ & 1.92 & -2.10 & -0.64 & -0.26 & 0.97 & 0.77 & 0.46 & 0.49 & -0.15 & 0.25 & 0.29 & 0.73 & 0.76 & 0.51 & 1.35 & 1.27 & 1.29 & -1.03 & -1.12 & -0.59 & -0.36 & 0.04 & -0.52 & -0.54 & 0 & & \\
\hline $7^{b}$ & 1.58 & -1.54 & 0.17 & -0.14 & 0.56 & 0.48 & -1.19 & -0.24 & -0.53 & -0.34 & -0.80 & -0.19 & -0.67 & 0.10 & -0.04 & -0.49 & -1.03 & 0.17 & 0.69 & 0.21 & 0.38 & -1.27 & -1.09 & 0.57 & -0.00 & 0 & \\
\hline $9^{b}$ & 1.16 & -0.34 & 0.13 & 0.734 & -0.57 & -0.67 & -1.05 & -0.89 & -0.89 & -1.38 & 0.55 & -0.11 & 1.19 & 1.01 & 0.1 & 0.17 & -1.03 & 0.09 & -0.33 & 1.12 & 1.08 & -0.60 & -0.48 & 0.01 & -0.15 & 0.08 & 0 \\
\hline
\end{tabular}


Table S9

Frequency of standardized residual covariance among items from factors with excess items

\begin{tabular}{llllllllllllllllll}
\hline Item & $1^{\mathrm{b}}$ & $4^{\mathrm{b}}$ & $5^{\mathrm{b}}$ & $6^{\mathrm{b}}$ & $7^{\mathrm{b}}$ & $8^{\mathrm{b}}$ & $9^{\mathrm{b}}$ & $24^{\mathrm{c}}$ & $25^{\mathrm{c}}$ & $26^{\mathrm{c}}$ & $28^{\mathrm{c}}$ & $29^{\mathrm{c}}$ & $42^{\mathrm{d}}$ & $43^{\mathrm{d}}$ & $44^{\mathrm{d}}$ & $45^{\mathrm{d}}$ & $46^{\mathrm{d}}$ \\
\hline Frequency & 16 & 19 & 16 & 17 & 14 & 18 & 16 & 18 & 15 & 17 & 9 & 14 & 21 & 13 & 15 & 15 & 12 \\
Remove & & $\mathrm{x}$ & & $\mathrm{x}$ & & $\mathrm{x}$ & & $\mathrm{x}$ & & & & & $\mathrm{x}$ & & & & \\
\hline
\end{tabular}

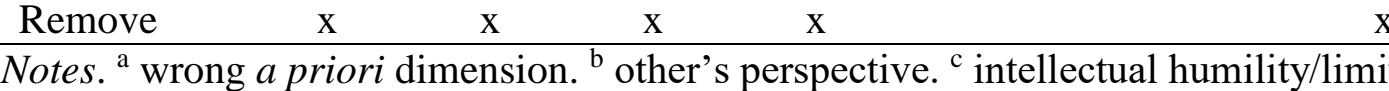

the 


\section{Confirmatory analyses on new samples}

After removing items 4, 6, 8, 14, 24 and 42 we conducted another CFA on the remaining 21 items, as shown below. This model yield an excellent model fit. Thus, we settled on the remaining 21 items for the final scale, finalizing the scale development procedure. In the next sections we detail further tests on subsequent samples (after Sample A):

- In Sample B, we conducted another confirmatory test of the factor structure, simultaneously using distractor items;

- Upon collecting Samples C-G, we conducted another confirmatory factor analysis to test model fit over a much larger, new sample. We did this analysis both on the total C-G sample, as well as using a lock-box approach: splitting the total sample into two random sub-sets and testing model fit on each.

Figure S3. Confirmatory factor analysis on Sample A CMIN/df $\quad 1.516$

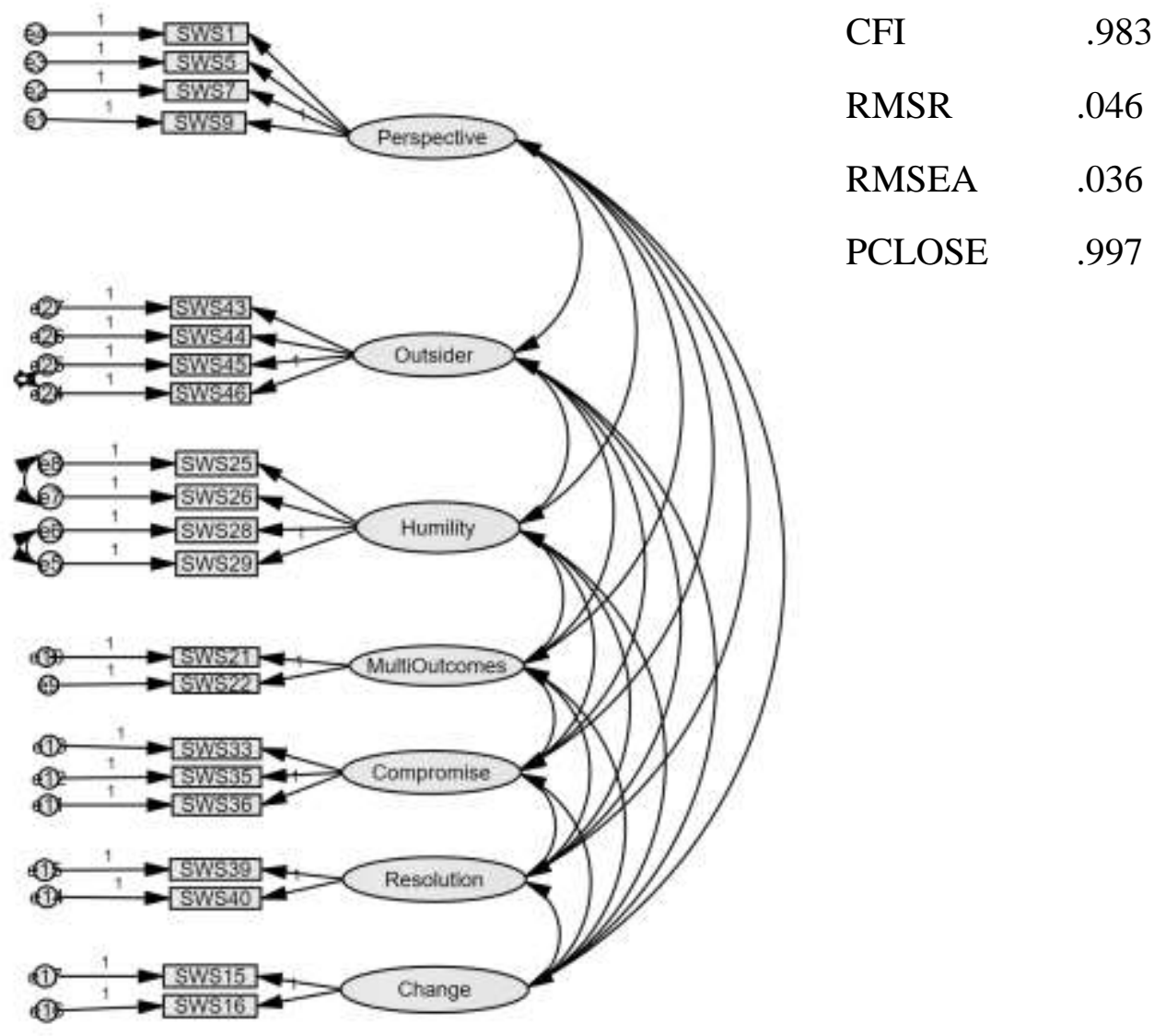




\section{$\underline{\text { Sample B Distractor item test }}$}

We developed a set of distractor items. These items appeared to be plausible conflict processing strategies (e.g., they asked questions concerning conflict behaviors, but did not theoretically belong to any aspects of wise reasoning as discussed in the main text) for inclusion in Sample B. We wanted to test whether these items grouped with the aspects of wise reasoning outlined in prior scholarship on wisdom (see Baltes \& Smith, 2008; Grossmann, 2017, for reviews).

Distractor items are listed in Table S10 below.

Table S10. Distractor items used in Sample B.

Avoidance

1. Tried to pretend the situation wasn't happening

2. Tried to get the person to accept the blame for the situation

3. Admitted to myself that I couldn't solve the problem and quit trying

Trust

4. Kept in mind that it would be wise to not trust the other person

Worry

5. Worried a great deal about the consequences of this issue for myself

6. Thought over and over about how the situation could have been prevented

7. Kept trying to figure out what the other person must have been thinking to do the things they did

We conducted a Principal Components Analysis (PCA) with eigenvalues > .1 and promax rotation to allow components to correlate to test how inclusion of these items affected the component structure. We expected that distractor items would cluster together, producing their own component, or to load weakly or cross-load substantially onto multiple components. As indicated in Table S11 below, distractor items either clustered into a separate component or loaded weakly with other wise reasoning items but cross-loaded substantially (voiding clear identification with any concrete aspect of wise reasoning). Distractor items are marked with a D (refer to Table S10) and highlighted in Table S11. Follow-up PCA without distractor items showed that item loadings for the wise reasoning scale items from Sample B were nearly identical to those from Sample A (e.g., only item 12 cross-loaded onto multiple components), see Table S12. 
Sample B PCA (eigenvalues > 1, promax) including distractor items

Table S11

Principal components analysis on Sample B, including distractor items

\begin{tabular}{|c|c|c|c|c|c|c|c|}
\hline \multirow[b]{2}{*}{ Item \# } & \multicolumn{7}{|c|}{ Component } \\
\hline & 1 & 2 & 3 & 4 & 5 & 6 & 7 \\
\hline 1 & .799 & .118 & & & & & \\
\hline 3 & .785 & & & & & & \\
\hline 4 & .753 & & & & & & .104 \\
\hline 2 & .743 & & & & & & \\
\hline 19 & .110 & .800 & & & & & \\
\hline 20 & & .772 & & & & & \\
\hline 21 & -.130 & .716 & & .142 & .108 & & \\
\hline 18 & & .688 & & & & .124 & .117 \\
\hline 7 & & & .918 & & -.144 & & \\
\hline 8 & & & .910 & & -.148 & & \\
\hline 9 & .182 & -.103 & .564 & & .203 & & -.145 \\
\hline D5 & -.430 & & .454 & & .440 & & \\
\hline 5 & .300 & -.117 & .452 & .184 & .104 & & \\
\hline D6 & & .246 & .384 & & .278 & & \\
\hline 9 & & & & .896 & & & \\
\hline 10 & & & & .877 & & -.131 & \\
\hline 11 & .189 & .179 & & .613 & & -.109 & \\
\hline 12 & & & & .593 & .179 & .167 & \\
\hline 16 & & & & & .842 & -.359 & .113 \\
\hline 17 & & .152 & & & .765 & -.226 & \\
\hline 14 & .197 & & -.106 & & .702 & .222 & -.147 \\
\hline 13 & .218 & & & & .552 & .317 & -.105 \\
\hline 15 & .313 & & -.118 & .112 & .509 & & \\
\hline D1 & & .165 & & & & .753 & \\
\hline D3 & & & & & -.100 & .721 & .276 \\
\hline D4 & -.219 & & & .247 & & .218 & .770 \\
\hline D7 & .362 & -.121 & & -.129 & .249 & & .618 \\
\hline D2 & .119 & .342 & -.130 & -.124 & & & .489 \\
\hline
\end{tabular}

Note. $\mathrm{D}=$ distractor item; highlights indicate items with high modification indices. 


\section{Sample B PCA (forced 7-factor, promax) without distractor items}

Table S12. Principal components analysis on Sample B, without distractor items.

\begin{tabular}{|c|c|c|c|c|c|c|c|}
\hline \multirow[b]{2}{*}{ Item \# } & \multicolumn{7}{|c|}{ Component } \\
\hline & 1 & 2 & 3 & 4 & 5 & 6 & 7 \\
\hline 3 & .856 & & & & & & \\
\hline 4 & .824 & & & & & & \\
\hline 2 & .815 & & & & & & \\
\hline 1 & .792 & & & & & & \\
\hline 20 & & .868 & & & & & \\
\hline 19 & & .824 & & & & & \\
\hline 18 & & .787 & & & & & \\
\hline 21 & & .713 & & & & & \\
\hline 9 & & & .994 & & & & \\
\hline 10 & & & .977 & & & & \\
\hline 11 & & & .594 & & & & \\
\hline 12 & & & .466 & .399 & & & -.344 \\
\hline 13 & & & & .973 & & & \\
\hline 14 & & & & .950 & & & \\
\hline 15 & .225 & & & .464 & & .224 & \\
\hline 8 & & & & & .969 & & \\
\hline 7 & & & & & .931 & & .205 \\
\hline 16 & & & & & & .932 & \\
\hline 17 & & & & & & .842 & \\
\hline 6 & & & & .261 & .299 & & .607 \\
\hline 5 & & & .236 & & .211 & & .598 \\
\hline
\end{tabular}


Table S13: Wave 2 Sample Characteristics

\begin{tabular}{lc}
\hline Recruited $N$ & 293 \\
Valid $N$ & 290 \\
Age $M(S D)$ & $38.83(13.99)$ \\
Gender $(\%$ \% $)$ & 58.80 \\
Ethnicity (\%) & \\
$\quad$ Asian-American & 7.20 \\
African-American & 8.00 \\
White & 77.90 \\
Latino & 5.80 \\
Other & 1.10 \\
Edu (\%) & \\
High school & 12.00 \\
Some college & 28.50 \\
College & 45.80 \\
Post-grad & 13.70 \\
Median Income & $\$ 50,001-\$ 75,000$ \\
Median Time on & 15.36 \\
Survey & \\
\hline
\end{tabular}




\section{Tests of model fit over larger sample (Samples C-G)}

\section{Confirmatory factor analysis: Single factor model.}

Note: Post-peer review feedback suggested that these initial tests were potentially problematic. To be consistent with the CFAs on Sample A and B, we used the same cutoff criterion to identify error terms to covary in the models. However, in the subsequent replication sample $\mathrm{C}-\mathrm{G}$, this criterion resulted in a high number of covaried error terms, potentially overfitting the data and inflating goodness of fit estimates. See the subsequent "Post-peer review Factor Analytic Tests" section below for supplementary confirmatory tests using more rigorous tests without/with fewer covaried residuals. Further information is on Open Science Framework (https://osf.io/a2sxh/).

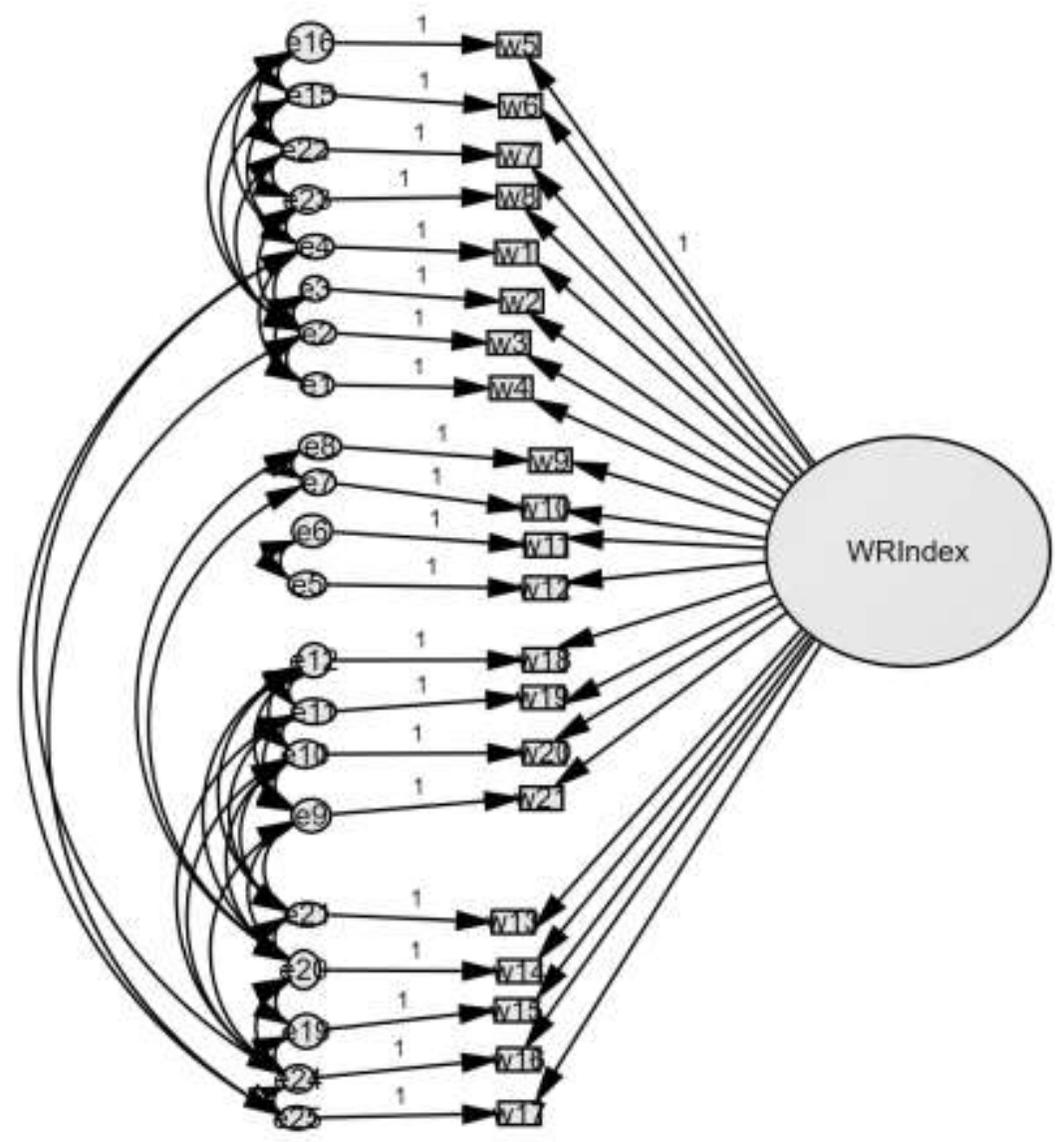

Figure S4. Single-factor confirmatory factor analysis on Samples C-F, using modification indices $>20$ to identify error terms to be covaried, consistent with Sample A and B CFAs. WRIndex - single-order wise reasoning factor.

$\begin{array}{ll}\text { CMIN/df } & 6.704 \\ \text { CFI } & .972 \\ \text { RMSR } & .052 \\ \text { RMSEA } & .047 \\ \text { PCLOSE } & .974\end{array}$




\section{Confirmatory factor analysis: Social-cognitive 2-factor model of wise reasoning}

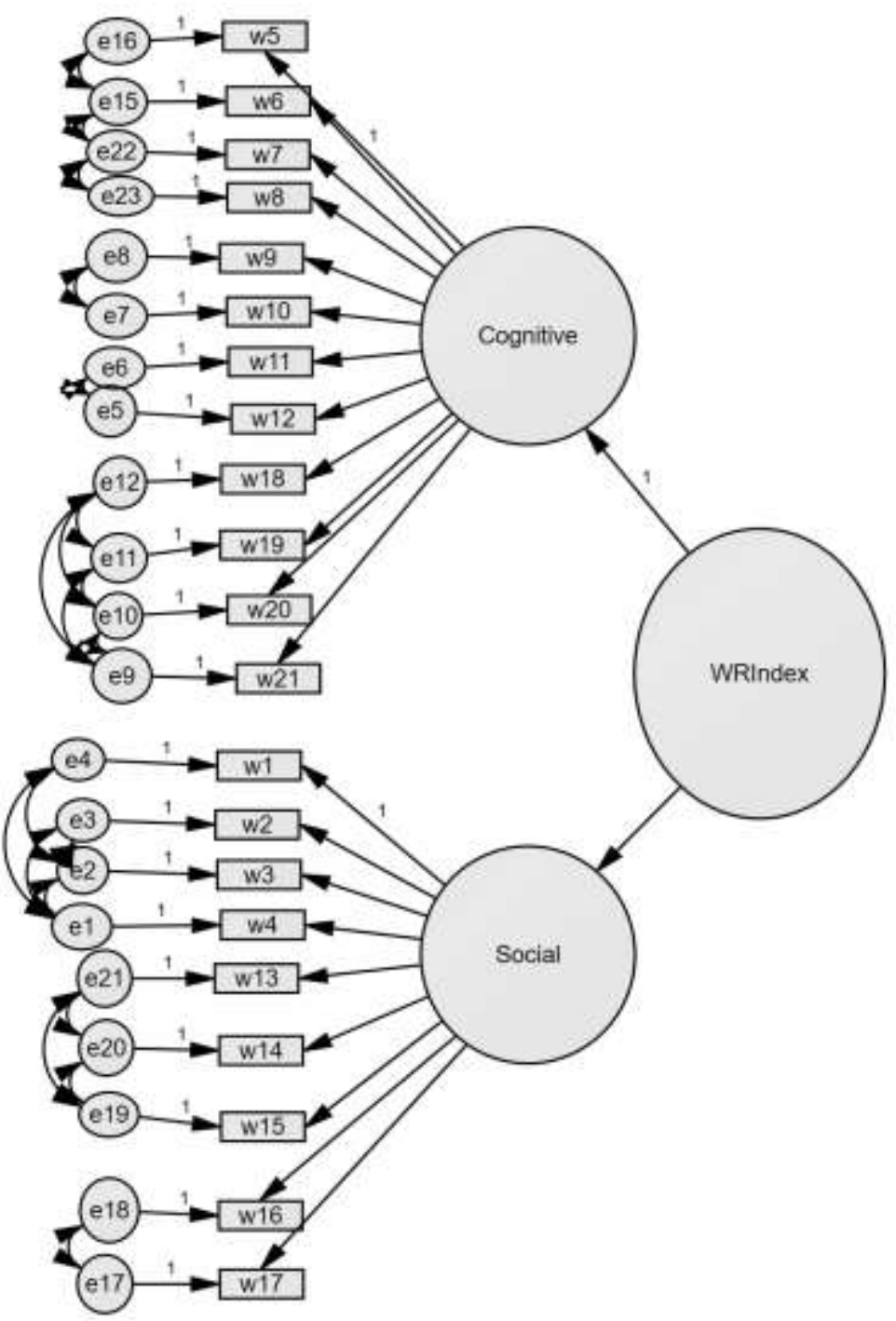

Figure S5. 2-factor CFA on Samples C-F, using modification indices $>20$ to identify error terms to be covaried. WRIndex- second-order wise reasoning factor. Cognitive first-order factor consists of items covering intellectual humility, change, multiple outcomes, and outsider's vantage point. Social first-order factor consists of items covering recognition of others' perspectives and search for a compromise and conflict resolution.

$\begin{array}{ll}\text { CMIN/df } & 7.165 \\ \text { CFI } & .965 \\ \text { RMSR } & .061 \\ \text { RMSEA } & .049 \\ \text { PCLOSE } & .822\end{array}$


Confirmatory factor analysis: 5-factor model.

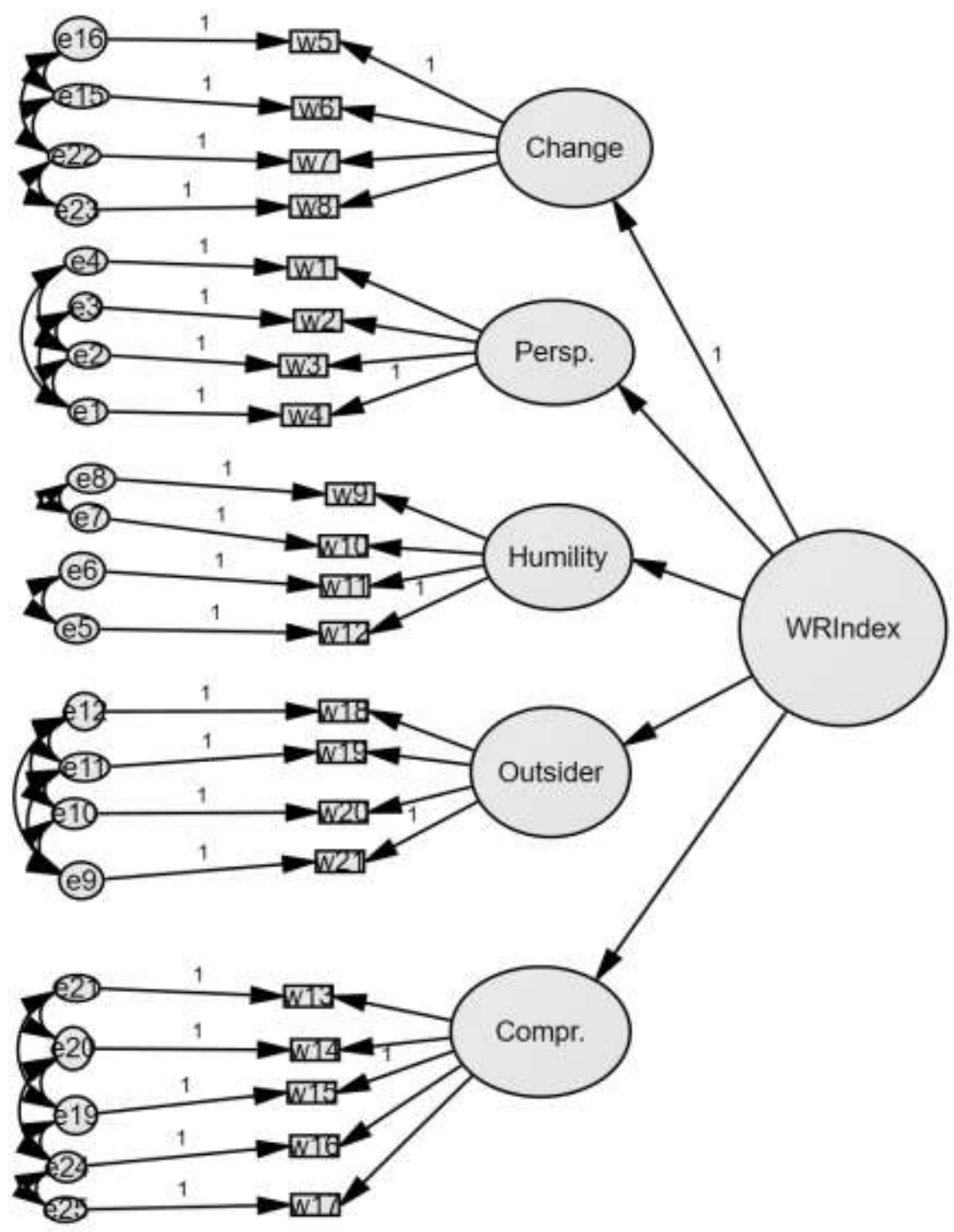

Figure S6. 5-factor confirmatory factor analysis on Samples C-F, using modification indices >20 to identify error terms to be covaried. WRIndex- second-order wise reasoning factor.

$\begin{array}{ll}\text { CMIN/df } & 6.617 \\ \text { CFI } & .969 \\ \text { RMSR } & .057 \\ \text { RMSEA } & .046 \\ \text { PCLOSE } & .990\end{array}$




\section{Confirmatory factor analysis: 6-factor model, Version A}

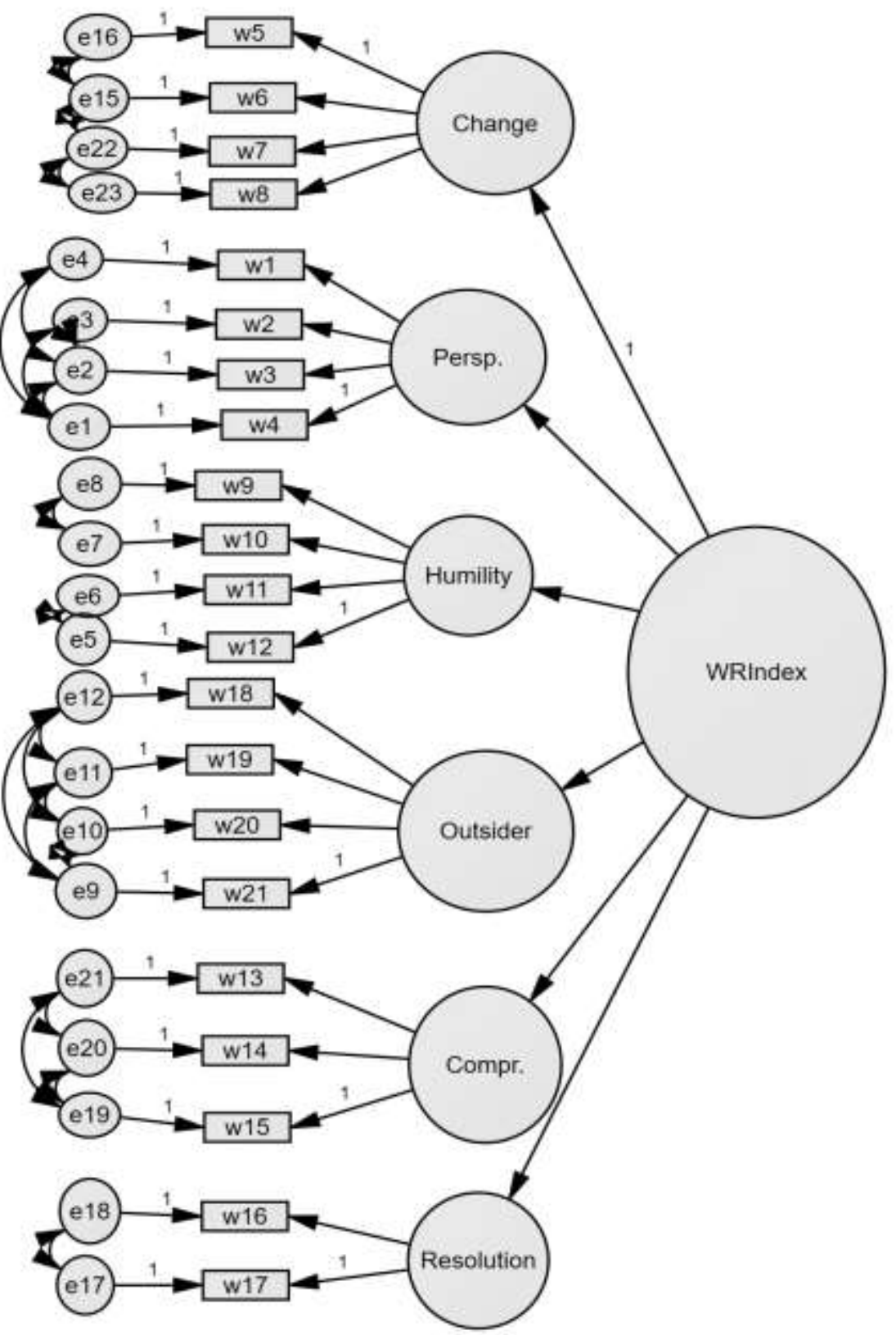

Figure S7. 6-factor (version A) confirmatory factor analysis on Samples C-F, using modification indices $>20$ to identify error terms to be covaried. WRIndex- second-order wise reasoning factor.

$\begin{array}{ll}\text { CMIN/df } & 7.165 \\ \text { CFI } & .965 \\ \text { RMSR } & .061 \\ \text { RMSEA } & .049 \\ \text { PCLOSE } & .822\end{array}$




\section{Confirmatory factor analysis: 6-factor model, Version B}

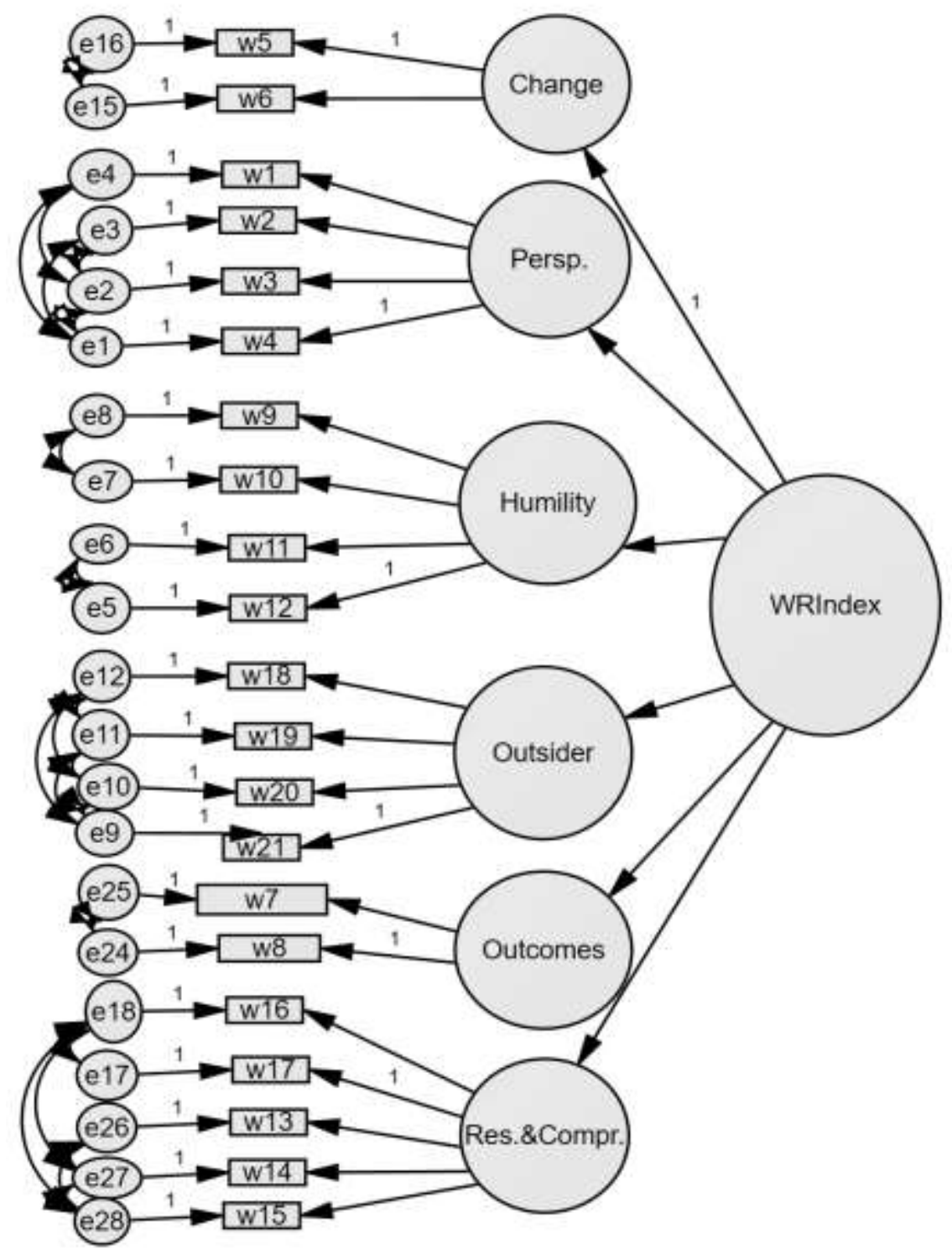

Figure S8. 6-factor (version B) confirmatory factor analysis on Samples C-F, using modification indices $>20$ to identify error terms to be covaried. WRIndex- second-order wise reasoning factor.

$\begin{array}{ll}\text { CMIN/df } & 7.143 \\ \text { CFI } & .966 \\ \text { RMSR } & .060 \\ \text { RMSEA } & .048 \\ \text { PCLOSE } & .835\end{array}$




\section{Confirmatory factor analysis: 7-factor model}

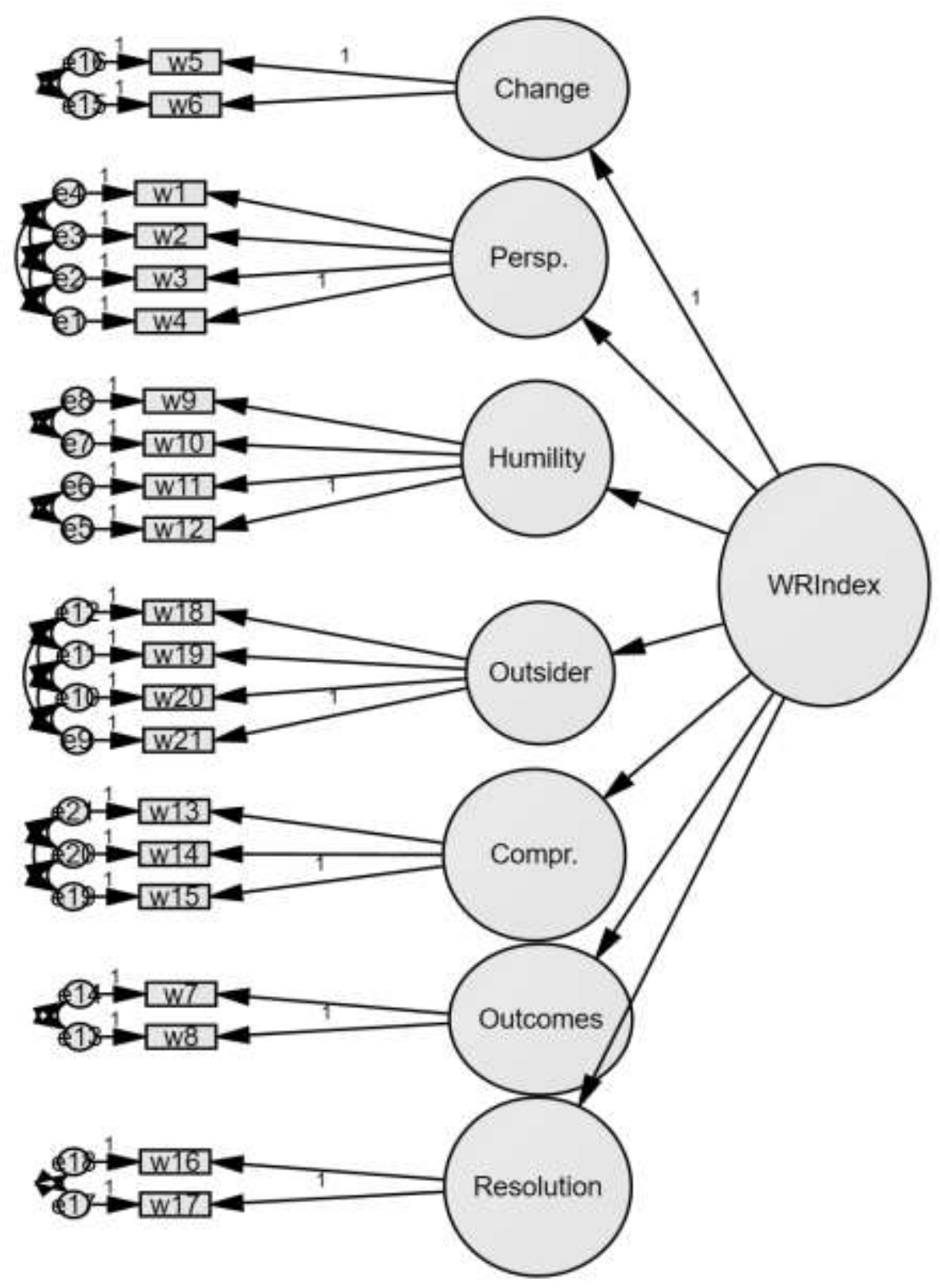

Figure S9. 7-factor confirmatory factor analysis on Samples C-F, using modification indices >20 to identify error terms to be covaried. WRIndex-second-order wise reasoning factor.

CMIN/df

CFI

RMSR

RMSEA

PCLOSE
7.072

.966

.061

.048

.876 


\section{Post-peer review Factor Analytic Tests}

Post-peer review feedback suggested that the second confirmatory factor analysis on the samples C-G could be improved. ${ }^{8}$ Namely, our confirmatory factor analyses (CFA) on samples C-G used the stringent cutoff point for modification indices ( $>20$; consistent with Sample A and B CFAs). Retaining this cutoff resulted in frequent covaried error terms in the large-scale sample C-G, which may have resulted in overfitting the model to the data and inflated goodness of fit. Therefore, here we re-examine the larger replication sample using a different, train-test (or lockbox) method (e.g., Yarkoni \& Westfall, 2017) to determine the most appropriate factor structure of wise reasoning. To ensure further, program-independent robustness of the model, we conducted these analyses in $\mathrm{R}$ via psych v. 1.8.4 (Revelle, 2011) and lavaan v 0.6-1 (Rosseel 2012) packages (see script and full results at https://osf.io/9h5dp/). We applied this method to confirmatory samples C-G.

In the train-test approach, we first split the full set of responses into halves, randomly assigning each response to either a "training" half-set or a "test" half-set. The analyses were conducted in two phases. In Phase 1, we conducted exploratory factor analyses (EFA) using the "training" set, followed by a CFA and inspection of top 3 possible modification indices.

Subsequently, in Phase 2, we conducted an identical CFA on the "test" set, to determine whether the factor structure established on the training set would replicate, and generalize to the test set. Notably, this method can be viewed as an extention of the general approach taken earlier, as CFA on samples $\mathrm{C}-\mathrm{G}$ represents a confirmatory replication of the structure identified in Samples A-B.

Phase 1. On the "training" half-set of the samples C-G, we tested single-factor, 2-factor, 5-factor, 6-factor, and 7-factor models. As we expected that the factors would correlate, we used principal axis factor analysis with oblique rotation. For each model, we examined the percent of cumulative variance explained by the model. Ultimately, our selection of which model to test in Phase 2 confirmatory analyses would rest on striking a balance between i) maximizing the cumulative variance explained by principal axis factoring, while also ii) maintaining parsimony and approximately equivalent item-weighting per factor. Ideally, we would opt for a factor structure that allowed for a minimum of three items per factor, to allow for more aqequate and reliable coverage of the latent constructs (e.g., Kenny, 2012).

Exploratory factor analysis. First, we examined the most parsimonious single-factor model, which explained only $35 \%$ of the variance in responses. The 2 -factor model explained $44 \%$ of the variance, for a $9 \%$ increment over the single-factor model. The 5-factor model explained $59 \%$ of the variance, for a $15 \%$ increment over the 2 -factor model. The 6 -factor model explained $63 \%$ of the variance, and the 7 -factor model explained $65 \%$ of the variance, for $4 \%$ and $6 \%$ increments over the 5-factor model. Notably, opting for either the 6- or 7-factor model would result in dimensions which included only two items, and therefore sacrifice parsimony, even weighting of items, and more adequate and reliable coverage of the latent constructs for the sake of negligible increments in variance explained. Therefore, we settled on the 5-factor model, which includes i) taking others' perspectives, ii) recognizing change and multiple outcomes, iii) intellectual humility, iv) searching for compromise/resolution, and v) considering outsider's

\footnotetext{
${ }^{8}$ We would like to thank Patrick Forscher for his insightful feedback, which initiated this post-peer review examination and subsequent tests.
} 
vantage point. Then we proceeded to confirmatory tests analyzing the 5-factor model for goodness of fit.

Confirmatory factor analysis. Following insights gained on the factor structure of wise reasoning from the EFA, we applied a confirmatory factor analyses using the lavaan package for $\mathrm{R}$ to the "training" set. We organized the items by factors: Items 1-4: others' perspectives; items 5-9: consideration of change and multiple ways situation may unfold; items 10-13: intellectual humility/recognition of limits of knowledge; items 14-18: search for a compromise / conflict resolution; items 19-21: view of the event through the vantage point of an outsider. This model did not include covariances among the residuals and showed an adequate fit, CMIN/df $=11.320$, $\mathrm{CFI}=.881, \mathrm{TLI}=.860, \mathrm{RMSEA}=.088$. In this model, all item loadings were $>.43$ on their respective factors, and all latent factors correlated $\geq .50$, with the exception of outsider's viewpoint, which correlated $\geq .33$.

We inspected possible improvements to the model by examining modification indices, a priori selecting only the top three highest modification indices, and selecting only one set of error terms to correlate per dimension of wise reasoning (items 7-8, 9-10, and 16-17) - a more conservative cutoff for covarying error terms than our previous tests. Covarying only three error terms, model fit was improved, CMIN/df $=4.275, \mathrm{CFI}=.963$, TLI $=.956$, RMSEA $=.050$. Next, we compared this 5-factor model with the single-, 2-, 6-, and 7-factor models with the same correlated error terms as above, using Chi Square Difference tests. Again, we found that the 5factor model showed much better fit than the single- and 2-factor models, and nearly identical goodness of fit as the 6- and 7-factor models (see full results at https://osf.io/95z8k/). Thus, as above, we opted for parsimony, even weighting of items per factor, and $\geq 3$ items per factor, selecting to move ahead with replication tests of the 5-factor model on the second half of the data, the "test" half-set of Sample C-G.

Phase II. We conducted a CFA on the "test" half-set, using the same factor structure and covarying the same three error terms as those in the "training" half-set. This test showed a good model fit, $\mathrm{CMIN} / \mathrm{df}=4.489, \mathrm{CFI}=.958, \mathrm{TLI}=.950, \mathrm{RMSEA}=.051$, comparable to that of the "training" half-set. Item loadings and covariances are shown in Figure S10. As a final test, we compared the 5-factor model with the single-, 2-, 6-, and 7-factor models, as above. Again, we found the 5-factor model showed much better fit than the single- and 2-factor models. The 6- and 7-factor models showed better fit than the 5-factor model (i.e., slightly lower AIC indices; see full results at https://osf.io/95z8k/), however again we opted for the 5-factor model to avoid sacrificing parsimony, even weighting of items, and $\geq 3$ items per factor for the sake of negligible improvement in model fit.

Altogether, replication of the factor structure from the initial samples A to B and B to C$\mathrm{G}$, as well a train-/test-replication of the structure on random sub-sets of samples $\mathrm{C}-\mathrm{G}$, suggest that the 5-factor model is acceptable as our current model of wise reasoning in interpersonal conflicts. ${ }^{9}$

\footnotetext{
${ }^{9}$ It is noteworthy that the factor structure and goodness of fit indices reported in the current analyses have been further replicated in separate studies of wise reasoning about intergroup conflict (vs. interpersonal/workplace conflicts) presently under review.
} 


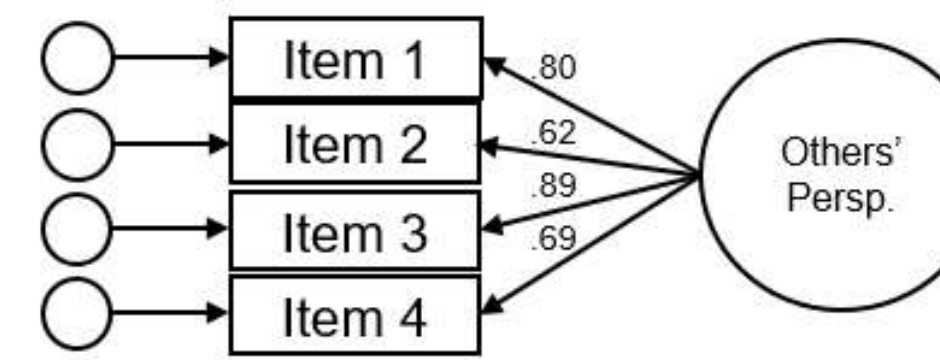

68

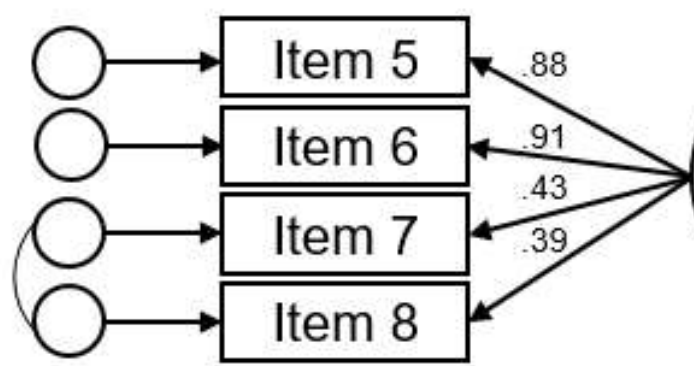

53

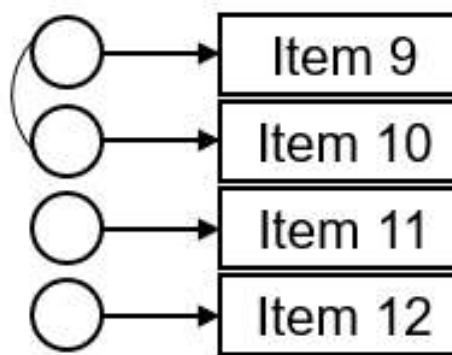

47
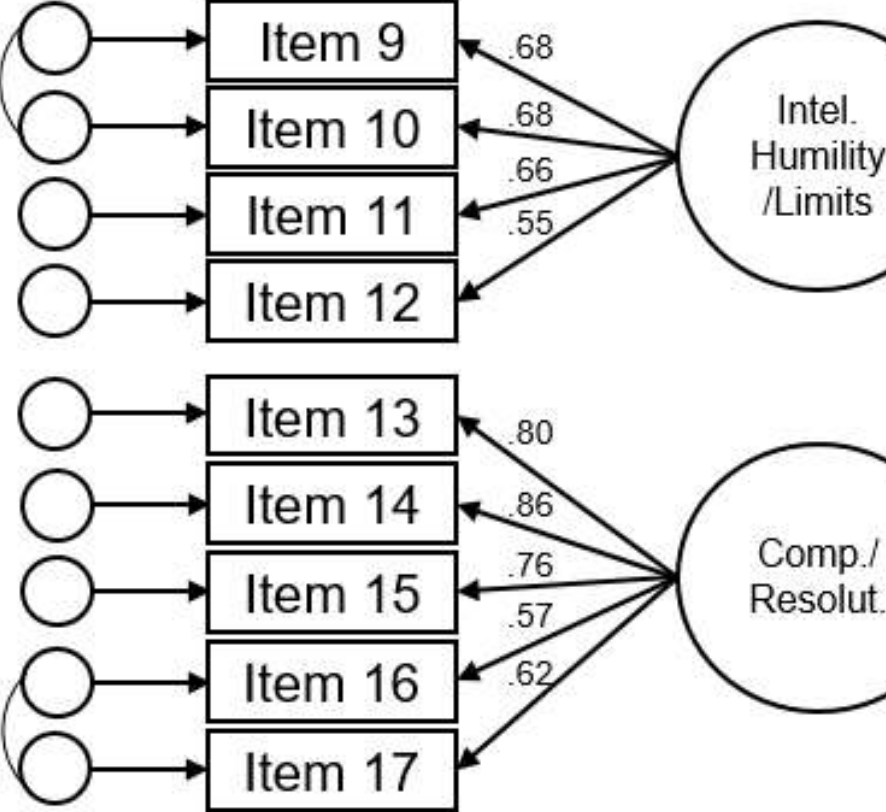

Change/ Multiple

Outcom.
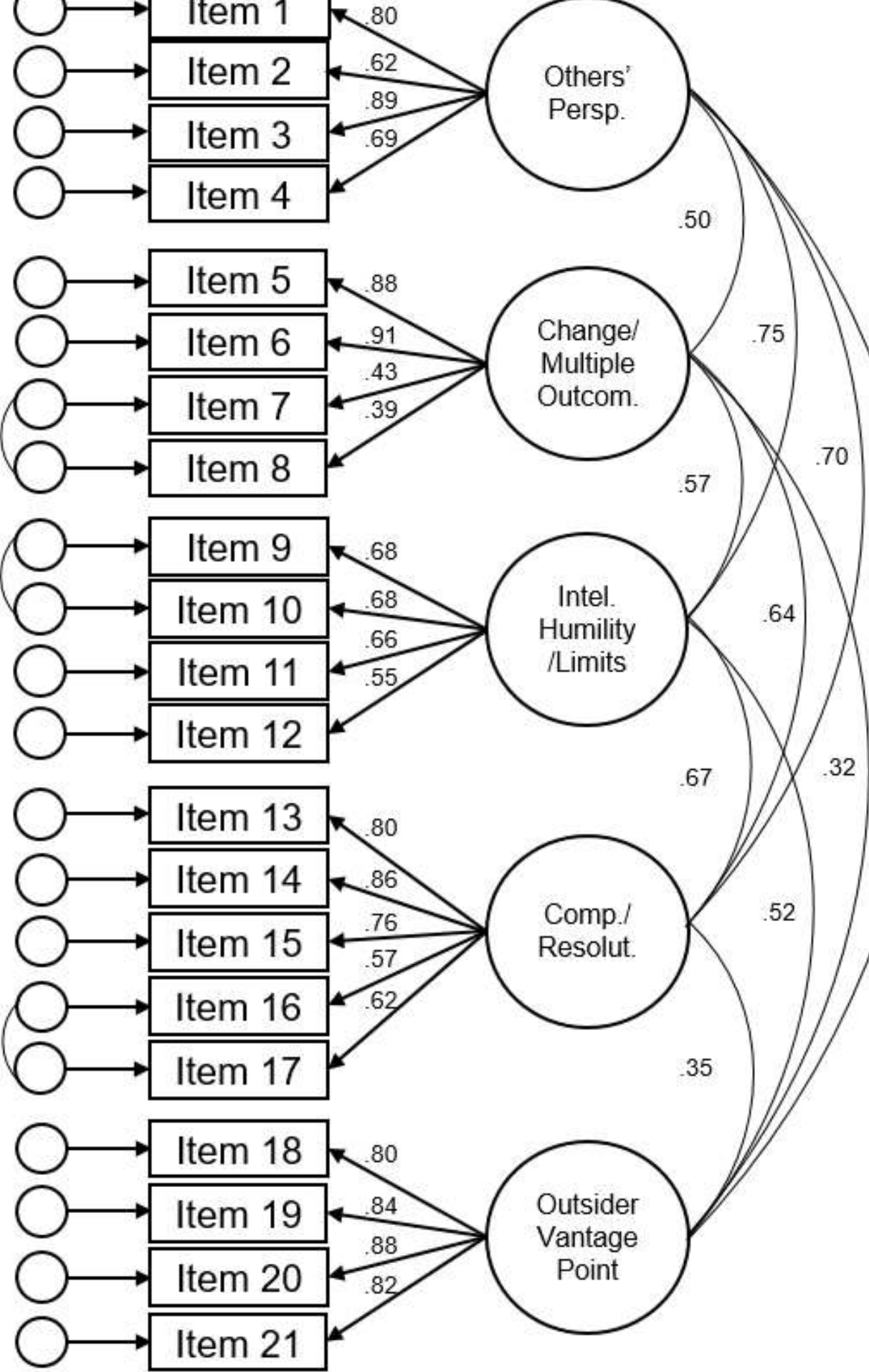

.57

.67
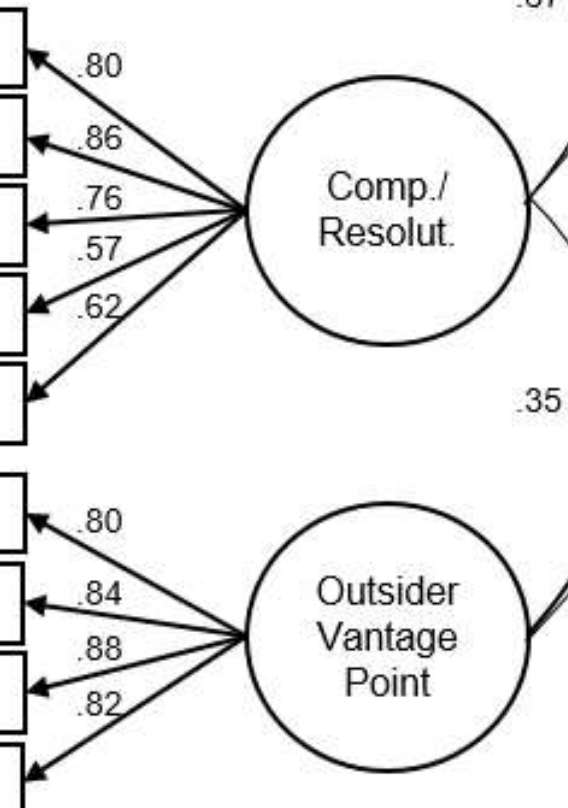

Figure S10. 5-factor confirmatory factor analysis on Samples C-F (the "test" half-set), covarying error terms for the three highest modification indices, and selecting only one set of error terms to correlate per dimension of wise reasoning. 
Wisdom_Bias_and_Balance_Analysis_Studies_1-8_Syntax.R

File Preparation: SPSS files with final samples were merged and saved as csv (then all NA's were replaced with "' in csv file) String and qualitative data were removed to avoid issues with tabs, line breaks, and carriage returns when reading csv Variables were already recoded, computed, and cleaned

WIS.RAW<-read.csv(file.choose(), header=TRUE, na.strings=" ", stringsA sFactors=FALSE) \# Select Wisdom Bias and Balance Clean Final Data.csv, should have 4168 obs. of 1488 variables

WIS.PA.RAW<-read.csv(file.choose(), header=TRUE, na.strings=" ", strin gsAsFactors=FALSE) \#Select Wisdom Bias and Balance Prolific Final Data . cSV (SPSS file resaved without row 2 [Labels]), should have 330 obs. of 306 variables

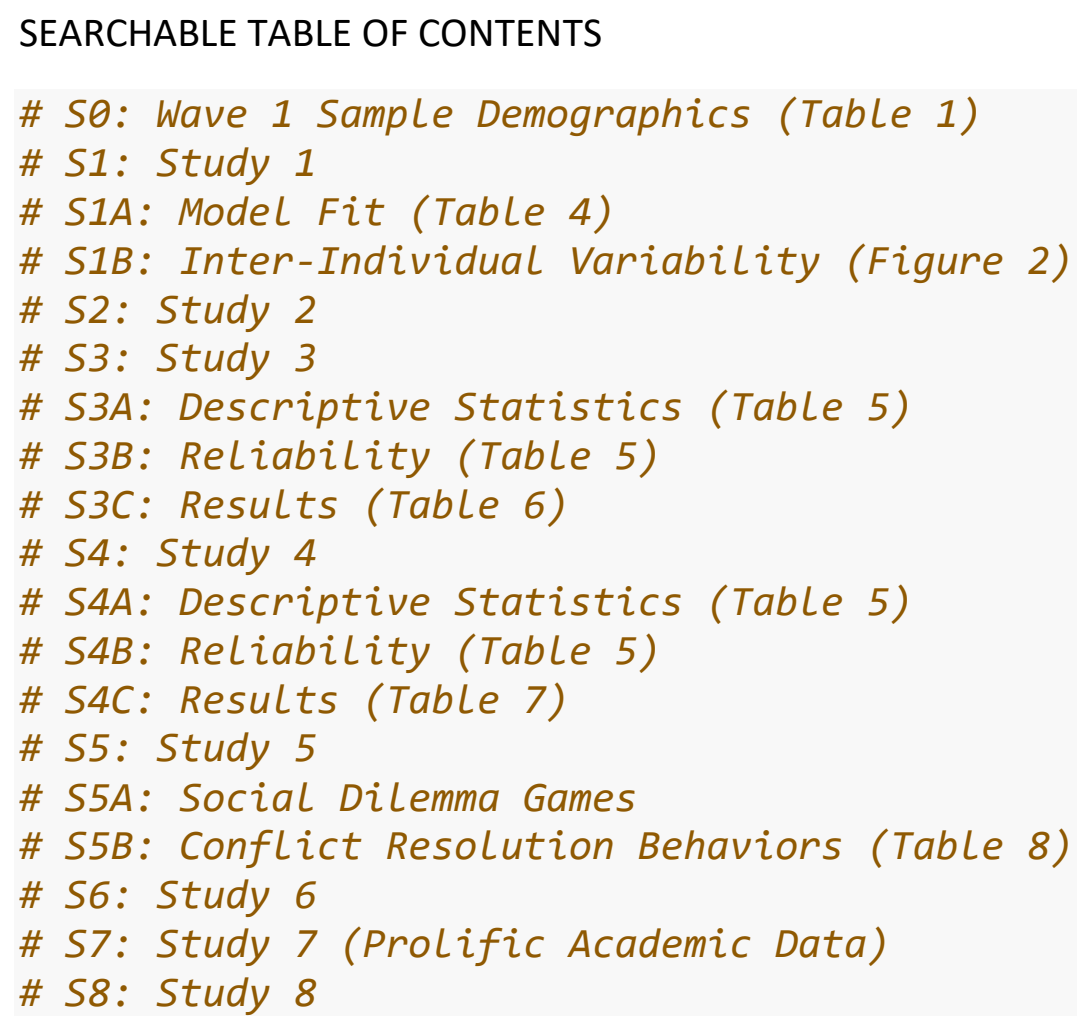


\#\# Loading required package: plyr

\#\# Warning: package 'plyr' was built under $\mathrm{R}$ version 3.3.2

require(ggplot2)

\#\# Loading required package: ggplot2

\#\# Warning: package 'ggplot2' was built under $\mathrm{R}$ version 3.3 .2

\#\#

\#\# Attaching package: 'ggplot2'

\#\# The following objects are masked from 'package:psych':

\#\#

\#\# $\%+\%$, alpha

require( $\mathrm{sm})$

\#\# Loading required package: sm

\#\# Warning: package 'sm' was built under $\mathrm{R}$ version 3.3.2

\#\# Package 'sm', version 2.2-5.4: type help(sm) for summary informatio $\mathrm{n}$

require(ppcor)

\#\# Loading required package: ppcor

\#\# Warning: package 'ppcor' was built under $\mathrm{R}$ version 3.3 .2

\#\# Loading required package: MASS

\#\#

\#\# Attaching package: 'MASS'

\#\# The following object is masked from 'package:sm':

\#\#

\#\# muscle

require(reshape2)

\#\# Loading required package: reshape2

\#\# Warning: package 'reshape2' was built under $\mathrm{R}$ version 3.3 .2

require(lme4)

\#\# Loading required package: Ime4 
\#\# Warning: package 'lme4' was built under $\mathrm{R}$ version 3.3 .2

\#\# Loading required package: Matrix

require(lmerTest)

\#\# Loading required package: ImerTest

\#\# Warning: package 'lmerTest' was built under $\mathrm{R}$ version 3.3 .2

\#\#

\#\# Attaching package: 'ImerTest'

\#\# The following object is masked from 'package:lme4':

\#\#

\#\# Imer

\#\# The following object is masked from 'package:stats':

\#\#

\#\# $\quad$ step

PREPARING DATA FOR WAVE 1 STUDIES (STUDIES 1, 3, 4, 5)

WIS <-WIS . RAW

WIS [WIS $==-99]<-N A$ \# Removing missing values

WIS [WIS=='NA']<-NA \# Removing NA string

options(scipen=999) \# Disabling scientific notation

WIS\$IDvar<-rownames(WIS) \# Creating ID variable for long format analys es

COMPUTING WISE REASONING FOR WAVE 1 (PCA)

WIS\$PCAsort<-car: : recode(WIS\$sample, " ' a '=1; ' b' =2; 'c '=3; 'd '=4; 'e '=5; ' f' $=6$; ' g ' $=7$; ' $\mathrm{x}$ '=8; ' $h$ ' $=9$; ' $W 2$ ' =10")

WIS<-WIS[order(WIS\$PCAsort), ] \#Resorting to be ordered by sample WIS\$wisdom.pca<-c(rep(NA, nrow(subset(WIS, sample \%in\% c("a", "b")))), principal(WIS [which (WIS\$sample\%in\%c("c", "d", "e", "f" , "g", "x")) , c("Perspective.ave", "ChangeOutcomes.ave", "Limits.ave", "Comp Resolve.ave", "Outsider.ave")],

nfactors $=1$, residuals=TRUE, rotate="oblimin ", missing=TRUE, impute="median", scores=TRUE) $\$$ scores [ , 1], $\operatorname{rep}($ NA, nrow(subset (WIS, sample \%in\% c("h", "w2")))))

\# Getting PCA scores (2nd order factor across means of 5 dimensions) $p$ er sample (except Samples A \& B (scale construction), H (no data), I \& W2 (Wave 2 study))

WIS[WIS\$sample=="a", ]\$wisdom.pca<-principal(WIS[WIS\$sample=="a", c("Per spective.ave", "ChangeOutcomes.ave", "Limits.ave", "CompResolve.ave", "Out 
sider. ave") ], nfactors=1, residuals=TRUE, rotate="oblimin", missing=TRUE, i mpute="median", scores=TRUE) \$scores $[, 1]$

WIS [WIS\$sample=="b", ]\$wisdom.pca<-principal(WIS[WIS\$sample=="b", c("Per spective.ave", "ChangeOutcomes.ave", "Limits.ave", "CompResolve.ave", "Out sider.ave") ], nfactors=1, residuals=TRUE, rotate="oblimin", missing=TRUE, i mpute="median", scores=TRUE)\$scores $[, 1]$

plyr:: count(WIS[! is.na(WIS\$wisdom.pca), ], "sample") \#Checking that vari able exists across samples / Samples A, B, H, W2 missing

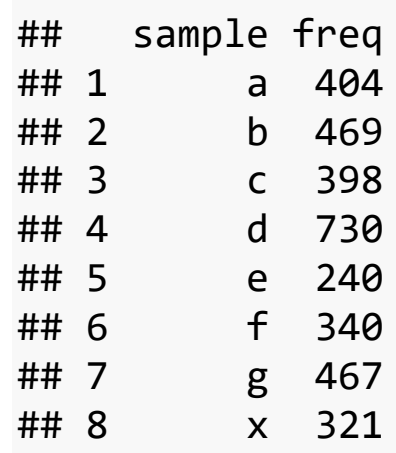

WIS\$PCAsort2<-car: : recode(WIS\$sample, " ' a '=1; ' b '=2; 'c ' =3; ' d '=4; 'e '=5; ' f '=6; ' g' $=7$; ' $h$ '=8; ' $x$ ' =9; ' $w 2$ ' =10")

WIS<-WIS[order(WIS\$PCAsort2), ] \#Resorting to be ordered by sample WIS\$wisdom. IG.pca<-c(rep(NA, nrow(subset(WIS, sample \%in\% c("a", "b", "c", "d")))),

principal(WIS[WIS\$sample=="e", c("Perspective.IG. ave", "ChangeOutcomes.IG.ave", "Limits.IG.ave", "CompResolve.IG.ave", "Out sider.IG.ave")],

nfactors $=1$, residuals $=$ TRUE, rotate $="$ obli min", missing=TRUE, impute="median", scores=TRUE) $\$$ scores $[, 1]$, rep(NA, nrow(subset(WIS, sample \%in\% c("f", "g", "h" , "x", "w2"))))) \# Getting PCA scores (2nd order factor across means of 5 dimensions) for intergroup wise reasoning (Sample E)

plyr:: count(WIS[! is.na(WIS\$wisdom.IG.pca), ], "sample") \#Checking that $v$ ariable exists across samples / Only sample E

\section{\#\# sample freq \\ \#\# 1 e 240}

WIS\$WiseObs.pca<-c(rep(NA, nrow(subset(WIS, sample \%in\% c("a", "b", "c", "d "))),

principal(WIS[WIS\$sample=="e", c("Perspectives", "Ch ange", "Limits", "Compromise", "Resolution")] , nfactors $=1$, residuals=TRUE, rotate $=$ "oblimi $n "$, missing=TRUE, impute="median", scores=TRUE) $\$$ scores $[, 1]$, rep(NA, nrow(subset(WIS, sample \%in\% c("f", "g", "h", " 
x", "w2"))))) \# Getting PCA scores (2nd order factor across means of 5 rating dimensions) for observer ratings (Sample E)

plyr:: count(WIS[! is.na(WIS\$WiseObs.pca), ], "sample") \#Checking that var iable exists across samples / Only sample E

\#\# sample freq

\#\# 1 e 240

WIS\$WiseObs.pca[WIS\$IncludeObs==0]<-NA \# Deleting excluded data from 0 bserver rating $P C A$

For supplementary analyses: Recomputing wise reasoning composite without perspective aspect

WIS\$wisdom.pca.NoP<-c(rep(NA, nrow(subset(WIS, sample \%in\% c("a", "b"))))

,

principal(WIS [which(WIS\$sample\%in\%c("c", "d", "e" , "f" , "g", "x")) , c("ChangeOutcomes.ave", "Limits.ave", "CompResolve.ave", "Outs ider.ave")],

", missing=TRUE, impute="median", scores=TRUE) $\$$ scores $[, 1]$, $\operatorname{rep}($ NA, nrow(subset(WIS, sample \%in\% c("h", "w2")))))

\# Getting PCA scores (2nd order factor across means of 5 dimensions) $p$ er sample (except Samples A \& B (scale construction), H (no data), I \& W2 (Wave 2 study))

WIS[WIS\$sample=="a", ]\$wisdom.pca. NoP<-principal(WIS[WIS\$sample=="a", c( "ChangeOutcomes.ave", "Limits.ave", "CompResolve.ave", "Outsider.ave") ], n factor $s=1$, residuals=TRUE, rotate="oblimin", missing=TRUE, impute="median" , scores=TRUE) $\$$ scores $[, 1]$

WIS [WIS\$sample=="b", ]\$wisdom.pca. NoP<-principal(WIS[WIS\$sample=="b", c( "ChangeOutcomes .ave", "Limits.ave", "CompResolve.ave", "Outsider.ave") ], n factors=1, residuals=TRUE, rotate="oblimin", missing=TRUE, impute="median" , scores=TRUE) $\$$ scores $[, 1]$

Subsetting by sample

WIS.A<-WIS[WIS\$sample $==" a ",] \quad \# n=404$

WIS. B<-WIS[WIS\$sample $==" b "$,] \# $n=469$

WIS.C<-WIS[WIS\$sample $==" c "$ "] \# $n=398$

WIS.D $<$-WIS[WIS $\$$ sample $==" d "$, ] \# $n=730$

WIS.E<-WIS[WIS $\$$ sample $==" e "$, ] \# $n=240$

WIS.F $<$-WIS[WIS $\$$ sample $==" f "$, ] \# $n=340$

WIS.G<-WIS[WIS $\$$ sample $==" g ",] \quad \# n=467$

WIS.H<-WIS[WIS\$sample=="h",] \# $n=501$

\#\#\#\# S0: WAVE 1 SAMPLE DEMOGRAPHICS (Table 1) \#\#\#\# 
For samples w2 and $\mathrm{x}$, look at the Demographic analyses (both of these make up sample I);

Prolific Academic samples separate Mean Age

$\begin{array}{lrrrr}\text { ddply(WIS, "sample", function(x) descr } \\ \text { \#\# } & \text { sample } & \mathrm{n} & \text { mean } & \text { sd } \\ \text { \#\# } 1 & \text { a } 404 & 32.25248 & 10.257993 \\ \text { \#\# } 2 & \text { b } 469 & 32.08955 & 10.196388 \\ \text { \#\# } 3 & \text { c } 398 & 31.51005 & 10.188582 \\ \text { \#\# } 4 & \text { d } 730 & 33.37808 & 11.734234 \\ \text { \#\# } 5 & \text { e } 200 & 29.90000 & 7.814431 \\ \text { \#\# } 6 & \text { f } 338 & 34.35503 & 12.445656 \\ \text { \#\# } 7 & \text { g } 421 & 20.04038 & 3.508599 \\ \text { \#\# } 8 & \text { h } 315 & 20.05397 & 1.832198 \\ \text { \#\# } 9 & \text { w2 } 298 & 38.74497 & 12.781855 \\ \text { \#\# } 10 & \text { x } 321 & 36.90031 & 12.155607\end{array}$

Gender Frequency

ddply(WIS, "sample",function(x) count(x\$gender)) \# No data for Sample G \#\# sample $x$ freq

\#\# 1 a Female 195

\#\# 2 a Male 209

\#\# 3 b Female 231

\#\# 4 b Male 238

\#\# $5 \quad$ c Female 233

\#\# $6 \quad$ c $\quad$ Male 163

\#\# 7 c $\quad\langle N A\rangle \quad 2$

\#\# 8 d Female 474

\#\# 9 d Male 256

\#\# $10 \quad$ e Female 126

\#\# 11 e Male 75

\#\# 12 e $\quad$ NA> 39

\#\# $13 \quad f$ Female 189

\#\# $14 \quad f$ Male 150

\#\# $15 \quad f \quad\langle N A\rangle \quad 1$

\#\# $16 \quad$ g Female 256

\#\# 17 g Male 181

\#\# 18 g $\quad$ 〈NA $\quad 30$

\#\# $19 \quad$ h Female 197

\#\# $20 \quad$ h Male 121

\#\# 21 h $\langle$ NA 183

\#\# 22 w2 Female 174

\#\# 23 w2 Male 122

$\begin{array}{llll}\# \# 24 & \text { w2 } & \langle\mathrm{NA}\rangle & 2\end{array}$ 


\begin{tabular}{|c|c|c|c|}
\hline \#\# 25 & $x$ & Female & 17 \\
\hline \#\# 26 & $x$ & Male & 14 \\
\hline \#\# 27 & $x$ & $\langle N A\rangle$ & \\
\hline
\end{tabular}

Ethnicity Frequency

ddply(WIS, "sample",function(x) count(x\$Ethnicity)) \# No data for Sampl es $G$ and $H$

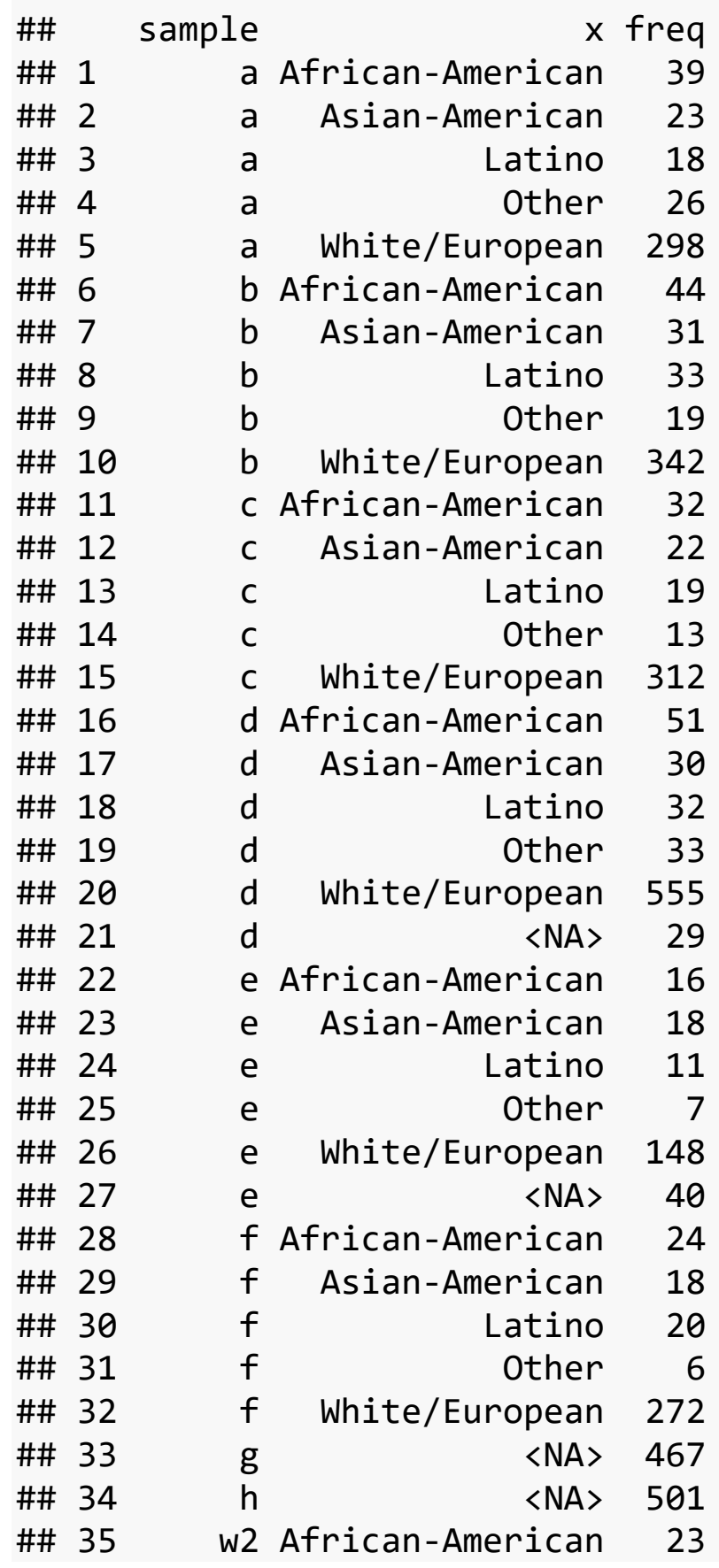




$\begin{array}{rrrrr}\text { \#\# } 36 & \text { w2 } & \text { Asian-American } & 22 \\ \text { \#\# } 37 & \text { w2 } & \text { Latino } & 16 \\ \text { \#\# } 38 & \text { w2 } & \text { Other } & 12 \\ \text { \#\# 39 } & \text { w2 } & \text { White/European } & 216 \\ \text { \#\# 40 } & \text { w2 } & \text { <NA } & 9 \\ \text { \#\# } 41 & \text { x } & \text { African-American } & 31 \\ \text { \#\# 42 } & \text { x } & \text { Asian-American } & 17 \\ \text { \#\# 43 } & \text { x } & \text { Latino } & 22 \\ \text { \#\# 44 } & \text { x } & \text { Other } & 21 \\ \text { \#\# 45 } & \text { x } & \text { White/European } & 230\end{array}$

Median Income

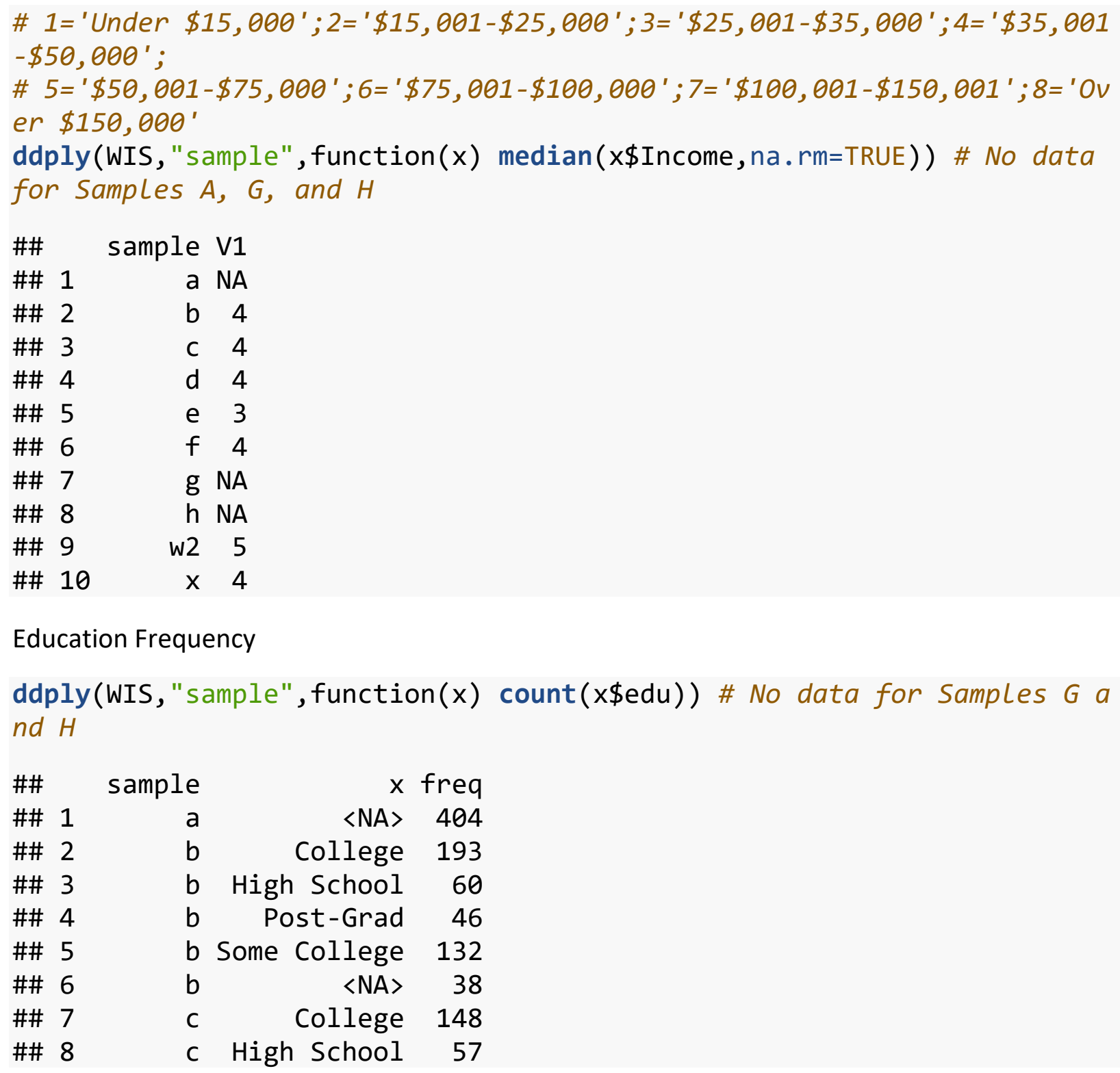

Education Frequency

ddply(WIS,"sample", function(x) count(x\$edu)) \# No data for Samples $G$ a nd $H$

\begin{tabular}{|c|c|c|c|}
\hline \#\# & sample & $x$ & freq \\
\hline \#\# 1 & $a$ & $\langle N A\rangle$ & 404 \\
\hline \#\# 2 & $b$ & College & 193 \\
\hline \#\# 3 & $b$ & High School & $6 e$ \\
\hline \#\# 4 & b & Post-Grad & 46 \\
\hline \#\# 5 & b & Some College & 132 \\
\hline \#\# 6 & & $\langle N A\rangle$ & 38 \\
\hline \#\# 7 & & College & 148 \\
\hline \# 8 & & High School & \\
\hline
\end{tabular}




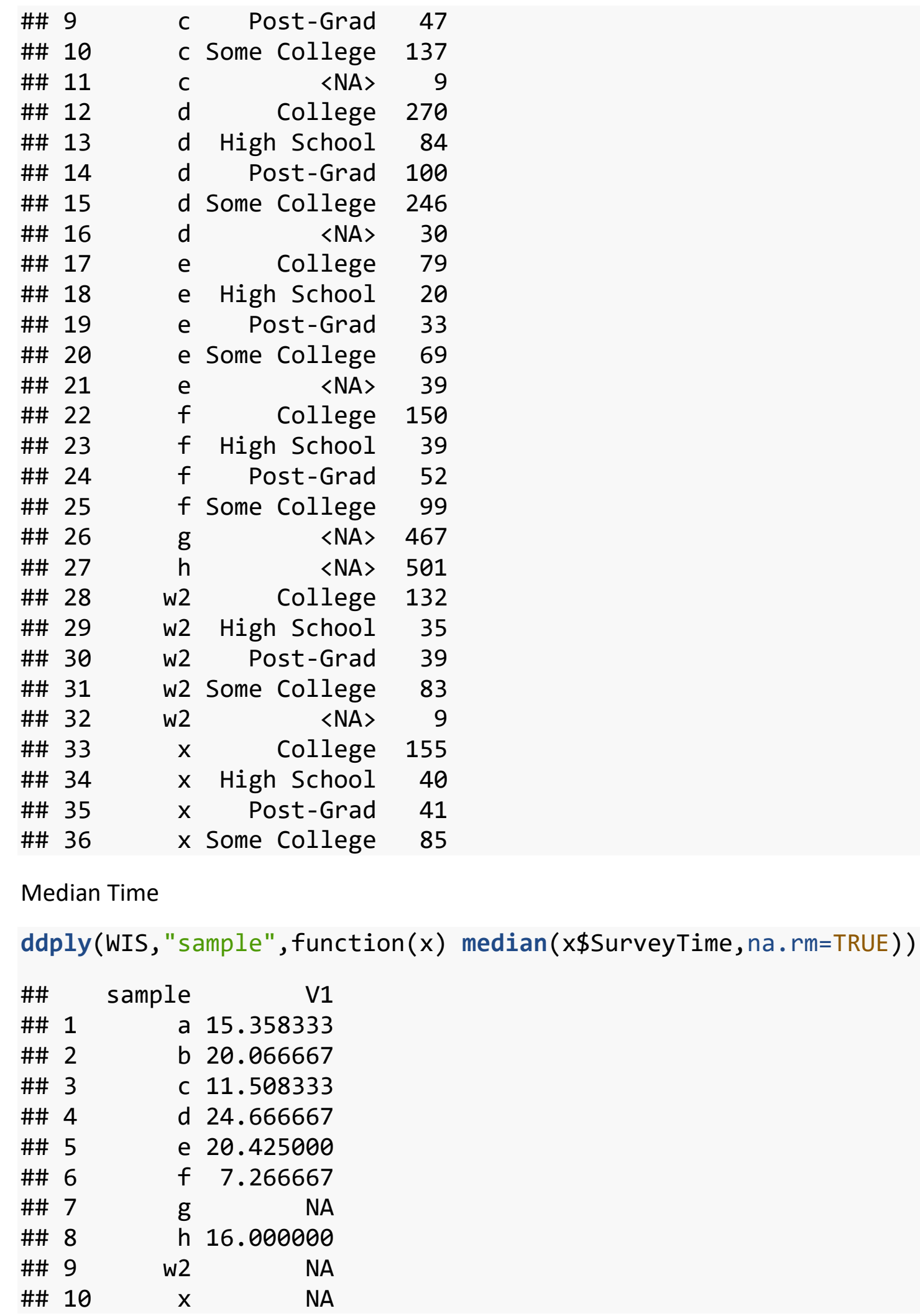




\section{\#\#\#\# S1: STUDY 1 \#\#\#\#}

S1A: MODEL FIT - WISE REASONING ASPECT LOADINGS IN DIFFERENT SAMPLES Creating different subsets based on sample location for PCA

WIS.work<-subset(WIS, sample \%in\% c("a","b")) \# Workplace conflict sam ple

WIS.personal<-subset(WIS, sample \%in\% c("c","d","e","f")) \# Interperso nal conflict sample

PCA loadings of 5 aspects of wise reasoning on 2nd order latent component of wise reasoning across each sample (Table 4)

\# Look at PC1 column. All loadings are .60 and above, which is good. principal(WIS.work [, c("Perspective.ave", "ChangeOutcomes.ave", "Limits.a ve", "CompResolve.ave", "Outsider.ave")], nfactors=1, residuals=TRUE, rotat e="oblimin", missing=TRUE, impute="median") \# Workplace conflict sample

\#\# Principal Components Analysis

\#\# Call: principal( $r=$ WIS.work [, c("Perspective.ave", "ChangeOutcomes . ave",

\#\# "Limits.ave", "CompResolve.ave", "Outsider.ave")], nfactors = 1 \#\# residuals $=$ TRUE, rotate $=$ "oblimin", missing $=$ TRUE, impute $=$ "median")

\#\# Standardized loadings (pattern matrix) based upon correlation matri $\mathrm{X}$

\#\# PC1 h2 u2 com

$\begin{array}{llllll}\text { \#\# Perspective.ave } \quad 0.81 & 0.66 & 0.34 & 1\end{array}$

\#\# ChangeOutcomes.ave $0.76 \quad 0.57 \quad 0.43 \quad 1$

\#\# Limits.ave $\quad 0.800 .650 .35 \quad 1$

\#\# CompResolve.ave $\quad 0.84 \quad 0.71 \quad 0.29 \quad 1$

$\begin{array}{llllll}\text { \#\# Outsider.ave } \quad 0.70 & 0.49 & 0.51 & 1\end{array}$

\#\#

\#\# PC1

\#\# SS loadings 3.08

\#\# Proportion Var 0.62

\#\#

\#\# Mean item complexity $=1$

\#\# Test of the hypothesis that 1 component is sufficient.

\#\#

\#\# The root mean square of the residuals (RMSR) is 0.1

\#\# with the empirical chi square 177.13 with prob $<0.000000000000$ 0000000000000000000000022

\#\#

\#\# Fit based upon off diagonal values $=0.96$ 
principal(WIS.personal [, c("Perspective.ave", "ChangeOutcomes.ave", "Limi ts.ave", "CompResolve.ave", "Outsider.ave") ], nfactors=1, residuals=TRUE, $r$ otate="oblimin", missing=TRUE, impute="median") \# Interpersonal conflict sample

\#\# Principal Components Analysis

\#\# Call: principal( $r$ = WIS.personal[, c("Perspective.ave", "ChangeOutc omes.ave",

\#\# "Limits.ave", "CompResolve.ave", "Outsider.ave")], nfactors = 1

\#\# $\quad$ residuals $=$ TRUE, rotate $=$ "oblimin", missing $=$ TRUE, impute $=$ "median")

\#\# Standardized loadings (pattern matrix) based upon correlation matri $x$

\#\# $\quad$ PC1 1 h2 u2 com

$\begin{array}{llllll}\text { \#\# Perspective.ave } \quad 0.82 & 0.67 & 0.33 & 1\end{array}$

\#\# ChangeOutcomes.ave $0.72 \quad 0.53 \quad 0.47 \quad 1$

$\begin{array}{llllll}\text { \#\# Limits.ave } \quad 0.81 & 0.66 & 0.34 & 1\end{array}$

\#\# CompResolve.ave $\quad 0.82 \quad 0.68 \quad 0.32 \quad 1$

$\begin{array}{llllll}\text { \#\# Outsider.ave } \quad 0.60 & 0.37 & 0.63 & 1\end{array}$

\#\#

\#\# PC1

\#\# SS loadings 2.90

\#\# Proportion Var 0.58

\#\#

\#\# Mean item complexity $=1$

\#\# Test of the hypothesis that 1 component is sufficient.

\#\#

\#\# The root mean square of the residuals (RMSR) is 0.11

\#\# with the empirical chi square 442.54 with prob $<0.000000000000$ 0000000000000000000000000000000000000000000000000000000000000000000000 00000000002

\#\#

\#\# Fit based upon off diagonal values $=0.94$

principal(WIS.E[ , c("Perspective.IG.ave", "ChangeOutcomes.IG.ave", "Limit s.IG.ave", "CompResolve.IG.ave", "Outsider.IG.ave")], nfactors=1, residual $\mathrm{s}=$ TRUE, rotate="oblimin", missing=TRUE, impute="median") \# Intergroup con flict sample

\#\# Principal Components Analysis

\#\# Call: principal( $r=$ WIS.E[, c("Perspective.IG.ave", "ChangeOutcomes .IG.ave",

\#\# "Limits.IG.ave", "CompResolve.IG.ave", "Outsider.IG.ave")],

\#\# $\quad$ nfactors $=1$, residuals $=$ TRUE, rotate $=$ "oblimin", missing $=\mathrm{T}$ 


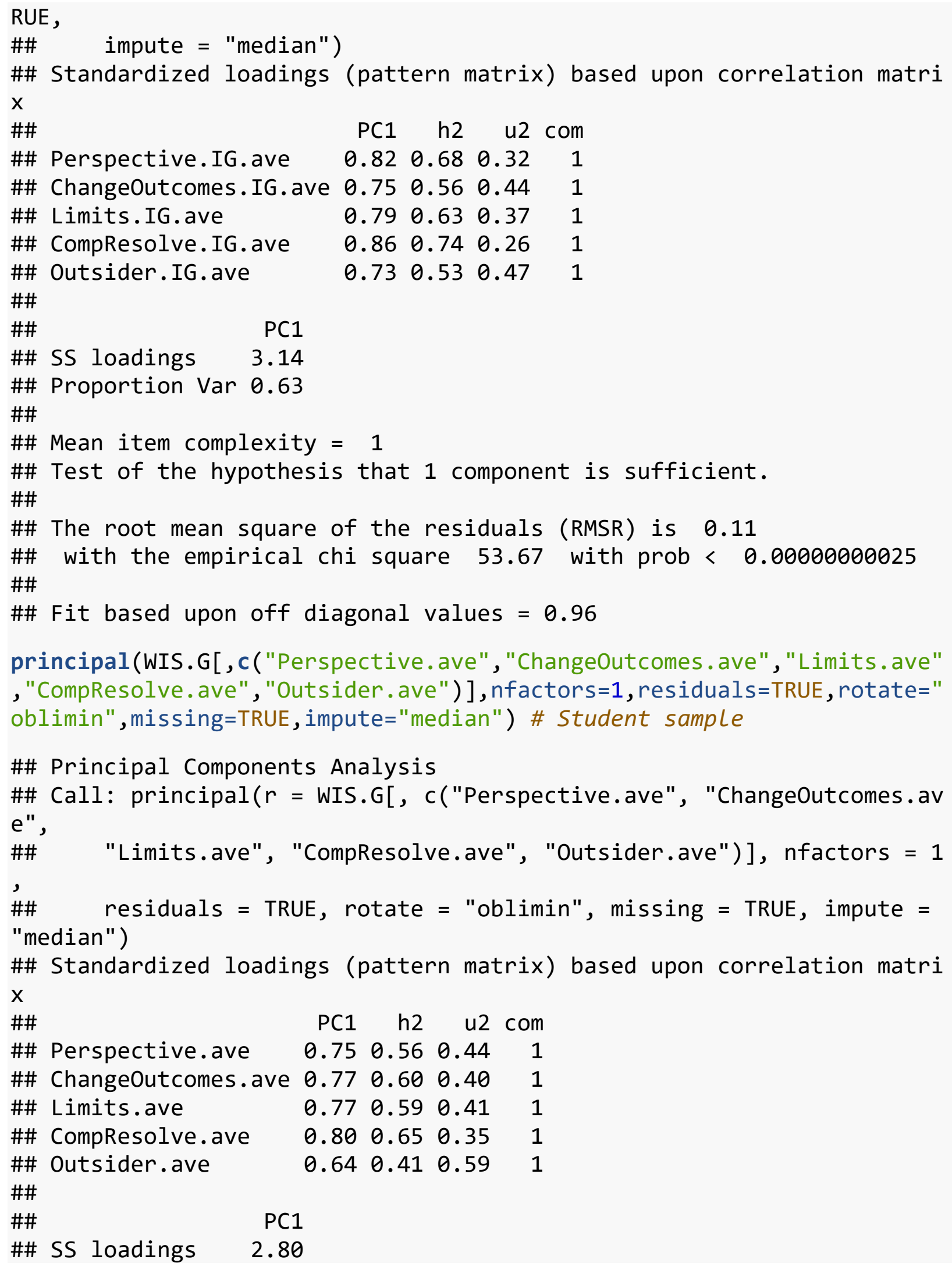




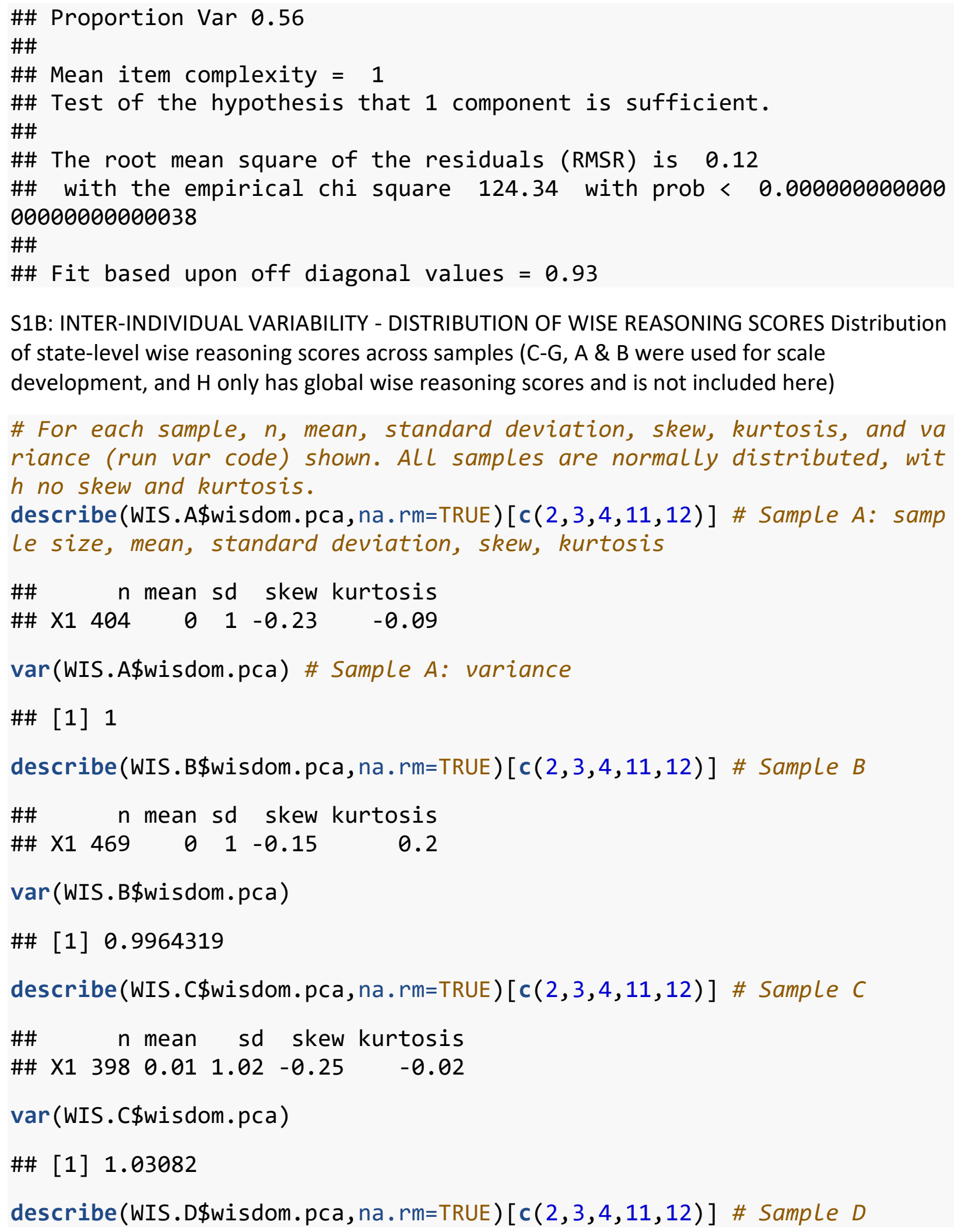




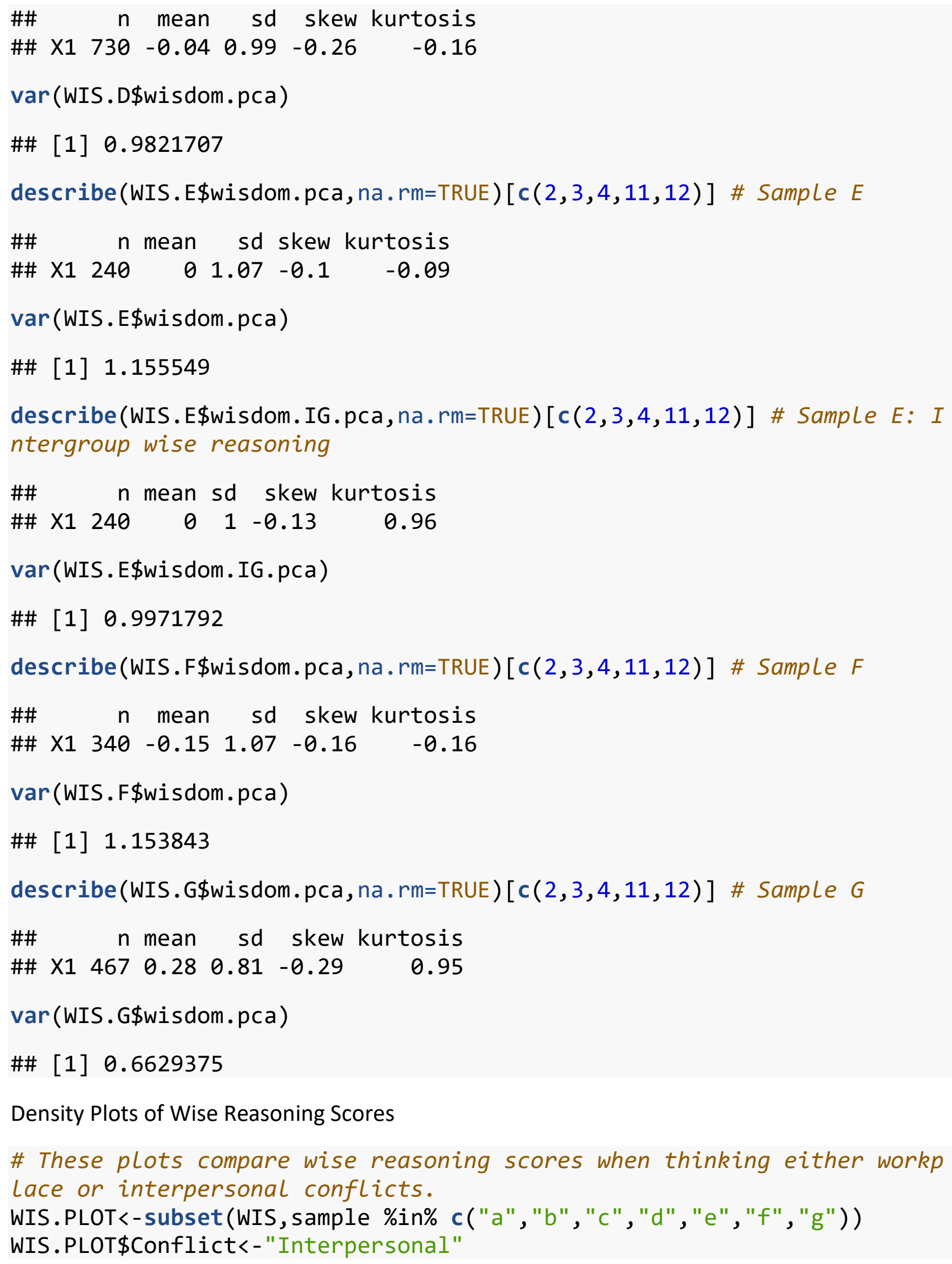


WIS.PLOT\$Conflict [WIS.PLOT\$sample=="a"]<-"Work-related" WIS.PLOT\$Conflict[WIS.PLOT\$sample=="b"]<-"Work-related"

\# The plots are similar across different kinds of conflicts and are no rmally distributed, suggesting that wisdom is not rare

ggplot (WIS.PLOT, aes ( $x=$ wisdom . ave, colour=Conflict, linetype=Conflict $)$ ) $+g$

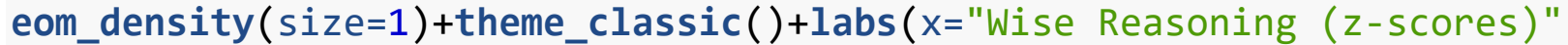
, $y=$ "Density")+scale_color_manual(values=c("royalblue1", "red1"))+theme( legend.position="top") \#Export as 600x400

\section{Conflict $\square$ Interpersonal}

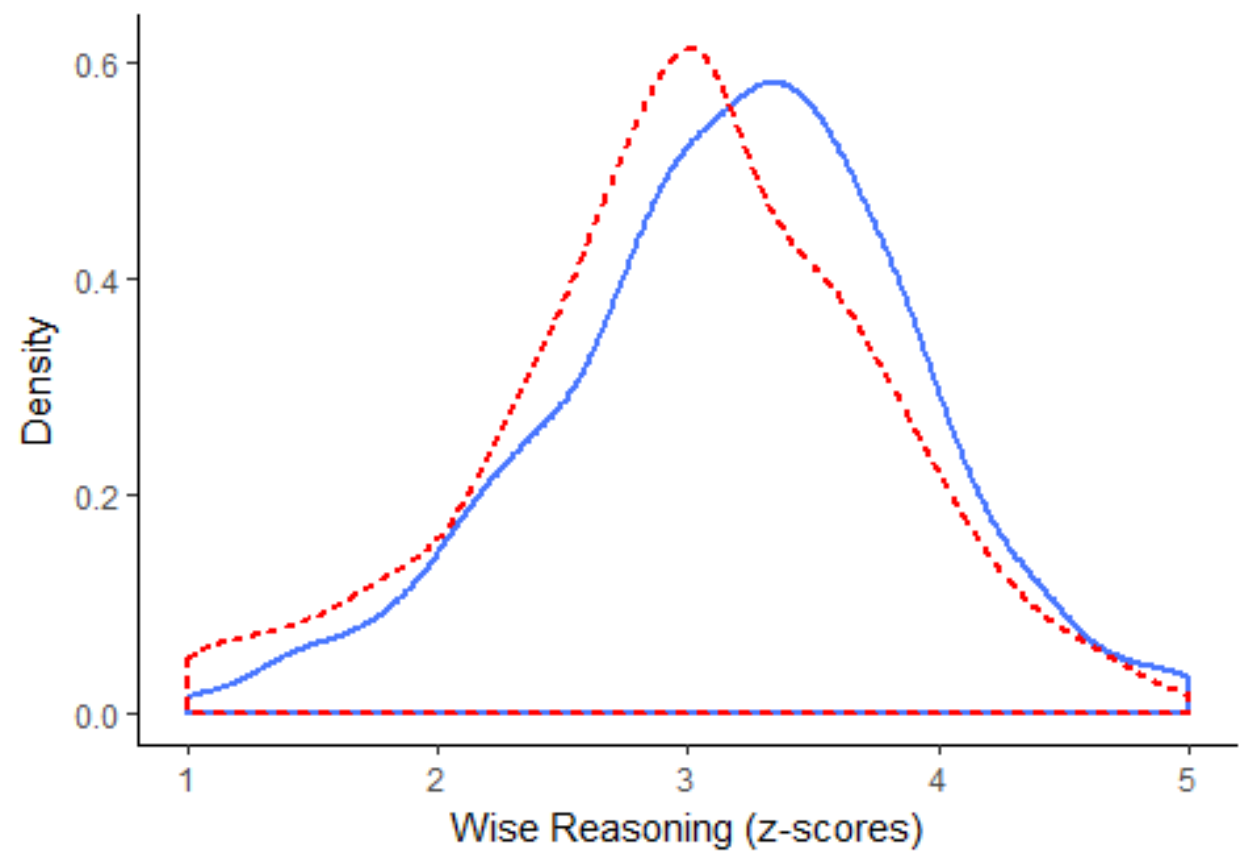

t-test comparing wise reasoning in interpersonal and work-related conflicts - As expected,

people report greater wise reasoning in interpersonal conflicts vs. work-related ones

\section{t.test (WIS.PLOT\$wisdom. ave WIS.PLOT\$Conflict)}

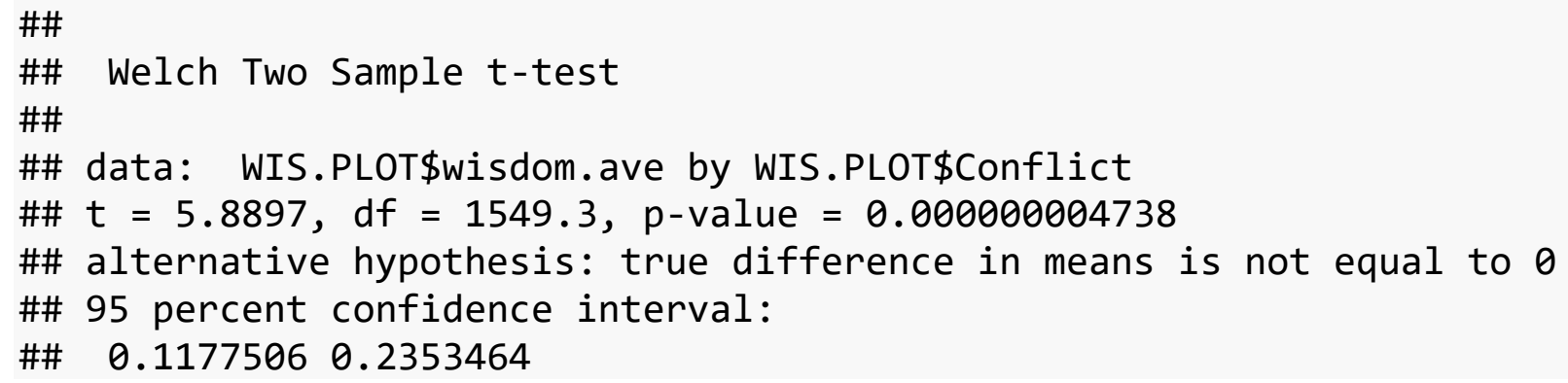


\#\# sample estimates:

\#\# mean in group Interpersonal mean in group Work-related

\#\# $\quad 3.173918 \quad 2.997369$

dlply (WIS. PLOT, "Conflict", summarise, mean=mean (wisdom. ave), sem=sd (wisdo m.ave)/sqrt(length(wisdom.ave)))[1:2] \# Means and standard error inter personal and work-related samples

\#\# \$Interpersonal

\#\# mean sem

\#\# $13.173918 \quad 0.01556033$

\#\#

\#\# \$`Work-related

\#\# mean sem

\#\# 12.9973690 .02562108

Reliability of Wise Reasoning Measure and Its Five Aspects across Samples Overall Sample (Samples C-F)

psych: :alpha(WIS[, c('w1', 'W2', 'W3', 'w4')], check.keys=FALSE)[1] \# Wise reasoning (Interpersonal) - Perspective Seeking Aspect

\#\# \$total

\#\# raw_alpha std.alpha $\mathrm{G6}(\mathrm{smc})$ average_r $\mathrm{S} / \mathrm{N}$ ase $\mathrm{m}$ ean

$\begin{array}{lllllllll}\text { \#\# } & 0.8274878 & 0.8283342 & 0.7987936 & 0.5467561 & 4.825271 & 0.004407379 & 3.024\end{array}$ 914

\#\# sd

\#\# $\quad 0.9415263$

psych: :alpha(WIS[,c('w5', 'w6', 'W7', 'w8')], check.keys=FALSE)[1] \# Wise reasoning (Interpersonal) - Change and Multiple Outcomes Aspect

\#\# \$total

\#\# raw_alpha std.alpha $\mathrm{G6}(\mathrm{smc})$ average_r $\mathrm{S} / \mathrm{N}$ ase me

an

$\begin{array}{llllllll}\text { \#\# } & 0.8119176 & 0.8118874 & 0.8414352 & 0.5189975 & 4.315965 & 0.005048865 & 3.313\end{array}$

87

\#\# $\quad$ sd

\#\# $\quad 0.9308932$

psych: :alpha(WIS [,c('w9', 'W10', 'W11', 'W12')], check.keys=FALSE)[1] \#W ise reasoning (Interpersonal) - Intellectual Humility Aspect

\#\# \$total

\#\# raw_alpha std.alpha $\mathrm{G6}(\mathrm{smc})$ average_r $\mathrm{S} / \mathrm{N}$ ase me an 


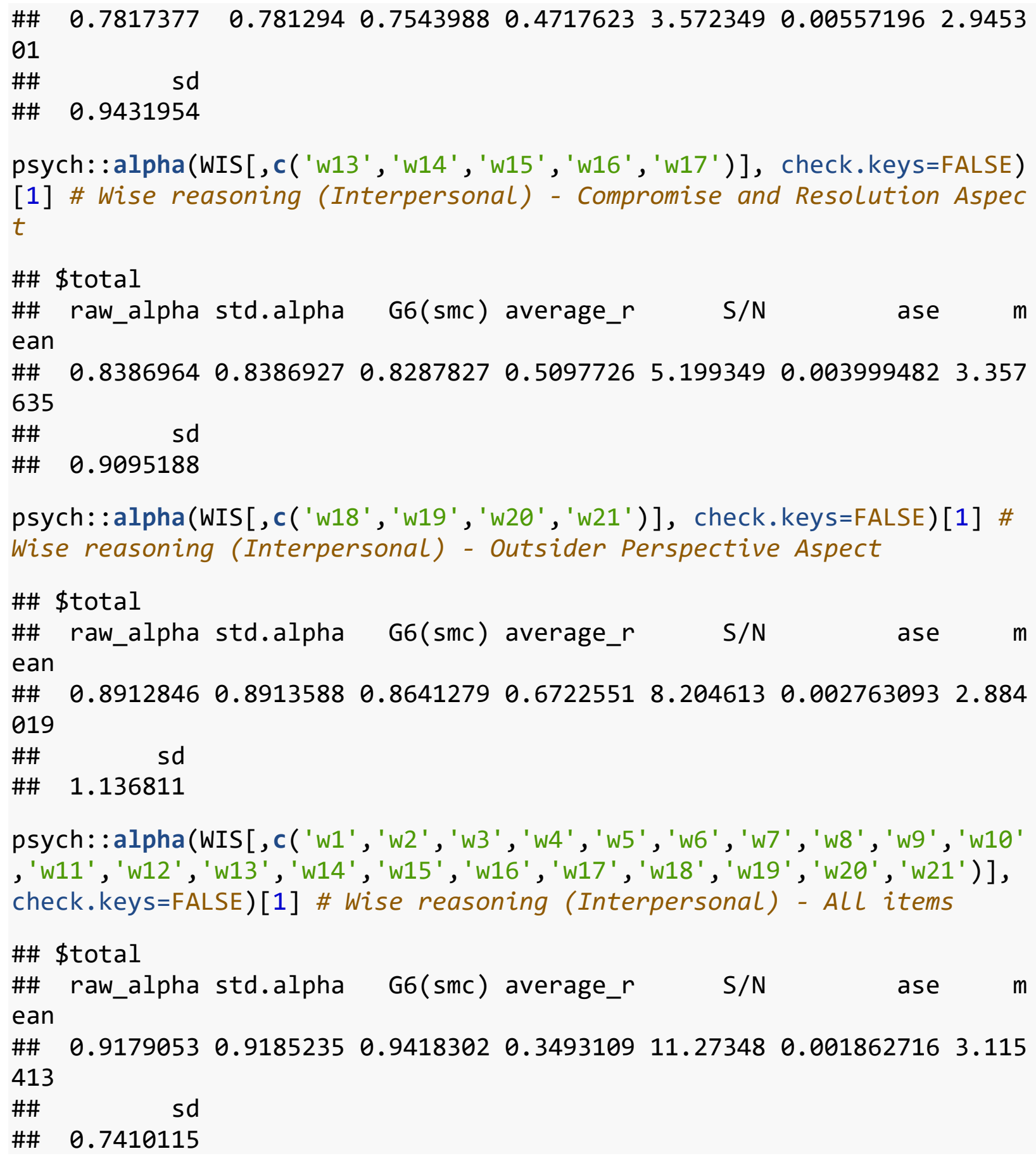

Sample C

psych: :alpha(WIS.C[, c('w1','w2', 'w3', 'w4')], check.keys=FALSE)[1] \# wi se reasoning (Interpersonal) - Perspective Seeking Aspect

\#\# \$total

\#\# raw_alpha std.alpha G6(smc) average_r S/N ase me 


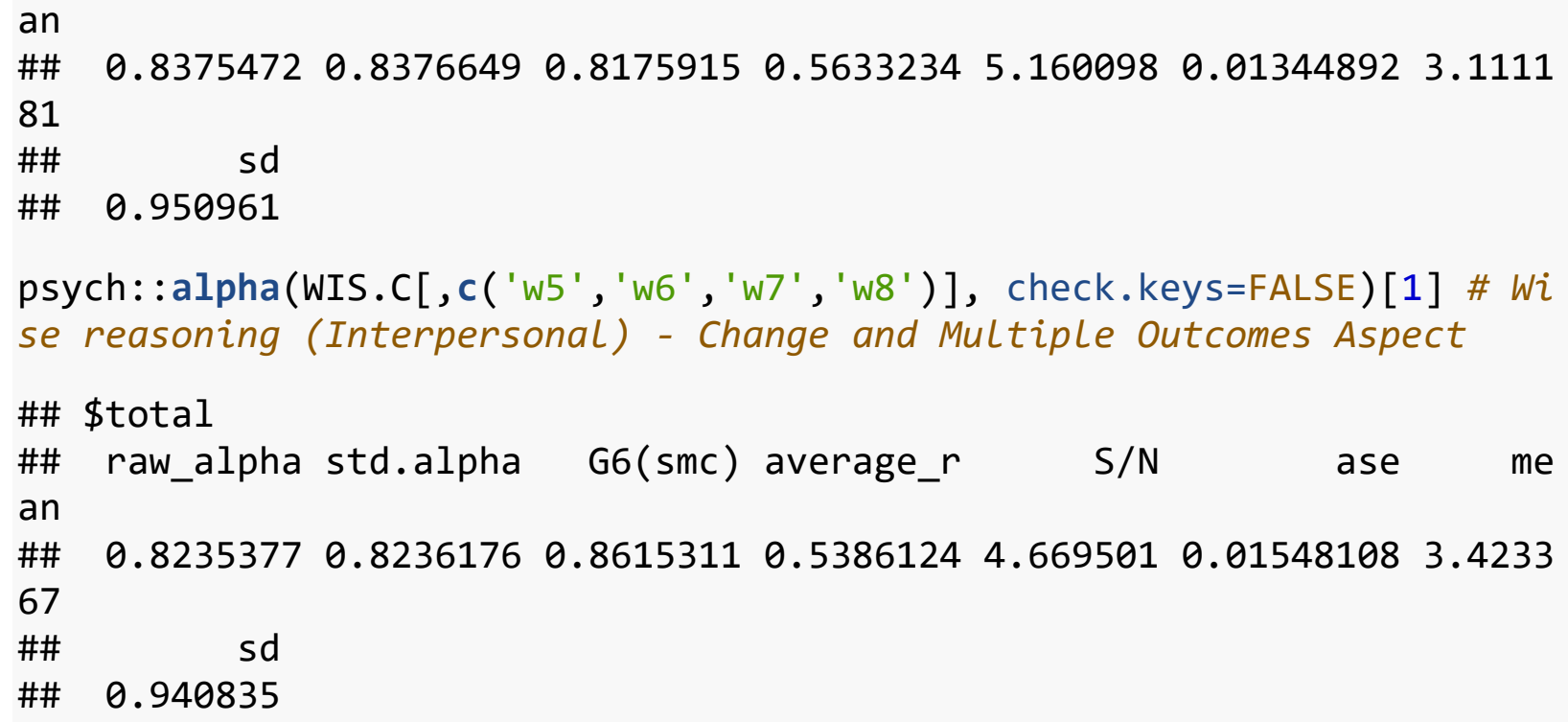

psych: :alpha(WIS.C[, c( 'w13', 'w14', 'w15', 'w16', 'w17')], check.keys=FALS E)[1] \# Wise reasoning (Interpersonal) - Compromise and Resolution Asp ect

\#\# \$total

\#\# raw_alpha std.alpha G6(smc) average_r S/N ase me

an 0.86957710 .86983830 .86091610 .57201876 .6827530 .010492823 .4568

$\begin{array}{llllllllll}\text { \#\# } & 0.8695771 & 0.8698383 & 0.8609161 & 0.5720187 & 6.682753 & 0.01049282 & 3.4568\end{array}$

53

\#\# sd

\#\# 0.955507

psych: :alpha(WIS.C[, c('w18', 'w19', 'w20', 'w21')], check.keys=FALSE)[1] \# Wise reasoning (Interpersonal) - Outsider Perspective Aspect

\#\# \$total

\#\# raw_alpha std.alpha G6(smc) average_r $\mathrm{S} / \mathrm{N}$ ase ean

$\begin{array}{llllllllll}\text { \#\# } & 0.8891256 & 0.8892189 & 0.8603011 & 0.6674097 & 8.026808 & 0.009100973 & 2.826\end{array}$ 


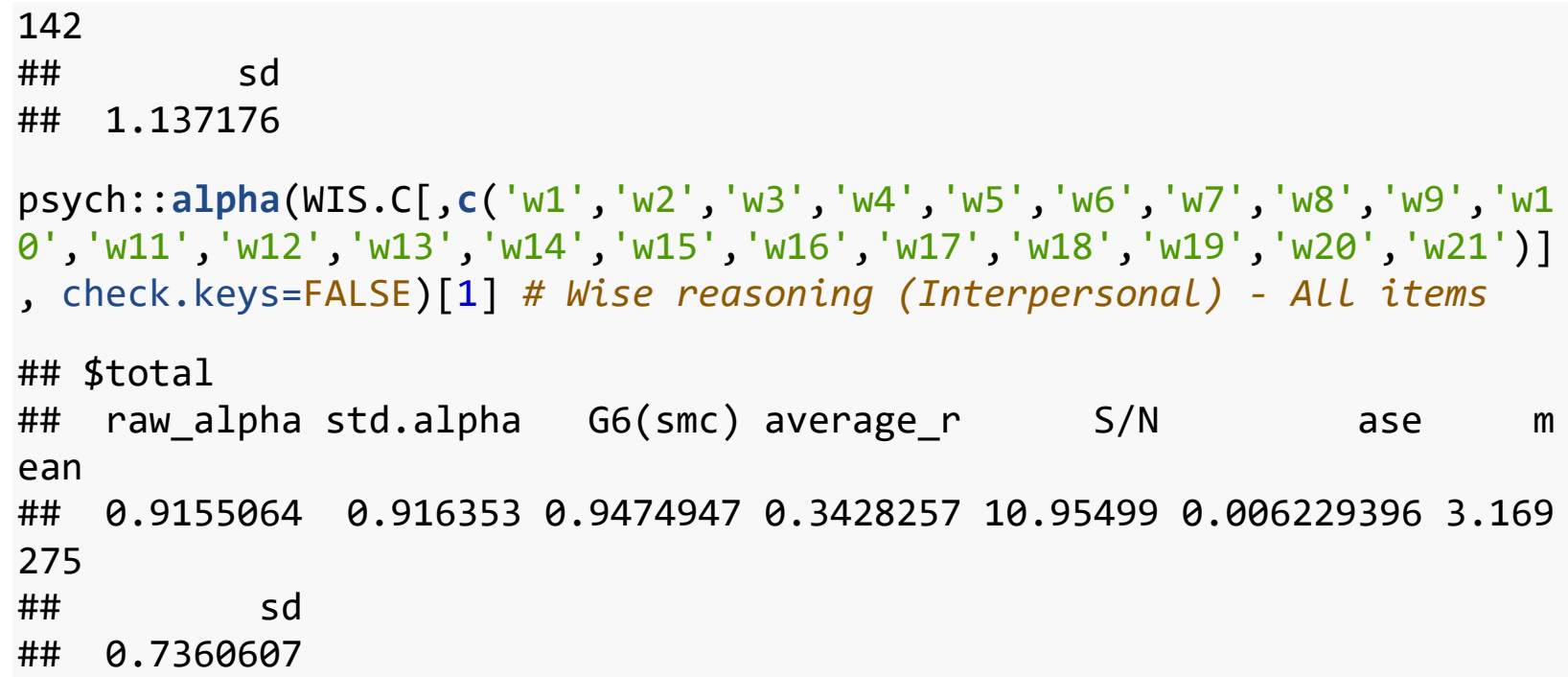

Sample D

psych: :alpha(WIS.D[, c('w1', 'w2', 'w3', 'w4')], check.keys=FALSE)[1] \# wi se reasoning (Interpersonal) - Perspective Seeking Aspect

\#\# \$total

\#\# raw_alpha std.alpha G6(smc) average_r $\mathrm{S} / \mathrm{N}$ ase me

an

$\begin{array}{llllllll}\text { \#\# } & 0.8201139 & 0.8213663 & 0.7960747 & 0.534778 & 4.598047 & 0.01101458 & 3.0534\end{array}$

98

\#\# sd

\#\# 0.9446666

psych: :alpha(WIS.D[, c('w5', 'w6', 'w7', 'w8')], check.keys=FALSE)[1] \# wi se reasoning (Interpersonal) - Change and Multiple Outcomes Aspect

\#\# \$total

\#\# raw_alpha std.alpha G6(smc) average_r $\mathrm{S} / \mathrm{N}$ ase me

$\begin{array}{llllllll}\text { an \# } & 0.803614 & 0.8035678 & 0.8682812 & 0.5056122 & 4.090814 & 0.01296278 & 3.3731\end{array}$

14

\#\# sd

\#\# $\quad 0.9555815$

psych: :alpha(WIS.D[, c('w9', 'w10', 'w11', 'w12')], check.keys=FALSE)[1] \# Wise reasoning (Interpersonal) - Intellectual Humility Aspect

\#\# \$total

\#\# raw_alpha std.alpha G6(smc) average_r $\mathrm{S} / \mathrm{N}$ ase mean

$\begin{array}{llllllll}\text { \#\# } & 0.7 \overline{5} 36975 & 0.7525741 & 0.7462699 & 0.4319484 & 3.041613 & 0.0151042 & 2.94726\end{array}$ 


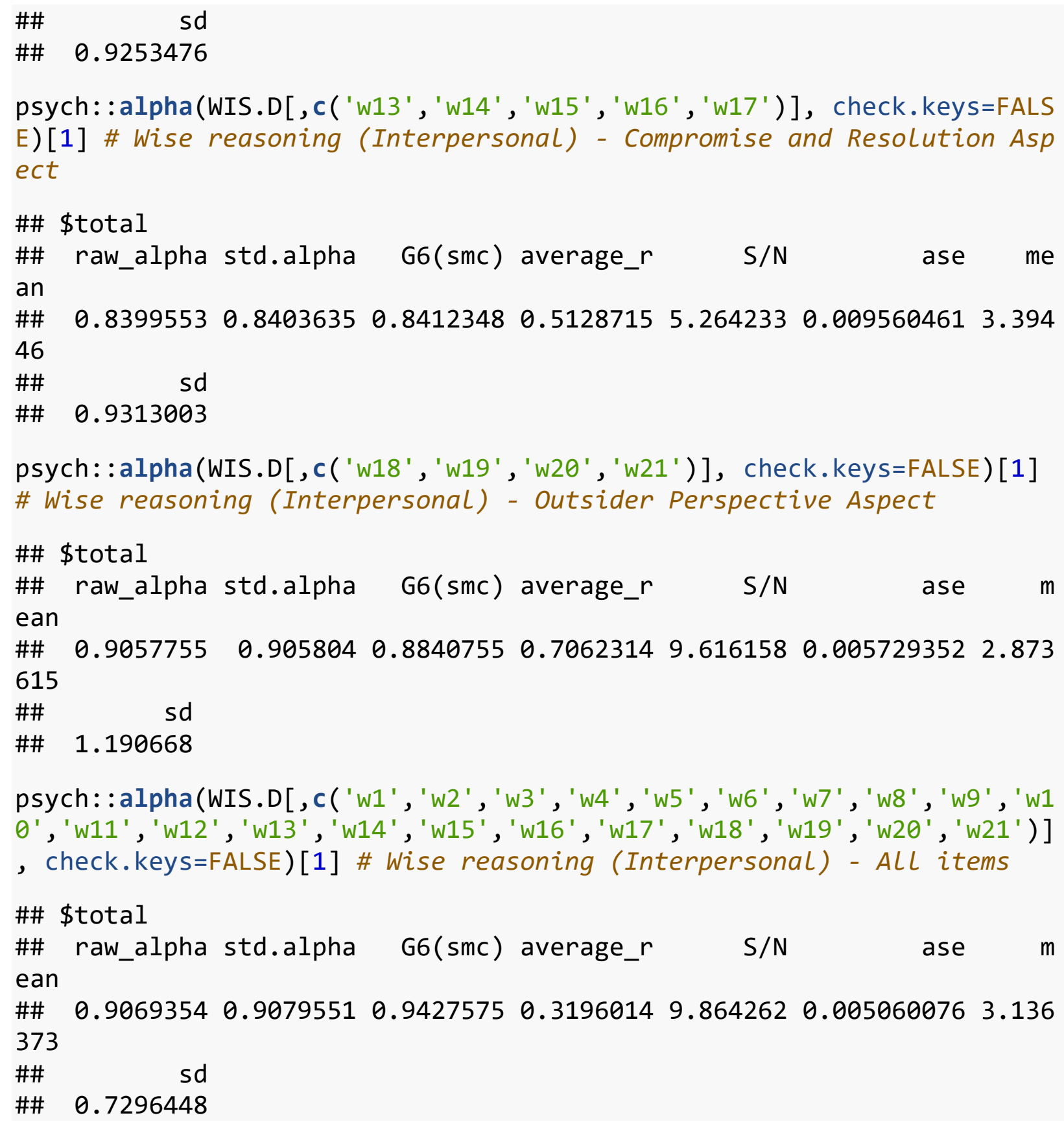

Sample E

psych: :alpha(WIS.E[,c('w1','w2', 'w3', 'w4')], check.keys=FALSE)[1] \# wi se reasoning (Interpersonal) - Perspective Seeking Aspect

\#\# \$total

\#\# raw_alpha std.alpha G6(smc) average_r $\mathrm{S} / \mathrm{N}$ ase me

$\begin{array}{llllllllll}\text { an } & & 0.843744 & 0.8452282 & 0.8194106 & 0.5772174 & 5.461127 & 0.01667525 & 3.0520\end{array}$ 


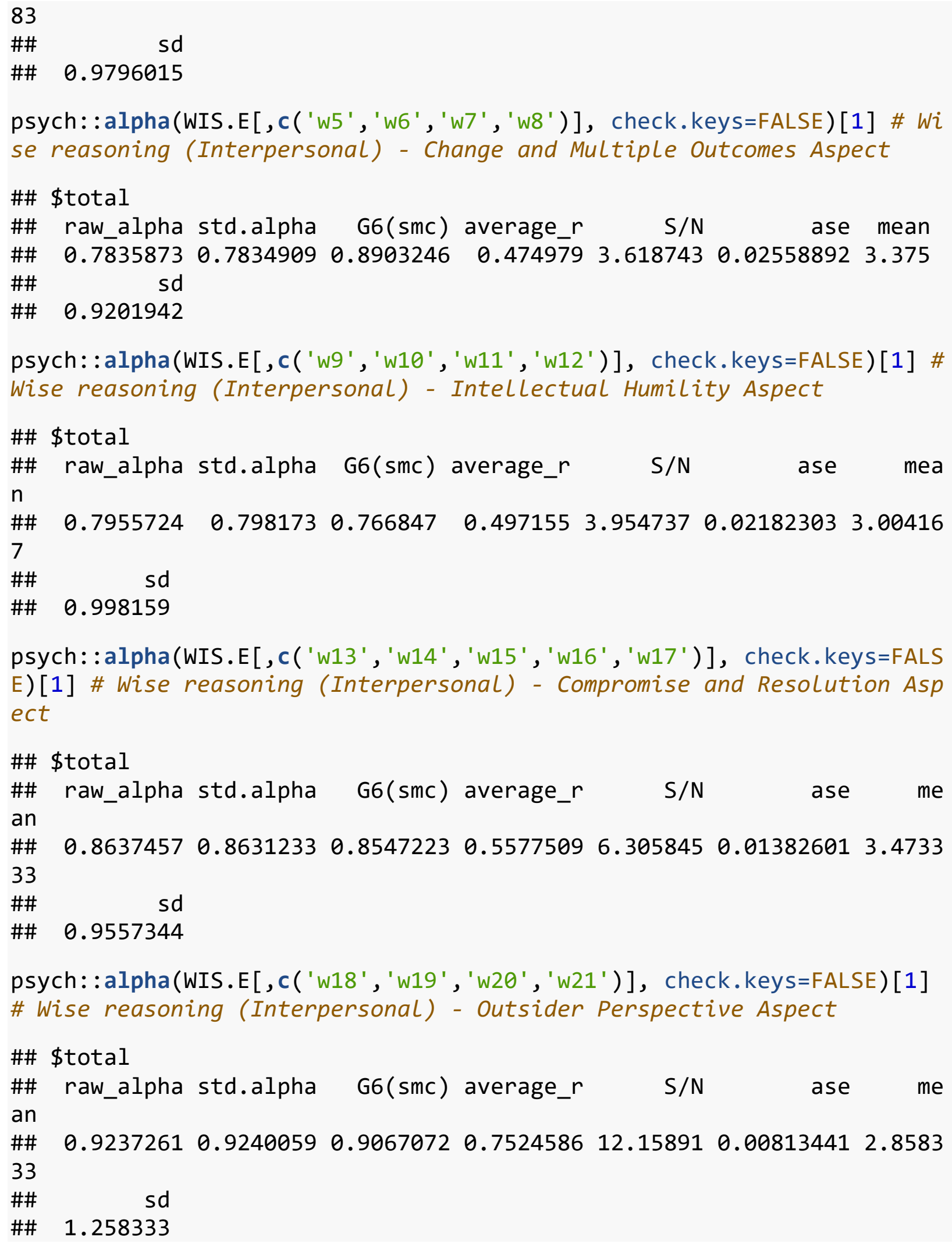


psych: :alpha(WIS.E[ , c( 'w1' , 'w2', 'w3', 'w4' , 'w5' ', 'w6', 'w7' , 'w8', 'w9' , 'w1 0', 'w11', 'w12', 'w13', 'w14' , 'w15', 'w16', 'w17', 'w18', 'w19' , 'w20', 'w21')] , check.keys=FALSE)[1] \# Wise reasoning (Interpersonal) - ALL items

\#\# \$total

\#\# raw_alpha std.alpha $\mathrm{G6}(\mathrm{smc})$ average_r $\mathrm{S} / \mathrm{N}$ ase $\mathrm{m}$ ean

$\begin{array}{llllllll}\text { \#\# } & 0.9242289 & 0.9250221 & 0.9571428 & 0.3700743 & 12.33726 & 0.007153152 & 3.167\end{array}$

857

\#\# sd

\#\# $\quad 0.7842887$

Sample F

psych: :alpha(WIS.F[, c('w1', 'W2', 'W3', 'W4')], check.keys=FALSE)[1] \# Wi se reasoning (Interpersonal) - Perspective Seeking Aspect

\#\# \$total

\#\# raw_alpha std.alpha $\mathrm{G6}(\mathrm{smc})$ average_r $\mathrm{S} / \mathrm{N}$ ase me

an

$\begin{array}{lllllllll}\text { \#\# } & 0.8298454 & 0.8312035 & 0.8145137 & 0.5517853 & 4.924295 & 0.01537535 & 2.9058\end{array}$

82

\#\# sd

\#\# $\quad 0.9399181$

psych: :alpha(WIS.F[, c('w5', 'w6', 'W7', 'W8')], check.keys=FALSE)[1] \# Wi se reasoning (Interpersonal) - Change and Multiple Outcomes Aspect

\#\# \$total

\#\# raw_alpha std.alpha $\mathrm{G6}(\mathrm{smc})$ average_r $\mathrm{S} / \mathrm{N}$ ase mea n

$\begin{array}{llllllll}\text { \#\# } & 0.8343392 & 0.8342274 & 0.8738546 & 0.5571479 & 5.03236 & 0.01576481 & 3.28504\end{array}$ 9

\#\# sd

\#\# 0.9520938

psych: :alpha(WIS.F[, c('w9', 'W10', 'W11', 'W12')], check.keys=FALSE)[1] \# Wise reasoning (Interpersonal) - Intellectual Humility Aspect

\#\# \$total

\#\# raw_alpha std.alpha $\mathrm{G6}(\mathrm{smc})$ average_r $\mathrm{S} / \mathrm{N}$ ase me

$\begin{array}{llllllll}\text { an } & & & & \end{array}$

71

\#\# $\quad$ sd

\#\# $\quad 0.9352506$ 
psych: : alpha(WIS.F[, c('W13', 'W14', 'W15', 'w16', 'w17')], check.keys=FALS E)[1] \# Wise reasoning (Interpersonal) - Compromise and Resolution Asp ect

\#\# \$total

\#\# raw_alpha std.alpha $\mathrm{G6}(\mathrm{smc})$ average_r $\mathrm{S} / \mathrm{N}$ ase me

an

$\begin{array}{lllllllll}\# \# & 0.8665463 & 0.865917 & 0.8583707 & 0.5636262 & 6.458066 & 0.01154731 & 3.3263\end{array}$

24

\#\# sd

\#\# $\quad 0.9353385$

psych: :alpha(WIS.F [,c('w18', 'w19' , 'w20', 'w21')], check.keys=FALSE)[1] \# Wise reasoning (Interpersonal) - Outsider Perspective Aspect

\#\# \$total

\#\# raw_alpha std.alpha G6(smc) average_r $\quad \mathrm{S} / \mathrm{N}$ ase $\begin{array}{llllllllll}\text { ean } & 0.8949097 & 0.8952349 & 0.8657723 & 0.681152 & 8.545164 & 0.009307127 & 2.894\end{array}$ 118

\#\# sd

\#\# 1.12371

psych: :alpha(WIS.F[, c( 'w1', 'w2', 'w3 ', 'w4 ', 'w5 ', 'w6 ', 'w7 ', 'w8 ', 'w9 ', 'w1 0', 'w11', 'w12', 'w13', 'w14', 'w15 ', 'w16', 'w17', 'w18', 'w19 ', 'w20', 'w21 ') ] , check.keys=FALSE)[1] \# Wise reasoning (Interpersonal) - ALL items

\#\# \$total

\#\# raw_alpha std.alpha G6(smc) average_r $\quad$ S/N $\quad$ ase ean

$\begin{array}{lllllllll}\# \# & 0.9329474 & 0.9334466 & 0.9560481 & 0.4004373 & 14.02553 & 0.005313516 & 3.067\end{array}$ 871

\#\# sd

\#\# $\quad 0.7813972$

\#\#\#\# S2: STUDY 2 \#\#\#\#

LOADING PACKAGES

require(psych)

require(car)

\#\# Loading required package: car

\#\# Warning: package 'car' was built under $\mathrm{R}$ version 3.3 .3 


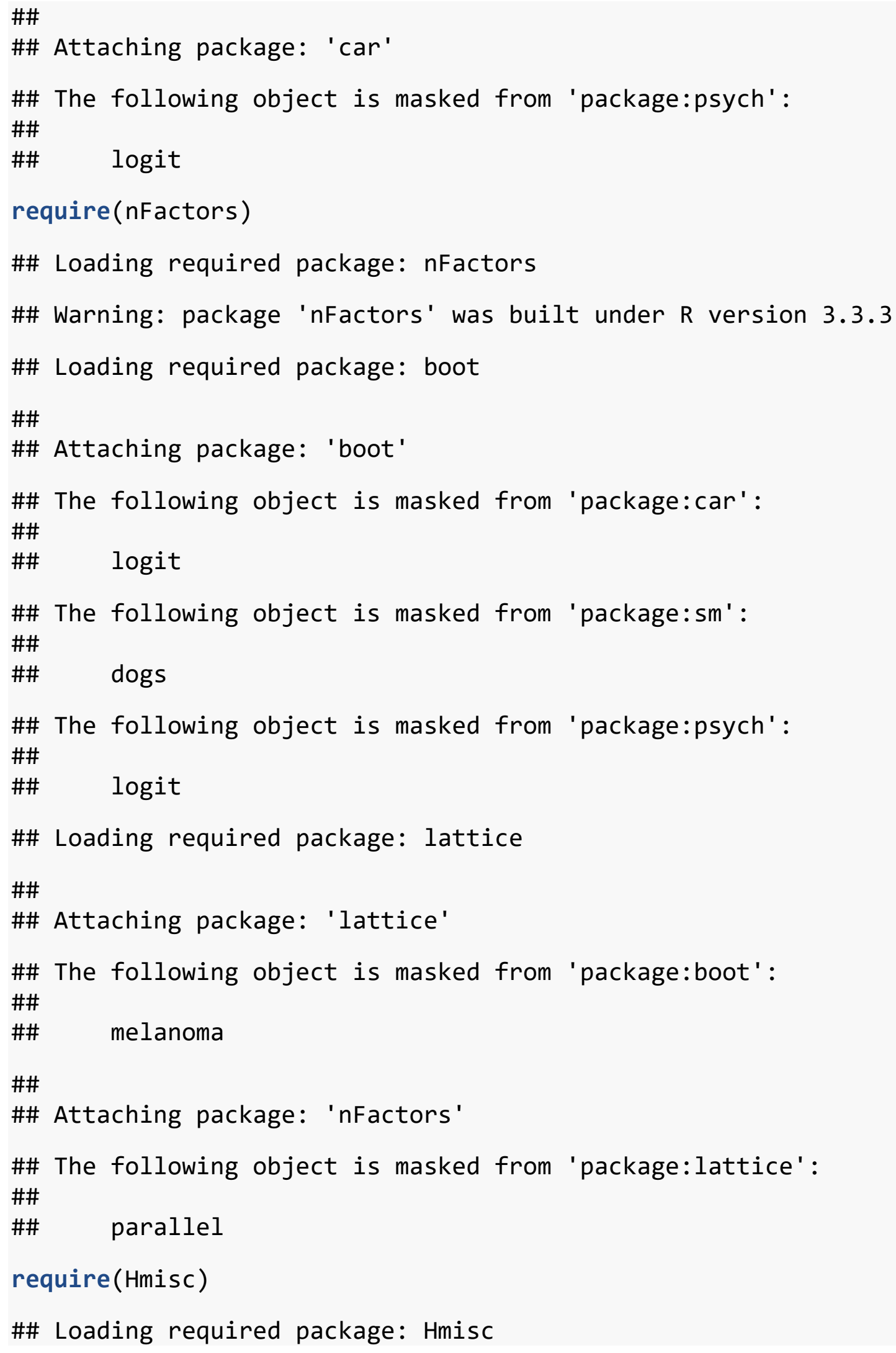


\#\# Warning: package 'Hmisc' was built under $\mathrm{R}$ version 3.3 .2

\#\# Loading required package: survival

\#\# Warning: package 'survival' was built under $\mathrm{R}$ version 3.3 .2

\#\#

\#\# Attaching package: 'survival'

\#\# The following object is masked from 'package:boot':

\#\#

\#\# aml

\#\# Loading required package: Formula

\#\# Warning: package 'Formula' was built under $\mathrm{R}$ version 3.3 .2

\#\#

\#\# Attaching package: 'Hmisc'

\#\# The following objects are masked from 'package:plyr':

\#\#

\#\# is.discrete, summarize

\#\# The following object is masked from 'package:psych':

\#\#

\#\# describe

\#\# The following objects are masked from 'package:base':

\#\#

\#\# format.pval, round.POSIXt, trunc.POSIXt, units

require(tidyr)

\#\# Loading required package: tidyr

\#\# Warning: package 'tidyr' was built under $\mathrm{R}$ version 3.3.3

\#\#

\#\# Attaching package: 'tidyr'

\#\# The following object is masked from 'package:Matrix':

\#\#

\#\# expand

\#\# The following object is masked from 'package:reshape2':

\#\#

\#\# smiths 


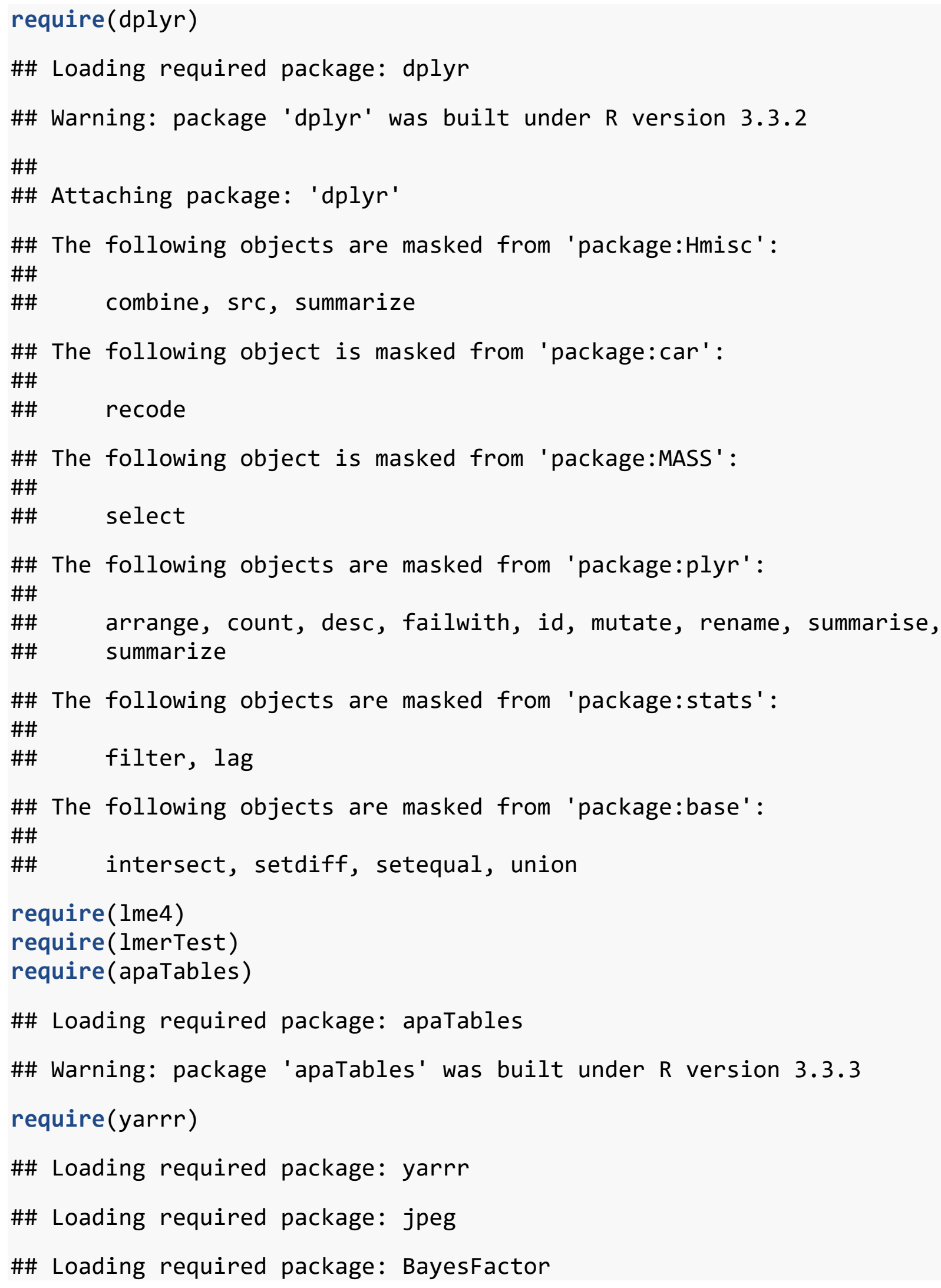




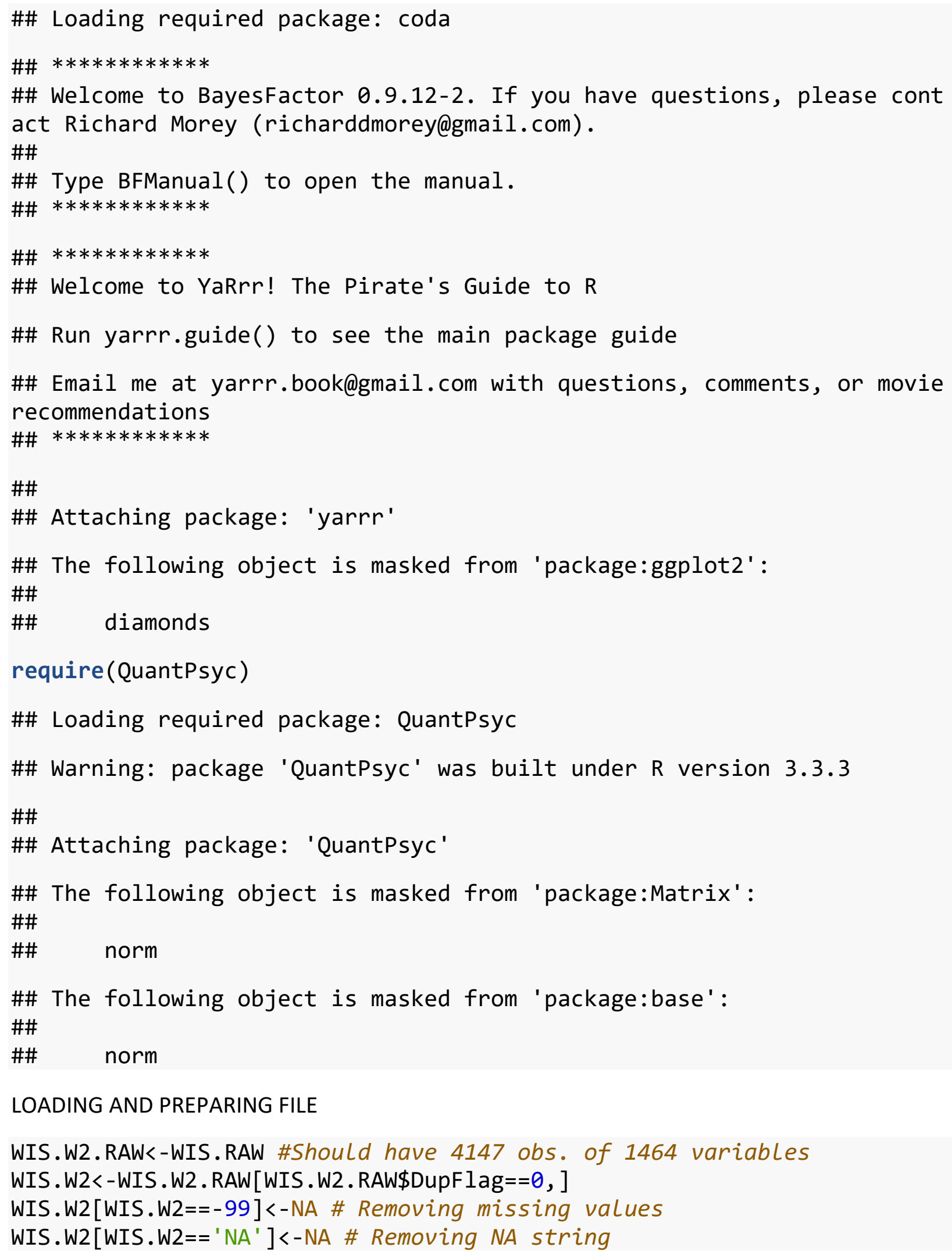


WIS.W2.I<-WIS.W2[WIS.W2\$sample=="W2", ] \# Sample W2 (Study 6); $n=293$ \#demographics (just descriptives, no analyses)

describe(WIS.W2.I [,c('Age', 'Ethnicity', 'gender', 'edu', 'Employment')]) \#\# WIS.W2.I[, c("Age", "Ethnicity", "gender", "edu", "Employment")] \#\#

\#\# 5 Variables 293 Observations

\#\#

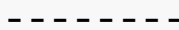

\#\# Age

\#\# $\mathrm{n}$ missing distinct

Info

Mean

Gmd

.05

.10

\#\# 293

0

53

0.999

38.83

13.99

23.6

26.0

\#\#

.25

.50

.75

.90

.95

\#\#

29.0

36.0

46.0

60.0

63.4

\#\#

\#\# lowest : 20212223 24, highest: 7274767790

\#\#

\#\# Ethnicity

\#\# $\quad n$ missing distinct

\#\# $\quad 284 \quad 9 \quad 5$

\#\#

\#\# Value African-American

\#\# Frequency

22

\#\# Proportion

0.077

Asian-American

Latino

20

16

\#\#

\#\# Value

Other White/European

\#\# Frequency

12

214

\#\# Proportion

0.042

0.754

\#\#

- - - - - -

\#\# gender

\#\# $n$ missing distinct

\#\# $291 \quad 2$

\#\#

\#\# Value Female Male

\#\# Frequency $171 \quad 120$

\#\# Proportion $0.588 \quad 0.412$

\#\# - - - -

\#\# edu 


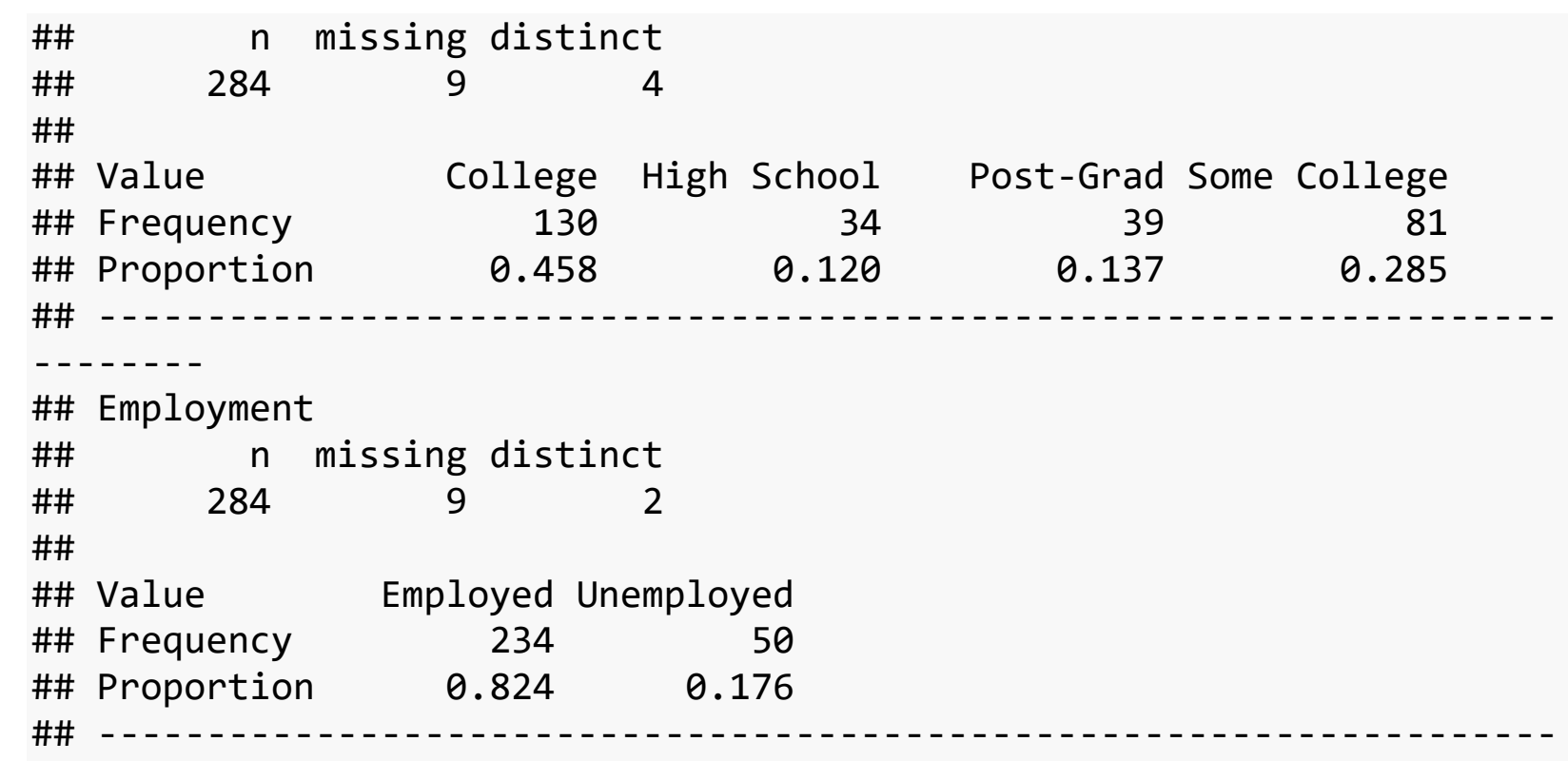

Median Income

\# 1='Under $\$ 15,000 ' ; 2=' \$ 15,001-\$ 25,000 ' ; 3=' \$ 25,001-\$ 35,000 ' ; 4=' \$ 35,001$ $-\$ 50,000 '$;

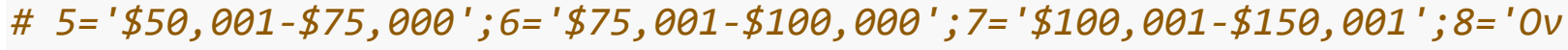
er $\$ 150,000$ '

median (WIS.W2.I\$Income, na.rm=TRUE)

\#\# [1] 5

\#time taking survey

dtparts = as.data.frame(t(as.data.frame(strsplit(WIS.W2.I\$V8, ' ')))) \# isolating date and time

tparts = as.data.frame(t(as.data.frame(strsplit(as.character(dtparts [, 2]), '\(:')))) \#isolating hours and minutes

row.names (tparts) $=$ NULL

WIS.W2. I\$Start<- (as . numeric (as . character (tparts $\$ V 1)$ ) *60) +as . numeric (as - character(tparts\$V2)) \#start time in minutes from midnight

dtparts = as.data.frame(t(as.data.frame(strsplit(WIS.W2.I\$V9, ' '))) $\#$ isolating date and time

tparts = as.data.frame(t(as.data.frame(strsplit(as.character (dtparts $[$, 2]), "(\:")))) \#isolating hours and minutes

row.names (dtparts) $=$ NULL

WIS.W2. I $\$$ End<- (as . numeric (as . character (tparts $\$ V 1)$ ) *60) +as . numeric (as . c haracter(tparts\$V2)) \#End time in minutes from midnight

WIS.W2. I\$End[WIS.W2. I\$End<WIS.W2. I\$Start]<-WIS.W2. I\$End[WIS.W2. I\$End<W IS.W2.I\$Start]+1440 \#Fixing times that went over midnight 
WIS.W2.I\$SurveyTime<-WIS.W2.I\$End-WIS.W2.I\$Start \#Time spent on survey WIS.W2.I\$SurveyTime[is.na(WIS.W2.I\$End)]<-NA \#Removing incomplete median (WIS.W2.I\$SurveyTime)

\section{\#\# [1] 17}

within-person variability wise reasoning over time

WRList<-WIS.W2[ ! is.na(WIS.W2\$PID)\&WIS.W2\$sample!="i" , c ("PID", "Session" , "wisdom.ave")] \#Selecting participants who have a PID (for within-sub ject purposes) and not from sample $i$

WRList. long<-reshape(WRList, idvar="PID", timevar="Session", direction="w ide") \#Transforming to wide format

WRList.long\$WRcount<-rowSums(! is.na(WRList.long[,2:5])) \#Counting numb er of datapoints

sum(WRList.long\$WRcount>1) \# 290 with 2 or more datapoints

\#\# [1] 290

WIS.W2<-merge(WIS.W2, WRList. long [ , c ("PID", "WRcount") ], by="PID", all . x=T RUE) \#Merging datapoint count to main file if needed WIS.W2I<-WIS.W2 [WIS.W2\$sample! =" i"\&WIS.W2\$WRcount >1, ]

total score

summary (1mer (wisdom.ave (1|PID), data=WIS.W2I))

\#\# summary from lme4 is returned

\#\# some computational error has occurred in lmerTest

\#\# Linear mixed model fit by REML ['lmerMod']

\#\# Formula: wisdom.ave (1 | PID)

\#\# Data: WIS.W2I

\#\#

\#\# REML criterion at convergence: 1277.4

\#\#

\#\# Scaled residuals:

\#\# Min $1 Q$ Median $3 Q \quad$ Max

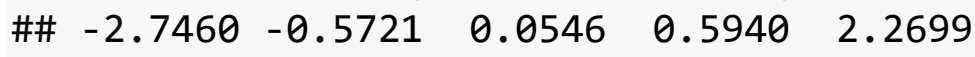

\#\#

\#\# Random effects:

\#\# Groups Name Variance Std.Dev.

$\begin{array}{llll}\text { \#\# PID (Intercept) } & 0.2313 & 0.4809\end{array}$

\#\# Residual $\quad 0.32140 .5669$

\#\# Number of obs: 594, groups: PID, 290

\#\#

\#\# Fixed effects: 


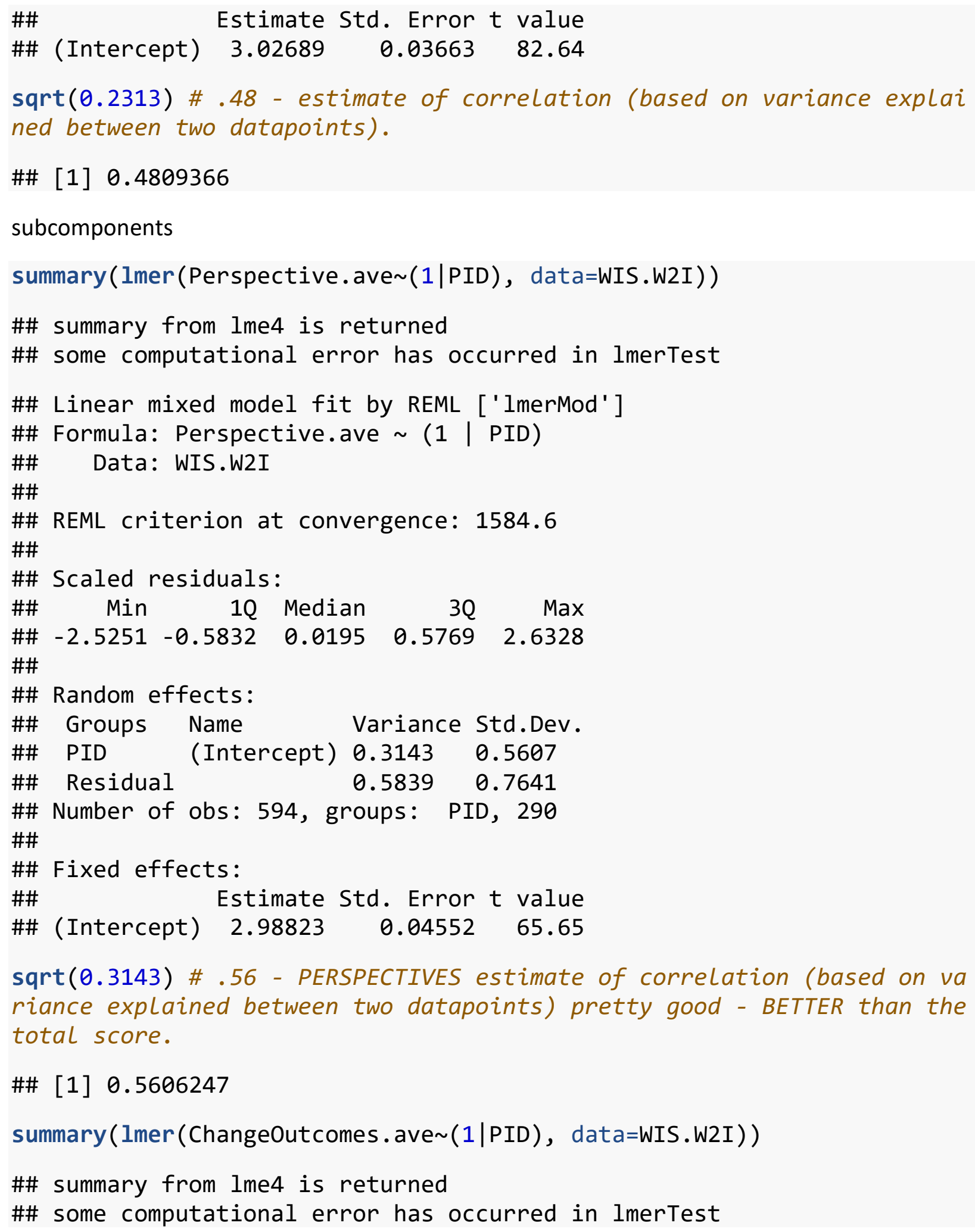




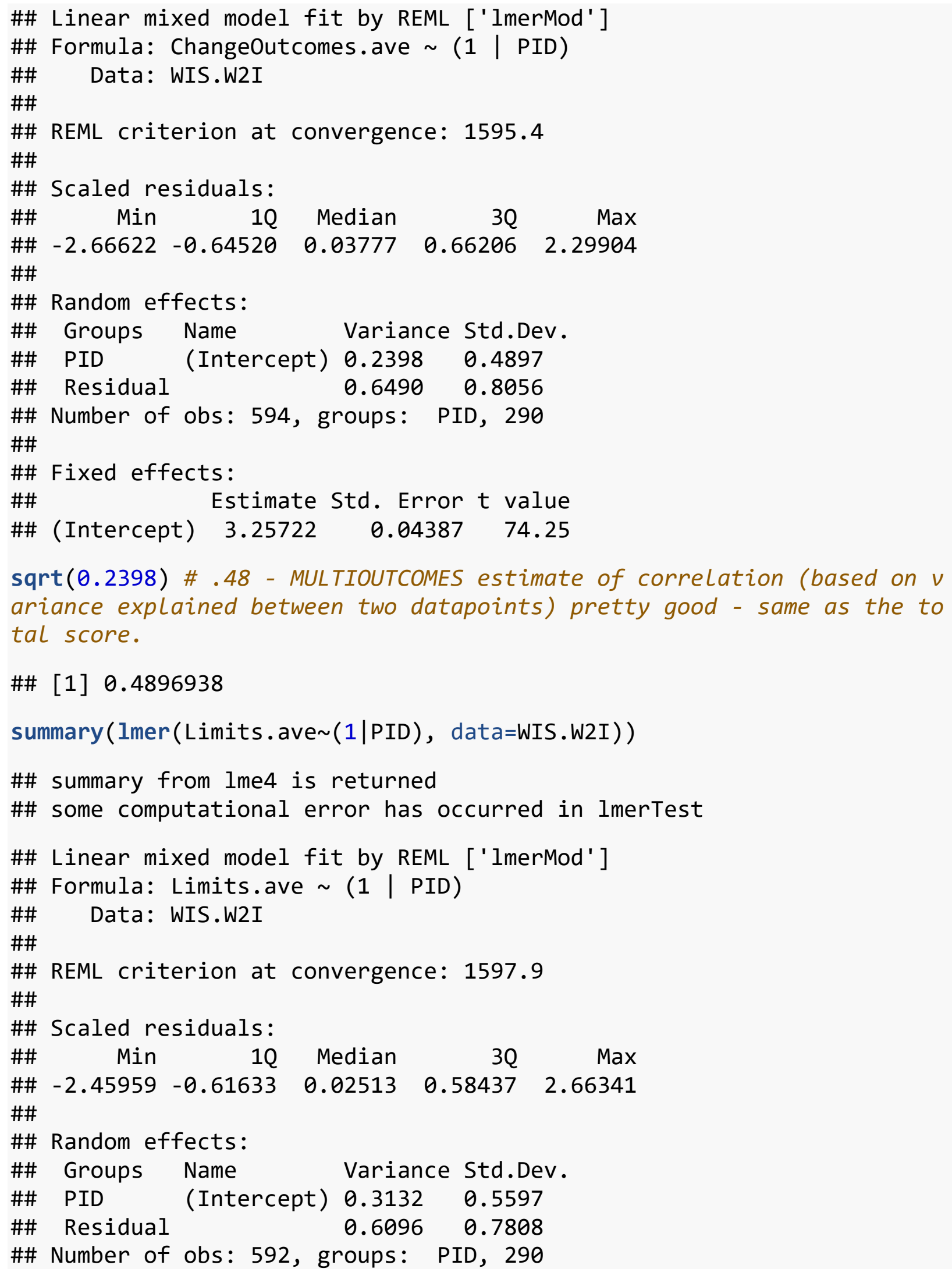




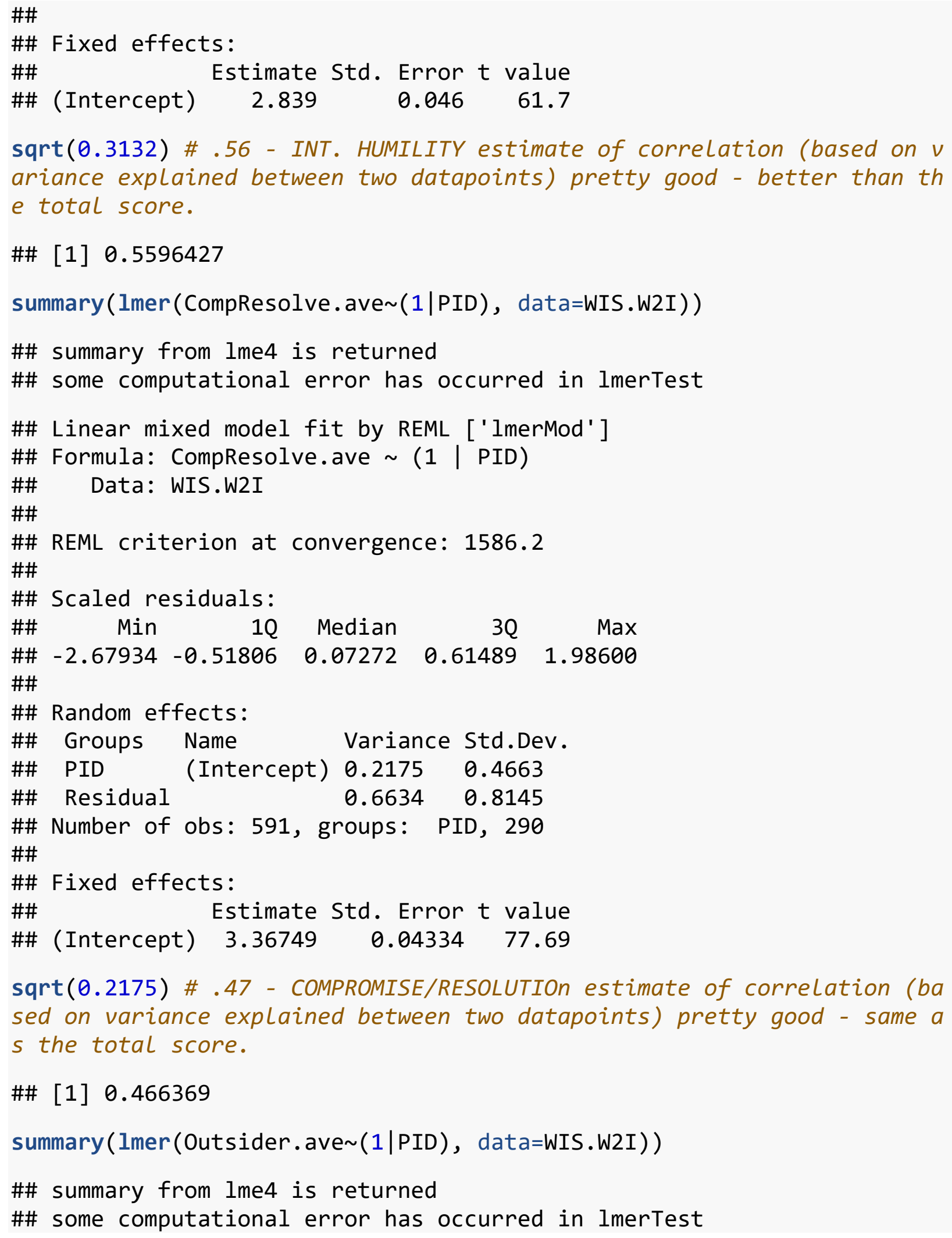




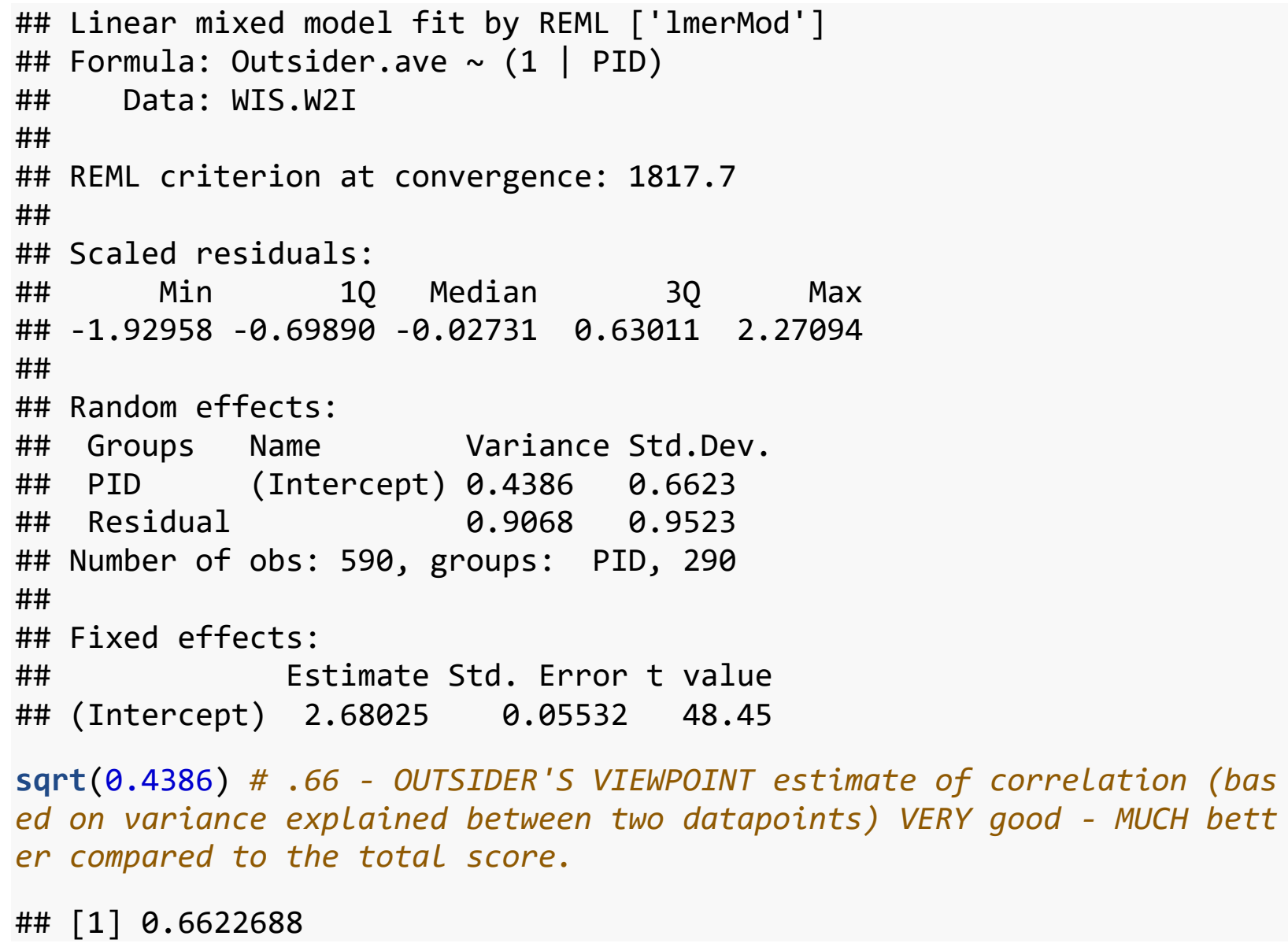

ASTIList<-WIS.W2 [ ! is .na(WIS.W2\$PID)\&WIS.W2\$sample!="i ", c( "PID", "Sessio n", "ASTISelfTrans")] \#Selecting participants who have a PID (for withi $n$-subject purposes) and not from sample $i$

ASTIList. long<-reshape(ASTIList, idvar="PID", timevar="Session", directio $\mathrm{n}=$ "wide") \#Transforming to wide format

ASTIList.long\$ASTIcount<-rowSums(! is.na(ASTIList.long[, 2:5])) \#Countin $g$ number of datapoints sum(ASTIList.long\$ASTIcount>1) \# 40 with 2 or more datapoints

\#\# [1] 40

WIS.W2<-merge(WIS.W2, ASTIList. long [ , c("PID", "ASTIcount") ] , by="PID", all . $x=$ TRUE) \#Merging datapoint count to main file if needed 


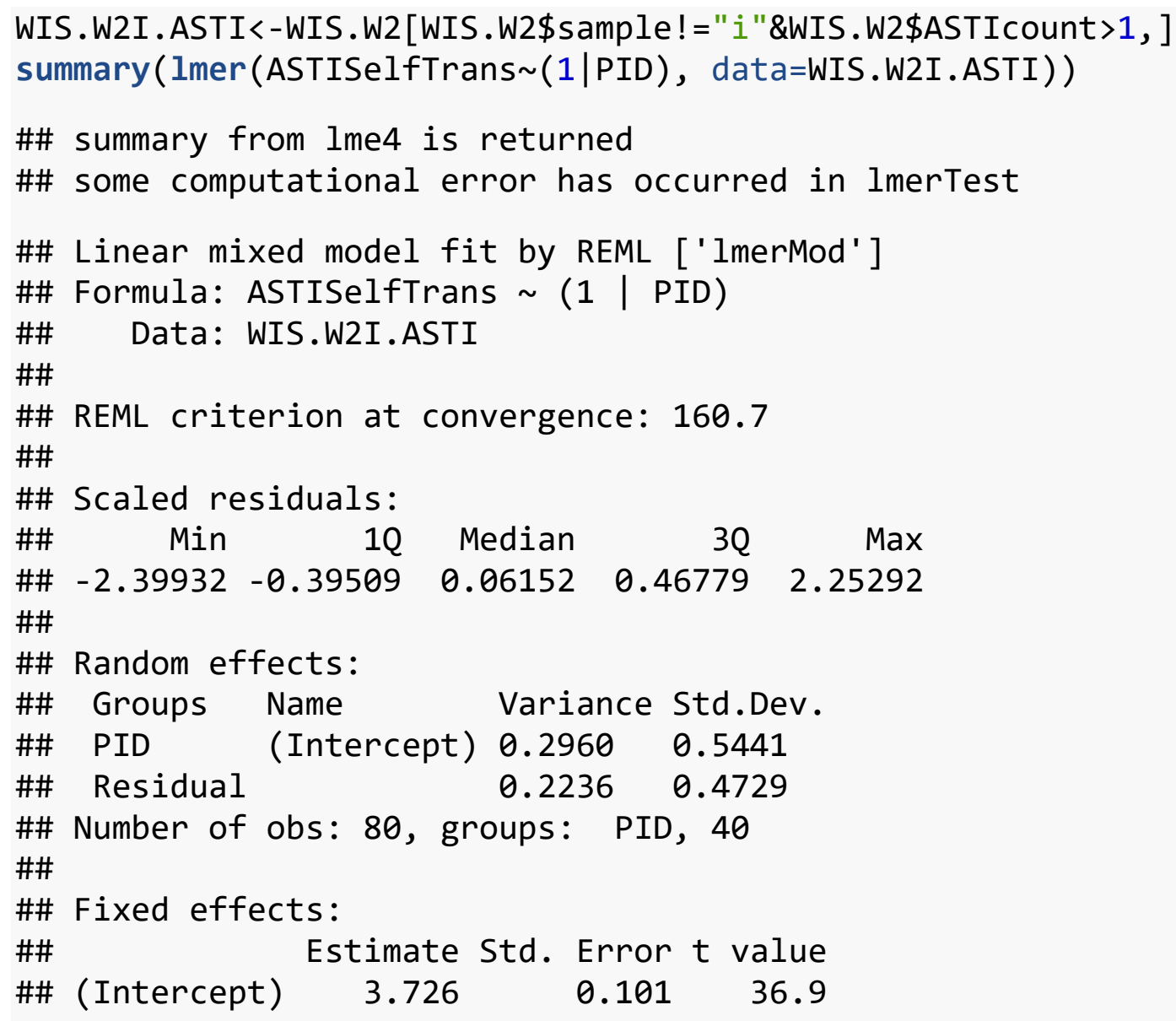
ession","TDWSBrief")] \#Selecting participants who have a PID (for with in-subject purposes) and not from sample $i$

TDWSBriefList. long<-reshape(TDWSBriefList, idvar="PID", timevar="Session ", direction="wide") \#Transforming to wide format

TDWSBriefList. long\$TDWSBriefcount<-rowSums (! is . na (TDWSBriefList. long [, 2:5])) \#Counting number of datapoints sum(TDWSBriefList.long\$TDWSBriefcount>1) \# 41 with 2 or more datapoint S

\#\# [1] 41

WIS.W2<-merge(WIS.W2, TDWSBriefList.long[, c("PID", "TDWSBriefcount") ], by ="PID",all.x=TRUE) \#Merging datapoint count to main file if needed 


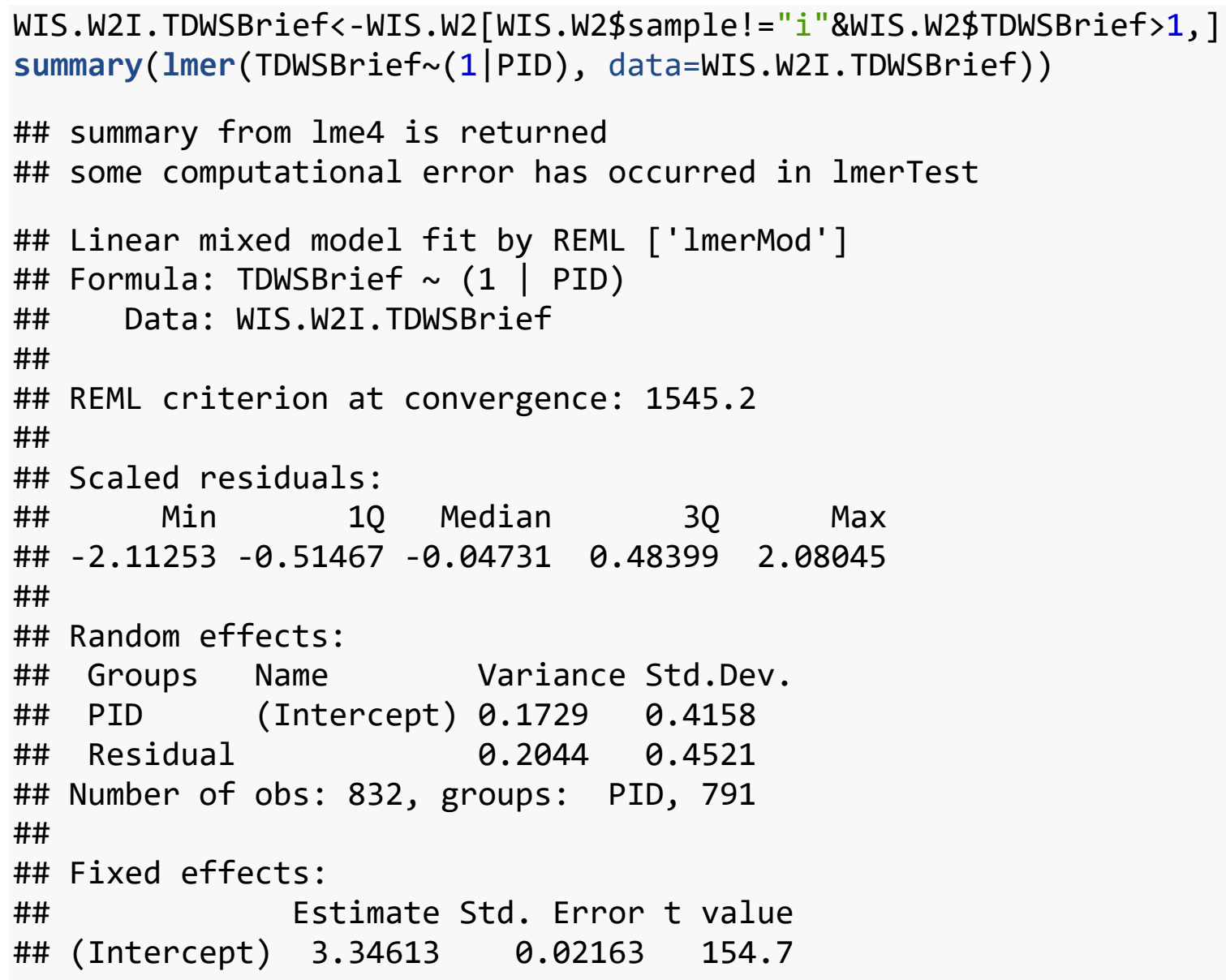




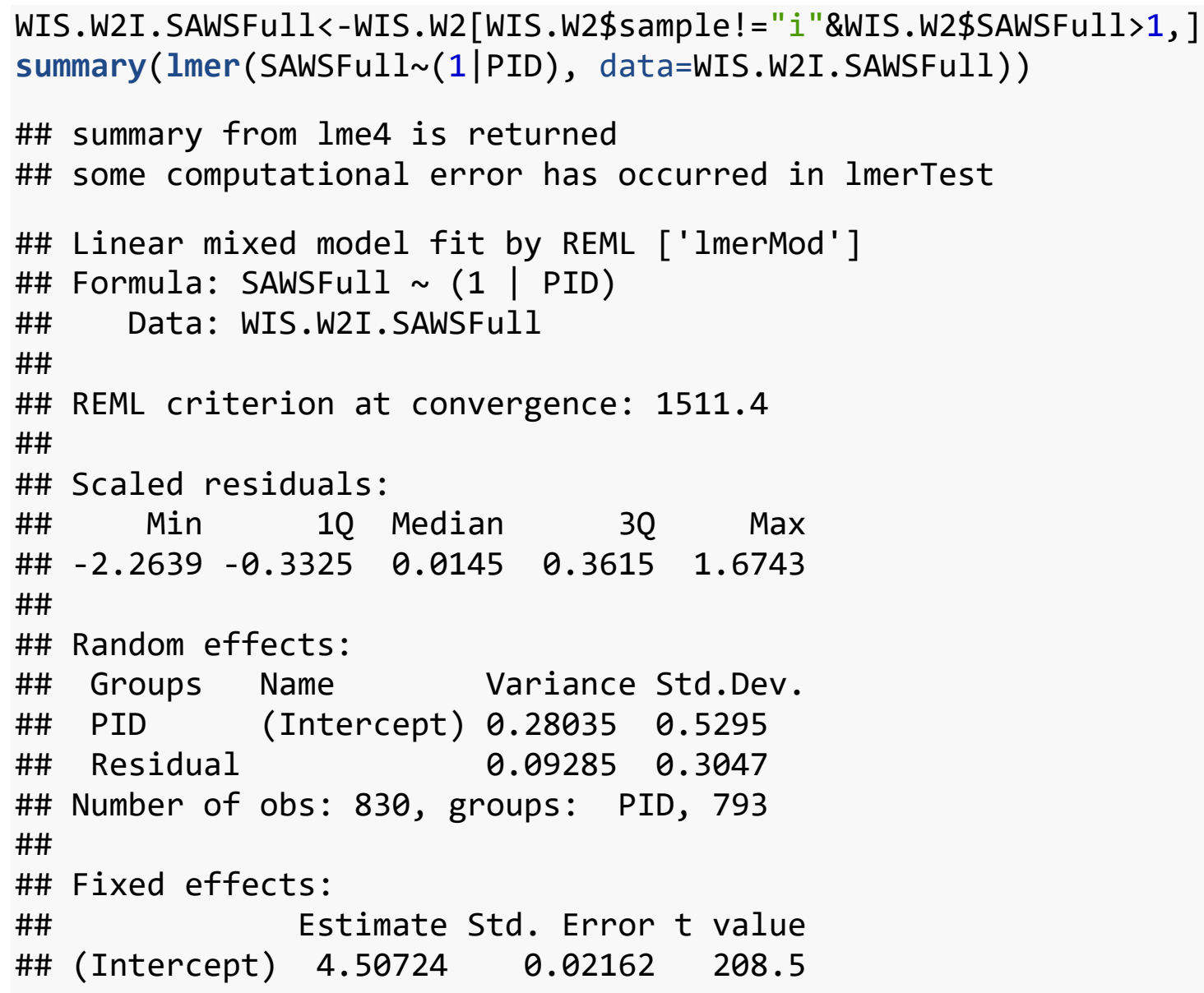


goals. long<-goals. long[ goals. long \$wiscount $>1$ \&goals. long $\$$ goalscount $==1$, ] goals.long \$goal.balance<-rowMeans (goals.long[c('goals.balance.2' , 'goal s.balance. 3', 'goals.balance.4') ], na.rm=TRUE)

\#test

summary(1m(goal.balance wisdom.ave.1, data=goals.long)) \#this is IMPRE SSIVE (though, maybe just a footnote)!

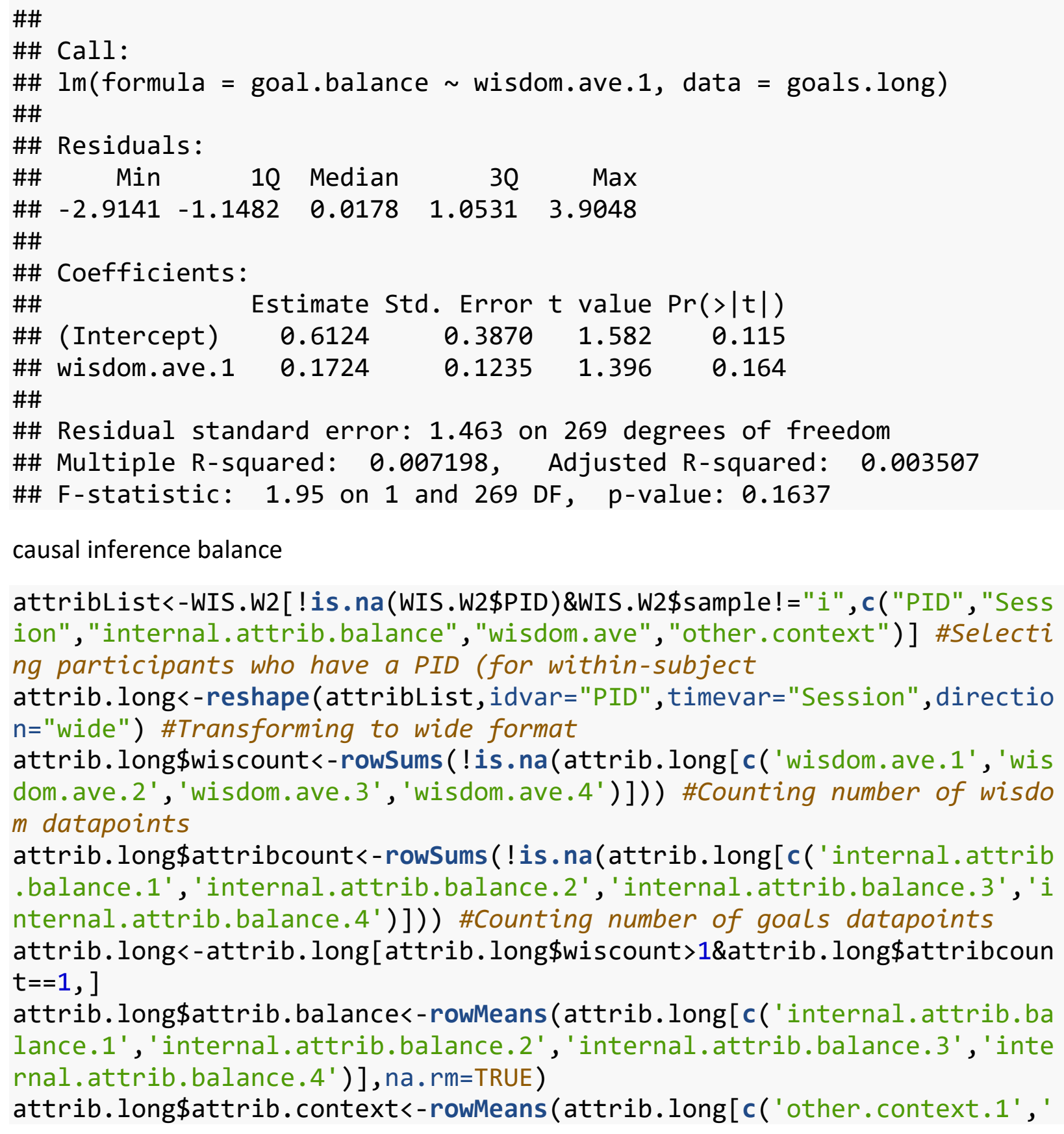
lance.1', 'internal.attrib.balance.2', 'internal.attrib.balance.3', 'inte rnal.attrib.balance. $\left.4^{\prime}\right)$ ], na.rm=TRUE) attrib.long\$attrib.context<-rowMeans (attrib.long[c('other.context.1 ' , ' 


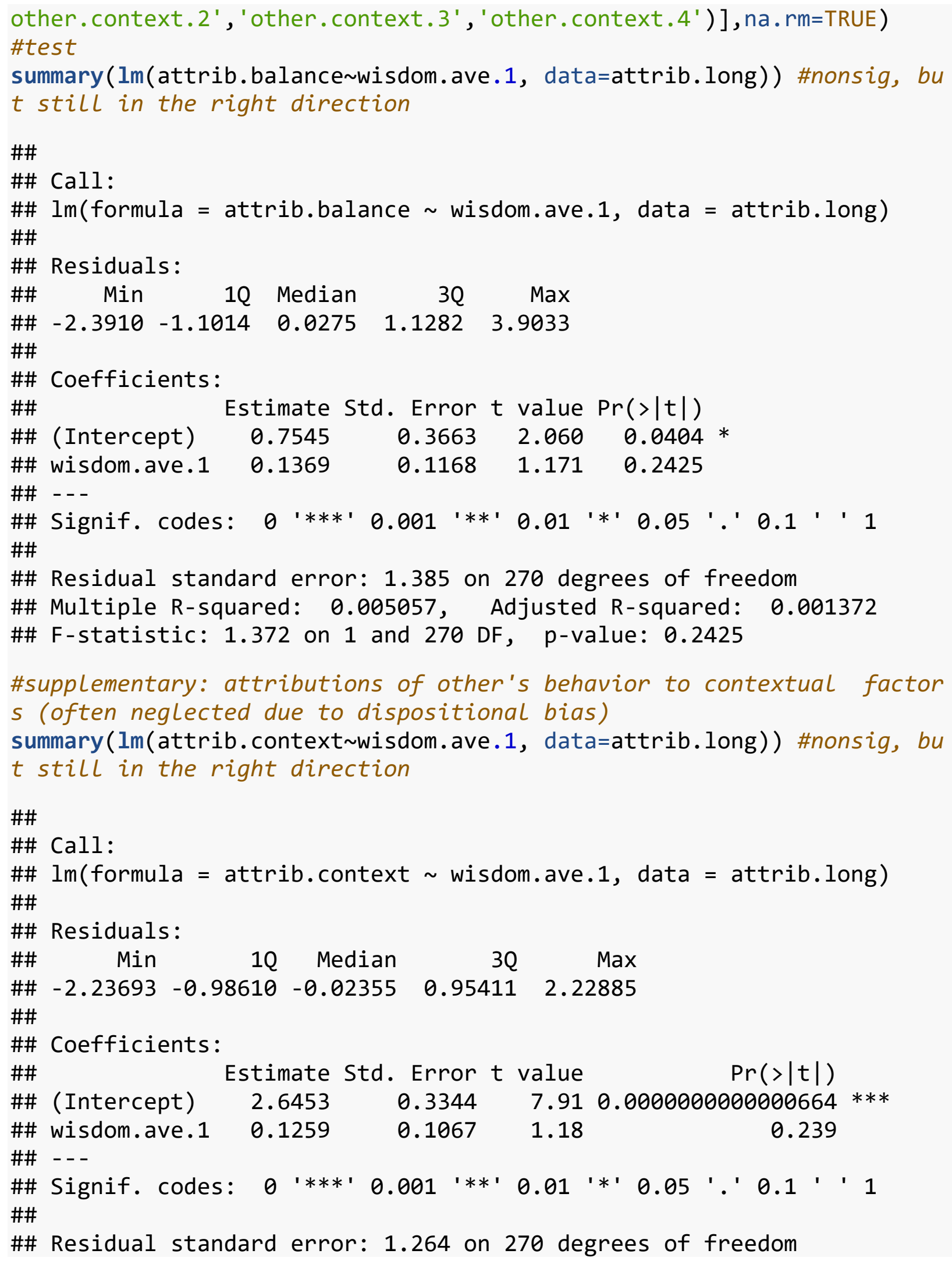


\#\# Multiple R-squared: 0.005132 , Adjusted R-squared: 0.001447

\#\# F-statistic: 1.393 on 1 and 270 DF, p-value: 0.239

\section{\#Gender Interaction}

WIS.W2\$GenderX<-car: : recode(WIS.W2\$gender, " ' Female ' = ' Female ' ; 'Male ' = ' M ale'; 'Other'=NA") \#delete "other" gender for the sake of analyses WIS.W2\$OtherGenderX<-car : : recode(WIS. W2\$OtherGender, " ' Female ' = ' Female ' ; 'Male'='Male';3=NA;'Other'=NA") \#delete "other" gender for the sake 0 $f$ analyses

apaTables: : apa.2way.table(GenderX, OtherGenderX, wisdom.ave,WIS.W2, land scape $=\mathrm{T}$ )

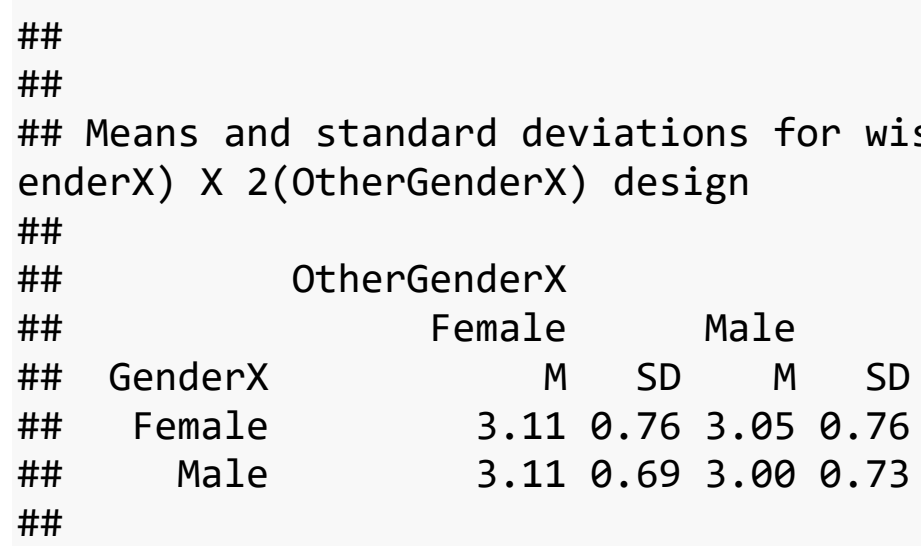

\#\#

\#\# Note. M and SD represent mean and standard deviation, respectively. \#Removing conflicting packages 
S3A: DESCRIPTIVE STATISTICS OF STUDY 3 MEASURES (Table 5)

\# For each measure, sample size, mean, and standard deviation reported in Table 4

as.data.frame(psych::describe(WIS.D\$SAWSFull))[2:4] \# Self-Assessed Wi sdom Scale (SAWS)

\#\# $n$ mean sd

\#\# X1 $238 \quad 4.576786 \quad 0.6080783$

as.data.frame(psych::describe(WIS.D\$TDWSFull))[2:4] \# Three Dimensiona L Wisdom Scale (3DWS)

\#\# $\quad \mathrm{n} \quad$ mean sd

$\begin{array}{lllll}\text { \#\# X1 } & 240 & 3.279514 & 0.4682287\end{array}$

as.data.frame(psych::describe(WIS.D\$ASTISelfTrans))[2:4] \# Adult SelfTranscendence Inventory (ASTI): Self-Transcendence

\#\# $\quad \mathrm{n} \quad$ mean $\quad \mathrm{sd}$

\#\# X1 $2403.668333 \quad 0.6902772$

as.data.frame(psych::describe(WIS.H\$GlobalW))[2:4] \# Global scale of $w$ ise reasoning

\#\# $\quad n$ mean sd

\#\# X1 $501 \quad 4.361142 \quad 0.6783195$

as.data.frame(psych::describe(WIS.E\$wisdom.IG.pca))[2:4] \# Wise reason ing (intergroup)

\#\# $\mathrm{n} \quad$ mean sd

\#\# X1 $240-0.000000000000000034164220 .9985886$

as.data.frame(psych::describe(WIS.E\$WiseObs.pca))[2:4] \# Observer-rate $d$ wise reasoning

\#\# $\quad \mathrm{n} \quad$ mean sd

$\begin{array}{lllll}\# \text { X1 } & 202 & 0.06596494 & 1.071327\end{array}$

as.data.frame(psych::describe(WIS.D\$BIDRImpMGMT))[2:4] \# BIDR: Impress ion management (Sample D)

\#\# $\quad \mathrm{n} \quad$ mean sd

$\begin{array}{lllll}\text { \#\# X1 } 730 & 0.1209589 & 0.1135494\end{array}$

as.data.frame(psych: :describe(WIS.H\$BIDRImpMGMTH))[2:4] \# BIDR: Impres sion management, brief measure (Sample H) 
\#\# $\quad \mathrm{n} \quad$ mean sd

$\begin{array}{lllll}\text { \#\# X1 } 497 & 0.2508384 & 0.3328489\end{array}$

as.data.frame(psych: :describe(WIS.D\$BIDRSelfDec))[2:4] \# BIDR: Self-de ception (Sample D)

\#\# $\quad \mathrm{n} \quad$ mean sd

\#\# X1 $730 \quad 0.09575342 \quad 0.1030524$

as.data.frame(psych::describe(WIS.H\$BIDRSelfDecH))[2:4] \# BIDR: Self-d eception, brief measure (Sample $H$ )

\#\# $n$ mean sd

\#\# X1 4970.086519110 .1934392

as.data.frame(psych: :describe(WIS.D\$BiasBlindSpot))[2:4] \# Bias BLind spot

\#\# $\quad \mathrm{n} \quad$ mean $\quad$ sd

\#\# X1 7051.4198581 .805815

as.data.frame(psych: :describe(WIS.D\$BiasAttrib.comp)) [2:4] \# Balanced attributions

\#\# $\quad \mathrm{n} \quad$ mean $\quad \mathrm{sd}$

\#\# X1 708 -0.565678 0.333896

S3B: RELIABILITY OF STUDY 3 MEASURES (Table 5)

\# Look at raw_alpha for Cronbach's Alpha or \$kappa for Cohen's Kappa psych: :alpha(WIS.D[, c('saws1', 'saws2', 'saws3', 'saws4', 'saws5',

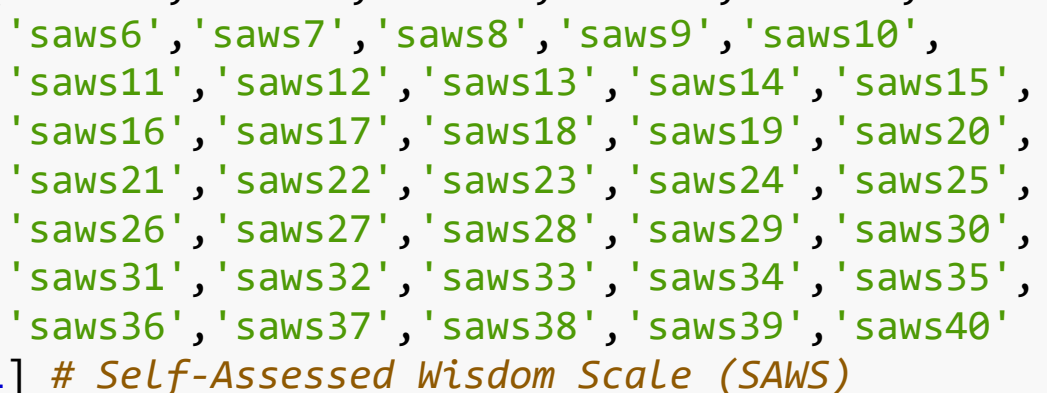

)], check.keys=FALSE)[1] \# Self-Assessed Wisdom Scale (SAWS)

\#\# \$total

\#\# raw_alpha std.alpha G6(smc) average_r S/N ase me

an

$\begin{array}{llllllll}\text { \#\# } & 0.9140115 & 0.9184439 & 0.9521244 & 0.2196872 & 11.2615 & 0.004567647 & 4.5767\end{array}$

86

\#\# sd

\#\# 0.6080783 
psych: :alpha(WIS.D[, c('a3dws1', 'a3dws2' , 'a3dws3', 'a3dws4' , 'a3dws5' , 'a 3dws6', 'a3dws7', 'a3dws8', 'a3dws9' , 'a3dws10' , 'a3dws11', 'a3dws12' , 'a3dws13 ' , 'a3dws14' ,

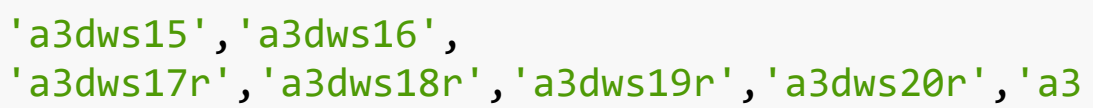

\#\# raw_alpha std.alpha $\mathrm{G6}(\mathrm{smc})$ average_r $\mathrm{S} / \mathrm{N}$ ase me an

$\begin{array}{lllllllll}\text { \#\# } & 0.8715957 & 0.8720451 & 0.918339 & 0.1487551 & 6.815252 & 0.006777252 & 3.2795\end{array}$

14

\#\# sd

\#\# $\quad 0.4682287$

psych: :alpha(WIS.D[, c('asti1', 'asti2', 'asti3', 'asti4', 'asti5', 'asti6', 'asti7', 'asti8' , 'asti9', 'asti10' )], check.keys=FALSE)[1] \# Adult Self-Transcendence Inventory (ASTI): Self-Transcendence

\#\# \$total

\#\# raw_alpha std.alpha $\mathrm{G6}(\mathrm{smc})$ average_r $\mathrm{S} / \mathrm{N}$ ase mean $\begin{array}{llllllll}\text { \#\# } & 0.803121 & 0.798312 & 0.817431 & 0.2835728 & 3.958153 & 0.0106486 & 3.668333\end{array}$

\#\# sd

\#\# 0.6902772

psych: :alpha(WIS.H[, c('edrT1', 'edrT2' , 'edrT3', 'edrT4', 'edrT5', 'edrT6' , 'edrT8', 'edrT9', 'edrT10', ' edrT11', 'edrT12', 'edrT13' , 'edrT14' )], check.keys=FALSE)[1] \# Global scale of wise reasoning (item 7 dropped)

\#\# \$total

\#\# raw_alpha std.alpha G6(smc) average_r $\mathrm{S} / \mathrm{N}$ ase $\mathrm{m}$ ean

$\begin{array}{lllllllll}\text { \#\# } & 0.8893118 & 0.8898267 & 0.9089029 & 0.3832024 & 8.076606 & 0.007301139 & 4.361\end{array}$ 142

\#\# sd

\#\# 0.6783195 
\# Item 7 was dropped for not hanging well together with other items; i

$n$ the code below, look at Item Statistics, r.drop column, row: edrT7 $t$ o see corrected item-total $r$

psych: :alpha(WIS.H[, c('edrT1', 'edrT2', 'edrT3', 'edrT4', 'edrT5', 'edrT6' , 'edrT7', ' 'edrT8', 'edrT9', 'edrT10', ' edrT11', 'edrT12' , 'edrT13' , 'edrT14' )], check . keys=FALSE)

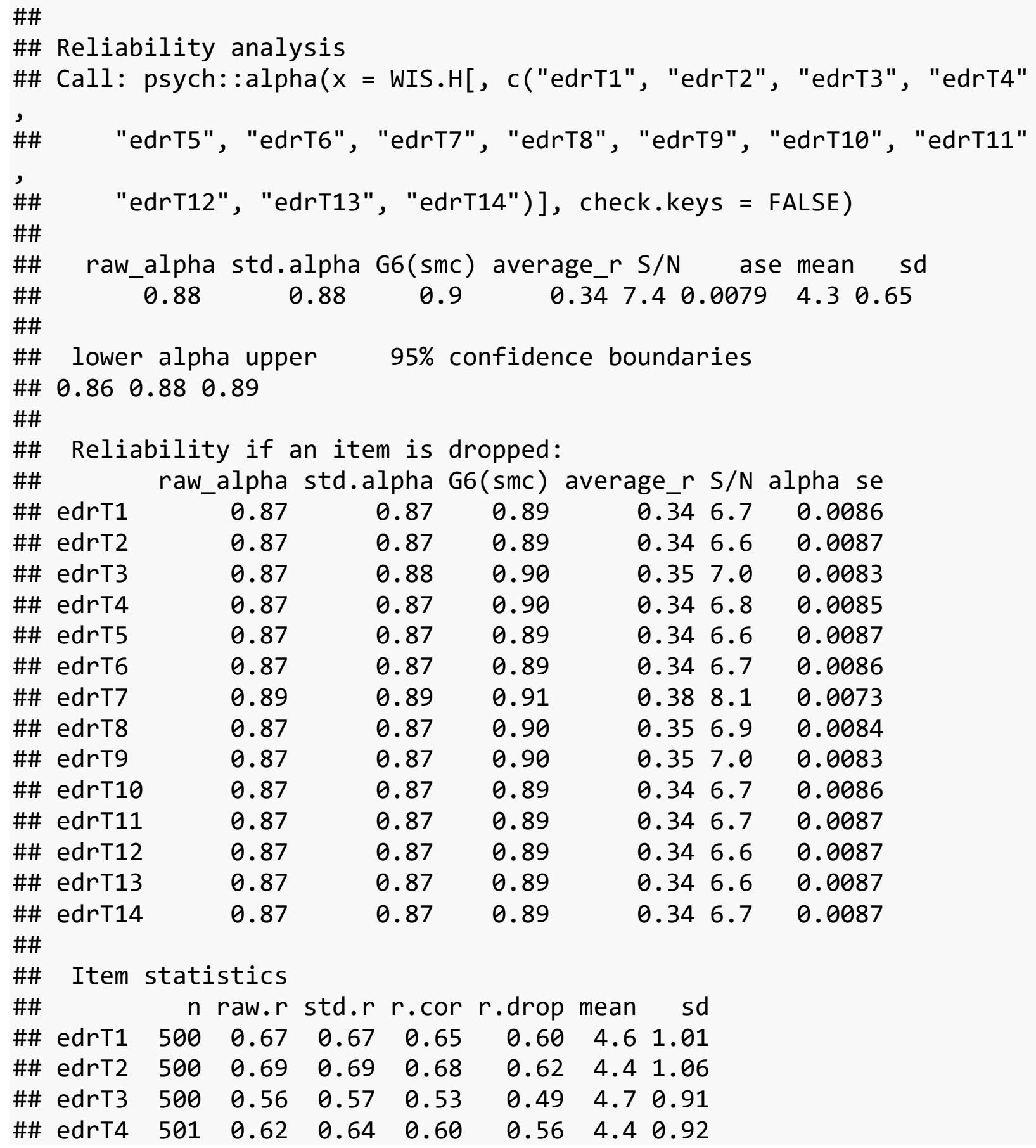




$\begin{array}{llllllll}\text { \#\# edrT5 } & 499 & 0.68 & 0.68 & 0.66 & 0.61 & 4.5 & 1.05 \\ \text { \#\# edrT6 } & 500 & 0.65 & 0.65 & 0.63 & 0.58 & 4.2 & 1.06 \\ \text { \#\# edrT7 } & 499 & 0.30 & 0.28 & 0.19 & 0.17 & 3.4 & 1.13 \\ \text { \#\# edrT8 } & 499 & 0.60 & 0.60 & 0.55 & 0.52 & 3.9 & 1.08 \\ \text { \#\# edrT9 } & 498 & 0.58 & 0.58 & 0.53 & 0.49 & 4.3 & 1.02 \\ \text { \#\# edrT10 } & 499 & 0.67 & 0.67 & 0.64 & 0.60 & 4.5 & 1.05 \\ \text { \#\# edrT11 } & 498 & 0.68 & 0.68 & 0.66 & 0.61 & 4.4 & 1.03 \\ \text { \#\# edrT12 } & 499 & 0.69 & 0.70 & 0.68 & 0.63 & 4.4 & 0.93 \\ \text { \#\# edrT13 } & 499 & 0.70 & 0.68 & 0.68 & 0.62 & 4.2 & 1.13 \\ \text { \#\# edrT14 } 499 & 0.68 & 0.67 & 0.65 & 0.60 & 4.2 & 1.15 \\ \text { \#\# } & & & & & & & \end{array}$

\#\# Non missing response frequency for each item

$\begin{array}{lllllll}\text { \#\# } & 1 & 2 & 3 & 4 & 5 & 6 \text { miss }\end{array}$

$\begin{array}{llllllll}\text { \#\# edrT1 } & 0.01 & 0.02 & 0.07 & 0.28 & 0.43 & 0.18 & 0.00\end{array}$

$\begin{array}{lllllllllll}\text { \#\# edrT2 } & 0.01 & 0.05 & 0.10 & 0.34 & 0.34 & 0.16 & 0.00\end{array}$

$\begin{array}{lllllllllll}\text { \#\# edrT3 } & 0.01 & 0.01 & 0.09 & 0.27 & 0.47 & 0.15 & 0.00\end{array}$

$\begin{array}{lllllllllll}\text { \#\# edrT4 } & 0.00 & 0.03 & 0.10 & 0.41 & 0.34 & 0.11 & 0.00\end{array}$

$\begin{array}{llllllllll}\text { \#\# edrT5 } & 0.00 & 0.04 & 0.12 & 0.32 & 0.36 & 0.16 & 0.00\end{array}$

$\begin{array}{llllllllll}\text { \#\# edrT6 } & 0.01 & 0.06 & 0.18 & 0.34 & 0.32 & 0.09 & 0.00\end{array}$

$\begin{array}{llllllllll}\text { \#\# edrT7 } & 0.04 & 0.16 & 0.34 & 0.28 & 0.14 & 0.03 & 0.00\end{array}$

$\begin{array}{lllllllll}\text { \#\# edrT8 } & 0.02 & 0.08 & 0.25 & 0.38 & 0.21 & 0.06 & 0.00\end{array}$

$\begin{array}{llllllll}\text { \#\# edrT9 } & 0.00 & 0.05 & 0.16 & 0.34 & 0.35 & 0.10 & 0.01\end{array}$

$\begin{array}{llllllllll}\text { \#\# edrT10 } & 0.01 & 0.04 & 0.11 & 0.29 & 0.42 & 0.13 & 0.00\end{array}$

$\begin{array}{llllllllll}\text { \#\# edrT11 } & 0.01 & 0.03 & 0.14 & 0.31 & 0.40 & 0.11 & 0.01\end{array}$

$\begin{array}{llllllllll}\text { \#\# edrT12 } & 0.00 & 0.02 & 0.14 & 0.34 & 0.38 & 0.11 & 0.00\end{array}$

$\begin{array}{lllllllll}\text { \#\# edrT13 } & 0.01 & 0.07 & 0.16 & 0.34 & 0.30 & 0.12 & 0.00\end{array}$

$\begin{array}{lllllllllll}\text { \#\# edrT14 } & 0.01 & 0.08 & 0.13 & 0.32 & 0.34 & 0.12 & 0.00\end{array}$

\# Also, for dropping Item 7, Look at the code below for a PCA (in the code below, Look at column PC1 and edrT7, which is low)

principal(WIS.H[, c( 'edrT1' , 'edrT2' , 'edrT3' , 'edrT4' , 'edrT5 ' , 'edrT6' , 'ed rT7', ' 'edrT8', ' edrT9',

'edrT10', 'edrT11', 'edrT12' , 'edrT13' , 'edrT14' ) ], nfac tors $=1$, residuals=TRUE, rotate="oblimin", missing=TRUE, impute="median")

\#\# Principal Components Analysis

\#\# Call: principal(r = WIS.H[, c("edrT1", "edrT2", "edrT3", "edrT4", " edrT5",

\#\# "edrT6", "edrT7", "edrT8", "edrT9", "edrT10", "edrT11", "edrT12

", $\quad$ "edrT13", "edrT14")],

\#\# "edrT13", "edrT14")], nfactors $=1$, residuals $=$ TRUE, rotate $=$ "oblimin",

\#\# $\quad$ missing = TRUE, impute = "median")

\#\# Standardized loadings (pattern matrix) based upon correlation matri $\mathrm{x}$ 


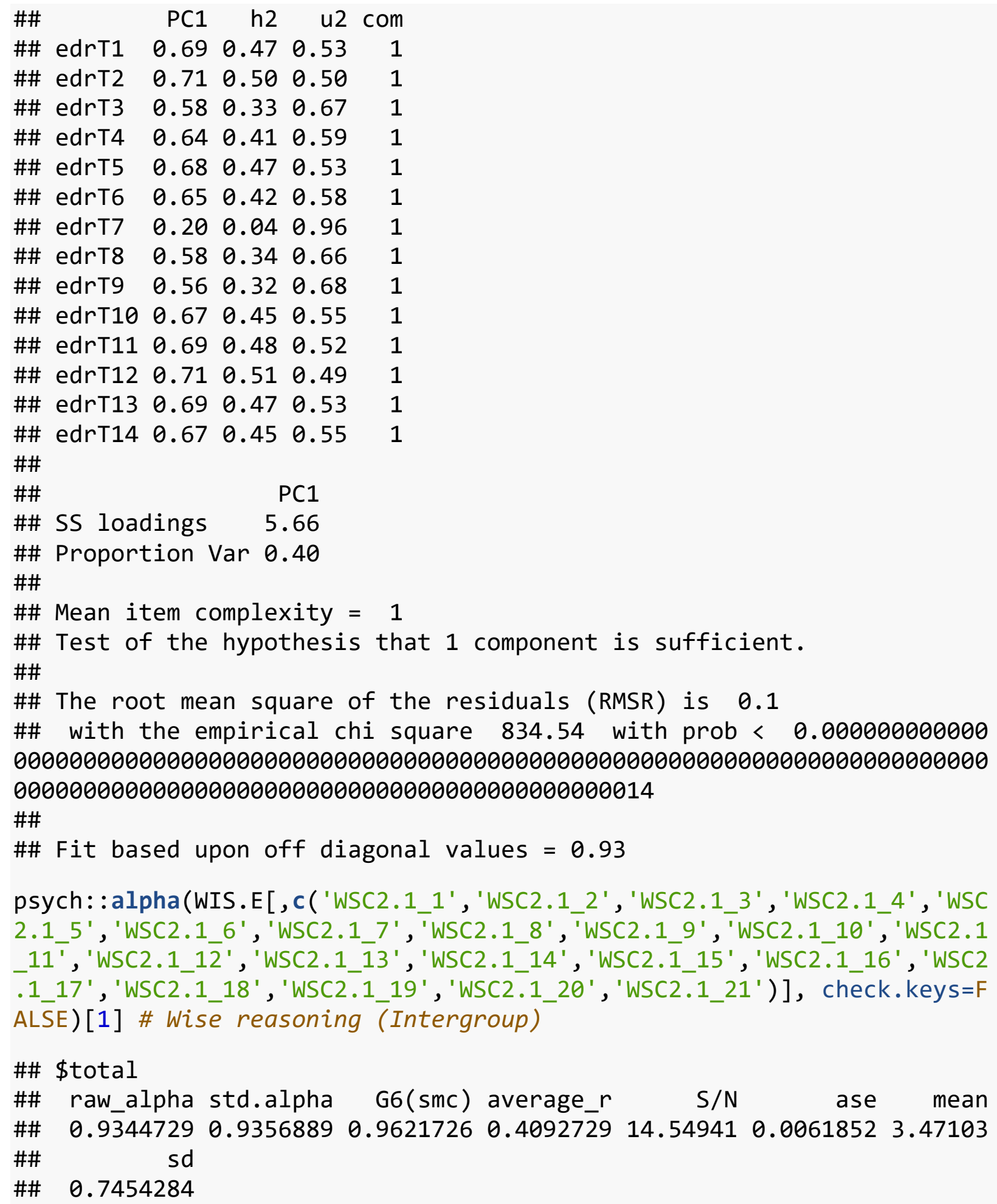
2.1_5', 'WSC2.1_6', 'WSC2.1_7', 'WSC2.1_8', 'WSC2.1_9' , 'WSC2.1_10', 'WSC2.1 11 ', 'WSC2.1_12', 'WSC2.1_13 ', 'WSC2.1_14', 'WSC2.1_15 ', 'WSC2.1_16 ' , 'WSC2 .1_17', 'WSC2.1_18', 'WSC2.1_19', 'WSC2.1_20', 'WSC2.1_21')], check. keys=F ALSE)[1] \# Wise reasoning (Intergroup)

\#\# \$total

\#\# raw_alpha std.alpha G6(smc) average_r S/N ase mean

$\begin{array}{lllllllll}\text { \#\# } & 0.9344729 & 0.9356889 & 0.9621726 & 0.4092729 & 14.54941 & 0.0061852 & 3.47103\end{array}$

\#\# sd

\#\# 0.7454284

cohen.kappa(WIS.E[, c('Perspective.R', 'Perspective.S')])[1] \# Observer ratings: Perspectives 


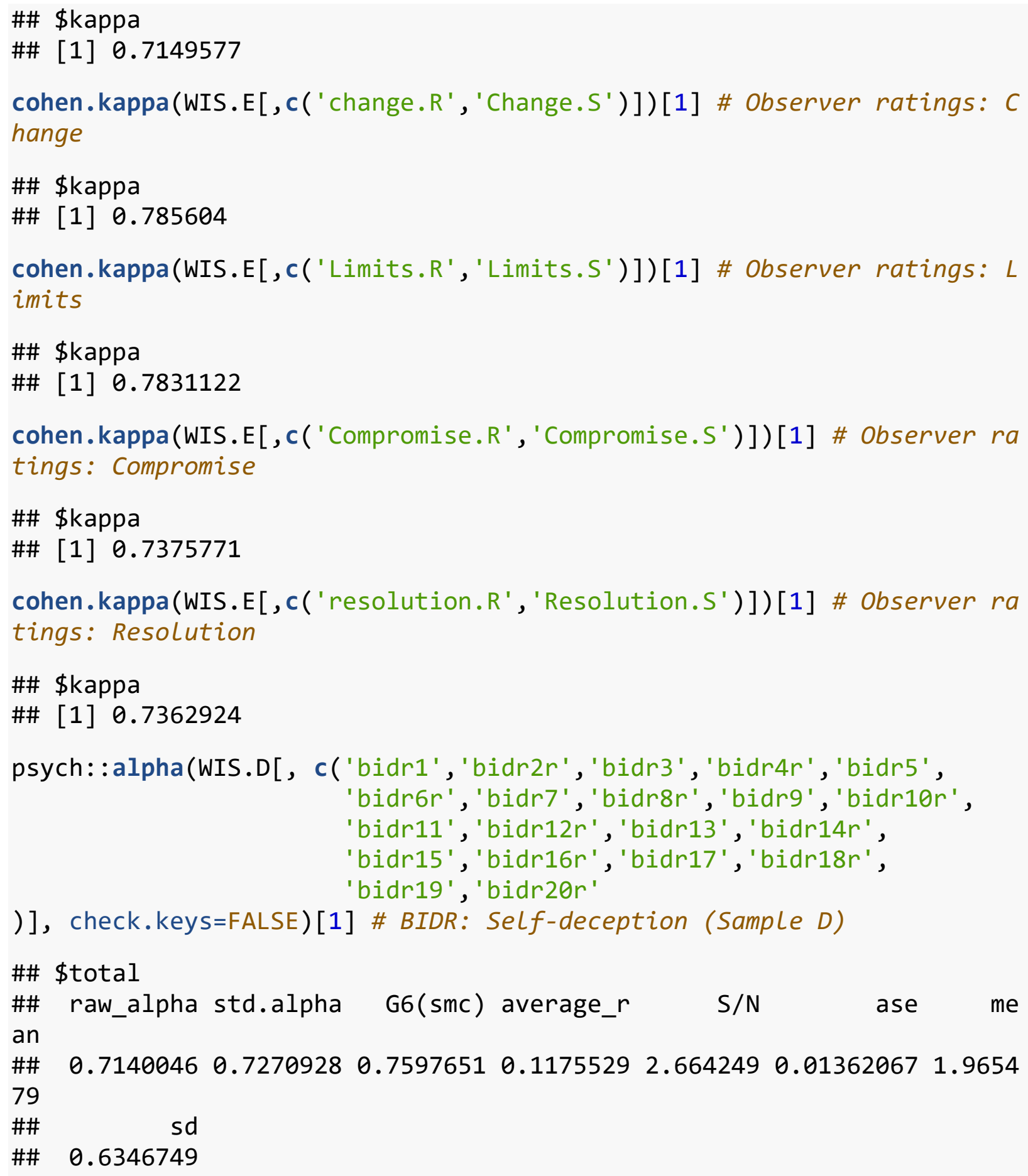

cohen.kappa(WIS.E[, c('Compromise.R', 'Compromise.S')])[1] \# Observer ra tings: Compromise

\#\# \$kappa

\#\# [1] 0.7375771

cohen.kappa(WIS.E[,c('resolution.R', 'Resolution.S')])[1] \# Observer ra tings: Resolution

\#\# \$kappa

\#\# [1] 0.7362924

psych: :alpha(WIS.D[, c('bidr1', 'bidr2r', 'bidr3', 'bidr4r', 'bidr5',

'bidr6r', 'bidr7', 'bidr8r', 'bidr9', 'bidr10r',

'bidr11', 'bidr12r', 'bidr13 ', 'bidr14r',

'bidr15 ', 'bidr16r', 'bidr17', 'bidr18r',

'bidr19', 'bidr20r'

)], check.keys=FALSE)[1] \# BIDR: Self-deception (Sample D)

\#\# \$total

\#\# raw_alpha std.alpha G6(smc) average_r $\mathrm{S} / \mathrm{N}$ ase me

an

$\begin{array}{lllllllll}\text { \#\# } & 0.7140046 & 0.7270928 & 0.7597651 & 0.1175529 & 2.664249 & 0.01362067 & 1.9654\end{array}$

79

\#\# sd

\#\# 0.6346749

psych: :alpha(WIS.H[, c('bidr1', 'bidr2', 'bidr3 ', 'bidr4' , 'bidr5 '

)], check.keys=FALSE)[1] \# BIDR: Self-deception, brief measure (Sample H)

\#\# \$total

\#\# raw_alpha std.alpha G6(smc) average_r S/N ase me 


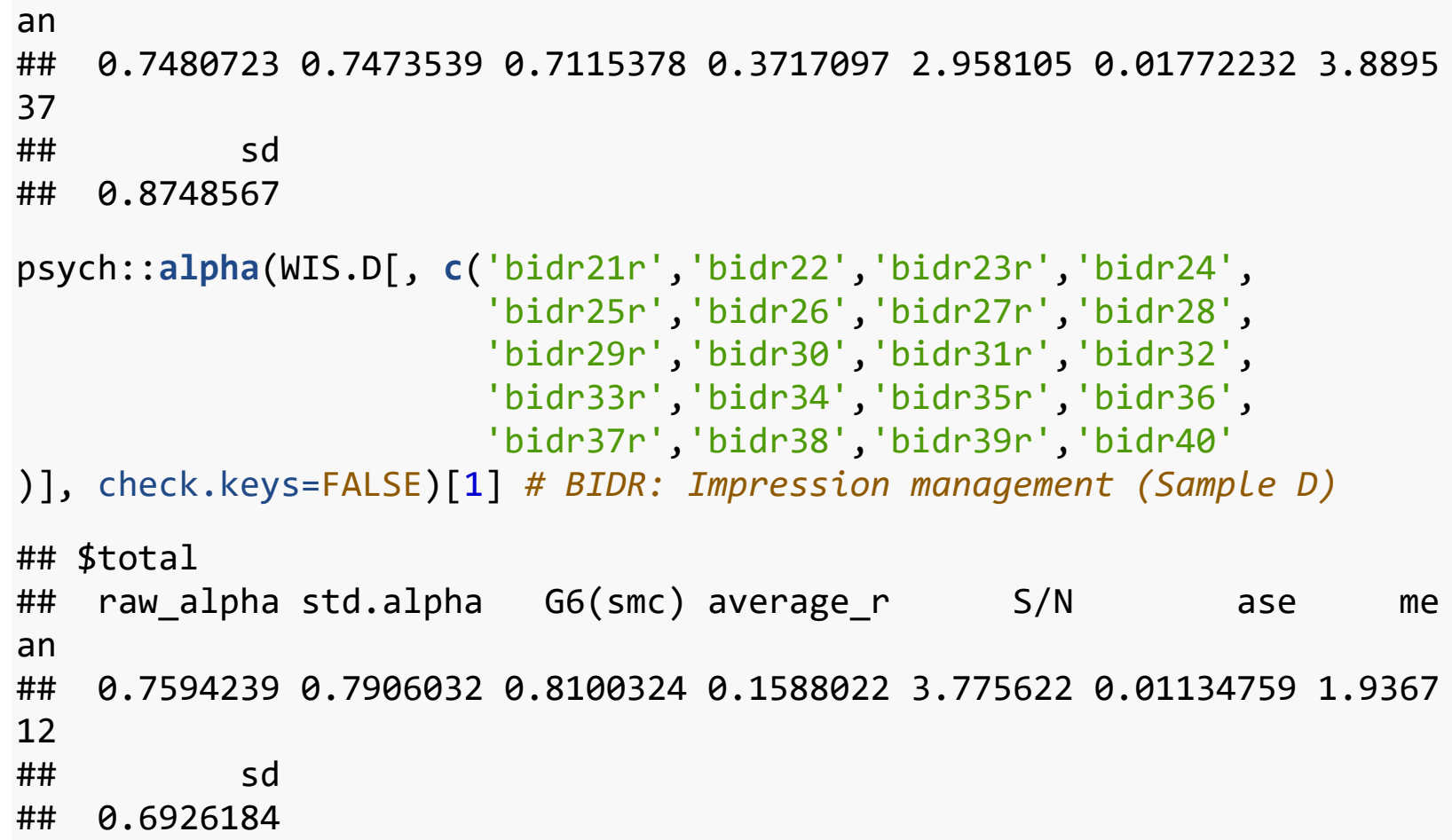

\# Items 9 and 10 were dropped for not hanging well together with other items in a PCA (in the code below, look at column PC1 and bidr9 and bi dr10, which are Low)

principal(WIS.H[, c( 'bidr6', 'bidr7', 'bidr8', 'bidr9' , 'bidr10')], nfactors $=1$, residuals=TRUE, rotate="oblimin", missing=TRUE, impute="median")

\#\# Principal Components Analysis

\#\# Call: principal(r = WIS.H[, c("bidr6", "bidr7", "bidr8", "bidr9", " bidr10")],

\#\# $\quad$ nfactors $=1$, residuals $=$ TRUE, rotate $=$ "oblimin", missing $=\mathrm{T}$

RUE,

\#\# $\quad$ impute = "median")

\#\# Standardized loadings (pattern matrix) based upon correlation matri $\mathrm{x}$

\#\# $\quad$ PC1 $\quad$ h2 $\quad$ u2 com 


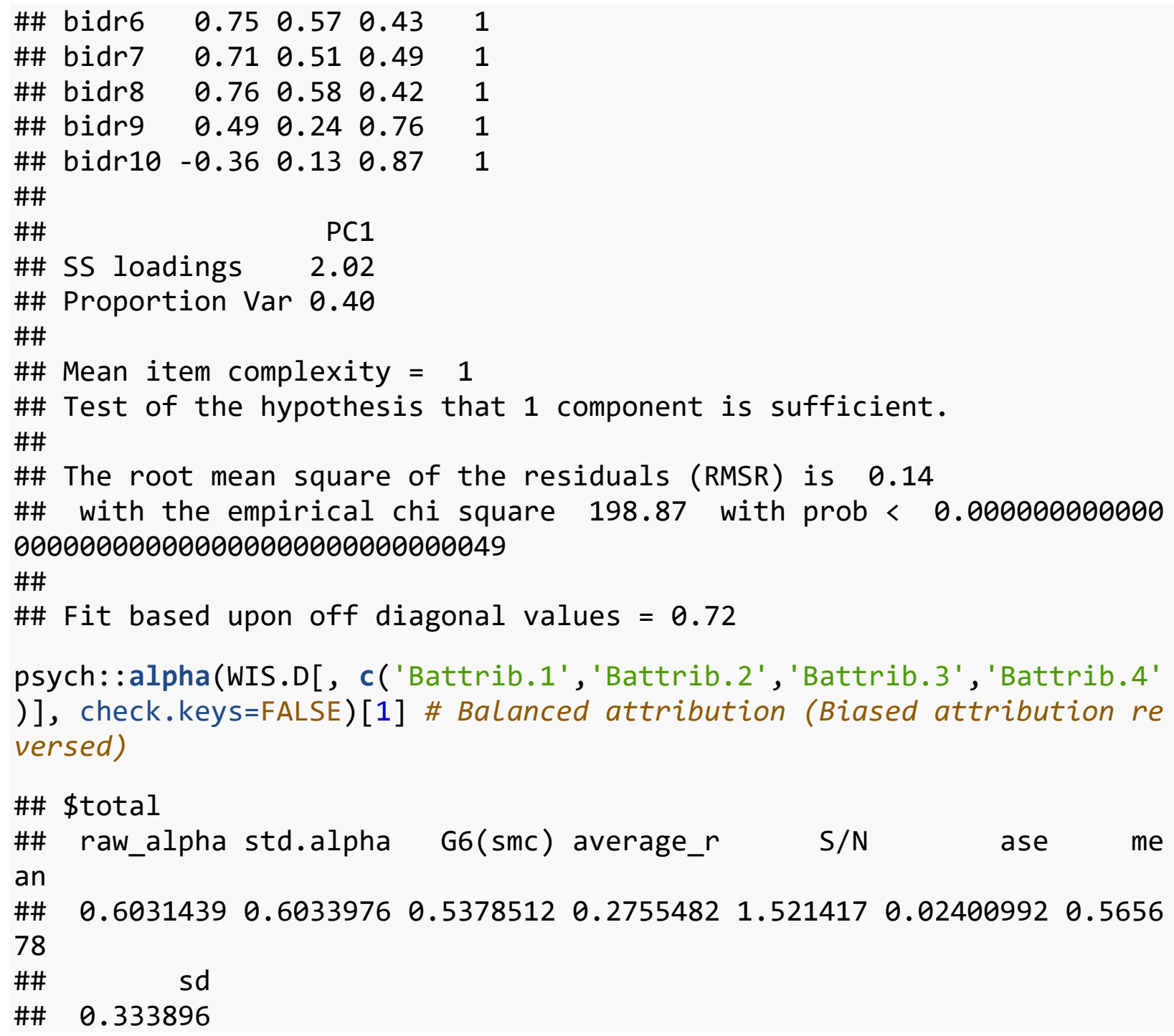

RESULTS FOR STUDY 3 Pearson correlations with wise reasoning and its five aspects (see Table 6)

\# Each Line of code presents 3 Lists (Pearson's r, sample size, p-valu e)

\# Wise reasoning variable Labels: Wise reasoning latent component (wis dom.pca), Wise reasoning intergroup (wisdom.IG.pca), Global measure of wise reasoning (GlobalW)

\# Wise reasoning aspects variable Labels: Perspective (Perspective.ave ), Change/outcomes (ChangeOutcomes.ave), Limits (Limits.ave), Compromi se/resolution (CompResolve.ave), Outsider (Outsider.ave). Wise reasoni ng intergroup aspects all end with. IG.ave

\# Global wisdom variable labels: SAWS (SAWSFuLL), 3DWS (TDWSFuLL), AST I - Self-transcendence subscale (ASTISelfTrans) 
\# Bias variable Labels: BIDR: Impression management, long and brief ve rsions (BIDRImpMGMT, BIDRImpMGMTH), BIDR: Self-deception, long and bri ef versions (BIDRSelfDec, BIDRSelfDecH), Bias Blind Spot (BiasBlindSpo t), Biased Attribution (BiasAttrib.comp)

Convergent validity (Global wisdom measures): Wise reasoning (state-level) has a small-tomedium positive association between global measures of wisdom (SAWS, 3DWS, ASTI) as expected

Hmisc::rcorr(as.matrix(WIS.D[, c('wisdom.pca', 'Perspective.ave', 'Chang eOutcomes.ave', 'Limits.ave' , 'CompResolve.ave', ' Outsider.ave' , 'SAWSFull ' , 'TDWSFull' , 'ASTISelfTrans')]))

\#\# Warning in sqrt(npair - 2): NaNs produced

\begin{tabular}{|c|c|c|c|c|c|}
\hline \#\# & & wisdom.pca & Perspective.ave & ChangeOutcom & es.ave \\
\hline \#\# & wisdom.pca & 1.00 & 0.80 & & 0.71 \\
\hline \#\# & Perspective.ave & 0.80 & 1.00 & & 0.43 \\
\hline \#\# & ChangeOutcomes ave & 0.71 & 0.43 & & 1.00 \\
\hline \#\# & Limits.ave & 0.78 & 0.53 & & 0.41 \\
\hline \#\# & CompResolve.ave & 0.81 & 0.58 & & 0.53 \\
\hline \#\# & Outsider.ave & 0.59 & 0.35 & & 0.25 \\
\hline \#\# & SAWSFull & 0.39 & 0.26 & & 0.30 \\
\hline \#\# & TDWSFull & 0.21 & 0.17 & & 0.14 \\
\hline \#\# & ASTISelfTrans & 0.19 & 0.22 & & 0.17 \\
\hline \#\# & & Limits.ave & CompResolve.ave & Outsider.ave & SAWSFull \\
\hline \#\# & wisdom.pca & 0.78 & 0.81 & 0.59 & 0.39 \\
\hline \#\# & Perspective.ave & 0.53 & 0.58 & 0.35 & 0.26 \\
\hline \#\# & ChangeOutcomes.ave & 0.41 & 0.53 & 0.25 & 0.30 \\
\hline \#\# & Limits.ave & 1.00 & 0.53 & 0.40 & 0.35 \\
\hline \#\# & CompResolve.ave & 0.53 & 1.00 & 0.29 & 0.36 \\
\hline \#\# & Outsider.ave & 0.40 & 0.29 & 1.00 & 0.28 \\
\hline \#\# & SAWSFull & 0.35 & 0.36 & 0.28 & 1.00 \\
\hline \#\# & TDWSFull & 0.15 & 0.26 & 0.01 & NA \\
\hline \#\# & ASTISelfTrans & 0.03 & 0.16 & 0.13 & NA \\
\hline \#\# & & \multicolumn{4}{|c|}{ TDWSFull ASTISelfTrans } \\
\hline \#\# & wisdom.pca & 0.21 & 0.19 & & \\
\hline \#\# & Perspective.ave & 0.17 & 0.22 & & \\
\hline \#\# & ChangeOutcomes.ave & 0.14 & 0.17 & & \\
\hline \#\# & Limits.ave & 0.15 & 0.03 & & \\
\hline \#\# & CompResolve.ave & 0.26 & 0.16 & & \\
\hline \#\# & Outsider.ave & 0.01 & 0.13 & & \\
\hline \#\# & SAWSFull & NA & NA & & \\
\hline \#\# & TDWSFull & 1.00 & NA & & \\
\hline \#\# & ASTISelfTrans & NA & 1.00 & & \\
\hline
\end{tabular}




\begin{tabular}{|c|c|c|c|c|c|}
\hline \multicolumn{6}{|l|}{ \#\# } \\
\hline \#\# & $\mathrm{n}$ & & & & \\
\hline \#\# & & wisdom.pca & Perspective.ave & \multicolumn{2}{|c|}{ ChangeOutcomes.ave } \\
\hline \#\# & wisdom.pca & 730 & 729 & & 729 \\
\hline \#\# & Perspective.ave & 729 & 729 & & 729 \\
\hline \#\# & Changeoutcomes.ave & 729 & 729 & & 729 \\
\hline \#\# & Limits.ave & 730 & 729 & & 729 \\
\hline \#\# & CompResolve.ave & 722 & 722 & & 722 \\
\hline \#\# & Outsider.ave & 722 & 722 & & 722 \\
\hline \#\# & SAWSFull & 238 & 237 & & 237 \\
\hline \#\# & TDWSFull & 240 & 240 & & 240 \\
\hline \#\# & ASTISelfTrans & 240 & 240 & & 240 \\
\hline \#\# & & Limits.ave & CompResolve.ave & Outsider.ave $S$ & SAWSFull \\
\hline \#\# & wisdom.pca & 730 & 722 & 722 & 238 \\
\hline \#\# & Perspective.ave & 729 & 722 & 722 & 237 \\
\hline \#\# & ChangeOutcomes.ave & 729 & 722 & 722 & 237 \\
\hline \#\# & Limits.ave & 730 & 722 & 722 & 238 \\
\hline \#\# & CompResolve.ave & 722 & 722 & 722 & 237 \\
\hline \#\# & Outsider.ave & 722 & 722 & 722 & 237 \\
\hline \#\# & SAWSFull & 238 & 237 & 237 & 238 \\
\hline \#\# & TDWSFull & 240 & 240 & 240 & 0 \\
\hline \#\# & ASTISelfTrans & 240 & 240 & 240 & 0 \\
\hline \#\# & & \multicolumn{4}{|c|}{ TDWSFull ASTISelfTrans } \\
\hline \#\# & wisdom.pca & \multicolumn{2}{|c|}{$240 \quad 240$} & & \\
\hline \#\# & Perspective.ave & 240 & 240 & & \\
\hline \#\# & ChangeOutcomes.ave & 240 & 240 & & \\
\hline \#\# & Limits.ave & 240 & 240 & & \\
\hline \#\# & CompResolve.ave & 240 & 240 & & \\
\hline \#\# & Outsider.ave & 240 & 240 & & \\
\hline \#\# & SAWSFull & 0 & 0 & & \\
\hline \#\# & TDWSFull & 240 & 0 & & \\
\hline \#\# & ASTISelfTrans & \multirow[t]{2}{*}{0} & \multirow[t]{2}{*}{240} & & \\
\hline \multicolumn{4}{|c|}{ \#\# } & & \\
\hline \#\# & $P$ & & & & \\
\hline \#\# & & \multirow[t]{2}{*}{ wisdom.pca } & Perspective.ave & ChangeOutcomes & s.ave \\
\hline \#\# & wisdom.pca & & 0.0000 & 0.0000 & \\
\hline \#\# & Perspective.ave & 0.0000 & & 0.0000 & \\
\hline \#\# & ChangeOutcomes.ave & 0.0000 & 0.0000 & & \\
\hline \#\# & Limits.ave & 0.0000 & 0.0000 & 0.0000 & \\
\hline \#\# & CompResolve.ave & 0.0000 & 0.0000 & 0.0000 & \\
\hline \#\# & Outsider.ave & 0.0000 & 0.0000 & 0.0000 & \\
\hline \#\# & SAWSFull & 0.0000 & 0.0000 & 0.0000 & \\
\hline \#\# & TDWSFull & 0.0012 & 0.0094 & 0.0300 & \\
\hline \#\# & ASTISelfTrans & 0.0035 & 0.0005 & 0.0100 & \\
\hline
\end{tabular}




$\begin{array}{llccc}\text { \#\# } & \text { Limits.ave CompResolve.ave Outsider.ave SAWSFull } \\ \text { \#\# wisdom.pca } & 0.0000 & 0.0000 & 0.0000 & 0.0000 \\ \text { \#\# Perspective.ave } & 0.0000 & 0.0000 & 0.0000 & 0.0000 \\ \text { \#\# ChangeOutcomes.ave } & 0.0000 & 0.0000 & 0.0000 & 0.0000 \\ \text { \#\# Limits.ave } & & 0.0000 & 0.0000 & 0.0000 \\ \text { \#\# CompResolve.ave } & 0.0000 & & 0.0000 & 0.0000 \\ \text { \#\# Outsider.ave } & 0.0000 & 0.0000 & 0.0000 \\ \text { \#\# SAWSFull } & 0.0000 & 0.0000 & 0.0000 & \\ \text { \#\# TDWSFull } & 0.0199 & 0.0000 & 0.8360 & \\ \text { \#\# ASTISelfTrans } & 0.6373 & 0.0107 & \\ \text { \#\# } & \text { TDWSFull } & \text { ASTISelfTrans } & \\ \text { \#\# wisdom.pca } & 0.0012 & 0.0035 & \\ \text { \#\# Perspective.ave } & 0.0094 & 0.0005 & \\ \text { \#\# ChangeOutcomes.ave } & 0.0300 & 0.0100 & \\ \text { \#\# Limits.ave } & 0.0199 & 0.6373 & \\ \text { \#\# CompResolve.ave } & 0.0000 & 0.0107 & \\ \text { \#\# Outsider.ave } & 0.8360 & 0.0408 & \\ \text { \#\# SAWSFull } & & & \\ \text { \#\# TDWSFull } & & & \end{array}$

Divergent validity (Biased Responding): Global measures of wisdom (SAWS, 3DWS, ASTI) are associated with self-deception and impression management. Wise reasoning (state-level) is not, as expected.

Hmisc::rcorr(as.matrix(WIS.D[, c('wisdom.pca', 'Perspective.ave ', 'Chang eOutcomes.ave', 'Limits.ave ', 'CompResolve.ave ', 'Outsider.ave ', 'SAWSFull ', 'TDWSFull ', 'ASTISelfTrans ', 'BIDRSelfDec ', 'BIDRImpMGMT' )] ))

\#\# Warning in sqrt(npair - 2): NaNs produced

$\begin{array}{lrrr}\text { \#\# } & \text { wisdom.pca } & \text { Perspective.ave } & \text { ChangeOutcomes.ave } \\ \text { \#\# wisdom.pca } & 1.00 & 0.80 & 0.71 \\ \text { \#\# Perspective.ave } & 0.80 & 1.00 & 0.43 \\ \text { \#\# ChangeOutcomes.ave } & 0.71 & 0.43 & 1.00 \\ \text { \#\# Limits.ave } & 0.78 & 0.53 & 0.41 \\ \text { \#\# CompResolve.ave } & 0.81 & 0.58 & 0.53 \\ \text { \#\# Outsider.ave } & 0.59 & 0.35 & 0.25 \\ \text { \#\# SAWSFull } & 0.39 & 0.26 & 0.30 \\ \text { \#\# TDWSFull } & 0.21 & 0.17 & 0.14 \\ \text { \#\# ASTISelfTrans } & 0.19 & 0.22 & 0.17 \\ \text { \#\# BIDRSelfDec } & -0.05 & -0.03 & 0.02 \\ \text { \#\# BIDRImpMGMT } & 0.07 & 0.02 & 0.05 \\ \text { \#\# } & \text { Limits.ave CompResolve.ave Outsider.ave SAWSFull } \\ \text { \#\# wisdom.pca } & 0.78 & 0.81 & 0.59\end{array}$




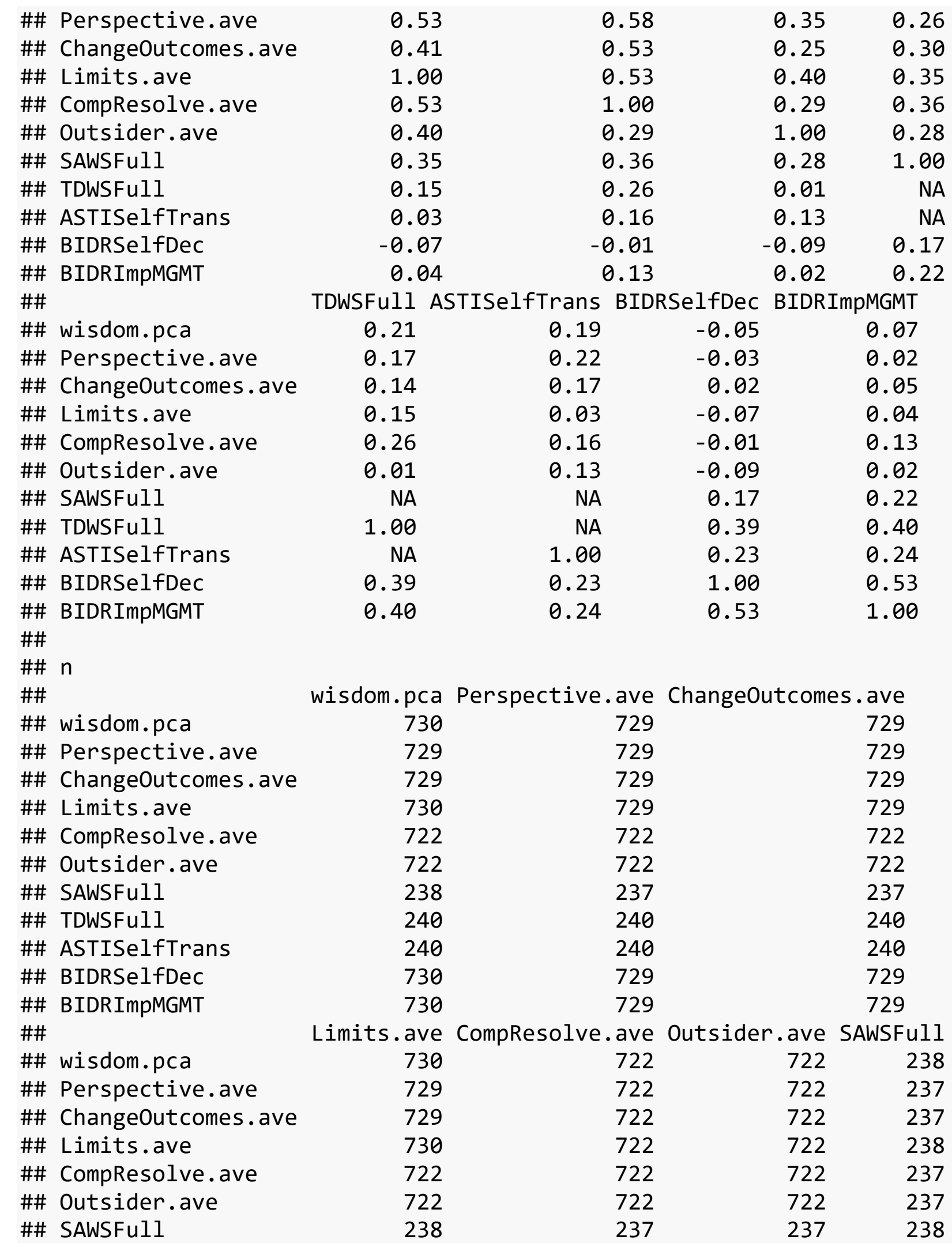




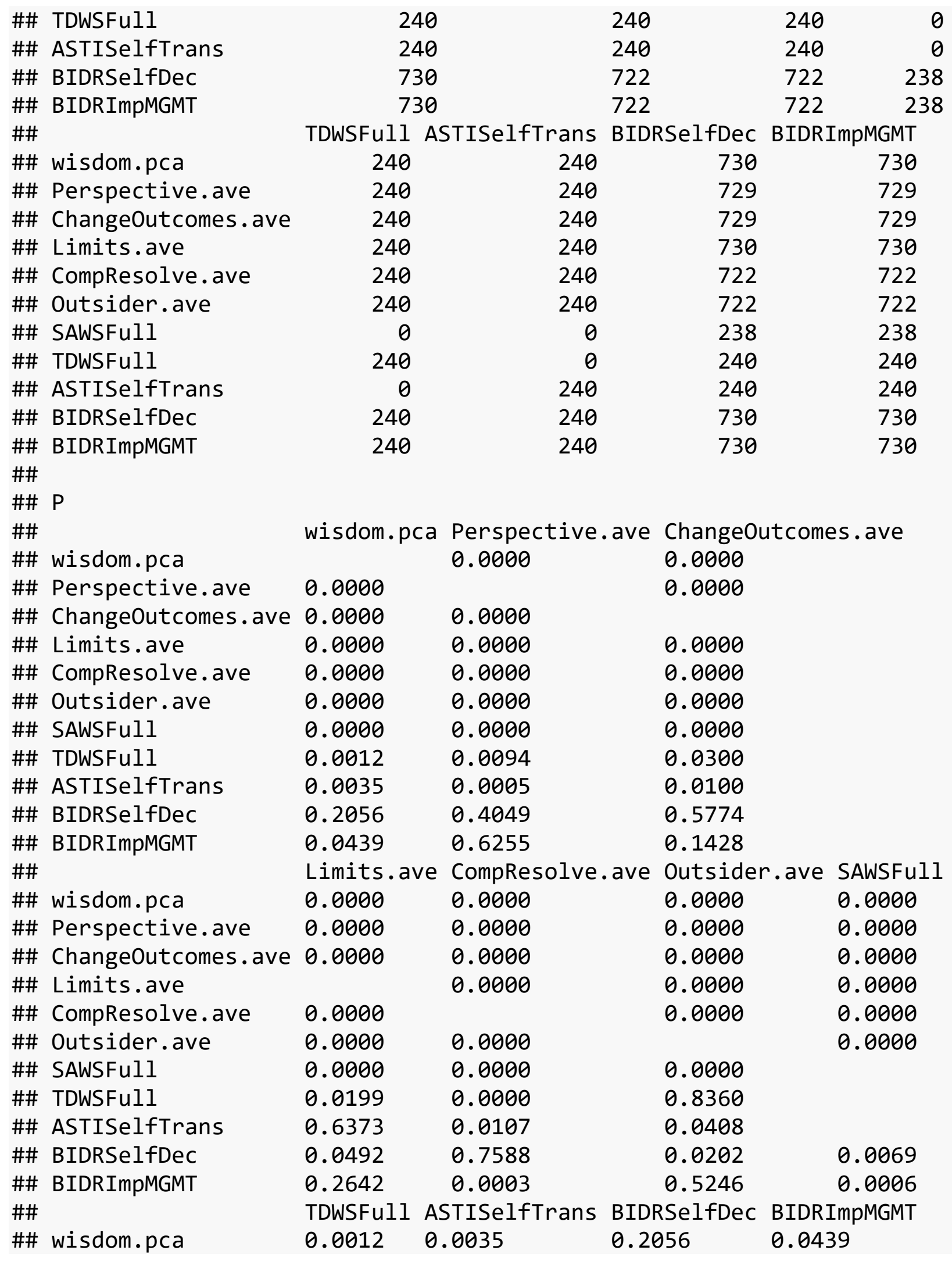




$\begin{array}{lllll}\text { \#\# Perspective.ave } & 0.0094 & 0.0005 & 0.4049 & 0.6255 \\ \text { \#\# ChangeOutcomes.ave } & 0.0300 & 0.0100 & 0.5774 & 0.1428 \\ \text { \#\# Limits.ave } & 0.0199 & 0.6373 & 0.0492 & 0.2642 \\ \text { \#\# CompResolve.ave } & 0.0000 & 0.0107 & 0.7588 & 0.0003 \\ \text { \#\# Outsider.ave } & 0.8360 & 0.0408 & 0.0202 & 0.5246 \\ \text { \#\# SAWSFull } & & & 0.0069 & 0.0006 \\ \text { \#\# TDWSFull } & & & 0.0000 & 0.0000 \\ \text { \#\# ASTISelfTrans } & & & 0.0003 & 0.0002 \\ \text { \#\# BIDRSelfDec } & 0.0000 & 0.0003 & & 0.0000 \\ \text { \#\# BIDRImpMGMT } & 0.0000 & 0.0002 & 0.0000 & \end{array}$

Divergent validity (Biased Responding): Global measure of wise reasoning is also associated with self-deception and impression management, as expected.

Hmisc: : rcorr(as.matrix(WIS.H[, c('GlobalW' , 'BIDRSelfDecH ' , 'BIDRImpMGMT $\left.\left.\left.H^{\prime}\right)\right]\right)$ )

\begin{tabular}{|c|c|c|c|c|}
\hline \#\# & & GlobalW & BIDRSelfDecH & BIDRImpMGMTH \\
\hline \#\# & GlobalW & 1.00 & 0.17 & 0.30 \\
\hline \#\# & BIDRSelfDecH & 0.17 & 1.00 & 0.18 \\
\hline \#\# & BIDRImpMGMTH & 0.30 & 0.18 & 1.00 \\
\hline \multicolumn{5}{|c|}{ \#\# } \\
\hline \multicolumn{5}{|c|}{$\# \#$ n } \\
\hline \#\# & & GlobalW & BIDRSelfDecH & BIDRImpMGMTH \\
\hline \#\# & GlobalW & 501 & 497 & 497 \\
\hline \#\# & BIDRSelfDecH & 497 & 497 & 497 \\
\hline \#\# & BIDRImpMGMTH & 497 & 497 & 497 \\
\hline \multicolumn{5}{|c|}{ \#\# } \\
\hline \multicolumn{5}{|c|}{ \#\# P } \\
\hline \#\# & & GlobalW & BIDRSelfDecH & BIDRImpMGMTH \\
\hline \#\# & GlobalW & & 0.0002 & 0.0000 \\
\hline \#\# & BIDRSelfDecH & 0.0002 & & 0.0000 \\
\hline \#\# & BIDRImpMGMTH & 0.0000 & 0.0000 & \\
\hline
\end{tabular}

Divergent validity (Social Biases): Global measures of wisdom (SAWS, 3DWS, ASTI) are associated with bias blindspot. Wise reasoning (state-level) is not and is associated with less biased attribution, as expected. SAWS also has less biased attribution.

Hmisc::rcorr(as.matrix(WIS.D[, c('wisdom.pca', ' Perspective.ave', 'Chang eOutcomes.ave', 'Limits.ave' , 'CompResolve.ave' , ' Outsider.ave' , 'SAWSFull ', ' TDWSFull', 'ASTISelfTrans ', 'BiasBlindSpot ', 'BiasAttrib.comp' )]))

\#\# Warning in sqrt(npair - 2): NaNs produced

\#\# wisdom.pca Perspective.ave ChangeOutcomes.ave

$\begin{array}{llll}\text { \#\# wisdom.pca } & 1.00 & 0.80 & 0.71\end{array}$ 


\begin{tabular}{|c|c|c|c|}
\hline \#\# Perspective.ave & 0.80 & 1.00 & \\
\hline \#\# ChangeOutcomes.ave & 0.71 & 0.43 & \\
\hline \#\# Limits.ave & 0.78 & 0.53 & \\
\hline \#\# CompResolve.ave & 0.81 & 0.58 & \\
\hline \#\# Outsider.ave & 0.59 & 0.35 & \\
\hline \#\# SAWSFull & 0.39 & 0.26 & \\
\hline \#\# TDWSFull & 0.21 & 0.17 & \\
\hline \#\# ASTISelfTrans & 0.19 & 0.22 & \\
\hline \#\# BiasBlindSpot & 0.00 & 0.03 & \\
\hline \#\# BiasAttrib.comp & -0.11 & -0.09 & \\
\hline 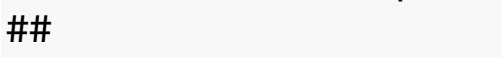 & Limits.ave & CompResolve.ave & Outsider. \\
\hline \#\# wisdom.pca & 0.78 & 0.81 & 0 \\
\hline \#\# Perspective.ave & 0.53 & 0.58 & 0 \\
\hline \#\# ChangeOutcomes.ave & 0.41 & 0.53 & 0 \\
\hline \#\# Limits.ave & 1.00 & 0.53 & 0 \\
\hline \#\# CompResolve.ave & 0.53 & 1.00 & 0 \\
\hline \#\# Outsider.ave & 0.40 & 0.29 & 1 \\
\hline \#\# SAWSFull & 0.35 & 0.36 & \\
\hline \#\# TDWSFull & 0.15 & 0.26 & 0 \\
\hline \#\# ASTISelfTrans & 0.03 & 0.16 & 0 \\
\hline \#\# BiasBlindSpot & -0.05 & 0.02 & -0 \\
\hline \#\# BiasAttrib.comp & -0.04 & -0.11 & -0 \\
\hline \#\# & \multicolumn{3}{|c|}{ TDWSFull ASTISelfTrans BiasBlindSpot } \\
\hline \multicolumn{4}{|l|}{ comp } \\
\hline $\begin{array}{l}\text { \#\# wisdom.pca } \\
0.11\end{array}$ & 0.21 & 0.19 & 0.00 \\
\hline $\begin{array}{l}\text { \#\# Perspective.ave } \\
0.09\end{array}$ & 0.17 & 0.22 & 0.03 \\
\hline $\begin{array}{l}\text { \#\# ChangeOutcomes.ave } \\
0.08\end{array}$ & 0.14 & 0.17 & -0.01 \\
\hline $\begin{array}{l}\text { \#\# Limits.ave } \\
0.04\end{array}$ & 0.15 & 0.03 & -0.05 \\
\hline $\begin{array}{l}\text { \#\# CompResolve.ave } \\
0.11\end{array}$ & 0.26 & 0.16 & 0.02 \\
\hline $\begin{array}{l}\# \# \text { Outsider.ave } \\
0.10\end{array}$ & 0.01 & 0.13 & -0.01 \\
\hline $\begin{array}{l}\text { \#\# SAWSFull } \\
0.13\end{array}$ & NA & NA & 0.25 \\
\hline $\begin{array}{l}\text { \#\# TDWSFull } \\
0.04\end{array}$ & 1.00 & NA & 0.18 \\
\hline $\begin{array}{l}\text { \#\# ASTISelfTrans } \\
0.02\end{array}$ & NA & 1.00 & 0.19 \\
\hline $\begin{array}{l}\text { \#\# BiasBlindSpot } \\
0.01\end{array}$ & 0.18 & 0.19 & 1.00 \\
\hline
\end{tabular}




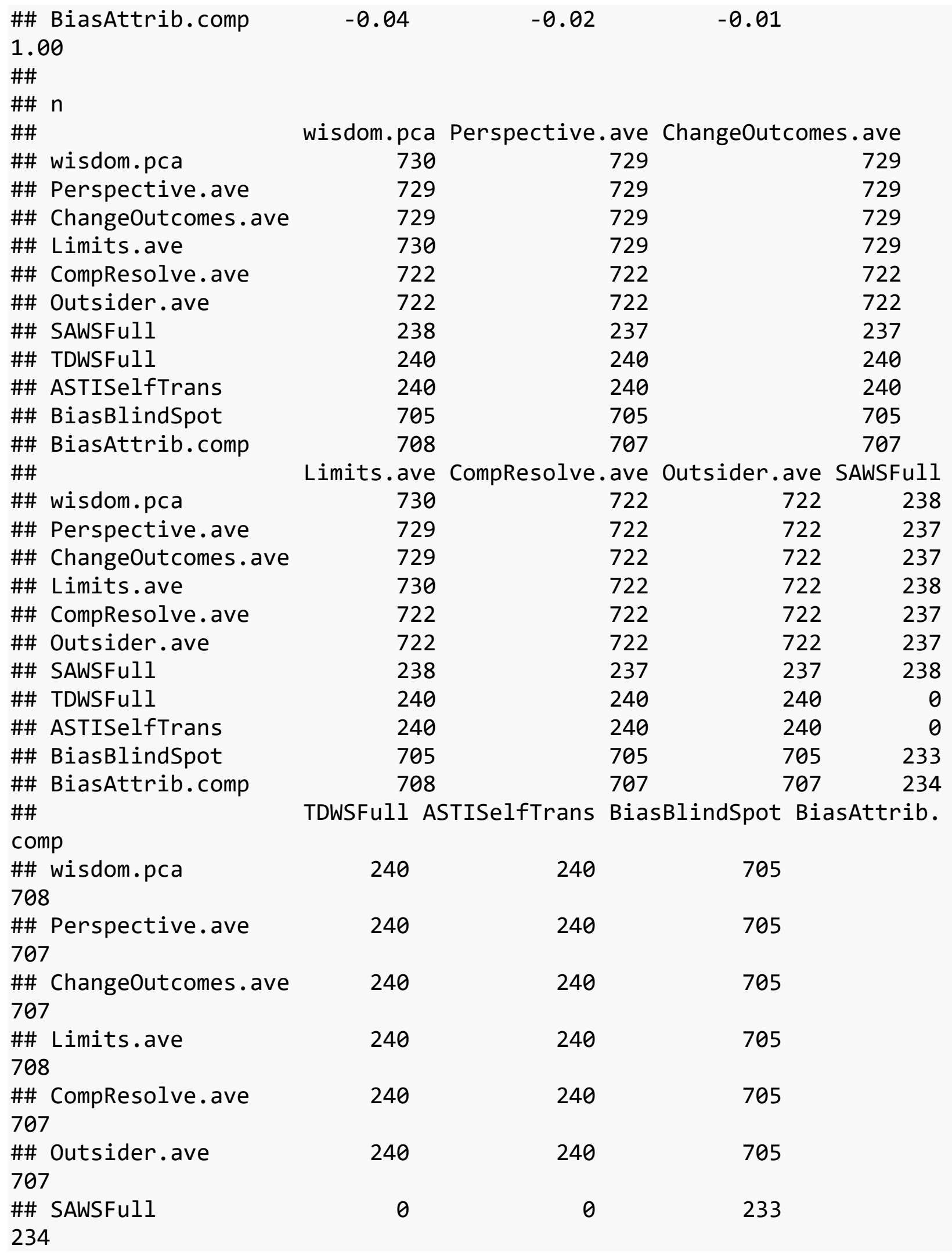




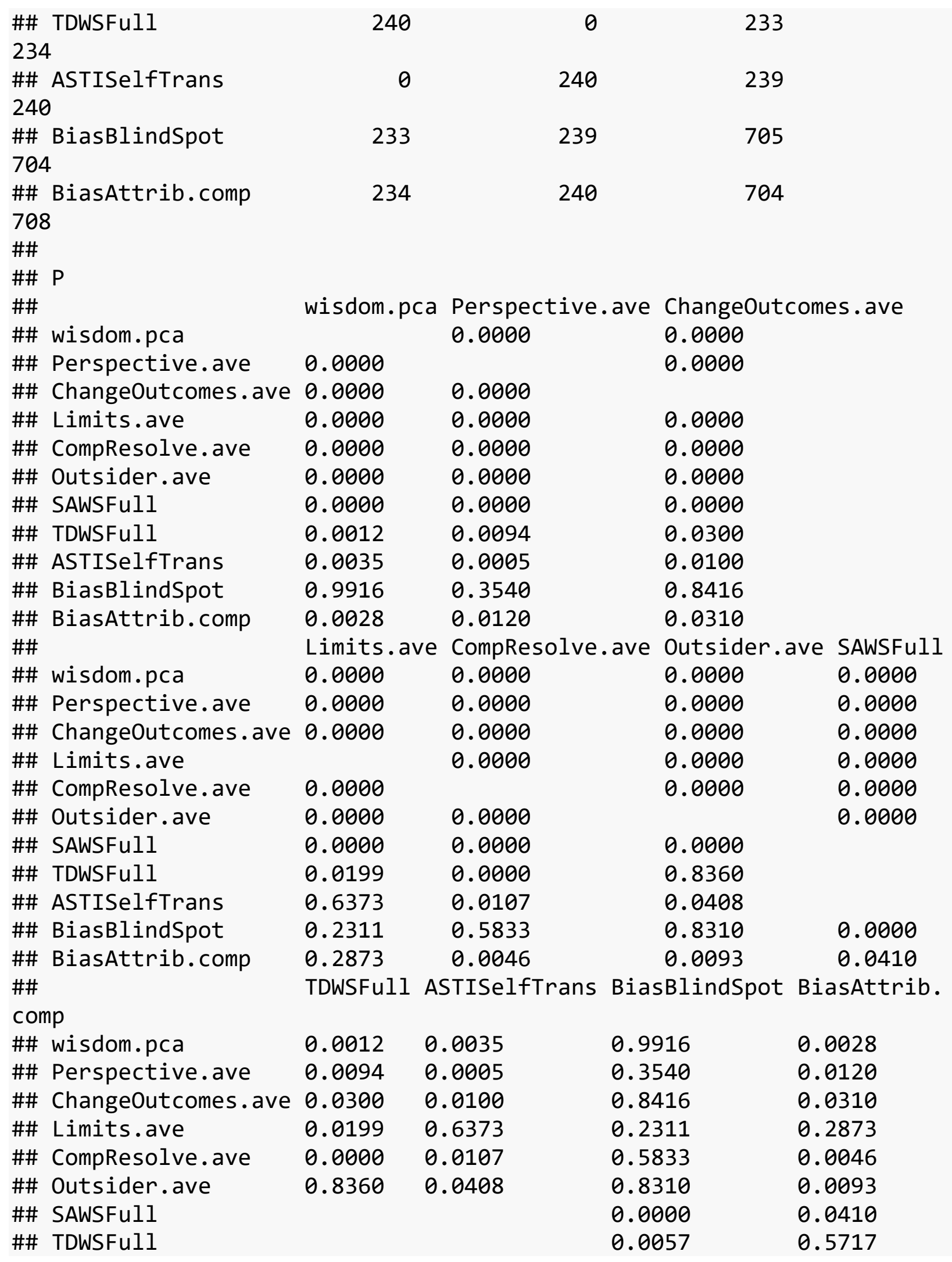




$\begin{array}{lllll}\text { \#\# ASTISelfTrans } & & & 0.0036 & 0.7237 \\ \text { \#\# BiasBlindSpot } & 0.0057 & 0.0036 & & 0.6991 \\ \text { \#\# BiasAttrib.comp } & 0.5717 & 0.7237 & 0.6991 & \end{array}$

Convergent validity (Wise reasoning performance): Wise reasoning (state-level) and three its five aspects are associated with observer-rated wise reasoning, as expected

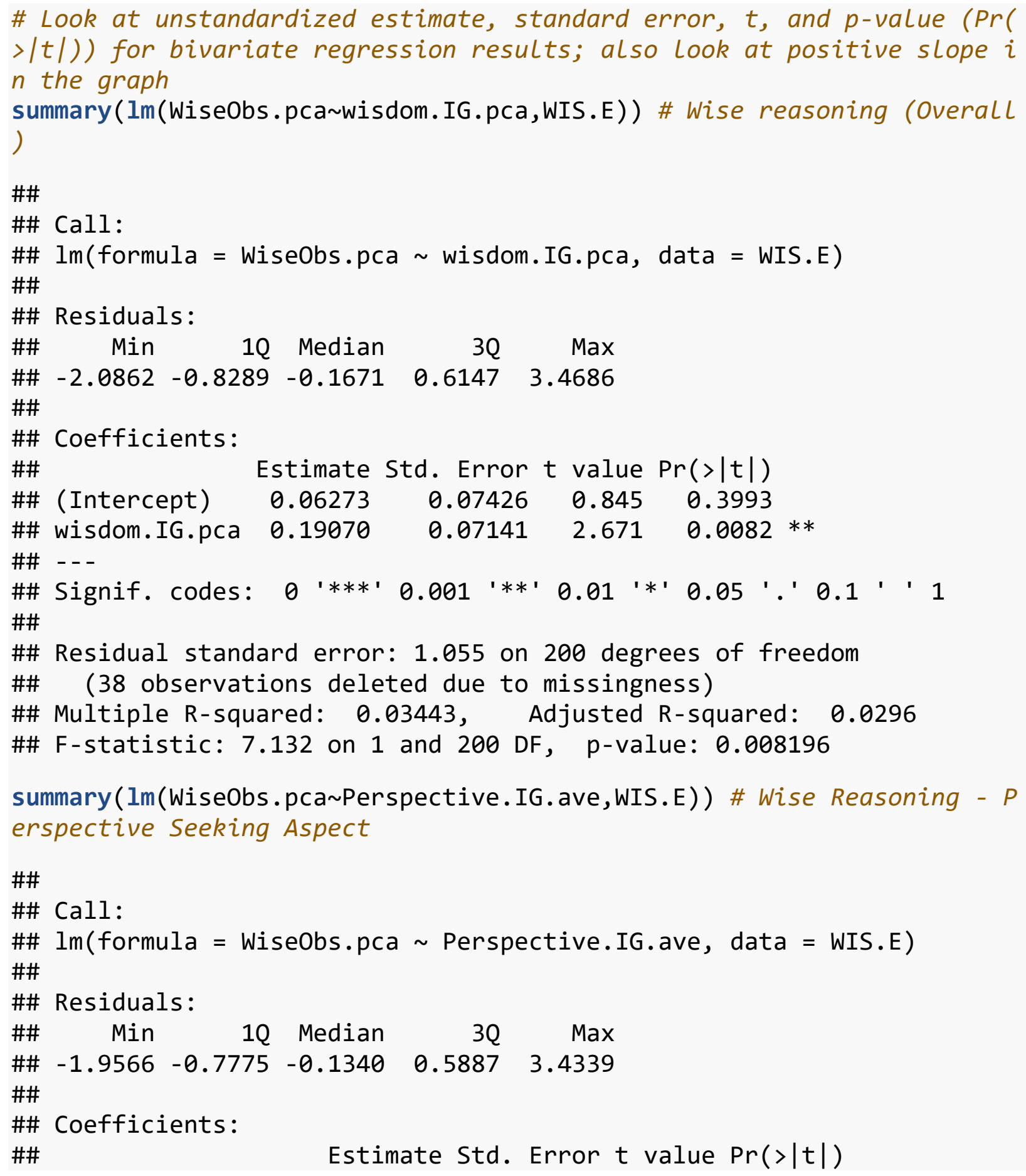




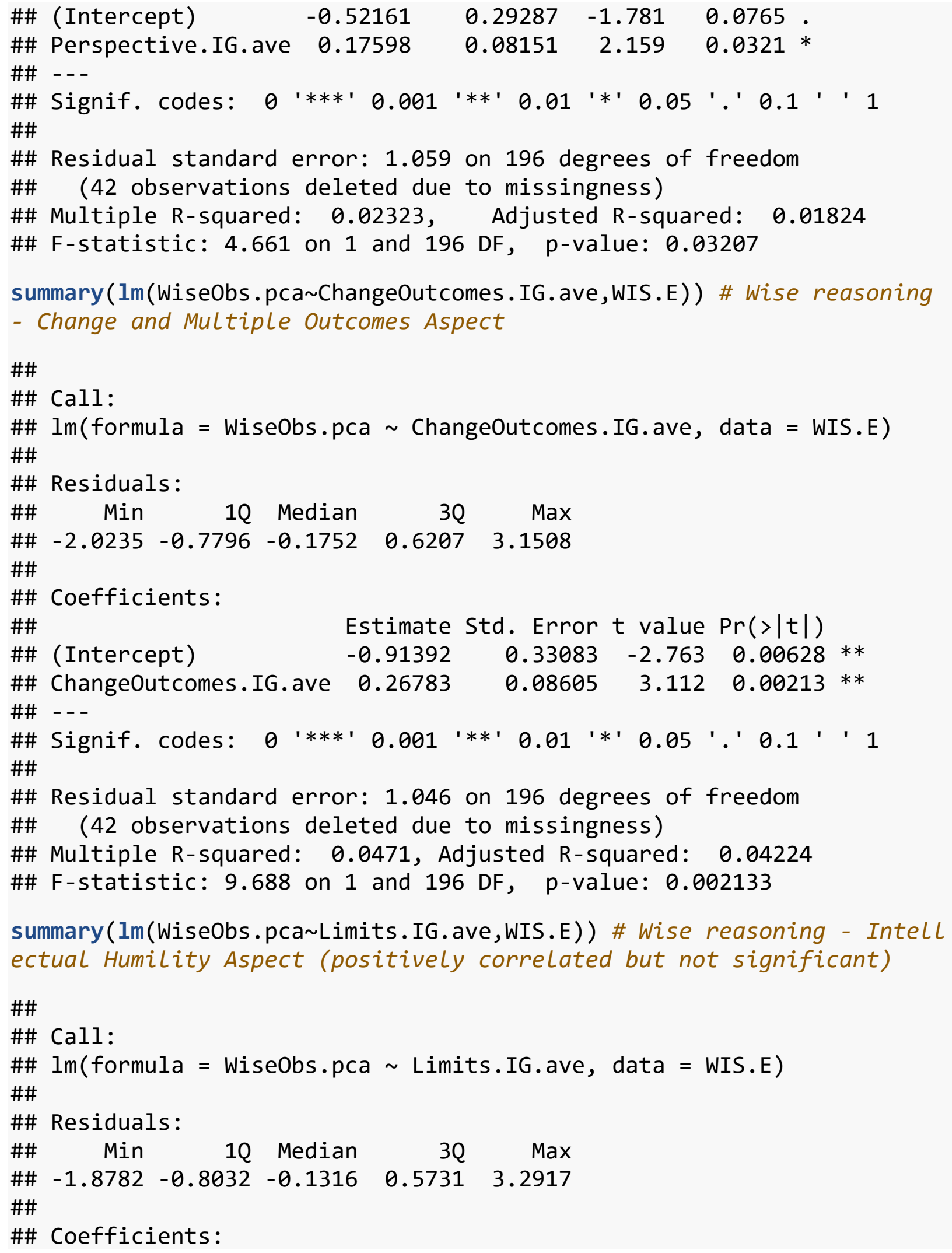




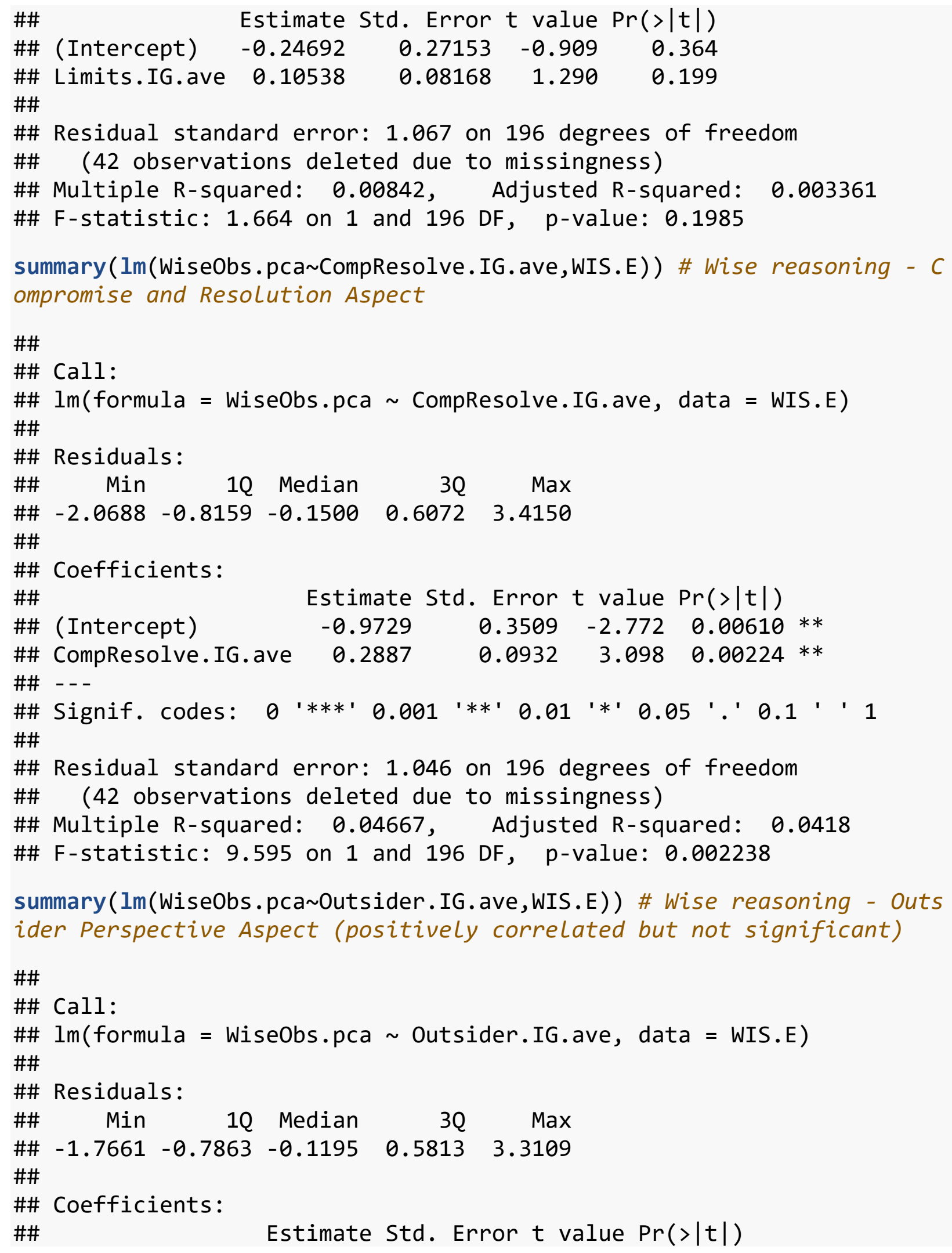




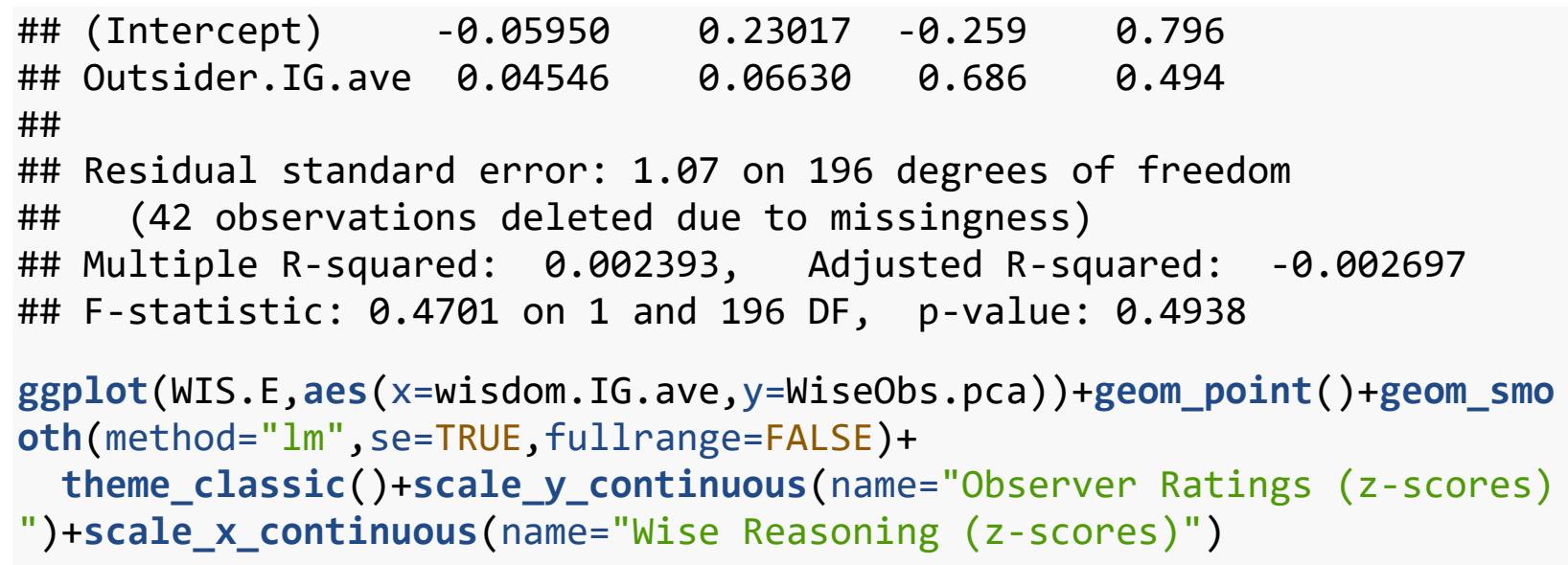

\#\# Warning: Removed 42 rows containing non-finite values (stat_smooth)

\#\# Warning: Removed 42 rows containing missing values (geom_point).

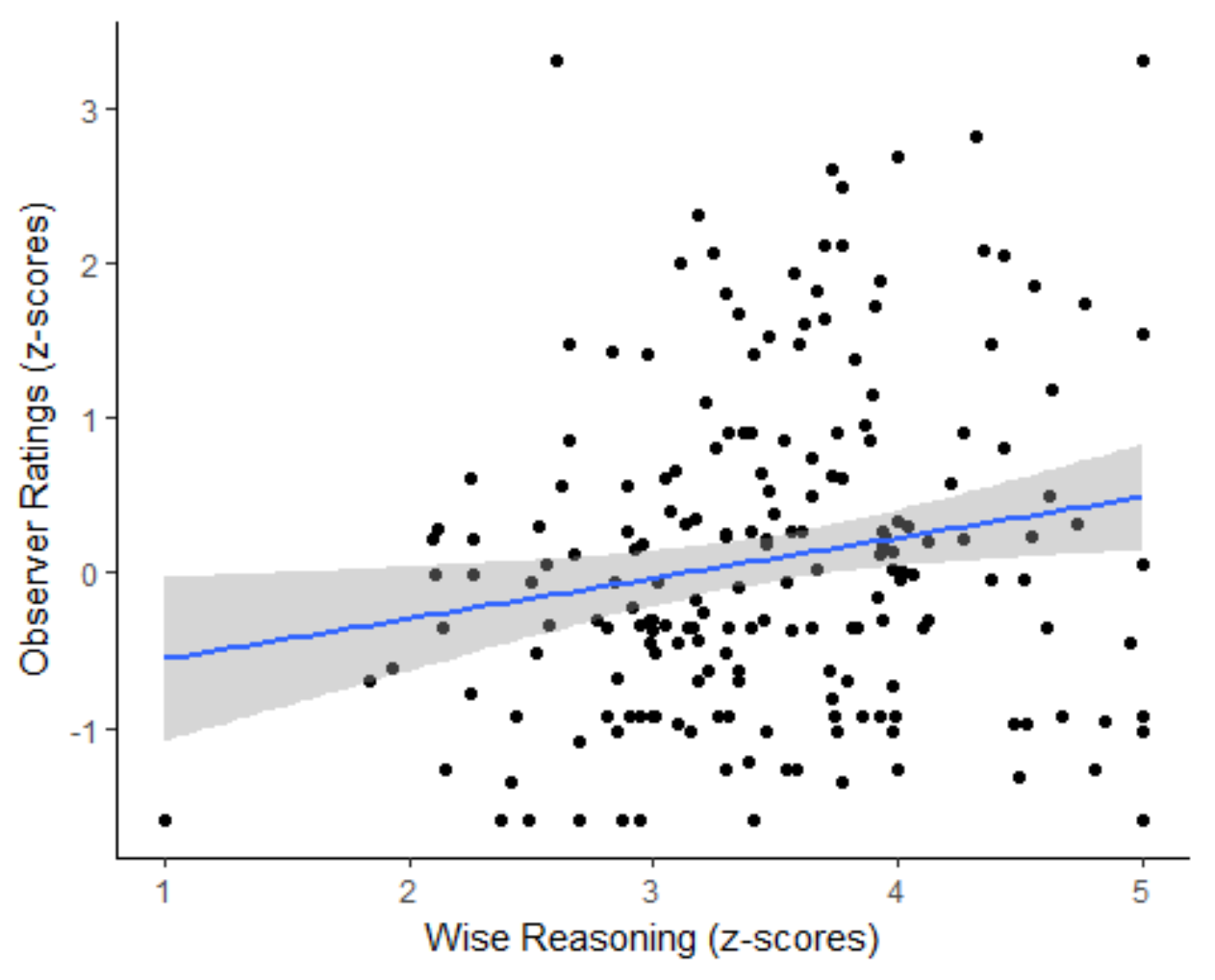

Convergent validity (Wise reasoning performance): Wise reasoning (state-level) about interpersonal and intergroup conflicts are positively correlated, as expected Hmisc::rcorr(as.matrix(WIS.E[, c('wisdom.pca', 'wisdom.IG.pca')])) 


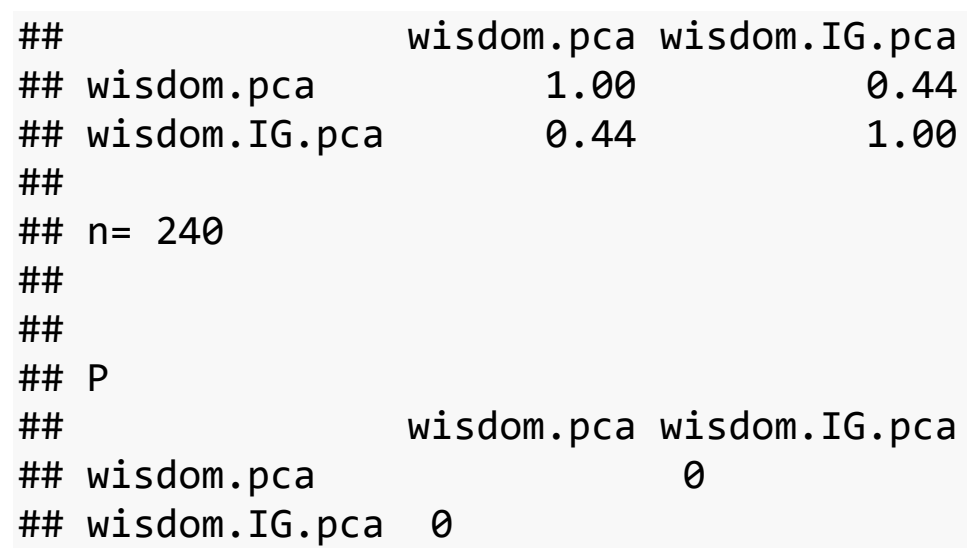

Partial correlations with wise reasoning and biased attributions controlling for SAWS (and vice versa)

\# Recall that in Divergent validity (Social Biases), both wise reasoni $n g$ and SAWS are associated with less biased reasoning. This analysis $t$ eases the two apart.

\# Run PCOR and ppcor::pcor.test Lines as a pair (to create the dataset and then run partial correlations)

PCOR<-na.omit(WIS [, c("wisdom.pca", "BiasAttrib.comp", "SAWSFull")])

ppcor: : pcor . test (PCOR \$wisdom. pca, PCOR \$BiasAttrib. comp, PCOR \$SAWSFull, me thod="pearson") \# Wise reasoning and bias controlling for SAWS: The ef fect for wise reasoning holds, as expected.

\#\# estimate p.value statistic $\mathrm{n}$ gp Method

\#\# 1 - $0.1158946 \quad 0.07748072 \quad-1.773396234 \quad 1$ pearson

ppcor: : pcor . test (PCOR\$SAWSFull, PCOR\$BiasAttrib. comp, PCOR\$wisdom. pca, me thod="pearson") \# SAWS and bias controlling for wise reasoning: the ef fect for SAWS is no longer significant.

\#\# estimate p.value statistic n gp Method

\#\# 1 -0.07735107 0.2395446 -1.179167 $234 \quad 1$ pearson

Partial correlations with wise reasoning and biased attributions, controlling for individual differences (emotional intelligence and emotion regulation) - Findings hold in all cases, as expected.

PCOR<-na.omit(WIS [, c("wisdom.pca", "BiasAttrib.comp", "EISelfEmotions")] )

ppcor: : pcor . test (PCOR\$wisdom. pca, PCOR \$BiasAttrib.comp, PCOR \$EISelfEmoti ons,method="spearman") \# Emotional intelligence: Self-emotion appraisa L 
\#\# estimate p.value statistic $\mathrm{n}$ gp Method \#\# 1 -0.09186531 0.01497145 -2.439105 7021 spearman

PCOR<-na.omit(WIS [ , c("wisdom.pca", "BiasAttrib.comp", "EIOthersEmotions" )])

ppcor: : pcor . test (PCOR\$wisdom. pca, PCOR\$BiasAttrib.comp, PCOR\$EIOthersEmo tions, method="spearman") \# Emotional intelligence: Others-emotion appr aisal

\#\# estimate p.value statistic $\mathrm{n}$ gp Method \#\# 1 -0.08164516 0.0307824 -2.164265 $701 \quad 1$ spearman

PCOR<-na.omit(WIS [, c("wisdom.pca", "BiasAttrib.comp", "EIEmotionUse")]) ppcor: : pcor .test (PCOR\$wisdom. pca, PCOR\$BiasAttrib.comp, PCOR \$EIEmotionUs e,method="spearman") \# Emotional intelligence: Use of emotion

\#\# estimate p.value statistic $\mathrm{n}$ gp Method \#\# 1 -0.1019451 0.006945829 -2.707465 $701 \quad 1$ spearman

PCOR<-na.omit(WIS [, c("wisdom.pca", "BiasAttrib.comp", "EIEmotionReg")] ) ppcor: : pcor . test (PCOR\$wisdom.pca, PCOR\$BiasAttrib.comp, PCOR \$EIEmotionRe g,method="spearman") \# Emotional intelligence: Regulation of emotion

\#\# estimate p.value statistic $\mathrm{n}$ gp Method \#\# 1 -0.09519606 $0.01173967 \quad-2.526524701 \quad 1$ spearman

PCOR<-na.omit(WIS [, c ("wisdom.pca", "BiasAttrib.comp", "Reflection")]) ppcor: : pcor . test (PCOR\$wisdom.pca, PCOR\$BiasAttrib.comp, PCOR \$Reflection, method="spearman") \# Ruminative response: Reflection

\#\# estimate p.value statistic $\mathrm{n}$ gp Method \#\# 1 -0.09202499 $0.01486831 \quad-2.441632701 \quad 1$ spearman

PCOR<-na.omit(WIS [, c("wisdom.pca", "BiasAttrib.comp", "Brooding")]) ppcor: : pcor . test (PCOR\$wisdom. pca, PCOR\$BiasAttrib.comp, PCOR \$Brooding, me thod="spearman")\# Ruminative response: Brooding

\#\# estimate p.value statistic $\mathrm{n}$ gp Method \#\# 1 -0.09813666 0.009374518 -2.605316 $701 \quad 1$ spearman

Examining each attribution scenario (Footnote 3)

\# Here, we test if dispositional (variables FAE*_1) or situational (va riables FAE*_2) have different relationships with wise reasoning acros $s$ the 4 vignettes (FAE1_*-FAE4_*)

\# We restructured the file to be long and ran a multilevel model where the dispositional and situational attribution questions were nested wi thin participant 
\# To read the multilevel model analysis (Lmer code), look at the last Line of fixed effects to see the interaction ( $p$-value in the last colu $m n: \operatorname{Pr}(>|t|))$

WIS.ATT1<-melt(WIS.D[ , c("IDvar", "wisdom.pca", "FAE1_1", "FAE1_2")] , id=c( "IDvar", "wisdom.pca"))

WIS.ATT2<-melt(WIS.D[ , c("IDvar", "wisdom.pca", "FAE2_1", "FAE2_2")] , id=c( "IDvar", "wisdom.pca")) WIS.ATT3<-melt(WIS.D[ , c("IDvar", "wisdom.pca", "FAE3_1", "FAE3_2")] , id=c( "IDvar", "wisdom.pca"))

WIS.ATT4<-melt(WIS.D[, c("IDvar", "wisdom.pca", "FAE4_1", "FAE4_2")], id=c( "IDvar", "wisdom.pca"))

summary(Imer(value wisdom.pca*variable+(1|IDvar), WIS.ATT1, REML = FAL SE)) \# Significant interaction

\#\# Linear mixed model fit by maximum likelihood t-tests use Satterthwa ite

\#\# approximations to degrees of freedom [1merMod]

\#\# Formula: value wisdom.pca * variable + (1 | IDvar $)$

\#\# Data: WIS.ATT1

\#\#

\#\# AIC BIC logLik deviance df.resid

$\begin{array}{llllll}\# \# & 4958.6 & 4990.1 & -2473.3 & 4946.6 & 1403\end{array}$

\#\#

\#\# Scaled residuals:

\#\# Min 10 Median 30 Max

$\begin{array}{llllll}\# \# & -2.6039 & -0.6004 & 0.2687 & 0.9385 & 1.7870\end{array}$

\#\#

\#\# Random effects:

\#\# Groups Name Variance Std.Dev.

\#\# IDvar (Intercept) $0.00 \quad 0.0$

\#\# Residual $1.96 \quad 1.4$

\#\# Number of obs: 1409, groups: IDvar, 705

\#\#

\#\# Fixed effects:

\#\#

\#\# (Intercept)

Estimate Std. Error df $t$ value

\#\# wisdom.pca

$\begin{array}{llll}4.59872 & 0.05279 & 1409.00000 & 87.114\end{array}$

\#\# variableFAE1_2

$\begin{array}{llll}-0.02211 & 0.05331 & 1409.00000 & -0.415\end{array}$

\#\# wisdom.pca:variableFAE1_2

\#\#

\#\# (Intercept)

$\begin{array}{llll}-0.57319 & 0.07463 & 1409.00000 & -7.680\end{array}$

\#\# wisdom.pca

\#\# variableFAE1_2

0.23791

$0.075391409 .00000 \quad 3.156$

\#\# wisdom.pca:variableFAE1_2 $\operatorname{Pr}(>|t|)$

$<0.0000000000000002 * * *$

0.67842

\#\# ---

$0.00163 * *$ 


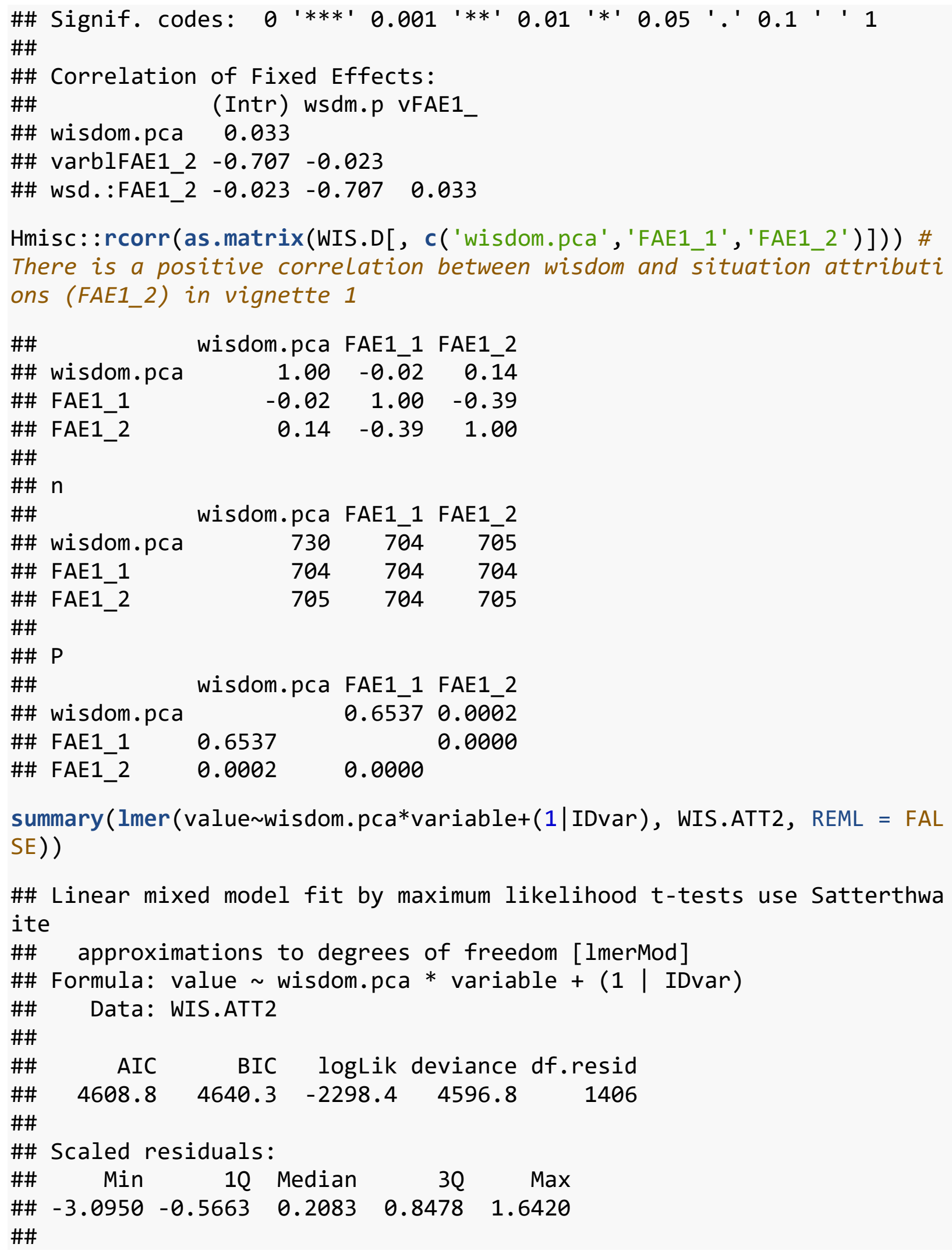




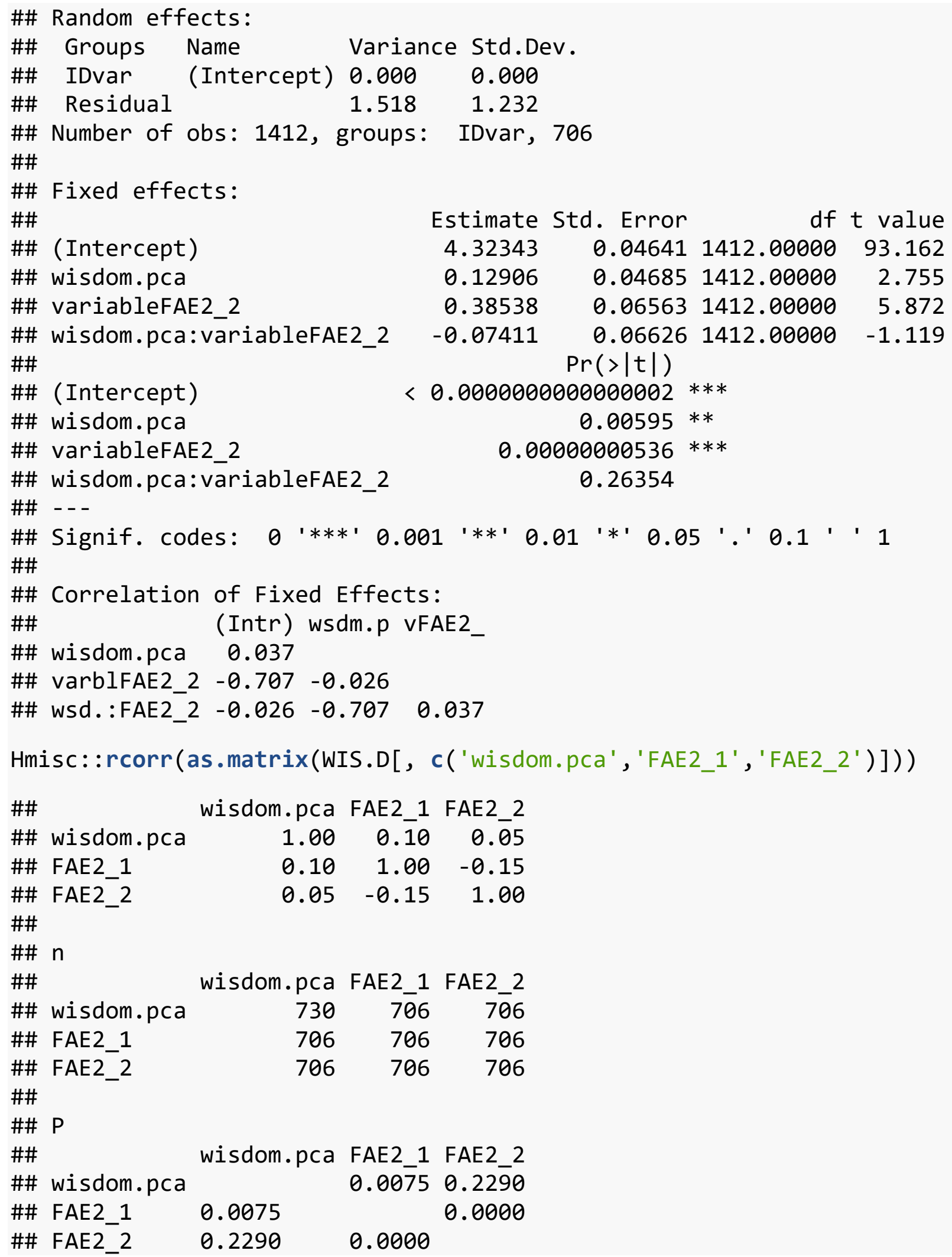


summary (Imer(value wisdom.pca*variable+(1|IDvar), WIS.ATT3, REML = FAL SE)) \# Marginally significant interaction

\#\# Linear mixed model fit by maximum likelihood t-tests use Satterthwa ite

\#\# approximations to degrees of freedom [1merMod]

\#\# Formula: value wisdom.pca * variable + (1 | IDvar)

\#\# Data: WIS.ATT3

\#\#

\#\# AIC BIC logLik deviance df.resid

$\begin{array}{llllll}\# \# & 4795.3 & 4826.8 & -2391.6 & 4783.3 & 1404\end{array}$

\#\#

\#\# Scaled residuals:

\#\# Min 10 Median 30 Max

$\begin{array}{llllll}\# \# & -2.8324 & -0.5126 & -0.0987 & 0.6556 & 1.4187\end{array}$

\#\#

\#\# Random effects:

\#\# Groups Name Variance Std.Dev.

\#\# IDvar (Intercept) $0.000 \quad 0.00$

\#\# Residual $\quad 1.741 \quad 1.32$

\#\# Number of obs: 1410, groups: IDvar, 705

\#\#

\#\# Fixed effects:

\#\#

Estimate Std. Error df $t$ val

ue

\#\# (Intercept)

$\begin{array}{llll}4.137702 & 0.049725 & 1410.000000 & 83.2\end{array}$

12

$\begin{array}{lllll}\text { \#\# wisdom.pca } & 0.003305 & 0.050286 & 1410.000000 & 0.0\end{array}$

66

$\begin{array}{lllll}\text { \#\# variableFAE3_2 } & 0.410370 & 0.070322 & 1410.000000 & 5.8\end{array}$

36

\#\# wisdom.pca:variableFAE3_2 $0.137489 \quad 0.0711151410 .000000 \quad 1.9$

33

\#\#

\#\# (Intercept)

$\operatorname{Pr}(>|t|)$
$<0.0000000000000002$$* * *$

\#\# wisdom.pca

0.9476

\#\# variableFAE3_2

\#\# wisdom.pca:variableFAE3_2

$0.00000000664 * * *$

\#\# ---

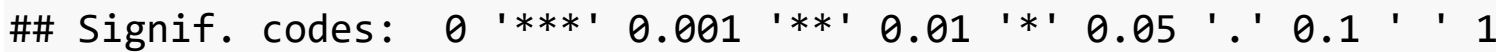

\#\#

\#\# Correlation of Fixed Effects:

\#\#

(Intr) wsdm.p VFAE3

\#\# wisdom.pca 0.035 


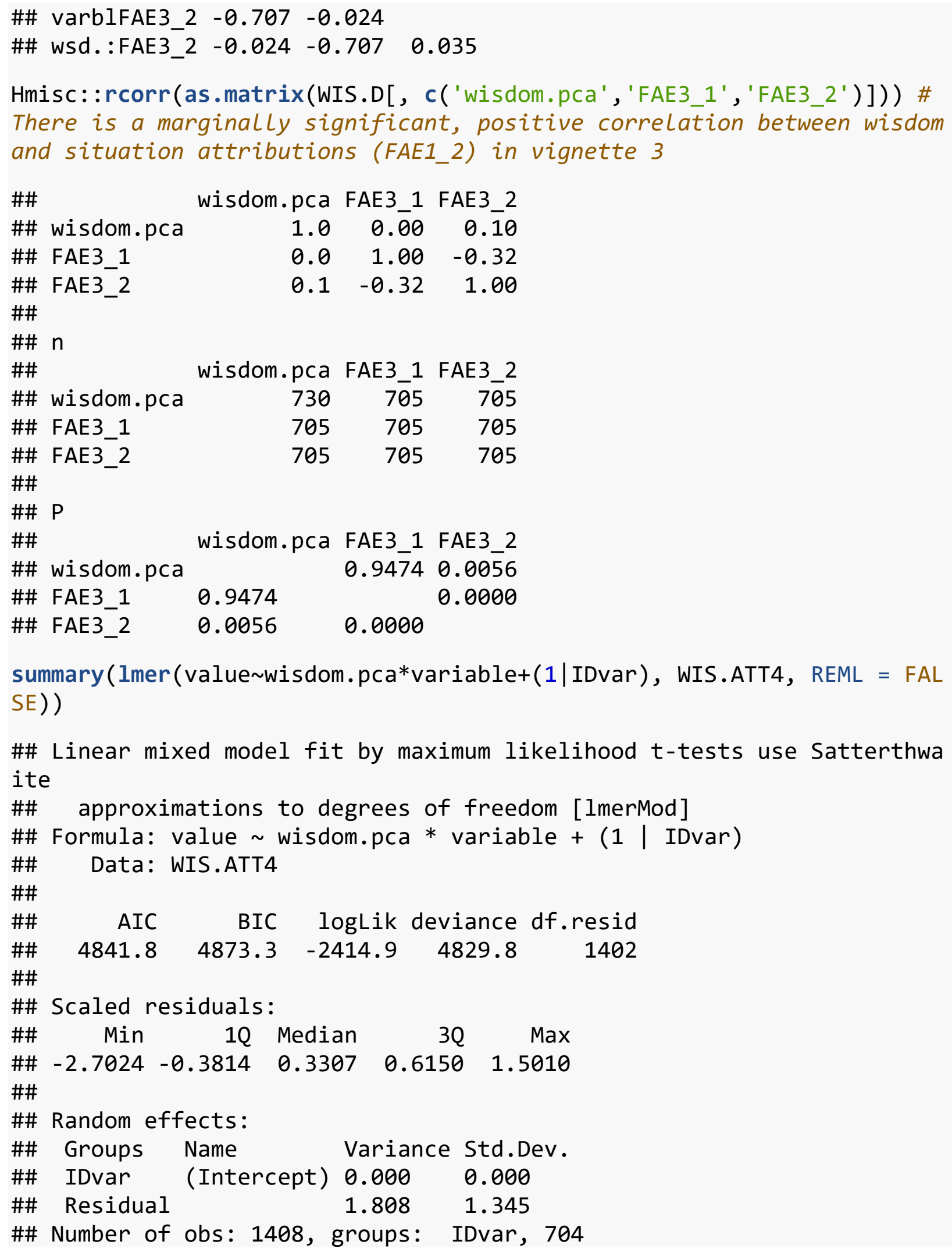




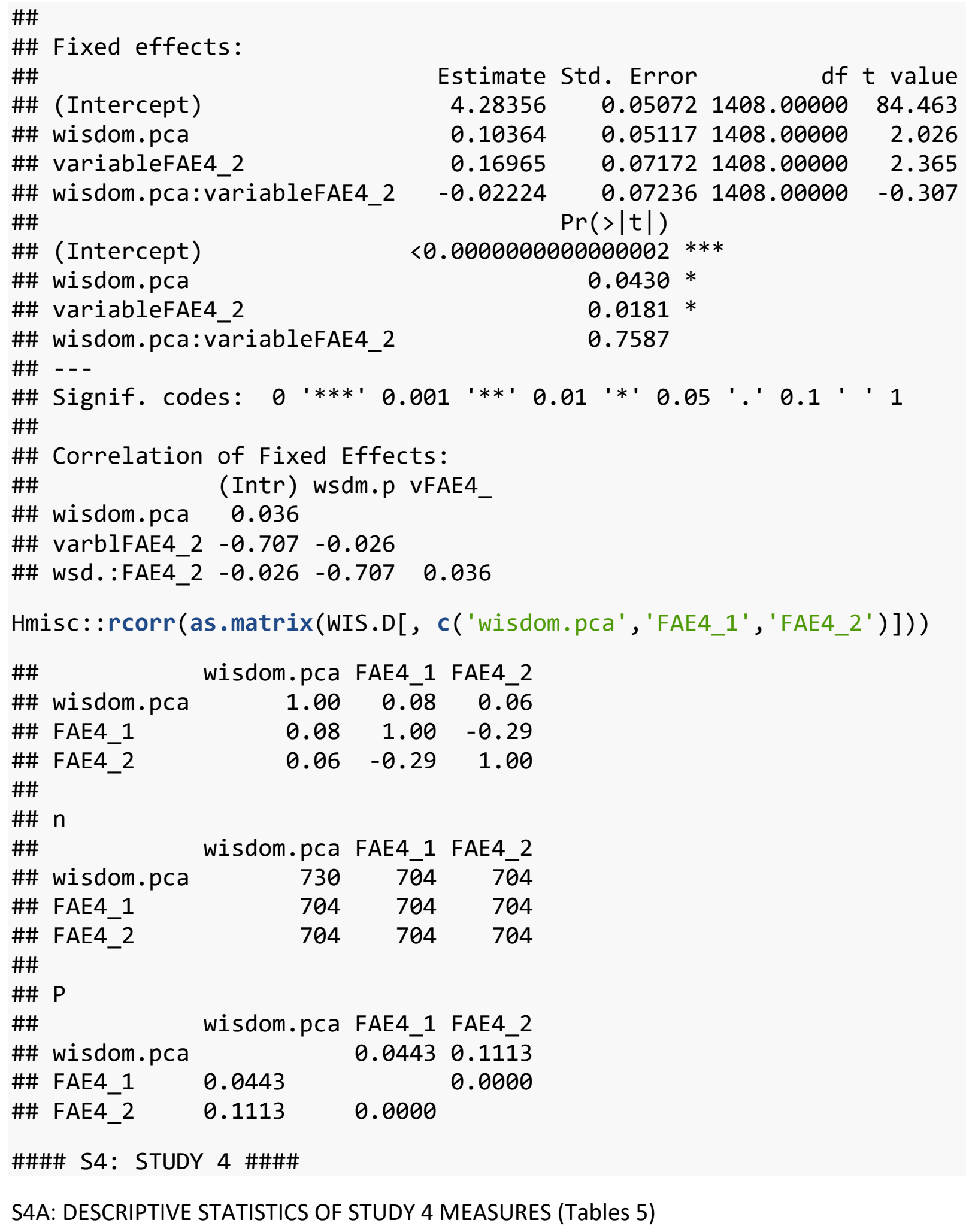


\# For each measure, sample size, mean, and standard deviation reported in Table 4 as.data.frame(psych::describe(WIS.B\$Openness)) [2:4] \# Openness

\#\# $\quad \mathrm{n}$ mean sd

\#\# X1 $220 \quad 3.717551 \quad 0.6362299$

as.data.frame(psych: :describe(WIS.B\$Conscientiousness)) [2:4] \# Conscie ntiousness

\#\# $\quad \mathrm{n}$ mean sd

\#\# X1 $220 \quad 3.877751 \quad 0.6885004$

as.data.frame(psych: :describe(WIS.B\$Extraversion))[2:4] \# Extraversion \#\# $\mathrm{n}$ mean sd

$\begin{array}{lllll}\text { \#\# X1 } & 220 & 3.143203 & 0.9102783\end{array}$

as.data.frame(psych: :describe(WIS.B\$Agreeableness)) [2:4] \# Agreeablene SS

\#\# $n$ mean sd

$\begin{array}{llll}\text { \#\# X1 } & 220 & 3.799729 & 0.7008132\end{array}$

as.data.frame(psych::describe(WIS.B\$Neuroticism))[2:4] \# Neuroticism

\#\# $\mathrm{n}$ mean sd

$\begin{array}{lllll}\text { \#\# X1 } & 220 & 2.630114 & 0.8275418\end{array}$

as.data.frame(psych::describe(WIS.B\$Intellect_1))[2:4] \# Intellect: Se ek

\#\# $n$ mean sd

\#\# X1 $220 \quad 5.391827 \quad 1.063865$

as.data.frame(psych::describe(WIS.B\$Intellect_2))[2:4] \# Intellect: Co nquer

\#\# $n$ mean sd

\#\# X1 $217 \quad 4.99623 \quad 1.151702$

as.data.frame(psych::describe(WIS.B\$ATT))[2:4] \# Attributional complex ity

\#\# $\quad \mathrm{n} \quad$ mean $\quad \mathrm{sd}$

\#\# X1 $218 \quad 4.685964 \quad 0.8677269$

as.data.frame(psych: :describe(WIS.A\$PerspTaking))[2:4] \# Perspective-t aking 
\#\# $\quad \mathrm{n} \quad$ mean sd

$\begin{array}{lllll}\text { \#\# X1 } & 404 & 3.407001 & 0.7273805\end{array}$

as.data.frame(psych: :describe(WIS.D\$EISelfEmotions))[2:4] \# Emotional intelligence: Self-emotion appraisal

\#\# $\quad \mathrm{n} \quad$ mean sd

\#\# X1 7025.2111821 .203451

as.data.frame(psych: :describe(WIS.D\$EIOthersEmotions)) [2:4] \# Emotiona L intelligence: Others-emotion appraisal

\#\# $\quad \mathrm{n} \quad$ mean sd

\#\# X1 7015.1972181 .0993

as.data.frame(psych: :describe(WIS.D\$EIEmotionUse))[2:4] \# Emotional in telligence: Use of emotion

\#\# $\quad \mathrm{n} \quad$ mean sd

\#\# X1 7015.2164761 .264254

as.data.frame(psych:: describe(WIS.D\$EIEmotionReg))[2:4] \# Emotional in telligence: Regulation of emotion

\#\# $\quad \mathrm{n} \quad$ mean $\quad \mathrm{sd}$

\#\# X1 $7014.82525 \quad 1.375276$

as.data.frame(psych::describe(WIS.B\$Mindful_1))[2:4] \# Mindfulness: No nreactivity

\#\# $\quad \mathrm{n} \quad$ mean sd

$\begin{array}{lllll}\text { \#\# X1 } & 223 & 3.265215 & 0.7512777\end{array}$

as.data.frame(psych::describe(WIS.B\$Mindful_2))[2:4] \# Mindfulness: Ob serving and attending

\#\# $\quad \mathrm{n} \quad$ mean $\quad \mathrm{sd}$

\#\# X1 $223 \quad 3.536542 \quad 0.7502282$

as.data.frame(psych::describe(WIS.B\$Mindful_3))[2:4] \# Mindfulness: Ac ting with awareness

\#\# $\quad \mathrm{n} \quad$ mean $\quad \mathrm{sd}$

\#\# X1 $223 \quad 3.3009290 .8818596$

as.data.frame(psych::describe(WIS.B\$Mindful_4))[2:4] \# MindfuLness: De scribing with words 
\#\# $\quad \mathrm{n} \quad$ mean sd

$\begin{array}{lllll}\text { \#\# X1 } & 221 & 3.390595 & 0.7533479\end{array}$

as.data.frame(psych::describe(WIS.B\$Mindful_5))[2:4] \# Mindfulness: No $n$-judging of experience

\#\# $\quad \mathrm{n} \quad$ mean sd

$\begin{array}{lllll}\text { \#\# X1 } & 220 & 3.036337 & 0.9593797\end{array}$

as.data.frame(psych: :describe(WIS.A\$Communal))[2:4] \# Communal relatio nship orientation

\#\# $\quad \mathrm{n} \quad$ mean sd

\#\# X1 $4043.47995 \quad 0.7889239$

as.data.frame(psych::describe(WIS.B\$EmoReg_1))[2:4] \# Emotion regulati on: Reappraisal

\#\# $\quad \mathrm{n}$ mean sd

\#\# X1 $216 \quad 4.875 \quad 1.220333$

as.data.frame(psych::describe(WIS.B\$EmoReg_2))[2:4] \# Emotion regulati on: Suppression

\#\# $\quad \mathrm{n} \quad$ mean $\quad \mathrm{sd}$

\#\# X1 $216 \quad 4.29321 \quad 1.375448$

as.data.frame(psych: :describe(WIS.D\$Reflection))[2:4] \# Ruminative res ponse: Reflection

\#\# $\quad \mathrm{n} \quad$ mean sd

\#\# X1 $701 \quad 2.5942940 .6756766$

as.data.frame(psych: :describe(WIS.D\$Brooding))[2:4] \# Ruminative respo nse: Brooding

\#\# $\quad \mathrm{n} \quad$ mean $\quad$ sd

\#\# X1 $7012.541227 \quad 0.7685037$

as.data.frame(psych: :describe(WIS.G\$ess.social))[2:4] \# Growth mindset about social relations: Changeable social relationships

\#\# $\quad n$ mean sd

\#\# X1 $467 \quad 4.750178 \quad 1.067097$

as.data.frame(psych::describe(WIS.G\$ess.conflict))[2:4] \# Growth minds et about social relations: Changeable social conflicts 
\#\# $\quad \mathrm{n} \quad$ mean sd

\#\# X1 $466 \quad 5.275751 \quad 0.9229056$

S4B: RELIABILITY OF STUDY 4 MEASURES (Table 5)

\# Look at raw_alpha for Cronbach's Alpha

psych: :alpha(WIS.B[, c('bfi5 ', 'bfi10', 'bfi15 ' , 'bfi20 ', 'bfi25 ', 'bfi30', 'BFI35R', 'bfi40', 'BFI41R', 'bfi44')], check.keys=FALSE)[1] \# Openness

\#\# \$total

\#\# raw_alpha std.alpha G6(smc) average_r $\mathrm{S} / \mathrm{N}$ ase me

$\begin{array}{llllllll}\text { \#\# } & 0.8191487 & 0.8350918 & 0.8509825 & 0.3361648 & 5.063981 & 0.01235782 & 3.7175\end{array}$

51

\#\# sd

\#\# 0.6362299

psych: :alpha(WIS.B[, c('bfi3', 'BFI8R' , 'bfi13 ' , 'BFI18R ' , 'BFI23R' , 'bfi28 ', 'bfi33', 'bfi38', 'BFI43R')], check.keys=FALSE)[1] \# Conscientiousness

\#\# \$total

\#\# raw_alpha std.alpha G6(smc) average_r S/N me

an

$\begin{array}{lllllllll}\text { \#\# } & 0.8458164 & 0.8521919 & 0.8622551 & 0.3904723 & 5.765531 & 0.01051806 & 3.8777\end{array}$

51

\#\# sd

\#\# 0.6885004

psych: :alpha(WIS.B[, c('bfi1', 'BFI6R', 'bfi11' , 'BFI21R' , 'bfi26' , 'BFI31R ', 'bfi36')], check.keys=FALSE)[1] \# Extraversion

\#\# \$total

\#\# raw_alpha std.alpha G6(smc) average_r S/N ase me

$\begin{array}{llllllll}\text { \#\# } & 0.8510113 & 0.8482772 & 0.8554877 & 0.4440458 & 5.590966 & 0.01035325 & 3.1432\end{array}$

03

\#\# sd

\#\# 0.9102783

psych: :alpha(WIS.B[, c('BFI2R', 'bfi7', 'BFI12R', 'bfi17' , 'bfi22' , 'BFI27R ', 'bfi32', 'BFI37R', 'bfi42')], check.keys=FALSE)[1] \# Agreeableness

\#\# \$total

\#\# raw_alpha std.alpha G6(smc) average_r $\mathrm{S} / \mathrm{N}$ ase me

an

$\begin{array}{llllllll}\text { \#\# } & 0.8264513 & 0.83067 & 0.8410222 & 0.35278 & 4.905628 & 0.01205039 & 3.7997\end{array}$

29 


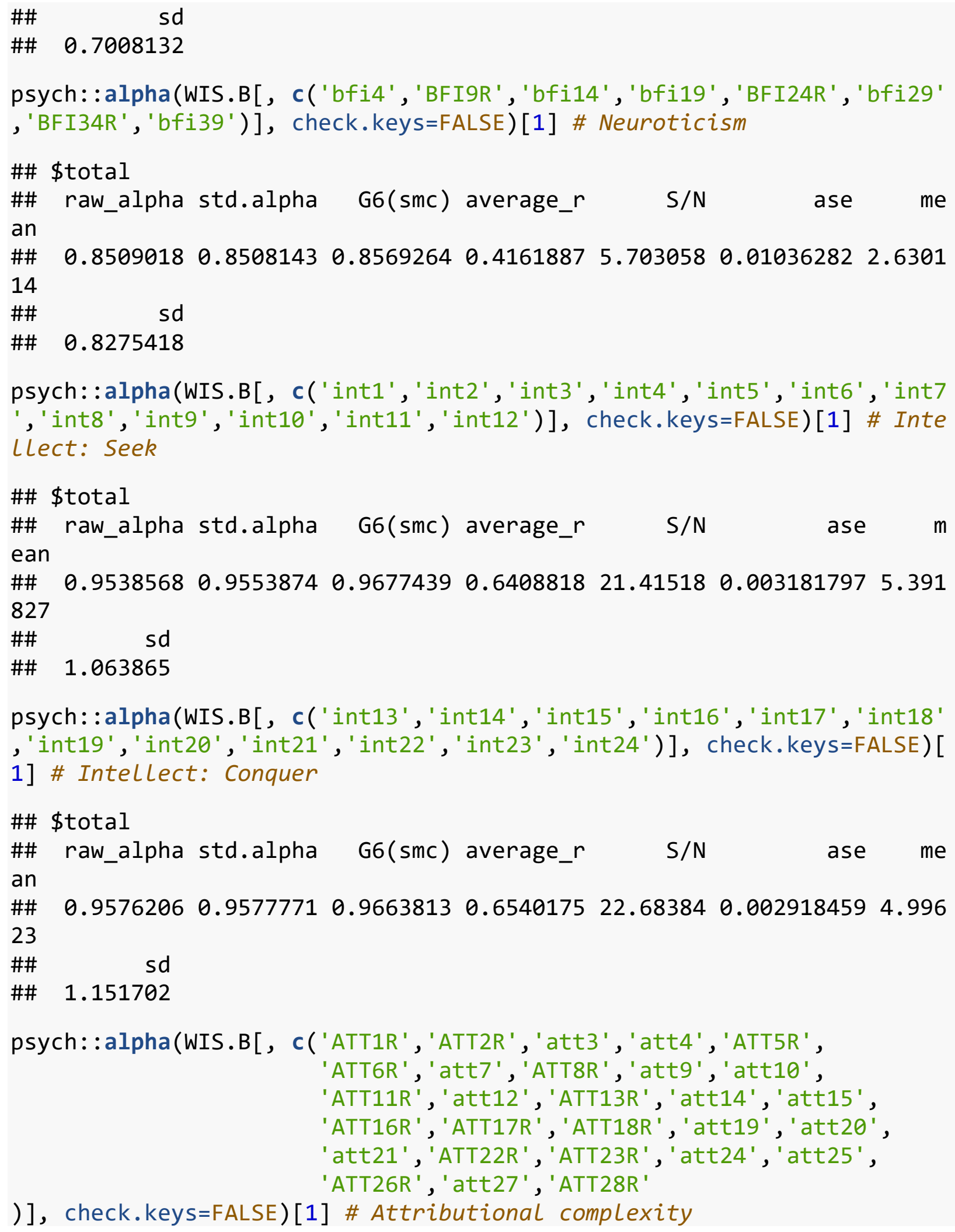




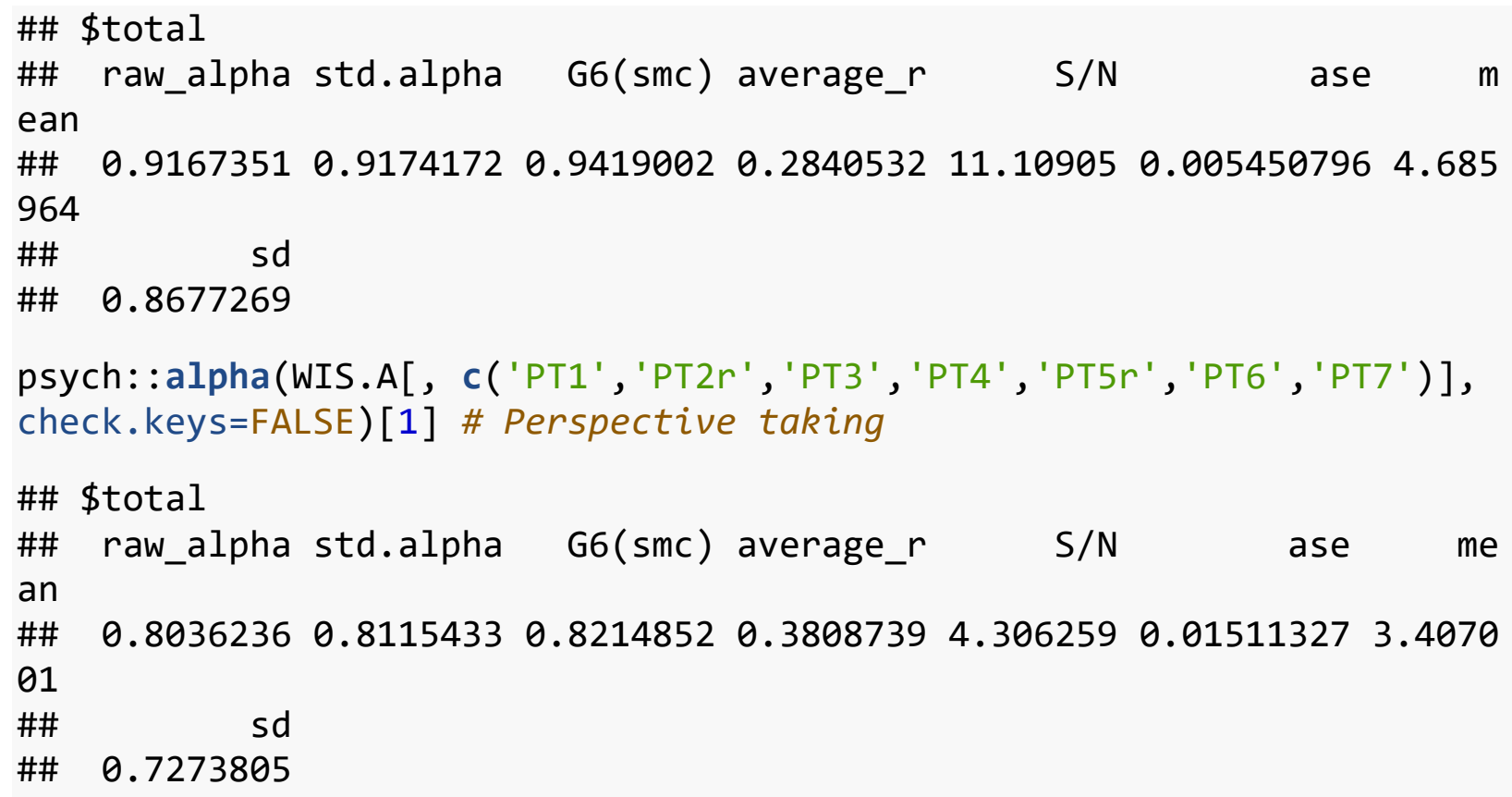




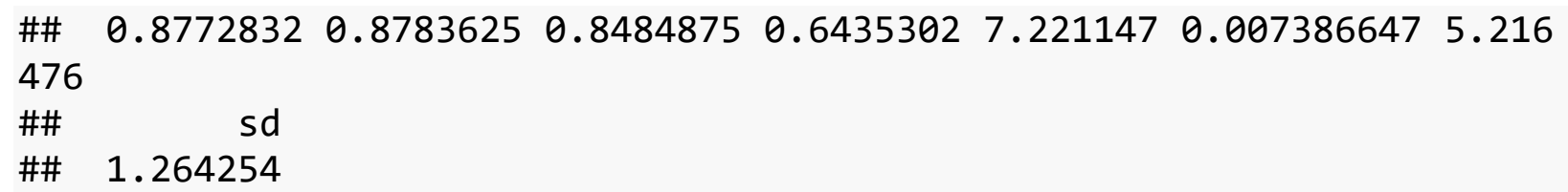

$\begin{array}{lllllllll}\text { \#\# } & 0.8772832 & 0.8783625 & 0.8484875 & 0.6435302 & 7.221147 & 0.007386647 & 5.216\end{array}$ 476

\#\# $\quad$ sd

\#\# 1.264254

psych: :alpha(WIS.D[, c('ei13', 'ei14', 'ei15', 'ei16')], check.keys=FALSE )[1] \# Emotional intelligence: Regulation of emotion

\#\# \$total

\#\# raw_alpha std.alpha G6(smc) average_r $\mathrm{S} / \mathrm{N}$ ase me

an

$\begin{array}{lllllllll}\text { \#\# } & 0.8990546 & 0.9005664 & 0.8881263 & 0.6936501 & 9.056963 & 0.006237353 & 4.825\end{array}$

25

\#\# $\quad$ sd

\#\# 1.375276

psych: :alpha(WIS.B[, c('mind1', 'mind2' , 'mind3' , 'mind4' ', 'mind5' , 'mind6' , 'mind7')], check.keys=FALSE)[1] \# Mindfulness: Nonreactivity

\#\# \$total

\#\# raw_alpha std.alpha G6(smc) average_r $\mathrm{S} / \mathrm{N}$ ase $\mathrm{m}$ ean

$\begin{array}{lllllllll}\text { \#\# } & 0.8724747 & 0.8729704 & 0.8647991 & 0.4953929 & 6.872179 & 0.008994699 & 3.265\end{array}$ 215

\#\# sd

\#\# $\quad 0.7512777$

psych: :alpha(WIS.B[, c('mind8', 'mind9', 'mind10', 'mind11', 'mind12', 'min d13', 'mind14','mind15')], check.keys=FALSE)[1] \# Mindfulness: Observin $g$ and attending

\#\# \$total

\#\# raw_alpha std.alpha G6(smc) average_r S/N ase me

an

$\begin{array}{llllllll}\text { \#\# } & 0.8679094 & 0.8679742 & 0.865664 & 0.4510876 & 6.574276 & 0.009152721 & 3.5365\end{array}$

42

\#\# $\quad$ sd

\#\# $\quad 0.7502282$

psych: :alpha(WIS.B [, c('mind16r', 'mind17r' , 'mind18r', 'mind19r', 'mind20 $r^{\prime}$, 'mind21r', 'mind22r', 'mind23r')], check.keys=FALSE)[1] \# Mindfulness : Acting with awareness

\#\# \$total

\#\# raw_alpha std.alpha G6(smc) average_r $\mathrm{S} / \mathrm{N}$ ase me an

$\begin{array}{lllllllll}\# \# & 0.9168177 & 0.9173111 & 0.9212219 & 0.5810096 & 11.09352 & 0.00587423 & 3.3009\end{array}$ 


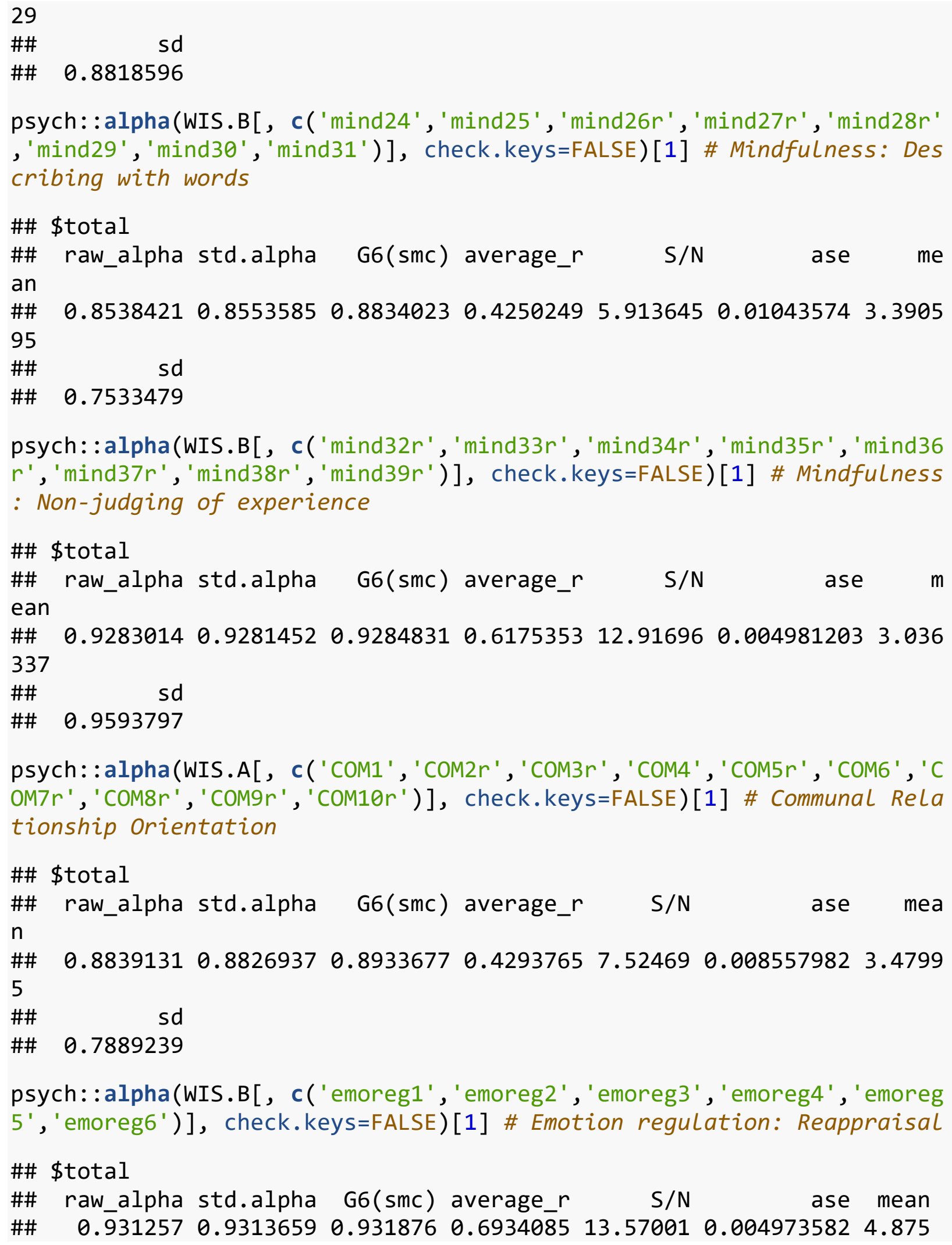
OM7r', 'COM8r', 'COM9r', 'COM10r')], check.keys=FALSE)[1] \# Communal Rela tionship Orientation

\#\# \$total

\#\# raw_alpha std.alpha G6(smc) average_r S/N ase mea

$\mathrm{n}$

$\begin{array}{llllllllll}\# \# & 0.8839131 & 0.8826937 & 0.8933677 & 0.4293765 & 7.52469 & 0.008557982 & 3.4799\end{array}$

5

\#\# $\quad$ sd

\#\# $\quad 0.7889239$

psych: :alpha(WIS.B[, c('emoreg1', 'emoreg2', 'emoreg3', 'emoreg4 ', 'emoreg 5', 'emoreg6')], check.keys=FALSE)[1] \# Emotion regulation: Reappraisal

\#\# \$total

\#\# raw_alpha std.alpha G6(smc) average_r S/N ase mean

$\begin{array}{llllllll}\text { \#\# } & 0.931257 & 0.9313659 & 0.931876 & 0.6934085 & 13.57001 & 0.004973582 & 4.875\end{array}$ 


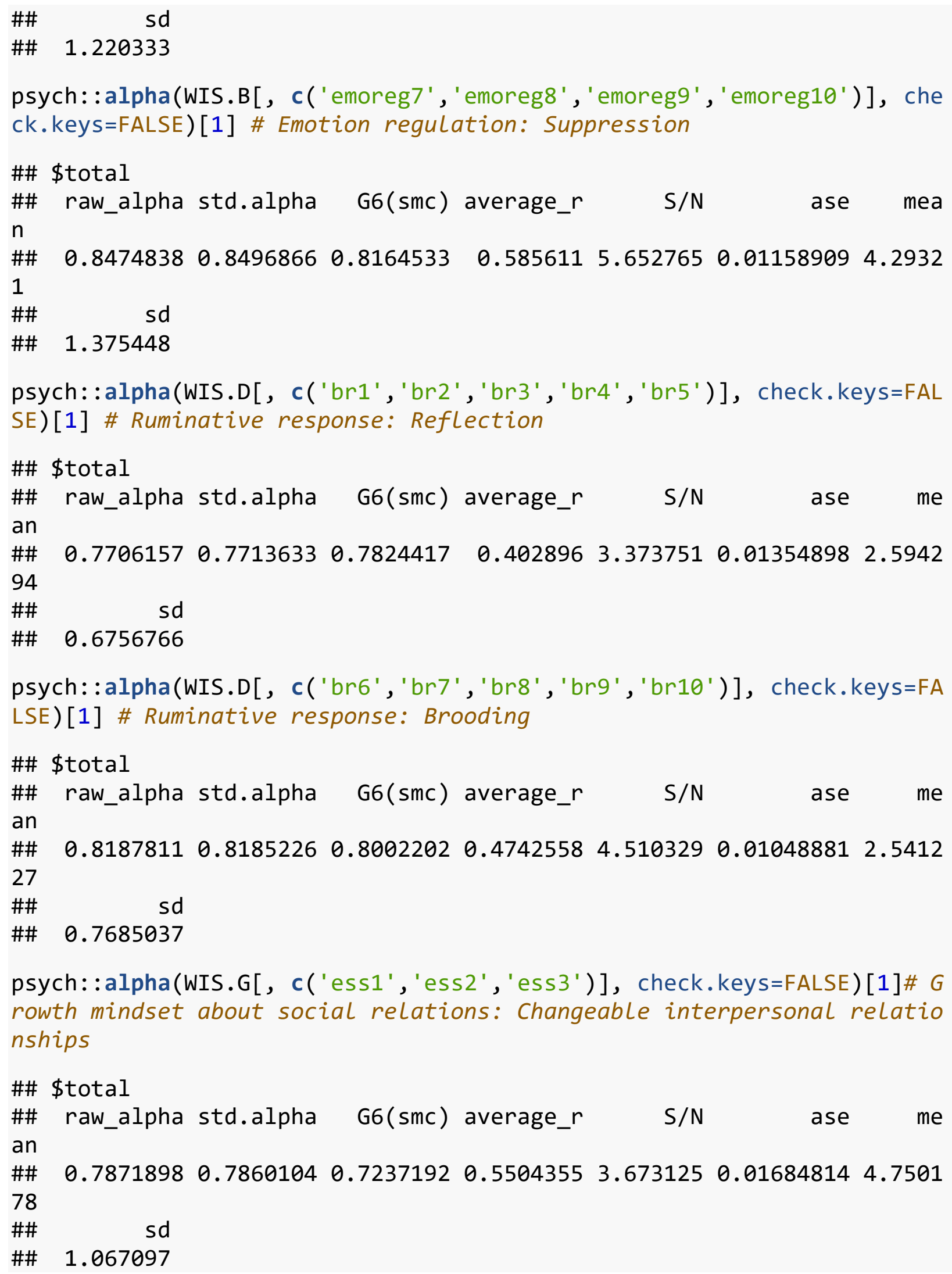
rowth mindset about social relations: Changeable interpersonal relatio nships

\#\# \$total

\#\# raw_alpha std.alpha G6(smc) average_r $\mathrm{S} / \mathrm{N}$ ase me

an

$\begin{array}{lllllllll}\# \# & 0.7871898 & 0.7860104 & 0.7237192 & 0.5504355 & 3.673125 & 0.01684814 & 4.7501\end{array}$

78

\#\# sd

\#\# 1.067097 
psych: :alpha(WIS.G[, c('ess4', 'ess5', 'ess6', 'ess7')], check.keys=FALSE )[1]\# Event-centered fixed mindset: Changeable social conflicts

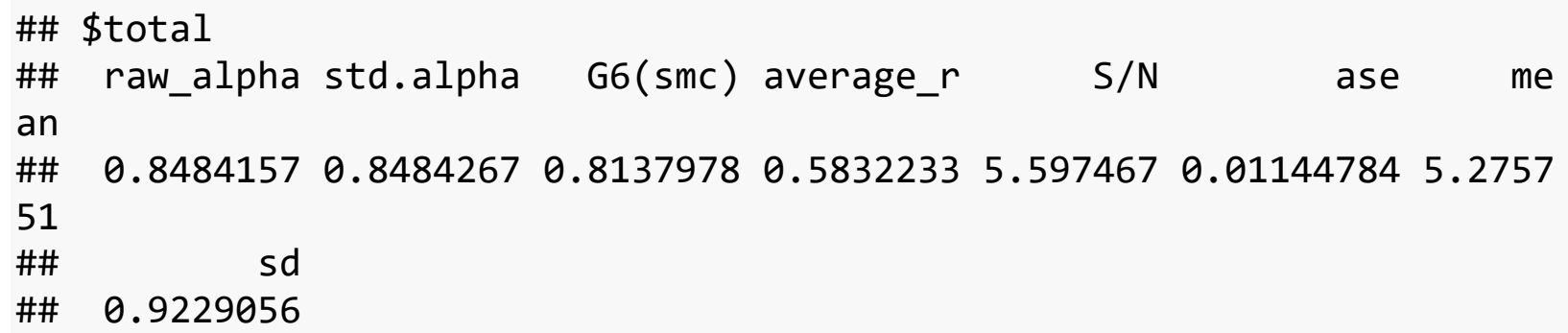

S4C: RESULTS FOR STUDY 4 Pearson correlations with wise reasoning and its five aspects (see Table 7)

\# Each Line of code presents 3 Lists (Pearson's r, sample size, p-valu e)

\# Wise reasoning variable labels: Wise reasoning latent component (wis dom.pca), Wise reasoning intergroup (wisdom.IG.pca), Global measure of wise reasoning (GlobalW)

\# Wise reasoning aspects variable Labels: Perspective (Perspective.ave ), Change/outcomes (ChangeOutcomes.ave), Limits (Limits.ave), Compromi se/resolution (CompResolve.ave), Outsider (Outsider.ave). Wise reasoni ng intergroup aspects all end with. IG.ave

\# Big 5 variable Labels: Openness, Conscientiousness, Extraversion, Ag reeableness, Neuroticism

\# Individual difference variable labels: Attributional complexity (ATT ), Intellect: Seek (Intellect_1), Intellect: Conquer (Intellect_2), Pe rspective-taking (PerspTaking), Communal Relationship Orientation (Com munal)

\# Emotional intelligence variable Labels: Self-emotions appraisal (EIS elfEmotions), Others-emotion appraisal (EIOthersEmotions), Use of emot ion (EIEmotionUse), Regulation of emotion (EIEmotionReg)

\# Mindfulness variable Labels: Nonreactivity (Mindful_1), Observing an d attending (Mindful_2), Acting with awareness (Mindful_3), Describing with words (Mindful_4), Non-judging of experience (Mindful_5)

\# Emotion regulation variable Labels: Reappraisal (EmoReg_1), Suppress ion (EmoReg_2)

\# Ruminative response variable Labels: Reflection, Brooding

\# Growth mindset about social relations variable Labels: Changeable so cial relations (ess.social). Changeable conflicts (ess.conflict)

Hmisc: :rcorr(as.matrix(WIS.B[, c('wisdom.pca', 'Perspective.ave', 'Chang eOutcomes.ave', 'Limits.ave', 'CompResolve.ave ', 'Outsider.ave ', 'Openness ', 'Conscientiousness ', 'Extraversion ', 'Agreeableness ', 'Neuroticism ' )])) 


\begin{tabular}{|c|c|c|c|c|c|}
\hline \#\# & & wisdom.pca $\mathrm{F}$ & \multicolumn{2}{|c|}{ Perspective.ave } & ChangeOutcomes.ave \\
\hline \#\# & wisdom.pca & 1.00 & & 0.82 & \\
\hline \#\# & Perspective.ave & 0.82 & & 1.00 & \\
\hline \#\# & Changeoutcomes.ave & 0.75 & & 0.48 & \\
\hline \#\# & Limits.ave & 0.77 & & 0.55 & \\
\hline \#\# & CompResolve.ave & 0.82 & & 0.63 & \\
\hline \#\# & Outsider.ave & 0.69 & & 0.45 & 0.4 \\
\hline \#\# & Openness & 0.19 & & 0.08 & \\
\hline \#\# & Conscientiousness & 0.02 & & -0.07 & \\
\hline \#\# & Extraversion & 0.24 & & 0.14 & \\
\hline \#\# & Agreeableness & 0.12 & & 0.04 & 0.2 \\
\hline \#\# & Neuroticism & -0.10 & & -0.09 & -0.1 \\
\hline \#\# & & Limits.ave & CompRe & solve.ave Outs & sider.ave Oper \\
\hline \#\# & wisdom.pca & 0.77 & & 0.82 & 0.69 \\
\hline \#\# & Perspective.ave & 0.55 & & 0.63 & 0.45 \\
\hline \#\# & ChangeOutcomes.ave & 0.43 & & 0.55 & 0.43 \\
\hline \#\# & Limits.ave & 1.00 & & 0.54 & 0.45 \\
\hline \#\# & CompResolve.ave & 0.54 & & 1.00 & 0.39 \\
\hline \#\# & Outsider.ave & 0.45 & & 0.39 & 1.00 \\
\hline \#\# & Openness & 0.15 & & 0.16 & 0.13 \\
\hline \#\# & Conscientiousness & 0.05 & & 0.12 & -0.13 \\
\hline \#\# & Extraversion & 0.19 & & 0.14 & 0.22 \\
\hline \#\# & Agreeableness & 0.00 & & 0.19 & 0.00 \\
\hline \#\# & Neuroticism & -0.12 & & -0.11 & 0.05 \\
\hline \#\# & & Conscientiol & usness & Extraversion & Agreeableness \\
\hline \#\# & wisdom.pca & & 0.02 & 0.24 & 0.12 \\
\hline \#\# & Perspective.ave & & -0.07 & 0.14 & 0.04 \\
\hline \#\# & ChangeOutcomes.ave & & 0.08 & 0.21 & 0.21 \\
\hline \#\# & Limits.ave & & 0.05 & 0.19 & 0.00 \\
\hline \#\# & CompResolve.ave & & 0.12 & 0.14 & 0.19 \\
\hline \#\# & Outsider.ave & & -0.13 & 0.22 & 0.00 \\
\hline \#\# & Openness & & 0.26 & 0.20 & 0.19 \\
\hline \#\# & Conscientiousness & & 1.00 & 0.07 & 0.46 \\
\hline \#\# & Extraversion & & 0.07 & 1.00 & 0.02 \\
\hline \#\# & Agreeableness & & 0.46 & 0.02 & 1.00 \\
\hline \#\# & Neuroticism & & -0.50 & -0.28 & -0.26 \\
\hline \#\# & & Neuroticism & & & \\
\hline \#\# & wisdom.pca & -0.10 & & & \\
\hline \#\# & Perspective.ave & -0.09 & & & \\
\hline \#\# & ChangeOutcomes.ave & -0.10 & & & \\
\hline \#\# & Limits.ave & -0.12 & & & \\
\hline \#\# & CompResolve.ave & -0.11 & & & \\
\hline \#\# & Outsider.ave & 0.05 & & & \\
\hline \#\# & Openness & -0.14 & & & \\
\hline
\end{tabular}




\begin{tabular}{|c|c|c|c|c|c|}
\hline \#\# & Conscientiousness & -0.50 & & & \\
\hline \#\# & Extraversion & -0.28 & & & \\
\hline \#\# & Agreeableness & -0.26 & & & \\
\hline \#\# & Neuroticism & 1.00 & & & \\
\hline \#\# & & & & & \\
\hline \#\# & $\mathrm{n}$ & & & & \\
\hline \#\# & & wisdom.pca & Perspective.ave & ChangeOutcome & s.ave \\
\hline \#\# & wisdom.pca & 469 & 469 & & 468 \\
\hline \#\# & Perspective.ave & 469 & 469 & & 468 \\
\hline \#\# & ChangeOutcomes.ave & 468 & 468 & & 468 \\
\hline \#\# & Limits.ave & 466 & 466 & & 466 \\
\hline \#\# & CompResolve.ave & 466 & 466 & & 466 \\
\hline \#\# & Outsider.ave & 461 & 461 & & 461 \\
\hline \#\# & Openness & 220 & 220 & & 220 \\
\hline \#\# & Conscientiousness & 220 & 220 & & 220 \\
\hline \#\# & Extraversion & 220 & 220 & & 220 \\
\hline \#\# & Agreeableness & 220 & 220 & & 220 \\
\hline \#\# & Neuroticism & 220 & 220 & & 220 \\
\hline \#\# & & Limits.ave & CompResolve.ave & Outsider.ave & Openness \\
\hline \#\# & wisdom.pca & 466 & 466 & 461 & 220 \\
\hline \#\# & Perspective.ave & 466 & 466 & 461 & 220 \\
\hline \#\# & ChangeOutcomes.ave & 466 & 466 & 461 & 220 \\
\hline \#\# & Limits.ave & 466 & 466 & 461 & 220 \\
\hline \#\# & CompResolve.ave & 466 & 466 & 461 & 220 \\
\hline \#\# & Outsider.ave & 461 & 461 & 461 & 220 \\
\hline \#\# & Openness & 220 & 220 & 220 & 220 \\
\hline \#\# & Conscientiousness & 220 & 220 & 220 & 220 \\
\hline \#\# & Extraversion & 220 & 220 & 220 & 220 \\
\hline \#\# & Agreeableness & 220 & 220 & 220 & 220 \\
\hline \#\# & Neuroticism & 220 & 220 & 220 & 220 \\
\hline \#\# & & Conscientio & ousness Extravers & sion Agreeabler & eness \\
\hline \#\# & wisdom.pca & & 220 & 220 & 220 \\
\hline \#\# & Perspective.ave & & 220 & 220 & 220 \\
\hline \#\# & ChangeOutcomes.ave & & 220 & 220 & 220 \\
\hline \#\# & Limits.ave & & 220 & 220 & 220 \\
\hline \#\# & CompResolve.ave & & 220 & 220 & 220 \\
\hline \#\# & Outsider.ave & & 220 & 220 & 220 \\
\hline \#\# & Openness & & 220 & 220 & 220 \\
\hline \#\# & Conscientiousness & & 220 & 220 & 220 \\
\hline \#\# & Extraversion & & 220 & 220 & 220 \\
\hline \#\# & Agreeableness & & 220 & 220 & 220 \\
\hline \#\# & Neuroticism & & 220 & 220 & 220 \\
\hline \#\# & & Neuroticism & & & \\
\hline \#\# & wisdom.pca & 220 & & & \\
\hline
\end{tabular}




\begin{tabular}{|c|c|c|c|c|c|c|}
\hline \multicolumn{3}{|c|}{ \# Perspective.ave } & \multicolumn{4}{|c|}{20} \\
\hline \#\# & \multicolumn{2}{|l|}{ ChangeOutcomes.ave } & \multicolumn{4}{|c|}{20} \\
\hline \#\# & \multicolumn{2}{|l|}{ Limits.ave } & \multicolumn{4}{|c|}{220} \\
\hline \#\# & \multicolumn{2}{|l|}{ CompResolve.ave } & \multicolumn{4}{|c|}{220} \\
\hline \#\# & \multicolumn{2}{|l|}{ Outsider.ave } & \multicolumn{4}{|c|}{220} \\
\hline \#\# & \multicolumn{2}{|l|}{ Openness } & \multicolumn{4}{|c|}{220} \\
\hline \#\# & \multicolumn{2}{|l|}{ Conscientiousness } & \multicolumn{4}{|c|}{220} \\
\hline \#\# & \multicolumn{2}{|l|}{ Extraversion } & \multicolumn{4}{|c|}{220} \\
\hline \#\# & \multicolumn{2}{|l|}{ Agreeableness } & \multicolumn{4}{|c|}{220} \\
\hline \#\# & \multicolumn{2}{|l|}{ Neuroticism } & \multicolumn{4}{|c|}{220} \\
\hline \multicolumn{7}{|c|}{ 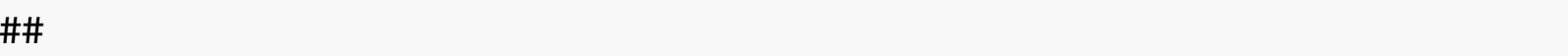 } \\
\hline \#\# & \multicolumn{6}{|c|}{$P$} \\
\hline \#\# & & \multirow[t]{2}{*}{ wisdom.pca } & \multicolumn{2}{|c|}{ Perspective.ave } & ChangeOutcome & es.ave \\
\hline \#\# & wisdom.pca & & 0.0000 & & 0.0000 & \\
\hline \#\# & Perspective.ave & 0.0000 & & & 0.0000 & \\
\hline \#\# & ChangeOutcomes.ave & 0.0000 & 0.0000 & & & \\
\hline \#\# & Limits.ave & 0.0000 & 0.0000 & & 0.0000 & \\
\hline \#\# & CompResolve.ave & 0.0000 & 0.0000 & & 0.0000 & \\
\hline \#\# & Outsider.ave & 0.0000 & 0.0000 & & 0.0000 & \\
\hline \#\# & Openness & 0.0045 & 0.2113 & & 0.0027 & \\
\hline \#\# & Conscientiousness & 0.7958 & 0.3287 & & 0.2492 & \\
\hline \#\# & Extraversion & 0.0004 & 0.0368 & & 0.0018 & \\
\hline \#\# & Agreeableness & 0.0749 & 0.5318 & & 0.0019 & \\
\hline \#\# & Neuroticism & 0.1413 & 0.2020 & & 0.1530 & \\
\hline \#\# & & Limits.ave & CompRes & olve.ave & Outsider.ave & Openness \\
\hline \#\# & wisdom.pca & 0.0000 & 0.0000 & & 0.0000 & 0.0045 \\
\hline \#\# & Perspective.ave & 0.0000 & 0.0000 & & 0.0000 & 0.2113 \\
\hline \#\# & Changeoutcomes.ave & 0.0000 & 0.0000 & & 0.0000 & 0.0027 \\
\hline \#\# & Limits.ave & & 0.0000 & & 0.0000 & 0.0305 \\
\hline \#\# & CompResolve.ave & 0.0000 & & & 0.0000 & 0.0189 \\
\hline \#\# & Outsider.ave & 0.0000 & 0.0000 & & & 0.0553 \\
\hline \#\# & Openness & 0.0305 & 0.0189 & & 0.0553 & \\
\hline \#\# & Conscientiousness & 0.4865 & 0.0811 & & 0.0637 & 0.0000 \\
\hline \#\# & Extraversion & 0.0044 & 0.0406 & & 0.0010 & 0.0033 \\
\hline \#\# & Agreeableness & 0.9415 & 0.0057 & & 0.9481 & 0.0045 \\
\hline \#\# & Neuroticism & 0.0786 & 0.1157 & & 0.4628 & 0.0338 \\
\hline \#\# & & Conscientio & busness & Extravers & sion Agreeable & eness \\
\hline \#\# & wisdom.pca & 0.7958 & & 0.0004 & 0.0749 & \\
\hline \#\# & Perspective.ave & 0.3287 & & 0.0368 & 0.5318 & \\
\hline \#\# & Changeoutcomes.ave & 0.2492 & & 0.0018 & 0.0019 & \\
\hline \#\# & Limits.ave & 0.4865 & & 0.0044 & 0.9415 & \\
\hline \#\# & CompResolve.ave & 0.0811 & & 0.0406 & 0.0057 & \\
\hline \#\# & Outsider.ave & 0.0637 & & 0.0010 & 0.9481 & \\
\hline \#\# & Openness & 0.0000 & & 0.0033 & 0.0045 & \\
\hline
\end{tabular}




\begin{tabular}{|c|c|c|c|c|}
\hline \#\# Conscientiousness & & 0.2722 & 0.0000 & \\
\hline \#\# Extraversion & 0.2722 & & 0.7575 & \\
\hline \#\# Agreeableness & 0.0000 & 0.7575 & & \\
\hline \#\# Neuroticism & 0.0000 & 0.0000 & 0.0000 & \\
\hline 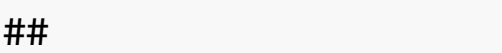 & Neuroticism & & & \\
\hline \#\# wisdom.pca & 0.1413 & & & \\
\hline \#\# Perspective.ave & 0.2020 & & & \\
\hline \#\# ChangeOutcomes.ave & 0.1530 & & & \\
\hline \#\# Limits.ave & 0.0786 & & & \\
\hline \#\# CompResolve.ave & 0.1157 & & & \\
\hline \#\# Outsider.ave & 0.4628 & & & \\
\hline \#\# Openness & 0.0338 & & & \\
\hline \#\# Conscientiousness & 0.0000 & & & \\
\hline \#\# Extraversion & 0.0000 & & & \\
\hline \#\# Agreeableness & 0.0000 & & & \\
\hline \#\# Neuroticism & & & & \\
\hline $\begin{array}{l}\text { Hmisc: : rcorr(as.matrix } \\
\text { eOutcomes.ave', 'Limits } \\
\text { t_1' , 'Intellect_2', 'AT }\end{array}$ & $\begin{array}{l}x(\text { WIS.B }[, \text { c }( \\
\text { s.ave', ' Comp } \\
\left.\left.\left.T^{\prime} T^{\prime}\right)\right]\right) \text { ) }\end{array}$ & $\begin{array}{l}\text { 'wisdom.pca' , 'Pe } \\
\text { Resolve.ave', 'Ou }\end{array}$ & $\begin{array}{l}\text { erspective.ave } \\
\text { utsider.ave', ' }\end{array}$ & $\begin{array}{l}\text { ' , 'Chang } \\
\text { 'Intellec }\end{array}$ \\
\hline \#\# Warning in sqrt(npa & air - 2): $\mathrm{Na}$ & Ns produced & & \\
\hline \#\# & wisdom.pca & Perspective.ave & ChangeOutcome & es.ave \\
\hline \#\# wisdom.pca & 1.00 & 0.82 & & 0.75 \\
\hline \#\# Perspective.ave & 0.82 & 1.00 & & 0.48 \\
\hline \#\# ChangeOutcomes.ave & 0.75 & 0.48 & & 1.00 \\
\hline \#\# Limits.ave & 0.77 & 0.55 & & 0.43 \\
\hline \#\# CompResolve.ave & 0.82 & 0.63 & & 0.55 \\
\hline \#\# Outsider.ave & 0.69 & 0.45 & & 0.43 \\
\hline \#\# Intellect_1 & 0.23 & 0.17 & & 0.23 \\
\hline \#\# Intellect_2 & 0.24 & 0.22 & & 0.22 \\
\hline \#\# АТT & 0.22 & 0.10 & & 0.16 \\
\hline \#\# & Limits.ave & CompResolve.ave & Outsider.ave & Intellec \\
\hline t_1 & & & & \\
\hline \#\# wisdom.pca & 0.77 & 0.82 & 0.69 & 0 \\
\hline .23 & & & & \\
\hline \#\# Perspective.ave & 0.55 & 0.63 & 0.45 & 0 \\
\hline .17 & & & & \\
\hline $\begin{array}{l}\# \# \text { ChangeOutcomes.ave } \\
.23\end{array}$ & 0.43 & 0.55 & 0.43 & 0 \\
\hline \#\# Limits.ave & 1.00 & 0.54 & 0.45 & 0 \\
\hline .13 & & & & \\
\hline $\begin{array}{l}\text { \#\# CompResolve.ave } \\
.20\end{array}$ & 0.54 & 1.00 & 0.39 & \\
\hline
\end{tabular}




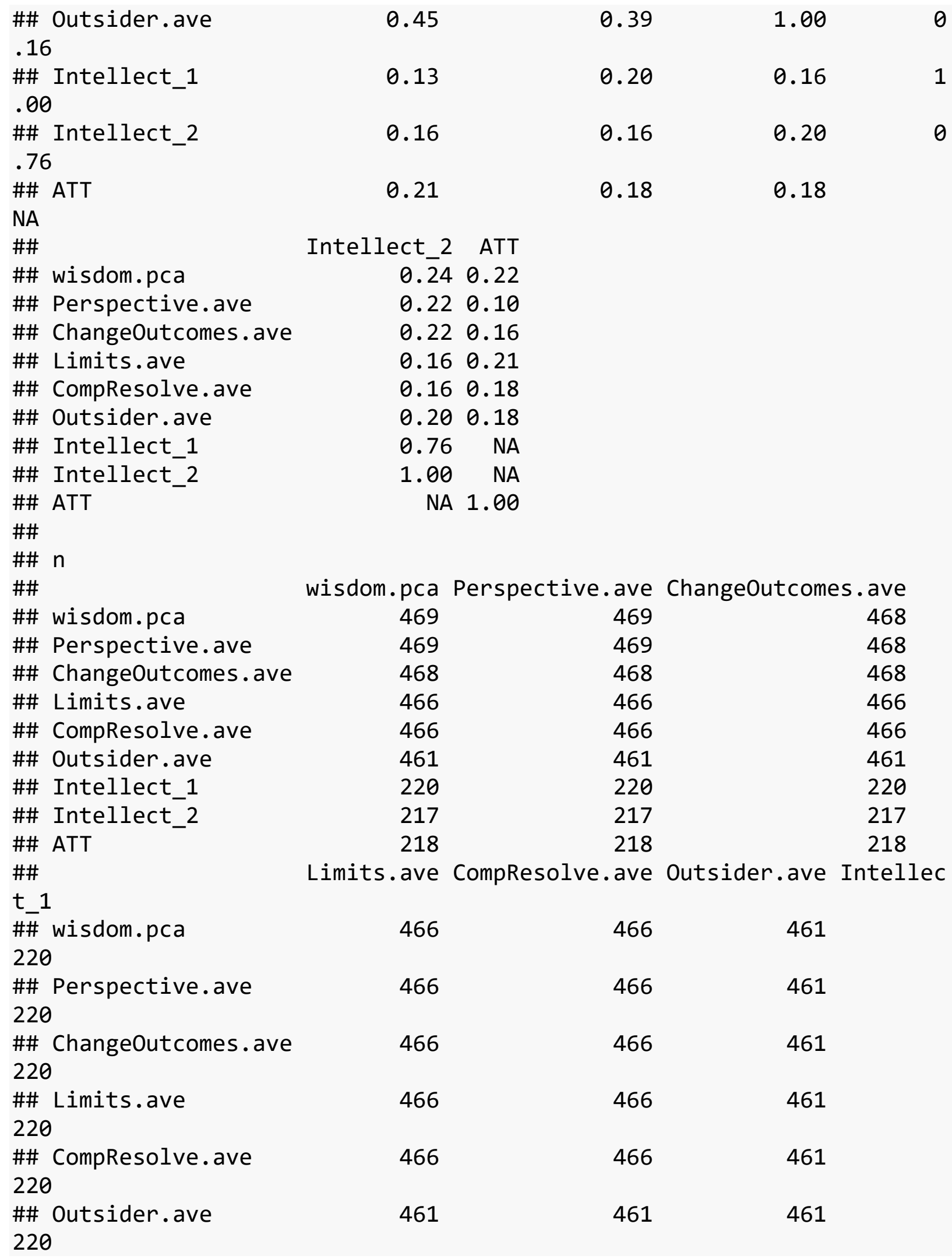




\begin{tabular}{|c|c|c|c|c|}
\hline \#\# Intellect_1 & 220 & 220 & 220 & \\
\hline 220 & & & & \\
\hline \#\# Intellect_2 & 217 & 217 & 217 & \\
\hline 217 & & & & \\
\hline \#\# АТТ & 218 & 218 & 218 & \\
\hline$\theta$ & & & & \\
\hline \#\# & Intellect_2 & ATT & & \\
\hline \#\# wisdom.pca & $2 \overline{1} \overline{7}$ & 218 & & \\
\hline \#\# Perspective.ave & 217 & 218 & & \\
\hline \#\# ChangeOutcomes.ave & 217 & 218 & & \\
\hline \#\# Limits.ave & 217 & 218 & & \\
\hline \#\# CompResolve.ave & 217 & 218 & & \\
\hline \#\# Outsider.ave & 217 & 218 & & \\
\hline \#\# Intellect_1 & 217 & 0 & & \\
\hline \#\# Intellect_2 & 217 & 0 & & \\
\hline \#\# АТT & & 218 & & \\
\hline \#\# & & & & \\
\hline \#\# P & & & & \\
\hline \#\# & wisdom.pca & Perspective.ave & ChangeOutcome & es.ave \\
\hline \#\# wisdom.pca & & 0.0000 & 0.0000 & \\
\hline \#\# Perspective.ave & 0.0000 & & 0.0000 & \\
\hline \#\# ChangeOutcomes.ave & 0.0000 & 0.0000 & & \\
\hline \#\# Limits.ave & 0.0000 & 0.0000 & 0.0000 & \\
\hline \#\# CompResolve.ave & 0.0000 & 0.0000 & 0.0000 & \\
\hline \#\# Outsider.ave & 0.0000 & 0.0000 & 0.0000 & \\
\hline \#\# Intellect_1 & 0.0007 & 0.0098 & 0.0005 & \\
\hline \#\# Intellect_2 & 0.0003 & 0.0013 & 0.0009 & \\
\hline \#\# АТT & 0.0010 & 0.1524 & 0.0161 & \\
\hline \#\# & Limits.ave & CompResolve.ave & Outsider.ave & Intellec \\
\hline t_1 & & & & \\
\hline \#\# wisdom.pca & 0.0000 & 0.0000 & 0.0000 & 0.0007 \\
\hline \#\# Perspective.ave & 0.0000 & 0.0000 & 0.0000 & 0.0098 \\
\hline \#\# ChangeOutcomes.ave & 0.0000 & 0.0000 & 0.0000 & 0.0005 \\
\hline \#\# Limits.ave & & 0.0000 & 0.0000 & 0.0500 \\
\hline \#\# CompResolve.ave & 0.0000 & & 0.0000 & 0.0029 \\
\hline \#\# Outsider.ave & 0.0000 & 0.0000 & & 0.0159 \\
\hline \#\# Intellect_1 & 0.0500 & 0.0029 & 0.0159 & \\
\hline \#\# Intellect_2 & 0.0150 & 0.0157 & 0.0035 & 0.0000 \\
\hline \#\# АТТ — & 0.0015 & 0.0074 & 0.0075 & \\
\hline 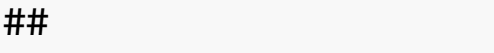 & Intellect_2 & ATT & & \\
\hline \#\# wisdom.pca & 0.0003 & 0.0010 & & \\
\hline \#\# Perspective.ave & 0.0013 & 0.1524 & & \\
\hline \#\# ChangeOutcomes.ave & 0.0009 & 0.0161 & & \\
\hline \#\# Limits.ave & 0.0150 & 0.0015 & & \\
\hline
\end{tabular}




\begin{tabular}{|c|c|c|c|c|}
\hline \#\# CompResolve.ave & 0.0157 & \multicolumn{3}{|l|}{0.0074} \\
\hline \#\# Outsider.ave & \multicolumn{4}{|l|}{0.0035} \\
\hline \#\# Intellect_1 & \multirow{2}{*}{\multicolumn{4}{|c|}{0.0000}} \\
\hline \multirow{2}{*}{\multicolumn{5}{|c|}{$\begin{array}{l}\text { \#\# Intellect_2 } \\
\text { \#\# ATT }\end{array}$}} \\
\hline & & & & \\
\hline \multicolumn{5}{|c|}{$\begin{array}{l}\text { Hmisc: :rcorr(as.matrix(WIS.A[, c('wisdom.pca' , 'Perspective.ave ', 'Chang } \\
\text { eOutcomes.ave', 'Limits.ave', 'CompResolve.ave', 'Outsider.ave' , 'PerspTak } \\
\text { ing')])) }\end{array}$} \\
\hline \#\# & wisdom.pca & Perspective.ave & ChangeOutcomes & s.ave \\
\hline \#\# wisdom.pca & 1.00 & 0.80 & & 0.76 \\
\hline \#\# Perspective.ave & 0.80 & 1.00 & & 0.48 \\
\hline \#\# ChangeOutcomes.ave & 0.76 & 0.48 & & 1.00 \\
\hline \#\# Limits.ave & 0.83 & 0.61 & & 0.51 \\
\hline \#\# CompResolve.ave & 0.86 & 0.63 & & 0.63 \\
\hline \#\# Outsider.ave & 0.72 & 0.45 & & 0.42 \\
\hline \#\# PerspTaking & 0.48 & 0.39 & & 0.38 \\
\hline $\begin{array}{l}\# \# \\
\text { ing }\end{array}$ & Limits.ave & CompResolve.ave & Outsider.ave $\mathrm{P}$ & PerspTak \\
\hline \#\# wisdom.pca & 0.83 & 0.86 & 0.72 & 0 \\
\hline \multicolumn{5}{|l|}{.48} \\
\hline \multicolumn{5}{|l|}{$\begin{array}{l}\text { \#\# Perspective.ave } \\
.39\end{array}$} \\
\hline \multicolumn{5}{|l|}{ \#\# ChangeOutcomes.ave } \\
\hline \multicolumn{5}{|l|}{.31} \\
\hline \multicolumn{5}{|l|}{$\begin{array}{l}\text { \#\# CompResolve.ave } \\
.48\end{array}$} \\
\hline \multicolumn{5}{|l|}{.37} \\
\hline & \multicolumn{3}{|c|}{.00} & \\
\hline \multicolumn{5}{|l|}{ 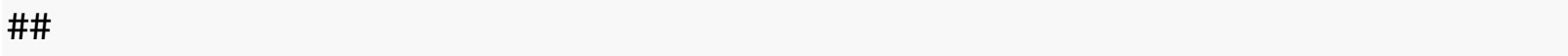 } \\
\hline \multicolumn{5}{|l|}{$\# \# n=404$} \\
\hline \multicolumn{5}{|l|}{ \#\# } \\
\hline \#\# P & & & & \\
\hline 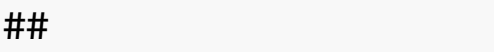 & wisdom.pca & Perspective.ave & ChangeOutcomes & s.ave \\
\hline \#\# wisdom.pca & & 0 & 0 & \\
\hline \#\# Perspective.ave & 0 & & 0 & \\
\hline \#\# ChangeOutcomes.ave & 0 & 0 & & \\
\hline \#\# Limits.ave & 0 & 0 & 0 & \\
\hline \#\# CompResolve.ave & 0 & $\theta$ & 0 & \\
\hline
\end{tabular}




\begin{tabular}{|c|c|c|c|c|}
\hline \#\# Outsider.ave & $\theta$ & 0 & $\theta$ & \\
\hline \#\# PerspTaking & 0 & 0 & 0 & \\
\hline \#\# - 1 - - & Limits.ave & CompResolve.ave & Outsider.ave & PerspTak \\
\hline \multicolumn{5}{|c|}{ - } \\
\hline \#\# wisdom.pca & $\theta$ & 0 & 0 & 0 \\
\hline \#\# Perspective.ave & 0 & 0 & 0 & 0 \\
\hline \#\# ChangeOutcomes.ave & $\theta$ & 0 & 0 & 0 \\
\hline \#\# Limits.ave & & 0 & 0 & 0 \\
\hline \#\# CompResolve.ave & 0 & & 0 & 0 \\
\hline \#\# Outsider.ave & 0 & 0 & & 0 \\
\hline \#\# PerspTaking & 0 & 0 & 0 & \\
\hline \multicolumn{5}{|c|}{$\begin{array}{l}\text { Hmisc: :rcorr(as.matrix(WIS.D[, c('wisdom.pca', 'Perspective.ave ', 'Chang } \\
\text { eOutcomes.ave', 'Limits.ave', 'CompResolve.ave', 'Outsider.ave ', 'EISelfEm } \\
\text { otions', 'EIOthersEmotions', 'EIEmotionUse', 'EIEmotionReg ')])) }\end{array}$} \\
\hline 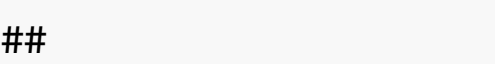 & wisdom.pca & Perspective.ave & ChangeOutcome & es.ave \\
\hline \#\# wisdom.pca & 1.00 & 0.80 & & 0.71 \\
\hline \#\# Perspective.ave & 0.80 & 1.00 & & 0.43 \\
\hline \#\# ChangeOutcomes.ave & 0.71 & 0.43 & & 1.00 \\
\hline \#\# Limits.ave & 0.78 & 0.53 & & 0.41 \\
\hline \#\# CompResolve.ave & 0.81 & 0.58 & & 0.53 \\
\hline \#\# Outsider.ave & 0.59 & 0.35 & & 0.25 \\
\hline \#\# EISelfEmotions & 0.10 & 0.07 & & 0.12 \\
\hline \#\# EIOthersEmotions & 0.21 & 0.19 & & 0.13 \\
\hline \#\# EIEmotionUse & 0.11 & 0.04 & & 0.11 \\
\hline \#\# EIEmotionReg & 0.12 & 0.11 & & 0.09 \\
\hline $\begin{array}{l}\# \# \\
\text { otions }\end{array}$ & Limits.ave & CompResolve.ave & Outsider.ave & EISelfEm \\
\hline $\begin{array}{l}\# \# \text { wisdom.pca } \\
0.10\end{array}$ & 0.78 & 0.81 & 0.59 & \\
\hline $\begin{array}{l}\# \# \text { Perspective.ave } \\
0.07\end{array}$ & 0.53 & 0.58 & 0.35 & \\
\hline $\begin{array}{l}\text { \#\# ChangeOutcomes.ave } \\
0.12\end{array}$ & 0.41 & 0.53 & 0.25 & \\
\hline $\begin{array}{l}\# \# \text { Limits.ave } \\
0.05\end{array}$ & 1.00 & 0.53 & 0.40 & \\
\hline $\begin{array}{l}\text { \#\# CompResolve.ave } \\
0.11\end{array}$ & 0.53 & 1.00 & 0.29 & \\
\hline $\begin{array}{l}\# \# \text { Outsider.ave } \\
0.00\end{array}$ & 0.40 & 0.29 & 1.00 & \\
\hline $\begin{array}{l}\text { \#\# EISelfEmotions } \\
1.00\end{array}$ & 0.05 & 0.11 & 0.00 & \\
\hline $\begin{array}{l}\text { \#\# EIOthersEmotions } \\
0.49\end{array}$ & 0.09 & 0.23 & 0.13 & \\
\hline
\end{tabular}




\begin{tabular}{|c|c|c|c|c|c|}
\hline $\begin{array}{l}\text { \#\# EIEmotionUse } \\
0.52\end{array}$ & 0.05 & & 0.13 & 0.07 & \\
\hline \#\# EIEmotionReg & 0.08 & & 0.11 & 0.07 & \\
\hline 0.51 & & & & & \\
\hline \#\# & EIOthersEmo & tions & EIEmotionU & Jse EIEmotion & $\operatorname{Reg}$ \\
\hline \#\# wisdom.pca & & 0.21 & 0 & $11 \quad 0$ & .12 \\
\hline \#\# Perspective.ave & & 0.19 & & 04 & .11 \\
\hline \#\# ChangeOutcomes.ave & & 0.13 & & 11 & .09 \\
\hline \#\# Limits.ave & & 0.09 & & .05 & .08 \\
\hline \#\# CompResolve.ave & & 0.23 & 0. & 13 & .11 \\
\hline \#\# Outsider.ave & & 0.13 & 0. & 07 & .07 \\
\hline \#\# EISelfEmotions & & 0.49 & & 52 & .51 \\
\hline \#\# EIOthersEmotions & & 1.00 & 0 . & 32 & .30 \\
\hline \#\# EIEmotionUse & & 0.32 & & 00 & .47 \\
\hline \#\# EIEmotionReg & & 0.30 & & 47 & 1.00 \\
\hline \#\# & & & & & \\
\hline$\# \# \mathrm{n}$ & & & & & \\
\hline \#\# & wisdom.pca & Perspe & ective.ave & ChangeOutcom & nes.ave \\
\hline \#\# wisdom.pca & 730 & & 729 & & 729 \\
\hline \#\# Perspective.ave & 729 & & 729 & & 729 \\
\hline \#\# ChangeOutcomes.ave & 729 & & 729 & & 729 \\
\hline \#\# Limits.ave & 730 & & 729 & & 729 \\
\hline \#\# CompResolve.ave & 722 & & 722 & & 722 \\
\hline \#\# Outsider.ave & 722 & & 722 & & 722 \\
\hline \#\# EISelfEmotions & 702 & & 701 & & 701 \\
\hline \#\# EIOthersEmotions & 701 & & 701 & & 701 \\
\hline \#\# EIEmotionUse & 701 & & 701 & & 701 \\
\hline \#\# EIEmotionReg & 701 & & 701 & & 701 \\
\hline $\begin{array}{l}\# \# \\
\text { otions }\end{array}$ & Limits.ave & CompRe & esolve.ave & Outsider.ave & EISelfEm \\
\hline \#\# wisdom.pca & 730 & & 722 & 722 & \\
\hline 702 & & & & & \\
\hline $\begin{array}{l}\text { \#\# Perspective.ave } \\
701\end{array}$ & 729 & & 722 & 722 & \\
\hline $\begin{array}{l}\text { \#\# ChangeOutcomes.ave } \\
701\end{array}$ & 729 & & 722 & 722 & \\
\hline $\begin{array}{l}\text { \#\# Limits.ave } \\
702\end{array}$ & 730 & & 722 & 722 & \\
\hline $\begin{array}{l}\# \# \text { CompResolve.ave } \\
701\end{array}$ & 722 & & 722 & 722 & \\
\hline $\begin{array}{l}\# \# \text { Outsider.ave } \\
701\end{array}$ & 722 & & 722 & 722 & \\
\hline $\begin{array}{l}\text { \#\# EISelfEmotions } \\
702\end{array}$ & 702 & & 701 & 701 & \\
\hline
\end{tabular}




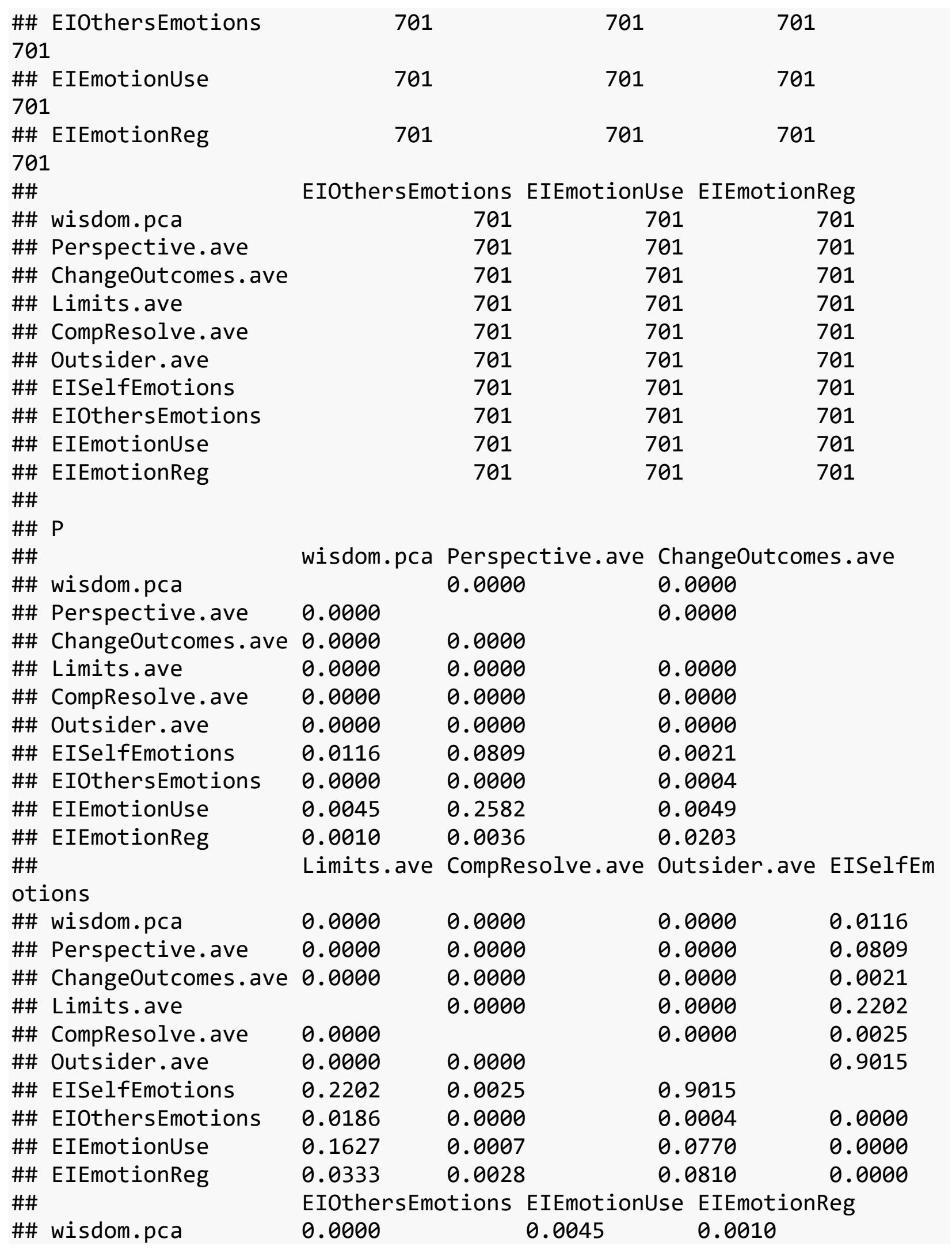




\begin{tabular}{|c|c|c|c|c|c|}
\hline \#\# & Perspective.ave & 0.0000 & 0.2582 & \multicolumn{2}{|l|}{0.0036} \\
\hline \#\# & ChangeOutcomes.ave & 0.0004 & 0.0049 & \multicolumn{2}{|l|}{0.0203} \\
\hline \#\# & Limits.ave & 0.0186 & 0.1627 & \multicolumn{2}{|l|}{0.0333} \\
\hline \#\# & CompResolve.ave & 0.0000 & 0.0007 & \multicolumn{2}{|l|}{0.0028} \\
\hline \#\# & Outsider.ave & 0.0004 & 0.0770 & \multicolumn{2}{|l|}{0.0810} \\
\hline \#\# & EISelfEmotions & 0.0000 & 0.0000 & \multicolumn{2}{|l|}{0.0000} \\
\hline \#\# & EIOthersEmotions & & 0.0000 & \multicolumn{2}{|l|}{0.0000} \\
\hline \#\# & EIEmotionUse & 0.0000 & \multirow{2}{*}{\multicolumn{3}{|c|}{0.0000}} \\
\hline \#\# & EIEmotionReg & 0.0000 & & & \\
\hline \multicolumn{6}{|c|}{$\begin{array}{l}\text { Hmisc: :rcorr(as.matrix(WIS.B[, c('wisdom.pca', 'Perspective.ave ', 'Chang } \\
\text { eOutcomes.ave', 'Limits.ave', 'CompResolve.ave', 'Outsider.ave ', 'Mindful_- } \\
\text { 1', 'Mindful_2', 'Mindful_3', 'Mindful_4', 'Mindful_5')])) }\end{array}$} \\
\hline \#\# & & wisdom.pca & Perspective.ave & \multicolumn{2}{|c|}{ ChangeOutcomes.ave } \\
\hline \#\# & wisdom.pca & 1.00 & 0.82 & \multicolumn{2}{|c|}{0.75} \\
\hline \#\# & Perspective.ave & 0.82 & 1.00 & \multicolumn{2}{|r|}{0.48} \\
\hline \#\# & ChangeOutcomes. ave & 0.75 & 0.48 & \multicolumn{2}{|r|}{1.00} \\
\hline \#\# & Limits.ave & 0.77 & 0.55 & \multicolumn{2}{|r|}{0.43} \\
\hline \#\# & CompResolve.ave & 0.82 & 0.63 & \multicolumn{2}{|r|}{0.55} \\
\hline \#\# & Outsider.ave & 0.69 & 0.45 & \multicolumn{2}{|r|}{0.43} \\
\hline \#\# & Mindful_1 & 0.19 & 0.17 & \multicolumn{2}{|r|}{0.16} \\
\hline \#\# & Mindful_2 & 0.42 & 0.38 & \multicolumn{2}{|r|}{0.36} \\
\hline \#\# & Mindful_3 & -0.12 & -0.13 & \multicolumn{2}{|r|}{-0.03} \\
\hline \#\# & Mindful_4 & 0.17 & 0.14 & \multicolumn{2}{|r|}{0.21} \\
\hline \#\# & Mindful_5 & -0.11 & -0.10 & \multicolumn{2}{|r|}{-0.11} \\
\hline \#\# & & Limits.ave & CompResolve.ave & \multicolumn{2}{|c|}{ Outsider.ave Mindful_ } \\
\hline \multicolumn{6}{|c|}{ ' } \\
\hline $\begin{array}{l}\# \# \\
9\end{array}$ & wisdom.pca & 0.77 & 0.82 & \multicolumn{2}{|l|}{0.69} \\
\hline $\begin{array}{l}\text { \#\# } \\
7\end{array}$ & Perspective.ave & 0.55 & 0.63 & 0.45 & 0.1 \\
\hline $\begin{array}{l}\text { \#\# } \\
6\end{array}$ & ChangeOutcomes.ave & 0.43 & 0.55 & 0.43 & 0.1 \\
\hline $\begin{array}{l}\# \# \\
6\end{array}$ & Limits.ave & 1.00 & 0.54 & 0.45 & 0.1 \\
\hline $\begin{array}{l}\text { \#\# } \\
8\end{array}$ & CompResolve.ave & 0.54 & 1.00 & 0.39 & 0.1 \\
\hline $\begin{array}{l}\# \# \\
7\end{array}$ & Outsider.ave & 0.45 & 0.39 & 1.00 & 0.0 \\
\hline $\begin{array}{l}\text { \#\# } \\
0\end{array}$ & Mindful_1 & 0.16 & 0.18 & 0.07 & 1.0 \\
\hline $\begin{array}{l}\# \# \\
0\end{array}$ & Mindful_2 & 0.32 & 0.34 & 0.27 & 0.2 \\
\hline & Mindful_3 & -0.09 & -0.04 & -0.20 & 0.1 \\
\hline
\end{tabular}




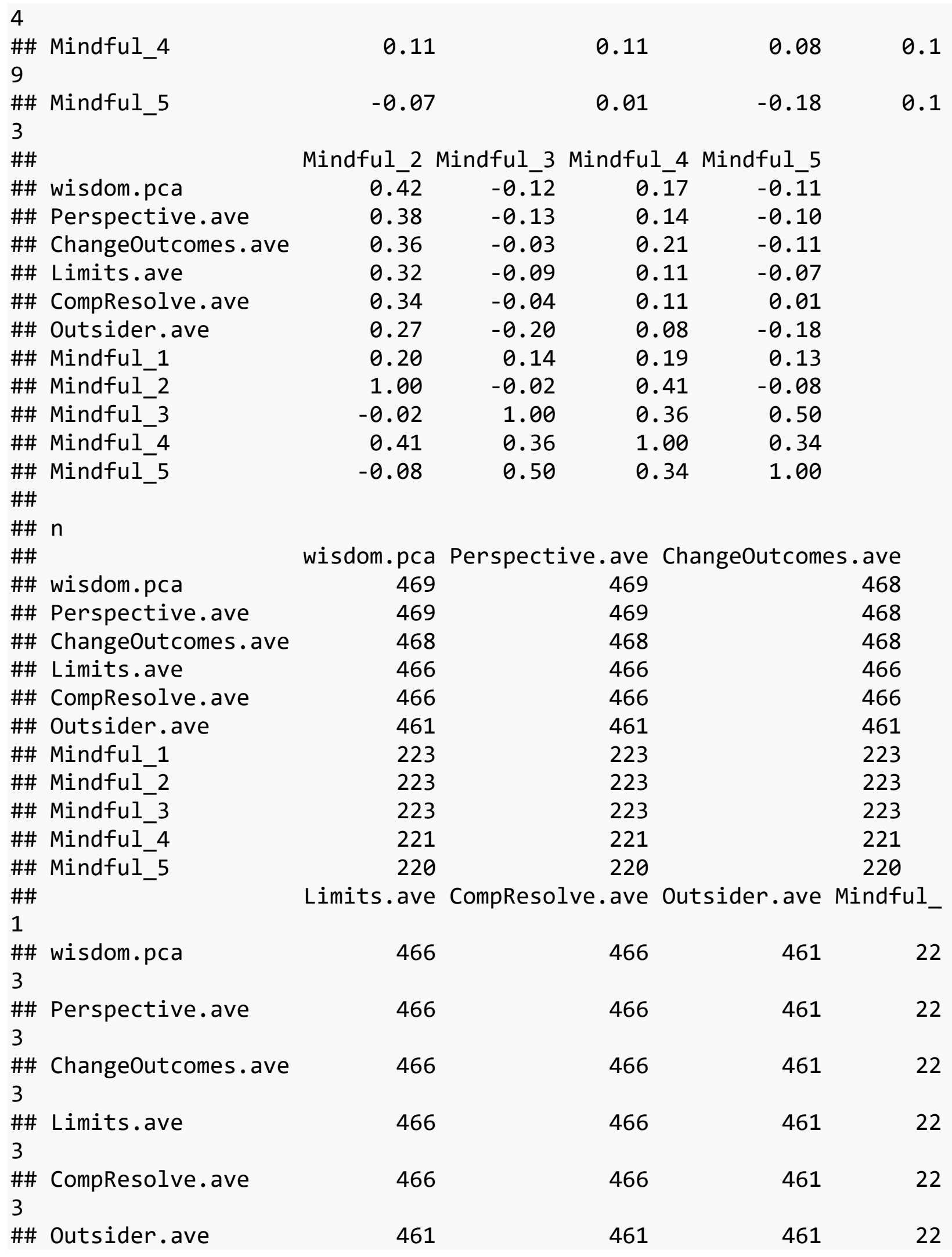




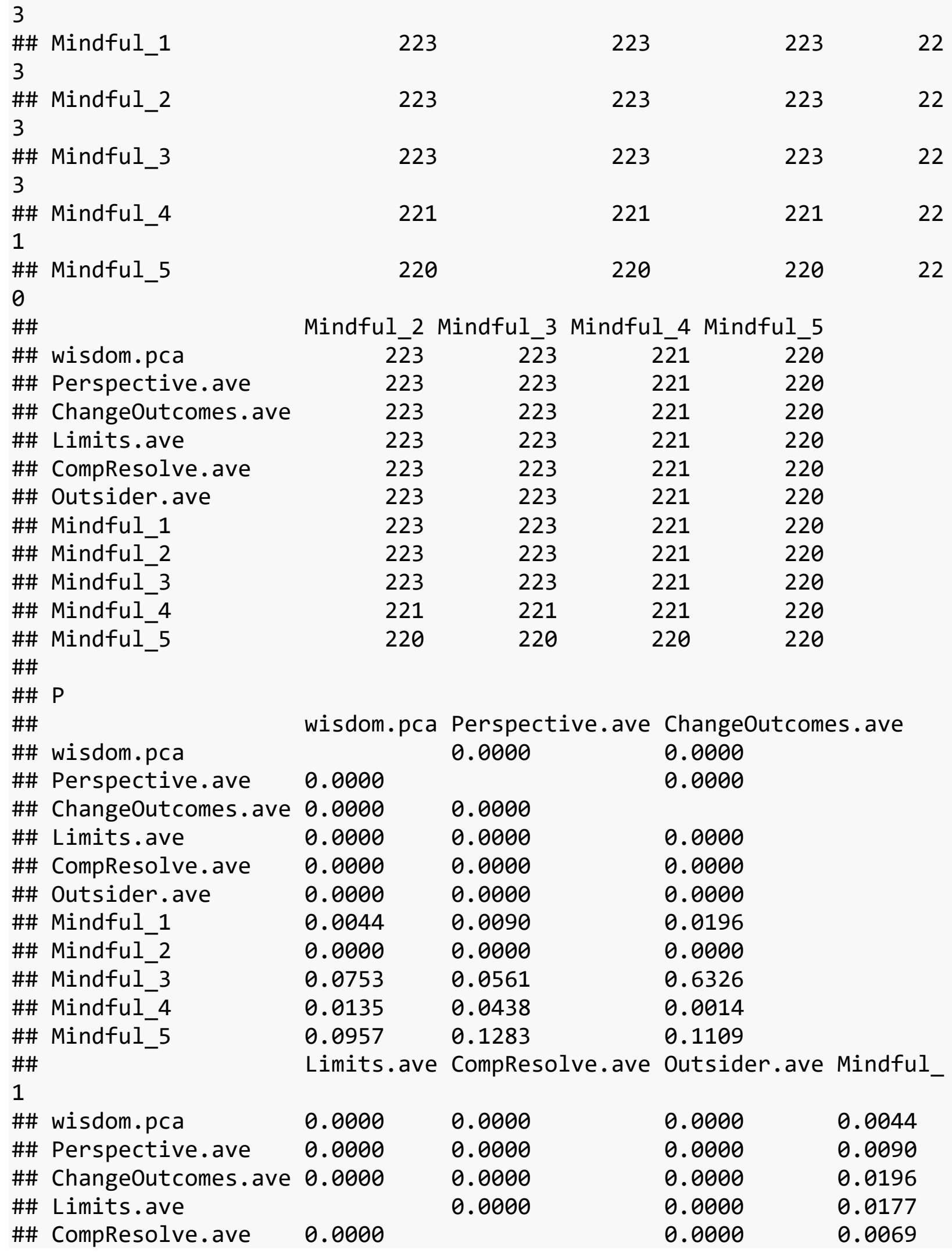




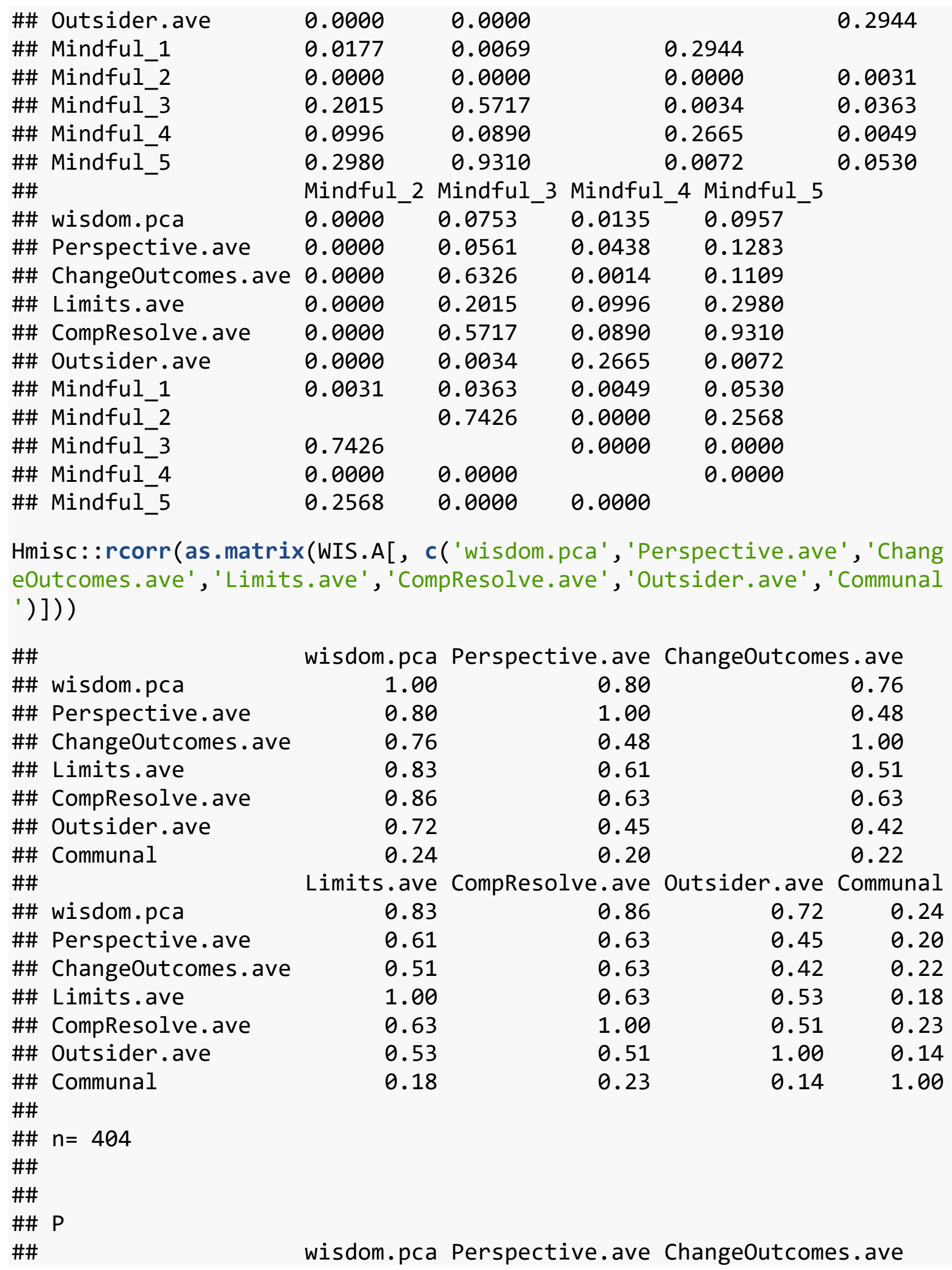




\begin{tabular}{|c|c|c|c|c|c|}
\hline \#\# & wisdom.pca & & 0.0000 & 0.0000 & \\
\hline \#\# & Perspective.ave & 0.0000 & & 0.0000 & \\
\hline \#\# & ChangeOutcomes. ave & 0.0000 & 0.0000 & & \\
\hline \#\# & Limits.ave & 0.0000 & 0.0000 & 0.0000 & \\
\hline \#\# & CompResolve.ave & 0.0000 & 0.0000 & 0.0000 & \\
\hline \#\# & Outsider.ave & 0.0000 & 0.0000 & 0.0000 & \\
\hline \#\# & Communal & 0.0000 & 0.0000 & 0.0000 & \\
\hline \#\# & & Limits.ave & CompResolve.ave & Outsider.ave & Communal \\
\hline \#\# & wisdom.pca & 0.0000 & 0.0000 & 0.0000 & 0.0000 \\
\hline \#\# & Perspective.ave & 0.0000 & 0.0000 & 0.0000 & 0.0000 \\
\hline \#\# & ChangeOutcomes. ave & 0.0000 & 0.0000 & 0.0000 & 0.0000 \\
\hline \#\# & Limits.ave & & 0.0000 & 0.0000 & 0.0003 \\
\hline \#\# & CompResolve.ave & 0.0000 & & 0.0000 & 0.0000 \\
\hline \#\# & Outsider.ave & 0.0000 & 0.0000 & & 0.0049 \\
\hline \#\# & Communal & 0.0003 & 0.0000 & 0.0049 & \\
\hline & $\begin{array}{l}\text { isc: :rcorr (as.matri } \\
\text { utcomes.ave', 'Limit } \\
\text { 'EmoReg_2')])) }\end{array}$ & $\begin{array}{l}\text { x(WIS.B }[, \text { c } \\
\text { s.ave', 'Comf }\end{array}$ & $\begin{array}{l}\text { ('wisdom.pca', 'P } \\
\text { oresolve.ave ', 'o }\end{array}$ & $\begin{array}{l}\text { erspective.ave } \\
\text { utsider.ave', ' }\end{array}$ & $\begin{array}{l}\text { e ', 'Chang } \\
\text { 'EmoReg_1 }\end{array}$ \\
\hline \#\# & & wisdom.pca & Perspective.ave & ChangeOutcome & es.ave \\
\hline \#\# & wisdom.pca & 1.00 & 0.82 & & 0.75 \\
\hline \#\# & Perspective.ave & 0.82 & 1.00 & & 0.48 \\
\hline \#\# & ChangeOutcomes. ave & 0.75 & 0.48 & & 1.00 \\
\hline \#\# & Limits.ave & 0.77 & 0.55 & & 0.43 \\
\hline \#\# & CompResolve.ave & 0.82 & 0.63 & & 0.55 \\
\hline \#\# & Outsider.ave & 0.69 & 0.45 & & 0.43 \\
\hline \#\# & EmoReg_1 & 0.23 & 0.20 & & 0.24 \\
\hline \#\# & EmoReg_2 & 0.05 & 0.11 & & 0.02 \\
\hline \#\# & & Limits.ave & CompResolve.ave & Outsider.ave & EmoReg_1 \\
\hline \#\# & wisdom.pca & 0.77 & 0.82 & 0.69 & 0.23 \\
\hline \#\# & Perspective.ave & 0.55 & 0.63 & 0.45 & 0.20 \\
\hline \#\# & ChangeOutcomes.ave & 0.43 & 0.55 & 0.43 & 0.24 \\
\hline \#\# & Limits.ave & 1.00 & 0.54 & 0.45 & 0.17 \\
\hline \#\# & CompResolve.ave & 0.54 & 1.00 & 0.39 & 0.21 \\
\hline \#\# & Outsider.ave & 0.45 & 0.39 & 1.00 & 0.10 \\
\hline \#\# & EmoReg_1 & 0.17 & 0.21 & 0.10 & 1.00 \\
\hline \#\# & EmoReg_2 & 0.11 & 0.02 & -0.06 & 0.14 \\
\hline \#\# & & EmoReg_2 & & & \\
\hline \#\# & wisdom.pca & 0.05 & & & \\
\hline \#\# & Perspective.ave & 0.11 & & & \\
\hline \#\# & ChangeOutcomes.ave & 0.02 & & & \\
\hline \#\# & Limits.ave & 0.11 & & & \\
\hline \#\# & CompResolve.ave & 0.02 & & & \\
\hline \#\# & Outsider.ave & -0.06 & & & \\
\hline
\end{tabular}




\begin{tabular}{|c|c|c|c|c|c|}
\hline \#\# & EmoReg_1 & 0.14 & & & \\
\hline \#\# & EmoReg_2 & 1.00 & & & \\
\hline \multicolumn{6}{|c|}{ \#\# } \\
\hline \multicolumn{6}{|c|}{ \#\# n } \\
\hline \#\# & & wisdom.pca & Perspective.ave & \multicolumn{2}{|c|}{ ChangeOutcomes.ave } \\
\hline \#\# & wisdom.pca & 469 & 469 & \multicolumn{2}{|c|}{468} \\
\hline \#\# & Perspective.ave & 469 & 469 & \multicolumn{2}{|r|}{468} \\
\hline \#\# & ChangeOutcomes ave & 468 & 468 & \multicolumn{2}{|r|}{468} \\
\hline \#\# & Limits.ave & 466 & 466 & \multicolumn{2}{|r|}{466} \\
\hline \#\# & CompResolve.ave & 466 & 466 & \multicolumn{2}{|r|}{466} \\
\hline \#\# & Outsider.ave & 461 & 461 & \multicolumn{2}{|r|}{461} \\
\hline \#\# & EmoReg_1 & 216 & 216 & \multicolumn{2}{|r|}{216} \\
\hline \#\# & EmoReg_2 & 216 & 216 & \multicolumn{2}{|r|}{216} \\
\hline \#\# & & Limits.ave & CompResolve.ave & \multicolumn{2}{|c|}{ Outsider.ave EmoReg_1 } \\
\hline \#\# & wisdom.pca & 466 & 466 & 461 & 216 \\
\hline \#\# & Perspective.ave & 466 & 466 & 461 & 216 \\
\hline \#\# & ChangeOutcomes.ave & 466 & 466 & 461 & 216 \\
\hline \#\# & Limits.ave & 466 & 466 & 461 & 216 \\
\hline \#\# & CompResolve.ave & 466 & 466 & 461 & 216 \\
\hline \#\# & Outsider.ave & 461 & 461 & 461 & 216 \\
\hline \#\# & EmoReg_1 & 216 & 216 & 216 & 216 \\
\hline \#\# & EmoReg_2 & 216 & 216 & 216 & 216 \\
\hline \multicolumn{6}{|c|}{ EmoReg_2 } \\
\hline \multicolumn{6}{|c|}{ \#\# wisdom.pca } \\
\hline \multicolumn{6}{|c|}{ \#\# Perspective.ave } \\
\hline \multicolumn{6}{|c|}{ \#\# ChangeOutcomes.ave } \\
\hline \multicolumn{6}{|c|}{ \#\# Limits.ave } \\
\hline \multicolumn{6}{|c|}{ \#\# CompResolve.ave } \\
\hline \multicolumn{6}{|c|}{ \#\# Outsider.ave } \\
\hline \multicolumn{6}{|c|}{ EmoReg_1 } \\
\hline \multicolumn{6}{|c|}{ \#\# EmoReg_2 } \\
\hline \multirow{2}{*}{\multicolumn{6}{|c|}{ \#\# }} \\
\hline \#\# & $P$ & & & & \\
\hline \#\# & & wisdom.pca & Perspective.ave & \multicolumn{2}{|c|}{ ChangeOutcomes.ave } \\
\hline \#\# & wisdom.pca & & 0.0000 & \multicolumn{2}{|c|}{0.0000} \\
\hline \#\# & Perspective.ave & 0.0000 & & 0.0000 & \\
\hline \#\# & ChangeOutcomes.ave & 0.0000 & 0.0000 & & \\
\hline \#\# & Limits.ave & 0.0000 & 0.0000 & 0.0000 & \\
\hline \#\# & CompResolve.ave & 0.0000 & 0.0000 & 0.0000 & \\
\hline \#\# & Outsider.ave & 0.0000 & 0.0000 & 0.0000 & \\
\hline \#\# & EmoReg_1 & 0.0006 & 0.0028 & 0.0005 & \\
\hline \#\# & EmoReg_2 & 0.4403 & 0.1195 & 0.7686 & \\
\hline \#\# & & Limits.ave & CompResolve.ave & Outsider.ave & EmoReg_1 \\
\hline \#\# & wisdom.pca & 0.0000 & 0.0000 & 0.0000 & 0.0006 \\
\hline
\end{tabular}




\begin{tabular}{|c|c|c|c|c|c|}
\hline \#\# & Perspective.ave & 0.0000 & 0.0000 & 0.0000 & 0.0028 \\
\hline \#\# & ChangeOutcomes.ave & 0.0000 & 0.0000 & 0.0000 & 0.0005 \\
\hline \#\# & Limits.ave & & 0.0000 & 0.0000 & 0.0140 \\
\hline \#\# & CompResolve.ave & 0.0000 & & 0.0000 & 0.0022 \\
\hline \#\# & Outsider.ave & 0.0000 & 0.0000 & & 0.1366 \\
\hline \#\# & EmoReg_1 & 0.0140 & 0.0022 & 0.1366 & \\
\hline \#\# & EmoReg_2 & 0.1225 & 0.7201 & 0.3692 & 0.0332 \\
\hline \#\# & & EmoReg_2 & & & \\
\hline \#\# & wisdom.pca & 0.4403 & & & \\
\hline \#\# & Perspective.ave & 0.1195 & & & \\
\hline \#\# & ChangeOutcomes.ave & 0.7686 & & & \\
\hline \#\# & Limits.ave & 0.1225 & & & \\
\hline \#\# & CompResolve.ave & 0.7201 & & & \\
\hline \#\# & Outsider.ave & 0.3692 & & & \\
\hline \#\# & EmoReg_1 & 0.0332 & & & \\
\hline \#\# & EmoReg_2 & & & & \\
\hline \multicolumn{6}{|c|}{$\begin{array}{l}\text { Hmisc: :rcorr(as.matrix(WIS.D[, c('wisdom.pca', 'Perspective.ave ', 'Chang } \\
\text { eOutcomes.ave', 'Limits.ave', 'CompResolve.ave', 'Outsider.ave ', 'Reflecti } \\
\text { on ', 'Brooding ')])) }\end{array}$} \\
\hline \#\# & & wisdom.pca & Perspective.ave & ChangeOutcome & es.ave \\
\hline \#\# & wisdom.pca & 1.00 & 0.80 & & 0.71 \\
\hline \#\# & Perspective.ave & 0.80 & 1.00 & & 0.43 \\
\hline \#\# & ChangeOutcomes.ave & 0.71 & 0.43 & & 1.00 \\
\hline \#\# & Limits.ave & 0.78 & 0.53 & & 0.41 \\
\hline \#\# & CompResolve.ave & 0.81 & 0.58 & & 0.53 \\
\hline \#\# & Outsider.ave & 0.59 & 0.35 & & 0.25 \\
\hline \#\# & Reflection & 0.26 & 0.21 & & 0.15 \\
\hline \#\# & Brooding & 0.13 & 0.08 & & 0.07 \\
\hline \#\# & & Limits.ave & CompResolve.ave & Outsider.ave & Reflecti \\
\hline \multicolumn{6}{|c|}{ 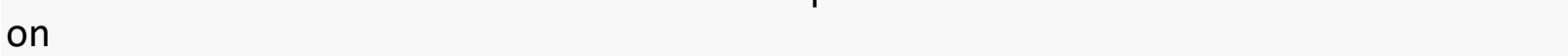 } \\
\hline $\begin{array}{l}\# \# \\
26\end{array}$ & wisdom.pca & 0.78 & 0.81 & 0.59 & 0 . \\
\hline $\begin{array}{l}\# \# \\
21\end{array}$ & Perspective.ave & 0.53 & 0.58 & 0.35 & 0 . \\
\hline $\begin{array}{l}\# \# \\
15\end{array}$ & ChangeOutcomes.ave & 0.41 & 0.53 & 0.25 & 0 . \\
\hline $\begin{array}{l}\# \# \\
21\end{array}$ & Limits.ave & 1.00 & 0.53 & 0.40 & 0 . \\
\hline $\begin{array}{l}\# \# \\
19\end{array}$ & CompResolve.ave & 0.53 & 1.00 & 0.29 & 0 . \\
\hline $\begin{array}{l}\# \# \\
22\end{array}$ & Outsider.ave & 0.40 & 0.29 & 1.00 & 0 . \\
\hline & Reflection & 0.21 & 0.19 & 0.22 & 1. \\
\hline
\end{tabular}




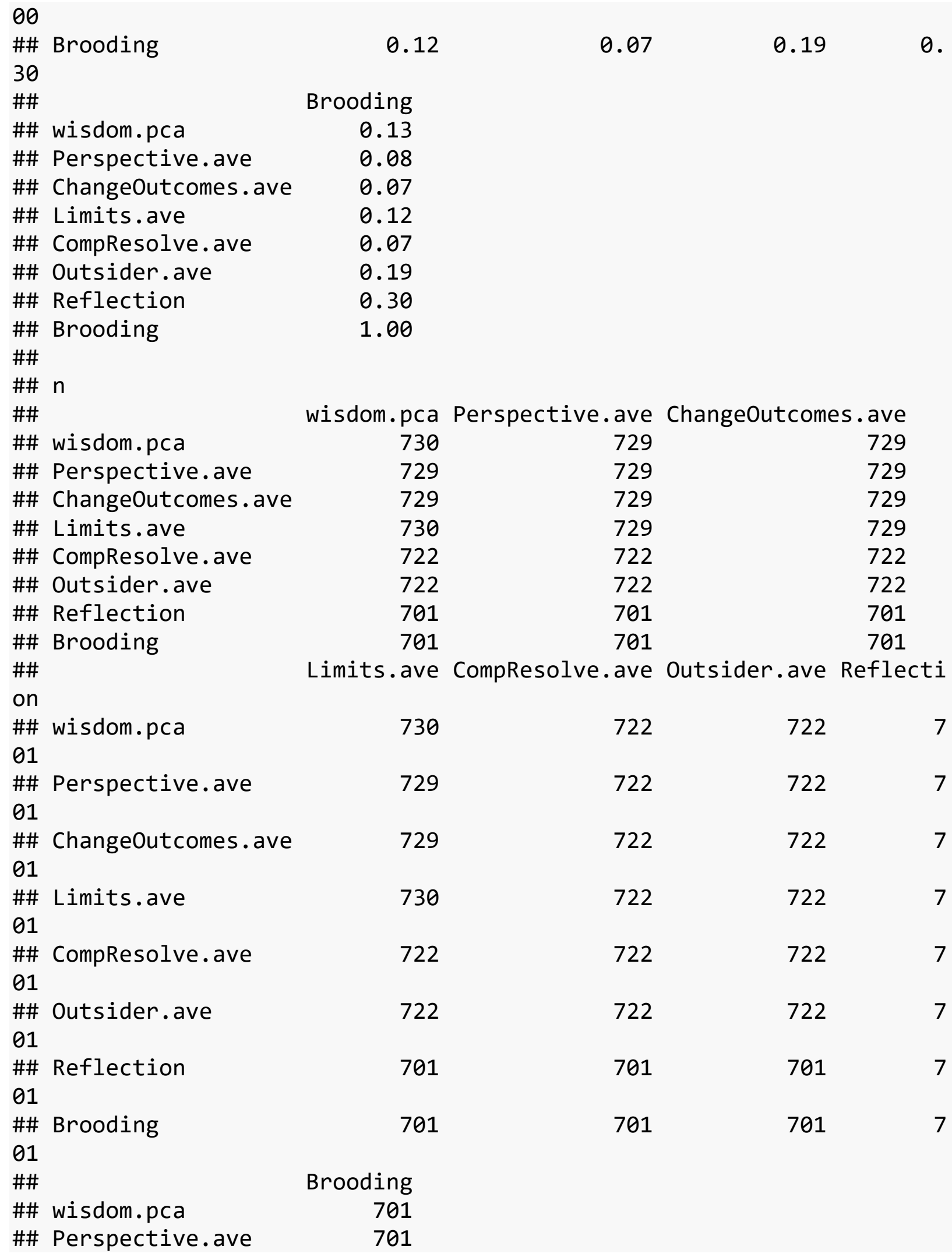




\begin{tabular}{|c|c|c|c|c|c|}
\hline \#\# & ChangeOutcomes.ave & 701 & & & \\
\hline \#\# & Limits.ave & 701 & & & \\
\hline \#\# & CompResolve.ave & 701 & & & \\
\hline \#\# & Outsider.ave & 701 & & & \\
\hline \#\# & Reflection & 701 & & & \\
\hline \#\# & Brooding & 701 & & & \\
\hline \#\# & & & & & \\
\hline \#\# & $\mathrm{P}$ & & & & \\
\hline \#\# & & wisdom.pca & Perspective.ave & ChangeOutcomes & s.ave \\
\hline \#\# & wisdom.pca & & 0.0000 & 0.0000 & \\
\hline \#\# & Perspective.ave & 0.0000 & & 0.0000 & \\
\hline \#\# & ChangeOutcomes.ave & 0.0000 & 0.0000 & & \\
\hline \#\# & Limits.ave & 0.0000 & 0.0000 & 0.0000 & \\
\hline \#\# & CompResolve.ave & 0.0000 & 0.0000 & 0.0000 & \\
\hline \#\# & Outsider.ave & 0.0000 & 0.0000 & 0.0000 & \\
\hline \#\# & Reflection & 0.0000 & 0.0000 & 0.0001 & \\
\hline \#\# & Brooding & 0.0003 & 0.0391 & 0.0538 & \\
\hline \#\# & & Limits.ave & CompResolve.ave & Outsider.ave $\mathrm{R}$ & Reflecti \\
\hline on & & & & & \\
\hline \#\# & wisdom.pca & 0.0000 & 0.0000 & 0.0000 & 0.0000 \\
\hline \#\# & Perspective.ave & 0.0000 & 0.0000 & 0.0000 & 0.0000 \\
\hline \#\# & ChangeOutcomes.ave & 0.0000 & 0.0000 & 0.0000 & 0.0001 \\
\hline \#\# & Limits.ave & & 0.0000 & 0.0000 & 0.0000 \\
\hline \#\# & CompResolve.ave & 0.0000 & & 0.0000 & 0.0000 \\
\hline \#\# & Outsider.ave & 0.0000 & 0.0000 & & 0.0000 \\
\hline \#\# & Reflection & 0.0000 & 0.0000 & 0.0000 & \\
\hline \#\# & Brooding & 0.0021 & 0.0802 & 0.0000 & 0.0000 \\
\hline \#\# & & Brooding & & & \\
\hline \#\# & wisdom.pca & 0.0003 & & & \\
\hline \#\# & Perspective.ave & 0.0391 & & & \\
\hline \#\# & ChangeOutcomes.ave & 0.0538 & & & \\
\hline \#\# & Limits.ave & 0.0021 & & & \\
\hline \#\# & CompResolve.ave & 0.0802 & & & \\
\hline \#\# & Outsider.ave & 0.0000 & & & \\
\hline \#\# & Reflection & 0.0000 & & & \\
\hline \#\# & Brooding & & & & \\
\hline $\begin{array}{l}\mathrm{Hm} \\
\mathrm{eO} \\
\mathrm{al}\end{array}$ & $\begin{array}{l}\text { isc: : rcorr (as.matrix } \\
\text { utcomes.ave ', 'Limits } \\
\text { ', 'ess.conflict')])) }\end{array}$ & $\begin{array}{l}\text { x(WIS.G[, c } \\
\text { s.ave ', 'Comf } \\
\text { ) }\end{array}$ & $\begin{array}{l}\text { ('wisdom.pca', 'Pe } \\
\text { oResolve.ave ', 'Ou }\end{array}$ & $\begin{array}{l}\text { erspective.ave ' } \\
\text { utsider.ave', 'e }\end{array}$ & $\begin{array}{l}\text { ', 'Chang } \\
\text { ess.soci }\end{array}$ \\
\hline \#\# & & wisdom.pca & Perspective.ave & ChangeOutcomes & s.ave \\
\hline \#\# & wisdom.pca & 1.00 & 0.76 & & 0.77 \\
\hline \#\# & Perspective.ave & 0.76 & 1.00 & & 0.43 \\
\hline \#\# & ChangeOutcomes.ave & 0.77 & 0.43 & & 1.00 \\
\hline
\end{tabular}




\begin{tabular}{|c|c|c|c|c|c|}
\hline \#\# & Limits.ave & 0.77 & 0.47 & & 0.50 \\
\hline \#\# & CompResolve.ave & 0.80 & 0.56 & & 0.54 \\
\hline \#\# & Outsider.ave & 0.63 & 0.31 & & 0.39 \\
\hline \#\# & ess.social & 0.16 & 0.11 & & 0.15 \\
\hline \#\# & ess.conflict & 0.15 & 0.08 & & 0.20 \\
\hline $\begin{array}{l}\# \# \\
\text { al }\end{array}$ & & Limits.ave & CompResolve.ave & Outsider.ave & ess.soc: \\
\hline $\begin{array}{l}\# \# \\
16\end{array}$ & wisdom.pca & 0.77 & 0.80 & 0.63 & 0 \\
\hline $\begin{array}{l}\# \# \\
11\end{array}$ & Perspective.ave & 0.47 & 0.56 & 0.31 & $\theta$ \\
\hline $\begin{array}{l}\# \# \\
15\end{array}$ & ChangeOutcomes.ave & 0.50 & 0.54 & 0.39 & $\theta$ \\
\hline $\begin{array}{l}\# \# \\
19\end{array}$ & Limits.ave & 1.00 & 0.48 & 0.40 & $\theta$ \\
\hline $\begin{array}{l}\# \# \\
11\end{array}$ & CompResolve.ave & 0.48 & 1.00 & 0.37 & $\theta$ \\
\hline $\begin{array}{l}\# \# \\
03\end{array}$ & Outsider.ave & 0.40 & 0.37 & 1.00 & 0 \\
\hline $\begin{array}{l}\# \# \\
00\end{array}$ & ess.social & 0.19 & 0.11 & 0.03 & 1 \\
\hline $\begin{array}{l}\# \# \\
43\end{array}$ & ess.conflict & 0.16 & 0.11 & 0.00 & 0 \\
\hline \#\# & & ess.conflic & & & \\
\hline \#\# & wisdom.pca & 0.1 & & & \\
\hline \#\# & Perspective.ave & 0.0 & & & \\
\hline \#\# & ChangeOutcomes.ave & 0.2 & & & \\
\hline \#\# & Limits.ave & 0.1 & & & \\
\hline \#\# & CompResolve.ave & 0.1 & & & \\
\hline \#\# & Outsider.ave & 0.0 & & & \\
\hline \#\# & ess.social & 0.4 & & & \\
\hline \#\# & ess.conflict & 1.0 & & & \\
\hline \#\# & $\mathrm{n}$ & & & & \\
\hline \#\# & & wisdom.pca & Perspective.ave & ChangeOutcomes & s.ave \\
\hline \#\# & wisdom.pca & 467 & 467 & & 467 \\
\hline \#\# & Perspective.ave & 467 & 467 & & 467 \\
\hline \#\# & ChangeOutcomes.ave & 467 & 467 & & 467 \\
\hline \#\# & Limits.ave & 467 & 467 & & 467 \\
\hline \#\# & CompResolve.ave & 466 & 466 & & 466 \\
\hline \#\# & Outsider.ave & 466 & 466 & & 466 \\
\hline \#\# & ess.social & 467 & 467 & & 467 \\
\hline \#\# & ess.conflict & 466 & 466 & & 466 \\
\hline \#\# & & Limits.ave & CompResolve.ave & Outsider.ave $\epsilon$ & ess.soc: \\
\hline
\end{tabular}




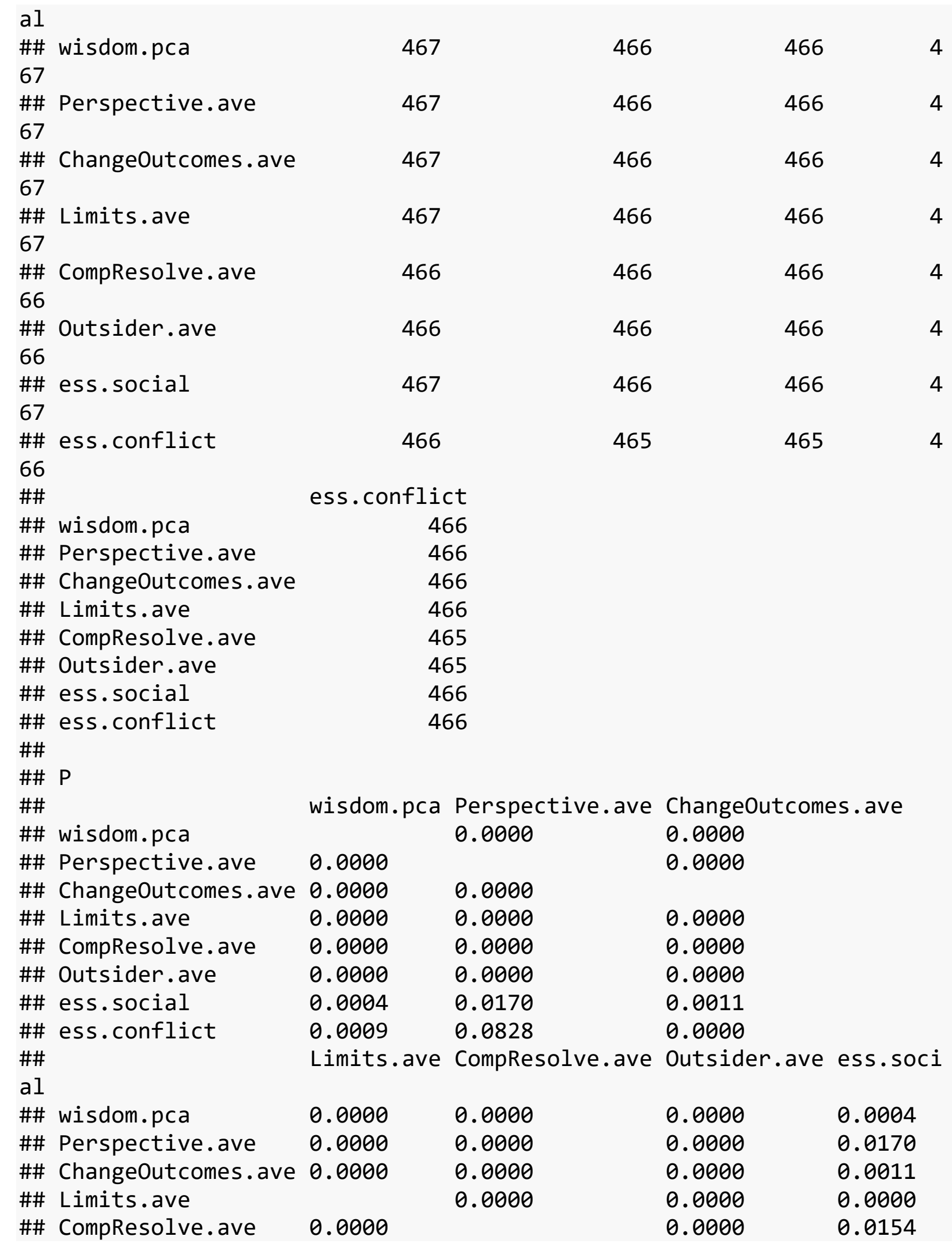




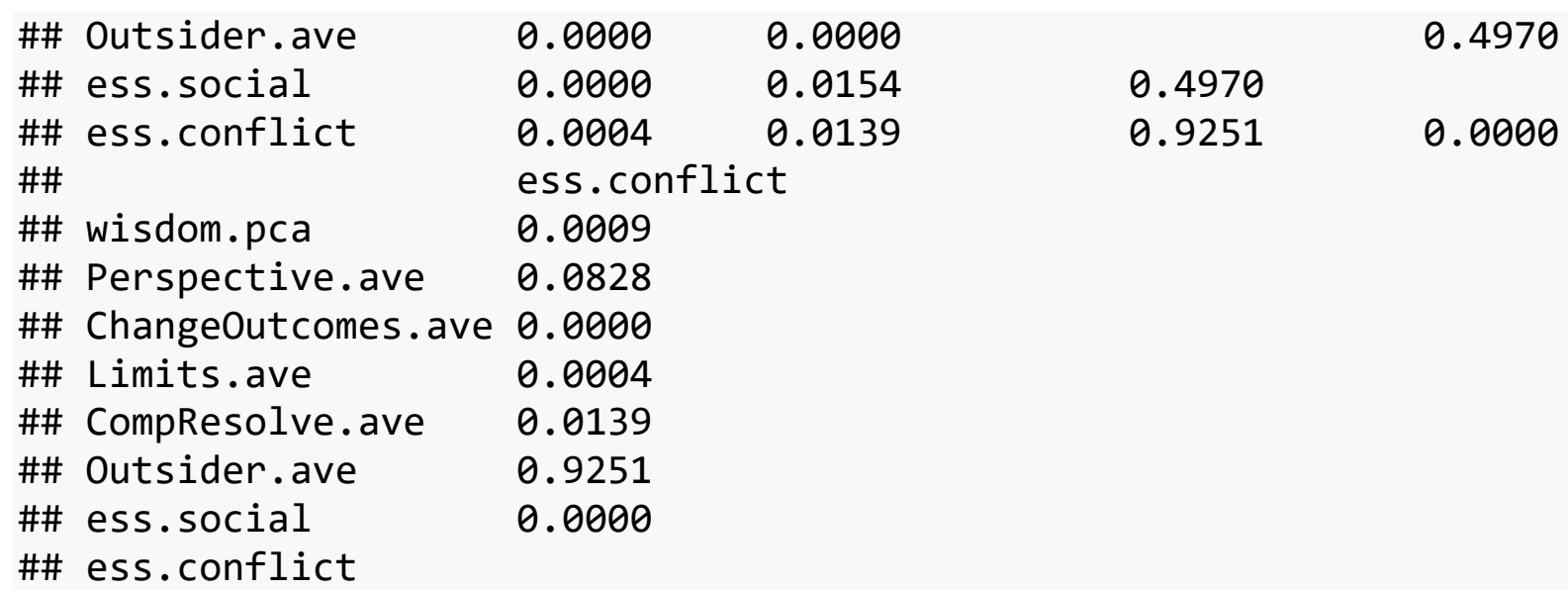

Partial correlation between wise reasoning and brooding, controlling for reflection, as brooding may involve some reflection

\# Run PCOR and ppcor::pcor.test Lines as a pair (to create the dataset and then run partial correlations)

PCOR<-na.omit(WIS.D[, c("wisdom.pca", "Brooding", "Reflection")]) ppcor: : pcor . test (PCOR\$wisdom. pca, PCOR\$Brooding, PCOR\$Reflection, method= "pearson") \# As expected, brooding is no longer correlated to wise rea soning when controlling for reflection

\#\# estimate p.value statistic $\mathrm{n}$ gp Method

\#\# 10.062622890 .097821111 .6577317011 pearson

ppcor: : pcor . test (PCOR\$wisdom. pca, PCOR\$Reflection, PCOR\$Brooding, method= "pearson") \# As expected, reflection is still correlated to wise reaso ning when controlling for brooding

\#\# estimate p.value statistic $\mathrm{n}$ gp Method

\#\# $10.231307 \quad 0.000000000590113 \quad 6.281407701 \quad 1$ pearson

Computing residual scores for Wise Reasoning Composite without Perspectives, Change/Outcomes, Limits, Compromise/Resolution, and Outsider Aspects after regressing Perspectives

WIS. A\$wisdom. pca.NoP. res<-resid( 1 m(wisdom. pca. NoP Perspective. ave, WIS. $A$, na.action=na.exclude))

WIS. A $\$$ ChangeOutcomes.res<-resid ( $\operatorname{lm}$ (ChangeOutcomes . ave Perspective.ave, WIS. A, na. action=na.exclude))

WIS. A\$Limits.res<-resid ( $1 \mathrm{~m}$ (Limits . ave Perspective. ave, WIS. A, na. action= na.exclude))

WIS.A\$CompResolve.res<-resid( $\operatorname{lm}$ (CompResolve.ave Perspective.ave, WIS.A, na. action=na.exclude))

WIS. A\$Outsider. res<-resid( $\operatorname{lm}$ (Outsider . ave Perspective . ave, WIS. A, na . act 
ion=na. exclude))

WIS. B\$Wisdom. pca.NoP. res<-resid( 1 m(wisdom. pca. NoP Perspective. ave, WIS. $B$, na.action=na. exclude))

WIS. B\$ChangeOutcomes . res<-resid ( $\operatorname{lm}$ (ChangeOutcomes . ave Perspective.ave, WIS. B, na. action=na. exclude))

WIS. $B \$$ Limits. res<-resid ( $1 \mathrm{~m}$ (Limits . ave Perspective . ave, WIS. $B$, na. action= na.exclude))

WIS.B\$CompResolve.res<-resid ( $\operatorname{lm}$ (CompResolve.ave Perspective.ave, WIS.B, na. action=na.exclude))

WIS. B\$Outsider. res<-resid( $\operatorname{lm}$ (Outsider . ave Perspective . ave, WIS. B, na . act ion=na. exclude))

WIS.D\$Wisdom. pca.NoP. res<-resid( 1 m(wisdom. pca. NoP Perspective. ave, WIS. $D$, na. action=na. exclude))

WIS.D\$ChangeOutcomes . res<-resid ( $\operatorname{lm}$ (ChangeOutcomes . ave Perspective .ave, WIS.D, na. action=na.exclude))

WIS.D\$Limits.res<-resid $(\operatorname{lm}$ (Limits . ave Perspective . ave, WIS.D, na. action= na.exclude))

WIS.D\$CompResolve.res<-resid( $\operatorname{lm}$ (CompResolve.ave Perspective.ave, WIS.D, na. action=na.exclude))

WIS.D\$Outsider. res<-resid( $\operatorname{lm}$ (Outsider . ave Perspective . ave, WIS.D, na . act ion=na. exclude))

WIS.G\$Wisdom. pca.NoP. res<-resid( $1 \mathrm{~m}$ (wisdom. pca. NoP Perspective.ave, WIS. $G$, na. action=na.exclude))

WIS.G\$ChangeOutcomes . res<-resid ( $\operatorname{lm}$ (ChangeOutcomes . ave Perspective.ave, WIS.G, na. action=na.exclude))

WIS.G\$Limits.res<-resid( $1 \mathrm{~m}$ (Limits . ave Perspective. ave, WIS.G, na. action= na.exclude))

WIS.G\$CompResolve.res<-resid ( $\operatorname{lm}$ (CompResolve.ave Perspective.ave, WIS.G, na.action=na.exclude))

WIS.G\$Outsider. res<-resid( $\operatorname{lm}$ (Outsider . ave Perspective . ave, WIS.G, na . act ion=na. exclude))

Rerunning analyses with residual scores

Hmisc: :rcorr(as.matrix(WIS.B[, c('wisdom.pca.NoP.res', 'ChangeOutcomes. res ', 'Limits.res', ' CompResolve.res', 'Outsider.res', 'Openness ', 'Conscie ntiousness', 'Extraversion', 'Agreeableness', 'Neuroticism')]))

\#\#

\#\# wisdom.pca.NoP.res

\#\# ChangeOutcomes.res

\#\# Limits.res

\#\# CompResolve.res

\#\# Outsider.res

\#\# Openness
wisdom.pca.NoP.res ChangeOutcomes.res Limits.res
1.00
0.70
0.67

0.70

1.00

0.22

0.67

0.22

1.00

0.67

0.36

0.29

0.64

0.28

0.28

0.20

0.12 


\begin{tabular}{|c|c|c|c|c|}
\hline \#\# Conscientiousness & \multicolumn{2}{|l|}{0.12} & 0.12 & 0.10 \\
\hline \#\# Extraversion & \multicolumn{2}{|l|}{0.20} & 0.15 & 0.14 \\
\hline \#\# Agreeableness & \multicolumn{2}{|l|}{0.14} & 0.20 & -0.02 \\
\hline \#\# Neuroticism & \multicolumn{2}{|c|}{-0.04} & -0.06 & -0.09 \\
\hline \#\# & \multicolumn{2}{|c|}{ CompResolve.res Outsider.res } & Openness & Conscienti \\
\hline \multicolumn{5}{|c|}{ 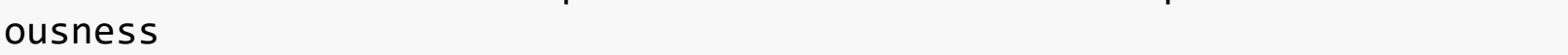 } \\
\hline $\begin{array}{l}\text { \#\# wisdom.pca.NoP.res } \\
0.12\end{array}$ & 0.67 & 0.64 & 0.20 & \\
\hline $\begin{array}{l}\text { \#\# ChangeOutcomes.res } \\
0.12\end{array}$ & 0.36 & \multicolumn{2}{|r|}{0.17} & \\
\hline $\begin{array}{l}\# \# \text { Limits.res } \\
0.10\end{array}$ & 0.29 & \multicolumn{2}{|r|}{0.12} & \\
\hline $\begin{array}{l}\text { \#\# CompResolve.res } \\
0.20\end{array}$ & 1.00 & \multicolumn{2}{|r|}{0.13} & \\
\hline $\begin{array}{l}\# \# \text { Outsider.res } \\
-0.10\end{array}$ & 0.16 & \multicolumn{2}{|r|}{0.10} & \\
\hline 0.26 & 0.13 & \multicolumn{2}{|r|}{1.00} & \\
\hline $\begin{array}{l}\# \# \text { Conscientiousness } \\
1.00\end{array}$ & 0.20 & \multicolumn{2}{|r|}{0.26} & \\
\hline $\begin{array}{l}\text { \#\# Extraversion } \\
0.07\end{array}$ & 0.06 & \multicolumn{2}{|r|}{0.20} & \\
\hline $\begin{array}{l}\text { \#\# Agreeableness } \\
0.46\end{array}$ & 0.20 & \multicolumn{2}{|r|}{0.19} & \\
\hline $\begin{array}{l}\# \# \text { Neuroticism } \\
-0.50\end{array}$ & -0.07 & 0.10 & -0.14 & \\
\hline \#\# & \multicolumn{3}{|c|}{ Extraversion Agreeableness Neuroticism } & \\
\hline \#\# wisdom.pca.NoP.res & 0.20 & 0.14 & -0.04 & \\
\hline \#\# ChangeOutcomes.res & 0.15 & 0.20 & -0.06 & \\
\hline \#\# Limits.res & 0.14 & -0.02 & -0.09 & \\
\hline \#\# CompResolve.res & 0.06 & 0.20 & -0.07 & \\
\hline \#\# Outsider.res & 0.17 & -0.02 & 0.10 & \\
\hline \#\# Openness & 0.20 & 0.19 & -0.14 & \\
\hline \#\# Conscientiousness & 0.07 & 0.46 & -0.50 & \\
\hline \#\# Extraversion & 1.00 & 0.02 & -0.28 & \\
\hline \#\# Agreeableness & 0.02 & 1.00 & -0.26 & \\
\hline \#\# Neuroticism & -0.28 & -0.26 & 1.00 & \\
\hline $\begin{array}{l}\# \# \\
\# \# n\end{array}$ & & & & \\
\hline \#\# & wisdom.pca. NoP.res & ChangeOutc & comes.res & Limits.res \\
\hline \#\# wisdom.pca.NoP.res & 469 & & 468 & 466 \\
\hline \#\# ChangeOutcomes.res & 468 & & 468 & 466 \\
\hline \#\# Limits.res & 466 & & 466 & 466 \\
\hline \#\# CompResolve.res & 466 & & 466 & 466 \\
\hline
\end{tabular}




\begin{tabular}{|c|c|c|c|c|}
\hline \#\# Outsider.res & \multicolumn{2}{|l|}{461} & 461 & 461 \\
\hline \#\# Openness & \multicolumn{2}{|l|}{220} & 220 & 220 \\
\hline \#\# Conscientiousness & \multicolumn{2}{|l|}{220} & 220 & 220 \\
\hline \#\# Extraversion & \multicolumn{2}{|l|}{220} & 220 & 220 \\
\hline \#\# Agreeableness & \multicolumn{2}{|l|}{220} & 220 & 220 \\
\hline \#\# Neuroticism & \multicolumn{2}{|l|}{220} & 220 & 220 \\
\hline \#\# & \multicolumn{2}{|c|}{ CompResolve.res Outsider.res } & Openness & Conscienti \\
\hline \multicolumn{5}{|l|}{ ousness } \\
\hline \#\# wisdom.pca.NoP.res & 466 & 461 & \multirow[t]{2}{*}{220} & \\
\hline \multicolumn{4}{|l|}{220} & \\
\hline \#\# ChangeOutcomes.res & 466 & \multirow{2}{*}{\multicolumn{2}{|c|}{220}} & \\
\hline \multicolumn{3}{|l|}{220} & & \\
\hline \#\# Limits.res & 466 & \multirow{2}{*}{\multicolumn{2}{|c|}{220}} & \\
\hline \multicolumn{4}{|l|}{220} & \\
\hline \#\# CompResolve.res & 466 & \multirow{2}{*}{\multicolumn{2}{|c|}{220}} & \\
\hline \multicolumn{3}{|l|}{220} & & \\
\hline \#\# Outsider.res & 461 & \multicolumn{2}{|r|}{220} & \\
\hline \multicolumn{5}{|l|}{220} \\
\hline \#\# Openness & 220 & 220 & 220 & \\
\hline 220 & & & & \\
\hline \#\# Conscientiousness & 220 & 220 & 220 & \\
\hline 220 & & & & \\
\hline \#\# Extraversion & 220 & 220 & 220 & \\
\hline 220 & & & & \\
\hline \#\# Agreeableness & 220 & 220 & 220 & \\
\hline 220 & & & & \\
\hline \#\# Neuroticism & 220 & 220 & 220 & \\
\hline 220 & & & & \\
\hline \#\# & Extraversion Agreea & ableness $\mathrm{Ne}$ & euroticism & \\
\hline \#\# wisdom.pca.NoP.res & 220 & 220 & 220 & \\
\hline \#\# ChangeOutcomes.res & 220 & 220 & 220 & \\
\hline \#\# Limits.res & 220 & 220 & 220 & \\
\hline \#\# CompResolve.res & 220 & 220 & 220 & \\
\hline \#\# Outsider.res & 220 & 220 & 220 & \\
\hline \#\# Openness & 220 & 220 & 220 & \\
\hline \#\# Conscientiousness & 220 & 220 & 220 & \\
\hline \#\# Extraversion & 220 & 220 & 220 & \\
\hline \#\# Agreeableness & 220 & 220 & 220 & \\
\hline \#\# Neuroticism & 220 & 220 & 220 & \\
\hline \#\# & & & & \\
\hline \#\# P & & & & \\
\hline \#\# & wisdom.pca.NoP.res & ChangeOutc & comes.res & Limits.res \\
\hline \#\# wisdom.pca.NoP.res & & 0.0000 & & 0.0000 \\
\hline \#\# ChangeOutcomes.res & 0.0000 & & & 0.0000 \\
\hline
\end{tabular}




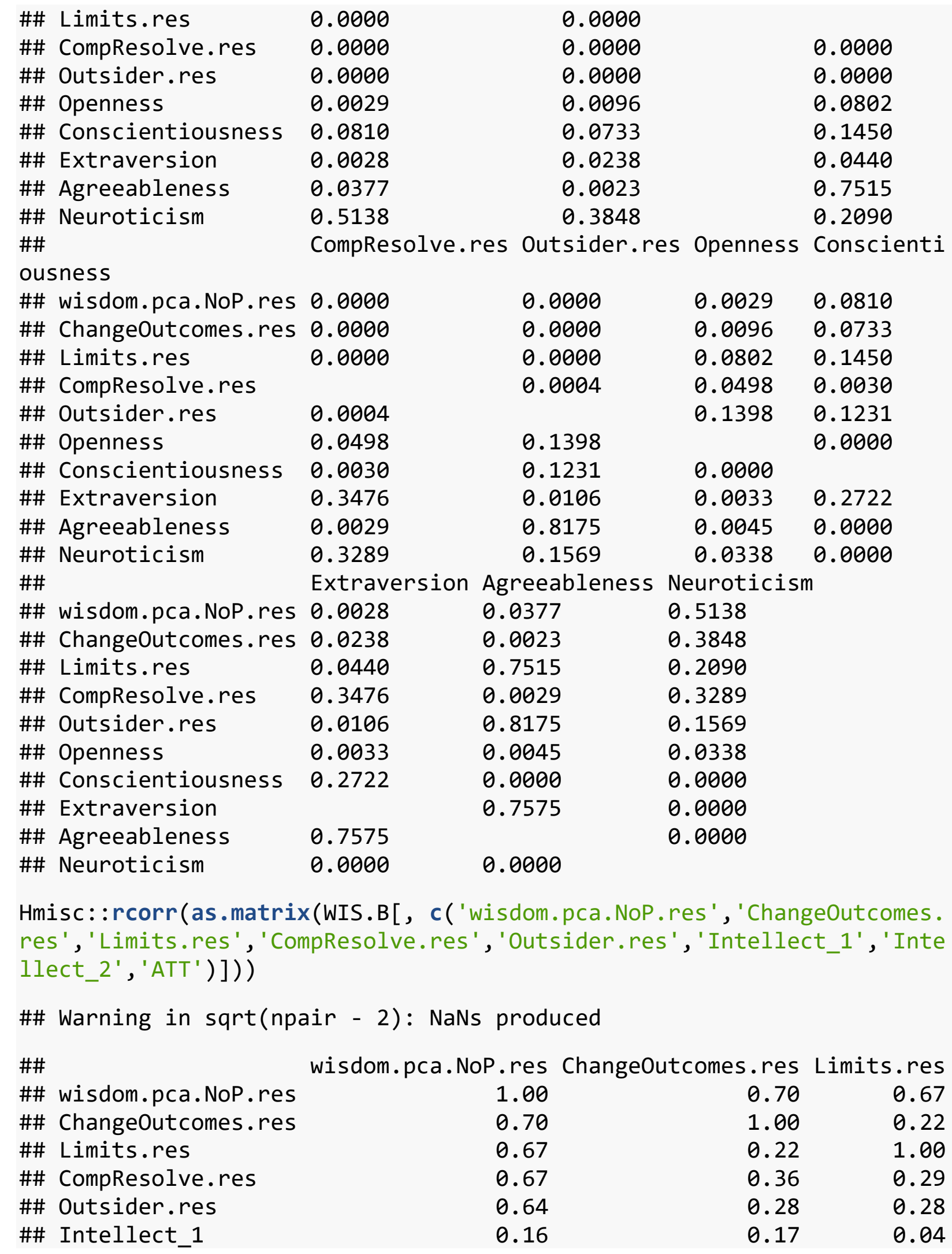




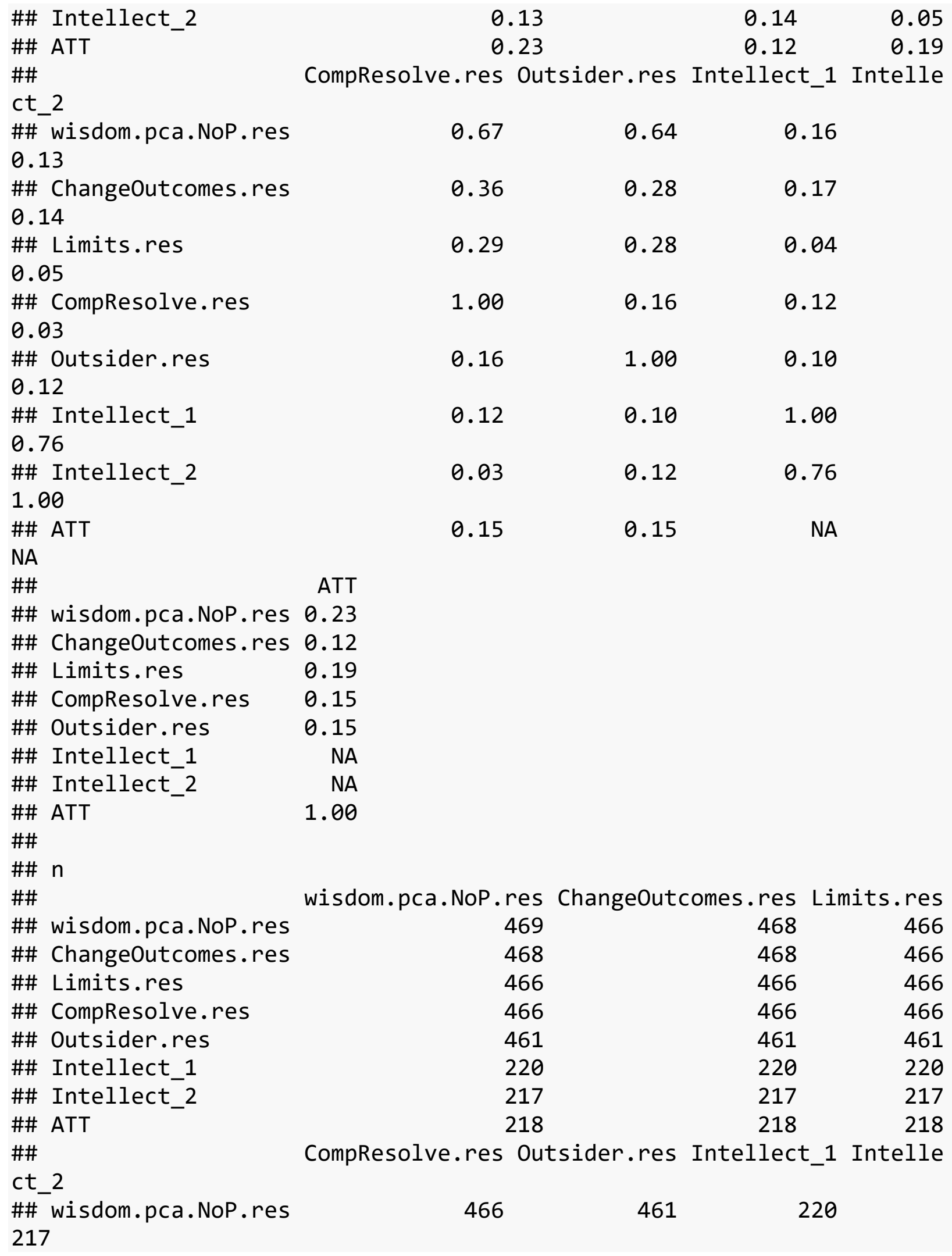

NA 
\#\# ChangeOutcomes.res

217

\#\# Limits.res

217

\#\# CompResolve.res

217

\#\# Outsider.res

217

\#\# Intellect_1

217

\#\# Intellect_2

217

\#\# ATT

0

\#\# ATT

\#\# wisdom.pca.NoP.res 218

\#\# ChangeOutcomes.res 218

\#\# Limits.res 218

\#\# CompResolve.res 218

\#\# Outsider.res 218

\#\# Intellect_1 0

\#\# Intellect_2 0

\#\# ATT 218

$\# \#$

\#\# P

\#\#

\#\# wisdom.pca.NoP.res

\#\# ChangeOutcomes.res

\#\# Limits.res

\#\# CompResolve.res

\#\# Outsider.res

\#\# Intellect_1

\#\# Intellect_2

\#\# ATT

\#\#

ct_2

\#\# wisdom.pca.NoP.res 0.0000

\#\# ChangeOutcomes.res 0.0000

\#\# Limits.res

\#\# CompResolve.res

\#\# Outsider.res

\#\# Intellect_1

\#\# Intellect_2

\#\# ATT

0.0000

0.0000

0.0000

0.0000

0.0167

0.0576

0.0006

0.0000
0.0000
0.0000

0.0000

0.0004

0.0793

0.6504

0.0246
466

461

220

466

461

220

466

461

220

461

461

220

220

220

220

217

217

217

218

218

0

wisdom.pca.NoP.res ChangeOutcomes.res Limits.res

$$
0.0000
$$

0.0000

0.0000

0.0000

0.0000

0.0000

0.0000

0.0000

0.0097

0.5348

0.0414

0.4395

0.0672

0.0053

CompResolve.res Outsider.res Intellect_1 Intelle

0.0000

0.0167

0.0576

0.0000

0.0097

0.0414

0.0000

0.5348

0.4395

0.0004

0.0793

0.6504

0.1473

0.1473

0.0878

0.0878

0.0000

0.0276

0.0000 


\begin{tabular}{|c|c|c|c|c|c|c|}
\hline \#\# & & ATT & & & & \\
\hline \#\# & wisdom.pca.NoP.res & 0.0006 & & & & \\
\hline \#\# & ChangeOutcomes.res & 0.0672 & & & & \\
\hline \#\# & Limits.res & 0.0053 & & & & \\
\hline \#\# & CompResolve.res & 0.0246 & & & & \\
\hline \#\# & Outsider.res & 0.0276 & & & & \\
\hline \#\# & Intellect_1 & & & & & \\
\hline \#\# & Intellect_2 & & & & & \\
\hline \#\# & ATT & & & & & \\
\hline & $\begin{array}{l}\text { isc: :rcorr(as.matrix } \\
\text { s', 'Limits.res ', 'Com }\end{array}$ & $\begin{array}{l}\text { x(WIS.A[, c( 'wisc } \\
\text { npResolve.res ', 'c }\end{array}$ & & $\begin{array}{l}\text {-pca.NoP.re } \\
\text { sider.res', }\end{array}$ & $\begin{array}{l}\text { es ', 'Chang } \\
\text { 'PerspTak }\end{array}$ & $\begin{array}{l}\text { geoutcomes. } \\
\text { king')])) }\end{array}$ \\
\hline \#\# & & wisdom.pca.NoP.r & res & ChangeOutc & comes.res & Limits.res \\
\hline \#\# & wisdom.pca.NoP.res & & .00 & & 0.72 & 0.71 \\
\hline \#\# & ChangeOutcomes.res & & .72 & & 1.00 & 0.31 \\
\hline \#\# & Limits.res & & .71 & & 0.31 & 1.00 \\
\hline \#\# & CompResolve.res & & .77 & & 0.47 & 0.40 \\
\hline \#\# & Outsider.res & & .68 & & 0.26 & 0.35 \\
\hline \#\# & PerspTaking & & .29 & & 0.22 & 0.09 \\
\hline \#\# & & CompResolve.res & Out & tsider.res & PerspTaki & ing \\
\hline \#\# & wisdom.pca.NoP.res & 0.77 & & 0.68 & & 29 \\
\hline \#\# & ChangeOutcomes.res & 0.47 & & 0.26 & & 22 \\
\hline \#\# & Limits.res & 0.40 & & 0.35 & & 09 \\
\hline \#\# & CompResolve.res & 1.00 & & 0.33 & & 30 \\
\hline \#\# & Outsider.res & 0.33 & & 1.00 & & 22 \\
\hline \#\# & PerspTaking & 0.30 & & 0.22 & & .00 \\
\hline \#\# & & & & & & \\
\hline \#\# & $n=404$ & & & & & \\
\hline \#\# & & & & & & \\
\hline \#\# & & & & & & \\
\hline \#\# & $P$ & & & & & \\
\hline \#\# & & wisdom.pca.NoP.r & res & ChangeOutc & comes.res & Limits.res \\
\hline \#\# & wisdom.pca.NoP.res & & & 0.0000 & & 0.0000 \\
\hline \#\# & ChangeOutcomes.res & 0.0000 & & & & 0.0000 \\
\hline \#\# & Limits.res & 0.0000 & & 0.0000 & & \\
\hline \#\# & CompResolve.res & 0.0000 & & 0.0000 & & 0.0000 \\
\hline \#\# & Outsider.res & 0.0000 & & 0.0000 & & 0.0000 \\
\hline \#\# & PerspTaking & 0.0000 & & 0.0000 & & 0.0591 \\
\hline \#\# & & CompResolve.res & Out & Esider.res & PerspTaki & ing \\
\hline \#\# & wisdom.pca.NoP.res & 0.0000 & 0.0 & 3000 & 0.0000 & \\
\hline \#\# & ChangeOutcomes.res & 0.0000 & 0.0 & 000 & 0.0000 & \\
\hline \#\# & Limits.res & 0.0000 & 0.0 & 3000 & 0.0591 & \\
\hline & CompResolve.res & & 0.0 & 000 & 0.0000 & \\
\hline
\end{tabular}




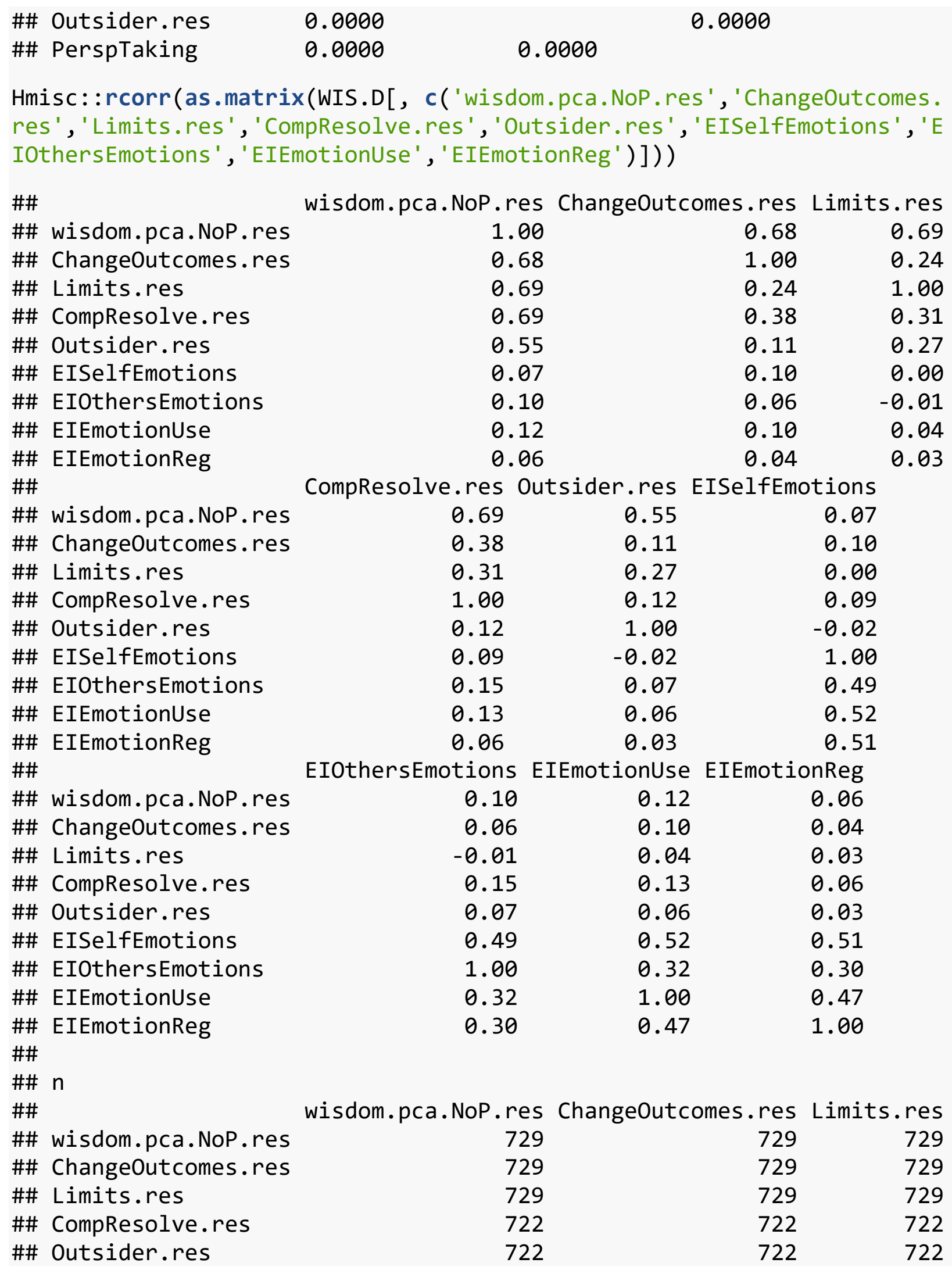




\begin{tabular}{|c|c|c|c|c|c|c|c|}
\hline \#\# & EISelfEmotions & \multicolumn{3}{|c|}{701} & 701 & & 701 \\
\hline \#\# & EIOthersEmotions & \multicolumn{3}{|c|}{701} & 701 & & 701 \\
\hline \#\# & EIEmotionUse & \multicolumn{3}{|c|}{701} & 701 & & 701 \\
\hline \#\# & EIEmotionReg & \multicolumn{3}{|c|}{701} & 701 & & 701 \\
\hline \#\# & & \multicolumn{3}{|c|}{ CompResolve.res Outsider.res } & \multicolumn{3}{|c|}{ EISelfEmotions } \\
\hline \#\# & wisdom.pca.NoP.res & \multicolumn{2}{|l|}{722} & 722 & \multicolumn{3}{|c|}{701} \\
\hline \#\# & ChangeOutcomes.res & \multicolumn{2}{|l|}{722} & 722 & \multicolumn{3}{|c|}{701} \\
\hline \#\# & Limits.res & \multicolumn{2}{|l|}{722} & 722 & \multicolumn{3}{|c|}{701} \\
\hline \#\# & CompResolve.res & \multicolumn{2}{|l|}{722} & 722 & \multicolumn{3}{|c|}{701} \\
\hline \#\# & Outsider.res & \multicolumn{2}{|l|}{722} & 722 & \multicolumn{3}{|c|}{701} \\
\hline \#\# & EISelfEmotions & \multicolumn{2}{|l|}{701} & 701 & \multicolumn{3}{|c|}{702} \\
\hline \#\# & EIOthersEmotions & \multicolumn{2}{|l|}{701} & 701 & \multicolumn{3}{|c|}{701} \\
\hline \#\# & EIEmotionUse & \multicolumn{2}{|l|}{701} & 701 & \multicolumn{3}{|c|}{701} \\
\hline \#\# & EIEmotionReg & \multicolumn{2}{|l|}{701} & 701 & & 701 & \\
\hline \#\# & & EIOthersEmotions & $S E I$ & EmotionUse & e EIEmotic & onReg & \\
\hline \#\# & wisdom.pca.NoP.res & 701 & & 70 & & 701 & \\
\hline \#\# & ChangeOutcomes.res & 701 & & 70 & & 701 & \\
\hline \#\# & Limits.res & 701 & & 70 & & 701 & \\
\hline \#\# & CompResolve.res & 701 & & 70 & & 701 & \\
\hline \#\# & Outsider.res & 701 & & 70 & & 701 & \\
\hline \#\# & EISelfEmotions & 701 & & 70 & & 701 & \\
\hline \#\# & EIOthersEmotions & 701 & & 70 & & 701 & \\
\hline \#\# & EIEmotionUse & 701 & & 70 & & 701 & \\
\hline \#\# & EIEmotionReg & 701 & & 70 & & 701 & \\
\hline \#\# & & & & & & & \\
\hline \#\# & $\mathrm{P}$ & & & & & & \\
\hline \#\# & & wisdom.pca.NoP.r & res & ChangeOut & comes.res & Limits & res \\
\hline \#\# & wisdom.pca.NoP.res & & & 0.0000 & & 0.0000 & \\
\hline \#\# & ChangeOutcomes.res & 0.0000 & & & & 0.0000 & \\
\hline \#\# & Limits.res & 0.0000 & & 0.0000 & & & \\
\hline \#\# & CompResolve.res & 0.0000 & & 0.0000 & & 0.0000 & \\
\hline \#\# & Outsider.res & 0.0000 & & 0.0021 & & 0.0000 & \\
\hline \#\# & EISelfEmotions & 0.0719 & & 0.0105 & & 0.9827 & \\
\hline \#\# & EIOthersEmotions & 0.0066 & & 0.1198 & & 0.7345 & \\
\hline \#\# & EIEmotionUse & 0.0013 & & 0.0101 & & 0.3521 & \\
\hline \#\# & EIEmotionReg & 0.1031 & & 0.2345 & & 0.5045 & \\
\hline \#\# & & CompResolve.res & Out & sider.res & EISelfEmc & otions & \\
\hline \#\# & wisdom.pca.NoP.res & 0.0000 & 0.0 & 3000 & 0.0719 & & \\
\hline \#\# & ChangeOutcomes.res & 0.0000 & 0.0 & 021 & 0.0105 & & \\
\hline \#\# & Limits.res & 0.0000 & 0.0 & 000 & 0.9827 & & \\
\hline \#\# & CompResolve.res & & 0.0 & 016 & 0.0137 & & \\
\hline \#\# & Outsider.res & 0.0016 & & & 0.5984 & & \\
\hline \#\# & EISelfEmotions & 0.0137 & 0.5 & 5984 & & & \\
\hline \#\# & EIOthersEmotions & 0.0000 & 0.0 & 544 & 0.0000 & & \\
\hline
\end{tabular}




$\begin{array}{llcc}\text { \#\# EIEmotionUse } & 0.0008 & 0.1424 & 0.0000 \\ \text { \#\# EIEmotionReg } & 0.1144 & 0.4409 & 0.0000 \\ \text { \#\# } & \text { EIOthersEmotions } & \text { EIEmotionUse } & \text { EIEmotionReg } \\ \text { \#\# wisdom.pca.NoP.res } & 0.0066 & 0.0013 & 0.1031 \\ \text { \#\# ChangeOutcomes.res } & 0.1198 & 0.0101 & 0.2345 \\ \text { \#\# Limits.res } & 0.7345 & 0.3521 & 0.5045 \\ \text { \#\# CompResolve.res } & 0.0000 & 0.0008 & 0.1144 \\ \text { \#\# Outsider.res } & 0.0544 & 0.1424 & 0.4409 \\ \text { \#\# EISelfEmotions } & 0.0000 & 0.0000 & 0.0000 \\ \text { \#\# EIOthersEmotions } & & 0.0000 & 0.0000 \\ \text { \#\# EIEmotionUse } & 0.0000 & & 0.0000 \\ \text { \#\# EIEmotionReg } & 0.0000 & 0.0000 & \end{array}$

Hmisc: :rcorr(as.matrix(WIS.B[, c('wisdom.pca.NoP.res', 'ChangeOutcomes. res', 'Limits.res', 'CompResolve.res ', 'Outsider.res ', 'Mindful_1', 'Mindfu 1_2', 'Mindful_3', 'Mindful_4', 'Mindful_5')]))

\begin{tabular}{|c|c|c|c|c|c|c|}
\hline \#\# & & wisdom.pca & . NoP.res & ChangeOutc & omes.res & Limits.res \\
\hline \#\# & wisdom.pca.NoP.res & & 1.00 & & 0.70 & 0.67 \\
\hline \#\# & ChangeOutcomes.res & & 0.70 & & 1.00 & 0.22 \\
\hline \#\# & Limits.res & & 0.67 & & 0.22 & 1.00 \\
\hline \#\# & CompResolve.res & & 0.67 & & 0.36 & 0.29 \\
\hline \#\# & Outsider.res & & 0.64 & & 0.28 & 0.28 \\
\hline \#\# & Mindful_1 & & 0.09 & & 0.09 & 0.07 \\
\hline \#\# & Mindful_2 & & 0.22 & & 0.21 & 0.13 \\
\hline \#\# & Mindful_3 & & -0.03 & & 0.04 & -0.02 \\
\hline \#\# & Mindful_-4 & & 0.10 & & 0.18 & 0.04 \\
\hline \#\# & Mindful_5 & & -0.06 & & -0.07 & -0.02 \\
\hline \#\# & & CompResolv & e.res out & zider.res I & Mindful_1 & Mindful_2 \\
\hline \#\# & wisdom.pca.NoP.res & & 0.67 & 0.64 & $0 . \overline{0} 9$ & $\quad 0 . \overline{2} 2$ \\
\hline \#\# & ChangeOutcomes.res & & 0.36 & 0.28 & 0.09 & 0.21 \\
\hline \#\# & Limits.res & & 0.29 & 0.28 & 0.07 & 0.13 \\
\hline \#\# & CompResolve.res & & 1.00 & 0.16 & 0.09 & 0.12 \\
\hline \#\# & Outsider.res & & 0.16 & 1.00 & -0.01 & 0.12 \\
\hline \#\# & Mindful_1 & & 0.09 & -0.01 & 1.00 & 0.20 \\
\hline \#\# & Mindful_2 & & 0.12 & 0.12 & 0.20 & 1.00 \\
\hline \#\# & Mindful_3 & & 0.06 & -0.16 & 0.14 & -0.02 \\
\hline \#\# & Mindful_4 & & 0.04 & 0.02 & 0.19 & 0.41 \\
\hline \#\# & Mindful_5 & & 0.10 & -0.15 & 0.13 & -0.08 \\
\hline \#\# & & Mindful_3 & Mindful_4 & Mindful_5 & & \\
\hline \#\# & wisdom.pca.NoP.res & -0.03 & 0.10 & $\quad-0.06$ & & \\
\hline \#\# & ChangeOutcomes.res & 0.04 & 0.18 & -0.07 & & \\
\hline \#\# & Limits.res & -0.02 & 0.04 & -0.02 & & \\
\hline \#\# & CompResolve.res & 0.06 & 0.04 & 0.10 & & \\
\hline \#\# & Outsider.res & -0.16 & 0.02 & -0.15 & & \\
\hline
\end{tabular}




\begin{tabular}{|c|c|c|c|c|c|c|}
\hline \#\# & Mindful_1 & 0.14 & 0.19 & 0.13 & & \\
\hline \#\# & Mindful_2 & -0.02 & 0.41 & -0.08 & & \\
\hline \#\# & Mindful_3 & 1.00 & 0.36 & 0.50 & & \\
\hline \#\# & Mindful_4 & 0.36 & 1.00 & 0.34 & & \\
\hline \#\# & Mindful_5 & 0.50 & 0.34 & 1.00 & & \\
\hline \multicolumn{7}{|c|}{ \#\# + ～－ - } \\
\hline \multicolumn{7}{|c|}{ \#\# n } \\
\hline \#\# & & \multicolumn{2}{|c|}{ wisdom.pca.NoP.res } & \multicolumn{2}{|c|}{ ChangeOutcomes.res } & Limits.res \\
\hline \#\# & wisdom.pca.NoP.res & \multicolumn{2}{|c|}{469} & & 468 & 466 \\
\hline \#\# & ChangeOutcomes.res & \multicolumn{2}{|r|}{468} & & 468 & 466 \\
\hline \#\# & Limits.res & \multicolumn{2}{|r|}{466} & & 466 & 466 \\
\hline \#\# & CompResolve.res & \multicolumn{2}{|r|}{466} & & 466 & 466 \\
\hline \#\# & Outsider.res & \multicolumn{2}{|r|}{461} & & 461 & 461 \\
\hline \#\# & Mindful_1 & \multicolumn{2}{|r|}{223} & & 223 & 223 \\
\hline \#\# & Mindful_2 & \multicolumn{2}{|r|}{223} & & 223 & 223 \\
\hline \#\# & Mindful_3 & \multicolumn{2}{|r|}{223} & & 223 & 223 \\
\hline \#\# & Mindful_4 & \multicolumn{2}{|r|}{221} & & 221 & 221 \\
\hline \#\# & Mindful_5 & \multicolumn{2}{|c|}{220} & & 220 & 220 \\
\hline \#\# & & \multicolumn{2}{|c|}{ CompResolve.res Outs } & zider.res I & Mindful_1 & Mindful_2 \\
\hline \#\# & wisdom.pca.NoP.res & \multicolumn{2}{|c|}{466} & 461 & 223 & 223 \\
\hline \#\# & ChangeOutcomes.res & \multicolumn{2}{|r|}{466} & 461 & 223 & 223 \\
\hline \#\# & Limits.res & \multicolumn{2}{|r|}{466} & 461 & 223 & 223 \\
\hline \#\# & CompResolve.res & \multicolumn{2}{|r|}{466} & 461 & 223 & 223 \\
\hline \#\# & Outsider.res & \multicolumn{2}{|r|}{461} & 461 & 223 & 223 \\
\hline \#\# & Mindful_1 & \multicolumn{2}{|r|}{223} & 223 & 223 & 223 \\
\hline \#\# & Mindful_2 & \multicolumn{2}{|r|}{223} & 223 & 223 & 223 \\
\hline \#\# & Mindful_3 & \multicolumn{2}{|r|}{223} & 223 & 223 & 223 \\
\hline \#\# & Mindful_4 & \multicolumn{2}{|r|}{221} & 221 & 221 & 221 \\
\hline \#\# & Mindful_5 & & 220 & 220 & 220 & 220 \\
\hline \#\# & & Mindful_3 & Mindful_4 & Mindful_5 & & \\
\hline \#\# & wisdom.pca.NoP.res & $2 \overline{2} 3$ & $2 \overline{21}$ & $2 \overline{2} 0$ & & \\
\hline \#\# & ChangeOutcomes.res & 223 & 221 & 220 & & \\
\hline \#\# & Limits.res & 223 & 221 & 220 & & \\
\hline \#\# & CompResolve.res & 223 & 221 & 220 & & \\
\hline \#\# & Outsider.res & 223 & 221 & 220 & & \\
\hline \#\# & Mindful_1 & 223 & 221 & 220 & & \\
\hline \#\# & Mindful_2 & 223 & 221 & 220 & & \\
\hline \#\# & Mindful_3 & 223 & 221 & 220 & & \\
\hline \#\# & Mindful_4 & 221 & 221 & 220 & & \\
\hline \#\# & Mindful_5 & 220 & 220 & 220 & & \\
\hline \#\# & & & & & & \\
\hline \#\# & $P$ & & & & & \\
\hline \#\# & & wisdom.pca & .NoP.res & ChangeOutc & comes.res & Limits.res \\
\hline \#\# & wisdom.pca.NoP.res & & & 0.0000 & & 0.0000 \\
\hline
\end{tabular}




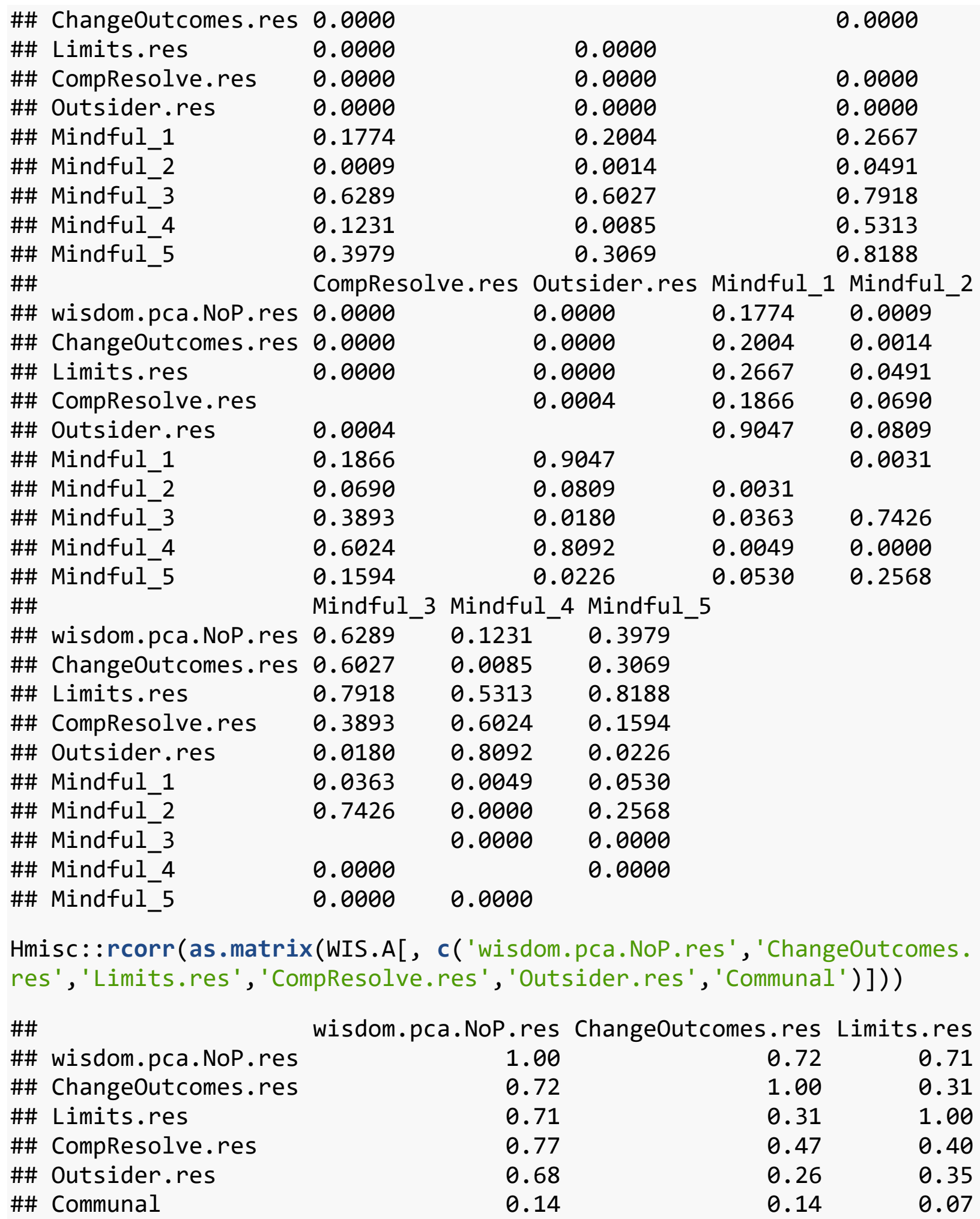

\#\#

CompResolve.res Outsider.res Communal

\#\# wisdom.pca.NoP.res
0.77
0.68
0.14

$\begin{array}{lll}0.47 & 0.26 & 0.14\end{array}$

\#\# ChangeOutcomes.res 


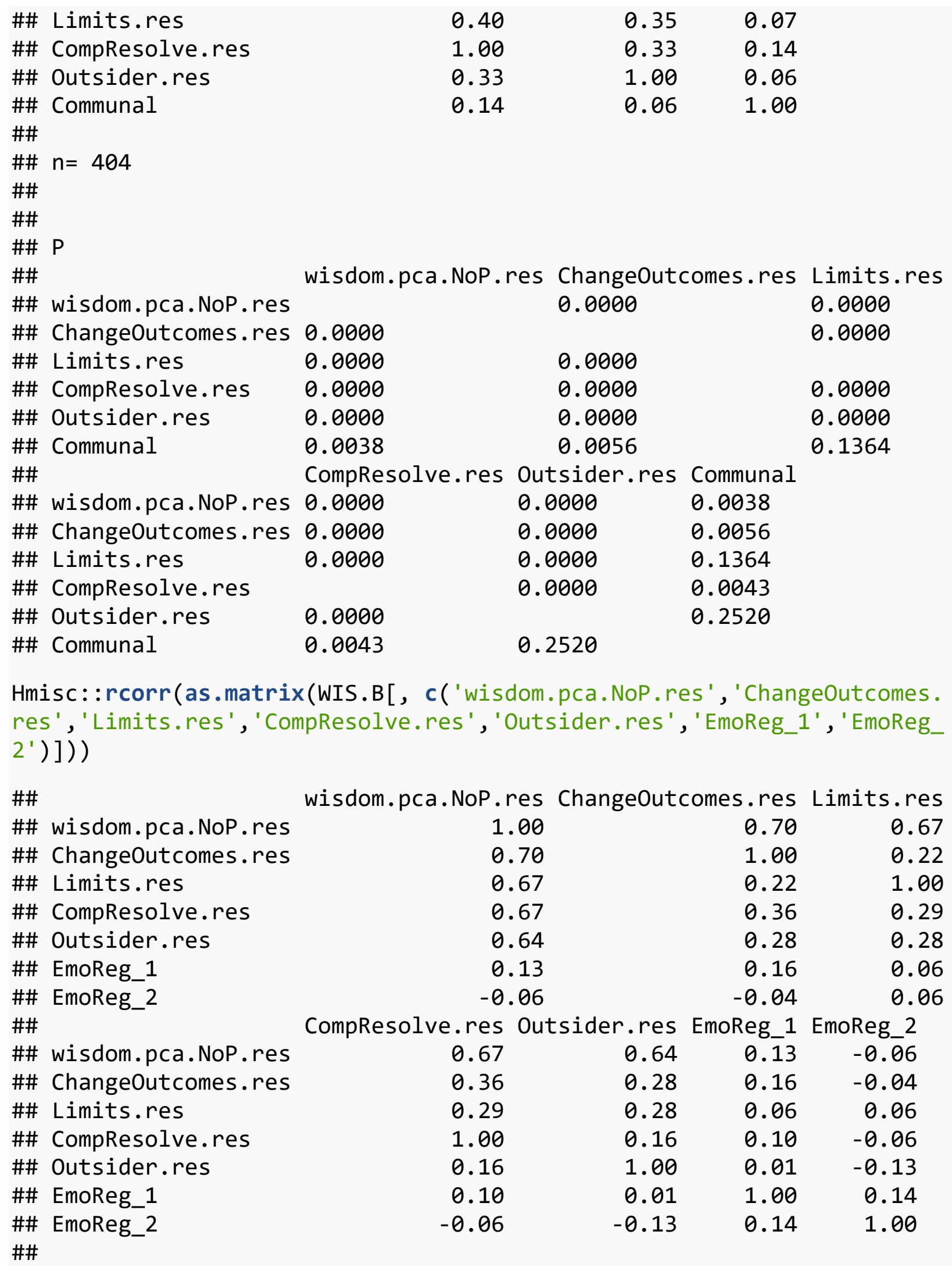




\begin{tabular}{|c|c|c|c|c|c|c|}
\hline \#\# & & wisdom.pca.NoP.r & res & ChangeOutc & comes.res & Limits.res \\
\hline \#\# & wisdom.pca.NoP.res & & 469 & & 468 & 466 \\
\hline \#\# & ChangeOutcomes.res & & 468 & & 468 & 466 \\
\hline \#\# & Limits.res & & 466 & & 466 & 466 \\
\hline \#\# & CompResolve.res & & 466 & & 466 & 466 \\
\hline \#\# & Outsider.res & & 461 & & 461 & 461 \\
\hline \#\# & EmoReg_1 & & 216 & & 216 & 216 \\
\hline \#\# & EmoReg_2 & & 216 & & 216 & 216 \\
\hline \#\# & & CompResolve.res & Out & tsider.res & EmoReg_1 & EmoReg_2 \\
\hline \#\# & wisdom.pca.NoP.res & 466 & & 461 & 216 & 216 \\
\hline \#\# & ChangeOutcomes.res & 466 & & 461 & 216 & 216 \\
\hline \#\# & Limits.res & 466 & & 461 & 216 & 216 \\
\hline \#\# & CompResolve.res & 466 & & 461 & 216 & 216 \\
\hline \#\# & Outsider.res & 461 & & 461 & 216 & 216 \\
\hline \#\# & EmoReg_1 & 216 & & 216 & 216 & 216 \\
\hline \#\# & EmoReg_2 & 216 & & 216 & 216 & 216 \\
\hline \#\# & & & & & & \\
\hline 讲 & $P$ & & & & & \\
\hline \#\# & & wisdom.pca.NoP.r & res & ChangeOutc & comes.res & Limits.res \\
\hline \#\# & wisdom.pca.NoP.res & & & 0.0000 & & 0.0000 \\
\hline \#\# & ChangeOutcomes.res & 0.0000 & & & & 0.0000 \\
\hline \#\# & Limits.res & 0.0000 & & 0.0000 & & \\
\hline \#\# & CompResolve.res & 0.0000 & & 0.0000 & & 0.0000 \\
\hline \#\# & Outsider.res & 0.0000 & & 0.0000 & & 0.0000 \\
\hline \#\# & EmoReg_1 & 0.0609 & & 0.0179 & & 0.3446 \\
\hline \#\# & EmoReg_2 & 0.3852 & & 0.5826 & & 0.4183 \\
\hline \#\# & & CompResolve.res & Out & Esider.res & EmoReg_1 & EmoReg_2 \\
\hline \#\# & wisdom.pca.NoP.res & 0.0000 & 0.0 & 3000 & 0.0609 & 0.3852 \\
\hline \#\# & ChangeOutcomes.res & 0.0000 & 0.0 & 000 & 0.0179 & 0.5826 \\
\hline \#\# & Limits.res & 0.0000 & 0.0 & 3000 & 0.3446 & 0.4183 \\
\hline \#\# & CompResolve.res & & 0.0 & 3004 & 0.1363 & 0.3720 \\
\hline \#\# & Outsider.res & 0.0004 & & & 0.8515 & 0.0658 \\
\hline & EmoReg_1 & 0.1363 & 0.8 & 3515 & & 0.0332 \\
\hline \#\# & EmoReg_2 & 0.3720 & 0.0 & 3658 & 0.0332 & \\
\hline $\begin{array}{l}\mathrm{Hm} \\
\text { re } \\
\text { in }\end{array}$ & $\begin{array}{l}\text { isc: : rcorr(as.matrix } \\
\left.\left.\text { s', 'Limits.res ', 'Com } \mathrm{g}^{\prime}\right)\right] \text { )) }\end{array}$ & $\begin{array}{l}\text { x(WIS.D [, c( 'wisd } \\
\text { npResolve.res ', '0 }\end{array}$ & $\begin{array}{l}\text { dom. } \\
\text { Duts }\end{array}$ & $\begin{array}{l}\text { pca.NoP.re } \\
\text { sider.res', }\end{array}$ & $\begin{array}{l}\text { es ', 'Chang } \\
\text { 'Reflecti }\end{array}$ & $\begin{array}{l}\text { Lon', 'Brood } \\
\text { Lon ' }\end{array}$ \\
\hline \#\# & & wisdom.pca.NoP.r & res & ChangeOutc & comes.res & Limits.res \\
\hline \#\# & wisdom.pca.NoP.res & & .00 & & 0.68 & 0.69 \\
\hline \#\# & ChangeOutcomes.res & & 68 & & 1.00 & 0.24 \\
\hline \#\# & Limits.res & & 69 & & 0.24 & 1.00 \\
\hline \#\# & CompResolve.res & & 69 & & 0.38 & 0.31 \\
\hline
\end{tabular}




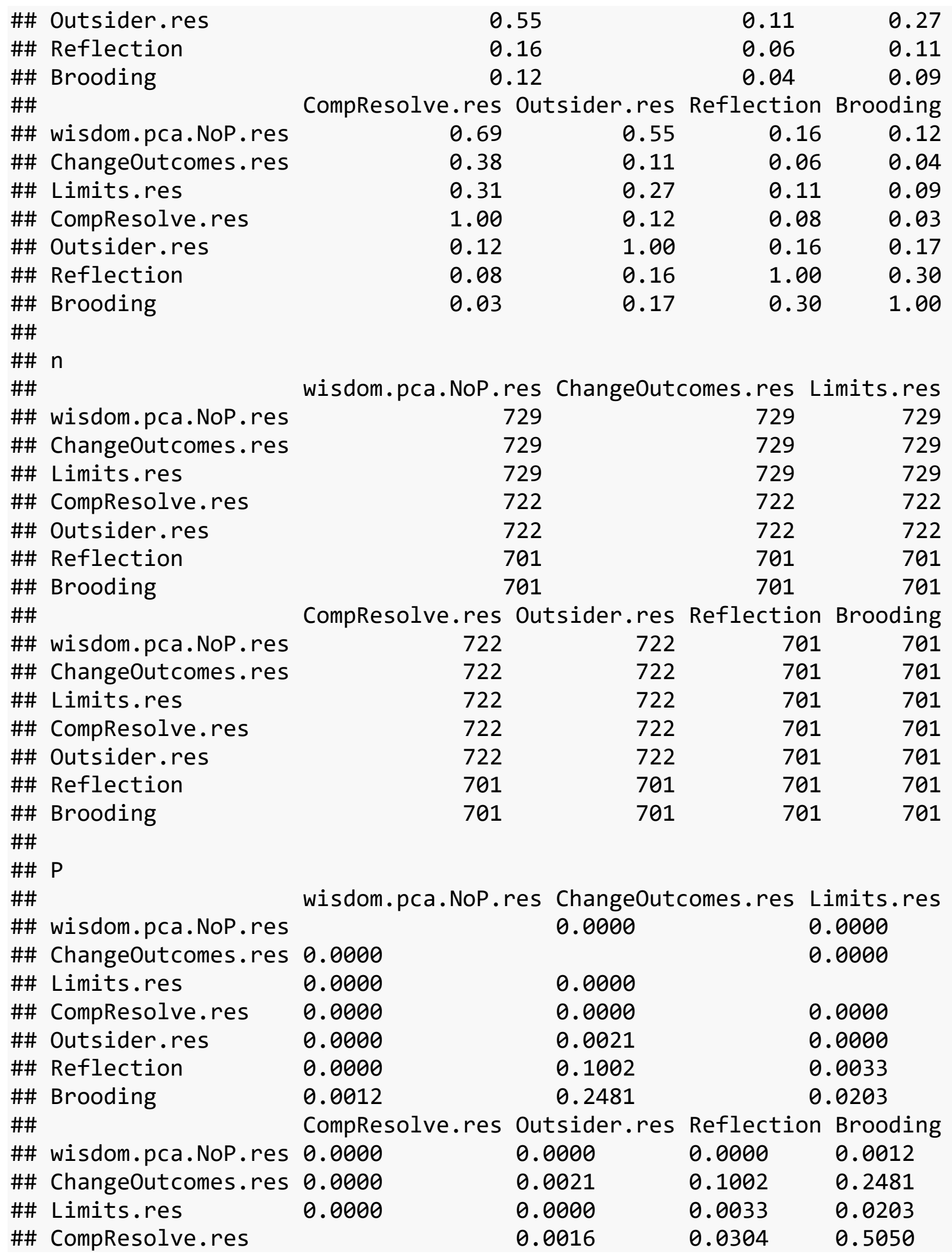




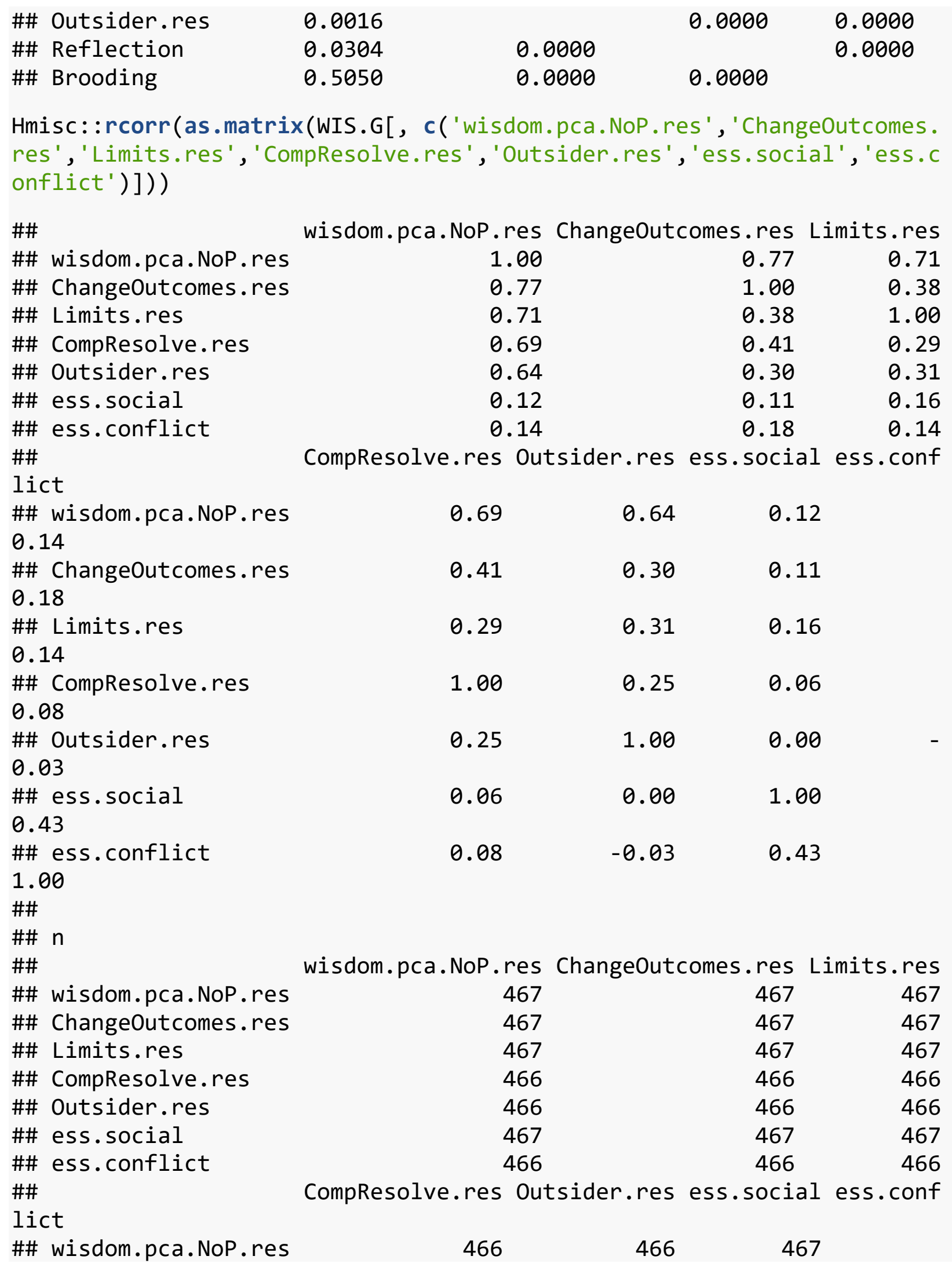




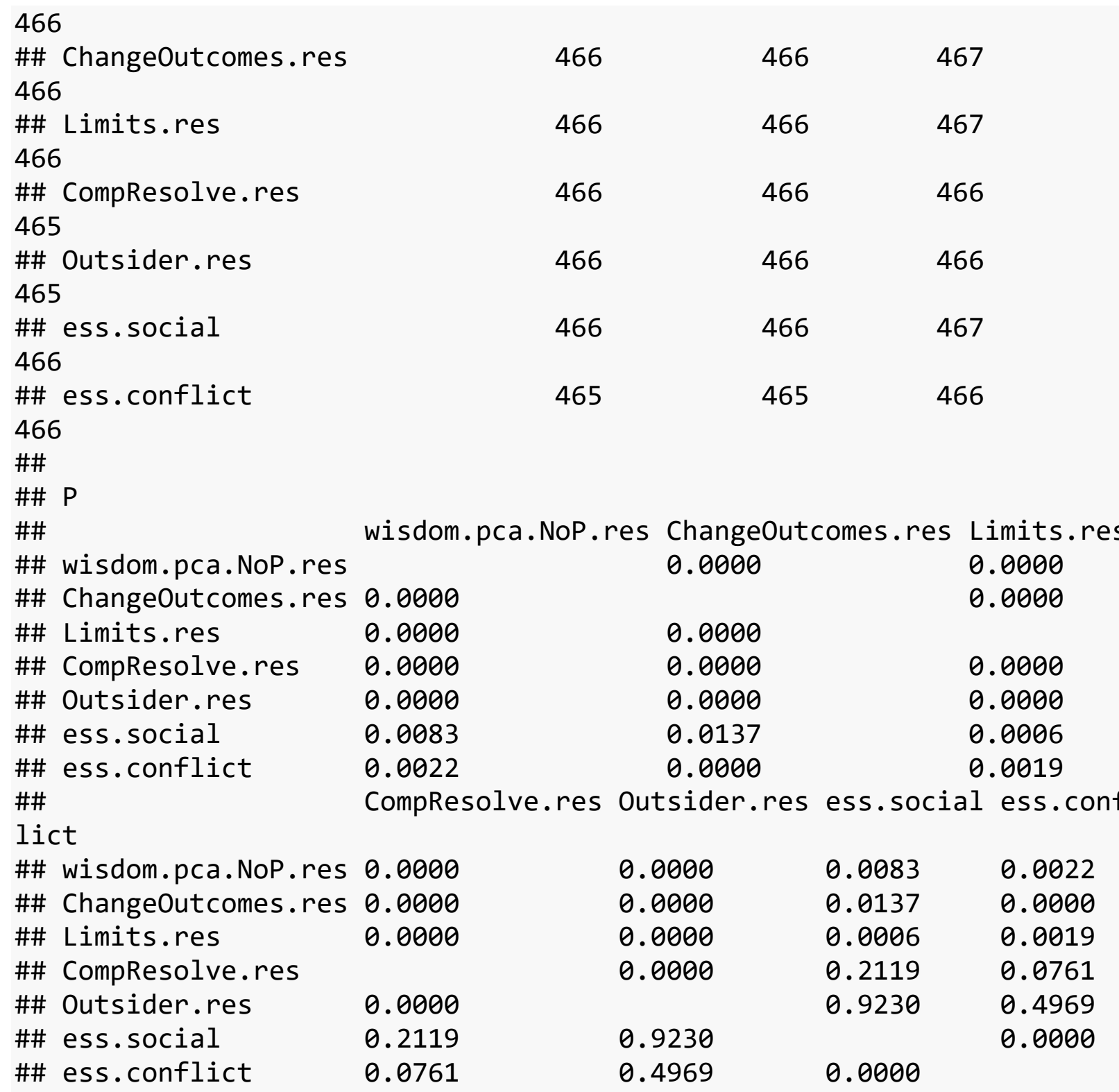

\#\#\#\# S5: STUDY 5 \#\#\#\#

\# Looking at number of Sample C participants who did the social dilemm a games

sum(! is.na(WIS.C\$commons)|! is.na(WIS.C\$prisoners)) \# Number who took o ne social dilemma game

\#\# [1] 379

sum(! is.na(WIS.C\$commons)\&! is.na(WIS.C\$prisoners)) \# Number who took b oth social dilemma games

\#\# [1] 109 
\# Preparing subsets for analysis

WIS.C $\$$ commonsRZ<-scale(WIS.C $\$$ commonsR, center=TRUE, scale=TRUE) \# Standa rdizing commons dilemma games variables - Higher scores means more coo peration

WIS.C\$PrisonerZ<-scale(WIS.C\$prisoner, center=TRUE, scale=TRUE) \# Standa rdizing prisoners dilemma games variables - Higher scores means more $c$ ooperation

WIS.GAME<-melt(WIS.C [ , c("IDvar", "wisdom.pca", "PrisonerZ", "commonsRZ") ] ,id=c("IDvar", "wisdom.pca")) \# Reshaping dataset to be long

\#\# Warning: attributes are not identical across measure variables; the $y$ will

\#\# be dropped

WIS.S5<-subset(WIS [, c( 'sample' ', 'wisdom.pca' , 'Perspective.ave ', 'Change0 utcomes.ave', ' Limits.ave', 'CompResolve.ave', 'Outsider.ave', 'seeklistener', 'seekadvice', ' solvetogether', ' for give', 'disengage ', 'retaliate', 'gangup')],

sample \%in\% c("a", "b", "c","d", "e","f")) \# Selecting sam ples a-f for conflict resolution behavior analysis

\section{S5A: SOCIAL DILEMMA GAMES}

\# We restructured the file to be long and ran a multilevel model where performance on the two games within participant

\# To read the multilevel model analysis (Lmer code), look at the last Line of fixed effects (wisdom.pca) to see the effect of wise reasoning on both games ( $p$-value in the last column: $\operatorname{Pr}(>|t|)$ )

summary (Imer(value wisdom.pca+(1|IDvar), WIS.GAME, REML = FALSE)) \# Mu Ltilevel model of effect of wisdom on cooperation - Wise reasoning is associated with more cooperation, as expected.

\#\# Linear mixed model fit by maximum likelihood t-tests use Satterthwa ite

\#\# approximations to degrees of freedom [lmerMod]

\#\# Formula: value $~$ wisdom.pca + (1 | IDvar $)$

\#\# Data: WIS.GAME

\#\#

\#\# AIC BIC logLik deviance df.resid

$\begin{array}{llllll}\# \# & 1366.2 & 1382.9 & -679.1 & 1358.2 & 482\end{array}$

\#\#

\#\# Scaled residuals:

\#\# Min 10 Median $3 Q \quad$ Max

$\begin{array}{llllll}\# \# & -2.0422 & -0.3203 & 0.3391 & 0.5159 & 1.5526\end{array}$

\#\#

\#\# Random effects: 


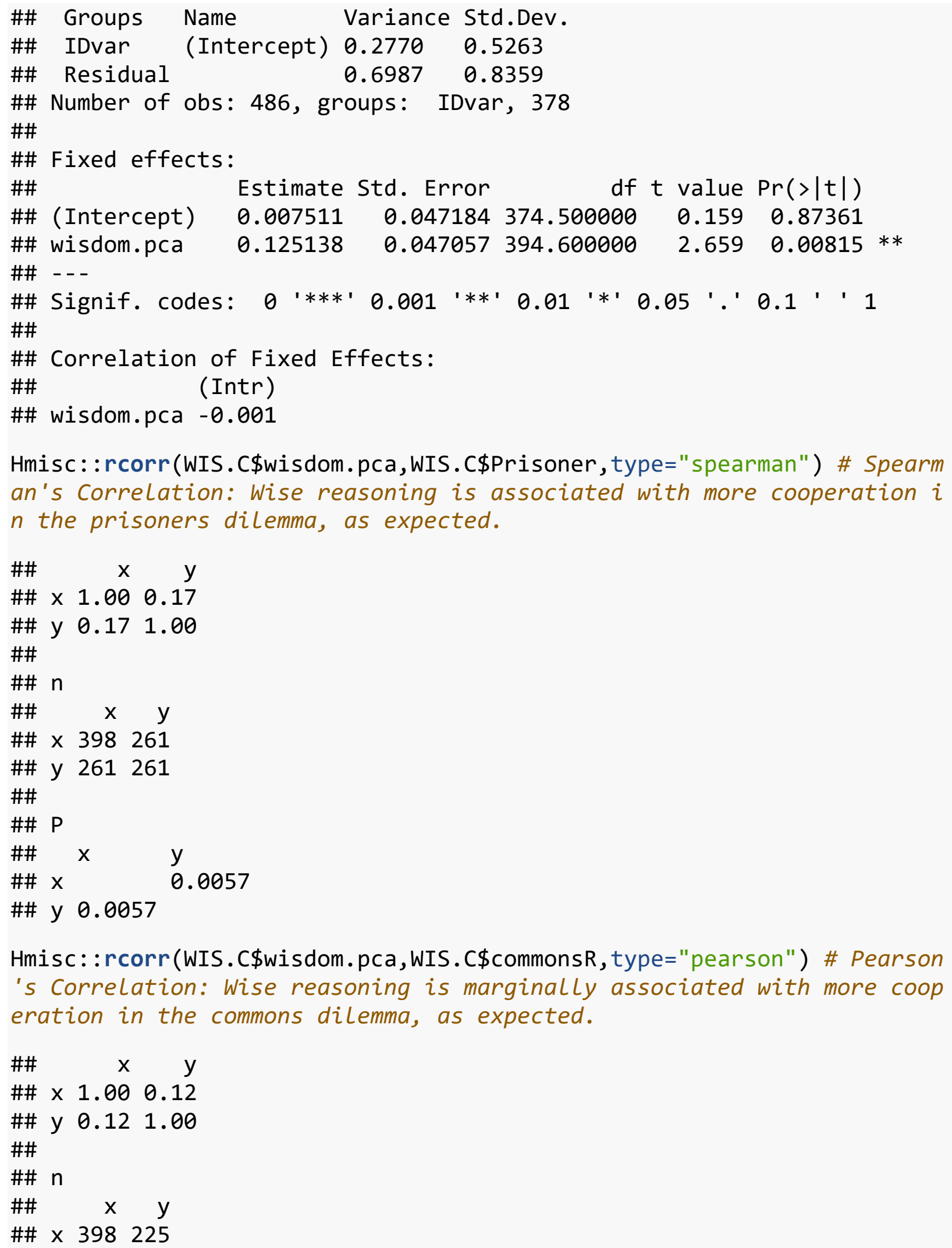




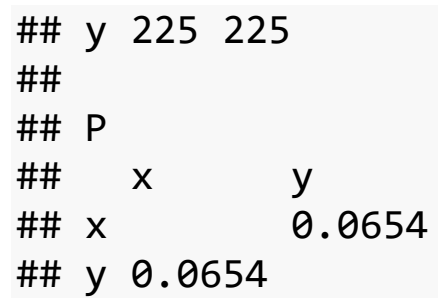

Plots for social dilemma games

ggplot(WIS.C, aes ( $x=$ wisdom.pca, $y=$ Prisoner $)$ ) +geom_point ( )+labs ( $x=$ "Wise R easoning", $\mathrm{y}=$ "Prisoner's Dilemma")+

stat_smooth (method="glm", se=TRUE, method.args=list(family="binomial") )+theme_classic()+ggtitle("A") \# Log Likelihood plot for Prisoner's Di Lemma - Look at positive slope, indicating greater cooperation with gr eater wise reasoning

\#\# Warning: Removed 137 rows containing non-finite values (stat_smooth ).

\#\# Warning: Removed 137 rows containing missing values (geom_point).

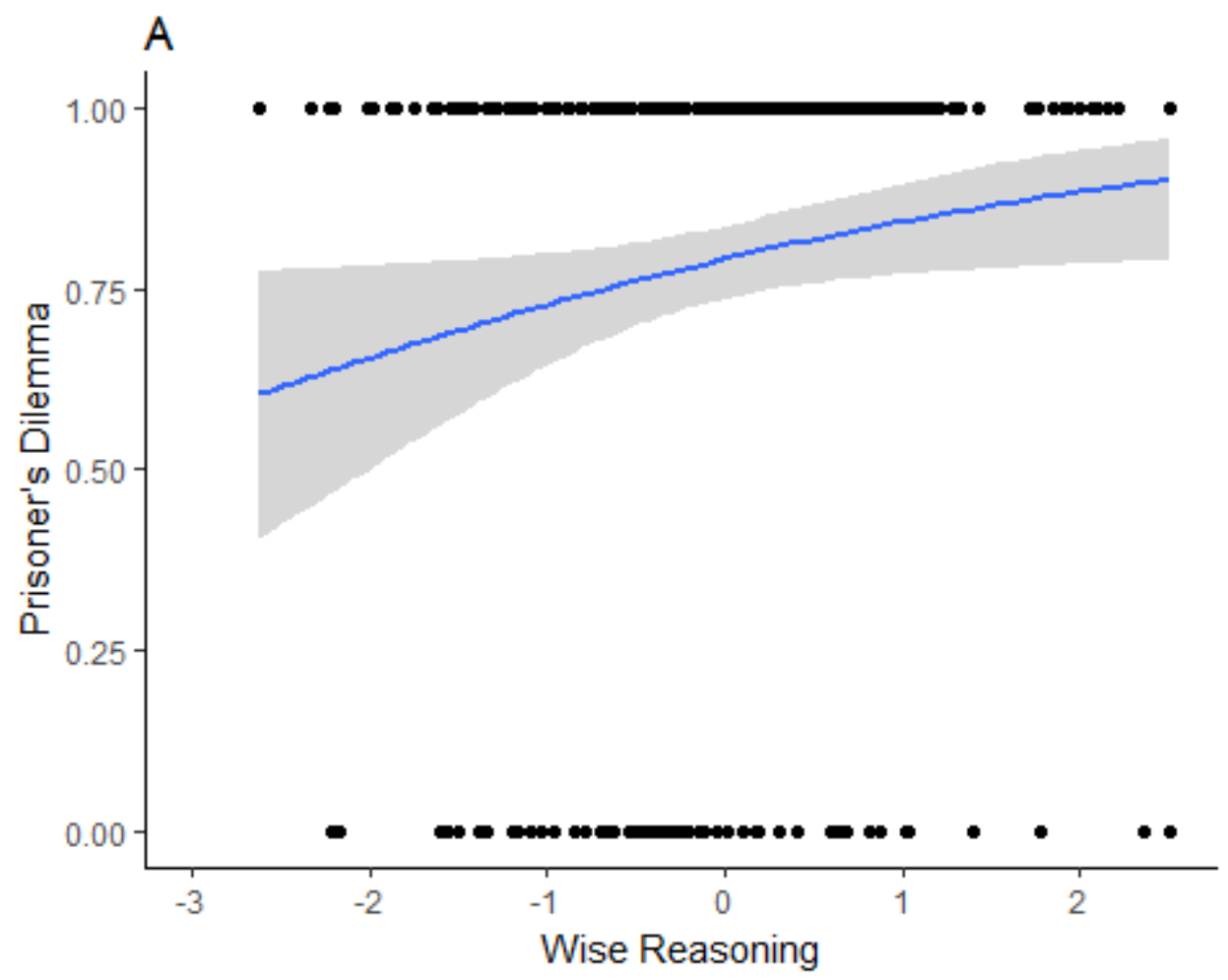

qplot (wisdom.pca, 400-commons, data=WIS.C, geom="hex")+stat_smooth (method ="lm", method.args=list (family="gaussian"), se=TRUE)+ theme_classic( )+ theme (legend. position $=c(0.89,0.27)$, legend. background=element_rect $(\mathrm{si}$ 


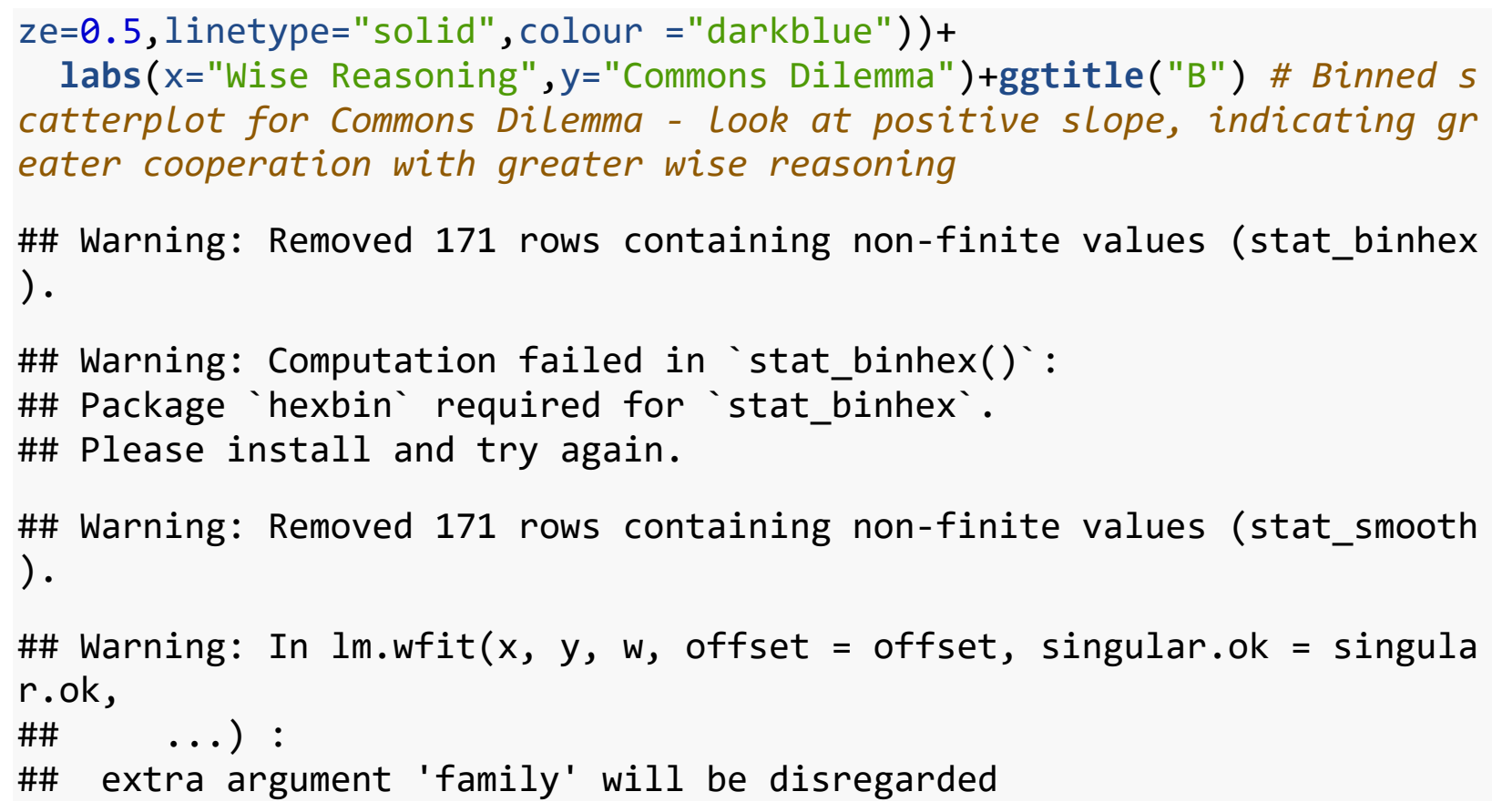

\#\# Warning: Removed 171 rows containing non-finite values (stat_binhex ) .

\#\# Warning: Computation failed in 'stat_binhex( $)^{\prime}$ :

\#\# Package 'hexbin' required for 'stat_binhex'.

\#\# Please install and try again.

\#\# Warning: Removed 171 rows containing non-finite values (stat_smooth ) .

\#\# Warning: In lm.wfit(x, $y, w$, offset $=$ offset, singular.ok = singula r.ok, \#\# (..) :

\#\# extra argument 'family' will be disregarded

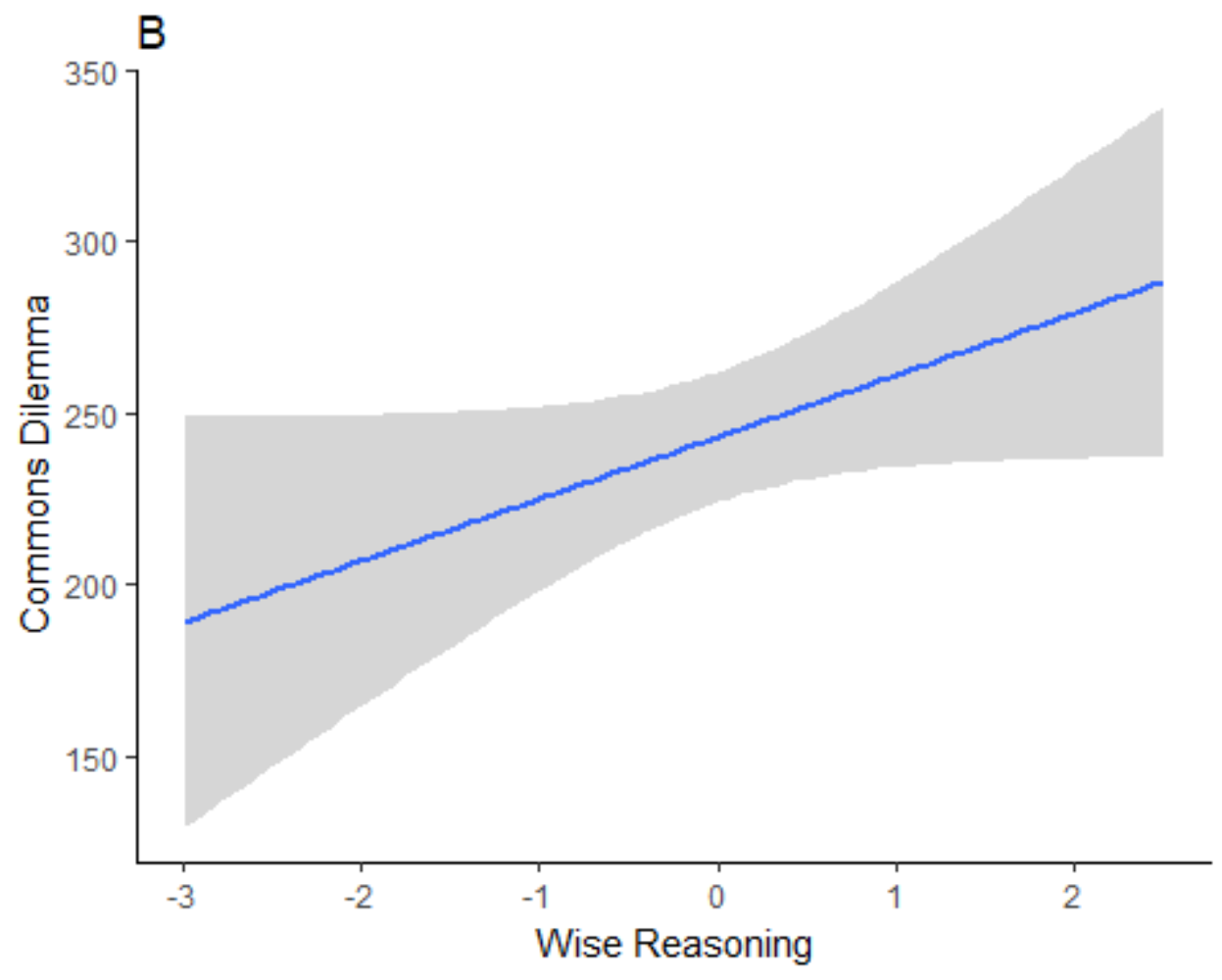

S5B: CONFLICT RESOLUTION BEHAVIORS Pearson correlations with wise reasoning and its five aspects (see Table 8) 
\# Each Line of code presents 3 Lists (Pearson's $r$, sample size, $p$-valu e)

\# Wise reasoning variable labels: Wise reasoning latent component (wis dom.pca), Wise reasoning intergroup (wisdom.IG.pca), Global measure of wise reasoning (GLobalW)

\# Wise reasoning aspects variable Labels: Perspective (Perspective.ave ), Change/outcomes (Changeoutcomes.ave), Limits (Limits.ave), Compromi se/resolution (CompResolve.ave), Outsider (Outsider.ave). Wise reasoni ng intergroup aspects all end with. IG.ave

\# Conflict resolution behavior variable labels: Find someone to hear $b$ oth sides (seeklistener), Find someone to give advice (seekadvice), So Lve problem together (solvetogether), Forgive, Disengage, Retaliate, $T$ ry to find ally (gangup)

\# As expected, wise reasoning is positively associated with seeklisten er, seekadvice, solvetogether, forgive

\# As expected, wise reasoning is negatively associated with disengage and retaliate. We expected a negative correlation with gangup, but the re was a negligible correlation

Hmisc: :rcorr(as.matrix(WIS.S5[, c('wisdom.pca', 'Perspective.ave', 'Chan geOutcomes.ave', 'Limits.ave', 'CompResolve.ave', ' 'Outsider.ave', 'seeklistener', 'seekadvice', ' solveto gether', 'forgive', 'disengage' , 'retaliate', 'gangup' )]), type="spearman") \# Correlation with wise reasoning

$\begin{array}{lrrr}\text { \#\# } & \text { wisdom.pca } & \text { Perspective.ave } & \text { ChangeOutcomes.ave } \\ \text { \#\# wisdom.pca } & 1.00 & 0.79 & 0.70 \\ \text { \#\# Perspective.ave } & 0.79 & 1.00 & 0.45 \\ \text { \#\# ChangeOutcomes.ave } & 0.70 & 0.45 & 1.00 \\ \text { \#\# Limits.ave } & 0.79 & 0.58 & 0.43 \\ \text { \#\# CompResolve.ave } & 0.79 & 0.61 & 0.56 \\ \text { \#\# Outsider.ave } & 0.62 & 0.37 & 0.33 \\ \text { \#\# seeklistener } & 0.15 & 0.07 & 0.09 \\ \text { \#\# seekadvice } & 0.17 & 0.11 & 0.12 \\ \text { \#\# solvetogether } & 0.22 & 0.21 & 0.13 \\ \text { \#\# forgive } & 0.07 & 0.11 & 0.06 \\ \text { \#\# disengage } & -0.11 & -0.17 & -0.08 \\ \text { \#\# retaliate } & -0.13 & -0.14 & -0.11 \\ \text { \#\# gangup } & 0.01 & -0.03 & 0.01 \\ \text { \#\# } & \text { Limits.ave CompResolve.ave Outsider.ave seeklist } \\ \text { ener } & & & \\ \text { \#\# wisdom.pca } & 0.79 & 0.79 & 0.62 \\ \text { 0.15 } & & & \\ \text { \#\# Perspective.ave } & 0.58 & 0.61 & 0.37 \\ \text { 0.07 } & & & \end{array}$




\begin{tabular}{|c|c|c|c|c|c|}
\hline \multicolumn{6}{|l|}{0.09} \\
\hline \#\# Limits.ave & 1.00 & \multicolumn{2}{|c|}{0.53} & \multicolumn{2}{|l|}{0.45} \\
\hline \multicolumn{6}{|l|}{0.12} \\
\hline \#\# CompResolve.ave & 0.53 & \multicolumn{2}{|c|}{1.00} & \multicolumn{2}{|l|}{0.33} \\
\hline \multicolumn{6}{|l|}{0.08} \\
\hline \#\# Outsider.ave & 0.45 & \multicolumn{2}{|c|}{0.33} & \multicolumn{2}{|l|}{1.00} \\
\hline \multicolumn{6}{|l|}{0.17} \\
\hline \#\# seeklistener & 0.12 & \multicolumn{2}{|c|}{0.08} & \multicolumn{2}{|l|}{0.17} \\
\hline \multicolumn{6}{|l|}{1.00} \\
\hline \#\# seekadvice & 0.15 & \multicolumn{2}{|c|}{0.12} & \multicolumn{2}{|l|}{0.16} \\
\hline \multicolumn{6}{|l|}{0.30} \\
\hline \#\# solvetogether & 0.14 & \multicolumn{2}{|c|}{0.29} & \multicolumn{2}{|l|}{0.05} \\
\hline \multicolumn{6}{|l|}{0.12} \\
\hline \#\# forgive & 0.04 & \multicolumn{2}{|c|}{0.09} & \multicolumn{2}{|l|}{-0.02} \\
\hline \multicolumn{6}{|l|}{0.05} \\
\hline \#\# disengage & -0.06 & \multicolumn{2}{|c|}{-0.13} & \multicolumn{2}{|l|}{0.03} \\
\hline \multicolumn{6}{|l|}{0.10} \\
\hline \#\# retaliate & -0.11 & \multicolumn{2}{|c|}{-0.13} & \multicolumn{2}{|l|}{-0.04} \\
\hline 0.05 & & & & & \\
\hline \#\# gangup & 0.00 & -0.01 & & 0.06 & \\
\hline 0.21 & & & & & \\
\hline \#\# & seekadvice & solvetogether $f$ & orgive & disengage & retal \\
\hline iate & & & & & \\
\hline \#\# wisdom.pca & 0.17 & 0.22 & 0.07 & -0.11 & - \\
\hline 0.13 & & & & & \\
\hline $\begin{array}{l}\text { \#\# Perspective.ave } \\
0.14\end{array}$ & 0.11 & 0.21 & 0.11 & -0.17 & - \\
\hline $\begin{array}{l}\text { \#\# ChangeOutcomes.ave } \\
0.11\end{array}$ & 0.12 & 0.13 & 0.06 & -0.08 & - \\
\hline $\begin{array}{l}\# \# \text { Limits.ave } \\
0.11\end{array}$ & 0.15 & 0.14 & 0.04 & -0.06 & - \\
\hline $\begin{array}{l}\text { \#\# CompResolve.ave } \\
0.13\end{array}$ & 0.12 & 0.29 & 0.09 & -0.13 & - \\
\hline $\begin{array}{l}\# \# \text { Outsider.ave } \\
0.04\end{array}$ & 0.16 & 0.05 & -0.02 & 0.03 & - \\
\hline $\begin{array}{l}\text { \#\# seeklistener } \\
0.05\end{array}$ & 0.30 & 0.12 & -0.05 & 0.10 & \\
\hline \#\# seekadvice & 1.00 & 0.16 & -0.02 & 0.10 & \\
\hline 0.02 & & & & & \\
\hline \#\# solvetogether & 0.16 & 1.00 & 0.01 & -0.19 & - \\
\hline 0.07 & & & & & \\
\hline \#\# forgive & -0.02 & 0.01 & 1.00 & -0.21 & - \\
\hline 0.09 & & & & & \\
\hline \#\# disengage & 0.10 & -0.19 & -0.21 & 1.00 & \\
\hline
\end{tabular}




\begin{tabular}{|c|c|c|c|c|}
\hline \multicolumn{5}{|l|}{0.00} \\
\hline \#\# retaliate & 0.02 & -0.07 & -0.09 & .00 \\
\hline \multicolumn{5}{|l|}{1.00} \\
\hline \#\# gangup & 0.18 & 0.05 & -0.05 & .08 \\
\hline \multicolumn{5}{|l|}{0.11} \\
\hline \#\# & \multicolumn{4}{|l|}{ gangup } \\
\hline \#\# wisdom.pca & \multicolumn{4}{|l|}{0.01} \\
\hline \#\# Perspective.ave & \multicolumn{4}{|l|}{-0.03} \\
\hline \#\# ChangeOutcomes.ave & \multicolumn{4}{|l|}{0.01} \\
\hline \#\# Limits.ave & \multicolumn{4}{|l|}{0.00} \\
\hline \#\# CompResolve.ave & \multicolumn{4}{|l|}{-0.01} \\
\hline \#\# Outsider.ave & \multicolumn{4}{|l|}{0.06} \\
\hline \#\# seeklistener & \multicolumn{4}{|l|}{0.21} \\
\hline \#\# seekadvice & \multicolumn{4}{|l|}{0.18} \\
\hline \#\# solvetogether & \multicolumn{4}{|l|}{0.05} \\
\hline \#\# forgive & \multicolumn{4}{|l|}{-0.05} \\
\hline \#\# disengage & \multicolumn{4}{|l|}{0.08} \\
\hline \#\# retaliate & \multicolumn{4}{|l|}{0.11} \\
\hline \#\# gangup & \multirow{2}{*}{\multicolumn{4}{|c|}{1.00}} \\
\hline \multicolumn{2}{|l|}{ \#\# } & & & \\
\hline \multicolumn{5}{|l|}{$\# \# \mathrm{n}$} \\
\hline \#\# & wisdom.pca & Perspective.ave & \multicolumn{2}{|c|}{ ChangeOutcomes.ave } \\
\hline \#\# wisdom.pca & 2581 & 2580 & & 2579 \\
\hline \#\# Perspective.ave & 2580 & 2580 & & 2579 \\
\hline \#\# ChangeOutcomes.ave & 2579 & 2579 & & 2579 \\
\hline \#\# Limits.ave & 2578 & 2577 & & 2577 \\
\hline \#\# CompResolve.ave & 2566 & 2566 & & 2566 \\
\hline \#\# Outsider.ave & 2561 & 2561 & & 2561 \\
\hline \#\# seeklistener & 1897 & 1896 & & 1896 \\
\hline \#\# seekadvice & 1897 & 1896 & & 1896 \\
\hline \#\# solvetogether & 1897 & 1896 & & 1896 \\
\hline \#\# forgive & 1897 & 1896 & & 1896 \\
\hline \#\# disengage & 1897 & 1896 & & 1896 \\
\hline \#\# retaliate & 1503 & 1502 & & 1502 \\
\hline \#\# gangup & 1897 & 1896 & & 1896 \\
\hline $\begin{array}{l}\# \# \\
\text { ener }\end{array}$ & Limits.ave & CompResolve.ave & Outsider.ave & seeklist \\
\hline \#\# wisdom.pca & 2578 & 2566 & 2561 & \\
\hline 1897 & & & & \\
\hline \#\# Perspective.ave & 2577 & 2566 & 2561 & \\
\hline 1896 & & & & \\
\hline \#\# ChangeOutcomes.ave & 2577 & 2566 & 2561 & \\
\hline 1896 & & & & \\
\hline \#\# Limits.ave & 2578 & 2566 & 2561 & \\
\hline
\end{tabular}




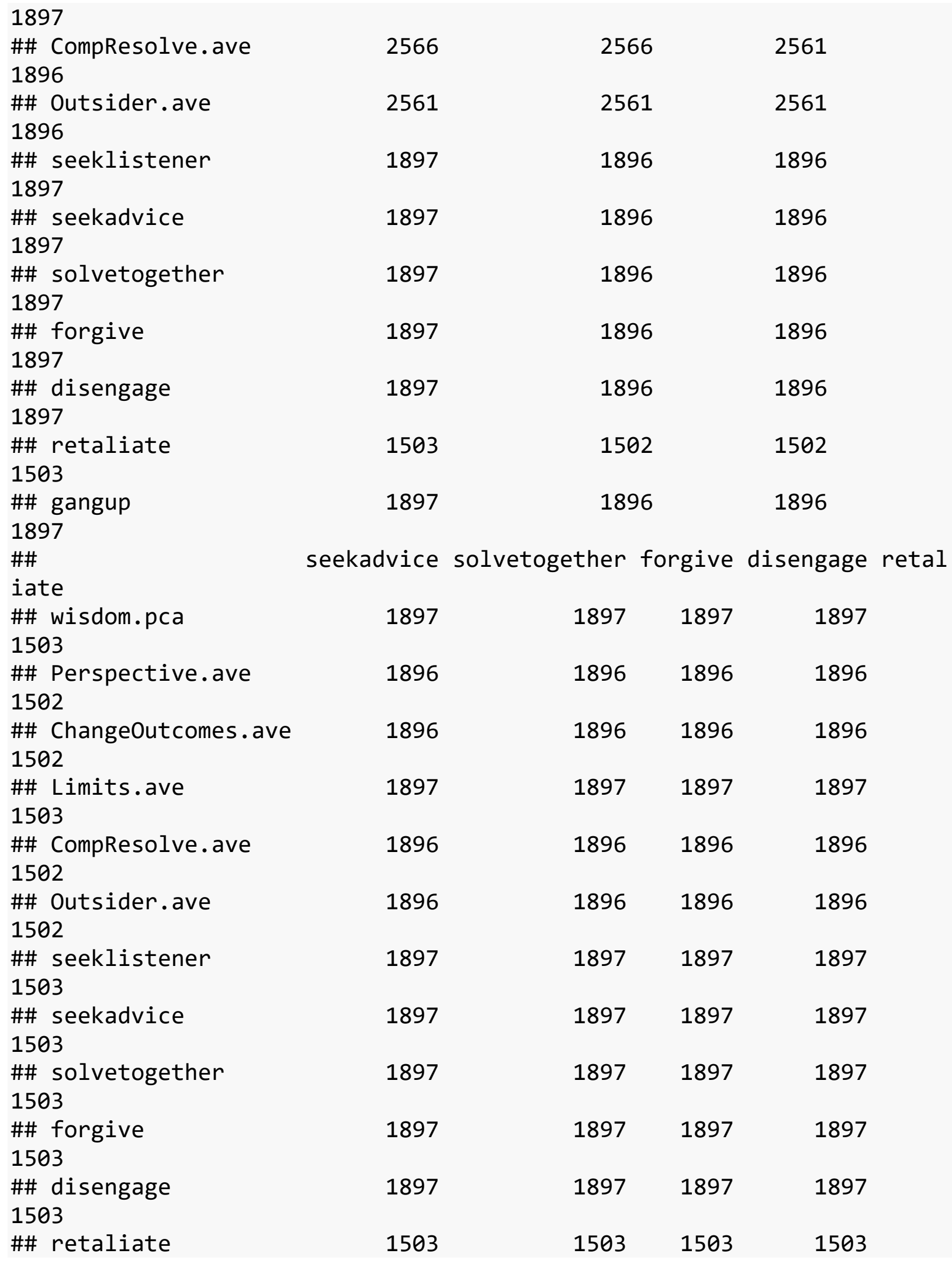




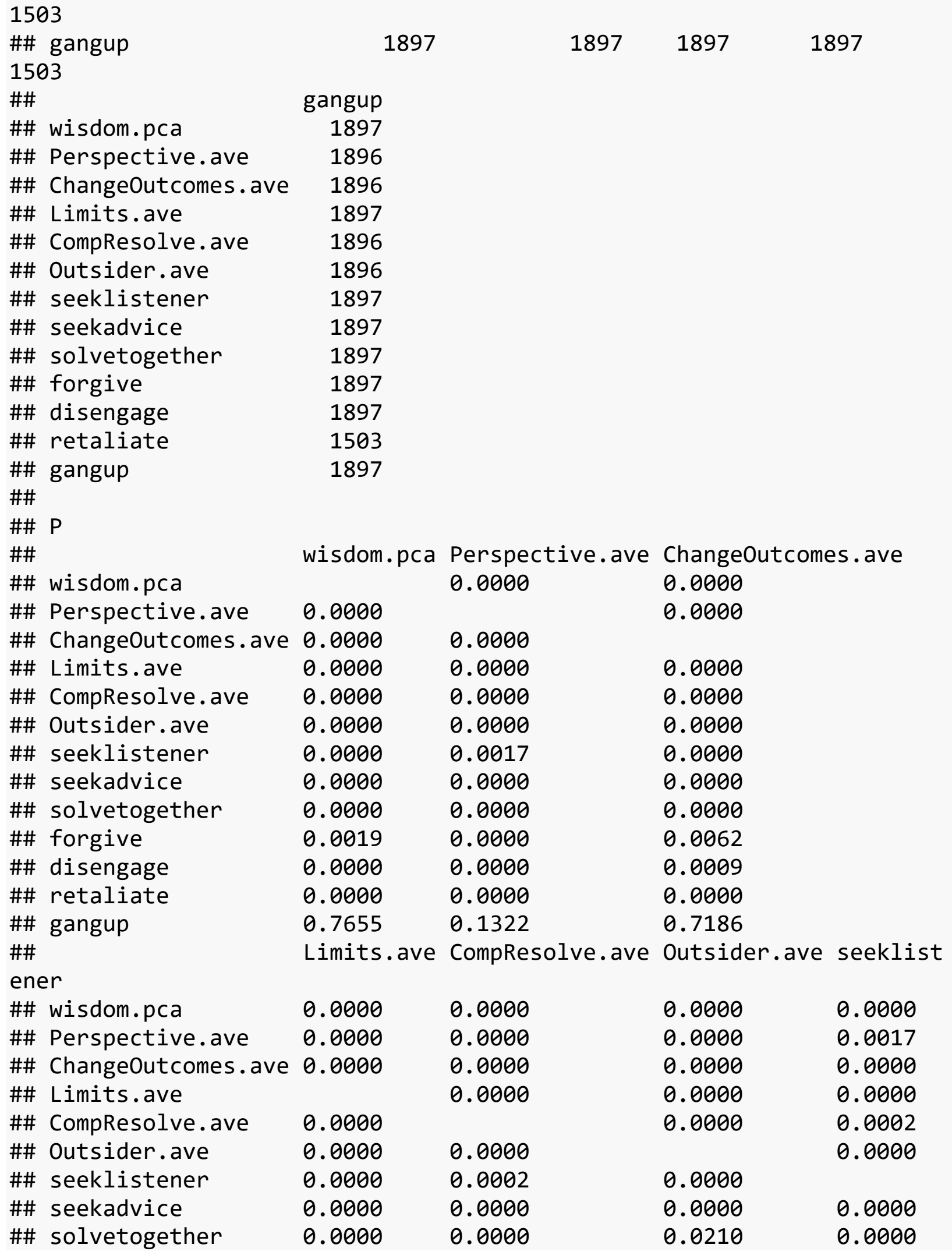




\begin{tabular}{|c|c|c|c|c|c|}
\hline \#\# forgive & 0.0653 & 0.0002 & 0.3769 & & .0457 \\
\hline \#\# disengage & 0.0114 & 0.0000 & $0.246 \epsilon$ & & .0000 \\
\hline \#\# retaliate & 0.0000 & 0.0000 & 0.1239 & & .0442 \\
\hline \#\# gangup & 0.9657 & 0.7634 & 0.0154 & & .0000 \\
\hline $\begin{array}{l}\# \# \\
\text { iate }\end{array}$ & seekadvice & solvetogether & forgive & disengage & e retal \\
\hline $\begin{array}{l}\text { \#\# wisdom.pca } \\
\theta\end{array}$ & 0.0000 & 0.0000 & 0.0019 & 0.0000 & 0.000 \\
\hline $\begin{array}{l}\text { \#\# Perspective.ave } \\
0\end{array}$ & 0.0000 & 0.0000 & 0.0000 & 0.0000 & 0.000 \\
\hline $\begin{array}{l}\# \# \text { ChangeOutcomes.ave } \\
\theta\end{array}$ & 0.0000 & 0.0000 & 0.0062 & 0.0009 & 0.000 \\
\hline $\begin{array}{l}\# \# \text { Limits.ave } \\
\theta\end{array}$ & 0.0000 & 0.0000 & 0.0653 & 0.0114 & 0.000 \\
\hline $\begin{array}{l}\text { \#\# CompResolve.ave } \\
\theta\end{array}$ & 0.0000 & 0.0000 & 0.0002 & 0.0000 & 0.000 \\
\hline $\begin{array}{l}\text { \#\# Outsider.ave } \\
9\end{array}$ & 0.0000 & 0.0210 & 0.3769 & 0.2466 & 0.123 \\
\hline $\begin{array}{l}\text { \#\# seeklistener } \\
2\end{array}$ & 0.0000 & 0.0000 & 0.0457 & 0.0000 & 0.044 \\
\hline $\begin{array}{l}\text { \#\# seekadvice } \\
6\end{array}$ & & 0.0000 & 0.2991 & 0.0000 & 0.506 \\
\hline $\begin{array}{l}\text { \#\# solvetogether } \\
5\end{array}$ & 0.0000 & & 0.7539 & 0.0000 & 0.006 \\
\hline $\begin{array}{l}\text { \#\# forgive } \\
9\end{array}$ & 0.2991 & 0.7539 & & 0.0000 & 0.000 \\
\hline $\begin{array}{l}\# \# \text { disengage } \\
8\end{array}$ & 0.0000 & 0.0000 & 0.0000 & & 0.986 \\
\hline \#\# retaliate & 0.5066 & 0.0065 & 0.0009 & 0.9868 & \\
\hline $\begin{array}{l}\# \# \text { gangup } \\
0\end{array}$ & 0.0000 & 0.0322 & 0.0365 & 0.0008 & 0.000 \\
\hline \#\# & gangup & & & & \\
\hline \#\# wisdom.pca & 0.7655 & & & & \\
\hline \#\# Perspective.ave & 0.1322 & & & & \\
\hline \#\# ChangeOutcomes.ave & 0.7186 & & & & \\
\hline \#\# Limits.ave & 0.9657 & & & & \\
\hline \#\# CompResolve.ave & 0.7634 & & & & \\
\hline \#\# Outsider.ave & 0.0154 & & & & \\
\hline \#\# seeklistener & 0.0000 & & & & \\
\hline \#\# seekadvice & 0.0000 & & & & \\
\hline \#\# solvetogether & 0.0322 & & & & \\
\hline \#\# forgive & 0.0365 & & & & \\
\hline \#\# disengage & 0.0008 & & & & \\
\hline
\end{tabular}


\#\# retaliate

0.0000

\#\# gangup

Partial correlations between conflict resolution behavior and wise reasoning, controlling for age, gender, socially desirable responding, and individual differences

\# Effects generally hold across all these analyses

Partial correlations between wise reasoning and seeking someone to listen to both sides (solvetogether)

\# Run PCOR and ppcor::pcor.test Lines as a pair (to create the dataset and then run partial correlations)

PCOR<-na.omit(WIS [, c("wisdom.pca", "seeklistener", "Age")]) ppcor: : pcor . test (PCOR \$wisdom.pca, PCOR \$seeklistener, PCOR \$Age, method="sp earman") \# Age

\#\# estimate p.value statistic $\mathrm{n}$ gp Method

\#\# $10.1467533 \quad 0.00000000013873596 .4532131895 \quad 1$ spearman

PCOR<-na.omit(WIS [, c("wisdom.pca", "seeklistener", "genderN")])

ppcor: : pcor . test (PCOR\$wisdom. pca, PCOR \$seeklistener, PCOR\$genderN, method ="spearman") \# Gender

\#\# estimate p.value statistic $\mathrm{n}$ gp Method

\#\# $10.1486858 \quad 0.00000000007978304 \quad 6.5383751894 \quad 1$ spearman

PCOR<-na.omit(WIS [, c("wisdom.pca", "seeklistener", "Order")])

ppcor: : pcor . test (PCOR\$wisdom . pca, PCOR \$seeklistener, PCOR \$Order, method=" spearman") \# Presentation Order

\#\# estimate p.value statistic $\mathrm{n}$ gp Method

\#\# $10.1503866 \quad 0.0000000000465913 \quad 6.620131 \quad 1897 \quad 1$ spearman

PCOR<-na.omit(WIS [, c ("wisdom.pca", "seeklistener", "BIDRImpMGMT")])

ppcor: : pcor .test (PCOR\$wisdom. pca, PCOR\$seeklistener, PCOR\$BIDRImpMGMT, me thod="spearman") \# BIDR: Impression management

\#\# estimate p.value statistic $\mathrm{n}$ gp Method

\#\# $10.14851740 .00006706716 \quad 4.010193716 \quad 1$ spearman

PCOR<-na.omit(WIS [, c ("wisdom.pca", "seeklistener", "BIDRSelfDec")])

ppcor: : pcor . test (PCOR\$wisdom. pca, PCOR\$seeklistener, PCOR\$BIDRSelfDec, me thod="spearman") \# BIDR: Self-deception

\#\# estimate p.value statistic $\mathrm{n}$ gp Method

\#\# $10.1449078 \quad 0.0001008566 \quad 3.910612716 \quad 1$ spearman 
PCOR<-na.omit(WIS [, c("wisdom.pca", "seeklistener", "Openness")])

ppcor: : pcor .test (PCOR\$wisdom.pca, PCOR\$seeklistener, PCOR\$Openness, metho $\mathrm{d}=$ "spearman") \# Openness

\#\# estimate p.value statistic $\mathrm{n}$ gp Method

\#\# $10.1492216 \quad 0.00000574628 \quad 4.562415917 \quad 1$ spearman

PCOR<-na.omit(WIS [, c("wisdom.pca", "seeklistener", "Conscientiousness")] )

ppcor: : pcor .test (PCOR\$wisdom.pca, PCOR\$seeklistener, PCOR \$Conscientiousn ess, method="spearman") \# Conscientiousness

\#\# estimate p.value statistic $\mathrm{n}$ gp Method

\#\# $10.14872120 .00000618005 \quad 4.546768917 \quad 1$ spearman

PCOR<-na.omit(WIS [, c("wisdom.pca", "seeklistener", "Extraversion")])

ppcor: : pcor .test (PCOR\$wisdom. pca, PCOR\$seeklistener, PCOR\$Extraversion, $m$ ethod="spearman") \# Extraversion

\#\# estimate p.value statistic $\mathrm{n}$ gp Method

\#\# $10.1503268 \quad 0.000004831114 \quad 4.599496918 \quad 1$ spearman

PCOR<-na.omit(WIS [, c ("wisdom.pca", "seeklistener", "Agreeableness")]) ppcor: : pcor.test (PCOR\$wisdom. pca, PCOR\$seeklistener, PCOR\$Agreeableness, method="spearman") \# Agreeableness

\#\# estimate p.value statistic $\mathrm{n}$ gp Method \#\# $10.1490096 \quad 0.00000592636 \quad 4.555786917 \quad 1$ spearman

PCOR<-na.omit(WIS [, c ("wisdom.pca", "seeklistener", "Neuroticism")]) ppcor: : pcor .test (PCOR\$wisdom. pca, PCOR\$seeklistener, PCOR\$Neuroticism, me thod="spearman") \# Neuroticism

\#\# estimate p.value statistic $\mathrm{n}$ gp Method

\#\# $10.1471805 \quad 0.000007720637 \quad 4.498617 \quad 917 \quad 1$ spearman

PCOR<-na.omit(WIS [, c("wisdom.pca", "seeklistener", "Mindful_1")])

ppcor: : pcor.test(PCOR\$wisdom.pca, PCOR\$seeklistener, PCOR\$Mindful_1, meth od="spearman") \# Mindfulness: Nonreactivity

\#\# estimate p.value statistic $\mathrm{n}$ gp Method

\#\# $10.12036690 .07348372 \quad 1.798405223 \quad 1$ spearman

PCOR<-na.omit(WIS [, c("wisdom.pca", "seeklistener", "Mindful_2")])

ppcor: : pcor.test(PCOR\$wisdom.pca, PCOR\$seeklistener, PCOR\$Mindful_2, meth od="spearman") \# MindfuLness: Observing and attending 
\#\# estimate p.value statistic $\mathrm{n}$ gp Method \#\# $10.06940524 \quad 0.30323591 .031935223 \quad 1$ spearman

PCOR<-na.omit(WIS [, c("wisdom.pca", "seeklistener", "Mindful_3")])

ppcor: : pcor.test(PCOR\$wisdom.pca, PCOR\$seeklistener, PCOR\$Mindful_3, meth od="spearman") \# Mindfulness: Acting with awareness

\#\# estimate p.value statistic $\mathrm{n}$ gp Method

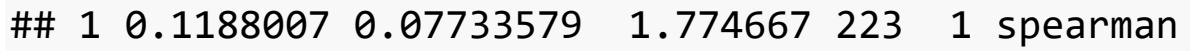

PCOR<-na.omit(WIS [, c("wisdom.pca", "seeklistener", "Mindful_4")])

ppcor: : pcor.test (PCOR\$wisdom.pca, PCOR\$seeklistener, PCOR\$Mindful_4, meth od="spearman") \# Mindfulness: Describing with words

\#\# estimate p.value statistic $\mathrm{n}$ gp Method

\#\# $10.098534990 .1451905 \quad 1.461966221 \quad 1$ spearman

PCOR<-na.omit(WIS [, c("wisdom.pca", "seeklistener", "Mindful_5")])

ppcor: : pcor .test (PCOR\$wisdom.pca, PCOR\$seeklistener, PCOR\$Mindful_5, meth od="spearman") \# Mindfulness: Non-judging of experience

\#\# estimate p.value statistic $\mathrm{n}$ gp Method

\#\# $10.1241092 \quad 0.06676821 \quad 1.842487220 \quad 1$ spearman

PCOR<-na.omit(WIS[, c("wisdom.pca", "seeklistener", "Intellect_1")])

ppcor: : pcor.test (PCOR\$wisdom.pca, PCOR\$seeklistener, PCOR $\$$ Intellect_1, me thod="spearman") \# Intellect: Seek

\#\# estimate p.value statistic $\mathrm{n}$ gp Method

\#\# $10.08078303 \quad 0.2338165 \quad 1.19391220 \quad 1$ spearman

PCOR<-na.omit(WIS [, c("wisdom.pca", "seeklistener", "Intellect_2")])

ppcor: : pcor . test (PCOR \$wisdom. pca, PCOR\$seeklistener, PCOR\$Intellect_2, me thod="spearman") \# Intellect: Conquer

\#\# estimate p.value statistic $\mathrm{n}$ gp Method

\#\# $10.067143850 .3260053 \quad 0.9844515 \quad 217 \quad 1$ spearman

PCOR<-na.omit(WIS [, c("wisdom.pca", "seeklistener", "ATT")])

ppcor: : pcor . test (PCOR\$wisdom.pca, PCOR\$seeklistener, PCOR \$ATT, method="sp earman") \# Attributional complexity

\#\# estimate p.value statistic $\mathrm{n}$ gp Method

\#\# $10.12088630 .07556758 \quad 1.785637218 \quad 1$ spearman

PCOR<-na.omit(WIS [, c("wisdom.pca", "seeklistener", "EmoReg_1")])

ppcor: : pcor .test (PCOR\$wisdom.pca, PCOR\$seeklistener, PCOR\$EmoReg_1, metho $\mathrm{d}=$ "spearman") \# Emotion regulation: Reappraisal 
\#\# estimate p.value statistic $\mathrm{n}$ gp Method

\#\# $10.125535 \quad 0.06617329 \quad 1.846732216 \quad 1$ spearman

PCOR<-na.omit(WIS [, c("wisdom.pca", "seeklistener", "EmoReg_2")])

ppcor: : pcor.test (PCOR\$wisdom.pca,PCOR\$seeklistener,PCOR\$EmoReg_2, metho $\mathrm{d}=$ "spearman") \# Emotion regulation: Suppression

\#\# estimate p.value statistic $\mathrm{n}$ gp Method

\#\# $10.12678890 .0634893 \quad 1.865479216 \quad 1$ spearman

PCOR<-na.omit(WIS [, c("wisdom.pca", "seeklistener", "EISelfEmotions")])

ppcor: : pcor . test (PCOR\$wisdom.pca, PCOR\$seeklistener, PCOR \$EISelfEmotions , method="spearman") \# Emotional intelligence: Self-emotion appraisal

\#\# estimate p.value statistic $\mathrm{n}$ gp Method

\#\# $10.1469096 \quad 0.00009799482 \quad 3.918254699 \quad 1$ spearman

PCOR<-na.omit(WIS [, c("wisdom.pca", "seeklistener", "EIOthersEmotions")]) ppcor: : pcor . test (PCOR\$wisdom.pca, PCOR \$seeklistener, PCOR\$EIOthersEmotio ns,method="spearman") \# Emotional intelligence: Others-emotion apprais al

\#\# estimate p.value statistic $\mathrm{n}$ gp Method \#\# $10.1386121 \quad 0.0002419542 \quad 3.689829698 \quad 1$ spearman

PCOR<-na.omit(WIS [, c("wisdom.pca", "seeklistener", "EIEmotionUse")]) ppcor: : pcor . test (PCOR \$wisdom. pca, PCOR \$seeklistener, PCOR \$EIEmotionUse, $m$ ethod="spearman") \# Emotional intelligence: Use of emotion

\#\# estimate p.value statistic $\mathrm{n}$ gp Method \#\# $10.1472585 \quad 0.00009538178 \quad 3.924943 \quad 698 \quad 1$ spearman

PCOR<-na.omit(WIS [, c("wisdom.pca", "seeklistener", "EIEmotionReg")]) ppcor: : pcor.test (PCOR\$wisdom.pca, PCOR \$seeklistener, PCOR \$EIEmotionReg, $m$ ethod="spearman") \# Emotional intelligence: Regulation of emotion

\#\# estimate p.value statistic $\mathrm{n}$ gp Method \#\# $10.156095 \quad 0.00003487837 \quad 4.166178698 \quad 1$ spearman

PCOR<-na.omit(WIS[, c("wisdom.pca", "seeklistener", "Reflection")]) ppcor: : pcor . test (PCOR\$wisdom.pca, PCOR\$seeklistener, PCOR \$Reflection, met hod="spearman") \# Ruminative response: Reflection

\#\# estimate p.value statistic $n$ gp Method \#\# $10.1388103 \quad 0.0002369853 \quad 3.695209698 \quad 1$ spearman 
PCOR<-na.omit(WIS [, c("wisdom.pca", "seeklistener", "Brooding")])

ppcor: : pcor .test (PCOR\$wisdom.pca, PCOR\$seeklistener, PCOR \$Brooding, metho $\mathrm{d}=$ "spearman")\# Ruminative response: Brooding

\#\# estimate p.value statistic $\mathrm{n}$ gp Method

\#\# $10.1317344 \quad 0.0004887396 \quad 3.503428698 \quad 1$ spearman

Partial correlations between wise reasoning and seeking advice (seekadvice)

\# Run PCOR and ppcor::pcor.test lines as a pair (to create the dataset and then run partial correlations)

PCOR<-na.omit(WIS [, c ("wisdom.pca", "seekadvice", "Age")])

ppcor: : pcor .test (PCOR\$wisdom. pca, PCOR\$seekadvice, PCOR\$Age, method="spea rman") \# Age

\#\# estimate p.value statistic $\mathrm{n}$ gp Method

\#\# $10.17059120 .00000000000007773692 \quad 7.5306121895 \quad 1$ spearman

PCOR<-na.omit(WIS [, c ("wisdom.pca", "seekadvice", "genderN")])

ppcor: : pcor . test (PCOR\$wisdom. pca, PCOR\$seekadvice, PCOR\$genderN, method=" spearman") \# Gender

\#\# estimate p.value statistic $\mathrm{n}$ gp Method

\#\# $10.17069960 .00000000000007607498 \quad 7.5335481894 \quad 1$ spearman

PCOR<-na.omit(WIS [, c("wisdom.pca", "seekadvice", "Order")])

ppcor: : pcor . test (PCOR\$wisdom. pca, PCOR\$seekadvice, PCOR\$Order, method="sp earman") \# Presentation Order

\#\# estimate p.value statistic $\mathrm{n}$ gp Method \#\# $10.17693530 .000000000000008457458 \quad 7.8236811897 \quad 1$ spearman

PCOR<-na.omit(WIS [, c("wisdom.pca", "seekadvice", "BIDRImpMGMT")]) ppcor: : pcor .test (PCOR\$wisdom. pca, PCOR\$seekadvice, PCOR\$BIDRImpMGMT, meth od="spearman") \# BIDR: Impression management

\#\# estimate p.value statistic $\mathrm{n}$ gp Method

\#\# $10.15701580 .00002471762 \quad 4.245305716 \quad 1$ spearman

PCOR<-na.omit(WIS [, c("wisdom.pca", "seekadvice", "BIDRSelfDec")]) ppcor: : pcor .test (PCOR\$wisdom. pca, PCOR\$seekadvice, PCOR\$BIDRSelfDec, meth od="spearman") \# BIDR: Self-deception

\#\# estimate p.value statistic $\mathrm{n}$ gp Method \#\# $10.15918990 .00001898493 \quad 4.305602716 \quad 1$ spearman 
PCOR<-na.omit(WIS [, c("wisdom.pca", "seekadvice", "Openness")]) ppcor: : pcor . test (PCOR\$wisdom.pca, PCOR\$seekadvice, PCOR\$Openness, method= "spearman") \# Openness

\#\# estimate p.value statistic $n$ gp Method \#\# $10.1641763 \quad 0.0000005852262 \quad 5.031725917 \quad 1$ spearman PCOR<-na.omit(WIS [, c("wisdom.pca", "seekadvice", "Conscientiousness")]) ppcor: : pcor . test (PCOR \$wisdom. pca, PCOR\$seekadvice, PCOR\$Conscientiousnes $\mathrm{s}$, method="spearman") \# Conscientiousness

\#\# estimate p.value statistic $\mathrm{n}$ gp Method \#\# $10.163987 \quad 0.0000006031999 \quad 5.025763 \quad 917 \quad 1$ spearman

PCOR<-na.omit(WIS [, c("wisdom.pca", "seekadvice", "Extraversion")]) ppcor: : pcor .test (PCOR\$wisdom. pca, PCOR\$seekadvice, PCOR\$Extraversion, met hod="spearman") \# Extraversion

\#\# estimate p.value statistic $\mathrm{n}$ gp Method \#\# $10.16521930 .0000004880245 \quad 5.067355918 \quad 1$ spearman PCOR<-na.omit(WIS [, c("wisdom.pca", "seekadvice", "Agreeableness")]) ppcor: : pcor .test (PCOR\$wisdom.pca, PCOR\$seekadvice, PCOR\$Agreeableness, me thod="spearman") \# Agreeableness

\#\# estimate p.value statistic $\mathrm{n}$ gp Method \#\# $10.1642596 \quad 0.0000005774843 \quad 5.034348917 \quad 1$ spearman

PCOR<-na.omit(WIS [, c("wisdom.pca", "seekadvice", "Neuroticism")]) ppcor: : pcor.test (PCOR\$wisdom.pca, PCOR\$seekadvice, PCOR\$Neuroticism, meth od="spearman") \# Neuroticism

\#\# estimate p.value statistic $\mathrm{n}$ gp Method \#\# $10.16452280 .0000005536555 \quad 5.04264917 \quad 1$ spearman PCOR<-na.omit(WIS [, c("wisdom.pca", "seekadvice", "Mindful_1")]) ppcor: : pcor.test (PCOR\$wisdom.pca, PCOR\$seekadvice, PCOR\$Mindful_1, method ="spearman") \# Mindfulness: Nonreactivity

\#\# estimate p.value statistic $\mathrm{n}$ gp Method \#\# $10.22230920 .0008515536 \quad 3.382008223 \quad 1$ spearman

PCOR<-na.omit(WIS [, c("wisdom.pca", "seekadvice", "Mindful_2")]) ppcor: : pcor.test (PCOR\$wisdom.pca, PCOR\$seekadvice, PCOR\$Mindful_2, method ="spearman") \# Mindfulness: Observing and attending

\#\# estimate p.value statistic $\mathrm{n}$ gp Method \#\# $10.1812665 \quad 0.00676832 \quad 2.733906223 \quad 1$ spearman 
PCOR<-na.omit(WIS [, c("wisdom.pca", "seekadvice", "Mindful_3")])

ppcor: : pcor.test (PCOR\$wisdom.pca, PCOR\$seekadvice, PCOR\$Mindful_3, method ="spearman") \# Mindfulness: Acting with awareness

\#\# estimate p.value statistic $\mathrm{n}$ gp Method

\#\# $10.2005525 \quad 0.002683414 \quad 3.036364223 \quad 1$ spearman

PCOR<-na.omit(WIS [ , c("wisdom.pca", "seekadvice", "Mindful_4")])

ppcor: : pcor.test (PCOR\$wisdom.pca, PCOR\$seekadvice, PCOR\$Mindful_4, method ="spearman") \# Mindfulness: Describing with words

\#\# estimate p.value statistic $\mathrm{n}$ gp Method \#\# $10.1878703 \quad 0.005179894 \quad 2.824158221 \quad 1$ spearman

PCOR<-na.omit(WIS [, c("wisdom.pca", "seekadvice", "MindfuI_5")])

ppcor: : pcor.test (PCOR\$wisdom.pca, PCOR\$seekadvice, PCOR\$Mindful_5, method ="spearman") \# Mindfulness: Non-judging of experience

\#\# estimate $\mathrm{p}$.value statistic $\mathrm{n}$ gp Method

\#\# $10.1835034 \quad 0.006464806 \quad 2.749869220 \quad 1$ spearman

PCOR<-na.omit(WIS [, c("wisdom.pca", "seekadvice", "Intellect_1")])

ppcor: : pcor.test (PCOR\$wisdom.pca, PCOR\$seekadvice, PCOR\$Intellect_1, meth od="spearman") \# Intellect: Seek

\#\# estimate p.value statistic $\mathrm{n}$ gp Method \#\# $10.1774542 \quad 0.0084889832 .656221220 \quad 1$ spearman

PCOR<-na.omit(WIS [, c("wisdom.pca", "seekadvice", "Intellect_2")])

ppcor: : pcor.test (PCOR\$wisdom.pca, PCOR\$seekadvice, PCOR\$Intellect_2, meth od="spearman") \# Intellect: Conquer

\#\# estimate p.value statistic $\mathrm{n}$ gp Method

\#\# $10.1848582 \quad 0.006437789 \quad 2.751667 \quad 217 \quad 1$ spearman

PCOR<-na.omit(WIS [, c("wisdom.pca", "seekadvice", "ATT")])

ppcor: : pcor . test (PCOR\$wisdom. pca, PCOR\$seekadvice, PCOR\$ATT, method=" spea rman") \# Attributional complexity

\#\# estimate p.value statistic $\mathrm{n}$ gp Method \#\# $10.1683046 \quad 0.01303915 \quad 2.503543218 \quad 1$ spearman

PCOR<-na.omit(WIS [, c("wisdom.pca", "seekadvice", "EmoReg_1")]) ppcor: : pcor .test (PCOR\$wisdom.pca, PCOR\$seekadvice, PCOR\$EmoReg_1, method= "spearman") \# Emotion regulation: Reappraisal

\#\# estimate p.value statistic $\mathrm{n}$ gp Method

\#\# $10.2120864 \quad 0.001764066 \quad 3.167353216 \quad 1$ spearman 
PCOR<-na.omit(WIS [, c("wisdom.pca", "seekadvice", "EmoReg_2")])

ppcor: : pcor.test (PCOR\$wisdom.pca, PCOR\$seekadvice, PCOR\$EmoReg_2, method= "spearman") \# Emotion regulation: Suppression

\#\# estimate p.value statistic $\mathrm{n}$ gp Method \#\# $10.18577850 .006295266 \quad 2.759385 \quad 216 \quad 1$ spearman

PCOR<-na.omit(WIS [, c("wisdom.pca", "seekadvice", "EISelfEmotions")]) ppcor: : pcor.test (PCOR\$wisdom.pca, PCOR\$seekadvice, PCOR\$EISelfEmotions, $m$ ethod="spearman") \# Emotional intelligence: Self-emotion appraisal

\#\# estimate p.value statistic $\mathrm{n}$ gp Method \#\# $10.14438760 .0001292339 \quad 3.849545699 \quad 1$ spearman

PCOR<-na.omit(WIS [, c("wisdom.pca", "seekadvice", "EIOthersEmotions")]) ppcor: : pcor .test (PCOR\$wisdom.pca, PCOR\$seekadvice, PCOR\$EIOthersEmotions , method="spearman") \# Emotional intelligence: Others-emotion appraisal

\#\# estimate p.value statistic $\mathrm{n}$ gp Method \#\# $10.14249490 .0001603293 \quad 3.795302698 \quad 1$ spearman

PCOR<-na.omit(WIS [, c("wisdom.pca", "seekadvice", "EIEmotionUse")]) ppcor: : pcor .test (PCOR\$wisdom. pca, PCOR\$seekadvice, PCOR\$EIEmotionUse, met hod="spearman") \# Emotional intelligence: Use of emotion

\#\# estimate p.value statistic $\mathrm{n}$ gp Method \#\# $10.14757290 .00009211564 \quad 3.93351698 \quad 1$ spearman

PCOR<-na.omit(WIS [, c("wisdom.pca", "seekadvice", "EIEmotionReg")]) ppcor: : pcor .test (PCOR\$wisdom. pca, PCOR\$seekadvice, PCOR\$EIEmotionReg, met hod="spearman") \# Emotional intelligence: Regulation of emotion

\#\# estimate p.value statistic $\mathrm{n}$ gp Method \#\# $10.1548168 \quad 0.00004048073 \quad 4.131223698 \quad 1$ spearman PCOR<-na.omit(WIS[, c("wisdom.pca", "seekadvice", "Reflection")]) ppcor: : pcor .test (PCOR\$wisdom. pca, PCOR\$seekadvice, PCOR\$Reflection, metho $\mathrm{d}=$ "spearman") \# Ruminative response: Reflection

\#\# estimate p.value statistic $\mathrm{n}$ gp Method \#\# $10.13422610 .0003802814 \quad 3.570898698 \quad 1$ spearman

PCOR<-na.omit(WIS [, c("wisdom.pca", "seekadvice", "Brooding")]) ppcor: : pcor .test (PCOR\$wisdom. pca, PCOR\$seekadvice, PCOR\$Brooding, method= "spearman")\# Ruminative response: Brooding

\#\# estimate p.value statistic $\mathrm{n}$ gp Method \#\# $10.1405553 \quad 0.0001971816 \quad 3.742591698 \quad 1$ spearman 
Partial correlations between wise reasoning and solving a problem together (solvetogether)

\# Run PCOR and ppcor::pcor.test lines as a pair (to create the dataset and then run partial correlations)

PCOR<-na.omit(WIS [, c("wisdom.pca", "solvetogether", "Age")])

ppcor: : pcor . test (PCOR\$wisdom. pca, PCOR\$solvetogether, PCOR\$Age, method="s pearman") \# Age

\#\# estimate p.value statistic $\mathrm{n}$ gp Meth

od

\#\# 1 0.2190727 $0.0000000000000000000005152772 \quad 9.76626918951$ spearm an

PCOR<-na.omit(WIS [, c("wisdom.pca", "solvetogether", "genderN")])

ppcor: : pcor . test (PCOR\$wisdom. pca, PCOR\$solvetogether, PCOR\$genderN, metho $\mathrm{d}=$ "spearman") \# Gender

\#\# estimate p.value statistic $\mathrm{n}$ gp Meth

od

\#\# 1 0.2224031 $0.0000000000000000000001207118 \quad 9.9197821894 \quad 1$ spearm an

PCOR<-na.omit(WIS [, c("wisdom.pca", "solvetogether", "Order")])

ppcor: : pcor . test (PCOR\$wisdom. pca, PCOR\$solvetogether, PCOR\$Order, method= "spearman") \# Presentation Order

\#\# estimate p.value statistic $\mathrm{n}$ gp Met hod

\#\# 1 0.2270943 $0.00000000000000000000001336845 \quad 10.148321897 \quad 1$ spear man

PCOR<-na.omit(WIS[, c("wisdom.pca", "solvetogether", "BIDRImpMGMT")])

ppcor: : pcor .test (PCOR\$wisdom. pca, PCOR\$solvetogether, PCOR\$BIDRImpMGMT, $m$ ethod="spearman") \# BIDR: Impression management

\#\# estimate p.value statistic $\mathrm{n}$ gp Method

\#\# $10.20199870 .00000005089673 \quad 5.50731716 \quad 1$ spearman

PCOR<-na.omit(WIS [, c("wisdom.pca", "solvetogether", "BIDRSelfDec")]) ppcor: : pcor .test (PCOR\$wisdom.pca, PCOR\$solvetogether, PCOR\$BIDRSelfDec, $m$ ethod="spearman") \# BIDR: Self-deception

\#\# estimate p.value statistic $\mathrm{n}$ gp Method

\#\# $10.20661320 .00000002470609 \quad 5.638665716 \quad 1$ spearman 
PCOR<-na.omit(WIS [, c ("wisdom.pca", "solvetogether", "Openness")]) ppcor: : pcor .test (PCOR\$wisdom. pca, PCOR\$solvetogether, PCOR\$Openness, meth od="spearman") \# Openness

\#\# estimate p.value statistic $\mathrm{n}$ gp Method $\begin{array}{lllllll}\text { \#\# } 1 & 0.2216237 & 0.00000000001177749 & 6.871094 & 917 & 1 & \text { spearman }\end{array}$ PCOR<-na.omit(WIS [ , c("wisdom.pca", "solvetogether", "Conscientiousness") ]) ppcor: : pcor .test (PCOR\$wisdom.pca, PCOR\$solvetogether, PCOR \$Conscientious ness, method="spearman") \# Conscientiousness

\#\# estimate p.value statistic $\mathrm{n}$ gp Method \#\# $10.2215825 \quad 0.00000000001188351 \quad 6.869749917 \quad 1$ spearman PCOR<-na.omit(WIS [, c ("wisdom.pca", "solvetogether", "Extraversion")]) ppcor: : pcor.test (PCOR\$wisdom. pca, PCOR\$solvetogether, PCOR\$Extraversion, method="spearman") \# Extraversion

\#\# estimate p.value statistic $\mathrm{n}$ gp Method \#\# $10.2215825 \quad 0.00000000001158198 \quad 6.873507918 \quad 1$ spearman

PCOR<-na.omit(WIS [, c("wisdom.pca", "solvetogether", "Agreeableness")]) ppcor: : pcor .test (PCOR\$wisdom.pca, PCOR\$solvetogether, PCOR \$Agreeableness , method="spearman") \# Agreeableness

\#\# estimate p.value statistic $\mathrm{n}$ gp Method \#\# $10.22277770 .000000000009158331 \quad 6.908735917 \quad 1$ spearman

PCOR<-na.omit(WIS[, c("wisdom.pca", "solvetogether", "Neuroticism")]) ppcor: : pcor .test (PCOR\$wisdom. pca, PCOR\$solvetogether, PCOR \$Neuroticism, $m$ ethod="spearman") \# Neuroticism

\#\# estimate p.value statistic $\mathrm{n}$ gp Method \#\# $10.2224443 \quad 0.000000000009850132 \quad 6.897855917 \quad 1$ spearman

PCOR<-na.omit(WIS [, c("wisdom.pca", "solvetogether", "Mindful_1")]) ppcor: : pcor.test (PCOR\$wisdom.pca, PCOR\$solvetogether, PCOR\$Mindful_1, met hod="spearman") \# Mindfulness: Nonreactivity

\#\# estimate p.value statistic $\mathrm{n}$ gp Method $\begin{array}{lllllll}\text { \#\# } 1 & 0.1743163 & 0.00925337 & 2.62573 & 223 & 1 & \text { spearman }\end{array}$

PCOR<-na.omit(WIS [, c("wisdom.pca", "solvetogether", "Mindful_2")]) ppcor: : pcor.test (PCOR\$wisdom.pca, PCOR\$solvetogether, PCOR\$Mindful_2, met hod="spearman") \# Mindfulness: Observing and attending 
\#\# estimate p.value statistic $\mathrm{n}$ gp Method \#\# $10.1555426 \quad 0.02041792 \quad 2.335494223 \quad 1$ spearman

PCOR<-na.omit(WIS [, c ("wisdom.pca", "solvetogether", "Mindful_3")])

ppcor: : pcor.test(PCOR\$wisdom.pca, PCOR\$solvetogether, PCOR\$Mindful_3, met hod="spearman") \# Mindfulness: Acting with awareness

\#\# estimate p.value statistic $\mathrm{n}$ gp Method \#\# $10.1871875 \quad 0.005141026 \quad 2.826399223 \quad 1$ spearman

PCOR<-na.omit(WIS [, c("wisdom.pca", "solvetogether", "Mindful_4")]) ppcor: : pcor.test (PCOR\$wisdom.pca, PCOR\$solvetogether, PCOR\$Mindful_4, met hod="spearman") \# Mindfulness: Describing with words

\#\# estimate p.value statistic $\mathrm{n}$ gp Method \#\# 1 0.165981 $0.01370158 \quad 2.485151221 \quad 1$ spearman

PCOR<-na.omit(WIS[, c("wisdom.pca", "solvetogether", "Mindful_5")]) ppcor: : pcor . test (PCOR\$wisdom. pca, PCOR\$solvetogether, PCOR\$Mindful_5, met hod="spearman") \# Mindfulness: Non-judging of experience

\#\# estimate p.value statistic $\mathrm{n}$ gp Method \#\# $10.1964618 \quad 0.003508919 \quad 2.951586 \quad 220 \quad 1$ spearman

PCOR<-na.omit(WIS[, c("wisdom.pca", "solvetogether", "Intellect_1")]) ppcor: : pcor.test (PCOR\$wisdom.pca, PCOR\$solvetogether, PCOR\$Intellect_1, m ethod="spearman") \# Intellect: Seek

\#\# estimate p.value statistic $\mathrm{n}$ gp Method \#\# $10.17440290 .009709506 \quad 2.6091022201$ spearman

PCOR<-na.omit(WIS[, c("wisdom.pca", "solvetogether", "Intellect_2")]) ppcor: : pcor.test(PCOR\$wisdom.pca, PCOR\$solvetogether, PCOR \$Intellect_2, $m$ ethod="spearman") \# Intellect: Conquer

\#\# estimate p.value statistic $\mathrm{n}$ gp Method \#\# $\begin{array}{llllll}1 & 0.1506135 & 0.02687327 & 2.228708 & 217 & 1\end{array}$ spearman

PCOR<-na.omit(WIS [, c ("wisdom.pca", "solvetogether", "ATT") ])

ppcor: : pcor .test (PCOR\$wisdom. pca, PCOR\$solvetogether, PCOR\$ATT, method="s pearman") \# Attributional complexity

\#\# estimate p.value statistic $\mathrm{n}$ gp Method \#\# $10.2502592 \quad 0.000195547 \quad 3.790127218 \quad 1$ spearman

PCOR<-na.omit(WIS [, c("wisdom.pca", "solvetogether", "EmoReg_1")]) ppcor: : pcor .test (PCOR\$wisdom.pca, PCOR\$solvetogether, PCOR\$EmoReg_1, meth od="spearman") \# Emotion regulation: Reappraisal 
\#\# estimate p.value statistic $\mathrm{n}$ gp Method

\#\# $10.1535819 \quad 0.02430937 \quad 2.268367216 \quad 1$ spearman

PCOR<-na.omit(WIS [, c ("wisdom.pca", "solvetogether", "EmoReg_2")])

ppcor: : pcor.test (PCOR\$wisdom.pca, PCOR\$solvetogether, PCOR\$EmoReg_2, meth od="spearman") \# Emotion regulation: Suppression

\#\# estimate p.value statistic $\mathrm{n}$ gp Method

\#\# $10.18089020 .0078401112 .684287216 \quad 1$ spearman

PCOR<-na.omit(WIS [, c("wisdom.pca", "solvetogether", "EISelfEmotions")] ) ppcor: : pcor.test (PCOR\$wisdom.pca, PCOR\$solvetogether, PCOR\$EISelfEmotion $\mathrm{s}$, method="spearman") \# Emotional intelligence: Self-emotion appraisal

\#\# estimate p.value statistic $\mathrm{n}$ gp Method

\#\# $10.20992930 .00000002158014 \quad 5.6645416991$ spearman

PCOR<-na.omit(WIS [ , c("wisdom.pca", "solvetogether", "EIOthersEmotions")] )

ppcor: : pcor . test (PCOR \$wisdom. pca, PCOR \$solvetogether, PCOR \$EIOthersEmoti ons,method="spearman") \# Emotional intelligence: Others-emotion apprai sal

\#\# estimate p.value statistic $\mathrm{n}$ gp Method \#\# $10.1956899 \quad 0.0000001913826 \quad 5.260655698 \quad 1$ spearman

PCOR<-na.omit(WIS [, c ("wisdom.pca", "solvetogether", "EIEmotionUse")]) ppcor: : pcor . test (PCOR\$wisdom.pca, PCOR\$solvetogether, PCOR\$EIEmotionUse, method="spearman") \# Emotional intelligence: Use of emotion

\#\# estimate p.value statistic $\mathrm{n}$ gp Method \#\# $10.2027083 \quad 0.00000006731722 \quad 5.457267 \quad 698 \quad 1$ spearman

PCOR<-na.omit(WIS [, c ("wisdom.pca", "solvetogether", "EIEmotionReg")]) ppcor: : pcor . test (PCOR\$wisdom.pca, PCOR\$solvetogether, PCOR\$EIEmotionReg, method="spearman") \# Emotional intelligence: Regulation of emotion

\#\# estimate p.value statistic $\mathrm{n}$ gp Method

\#\# 10.20072120 .00000009083695 .4015126981 spearman

PCOR<-na.omit(WIS [, c ("wisdom.pca", "solvetogether", "Reflection")]) ppcor: : pcor . test (PCOR\$wisdom. pca, PCOR\$solvetogether, PCOR $\$$ Reflection, me thod="spearman") \# Ruminative response: Reflection

\#\# estimate p.value statistic $\mathrm{n}$ gp Method

\#\# $10.2018945 \quad 0.00000007613432 \quad 5.434425 \quad 698 \quad 1$ spearman 
PCOR<-na.omit(WIS [, c ("wisdom.pca", "solvetogether", "Brooding")]) ppcor: : pcor .test (PCOR\$wisdom. pca, PCOR\$solvetogether, PCOR\$Brooding, meth od="spearman")\# Ruminative response: Brooding

\#\# estimate p.value statistic $\mathrm{n}$ gp Method

\#\# $10.2049783 \quad 0.00000004762803 \quad 5.521043698 \quad 1$ spearman

Partial correlations between wise reasoning and forgiveness (forgive)

\# Run PCOR and ppcor::pcor.test lines as a pair (to create the dataset and then run partial correlations)

PCOR<-na.omit(WIS [ , c ("wisdom.pca", "forgive", "Age")])

ppcor: : pcor . test (PCOR\$wisdom. pca, PCOR\$forgive, PCOR \$Age, method=" spearma n") \# Age

\#\# estimate p.value statistic $\mathrm{n}$ gp Method

\#\# $10.0675845 \quad 0.003253548 \quad 2.9464691895 \quad 1$ spearman

PCOR<-na.omit(WIS [, c("wisdom.pca", "forgive", "genderN")])

ppcor: : pcor . test (PCOR\$wisdom. pca, PCOR\$forgive, PCOR\$genderN, method="spe arman") \# Gender

\#\# estimate p.value statistic $\mathrm{n}$ gp Method \#\# $10.07311516 \quad 0.001456069 \quad 3.1879911894 \quad 1$ spearman

PCOR<-na.omit(WIS [, c("wisdom.pca", "forgive", "Order")]) ppcor: : pcor . test (PCOR\$wisdom. pca, PCOR\$forgive, PCOR\$Order, method=" spear man") \# Presentation Order

\#\# estimate p.value statistic $\mathrm{n}$ gp Method \#\# $10.07077576 \quad 0.002044919 \quad 3.0879131897 \quad 1$ spearman

PCOR<-na.omit(WIS [ , c("wisdom.pca", "forgive", "BIDRImpMGMT")] )

ppcor: : pcor . test (PCOR\$wisdom. pca, PCOR\$forgive, PCOR\$BIDRImpMGMT, method= "spearman") \# BIDR: Impression management

\#\# estimate p.value statistic $\mathrm{n}$ gp Method

\#\# $10.0575428 \quad 0.124233 \quad 1.539061716 \quad 1$ spearman

PCOR<-na.omit(WIS [, c("wisdom.pca", "forgive", "BIDRSelfDec")])

ppcor: : pcor . test (PCOR\$wisdom. pca, PCOR\$forgive, PCOR\$BIDRSelfDec, method= "spearman") \# BIDR: Self-deception

\#\# estimate p.value statistic $\mathrm{n}$ gp Method

$\begin{array}{lllllll}\text { \#\# } 1 & 0.05753814 & 0.1242635 & 1.538936 & 716 & 1 & \text { spearman }\end{array}$ 
PCOR<-na.omit(WIS [ , c("wisdom.pca", "forgive", "Openness")]) ppcor: : pcor .test (PCOR\$wisdom. pca, PCOR\$forgive, PCOR\$Openness, method="sp earman") \# Openness

\#\# estimate p.value statistic $\mathrm{n}$ gp Method \#\# $10.08823528 \quad 0.007538925 \quad 2.678012917 \quad 1$ spearman

PCOR<-na.omit(WIS [, c("wisdom.pca", "forgive", "Conscientiousness")]) ppcor: : pcor.test (PCOR\$wisdom.pca, PCOR\$forgive, PCOR\$Conscientiousness, $m$ ethod="spearman") \# Conscientiousness

\#\# estimate p.value statistic $\mathrm{n}$ gp Method \#\# $10.08925141 \quad 0.006872807 \quad 2.709099917 \quad 1$ spearman

PCOR<-na.omit(WIS [, c("wisdom.pca", "forgive", "Extraversion")]) ppcor: : pcor . test (PCOR\$wisdom. pca, PCOR\$forgive, PCOR\$Extraversion, method ="spearman") \# Extraversion

\#\# estimate p.value statistic $\mathrm{n}$ gp Method \#\# $10.08735237 \quad 0.0081291592 .652458918 \quad 1$ spearman PCOR<-na.omit(WIS [, c("wisdom.pca", "forgive", "Agreeableness")]) ppcor: : pcor .test (PCOR\$wisdom. pca, PCOR \$forgive, PCOR \$Agreeableness, metho $\mathrm{d}=$ "spearman") \# Agreeableness

\#\# estimate p.value statistic $\mathrm{n}$ gp Method \#\# $10.086348520 .008930668 \quad 2.620313917 \quad 1$ spearman

PCOR<-na.omit(WIS [, c("wisdom.pca", "forgive", "Neuroticism")]) ppcor: : pcor .test (PCOR\$wisdom.pca, PCOR\$forgive, PCOR\$Neuroticism, method= "spearman") \# Neuroticism

\#\# estimate p.value statistic $\mathrm{n}$ gp Method \#\# $10.088253830 .007526266 \quad 2.67858917 \quad 1$ spearman

PCOR<-na.omit(WIS[, c("wisdom.pca", "forgive", "Mindful_1")])

ppcor: : pcor.test (PCOR\$wisdom.pca, PCOR\$forgive, PCOR\$Mindful_1, method="s pearman") \# Mindfulness: Nonreactivity

\#\# estimate p.value statistic $\mathrm{n}$ gp Method \#\# $10.01486405 \quad 0.8256910 .2204939223 \quad 1$ spearman

PCOR<-na.omit(WIS[, c("wisdom.pca", "forgive", "Mindful_2")]) ppcor: : pcor.test (PCOR\$wisdom.pca, PCOR\$forgive, PCOR\$Mindful_2, method="s pearman") \# Mindfulness: Observing and attending

\#\# estimate p.value statistic $\mathrm{n}$ gp Method \#\# $1 \begin{array}{llllll} & 0.02669198 & 0.692454 & 0.3960472 & 223 & 1\end{array}$ spearman 
PCOR<-na.omit(WIS[, c("wisdom.pca", "forgive", "Mindful_3")])

ppcor: : pcor .test (PCOR\$wisdom. pca, PCOR\$forgive, PCOR\$Mindful_3, method="s pearman") \# Mindfulness: Acting with awareness

\#\# estimate p.value statistic $n$ gp Method

$\begin{array}{llllllll}\text { \#\# } 1 & 0.000007284017 & 0.9999139 & 0.0001080394 & 223 & 1 & \text { spearman }\end{array}$

PCOR<-na.omit(WIS [, c("wisdom.pca", "forgive", "Mindful_4")])

ppcor: : pcor.test (PCOR\$wisdom.pca, PCOR\$forgive, PCOR\$Mindful_4, method="s pearman") \# Mindfulness: Describing with words

\#\# estimate p.value statistic $\mathrm{n}$ gp Method

\#\# $10.039050860 .56452190 .5770192221 \quad 1$ spearman

PCOR<-na.omit(WIS [, c("wisdom.pca", "forgive", "Mindful_5")])

ppcor: : pcor.test (PCOR\$wisdom.pca, PCOR\$forgive, PCOR\$Mindful_5, method="s pearman") \# Mindfulness: Non-judging of experience

\#\# estimate p.value statistic $\mathrm{n}$ gp Method

$\begin{array}{llllll}\text { \#\# } 1 & -0.001744889 & 0.9795172 & -0.02570386 & 220 & 1\end{array}$ spearman

PCOR<-na.omit(WIS [, c("wisdom.pca", "forgive", "Intellect_1")])

ppcor: : pcor .test (PCOR\$wisdom.pca, PCOR\$forgive, PCOR\$Intellect_1, method= "spearman") \# Intellect: Seek

\#\# estimate p.value statistic $\mathrm{n}$ gp Method

\#\# $10.021728590 .74915690 .3201577220 \quad 1$ spearman

PCOR<-na.omit(WIS [, c("wisdom.pca", "forgive", "Intellect_2")])

ppcor: : pcor .test (PCOR\$wisdom.pca, PCOR\$forgive, PCOR\$Intellect_2, method= "spearman") \# Intellect: Conquer

\#\# estimate p.value statistic $\mathrm{n}$ gp Method

$\begin{array}{llllllll}\text { \#\# } 1 & 0.01250018 & 0.8550683 & 0.1828761 & 217 & 1 & \text { spearman }\end{array}$

PCOR<-na.omit(WIS [ , c ("wisdom.pca", "forgive", "ATT")])

ppcor: : pcor . test (PCOR\$wisdom. pca, PCOR\$forgive, PCOR\$ATT, method=" spearma n") \# Attributional complexity

\#\# estimate p.value statistic $\mathrm{n}$ gp Method

\#\# $10.2417467 \quad 0.0003255924 \quad 3.653054218 \quad 1$ spearman

PCOR<-na.omit(WIS [ , c("wisdom.pca", "forgive", "EmoReg_1")])

ppcor: : pcor.test (PCOR\$wisdom.pca, PCOR\$forgive, PCOR\$EmoReg_1, method="sp earman") \# Emotion regulation: Reappraisal

\#\# estimate p.value statistic $\mathrm{n}$ gp Method

\#\# 1 - $0.01162824 \quad 0.8653914 \quad-0.16972216 \quad 1$ spearman 
PCOR<-na.omit(WIS [ , c("wisdom.pca", "forgive", "EmoReg_2")])

ppcor: : pcor.test (PCOR\$wisdom.pca, PCOR\$forgive, PCOR\$EmoReg_2, method="sp earman") \# Emotion regulation: Suppression

\#\# estimate p.value statistic $\mathrm{n}$ gp Method

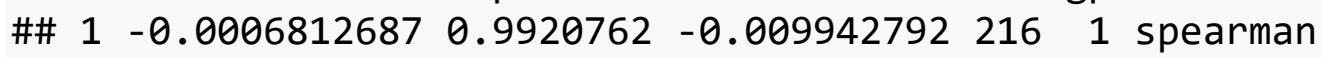

PCOR<-na.omit(WIS [, c("wisdom.pca", "forgive", "EISelfEmotions")])

ppcor: : pcor .test (PCOR\$wisdom.pca, PCOR\$forgive, PCOR\$EISelfEmotions, meth od="spearman") \# Emotional intelligence: Self-emotion appraisal

\#\# estimate p.value statistic $\mathrm{n}$ gp Method

\#\# $10.05005781 \quad 0.1865115 \quad 1.322273699 \quad 1$ spearman

PCOR<-na.omit(WIS [, c("wisdom.pca", "forgive", "EIOthersEmotions")])

ppcor: : pcor .test (PCOR\$wisdom. pca, PCOR\$forgive, PCOR\$EIOthersEmotions, me thod="spearman") \# Emotional intelligence: Others-emotion appraisal

\#\# estimate p.value statistic $\mathrm{n}$ gp Method

\#\# $10.05046085 \quad 0.1833005 \quad 1.331989698 \quad 1$ spearman

PCOR<-na.omit(WIS [ , c("wisdom.pca", "forgive", "EIEmotionUse")])

ppcor: : pcor . test (PCOR\$wisdom. pca, PCOR\$forgive, PCOR\$EIEmotionUse, method ="spearman") \# Emotional intelligence: Use of emotion

\#\# estimate p.value statistic $\mathrm{n}$ gp Method \#\# $10.04468327 \quad 0.2387394 \quad 1.179156 \quad 698 \quad 1$ spearman

PCOR<-na.omit(WIS [ , c("wisdom.pca", "forgive", "EIEmotionReg")])

ppcor: : pcor . test (PCOR\$wisdom. pca, PCOR\$forgive, PCOR\$EIEmotionReg, method ="spearman") \# Emotional intelligence: Regulation of emotion

\#\# estimate p.value statistic $\mathrm{n}$ gp Method

\#\# $10.0489887 \quad 0.1964288 \quad 1.293034 \quad 698 \quad 1$ spearman

PCOR<-na.omit(WIS [, c("wisdom.pca", "forgive", "Reflection")])

ppcor: : pcor . test (PCOR\$wisdom. pca, PCOR\$forgive, PCOR\$Reflection, method=" spearman") \# Ruminative response: Reflection

\#\# estimate p.value statistic $\mathrm{n}$ gp Method $\begin{array}{lllllll}\text { \#\# } 1 & 0.0320313 & 0.3984742 & 0.84487 & 698 & 1 & \text { spearman }\end{array}$

PCOR<-na.omit(WIS [ , c("wisdom.pca", "forgive", "Brooding")]) ppcor: : pcor . test (PCOR\$wisdom. pca, PCOR\$forgive, PCOR\$Brooding, method="sp earman")\# Ruminative response: Brooding

\#\# estimate p.value statistic $\mathrm{n}$ gp Method

\#\# $10.05753703 \quad 0.1291274 \quad 1.519357698 \quad 1$ spearman 
Partial correlations between wise reasoning and disengaging from the situation (disengage)

\# Run PCOR and ppcor::pcor.test lines as a pair (to create the dataset and then run partial correlations)

PCOR<-na.omit(WIS [, c ("wisdom.pca", "disengage", "Age")])

ppcor : : pcor . test (PCOR\$wisdom. pca, PCOR\$disengage, PCOR\$Age, method="spear man") \# Age

\#\# estimate p.value statistic $\mathrm{n}$ gp Method

\#\# 1 - $0.11205710 .000001013893 \quad-4.90505518951$ spearman

PCOR<-na.omit(WIS [ , c ("wisdom.pca", "disengage", "genderN")])

ppcor: : pcor .test (PCOR\$wisdom.pca, PCOR\$disengage, PCOR\$genderN, method="s pearman") \# Gender

\#\# estimate p.value statistic $\mathrm{n}$ gp Method

\#\# 1 -0.1174599 $0.0000002977061 \quad-5.1434241894 \quad 1$ spearman

PCOR<-na.omit(WIS [ , c("wisdom.pca", "disengage", "Order")])

ppcor: : pcor . test (PCOR\$wisdom. pca, PCOR\$disengage, PCOR\$Order, method="spe arman") \# Presentation Order

\#\# estimate p.value statistic $\mathrm{n}$ gp Method

\#\# $1-0.11106 \quad 0.000001248955 \quad-4.86343 \quad 1897 \quad 1$ spearman

PCOR<-na.omit(WIS [, c("wisdom.pca", "disengage", "BIDRImpMGMT")])

ppcor: : pcor .test (PCOR\$wisdom. pca, PCOR\$disengage, PCOR\$BIDRImpMGMT, metho $\mathrm{d}=$ "spearman") \# BIDR: Impression management

\#\# estimate p.value statistic $\mathrm{n}$ gp Method

\#\# 1 - $0.1012623 \quad 0.006729662 \quad-2.717884716 \quad 1$ spearman

PCOR<-na.omit(WIS [, c("wisdom.pca", "disengage", "BIDRSelfDec")])

ppcor: : pcor . test (PCOR\$wisdom.pca, PCOR\$disengage, PCOR\$BIDRSelfDec, metho $d=$ "spearman") \# BIDR: Self-deception

\#\# estimate p.value statistic $\mathrm{n}$ gp Method

\#\# 1 -0.09630609 $0.009975977 \quad-2.58358716 \quad 1$ spearman

PCOR<-na.omit(WIS [, c("wisdom.pca", "disengage", "Openness")])

ppcor: : pcor . test (PCOR\$wisdom. pca, PCOR\$disengage, PCOR\$Openness, method=" spearman") \# Openness

\#\# estimate p.value statistic $\mathrm{n}$ gp Method

\#\# 1 - $0.10012620 .002414482 \quad-3.042347917 \quad 1$ spearman 
PCOR<-na.omit(WIS [, c("wisdom.pca", "disengage", "Conscientiousness")]) ppcor: : pcor .test (PCOR\$wisdom.pca, PCOR\$disengage, PCOR\$Conscientiousness , method="spearman") \# Conscientiousness

\#\# estimate p.value statistic $\mathrm{n}$ gp Method \#\# 1 - $0.1005368 \quad 0.002316297 \quad-3.054949917 \quad 1$ spearman

PCOR<-na.omit(WIS [, c("wisdom.pca", "disengage", "Extraversion")]) ppcor: : pcor . test (PCOR\$wisdom. pca, PCOR\$disengage, PCOR\$Extraversion, meth od="spearman") \# Extraversion

\#\# estimate p.value statistic $\mathrm{n}$ gp Method $\begin{array}{llllll}\text { \#\# } 1 & -0.09885593 & 0.002728152 & -3.005009 & 918 & 1\end{array}$ spearman

PCOR<-na.omit(WIS [, c("wisdom.pca", "disengage", "Agreeableness")]) ppcor: : pcor .test (PCOR\$wisdom.pca, PCOR\$disengage, PCOR\$Agreeableness, met hod="spearman") \# Agreeableness

\#\# estimate p.value statistic $\mathrm{n}$ gp Method \#\# 1 -0.1010948 $0.002188682 \quad-3.072081917 \quad 1$ spearman PCOR<-na.omit(WIS [, c("wisdom.pca", "disengage", "Neuroticism")]) ppcor: : pcor . test (PCOR\$wisdom. pca, PCOR\$disengage, PCOR \$Neuroticism, metho $\mathrm{d}=$ "spearman") \# Neuroticism

\#\# estimate p.value statistic $\mathrm{n}$ gp Method \#\# 1 - $0.1027248 \quad 0.001851901 \quad-3.122137917 \quad 1$ spearman

PCOR<-na.omit(WIS[, c("wisdom.pca", "disengage", "Mindful_1")]) ppcor: : pcor.test (PCOR\$wisdom.pca, PCOR\$disengage, PCOR\$Mindful_1, method= "spearman") \# Mindfulness: Nonreactivity

\#\# estimate p.value statistic $\mathrm{n}$ gp Method \#\# 1 - $0.185875 \quad 0.005467926 \quad-2.805868223 \quad 1$ spearman PCOR<-na.omit(WIS [, c("wisdom.pca", "disengage", "Mindful_2")]) ppcor: : pcor .test (PCOR\$wisdom.pca, PCOR\$disengage, PCOR\$Mindful_2, method= "spearman") \# Mindfulness: Observing and attending

\#\# estimate p.value statistic $\mathrm{n}$ gp Method \#\# 1 - $0.20331890 .002333446 \quad-3.080041223 \quad 1$ spearman

PCOR<-na.omit(WIS [, c("wisdom.pca", "disengage", "Mindful_3")]) ppcor: : pcor .test (PCOR\$wisdom.pca, PCOR\$disengage, PCOR\$Mindful_3, method= "spearman") \# Mindfulness: Acting with awareness

\#\# estimate p.value statistic $\mathrm{n}$ gp Method

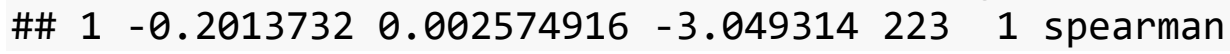


PCOR<-na.omit(WIS [, c("wisdom.pca", "disengage", "Mindful_4")])

ppcor: : pcor .test (PCOR\$wisdom.pca, PCOR\$disengage, PCOR\$Mindful_4, method= "spearman") \# Mindfulness: Describing with words

\#\# estimate p.value statistic $\mathrm{n}$ gp Method \#\# 1 - $0.194186 \quad 0.003835182 \quad-2.922757221 \quad 1$ spearman

PCOR<-na.omit(WIS [, c("wisdom.pca", "disengage", "Mindful_5")])

ppcor: : pcor.test (PCOR\$wisdom.pca, PCOR\$disengage, PCOR\$Mindful_5, method= "spearman") \# Mindfulness: Non-judging of experience

\#\# estimate p.value statistic $\mathrm{n}$ gp Method \#\# 1 - $0.1931356 \quad 0.004119677 \quad-2.89966220 \quad 1$ spearman

PCOR<-na.omit(WIS [, c("wisdom.pca", "disengage", "Intellect_1")])

ppcor: : pcor.test (PCOR\$wisdom.pca, PCOR\$disengage, PCOR $\$$ Intellect_1, metho $\mathrm{d}=$ "spearman") \# Intellect: Seek

\#\# estimate p.value statistic $\mathrm{n}$ gp Method

\#\# 1 - $0.2051494 \quad 0.00228022 \quad-3.087712220 \quad 1$ spearman

PCOR<-na.omit(WIS [, c("wisdom.pca", "disengage", "Intellect_2")])

ppcor: : pcor .test (PCOR\$wisdom.pca, PCOR\$disengage, PCOR\$Intellect_2, metho $\mathrm{d}=$ "spearman") \# Intellect: Conquer

\#\# estimate p.value statistic $\mathrm{n}$ gp Method

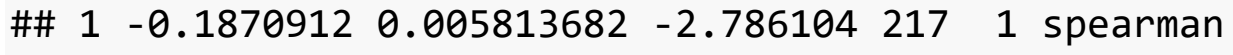

PCOR<-na.omit(WIS [, c ("wisdom.pca", "disengage", "ATT")])

ppcor: : pcor . test (PCOR\$wisdom. pca, PCOR\$disengage, PCOR\$ATT, method="spear man") \# Attributional complexity

\#\# estimate p.value statistic $\mathrm{n}$ gp Method \#\# 1 - $0.1595874 \quad 0.01865334 \quad-2.37039218 \quad 1$ spearman

PCOR<-na.omit(WIS [ , c("wisdom.pca", "disengage", "EmoReg_1")])

ppcor: : pcor . test (PCOR\$wisdom.pca, PCOR\$disengage, PCOR\$EmoReg_1, method=" spearman") \# Emotion regulation: Reappraisal

\#\# estimate p.value statistic $\mathrm{n}$ gp Method \#\# 1 - $0.1630678 \quad 0.01670461 \quad-2.412184216 \quad 1$ spearman

PCOR<-na.omit(WIS [ , c("wisdom.pca", "disengage", "EmoReg_2")]) ppcor: : pcor .test (PCOR\$wisdom.pca, PCOR\$disengage, PCOR\$EmoReg_2, method=" spearman") \# Emotion regulation: Suppression

\#\# estimate p.value statistic $\mathrm{n}$ gp Method \#\# 1 -0.1831173 $0.007098756 \quad-2.718475216 \quad 1$ spearman 
PCOR<-na.omit(WIS [, c ("wisdom.pca", "disengage", "EISelfEmotions")] ) ppcor: : pcor .test (PCOR\$wisdom.pca, PCOR\$disengage, PCOR\$EISelfEmotions, me thod="spearman") \# Emotional intelligence: Self-emotion appraisal

\#\# estimate p.value statistic $\mathrm{n}$ gp Method \#\# 1 - $0.11175160 .003112376 \quad-2.966792699 \quad 1$ spearman

PCOR<-na.omit(WIS [, c ("wisdom.pca", "disengage", "EIOthersEmotions")]) ppcor: : pcor . test (PCOR\$wisdom. pca, PCOR\$disengage, PCOR\$EIOthersEmotions, method="spearman") \# Emotional intelligence: Others-emotion appraisal

\#\# estimate p.value statistic $\mathrm{n}$ gp Method \#\# 1 - $0.1140005 \quad 0.002577173 \quad-3.025101698 \quad 1$ spearman

PCOR<-na.omit(WIS [, c("wisdom.pca", "disengage", "EIEmotionUse")]) ppcor: : pcor .test (PCOR\$wisdom. pca, PCOR\$disengage, PCOR\$EIEmotionUse, meth od="spearman") \# Emotional intelligence: Use of emotion

\#\# estimate p.value statistic $\mathrm{n}$ gp Method \#\# 1 - $0.1017505 \quad 0.007178483 \quad-2.696428698 \quad 1$ spearman PCOR<-na.omit(WIS [ , c("wisdom.pca", "disengage", "EIEmotionReg")]) ppcor: : pcor .test (PCOR\$wisdom. pca, PCOR\$disengage, PCOR\$EIEmotionReg, meth od="spearman") \# Emotional intelligence: Regulation of emotion

\#\# estimate p.value statistic $\mathrm{n}$ gp Method \#\# 1 - $0.092202410 .01489043 \quad-2.441117698 \quad 1$ spearman

PCOR<-na.omit(WIS [, c("wisdom.pca", "disengage", "Reflection")]) ppcor: : pcor . test (PCOR\$wisdom. pca, PCOR\$disengage, PCOR\$Reflection, method ="spearman") \# Ruminative response: Reflection

\#\# estimate p.value statistic $\mathrm{n}$ gp Method \#\# 1 - $0.10192880 .007077327 \quad-2.701201698 \quad 1$ spearman PCOR<-na.omit(WIS [ , c("wisdom.pca", "disengage", "Brooding")]) ppcor: : pcor . test (PCOR\$wisdom. pca, PCOR\$disengage, PCOR\$Brooding, method=" spearman")\# Ruminative response: Brooding

\#\# estimate p.value statistic $\mathrm{n}$ gp Method \#\# 1 - $0.10666390 .004817379 \quad-2.828098698 \quad 1$ spearman

Partial correlations between wise reasoning and retaliation (retaliate)

\# Run PCOR and ppcor::pcor.test Lines as a pair (to create the dataset and then run partial correlations)

PCOR<-na.omit(WIS [, c("wisdom.pca", "retaliate", "Age")]) 
ppcor: : pcor . test (PCOR\$wisdom. pca, PCOR\$retaliate, PCOR\$Age, method="spear man") \# Age

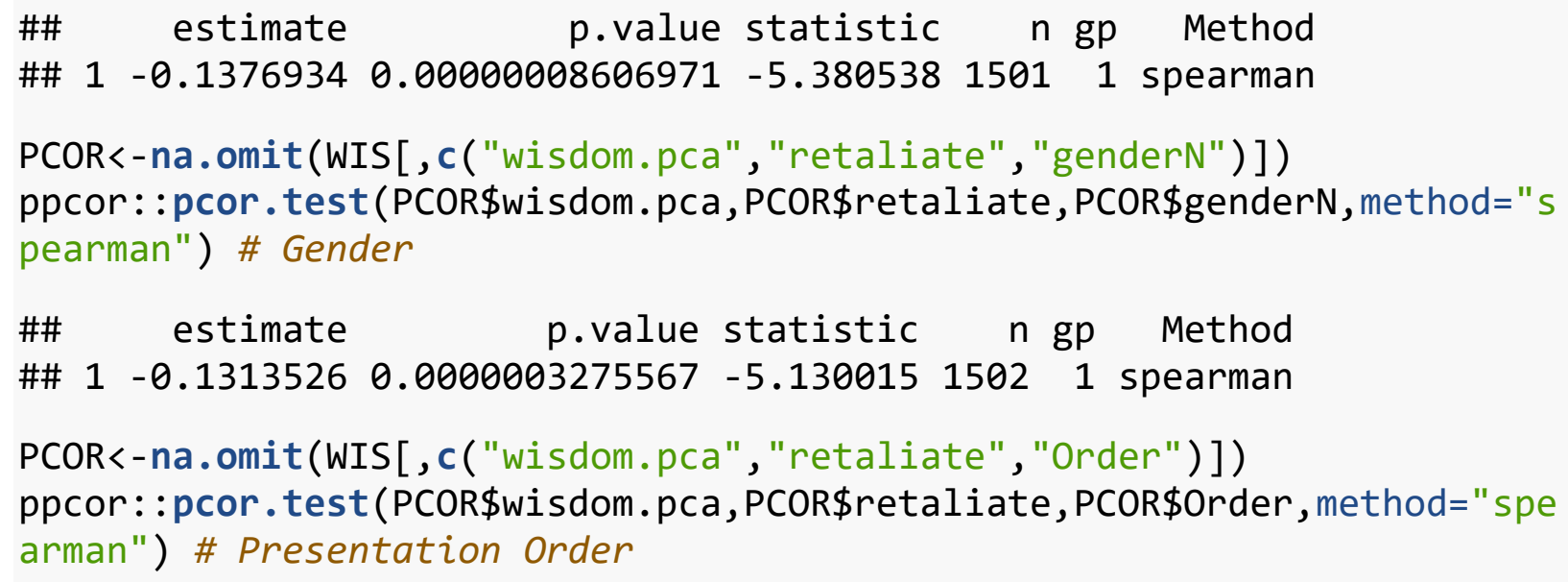


PCOR<-na.omit(WIS [, c("wisdom.pca", "retaliate", "Extraversion")]) ppcor: : pcor .test (PCOR\$wisdom. pca, PCOR\$retaliate, PCOR\$Extraversion, meth od="spearman") \# Extraversion

\#\# estimate p.value statistic $n$ gp Method

PCOR<-na.omit(WIS [, c("wisdom.pca", "retaliate", "Agreeableness")])

ppcor: : pcor .test (PCOR\$wisdom.pca, PCOR\$retaliate, PCOR\$Agreeableness, met hod="spearman") \# Agreeableness

\#\# estimate p.value statistic $\mathrm{n}$ gp Method \#\# 1 - $0.114648 \quad 0.0005077957 \quad-3.489095917 \quad 1$ spearman

PCOR<-na.omit(WIS [, c("wisdom.pca", "retaliate", "Neuroticism")]) ppcor: : pcor .test (PCOR\$wisdom. pca, PCOR\$retaliate, PCOR\$Neuroticism, metho $\mathrm{d}=$ "spearman") \# Neuroticism

\#\# estimate p.value statistic $\mathrm{n}$ gp Method

\#\# 1 -0.1159226 $0.0004388902 \quad-3.52841917 \quad 1$ spearman

PCOR<-na.omit(WIS [, c("wisdom.pca", "retaliate", "Mindful_1")])

ppcor: : pcor .test (PCOR\$wisdom.pca, PCOR\$retaliate, PCOR\$Mindful_1, method= "spearman") \# Mindfulness: Nonreactivity

\#\# estimate p.value statistic $\mathrm{n}$ gp Method \#\# $10.02890720 .66838490 .4289424223 \quad 1$ spearman

PCOR<-na.omit(WIS [, c("wisdom.pca", "retaliate", "Mindful_2")]) ppcor: : pcor .test (PCOR\$wisdom.pca, PCOR\$retaliate, PCOR\$Mindful_2, method= "spearman") \# Mindfulness: Observing and attending

\#\# estimate p.value statistic $\mathrm{n}$ gp Method

\#\# $1-0.0036376490 .9570197 \quad-0.05395541223 \quad 1$ spearman

PCOR<-na.omit(WIS [, c("wisdom.pca", "retaliate", "Mindful_3")])

ppcor: : pcor .test (PCOR\$wisdom. pca, PCOR\$retaliate, PCOR\$Mindful_3, method= "spearman") \# Mindfulness: Acting with awareness

\#\# estimate p.value statistic $\mathrm{n}$ gp Method

$\begin{array}{lllllll}\text { \#\# } 1 & 0.01707796 & 0.800239 & 0.253344 & 223 & 1 & \text { spearman }\end{array}$

PCOR<-na.omit(WIS [, c("wisdom.pca", "retaliate", "Mindful_4")])

ppcor: : pcor.test (PCOR\$wisdom.pca, PCOR\$retaliate, PCOR\$Mindful_4, method= "spearman") \# Mindfulness: Describing with words

\#\# estimate p.value statistic $\mathrm{n}$ gp Method

$\begin{array}{llllllll}\text { \#\# } 1 & 0.02649389 & 0.6959467 & 0.3913149 & 221 & 1 & \text { spearman }\end{array}$ 
PCOR<-na.omit(WIS [, c("wisdom.pca", "retaliate", "Mindful_5")])

ppcor: : pcor .test (PCOR\$wisdom.pca, PCOR\$retaliate, PCOR\$Mindful_5, method= "spearman") \# Mindfulness: Non-judging of experience

\#\# estimate p.value statistic $\mathrm{n}$ gp Method

$\begin{array}{lllllll}\text { \#\# } 1 & 0.01411591 & 0.8354544 & 0.207961 & 220 & 1 & \text { spearman }\end{array}$

PCOR<-na.omit(WIS[, c("wisdom.pca", "retaliate", "Intellect_1")])

ppcor: : pcor.test (PCOR\$wisdom.pca, PCOR\$retaliate, PCOR\$Intellect_1, metho $\mathrm{d}=$ "spearman") \# Intellect: Seek

\#\# estimate p.value statistic $\mathrm{n}$ gp Method

$\begin{array}{lllllll}\text { \#\# } 1 & 0.01627426 & 0.8107375 & 0.2397666 & 220 & 1 & \text { spearman }\end{array}$

PCOR<-na.omit(WIS [, c("wisdom.pca", "retaliate", "Intellect_2")])

ppcor: : pcor.test (PCOR\$wisdom.pca, PCOR\$retaliate, PCOR\$Intellect_2, metho $\mathrm{d}=$ "spearman") \# Intellect: Conquer

\#\# estimate p.value statistic $\mathrm{n}$ gp Method

\#\# $10.005532947 \quad 0.9355643 \quad 0.08094128 \quad 217 \quad 1$ spearman

PCOR<-na.omit(WIS [, c("wisdom.pca", "retaliate", "ATT")])

ppcor: : pcor . test (PCOR\$wisdom. pca, PCOR\$retaliate, PCOR\$ATT, method=" spear man") \# Attributional complexity

\#\# estimate p.value statistic $\mathrm{n}$ gp Method

$\begin{array}{llllll}\text { \#\# } 1 & -0.152636 & 0.02453317 & -2.264619 & 218 & 1\end{array}$ spearman

PCOR<-na.omit(WIS [, c("wisdom.pca", "retaliate", "EmoReg_1")])

ppcor: : pcor.test (PCOR\$wisdom.pca, PCOR\$retaliate, PCOR\$EmoReg_1, method=" spearman") \# Emotion regulation: Reappraisal

\#\# estimate p.value statistic $\mathrm{n}$ gp Method

\#\# $10.0054487920 .9366908 \quad 0.07952368216 \quad 1$ spearman

PCOR<-na.omit(WIS [, c("wisdom.pca", "retaliate", "EmoReg_2")])

ppcor: : pcor.test (PCOR\$wisdom.pca, PCOR\$retaliate, PCOR\$EmoReg_2, method=" spearman") \# Emotion regulation: Suppression

\#\# estimate p.value statistic $\mathrm{n}$ gp Method

\#\# $10.0256323 \quad 0.7086173 \quad 0.3742141216 \quad 1$ spearman

PCOR<-na.omit(WIS [ , c("wisdom.pca", "retaliate", "EISelfEmotions")]) ppcor: : pcor .test (PCOR\$wisdom.pca, PCOR\$retaliate, PCOR\$EISelfEmotions, me thod="spearman") \# Emotional intelligence: Self-emotion appraisal

\#\# estimate p.value statistic $\mathrm{n}$ gp Method

\#\# 1 -0.09366061 $0.01330525 \quad-2.481846699 \quad 1$ spearman 
PCOR<-na.omit(WIS [, c ("wisdom.pca", "retaliate", "EIOthersEmotions")]) ppcor: : pcor .test (PCOR\$wisdom.pca, PCOR\$retaliate, PCOR\$EIOthersEmotions, method="spearman") \# Emotional intelligence: Others-emotion appraisal

\#\# estimate p.value statistic $\mathrm{n}$ gp Method \#\# 1 - $0.08707868 \quad 0.02149441 \quad-2.304396 \quad 698 \quad 1$ spearman

PCOR<-na.omit(WIS [, c("wisdom.pca", "retaliate", "EIEmotionUse")]) ppcor: : pcor .test (PCOR\$wisdom.pca, PCOR\$retaliate, PCOR\$EIEmotionUse, meth od="spearman") \# Emotional intelligence: Use of emotion

\#\# estimate p.value statistic $\mathrm{n}$ gp Method \#\# 1 - $0.08849315 \quad 0.01945591 \quad-2.34212698 \quad 1$ spearman

PCOR<-na.omit(WIS [, c("wisdom.pca", "retaliate", "EIEmotionReg")]) ppcor: : pcor .test (PCOR\$wisdom. pca, PCOR\$retaliate, PCOR\$EIEmotionReg, meth od="spearman") \# Emotional intelligence: Regulation of emotion

\#\# estimate p.value statistic $\mathrm{n}$ gp Method

\#\# 1 -0.08886955 $0.01894269 \quad-2.352162698 \quad 1$ spearman

PCOR<-na.omit(WIS [ , c("wisdom.pca", "retaliate", "Reflection")])

ppcor: : pcor .test (PCOR\$wisdom.pca, PCOR\$retaliate, PCOR\$Reflection, method ="spearman") \# Ruminative response: Reflection

\#\# estimate p.value statistic $\mathrm{n}$ gp Method \#\# 1 - $0.1006753 \quad 0.007816512 \quad-2.667642698 \quad 1$ spearman

PCOR<-na.omit(WIS [, c("wisdom.pca", "retaliate", "Brooding")]) ppcor: : pcor .test (PCOR\$wisdom.pca, PCOR\$retaliate, PCOR\$Brooding, method=" spearman")\# Ruminative response: Brooding

\#\# estimate p.value statistic $\mathrm{n}$ gp Method

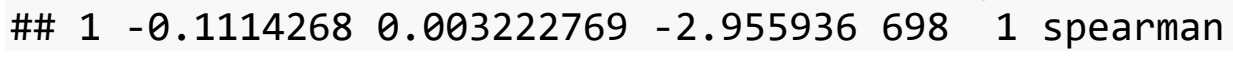

Partial correlations between wise reasoning and finding an ally against the other person (gangup)

\# Run PCOR and ppcor::pcor.test Lines as a pair (to create the dataset and then run partial correlations)

PCOR<-na.omit(WIS [ , c("wisdom.pca", "gangup", "Age")])

ppcor: : pcor .test (PCOR\$wisdom. pca, PCOR\$gangup, PCOR\$Age, method=" spearman ") \# Age

\#\# estimate p.value statistic $\mathrm{n}$ gp Method

\#\# $1 \begin{array}{llllll} & 0.003372165 & 0.8834 & 0.1466803 & 1895 & 1\end{array}$ spearman 
PCOR<-na.omit(WIS [ , c("wisdom.pca", "gangup", "genderN")])

ppcor: : pcor .test (PCOR\$wisdom. pca, PCOR\$gangup, PCOR\$genderN, method=" spea rman") \# Gender

\#\# estimate p.value statistic $\mathrm{n}$ gp Method \#\# $10.006243677 \quad 0.7860242 \quad 0.27151551894 \quad 1$ spearman PCOR<-na.omit(WIS [ , c("wisdom.pca", "gangup", "Order")]) ppcor: : pcor . test (PCOR\$wisdom. pca, PCOR\$gangup, PCOR\$Order, method="spearm an") \# Presentation Order

\#\# estimate p.value statistic $\mathrm{n}$ gp Method \#\# $10.005578197 \quad 0.80821180 .2427675 \quad 1897 \quad 1$ spearman PCOR<-na.omit(WIS [ , c ("wisdom.pca", "gangup", "BIDRImpMGMT")]) ppcor: : pcor . test (PCOR\$wisdom. pca, PCOR\$gangup, PCOR\$BIDRImpMGMT, method=" spearman") \# BIDR: Impression management

\#\# estimate p.value statistic $\mathrm{n}$ gp Method \#\# $10.0076690410 .83779840 .2047852716 \quad 1$ spearman PCOR<-na.omit(WIS [, c("wisdom.pca", "gangup", "BIDRSelfDec")]) ppcor: : pcor . test (PCOR\$wisdom. pca, PCOR\$gangup, PCOR\$BIDRSelfDec, method=" spearman") \# BIDR: Self-deception

\#\# estimate p.value statistic $\mathrm{n}$ gp Method \#\# $10.002122655 \quad 0.95481650 .05667939716 \quad 1$ spearman

PCOR<-na.omit(WIS[, c("wisdom.pca", "gangup", "Openness")]) ppcor: : pcor . test (PCOR\$wisdom. pca, PCOR\$gangup, PCOR\$Openness, method="spe arman") \# Openness

\#\# estimate p.value statistic $\mathrm{n}$ gp Method \#\# 1 -0.00266382 $0.9358301 \quad-0.08053405917 \quad 1$ spearman PCOR<-na.omit(WIS [ , c("wisdom.pca", "gangup", "Conscientiousness")]) ppcor: : pcor .test (PCOR\$wisdom.pca, PCOR\$gangup, PCOR\$Conscientiousness, me thod="spearman") \# Conscientiousness

\#\# estimate p.value statistic $\mathrm{n}$ gp Method \#\# 1 -0.003321246 0.9200409 -0.1004099 $917 \quad 1$ spearman

PCOR<-na.omit(WIS [, c("wisdom.pca", "gangup", "Extraversion")]) ppcor: : pcor . test (PCOR\$wisdom. pca, PCOR\$gangup, PCOR\$Extraversion, method= "spearman") \# Extraversion

\#\# estimate p.value statistic $\mathrm{n}$ gp Method \#\# 1 -0.001938149 0.9532619 -0.0586271 918 1 spearman 
PCOR<-na.omit(WIS [ , c("wisdom.pca", "gangup", "Agreeableness")])

ppcor: : pcor . test (PCOR\$wisdom. pca, PCOR\$gangup, PCOR \$Agreeableness, method ="spearman") \# Agreeableness

\#\# estimate p.value statistic $\mathrm{n}$ gp Method

\#\# $10.001001487 \quad 0.9758524 \quad 0.03027739917 \quad 1$ spearman

PCOR<-na.omit(WIS [ , c("wisdom.pca", "gangup", "Neuroticism")])

ppcor: : pcor . test (PCOR\$wisdom. pca, PCOR\$gangup, PCOR\$Neuroticism, method=" spearman") \# Neuroticism

\#\# estimate p.value statistic $\mathrm{n}$ gp Method \#\# $10.000048863260 .99882160 .001477255917 \quad 1$ spearman

PCOR<-na.omit(WIS [, c("wisdom.pca", "gangup", "Mindful_1")]) ppcor: : pcor.test (PCOR\$wisdom.pca, PCOR\$gangup, PCOR\$Mindful_1, method="sp earman") \# Mindfulness: Nonreactivity

\#\# estimate p.value statistic $\mathrm{n}$ gp Method

\#\# $1 \begin{array}{llllll} & 0.02992248 & 0.6574638 & 0.444021 & 223 & 1\end{array}$ spearman

PCOR<-na.omit(WIS [, c("wisdom.pca", "gangup", "Mindful_2")])

ppcor: : pcor .test (PCOR\$wisdom.pca, PCOR\$gangup, PCOR\$Mindful_2, method="sp earman") \# Mindfulness: Observing and attending

\#\# estimate p.value statistic $\mathrm{n}$ gp Method

\#\# $10.037434790 .57902350 .5556371223 \quad 1$ spearman

PCOR<-na.omit(WIS [, c("wisdom.pca", "gangup", "Mindful_3")])

ppcor: : pcor.test (PCOR\$wisdom. pca, PCOR\$gangup, PCOR\$Mindful_3, method="sp earman") \# Mindfulness: Acting with awareness

\#\# estimate p.value statistic $\mathrm{n}$ gp Method

\#\# $1 \begin{array}{llllll} & 0.01290392 & 0.8483793 & 0.191412 & 223 & 1\end{array}$ spearman

PCOR<-na.omit(WIS [ , c("wisdom.pca", "gangup", "Mindful_4")])

ppcor: : pcor .test (PCOR\$wisdom. pca, PCOR\$gangup, PCOR\$Mindful_4, method="sp earman") \# Mindfulness: Describing with words

\#\# estimate p.value statistic $\mathrm{n}$ gp Method \#\# $10.046024510 .49705870 .6802646221 \quad 1$ spearman

PCOR<-na.omit(WIS[ , c("wisdom.pca", "gangup", "Mindful_5")])

ppcor: : pcor .test(PCOR\$wisdom. pca, PCOR\$gangup, PCOR\$Mindful_5, method="sp earman") \# Mindfulness: Non-judging of experience

\#\# estimate p.value statistic $\mathrm{n}$ gp Method

$\begin{array}{llllllll}\text { \#\# } 1 & 0.02306417 & 0.7343006 & 0.3398468 & 220 & 1 & \text { spearman }\end{array}$ 
PCOR<-na.omit(WIS [, c("wisdom.pca", "gangup", "Intellect_1")])

ppcor: : pcor . test (PCOR\$wisdom. pca, PCOR\$gangup, PCOR\$Intellect_1, method=" spearman") \# Intellect: Seek

\#\# estimate p.value statistic $\mathrm{n}$ gp Method

$\begin{array}{lllllll}\text { \#\# } 1 & 0.03281402 & 0.6291283 & 0.4836411 & 220 & 1 & \text { spearman }\end{array}$

PCOR<-na.omit(WIS [, c("wisdom.pca", "gangup", "Intellect_2")])

ppcor: : pcor . test (PCOR\$wisdom.pca, PCOR\$gangup, PCOR\$Intellect_2, method=" spearman") \# Intellect: Conquer

\#\# estimate p.value statistic $\mathrm{n}$ gp Method

\#\# $10.032078210 .6391865 \quad 0.4695054217 \quad 1$ spearman

PCOR<-na.omit(WIS [, c("wisdom.pca", "gangup", "ATT")])

ppcor: : pcor . test (PCOR\$wisdom. pca, PCOR\$gangup, PCOR \$ATT, method=" spearman

") \# Attributional complexity

\#\# estimate p.value statistic $\mathrm{n}$ gp Method

\#\# $10.0039199290 .95421790 .05747788218 \quad 1$ spearman

PCOR<-na.omit(WIS [ , c("wisdom.pca", "gangup", "EmoReg_1")])

ppcor: : pcor .test (PCOR\$wisdom.pca, PCOR\$gangup, PCOR\$EmoReg_1, method="spe arman") \# Emotion regulation: Reappraisal

\#\# estimate p.value statistic $\mathrm{n}$ gp Method

\#\# $10.031115130 .6500537 \quad 0.4543304216 \quad 1$ spearman

PCOR<-na.omit(WIS [, c ("wisdom.pca", "gangup", "EmoReg_2")])

ppcor: : pcor.test (PCOR\$wisdom.pca, PCOR\$gangup, PCOR\$EmoReg_2, method="spe arman") \# Emotion regulation: Suppression

\#\# estimate p.value statistic $\mathrm{n}$ gp Method

\#\# $10.03411767 \quad 0.6188423 \quad 0.4982211216 \quad 1$ spearman

PCOR<-na.omit(WIS [, c("wisdom.pca", "gangup", "EISelfEmotions")])

ppcor: : pcor .test (PCOR\$wisdom.pca, PCOR\$gangup, PCOR\$EISelfEmotions, metho $\mathrm{d}=$ "spearman") \# Emotional intelligence: Self-emotion appraisal

\#\# estimate p.value statistic $\mathrm{n}$ gp Method

\#\# $10.0020380020 .9571368 \quad 0.05376629699 \quad 1$ spearman

PCOR<-na.omit(WIS [, c("wisdom.pca", "gangup", "EIOthersEmotions")])

ppcor: : pcor . test (PCOR\$wisdom. pca, PCOR\$gangup, PCOR \$EIOthersEmotions, met hod="spearman") \# Emotional intelligence: Others-emotion appraisal

\#\# estimate p.value statistic $\mathrm{n}$ gp Method

\#\# $10.0060212520 .8739196 \quad 0.1587402698 \quad 1$ spearman 
PCOR<-na.omit(WIS [ , c("wisdom.pca", "gangup", "EIEmotionUse")]) ppcor: : pcor . test (PCOR\$wisdom.pca, PCOR\$gangup, PCOR\$EIEmotionUse, method= "spearman") \# Emotional intelligence: Use of emotion

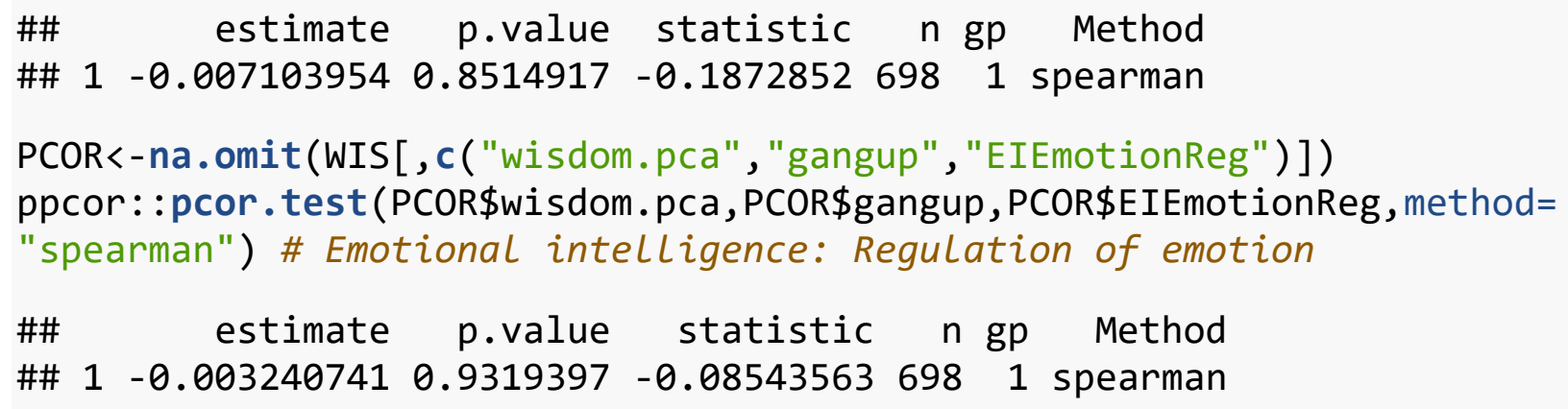


\#\# The following objects are masked from 'package:plyr':

\#\#

\#\# is.discrete, summarize

\#\# The following object is masked from 'package:psych':

\#\#

\#\# describe

\#\# The following objects are masked from 'package:base':

\#\#

\#\# format.pval, round.POSIXt, trunc.POSIXt, units

library(MASS)

library(tidyr)

library (dplyr)

library(lme4)

library(lmerTest)

library(apaTables)

\#\# Warning: package 'apaTables' was built under $\mathrm{R}$ version 3.3.3

library(yarrr)

library(QuantPsyc)

DISABLING SCIENTIFIC NOTATION

options ( scipen=999)

LOADING AND PREPARING FILE

WIS.MT . RAW $<-W I S$. RAW

WIS . MT <-WIS . MT . RAW [WIS.MT . RAW\$DupFlag $==0$, ]

WIS.MT[WIS.MT $==-99]<-N A$ \# Removing missing values

WIS.MT[WIS.MT $==$ 'NA'] $<-N A$ \# Removing NA string

WIS.MT.I<-WIS.MT[WIS.MT\$sample==" $x " \mid$ WIS.MT\$sample $==" W 2 "$, ] \# Study 6 (w isdom and goal/inteference balance); $n=614$

Influence/Adjustment Reliability (Study 6 only)

psych: :alpha(WIS.MT.I[, c('IA1', 'IA2', 'IA3', 'IA4' )], check.keys=FALSE) \#Influence alpha pretty good

\#\#

\#\# Reliability analysis

\#\# Call: psych::alpha(x = WIS.MT.I[, c("IA1", "IA2", "IA3", "IA4")], c heck.keys = FALSE)

\#\#

\#\# raw_alpha std.alpha G6(smc) average_r S/N ase mean sd 


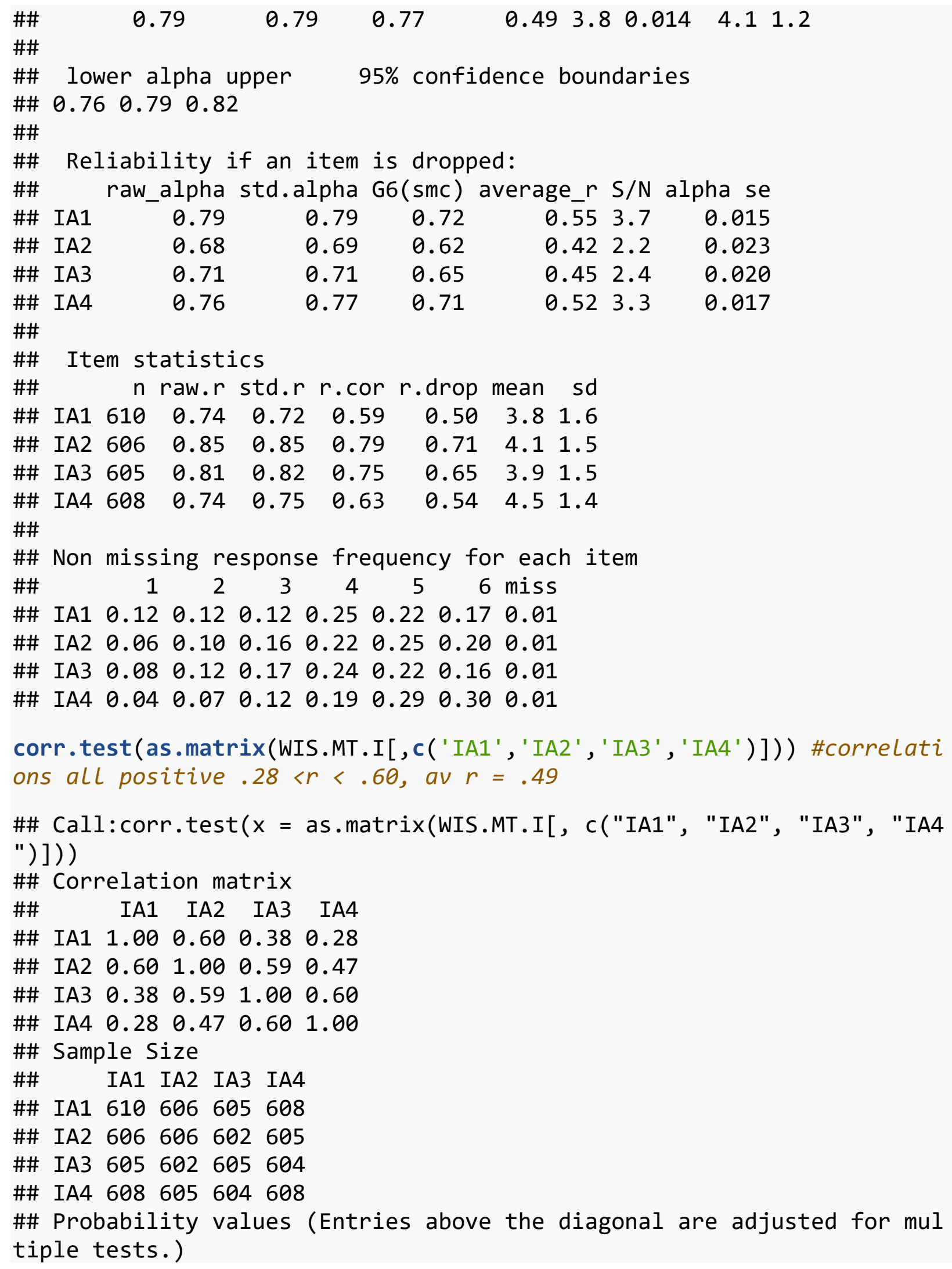




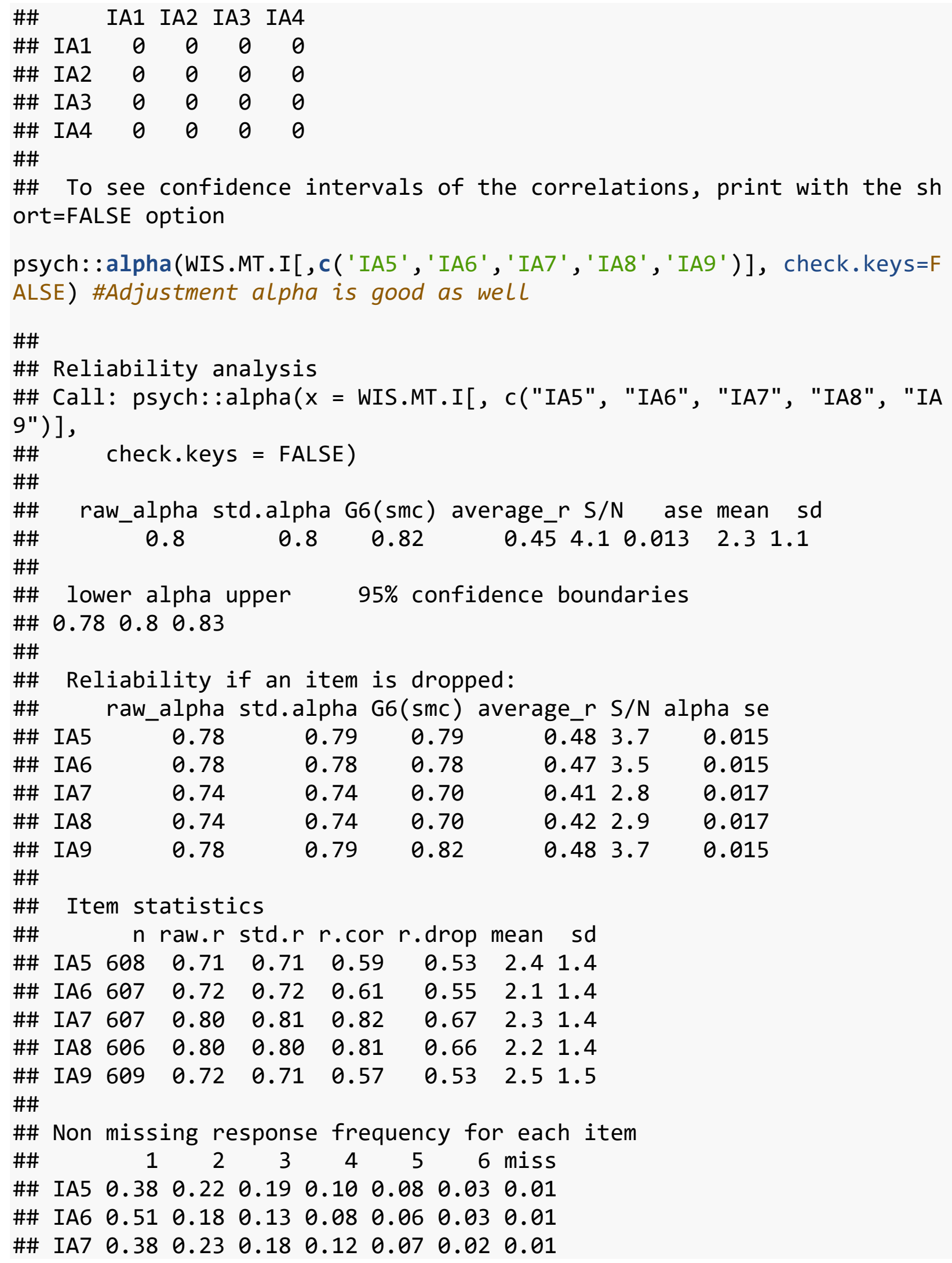




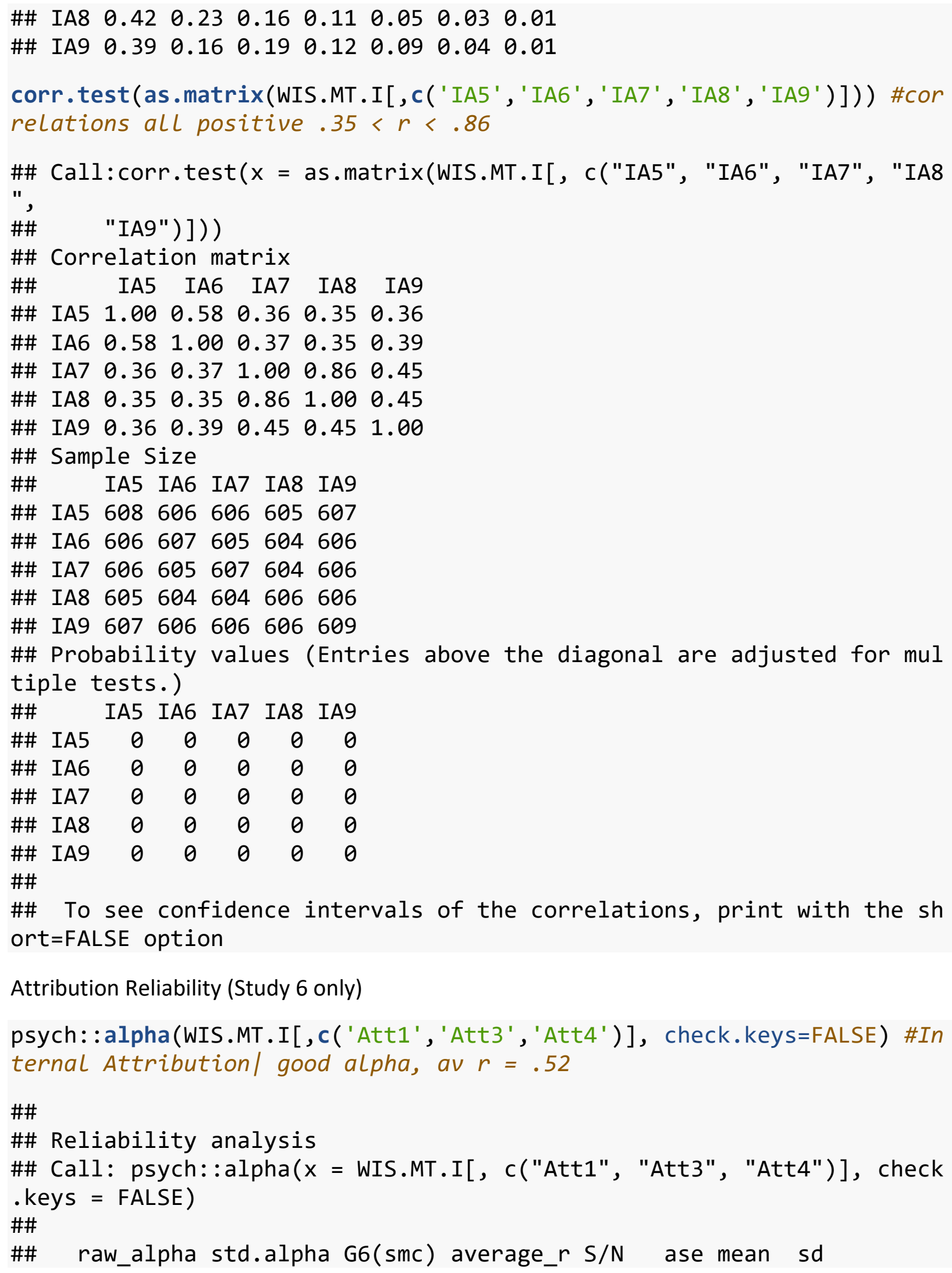




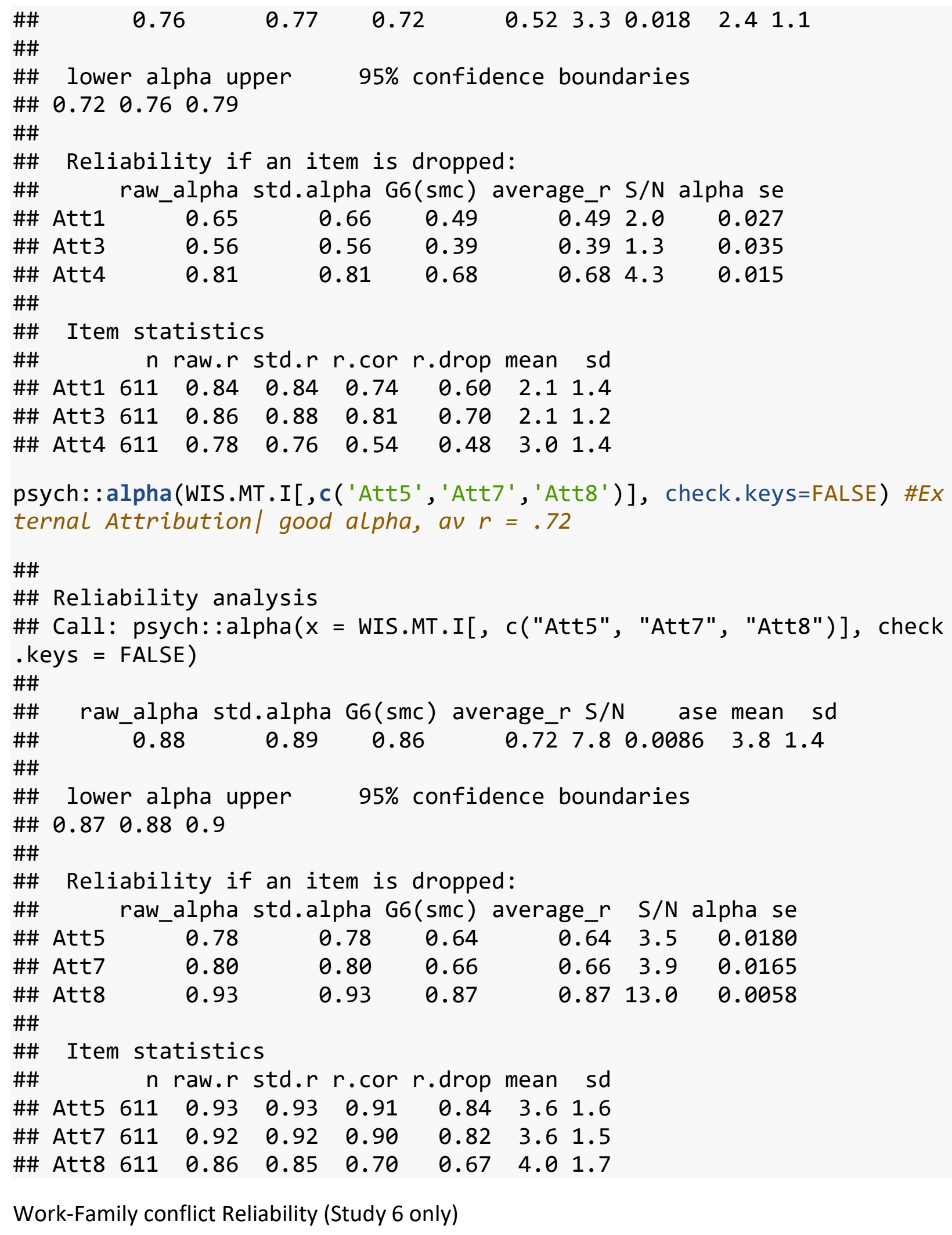

Work-Family conflict Reliability (Study 6 only) 
corr.test(as.matrix(WIS.MT.I [, c('WFB1', 'WFB2' , 'WFB3', 'WFB4' , 'WFB5' , 'WF $\left.\left.\left.\left(B 6^{\prime}\right)\right]\right)\right)$

\#\# Call:corr.test( $x$ = as.matrix(WIS.MT.I [, c("WFB1", "WFB2", "WFB3",

\#\# "WFB4", "WFB5", "WFB6")]))

\#\# Correlation matrix

\#\# WFB1 WFB2 WFB3 WFB4 WFB5 WFB6

$\begin{array}{llllllllll}\text { \#\# WFB1 } & 1.00 & 0.63 & 0.34 & 0.23 & 0.27 & 0.30\end{array}$

$\begin{array}{llllllllll}\text { \#\# WFB2 } & 0.63 & 1.00 & 0.49 & 0.29 & 0.34 & 0.40\end{array}$

$\begin{array}{llllllllllll}\text { \#\# WFB3 } & 0.34 & 0.49 & 1.00 & 0.17 & 0.24 & 0.46\end{array}$

$\begin{array}{lllllllll}\text { \#\# WFB4 } & 0.23 & 0.29 & 0.17 & 1.00 & 0.65 & 0.37\end{array}$

$\begin{array}{lllllllll}\text { \#\# WFB5 } & 0.27 & 0.34 & 0.24 & 0.65 & 1.00 & 0.41\end{array}$

$\begin{array}{lllllllllll}\text { \#\# WFB6 } & 0.30 & 0.40 & 0.46 & 0.37 & 0.41 & 1.00\end{array}$

\#\# Sample Size

\#\# [1] 610

\#\# Probability values (Entries above the diagonal are adjusted for mul tiple tests.)

\#\# WFB1 WFB2 WFB3 WFB4 WFB5 WFB6

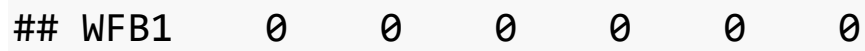

$\begin{array}{lllllll}\text { \#\# WFB2 } & 0 & 0 & 0 & 0 & 0 & 0\end{array}$

$\begin{array}{lllllll}\text { \#\# WFB3 } & 0 & 0 & 0 & 0 & 0 & 0\end{array}$

$\begin{array}{lllllll}\text { \#\# WFB4 } & 0 & 0 & 0 & 0 & 0 & 0\end{array}$

$\begin{array}{lllllll}\text { \#\# WFB5 } & 0 & 0 & 0 & 0 & 0 & 0\end{array}$

$\begin{array}{lllllll}\text { \#\# WFB6 } & 0 & 0 & 0 & 0 & 0 & 0\end{array}$

\#\#

\#\# To see confidence intervals of the correlations, print with the sh ort=FALSE option

\#correlations of items concerning work impacting family (1\&2): .63

\#correlations of items concerning family impacting work (4\&5): .65

\#correlations of items concerning specificity of family and work domai $n s(3 \& 6): .46$

SAWS Reliability (Sample 5 only)

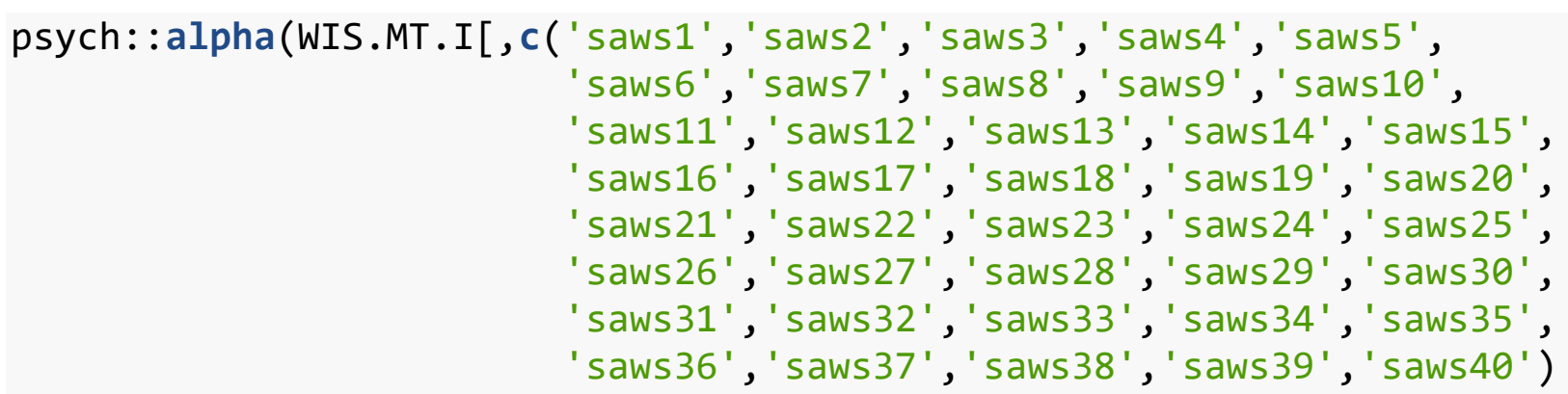

], check.keys=FALSE)[1] \#SAWS 
\#\# \$total

\#\# raw_alpha std.alpha G6(smc) average_r $\quad$ S/N $\quad$ ase ean

$\begin{array}{llllllll}\text { \#\# } & 0.9227884 & 0.9258522 & 0.9499376 & 0.2379003 & 12.48657 & 0.004479676 & 4.482\end{array}$ 261

\#\# sd

\#\# $\quad 0.6220203$

3DWS Brief Form Reliability (Study 6 only)

psych: :alpha(WIS.MT.I [ , c('TDWS1', 'TDWS2', 'TDWS3 ', 'TDWS4 ', 'TDWS5r' , 'TDW S6', 'TDWS7' , 'TDWS8', 'TDWS9r' , 'TDWS10r', 'TDWS11', 'TDWS12') ], check.keys =FALSE)[1] \#3DWS Brief Form with 12 items, all properly named.

\#\# \$total

\#\# raw_alpha std.alpha G6(smc) average_r S/N ase me

an

$\begin{array}{llllllll}\# \# & 0.7419097 & 0.738481 & 0.7813319 & 0.1904917 & 2.823814 & 0.01540106 & 3.4202\end{array}$

36

\#\# $\quad$ sd

\#\# $\quad 0.6252327$

ASTI Reliability (Study 6 only)

psych: :alpha(WIS.MT.I[, c('asti1', 'asti2', 'asti3', 'asti4' , 'asti5', 'asti6', 'asti7', 'asti8', 'asti9', 'asti10')], c heck.keys=FALSE) [1] \#ASTI

\#\# \$total

\#\# raw_alpha std.alpha G6(smc) average_r $\mathrm{S} / \mathrm{N}$ ase me

an

$\begin{array}{llllllll}\text { \#\# } & 0.8223154 & 0.8218883 & 0.8322988 & 0.3157459 & 4.614455 & 0.01072135 & 3.6112\end{array}$

03

\#\# sd

\#\# $\quad 0.7059668$

WISE REASONING PCA (Study 6 only)

principal(WIS.MT.I [, c("Perspective.ave", "ChangeOutcomes.ave", "Limits.a ve", "CompResolve.ave", "Outsider.ave")], nfactors=1, residuals=TRUE, rotat e="oblimin", missing=TRUE, impute="median") \#Conflict 1: Getting PCA sco res (2nd order factor across means of 5 dimensions) /58\% var explained

\#\# Principal Components Analysis

\#\# Call: principal( $r=$ WIS.MT.I[, c("Perspective.ave", "ChangeOutcomes .ave",

\#\# "Limits.ave", "CompResolve.ave", "Outsider.ave")], nfactors = 1 

\#\# $\quad$ residuals $=$ TRUE, rotate $=$ "oblimin", missing $=$ TRUE, impute $=$ "median")

\#\# Standardized loadings (pattern matrix) based upon correlation matri $x$

\#\# $\quad$ PC1 h2 u2 com

$\begin{array}{lllll}\text { \#\# Perspective.ave } \quad 0.79 & 0.62 & 0.38 & 1\end{array}$

\#\# ChangeOutcomes.ave $0.75 \quad 0.56 \quad 0.44 \quad 1$

\#\# Limits.ave $\quad 0.810 .650 .35 \quad 1$

\#\# CompResolve.ave $\quad 0.81 \quad 0.66 \quad 0.34 \quad 1$

$\begin{array}{lllll}\text { \#\# Outsider.ave } \quad 0.65 & 0.42 & 0.58 \quad 1\end{array}$

\#\#

\#\# PC1

\#\# SS loadings 2.91

\#\# Proportion Var 0.58

\#\#

\#\# Mean item complexity $=1$

\#\# Test of the hypothesis that 1 component is sufficient.

\#\#

\#\# The root mean square of the residuals (RMSR) is 0.12

\#\# with the empirical chi square 169.04 with prob < 0.000000000000 00000000000000000000012

\#\#

\#\# Fit based upon off diagonal values $=0.94$

"'wise reasoning sub-components PCA

principal(WIS.MT.I , c( 'w1' , 'w2' , 'w3 ', 'w4 ', 'w5 ', 'w6 ', 'w7' , 'w8 ', 'w9' , 'w1 $0^{\prime}$, 'w11', 'w12', 'w13', 'w14', 'w15', 'w16' , 'w17' , 'w18', 'w19' , 'w20', 'w21' )] , nfactors $=5$, residuals=TRUE, rotate="oblimin", missing=TRUE, impute="media n") \#58\% var explained

\#\# Loading required namespace: GPArotation

\#\# Principal Components Analysis

\#\# Call: principal( $r=$ WIS.MT.I[, c("w1", "w2", "w3", "w4", "w5", "w6"

\#\# "w7", "w8", "w9", "w10", "w11", "w12", "w13", "w14", "w15",

\#\# "w16", "w17", "w18", "w19", "w20", "w21")], nfactors = 5, \#\# $\quad$ residuals $=$ TRUE, rotate $=$ "oblimin", missing $=$ TRUE, impute $=$ "median")

\#\# Standardized loadings (pattern matrix) based upon correlation matri $x$

$\begin{array}{lllllllll}\text { \#\# } & \text { TC2 } & \text { TC1 } & \text { TC4 } & \text { TC3 } & \text { TC5 } & \text { h2 } & \text { u2 com }\end{array}$

$\begin{array}{llllllllll}\text { \#\# w1 } & 0.04 & 0.03 & 0.76 & -0.07 & 0.12 & 0.68 & 0.32 & 1.1\end{array}$ 


$\begin{array}{lrrrrrrrr}\text { \#\# w2 } & -0.01 & 0.00 & 0.74 & 0.14 & -0.13 & 0.55 & 0.45 & 1.1 \\ \text { \#\# w3 } & 0.06 & 0.00 & 0.75 & -0.04 & 0.20 & 0.73 & 0.27 & 1.2 \\ \text { \#\# w4 } & -0.02 & 0.10 & 0.65 & 0.00 & 0.12 & 0.57 & 0.43 & 1.1 \\ \text { \#\# w5 } & 0.01 & 0.49 & 0.28 & 0.28 & -0.19 & 0.59 & 0.41 & 2.6 \\ \text { \#\# w6 } & 0.03 & 0.44 & 0.25 & 0.40 & -0.14 & 0.64 & 0.36 & 2.8 \\ \text { \#\# w7 } & 0.05 & -0.02 & 0.00 & 0.90 & 0.03 & 0.83 & 0.17 & 1.0 \\ \text { \#\# w8 } & -0.02 & -0.01 & -0.04 & 0.91 & 0.09 & 0.82 & 0.18 & 1.0 \\ \text { \#\# w9 } & -0.01 & 0.10 & 0.01 & 0.06 & 0.83 & 0.78 & 0.22 & 1.0 \\ \text { \#\# w10 } & 0.01 & 0.03 & 0.14 & 0.08 & 0.79 & 0.80 & 0.20 & 1.1 \\ \text { \#\# w11 } & 0.32 & 0.09 & 0.07 & 0.07 & 0.40 & 0.46 & 0.54 & 2.2 \\ \text { \#\# w12 } & 0.31 & -0.07 & 0.13 & 0.16 & 0.37 & 0.43 & 0.57 & 2.7 \\ \text { \#\# w13 } & 0.02 & 0.67 & 0.25 & -0.07 & 0.05 & 0.68 & 0.32 & 1.3 \\ \text { \#\# w14 } & 0.03 & 0.73 & 0.17 & -0.05 & 0.07 & 0.71 & 0.29 & 1.1 \\ \text { \#\# w15 } & 0.11 & 0.69 & 0.07 & -0.06 & 0.13 & 0.65 & 0.35 & 1.2 \\ \text { \#\# w16 } & -0.07 & 0.85 & -0.19 & 0.00 & 0.07 & 0.61 & 0.39 & 1.1 \\ \text { \#\# w17 } & 0.11 & 0.74 & -0.09 & 0.12 & -0.01 & 0.62 & 0.38 & 1.1 \\ \text { \#\# w18 } & 0.89 & 0.03 & 0.01 & 0.05 & -0.10 & 0.79 & 0.21 & 1.0 \\ \text { \#\# w19 } & 0.86 & 0.00 & 0.11 & -0.01 & -0.02 & 0.79 & 0.21 & 1.0 \\ \text { \#\# w20 } & 0.94 & -0.01 & -0.03 & 0.00 & -0.02 & 0.85 & 0.15 & 1.0 \\ \text { \#\# w21 } & 0.88 & 0.01 & -0.08 & -0.03 & 0.13 & 0.81 & 0.19 & 1.1\end{array}$

\#\#

\#\#

TC2 TC1 TC4 TC3 TC5

$\begin{array}{llllllllll}\text { \#\# SS loadings } & 3.64 & 3.62 & 2.87 & 2.16 & 2.10\end{array}$

$\begin{array}{lllllllll}\text { \#\# Proportion Var } \quad 0.17 & 0.17 & 0.14 & 0.10 & 0.10\end{array}$

$\begin{array}{llllllllll}\text { \#\# Cumulative Var } & 0.17 & 0.35 & 0.48 & 0.59 & 0.69\end{array}$

\#\# Proportion Explained $0.25 \quad 0.25 \quad 0.20 \quad 0.15 \quad 0.15$

\#\# Cumulative Proportion $0.25 \quad 0.50 \quad 0.70 \quad 0.85 \quad 1.00$

\#\#

\#\# With component correlations of

\#\# $\quad$ TC2 TC1

$\begin{array}{lllllll}\text { \#\# TC2 } & 1.00 & 0.29 & 0.30 & 0.29 & 0.31\end{array}$

$\begin{array}{lllllll}\text { \#\# TC1 } & 0.29 & 1.00 & 0.46 & 0.37 & 0.33\end{array}$

$\begin{array}{llllllll}\text { \#\# TC4 } & 0.30 & 0.46 & 1.00 & 0.25 & 0.38\end{array}$

$\begin{array}{lllllll}\text { \#\# TC3 } & 0.29 & 0.37 & 0.25 & 1.00 & 0.18\end{array}$

$\begin{array}{llllllll}\text { \#\# TC5 } & 0.31 & 0.33 & 0.38 & 0.18 & 1.00\end{array}$

\#\#

\#\# Mean item complexity $=1.4$

\#\# Test of the hypothesis that 5 components are sufficient.

\#\#

\#\# The root mean square of the residuals (RMSR) is 0.05

\#\# with the empirical chi square 725.08 with prob < 0.000000000000 0000000000000000000000000000000000000000000000000000000000000000000000 00000032 


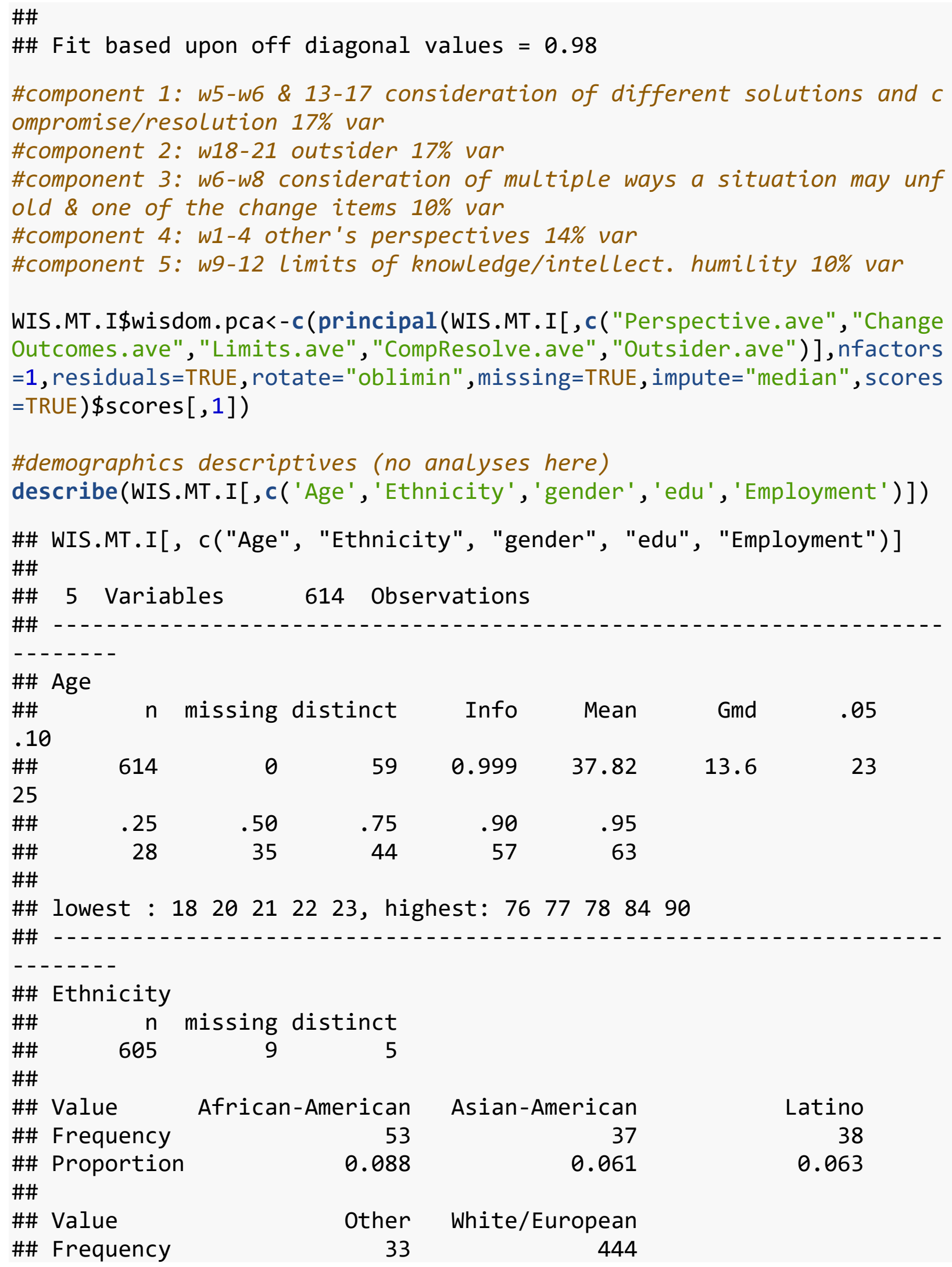




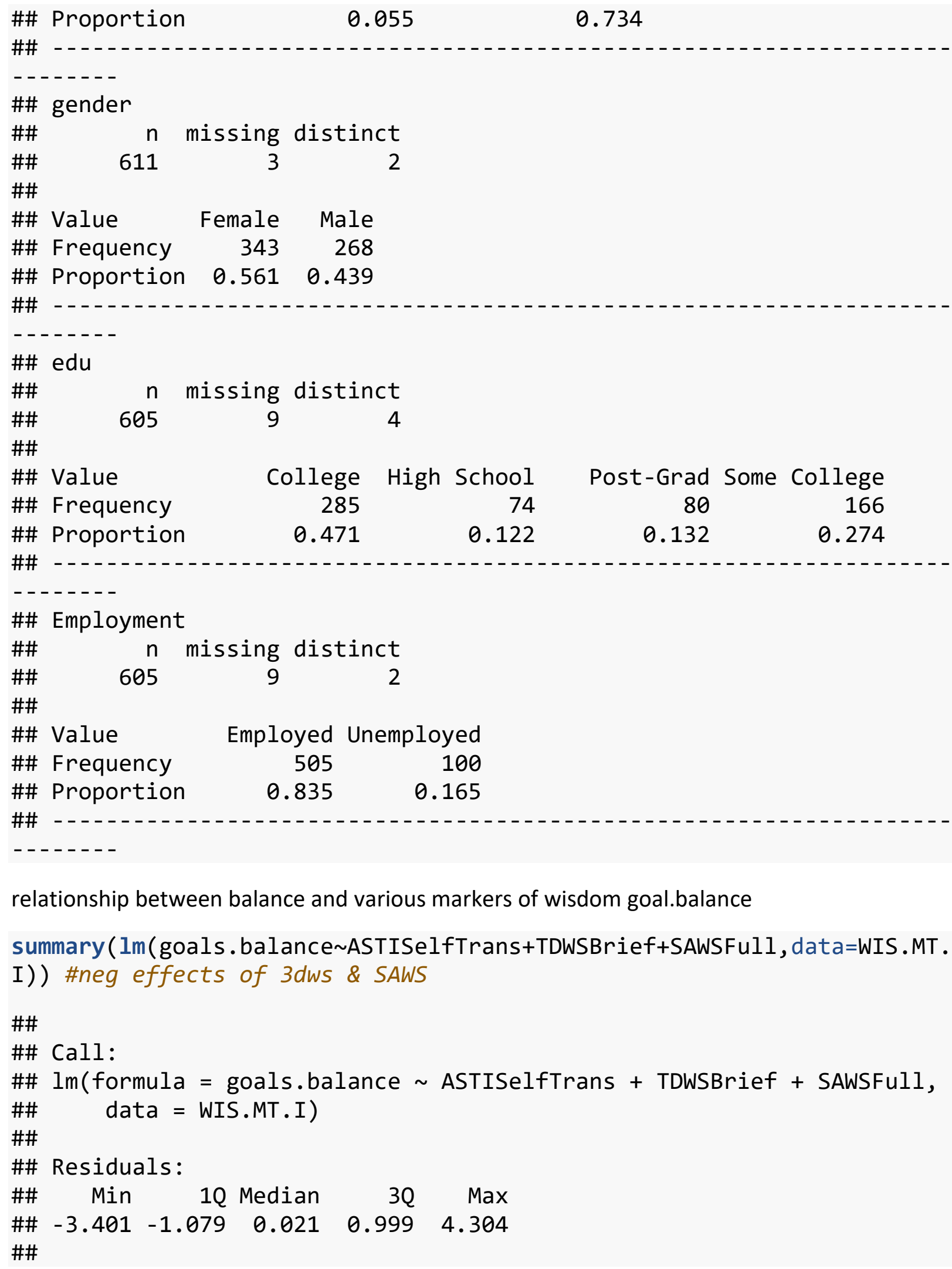

relationship between balance and various markers of wisdom goal.balance summary (lm (goals . balance ASTISelfTrans+TDWSBrief+SAWSFull, data=WIS .MT . I)) \#neg effects of $3 d w s$ \& SAWS 


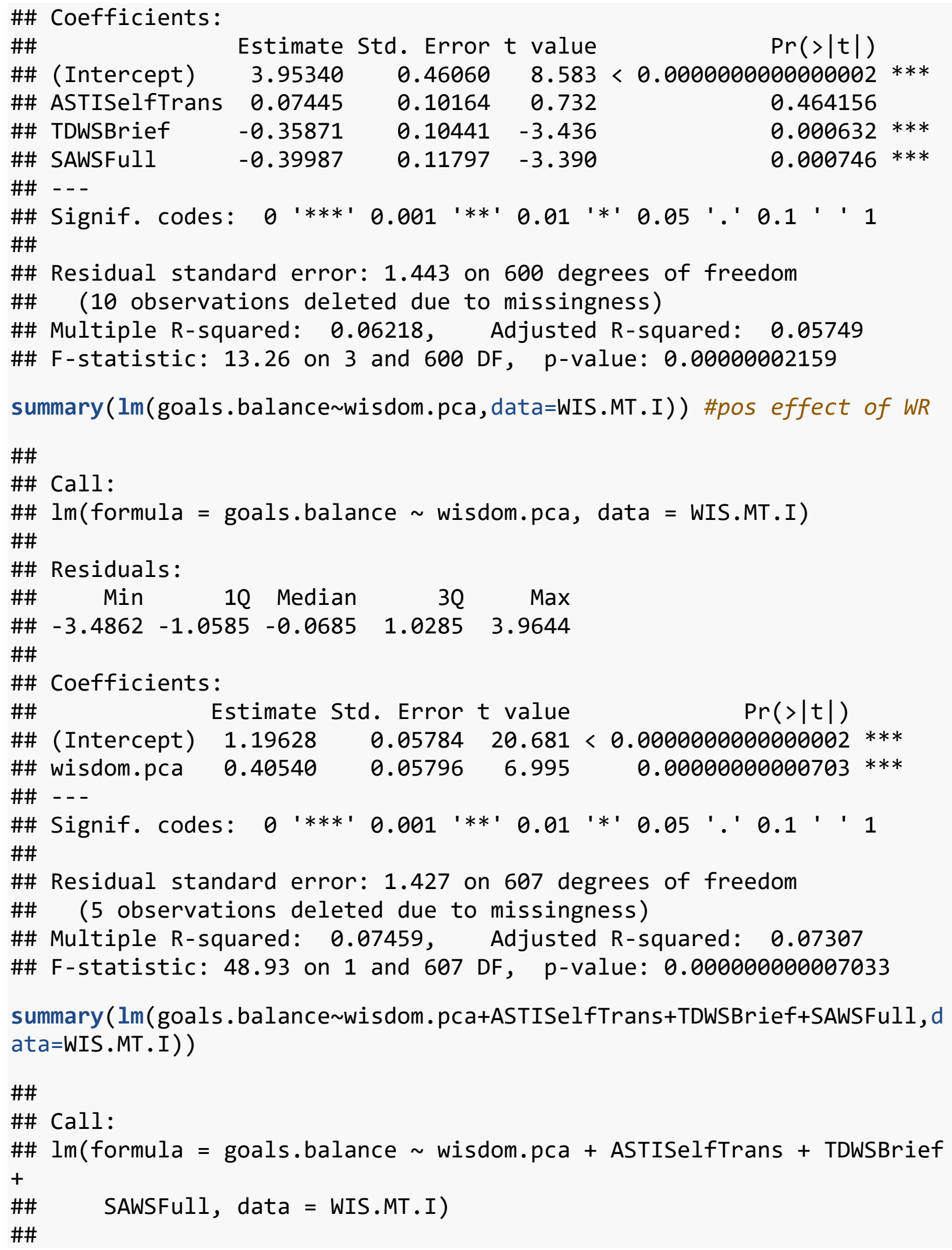




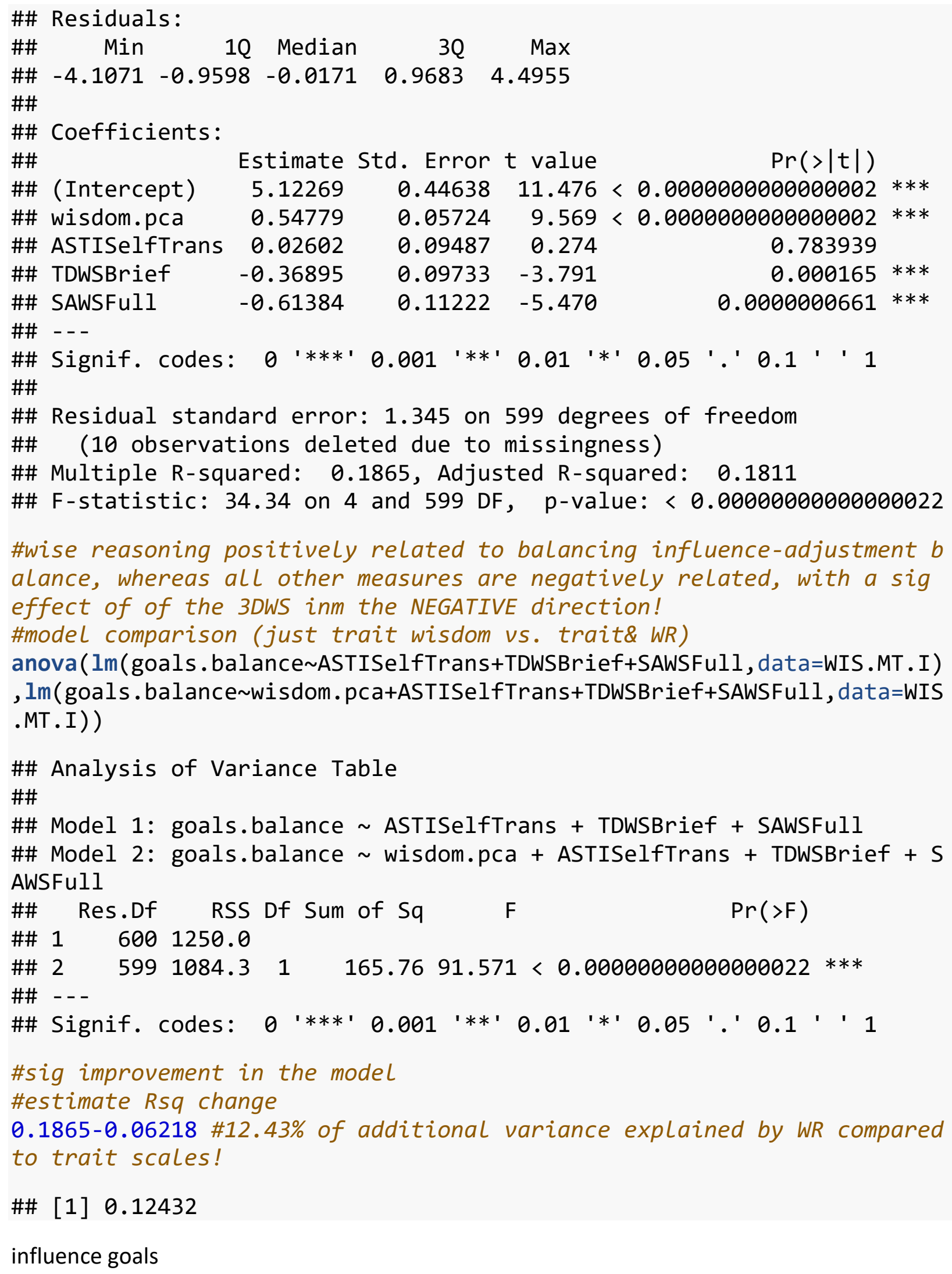


summary ( $1 \mathrm{~m}$ (Influence ASTISelfTrans+TDWSBrief+SAWSFull, data=WIS.MT.I)) \#3wds neg whereas saws pos related to influence goals

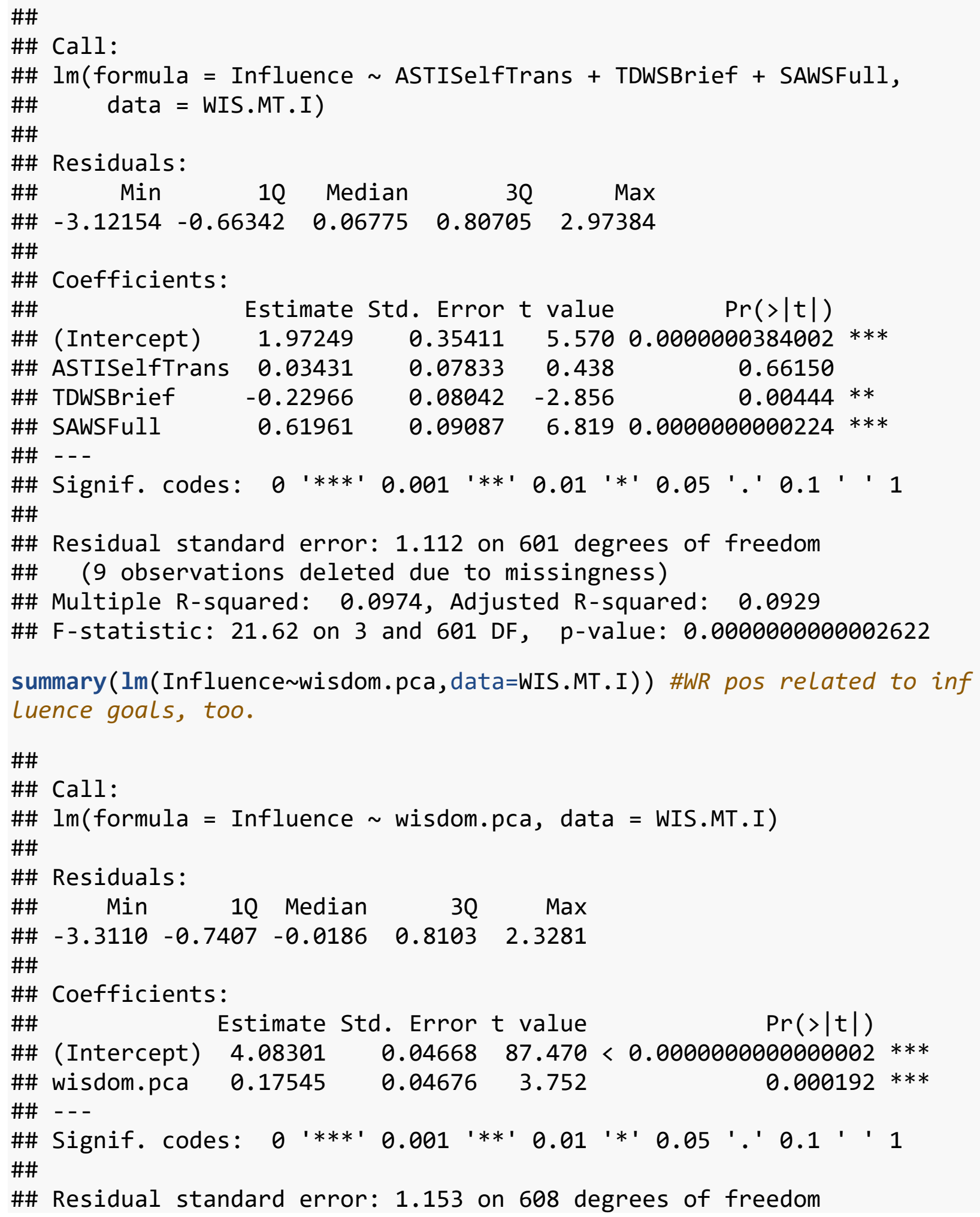


\#\# (4 observations deleted due to missingness)

\#\# Multiple R-squared: 0.02263, Adjusted R-squared: 0.02102

\#\# F-statistic: 14.08 on 1 and 608 DF, p-value: 0.0001924

summary ( $\operatorname{lm}$ (Influence wisdom.pca+ASTISelfTrans+TDWSBrief+SAWSFull, data= WIS.MT.I))

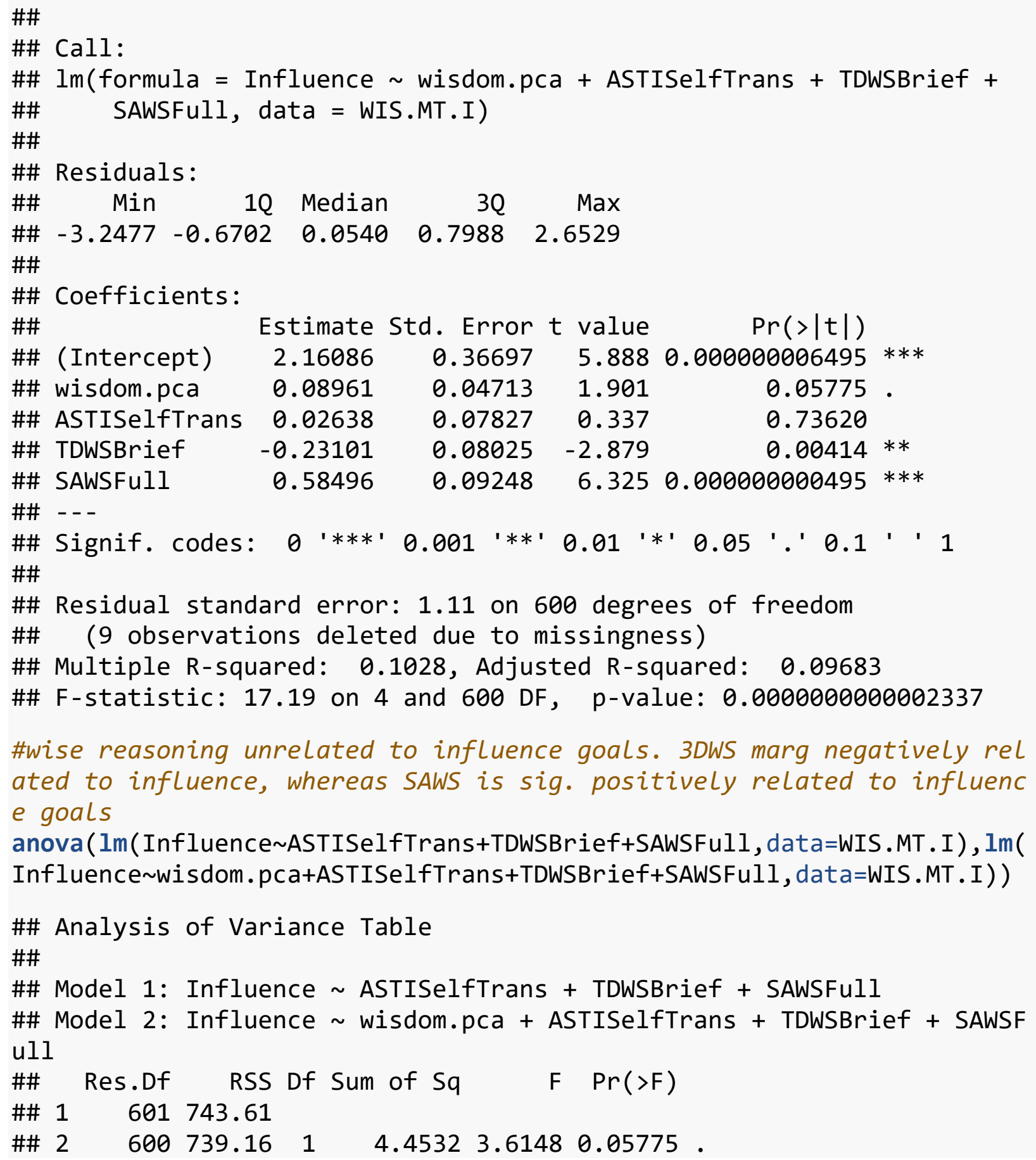
ated to influence, whereas SAWS is sig. positively related to influenc e goals anova ( Im( Influence ASTISelfTrans+TDWSBrief+SAWSFull, data=WIS.MT . I), Im( Influence wisdom.pca+ASTISelfTrans+TDWSBrief+SAWSFull, data=WIS.MT.I))

\#\# Analysis of Variance Table

\#\#

\#\# Model 1: Influence ASTISelfTrans + TDWSBrief + SAWSFull

\#\# Model 2: Influence $~$ wisdom.pca + ASTISelfTrans + TDWSBrief + SAWSF ul1

\#\# Res.Df RSS Df Sum of Sq $F \quad \operatorname{Pr}(>F)$

\#\# $1 \quad 601743.61$

$\begin{array}{llllllll}\# \# & 2 & 600 & 739.16 & 1 & 4.4532 & 3.6148 & 0.05775\end{array}$. 


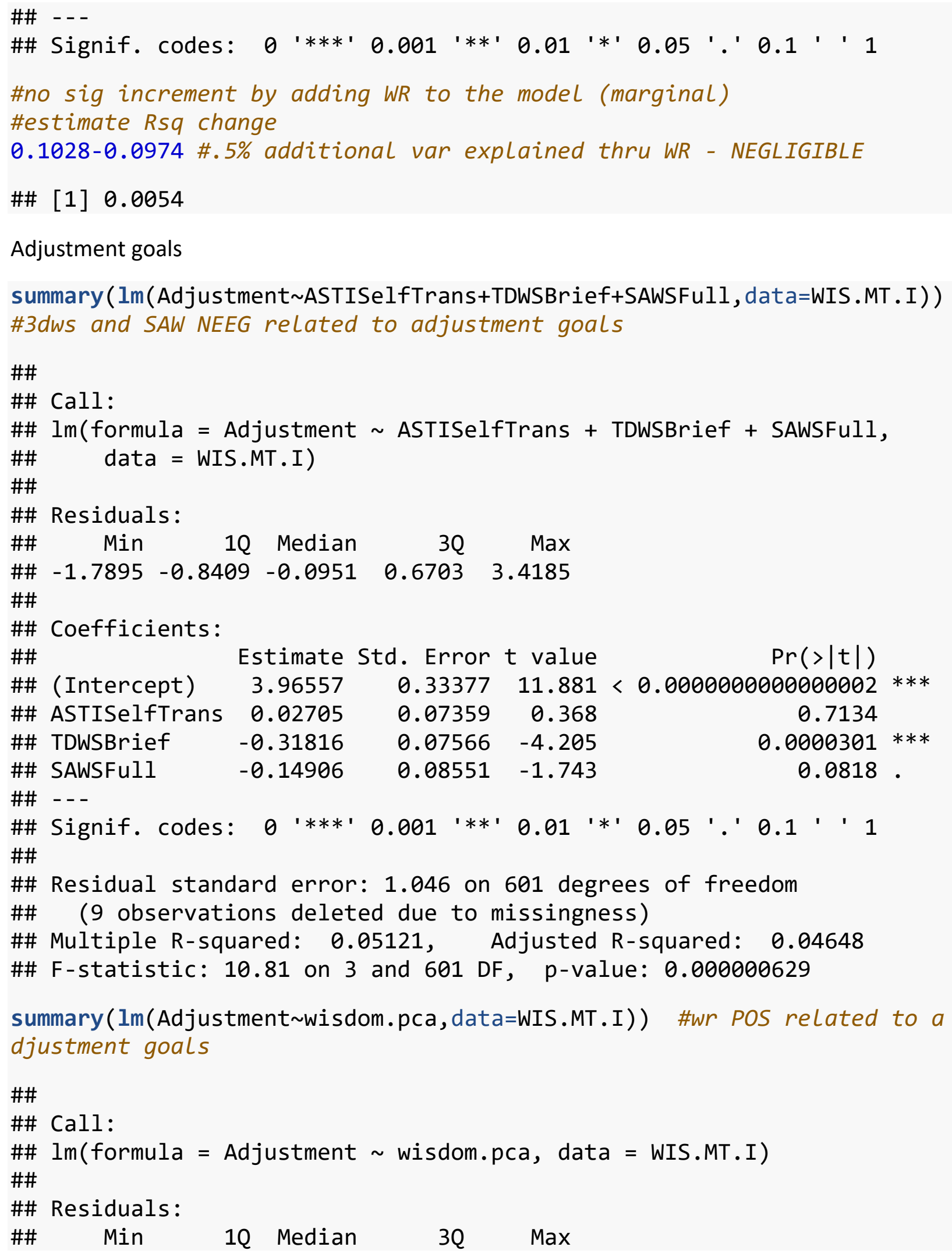




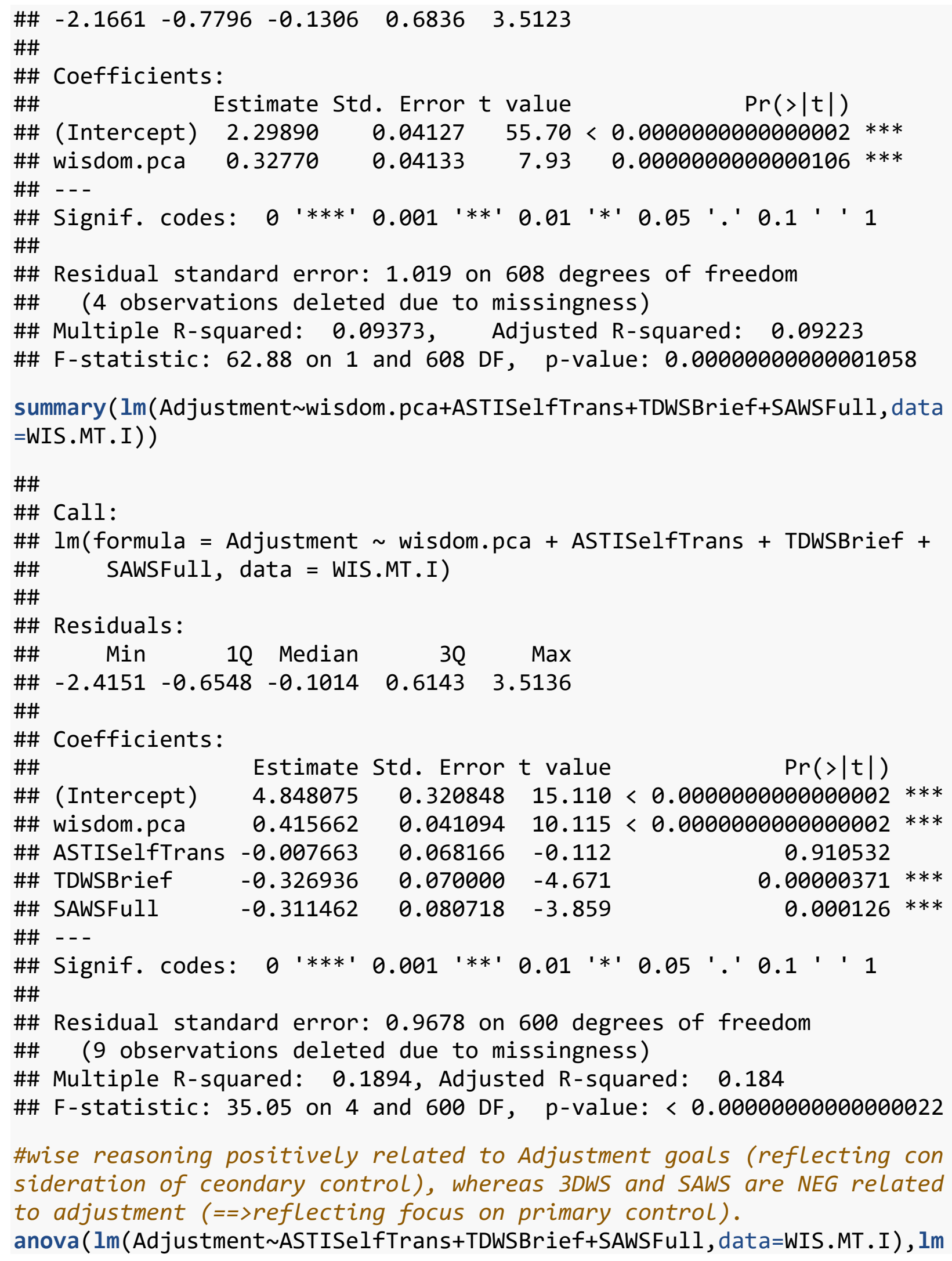


(Adjustment wisdom.pca+ASTISelfTrans+TDWSBrief+SAWSFull, data=WIS.MT.I) )

\#\# Analysis of Variance Table

\#\#

\#\# Model 1: Adjustment ASTISelfTrans + TDWSBrief + SAWSFull

\#\# Model 2: Adjustment wisdom.pca + ASTISelfTrans + TDWSBrief + SAWS Full

\#\# Res.Df RSS Df Sum of Sq $F \quad \operatorname{Pr}(>F)$

\#\# $1 \quad 601657.86$

\#\# $2 \quad 600562.03 \quad 1 \quad 95.835102 .31<0.00000000000000022$ ***

\#\# - - -

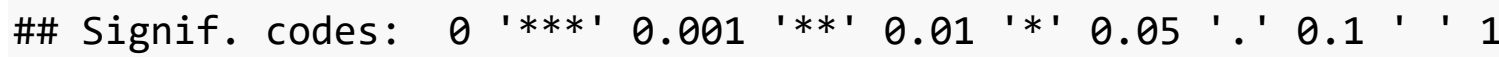

\#HUGE increment by adding WR to the model

\#estimate Rsq change

0.1894-0.05121 \#13.82\% additional var explained thru WR

\#\#[1] 0.13819

causal inference balance

summary ( $1 \mathrm{~m}$ (internal . attrib.balance ASTISelfTrans+TDWSBrief+SAWSFull, da ta=WIS.MT.I)) \#trait unrelated to causal inference balance

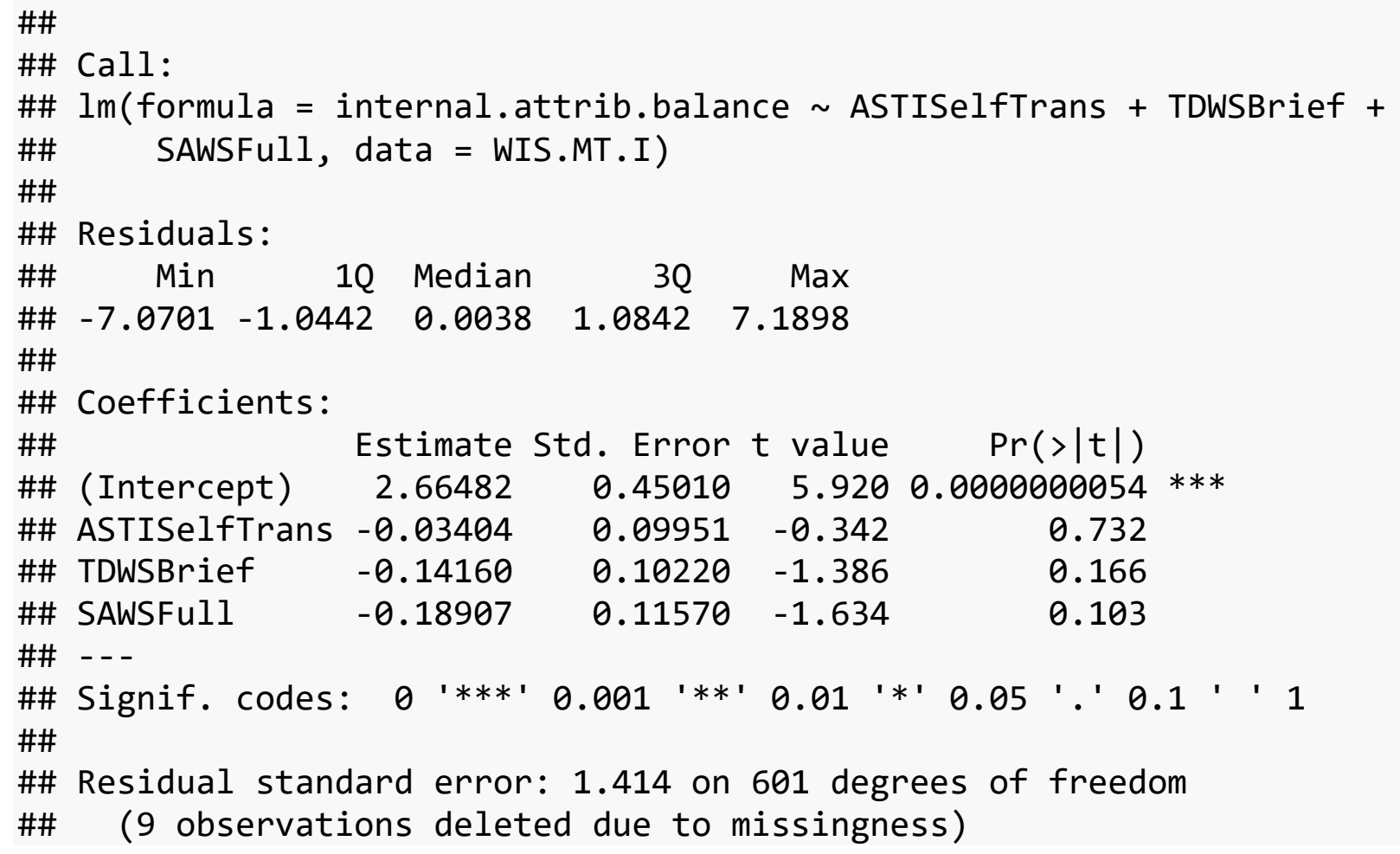


\#\# Multiple R-squared: 0.01753, Adjusted R-squared: 0.01262

\#\# F-statistic: 3.574 on 3 and 601 DF, p-value: 0.01386

summary (lm(internal.attrib.balance wisdom.pca, data=WIS.MT.I)) \#pos eff ect of WR on causal inference balance

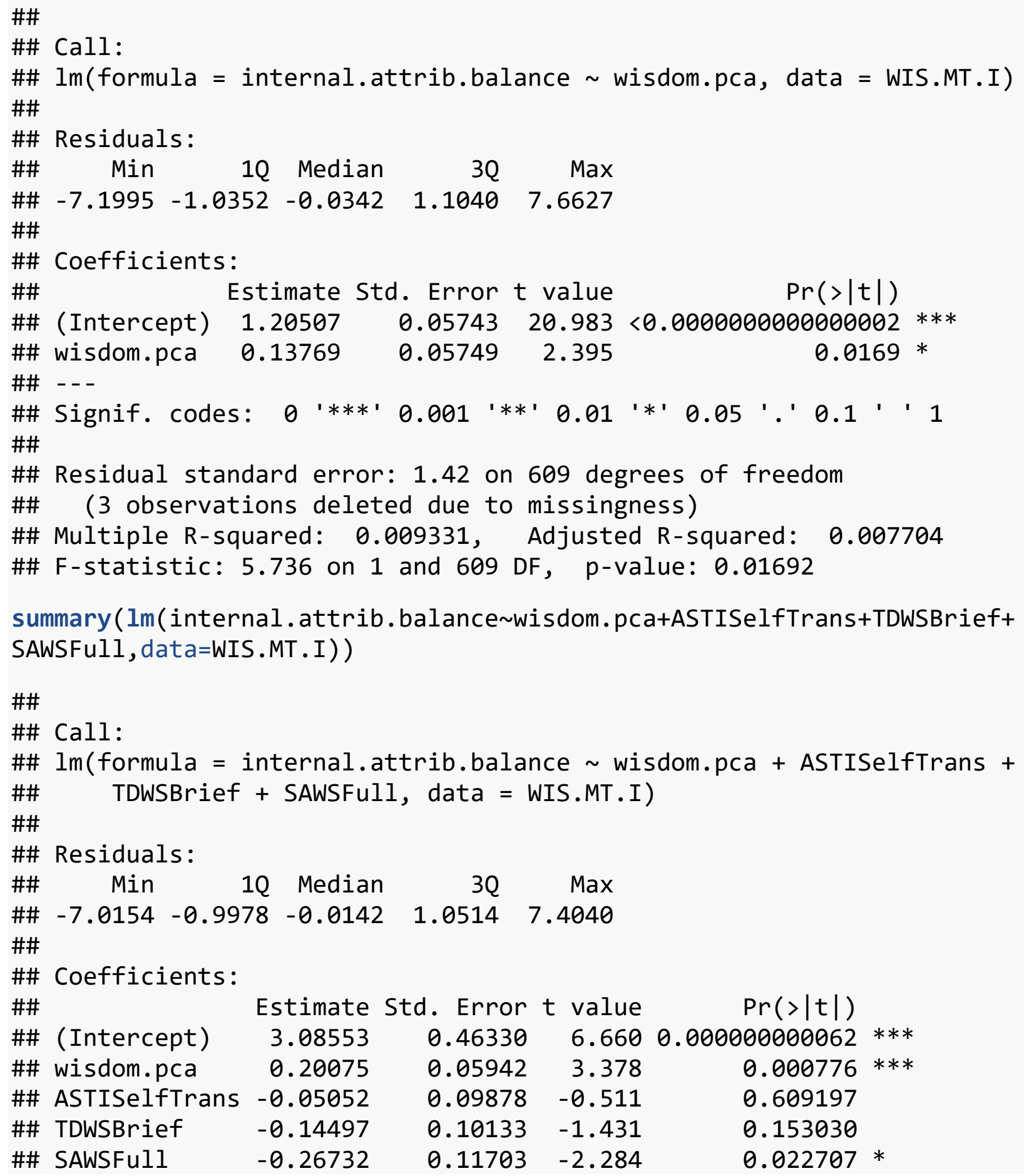




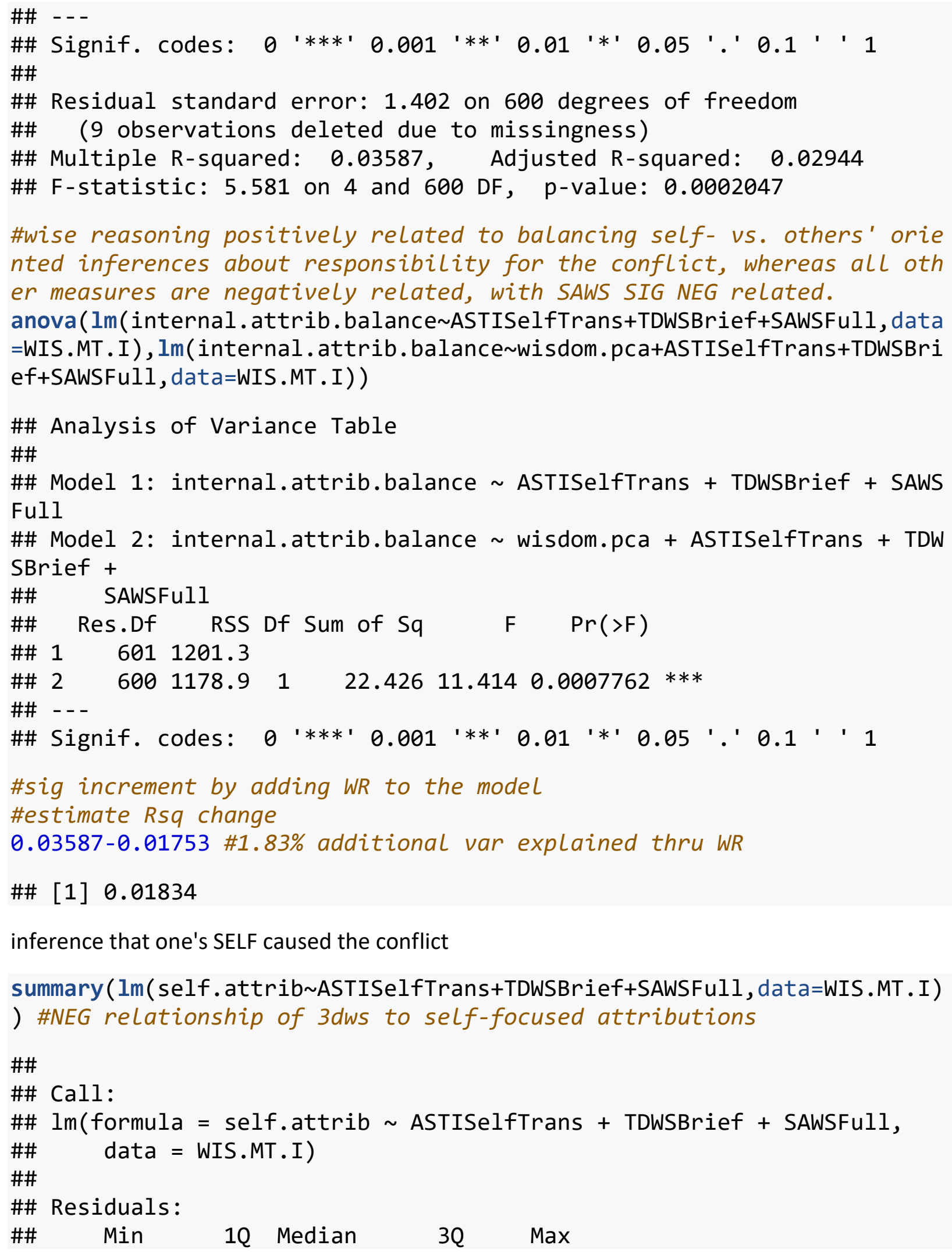




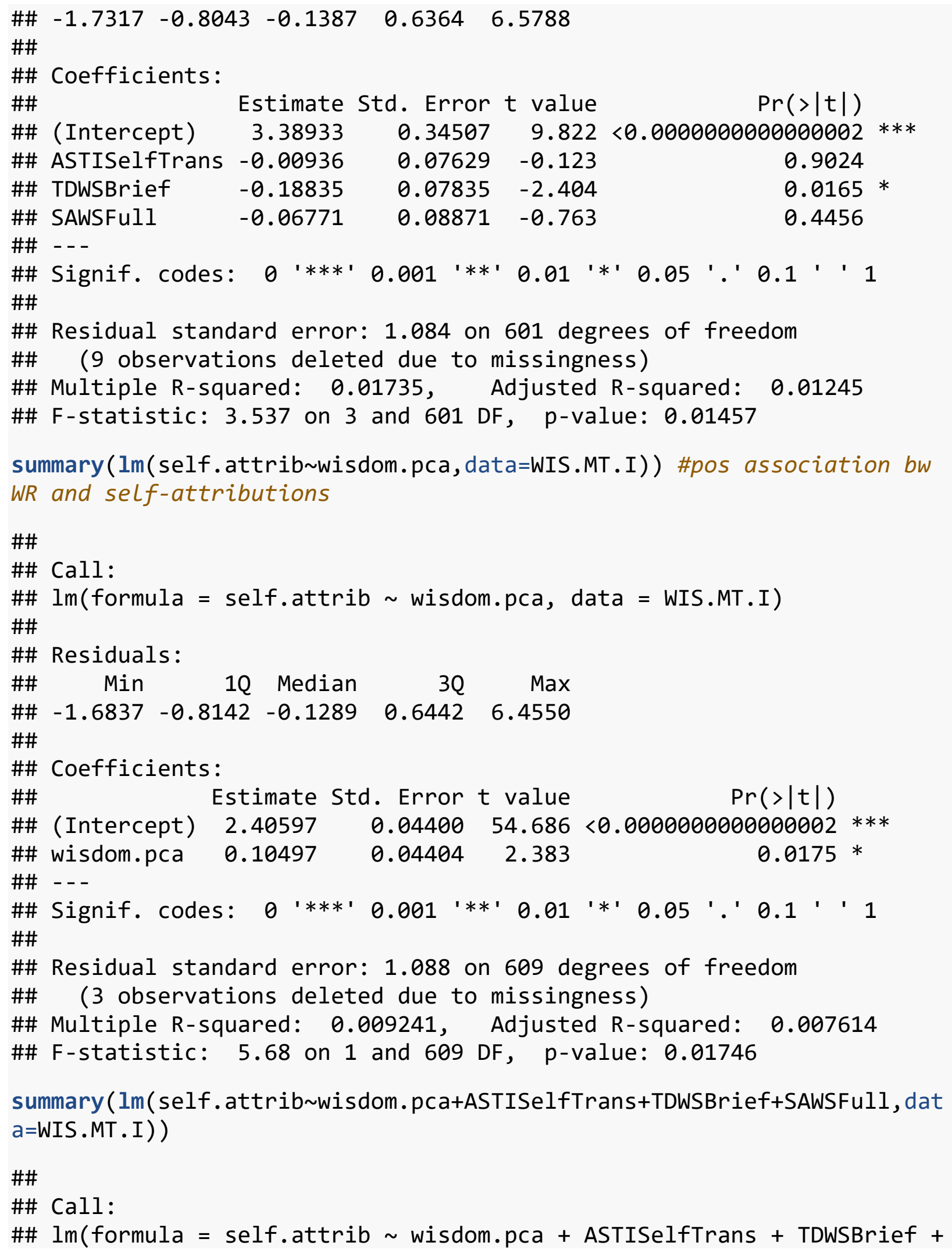




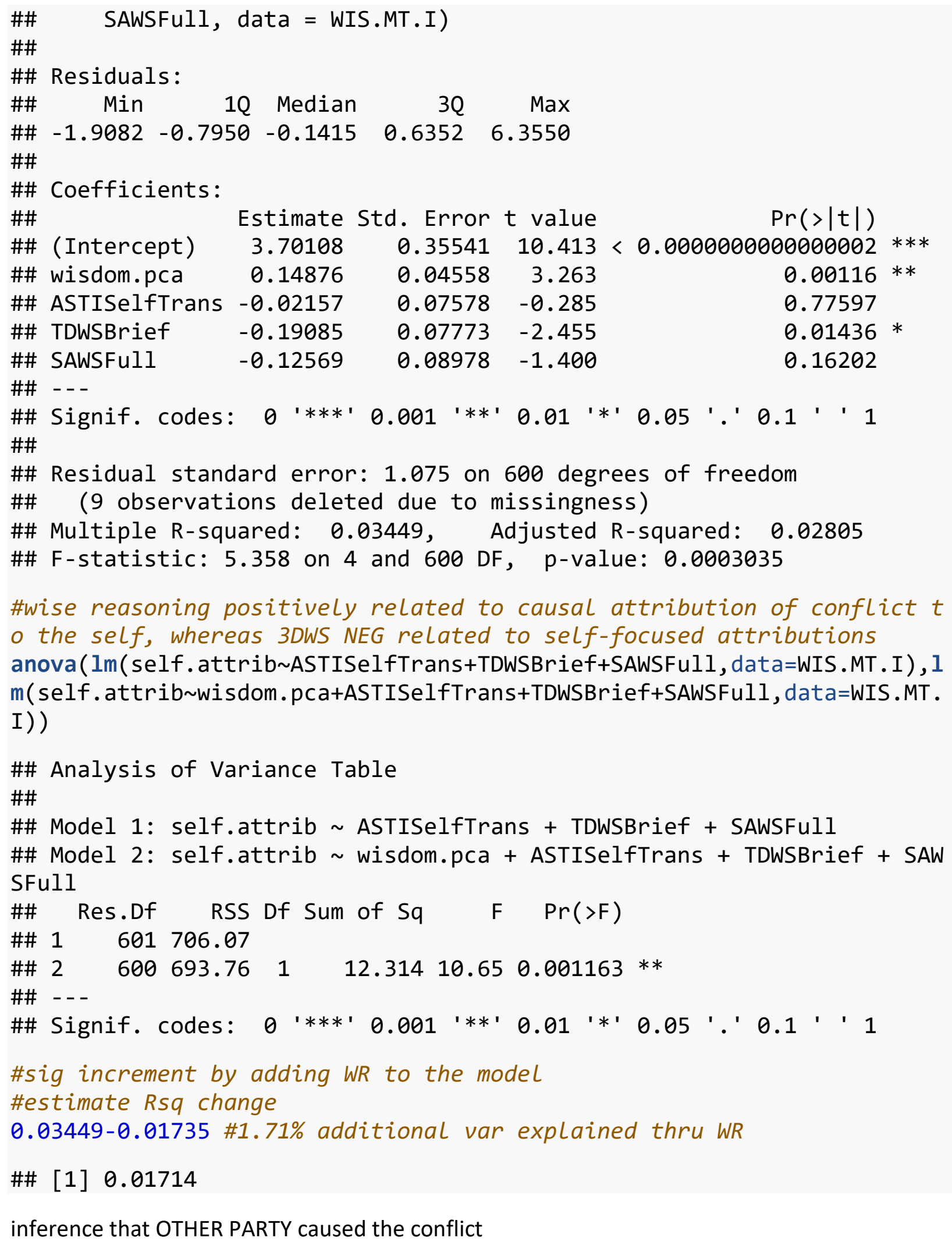


summary ( $1 \mathrm{~m}$ (other . attrib ASTISelfTrans+TDWSBrief+SAWSFull, data=WIS. MT . I )) \#3dws and saws POS Linked to other party attributions (i.e. blame)

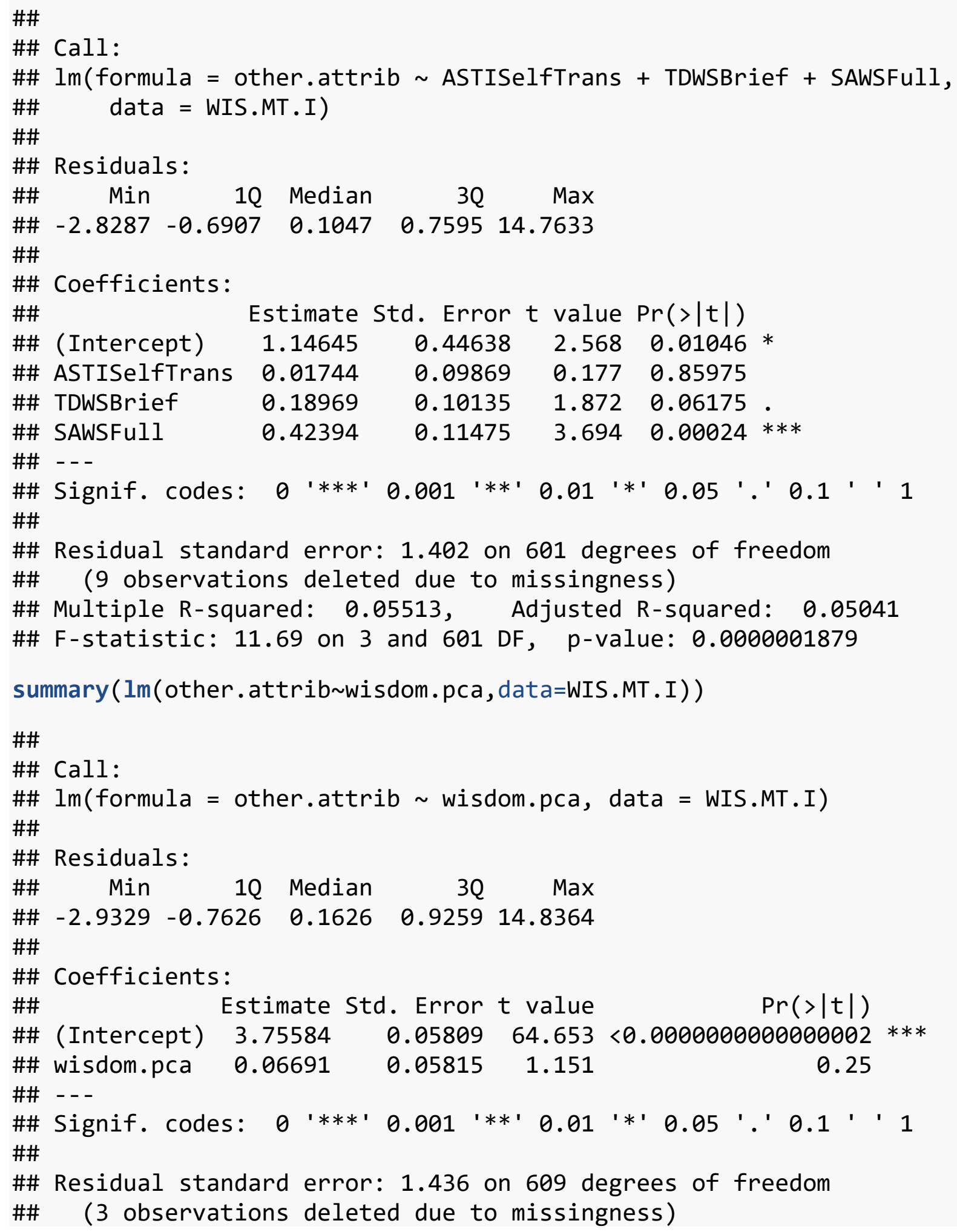


\#\# Multiple R-squared: 0.002169, Adjusted R-squared: 0.0005304 \#\# F-statistic: 1.324 on 1 and 609 DF, p-value: 0.2504

summary ( $1 \mathrm{~m}$ (other . attrib wi sdom. pca+ASTISelfTrans+TDWSBrief+SAWSFull, da ta $=$ WIS.MT.I))

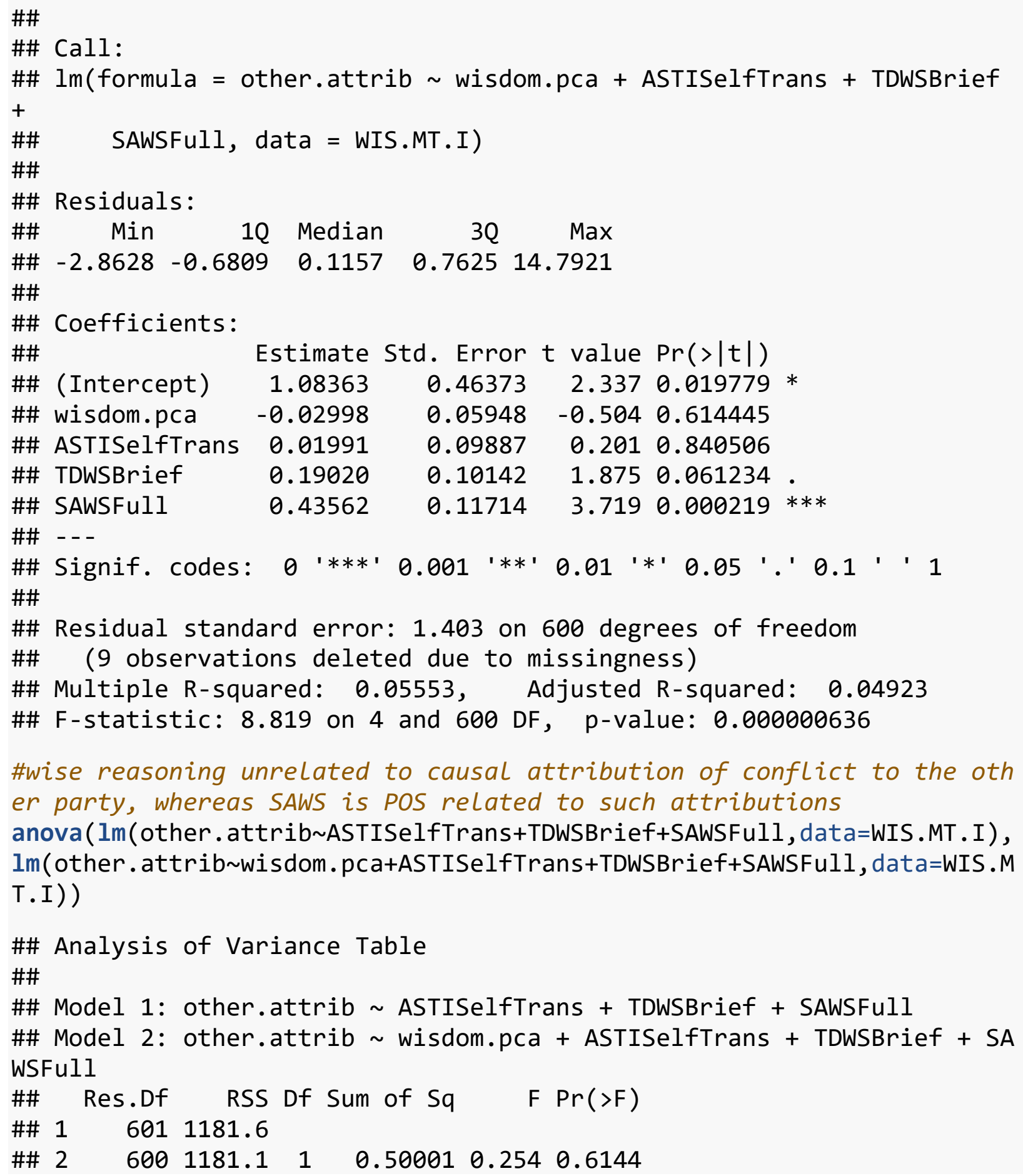


\#NO increment by adding WR to the model

\#estimate Rsq change

0.05553-0.05513 \#.04\% additional var explained thru WR

\#\#[1] 0.0004

supplementary: attributions of other's behavior to contextual factors (often neglected due to dispositional bias)

summary ( $1 \mathrm{~m}$ (other . context ASTISelfTrans+TDWSBrief+SAWSFull, data=WIS . MT . I)) \#saws pos related to situational attributions of the other party

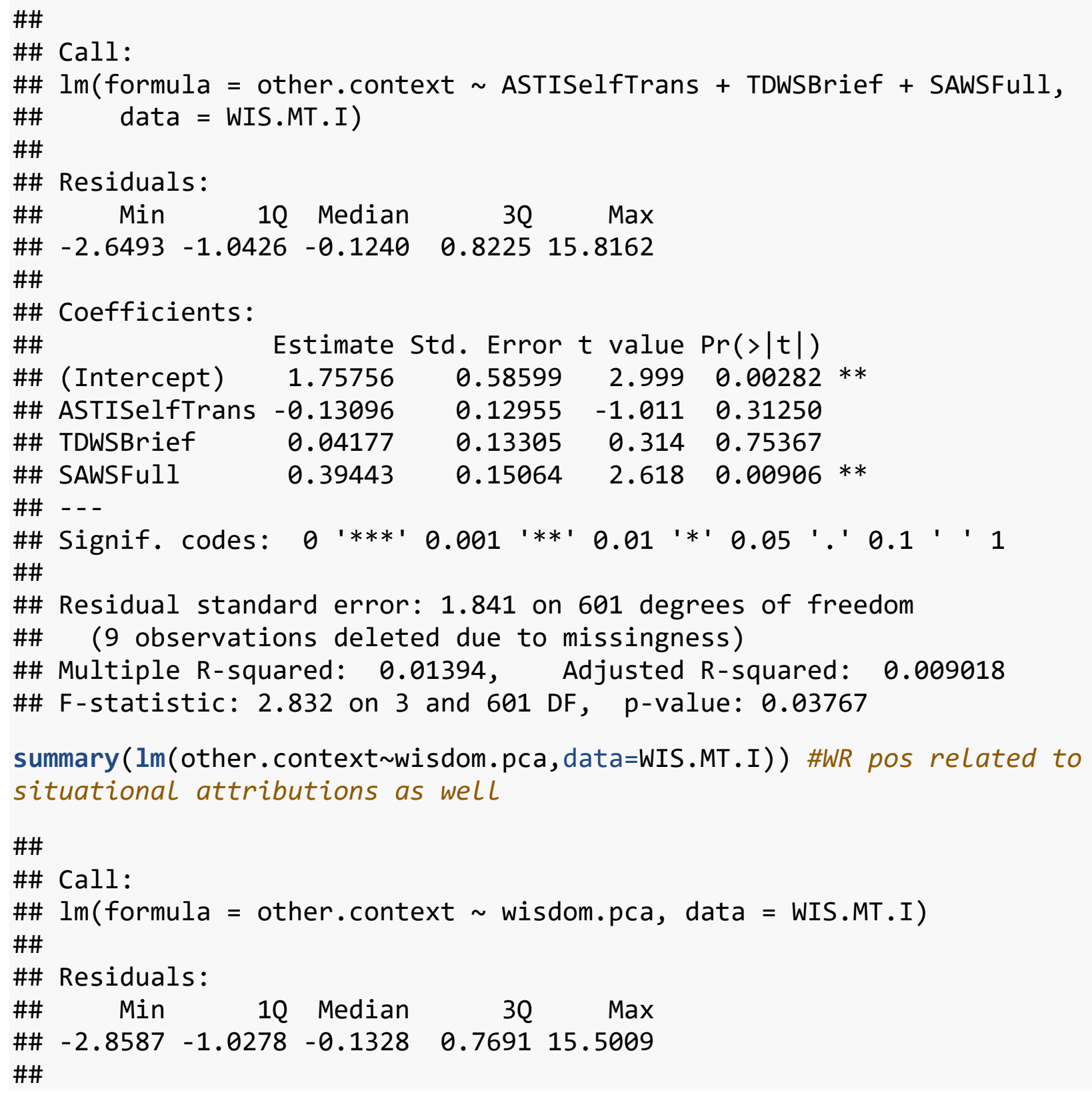




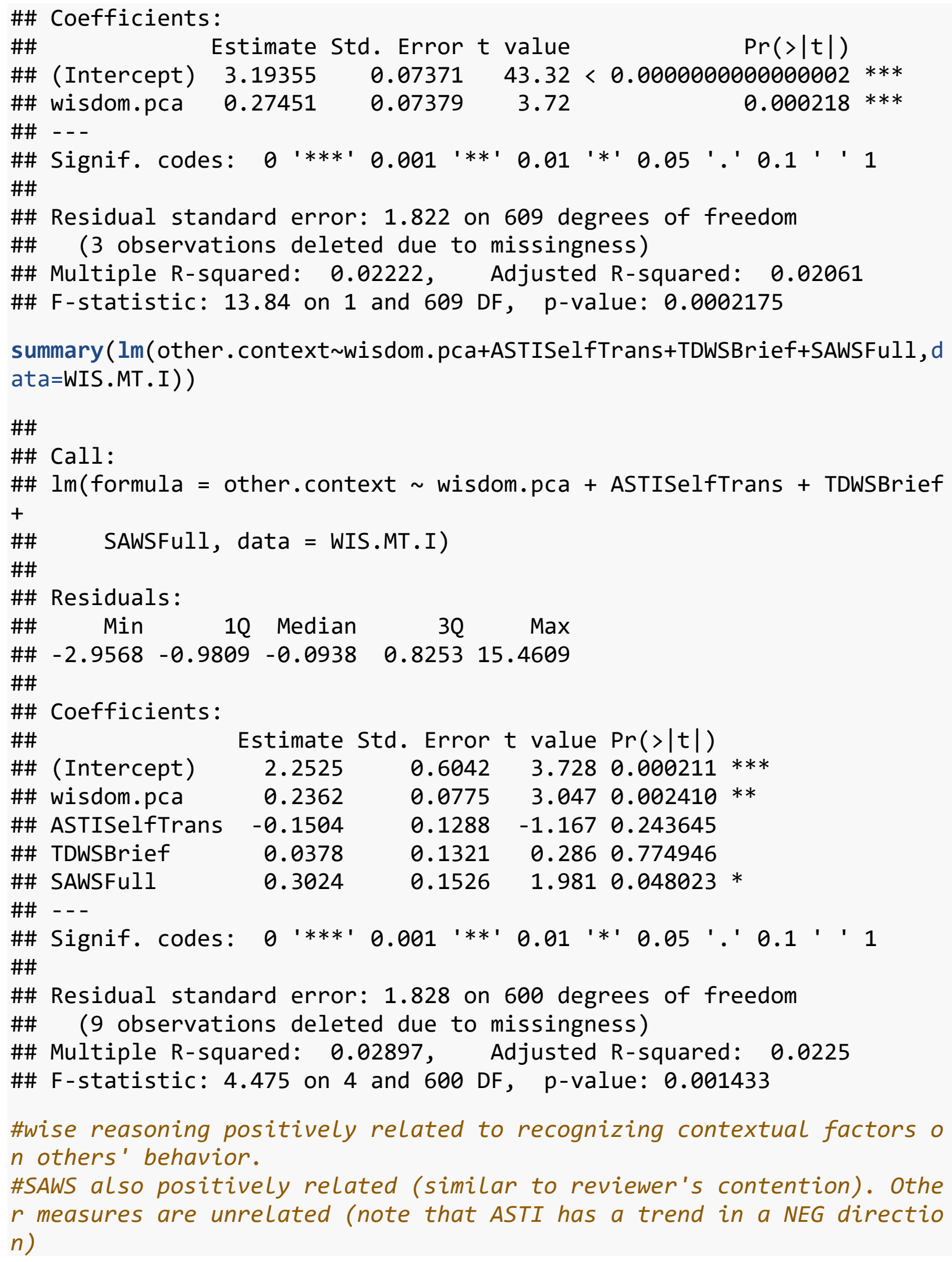


anova ( $1 \mathrm{~m}$ (other. context ASTISelfTrans+TDWSBrief+SAWSFull, data=WIS.MT. I) , $\operatorname{lm}$ (other. context wisdom. pca+ASTISelfTrans+TDWSBrief+SAWSFull, data=WIS .MT.I))

\#\# Analysis of Variance Table

\#\#

\#\# Model 1: other.context ASTISelfTrans + TDWSBrief + SAWSFull

\#\# Model 2: other.context $\sim$ wisdom.pca + ASTISelfTrans + TDWSBrief + S AWSFull

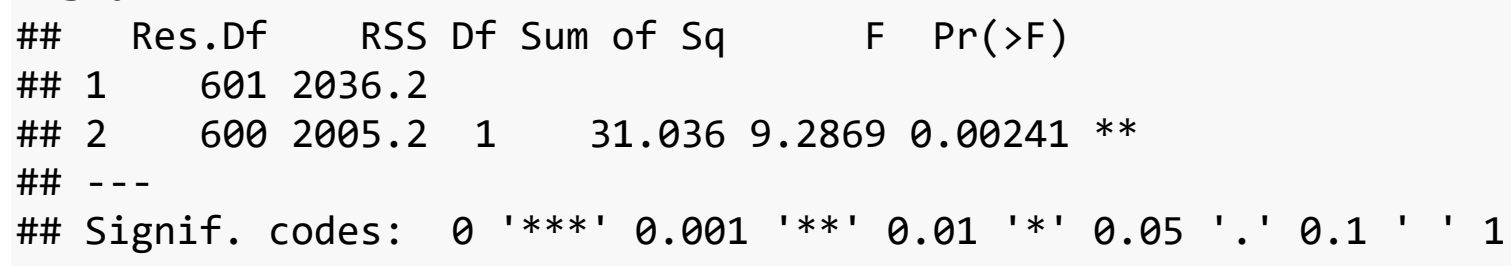

beliefs about work vs. family conflicts - only perform on a subsample of employed individuals. WIS.MT.I.working=subset(WIS.MT.I, Employment=='Employed' ) \#create subs ets of data with only employed people for work-family balance

describe(WIS.MT.I.working\$WorkTOFamilyConflict) \# WORK TO FAMILY: dist ribution from 1 strongly disagree (10\%) to 5 (moderately agree - 90\%)

\#\# WIS.MT.I.working\$WorkTOFamilyConflict

\#\# $\mathrm{n}$ missing distinct Info Mean 05

.10

$\begin{array}{llllllll}\# \# & 505 & 0 & 11 & 0.988 & 3.039 & 1.68 & 1.0\end{array}$

1.0

$\begin{array}{lllll}\# \# & .25 & .50 & .75 & .90\end{array}$

$\begin{array}{lllll}\# \# & 2.0 & 3.0 & 4.0 & 5.0\end{array}$

\#\#

$\begin{array}{llllllllll}\text { \#\# Value } & 1.0 & 1.5 & 2.0 & 2.5 & 3.0 & 3.5 & 4.0 & 4.5 & 5.0\end{array}$

5.5

$\begin{array}{llllllllll}\text { \#\# Frequency } & 80 & 43 & 52 & 50 & 52 & 67 & 49 & 37 & 35\end{array}$

14

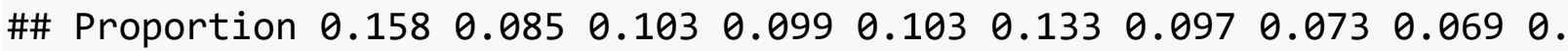
028

\#\#

\#\# Value $\quad 6.0$ 


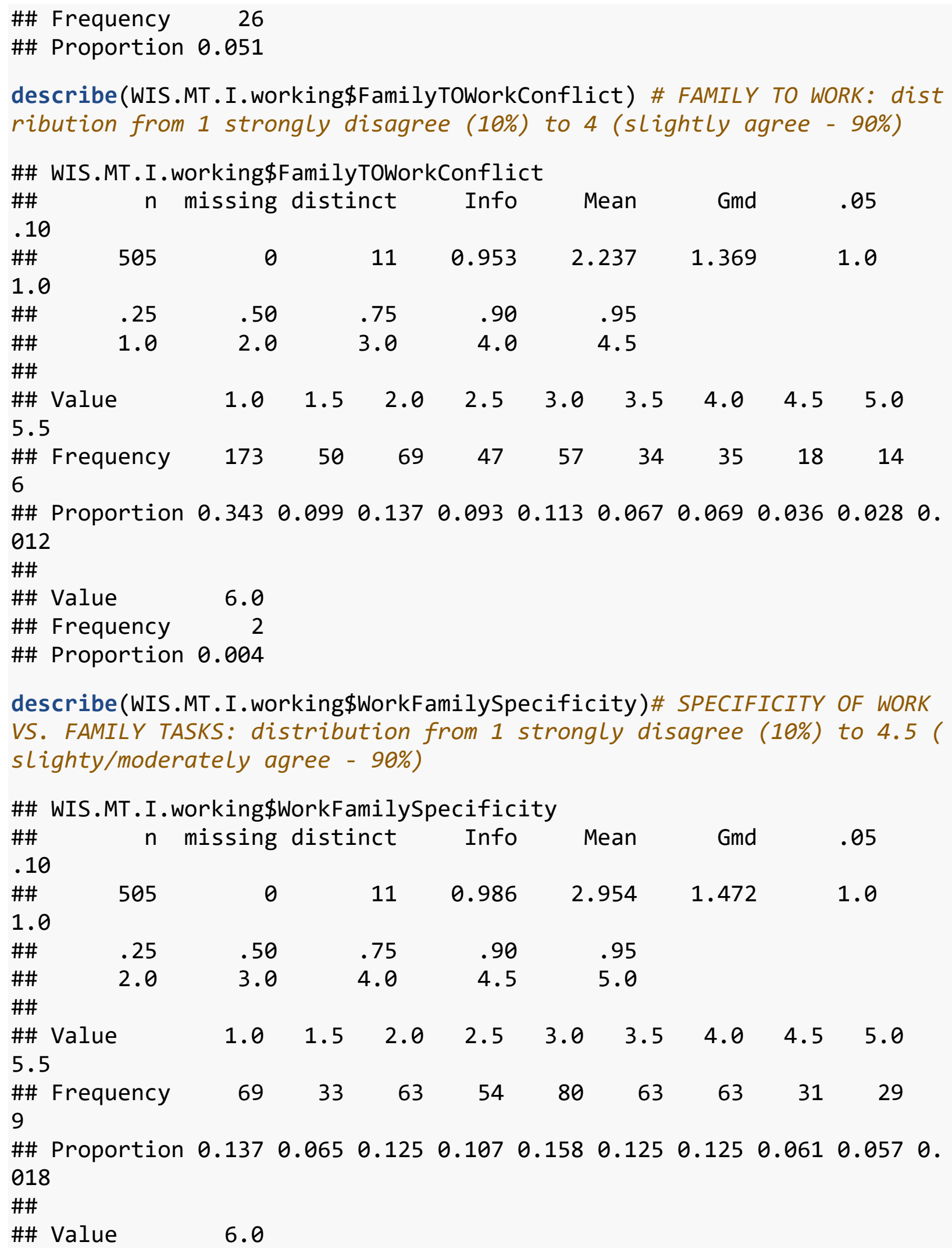




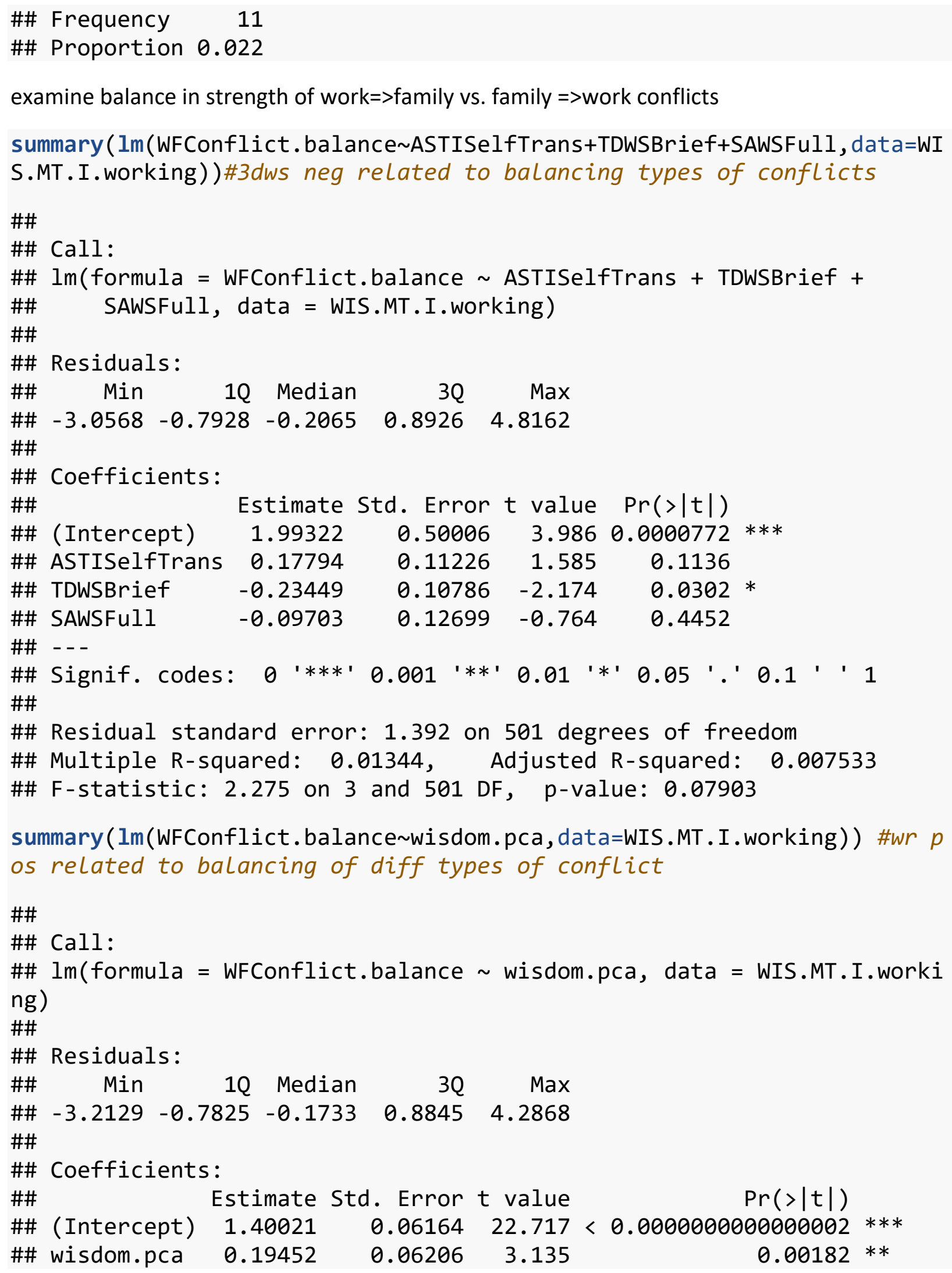




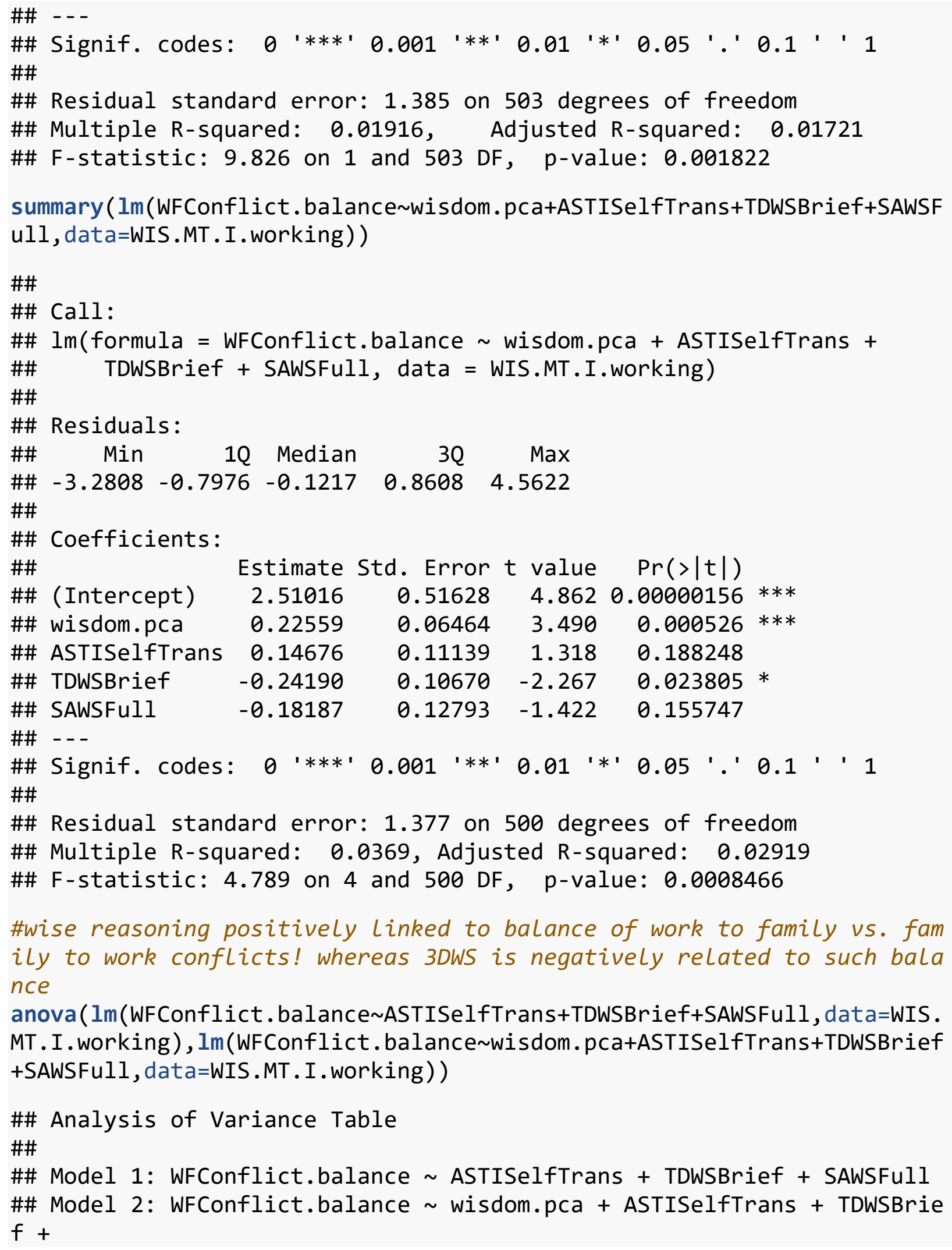
MT . I.working), lm(WFConflict.balance wisdom. pca+ASTISelfTrans+TDWSBrief +SAWSFull, data=WIS.MT . I.working))

\#\# Analysis of Variance Table

\#\#

\#\# Model 1: WFConflict.balance ASTISelfTrans + TDWSBrief + SAWSFull \#\# Model 2: WFConflict.balance wisdom.pca + ASTISelfTrans + TDWSBrie $f+$ 


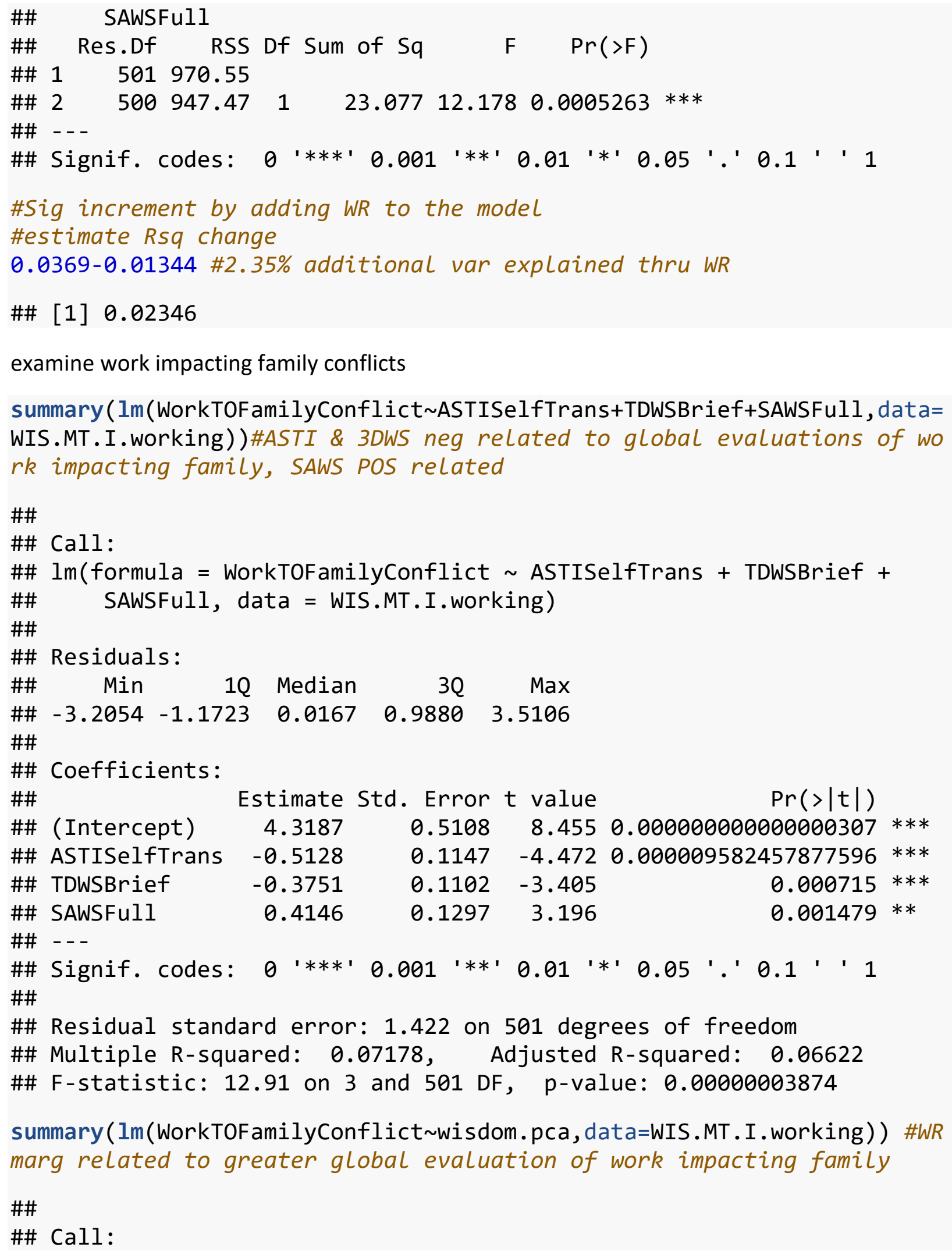


\#\# $\operatorname{lm}($ formula $=$ WorkTOFamilyConflict $\sim$ wisdom.pca, data $=$ WIS.MT.I.wor king)

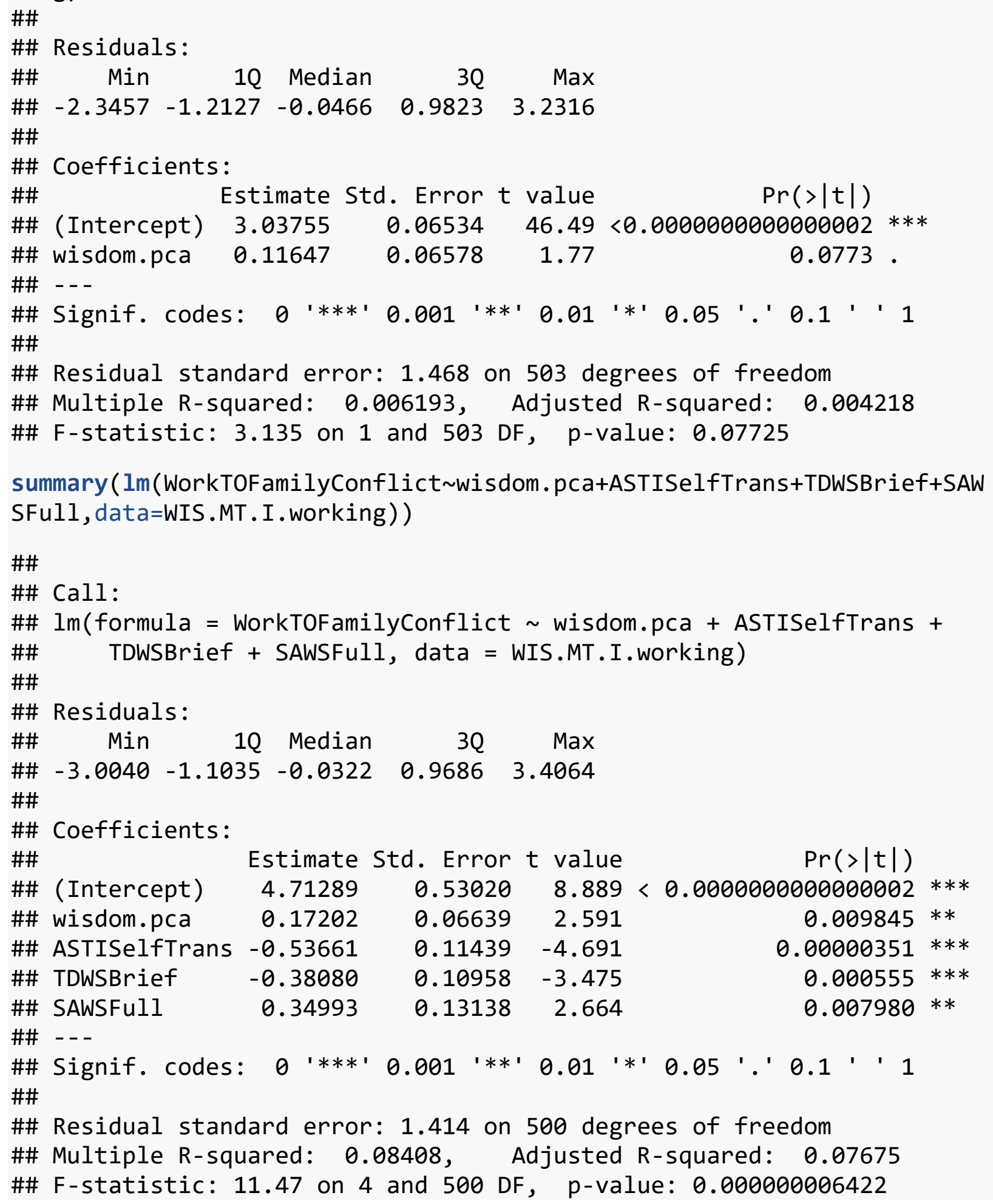


\#wise reasoning unrelated to the view that work negatively impacts fam ily life, whereas ASTI and 3dws are neg related to this belief, wherea $s$ SAWS is positively relaed to this belief

anova ( $1 \mathrm{~m}$ (WorkTOFamilyConflict ASTISelfTrans+TDWSBrief+SAWSFull, data=WI S.MT.I.working), $\operatorname{lm}$ (WorkTOFamilyConflict wisdom. pca+ASTISelfTrans+TDWSB rief+SAWSFull, data=WIS.MT . I.working))

\#\# Analysis of Variance Table

\#\#

\#\# Model 1: WorkTOFamilyConflict ASTISelfTrans + TDWSBrief + SAWSFul 1

\#\# Model 2: WorkTOFamilyConflict wisdom.pca + ASTISelfTrans + TDWSBr ief +

\#\# SAWSFull

\#\# Res.Df RSS Df Sum of Sq F $\operatorname{Pr}(>F)$

\#\# $1 \quad 501 \quad 1012.69$

$\begin{array}{lllllllll}\text { \#\# } 2 & 500 & 999.27 & 1 & 13.418 & 6.7141 & 0.009845 & * *\end{array}$

\#\# - - -

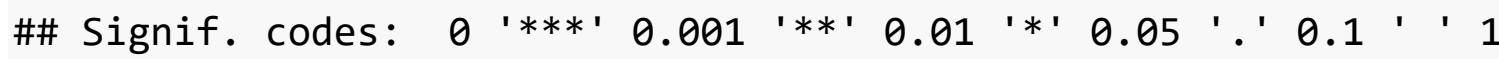

\#Sig increment by adding WR to the model

\#estimate Rsq change

0.08408-0.07178 \#1.23\% additional var explained thru WR

\#\#[1] 0.0123

examine family stress impacting work

summary ( $1 \mathrm{~m}$ (FamilyTOWorkConflict ASTISelfTrans+TDWSBrief+SAWSFull, data= WIS.MT.I.working))\#3dws neg related to claims that family stress impac ts work

\#\#

\#\# Call:

\#\# $\operatorname{lm}($ formula = FamilyTOWorkConflict ASTISelfTrans + TDWSBrief +

\#\# SAWSFull, data = WIS.MT.I.working)

\#\#

\#\# Residuals:

\#\# Min 10 Median $3 Q \quad$ Max

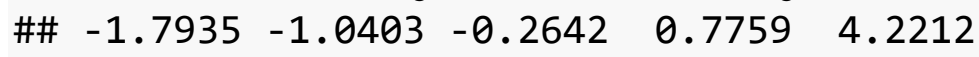

\#\#

\#\# Coefficients:

\#\#

\#\# (Intercept)

Estimate Std. Error t value

$\operatorname{Pr}(>|t|)$

H\# (Intercept) $\quad 3.53008$

0.44288

7.971

\#\# ASTISelfTrans -0.02050

$0.09942-0.206$

\#\# TDWSBrief

$-0.33896$

$0.09553-3.548$

0.836709

$0.000424 * * *$ 


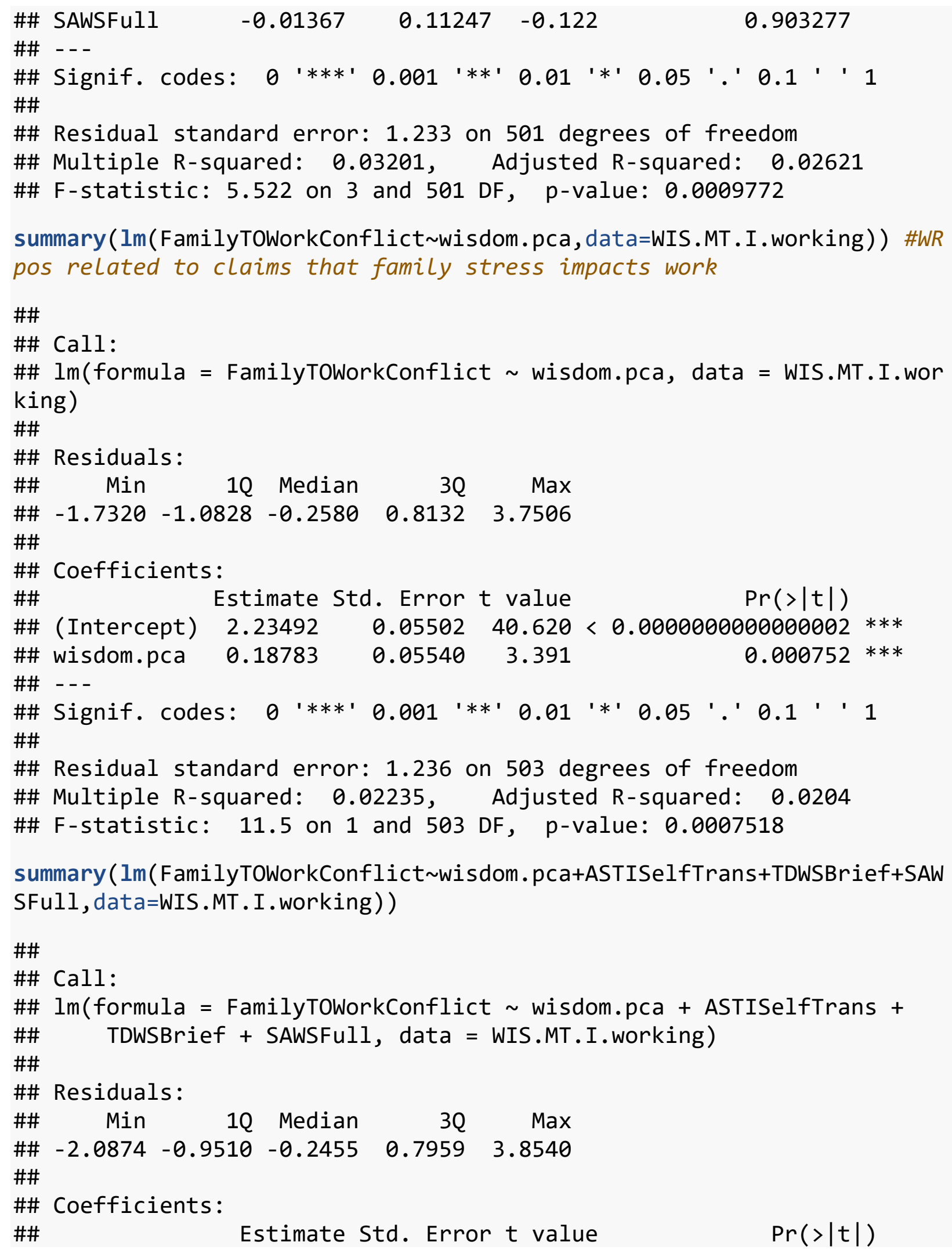




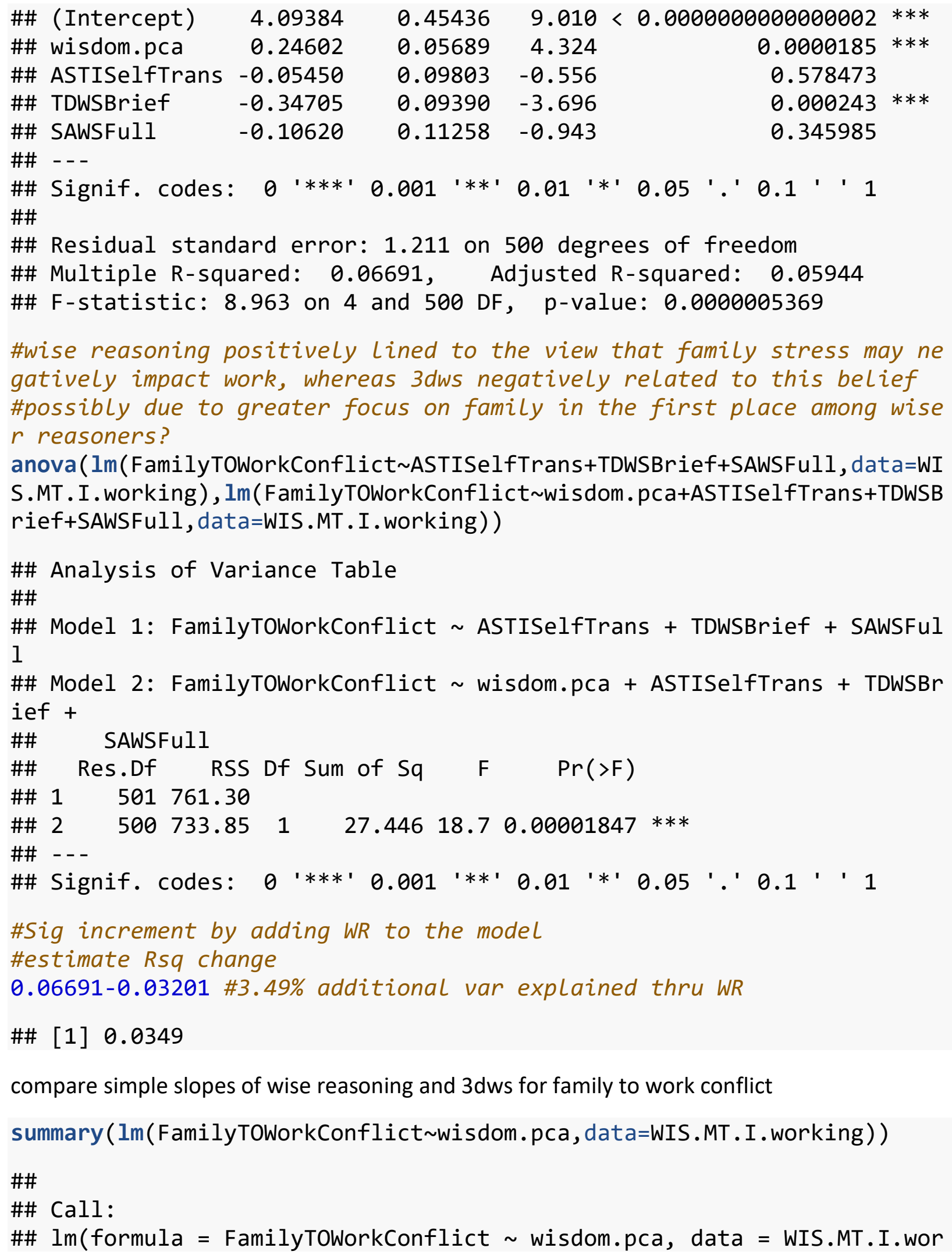




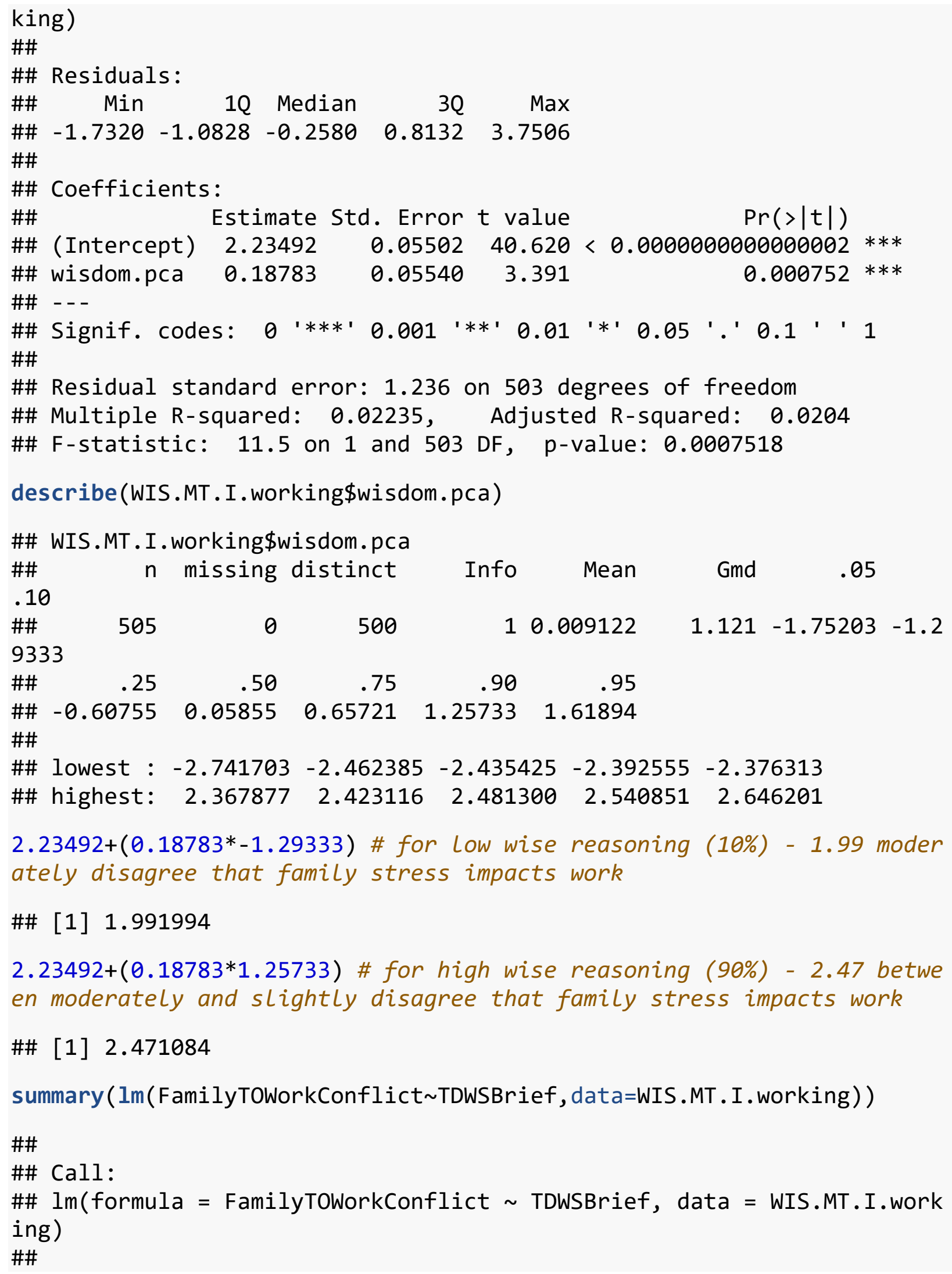




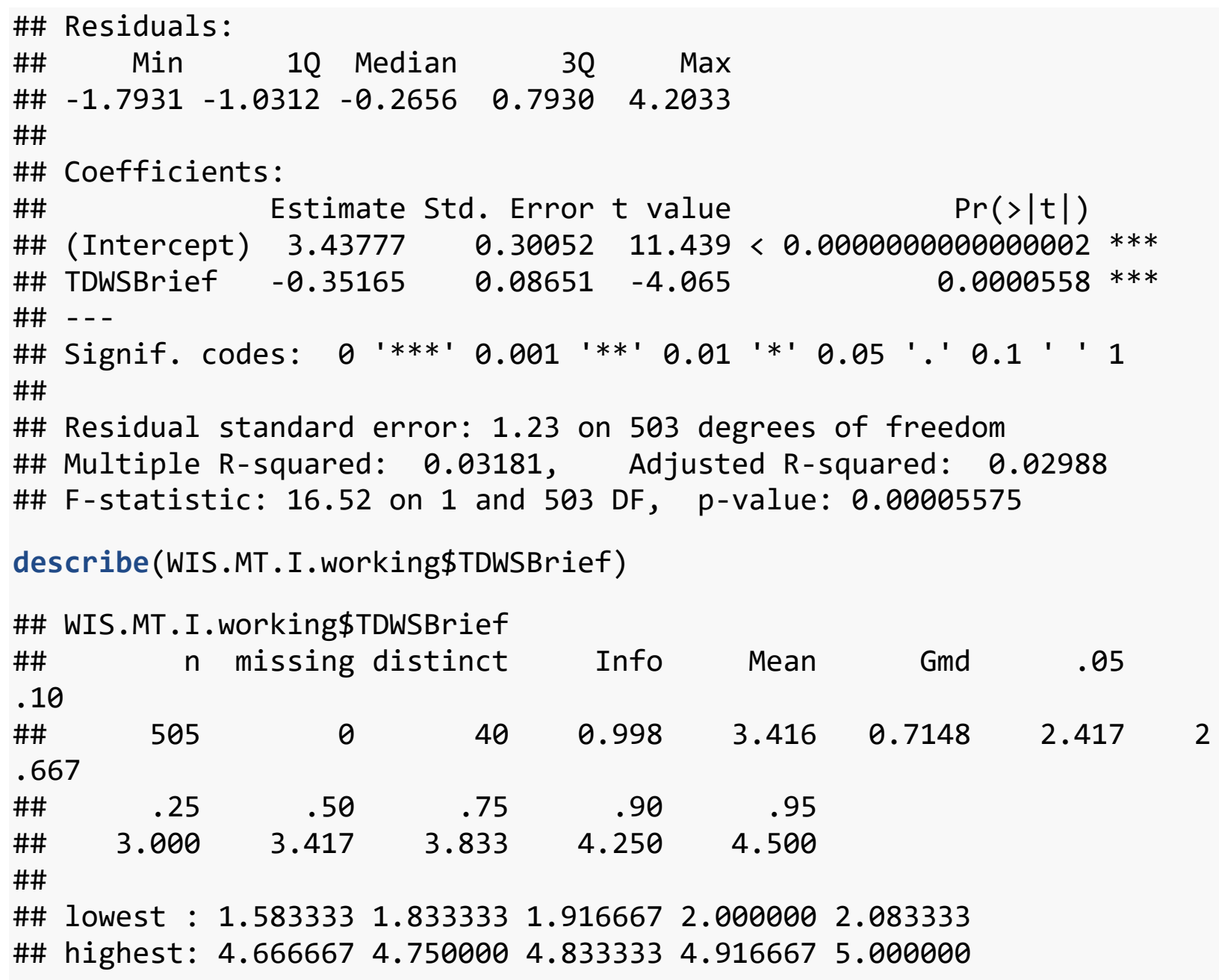

$3.43777+(-0.35165 * 2.667)$ \# for Low 3dws (10\%) - 2.50 between moderate ly and slightly disagree that family stress impacts work

\#\# [1] 2.499919

$3.43777+(-0.35165 * 4.250)$ \# for high 3dws (90\%) - 2.00 moderately disag ree that that family stress impacts work

\#\#[1] 1.943257

in short, the effect of wise reasoning for family to work conflicts is pretty small, and mainly concerns a healthy dose of realism, shifting from moderate disagreement that family stress impacts one's work to slight disagreement with this statement what is critical is that neither WR nor $3 \mathrm{~d}$ ws concern shifts ABOVE THE MIDPOINT of the scale supplemenmtary specificity of work and family-related tasks (not about conflict) 
summary (Im(WorkFamilySpecificity ASTISelfTrans+TDWSBrief+SAWSFull, data =WIS.MT.I.working)) \#asti \& 3dws neg related to separation of work and family tasks

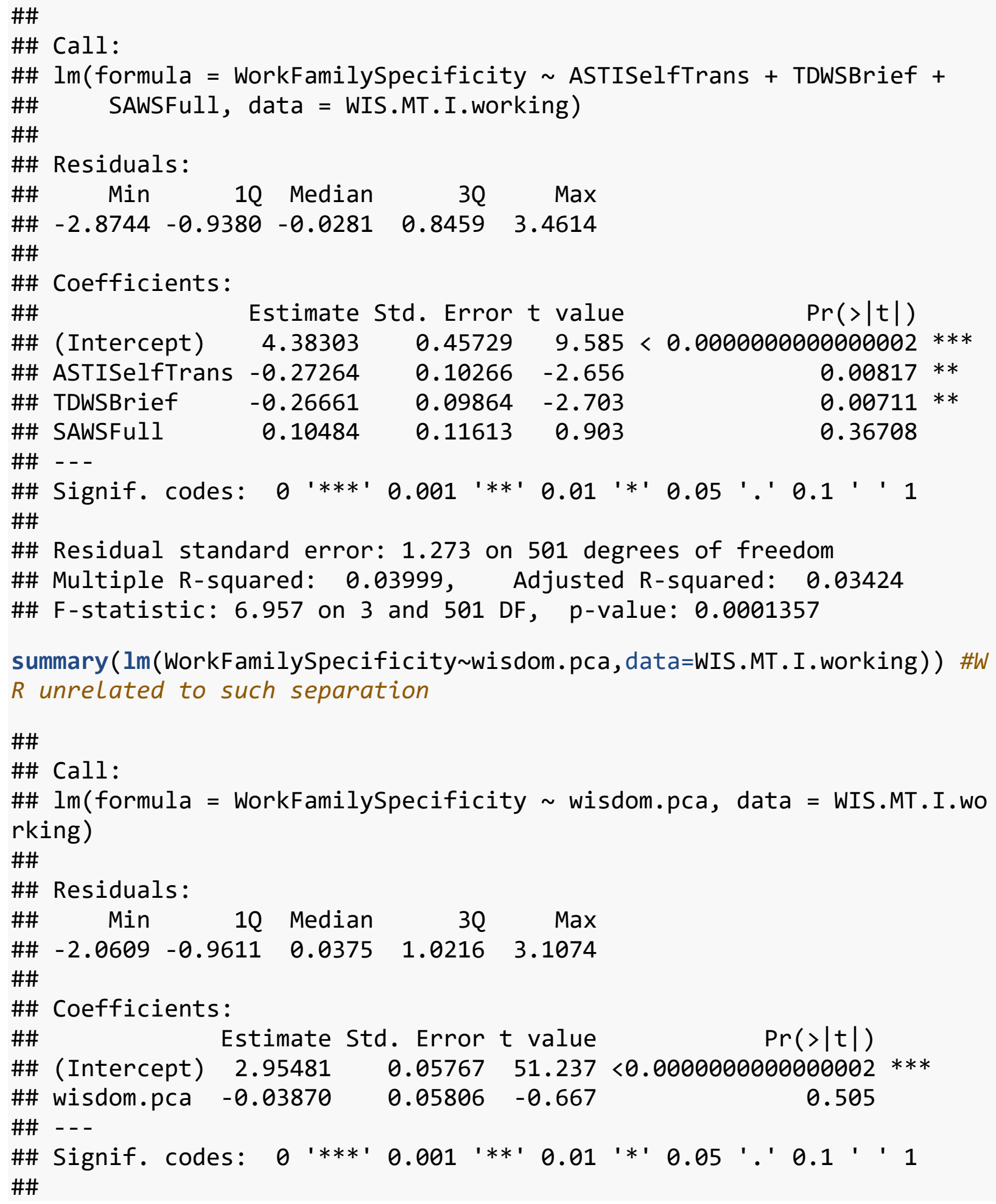


\#\# Residual standard error: 1.296 on 503 degrees of freedom \#\# Multiple R-squared: 0.0008827 , Adjusted R-squared: -0.001104 \#\# F-statistic: 0.4444 on 1 and 503 DF, p-value: 0.5053

summary ( $1 \mathrm{~m}$ (WorkFamilySpecificity wisdom.pca+ASTISelfTrans+TDWSBrief+SA WSFull, data=WIS.MT.I.working))

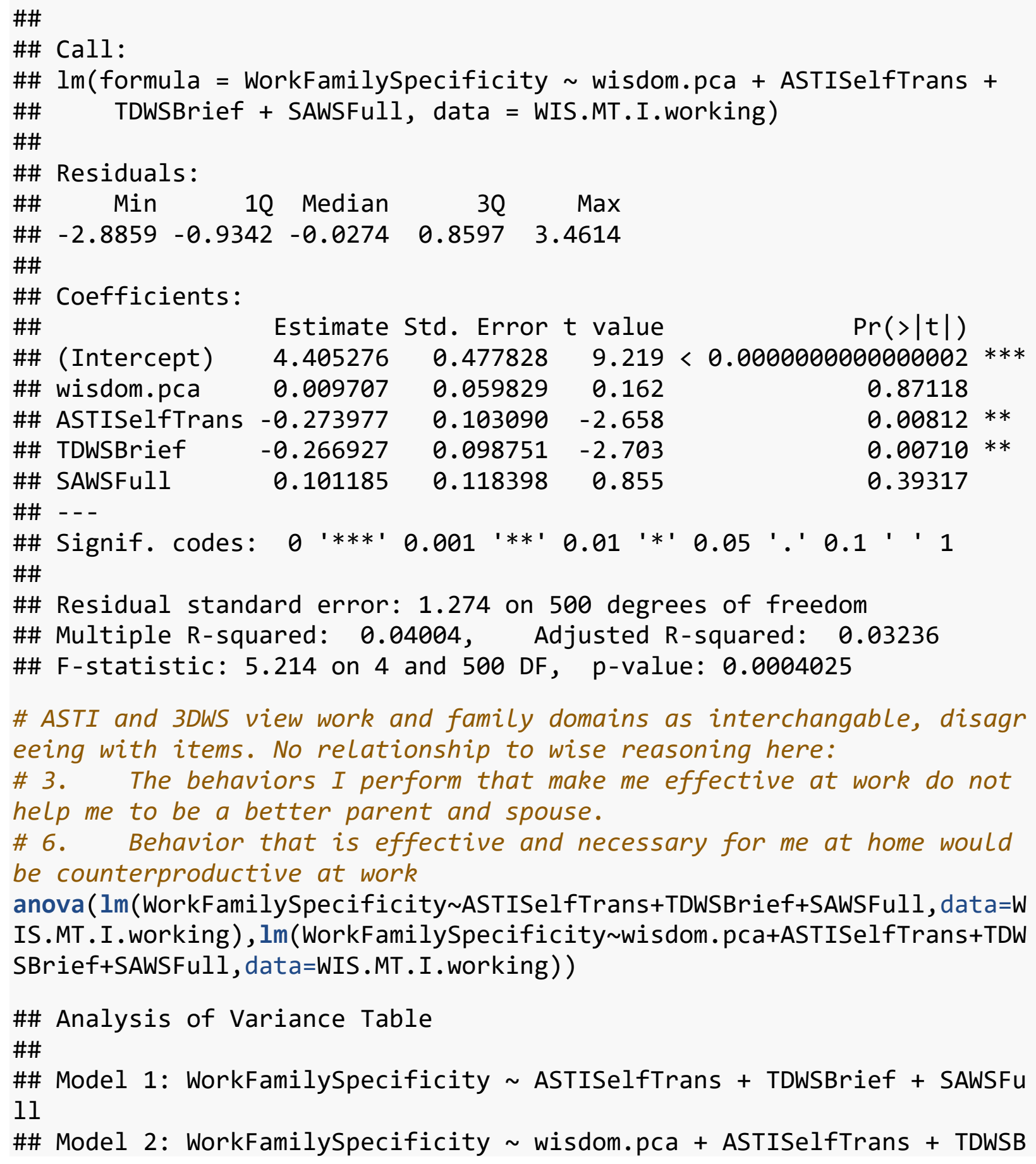
IS.MT.I.working), $\operatorname{lm}$ (WorkFamilySpecificity wisdom. pca+ASTISelfTrans+TDW SBrief+SAWSFull, data=WIS.MT.I.working)) 


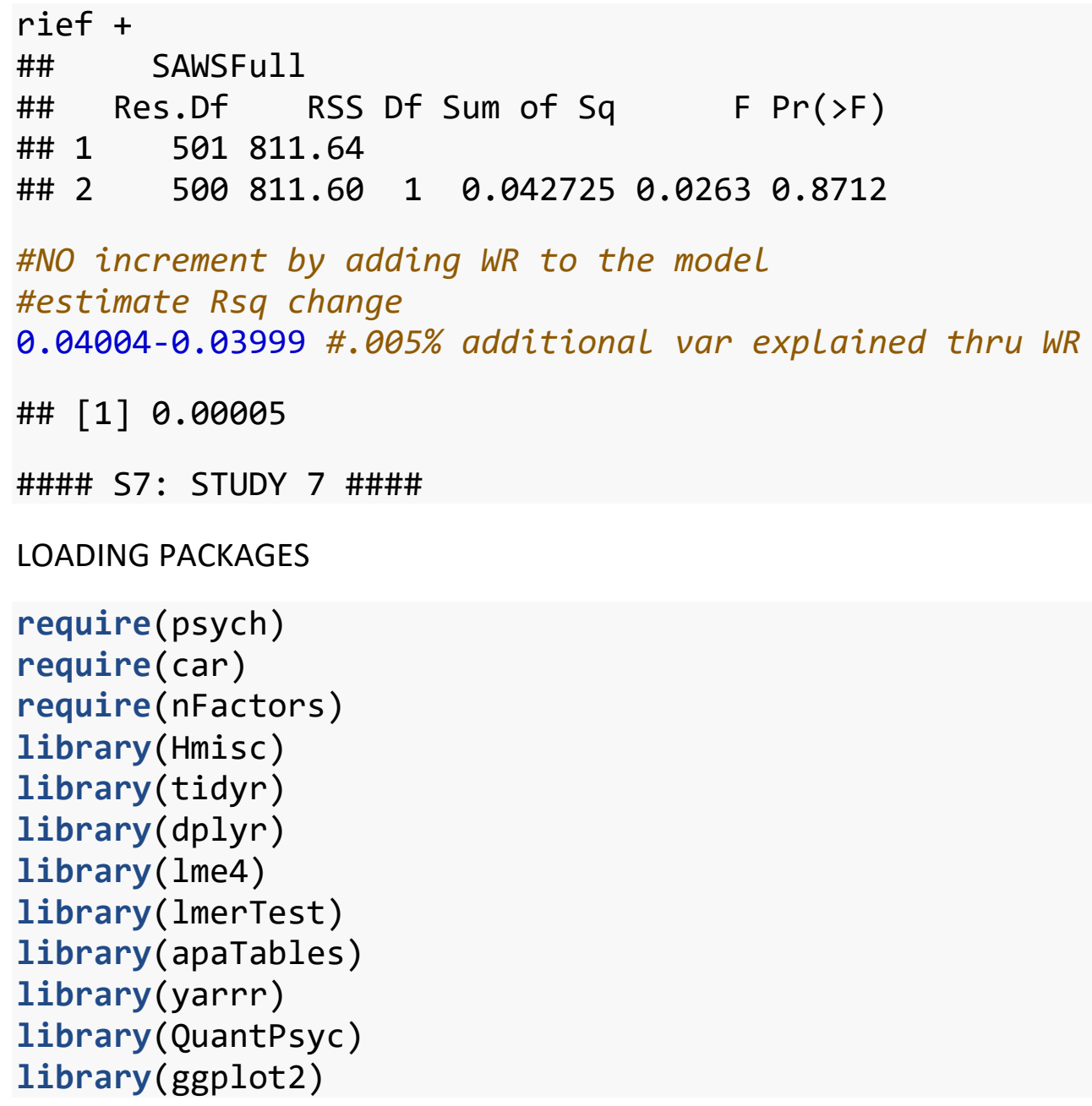

DISABLING SCIENTIFIC NOTATION

options (scipen=999)

LOADING AND PREPARING FILE

WIS.PA<-WIS.PA.RAW[WIS.PA.RAW\$joker==0, ] \#Removing bad responses, shou Ld have 300 obs. of 306 variables WIS.PA[WIS.PA==-99]<-NA \# Removing missing values WIS.PA[WIS.PA=='NA']<-NA \# Removing NA string WIS.PA\$id<-rownames(WIS.PA) \# Creating ID variable

RENAMING AND RECODING VARIABLES

names(WIS.PA)[names(WIS.PA)=='q3.2_1']<-'Conflict.Friend' \#Recent conf Lict with friend (multiple responses allowed)

names (WIS.PA) [names (WIS.PA) =='q3.2_2']<-'Conflict.Coworker' \#Recent co nflict with co-worker (multiple responses allowed)

names (WIS.PA) [names(WIS.PA)=='q3.2_3']<-'Conflict.Partner' \#Recent con 
flict with romantic partner (multiple responses allowed)

names (WIS.PA) [names (WIS.PA) =='q3.2_4']<-'Conflict.Family' \#Recent conf lict with family member (multiple responses allowed)

names (WIS.PA) [names(WIS.PA)=-'recall1']<-'C1. Person' \#Conflict 1: Pers on the conflict was with (displayed to participant)

names (WIS.PA) [names (WIS.PA)=='q4.4_3']<-'C1.Time' \#Conflict 1: Seconds thinking about conflict

WIS.PA\$C1.Time. $\log <-\log 10$ (WIS.PA\$C1.Time) \#Conflict 1: Seconds thinkin $g$ about conflict (Log)

WIS.PA\$C1. When<-car: : recode(WIS.PA\$q4.6, "1='This week'; $2=$ 'Last month '; 3='6 months'; 4='Last year';5='Over a year'") \#Conflict 1: When did the situation first begin

WIS. PA\$C1. Day<-car: : recode(WIS. PA\$4 . 7, "1= 'Monday ' ; $2=$ ' Tuesday ' ; $3=$ ' 'Wedn

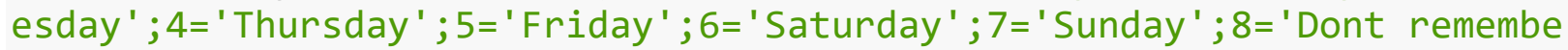
$r^{\prime \prime \prime) ~ \# C o n f l i c t ~ 1: ~ D a y ~ o f ~ t h e ~ w e e k ~}$

WIS.PA\$C1.TimeDay<-car: : recode(WIS.PA\$q4.8, "1= 'Morning ' ; $2=$ 'Afternoon '; 3='Evening';4='Dont remember'") \#Conflict 1: Time of day

names(WIS.PA) [names(WIS.PA)=='q4.9']<-'C1. Where' \#Conflict 1: Where th e situation happened

names(WIS.PA) [names(WIS.PA)=='q4.11']<-'C1. Doing' \#Conflict 1: What th $e$ person was doing when the situation happened

WIS.PA\$C1.OtherGender<-car: : recode(WIS.PA\$q4.12, "1=' Male '; $2=$ 'Female '; ' Other'") \#Conflict 1: Other person's gender

names (WIS.PA) [names(WIS.PA) =='q4.13']<-'C1. Thoughts' \#Conflict 1: Thou ghts and emotions about situation

names(WIS.PA) [names(WIS.PA)=='q5.2_1']<-'C1.W1' \#Conflict 1: Wise Reas oning

names(WIS.PA) [names(WIS.PA)=='q5.2_2']<-'C1.W2' \#Conflict 1: Wise Reas oning

names(WIS.PA) [names(WIS.PA)=='q5.2_3']<-'C1.W3' \#Conflict 1: Wise Reas oning

names(WIS.PA) [names(WIS.PA)=='q5.2_4']<-'C1.W4' \#Conflict 1: Wise Reas oning

names(WIS.PA)[names(WIS.PA)=='q5.2_5']<-'C1.W5' \#Conflict 1: Wise Reas oning

names(WIS.PA) [names(WIS.PA)=='q5.2_6']<-'C1.W6' \#Conflict 1: Wise Reas oning

names(WIS.PA) [names(WIS.PA)=='q5.2_7']<-'C1.W7' \#Conflict 1: Wise Reas oning

names (WIS.PA) [names(WIS.PA)=='q5.2_8']<-'C1.W8' \#Conflict 1: Wise Reas oning

names(WIS.PA) [names(WIS.PA)=='q5.2_9']<-'C1.W9' \#Conflict 1: Wise Reas oning

names(WIS.PA) [names(WIS.PA)=='q5.2_10']<-'C1.W10' \#Conflict 1: Wise Re 
asoning

names(WIS.PA) [names(WIS.PA)=='q5.4_1']<-'C1.W11' \#Conflict 1: Wise Rea soning

names(WIS.PA) [names (WIS.PA) =='q5.4_2']<-'C1.W12' \#Conflict 1: Wise Rea soning

names(WIS.PA) [names(WIS.PA)=='q5.4_3']<-'C1.W13' \#Conflict 1: Wise Rea soning

names(WIS.PA) [names (WIS.PA) =='q5.4_4']<-'C1.W14' \#Conflict 1: Wise Rea soning

names (WIS.PA) [names(WIS.PA) =='q5.4_5']<-'C1.W15' \#Conflict 1: Wise Rea soning

names(WIS.PA) [names (WIS.PA)=='q5.4_6']<-'C1.W16' \#Conflict 1: Wise Rea soning

names(WIS.PA) [names(WIS.PA) =='q5.4_7']<-'C1.W17' \#Conflict 1: Wise Rea soning

names(WIS.PA) [names(WIS.PA) ==' q5.4_8']<-'C1.W18' \#Conflict 1: Wise Rea soning

names(WIS.PA) [names(WIS.PA)=='q5.4_9']<-'C1.W19' \#Conflict 1: Wise Rea soning

names(WIS.PA) [names (WIS.PA) =='q5.4_10']<-'C1.W20' \#Conflict 1: Wise Re asoning

names(WIS.PA) [names(WIS.PA)=='q5.4_11']<-'C1.W21' \#Conflict 1: Wise Re asoning

names(WIS.PA) [names(WIS.PA)=='q6.3']<-'C1.Serious' \#Conflict 1: Seriou sness of situation

names(WIS.PA) [names (WIS.PA) == 'q6.4']<-'C1.OtherNum' \#Conflict 1: NUmbe $r$ of other people in situation

names(WIS.PA) [names (WIS.PA)=='q6.5_1']<-'C1.0therClose' \#Conflict 1: C lose friend involved in the situation

names (WIS.PA) [names(WIS.PA)=='q6.5_3']<-'C1.OtherCasual' \#Conflict 1: Casual friend involved in the situation

names (WIS.PA) [names (WIS.PA) ==' q6.5_4']<-'C1.OtherAcquaintance' \#Confli ct 1: Acquaintance involved in the situation

names(WIS.PA) [names(WIS.PA)=='q6.5_5']<-'C1.OtherRelative' \#Conflict 1 : Relative involved in the situation

names (WIS.PA) [names (WIS.PA)=='q6.5_6']<-'C1.0therPartner' \#Conflict 1: Romantic partner involved in the situation

names(WIS.PA) [names (WIS.PA) ==' q6.5_7']<-'C1.OtherEx' \#Conflict 1: Ex-p artner involved in the situation

names (WIS.PA) [names (WIS.PA) == 'q6.5_2' ]<-'C1.0therFriendPartner' \#Confl ict 1: Friend's romantic partner involved in the situation

names (WIS.PA) [names (WIS.PA) =='q6.5_9' ]<-'C1.OtherRelativePartner' \#Con flict 1: Relative's romantic partner involved in the situation names (WIS.PA) [names (WIS.PA) =='q6.5_10']<-'C1.0therCoworker' \#Conflict 
1: Co-worker involved in the situation names(WIS.PA) [names (WIS.PA)=='q6.5_12']<-'C1.0therHousemate' \#Conflict 1: Housemate involved in the situation names(WIS.PA) [names(WIS.PA)=='q6.5_13']<-'C1.0therFFriend' \#Conflict 1 : Friend of a friend involved in the situation names(WIS.PA)[names(WIS.PA)=='q6.5_14']<-'C1.0therClient' \#Conflict 1: $C$ lient or customer involved in the situation names (WIS.PA) [names (WIS.PA)=='q6.5_8']<-'C1.0therother' \#Conflict 1: 0 ther kind of person involved in the situation

WIS.PA\$C1. IA1<-car: : recode(WIS.PA $\$$ q7.2 $1, " 27=1 ; 28=2 ; 29=3 ; 33=4 ; 31=5 ; 20=$ 6") \#Conflict 1: Influence/Adjustment

WIS.PA $\$ C 1$. IA2<-car : : recode(WIS.PA $\$$ q7.2_ $21, " 27=1 ; 28=2 ; 29=3 ; 33=4 ; 31=5 ; 20$ =6") \#Conflict 1: Influence/Adjustment

WIS. PA\$C1. IA3<-car: : recode(WIS. PA $\$ 77.2 \_22, " 27=1 ; 28=2 ; 29=3 ; 33=4 ; 31=5 ; 20$ =6") \#Conflict 1: Influence/Adjustment

WIS. PA\$C1. IA4<-car: : recode(WIS.PA $\$$ q7.2_ $23, " 27=1 ; 28=2 ; 29=3 ; 33=4 ; 31=5 ; 20$ =6") \#Conflict 1: Influence/Adjustment

WIS. PA\$C1. IA5<-car: : recode(WIS.PA $\$$ q7.2_ $24, " 27=1 ; 28=2 ; 29=3 ; 33=4 ; 31=5 ; 20$ =6") \#Conflict 1: Influence/Adjustment

WIS. PA\$C1. IA6<-car: : recode(WIS.PA $\$$ q7.2_ $25, " 27=1 ; 28=2 ; 29=3 ; 33=4 ; 31=5 ; 20$ =6") \#Conflict 1: Influence/Adjustment

WIS. PA\$C1. IA7<-car: : recode(WIS.PA $\$$ q7.2_ $26, " 27=1 ; 28=2 ; 29=3 ; 33=4 ; 31=5 ; 20$ =6") \#Conflict 1: Influence/Adjustment

WIS. PA\$C1. IA8<-car: : recode(WIS.PA $\$$ q7.2_ $27, " 27=1 ; 28=2 ; 29=3 ; 33=4 ; 31=5 ; 20$ =6") \#Conflict 1: Influence/Adjustment

WIS. PA\$C1. IA9<-car: : recode(WIS. PA $\$$ q7.2_ $28, " 27=1 ; 28=2 ; 29=3 ; 33=4 ; 31=5 ; 20$ =6") \#Conflict 1: Influence/Adjustment

WIS.PA\$C1.Att1<-car: :recode(WIS.PA $\$$ q8.2_ $1, " 8=1 ; 14=2 ; 15=3 ; 16=4 ; 17=5 "$ ) \# Conflict 1: Attributions

WIS.PA\$C1.Att2<-car: :recode(WIS.PA $\$$ q8.2_2," $8=1 ; 14=2 ; 15=3 ; 16=4 ; 17=5 "$ ) \# Conflict 1: Attributions

WIS.PA\$C1.Att3<-car: : recode(WIS.PA $\$ 8.2 \_3, " 8=1 ; 14=2 ; 15=3 ; 16=4 ; 17=5 "$ ) \# Conflict 1: Attributions

WIS.PA\$C1.Att4<-car: :recode(WIS.PA\$q8.2_4," $8=1 ; 14=2 ; 15=3 ; 16=4 ; 17=5 "$ ) \# Conflict 1: Attributions

WIS.PA\$C1.Att5<-car: :recode(WIS.PA $\$ 8.2 \_5, " 8=1 ; 14=2 ; 15=3 ; 16=4 ; 17=5 "$ ) \# Conflict 1: Attributions

WIS.PA\$C1.Att6<-car: :recode(WIS.PA $\$$ q8.2_6, $8=1 ; 14=2 ; 15=3 ; 16=4 ; 17=5 "$ ) \# Conflict 1: Attributions

WIS.PA\$C1.Att7<-car: : recode(WIS.PA $\$ 8.2 \_7, " 8=1 ; 14=2 ; 15=3 ; 16=4 ; 17=5 "$ ) \# Conflict 1: Attributions

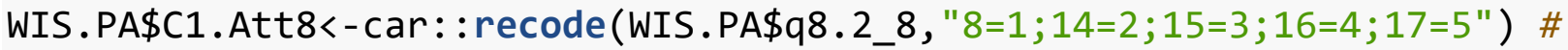
Conflict 1: Attributions

names(WIS.PA) [names(WIS.PA)=='q10.4_3']<-'C2. Time' \#Conflict 2: Second 
$S$ thinking about conflict

names(WIS.PA)[names(WIS.PA)=='recal12']<-'C2.Person' \#Conflict 2: Pers on the conflict was with (displayed to participant)

WIS.PA\$C2.Time. $\log <-\log 10$ (WIS.PA\$C2.Time) \#Conflict 2: Seconds thinkin $g$ about conflict (Log)

WIS.PA\$C2. When<-car: : recode(WIS.PA $\$ 10.8, " 1=$ 'This week'; $2=$ 'Last month ' ;3='6 months'; $4=$ 'Last year';5='Over a year'") \#Conflict 2: When did th e situation first begin

WIS. PA\$C2. Day<-car: : recode(WIS.PA\$10.9, " $1=$ 'Monday ' ; $2=$ ' Tuesday ' ; $3=$ ' Wed nesday '; $4=$ 'Thursday '; $5=$ ' Friday '; 6='Saturday '; $7=$ 'Sunday '; 8= 'Dont rememb er'") \#Conflict 2: Day of the week

WIS. PA\$C2. TimeDay<-car: : recode(WIS. PA $\$ 10.10$, "1= 'Morning ' ; $2=$ 'Afternoon ';3='Evening';4='Dont remember'") \#Conflict 2: Time of day

names(WIS.PA) [names(WIS.PA) =='q10.11']<-'C2. Where' \#Conflict 2: Where the situation happened

names (WIS.PA) [names(WIS.PA) =='q10.12_1']<-'C2.ConflictFriend' \#Recent conflict with friend (multiple responses allowed)

names (WIS.PA) [names(WIS.PA) =='q10.12_2']<-'C2.ConflictCoworker' \#Recen $t$ conflict with co-worker (multiple responses allowed)

names (WIS.PA) [names(WIS.PA) ==' q10.12_3']<-'C2. ConflictPartner' \#Recent conflict with romantic partner (multiple responses allowed)

names (WIS.PA) [names(WIS.PA) =='q10.12_4'] $<-'$ C2. ConflictFamily' \#Recent conflict with family member (multiple responses allowed)

names (WIS.PA) [names(WIS.PA) =='q10.12_5']<-'C2. Conflict0ther' \#Recent $c$ onflict with other type of person (multiple responses allowed)

names(WIS.PA)[names(WIS.PA)=='q10.15']<-'C2. Doing' \#Conflict 2: What $t$ he person was doing when the situation happened

WIS.PA\$C2.0therGender<-car: : recode(WIS.PA $\$ 10.16$, "1= 'Male ' ; $2=$ ' Female ' ; $3=$ 'Other'") \#Conflict 2: Other person's gender

names (WIS.PA) [names (WIS.PA)=='q10.17']<-'C2. Thoughts' \#Conflict 2: Tho ughts and emotions about situation

names (WIS.PA) [names(WIS.PA)=='q11.2_1']<-'C2.W1' \#Conflict 2: Wise Rea soning

names(WIS.PA) [names(WIS.PA)=='q11.2_2']<-'C2.W2' \#Conflict 2: Wise Rea soning

names(WIS.PA) [names(WIS.PA)=='q11.2_3']<-'C2.W3' \#Conflict 2: Wise Rea soning

names(WIS.PA) [names(WIS.PA)=='q11.2_4']<-'C2.W4' \#Conflict 2: Wise Rea soning

names(WIS.PA) [names(WIS.PA)=='q11.2_5']<-'C2.W5' \#Conflict 2: Wise Rea soning

names(WIS.PA) [names(WIS.PA)=='q11.2_6']<-'C2.W6' \#Conflict 2: Wise Rea soning

names(WIS.PA) [names(WIS.PA)=='q11.2_7']<-'C2.W7' \#Conflict 2: Wise Rea 
soning

names(WIS.PA) [names(WIS.PA) =='q11.2_8']<-'C2.W8' \#Conflict 2: Wise Rea soning

names(WIS.PA) [names (WIS.PA)=='q11.2_9' ]<-'C2.W9' \#Conflict 2: Wise Rea soning

names(WIS.PA) [names(WIS.PA)=='q11.2_10']<-'C2.W10' \#Conflict 2: Wise $R$ easoning

names(WIS.PA) [names (WIS.PA)=='q11.4_1']<-'C2.W11' \#Conflict 2: Wise Re asoning

names(WIS.PA) [names(WIS.PA) =='q11.4_2']<-'C2.W12' \#Conflict 2: Wise Re asoning

names(WIS.PA) [names(WIS.PA) =='q11.4_3']<-'C2.W13' \#Conflict 2: Wise Re asoning

names(WIS.PA) [names(WIS.PA) =='q11.4_4']<-'C2.W14' \#Conflict 2: Wise Re asoning

names(WIS.PA) [names(WIS.PA) =='q11.4_5']<-'C2.W15' \#Conflict 2: Wise Re asoning

names(WIS.PA) [names(WIS.PA)=='q11.4_6']<-'C2.W16' \#Conflict 2: Wise Re asoning

names(WIS.PA) [names (WIS.PA) =='q11.4_7']<-'C2.W17' \#Conflict 2: Wise Re asoning

names(WIS.PA) [names(WIS.PA)=='q11.4_8']<-'C2.W18' \#Conflict 2: Wise Re asoning

names(WIS.PA) [names (WIS.PA) =='q11.4_9' ]<-'C2.W19' \#Conflict 2: Wise Re asoning

names(WIS.PA) [names(WIS.PA) =='q11.4_10']<-'C2.W20' \#Conflict 2: Wise $R$ easoning

names(WIS.PA) [names (WIS.PA) =='q11.4_11']<-'C2.W21' \#Conflict 2: Wise $R$ easoning

names(WIS.PA) [names(WIS.PA)=='q12.3']<-'C2. Serious' \#Conflict 2: Serio usness of situation

names(WIS.PA) [names(WIS.PA)=='q12.4']<-'C2.0therNum' \#Conflict 2: Numb er of other people in situation

names (WIS.PA) [names(WIS.PA) =='q12.5_1']<-'C2.0therClose' \#Conflict 2: Close friend involved in the situation

names (WIS.PA) [names (WIS.PA) =='q12.5_3']<-'C2.0therCasual' \#Conflict 2: Casual friend involved in the situation

names (WIS.PA) [names (WIS.PA) =='q12.5_4' ]<-'C2.0therAcquaintance' \#Confl ict 2: Acquaintance involved in the situation

names (WIS.PA) [names (WIS.PA) =='q12.5_5']<-'C2.0therRelative' \#Conflict 2: Relative involved in the situation

names (WIS.PA) [names (WIS.PA) =='q12.5_6']<-'C2.0therPartner' \#Conflict 2 : Romantic partner involved in the situation names (WIS.PA) [names (WIS.PA) =='q12.5_7']<-'C2.OtherEx' \#Conflict 2: Ex- 
partner involved in the situation

names (WIS.PA) [names (WIS.PA) =='q12.5_2']<-'C2.0therFriendPartner' \#Conf Lict 2: Friend's romantic partner involved in the situation

names (WIS.PA) [names (WIS.PA) ==' q12.5_9']<-'C2.0therRelativePartner' \#CO nflict 2: Relative's romantic partner involved in the situation

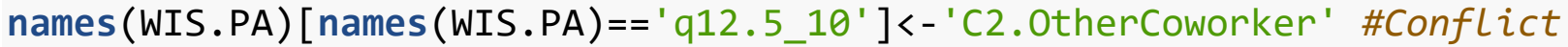
2: Co-worker involved in the situation

names(WIS.PA) [names(WIS.PA)=='q12.5_12']<-'C2.0therHousemate' \#Conflic $t$ 2: Housemate involved in the situation

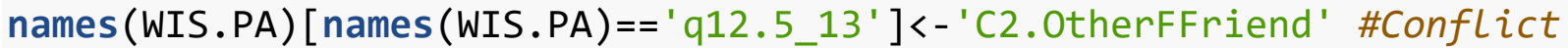

2: Friend of a friend involved in the situation

names(WIS.PA) [names(WIS.PA)=='q12.5_14']<-'C2.0therClient' \#Conflict 2 : Client or customer involved in the situation

names(WIS.PA) [names (WIS.PA) =='q12.5_8']<-'C2.0the0ther' \#Conflict 2: 0 ther kind of person involved in the situation

WIS. PA\$C2. IA1<-car: : recode(WIS. PA $\$ 13.2 \_1, " 27=1 ; 28=2 ; 29=3 ; 32=4 ; 30=5 ; 31$ =6") \#Conflict 2: Influence/Adjustment

WIS. PA\$C2. IA2<-car: : recode(WIS. PA $\$ 13.2$ 21, $" 27=1 ; 28=2 ; 29=3 ; 32=4 ; 30=5 ; 3$ 1=6") \#Conflict 2: Influence/Adjustment

WIS. PA\$C2. IA3<-car: : recode(WIS.PA $\$ 13.2 \_22, " 27=1 ; 28=2 ; 29=3 ; 32=4 ; 30=5 ; 3$ 1=6") \#Conflict 2: Influence/Adjustment

WIS. PA\$C2. IA4<-car: : recode(WIS.PA $\$ 13.2 \_23, " 27=1 ; 28=2 ; 29=3 ; 32=4 ; 30=5 ; 3$ 1=6") \#Conflict 2: Influence/Adjustment

WIS. PA\$C2. IA5<-car: : recode(WIS.PA $\$ 13.2 \_24, " 27=1 ; 28=2 ; 29=3 ; 32=4 ; 30=5 ; 3$ 1=6") \#Conflict 2: Influence/Adjustment

WIS. PA\$C2. IA6<-car: : recode(WIS. PA $\$ 13.2$ 25, $" 27=1 ; 28=2 ; 29=3 ; 32=4 ; 30=5 ; 3$ 1=6") \#Conflict 2: Influence/Adjustment

WIS. PA\$C2. IA7<-car: : recode(WIS.PA $\$ 13.2 \_26, " 27=1 ; 28=2 ; 29=3 ; 32=4 ; 30=5 ; 3$ 1=6") \#Conflict 2: Influence/Adjustment

WIS. PA $\$ C 2$. IA8<-car: : recode(WIS.PA $\$$ q13.2_ $27, " 27=1 ; 28=2 ; 29=3 ; 32=4 ; 30=5 ; 3$ 1=6") \#Conflict 2: Influence/Adjustment

WIS. PA\$C2. IA9<-car: : recode(WIS.PA $\$ 13.2 \_28, " 27=1 ; 28=2 ; 29=3 ; 32=4 ; 30=5 ; 3$ 1=6") \#Conflict 2: Influence/Adjustment

WIS.PA\$C2.Att1<-car: : recode(WIS.PA $\$ 14.2 \_1, " 8=1 ; 14=2 ; 15=3 ; 16=4 ; 17=5 "$ )

\#Conflict 2: Attributions

WIS.PA\$C2. Att2<-car: : recode(WIS.PA $\$ 14.2 \_2, " 8=1 ; 14=2 ; 15=3 ; 16=4 ; 17=5 "$ )

\#Conflict 2: Attributions

WIS.PA $\$ C 2$. Att $3<-$ car : : recode(WIS.PA $\$$ q14.2 $3, " 8=1 ; 14=2 ; 15=3 ; 16=4 ; 17=5 "$ )

\#Conflict 2: Attributions

WIS.PA\$C2. Att4<-car: : recode(WIS.PA $\$ 14.2 \_4, " 8=1 ; 14=2 ; 15=3 ; 16=4 ; 17=5 "$ )

\#Conflict 2: Attributions

WIS. PA $\$ C 2$. Att5<-car : : recode(WIS.PA $\$$ q14.2 5 , " $8=1 ; 14=2 ; 15=3 ; 16=4 ; 17=5 "$ )

\#Conflict 2: Attributions

WIS.PA\$C2. Att6<-car : : recode(WIS.PA $\$$ q14.2_6, "8=1; $14=2 ; 15=3 ; 16=4 ; 17=5 "$ ) 
\#Conflict 2: Attributions

WIS.PA $\$ C 2$. Att7<-car: : recode(WIS.PA $\$ 14.2 \_7, " 8=1 ; 14=2 ; 15=3 ; 16=4 ; 17=5 "$ )

\#Conflict 2: Attributions

WIS.PA\$C2.Att8<-car: : recode(WIS.PA\$q14.2_8, "8=1; 14=2; 15=3; 16=4;17=5")

\#Conflict 2: Attributions

WIS.PA\$GW1<-car: :recode(WIS.PA\$q15.3_1,"6=1;7=2;9=3;11=4;12=5") \#GLuck Wisdom

WIS.PA $\$$ GW2<-car: : recode(WIS.PA $\$$ q15.3_2, "6=1;7=2;9=3;11=4;12=5") \#GLuck Wisdom

WIS.PA $\$$ GW3<-car : : recode(WIS.PA\$q15.3_3, "6=1;7=2;9=3;11=4;12=5") \#GLuck Wisdom

WIS.PA $\$$ GW4<-car : : recode(WIS.PA $\$$ q15.3_4, "6=1;7=2;9=3;11=4;12=5") \#GLuck Wisdom

WIS.PA\$GW5<-car : : recode(WIS.PA\$q15.3_5, "6=1;7=2;9=3;11=4;12=5") \#GLuck Wisdom

WIS.PA $\$$ GW6r<-car: : recode(WIS.PA $\$ 115.3 \_6, " 6=5 ; 7=4 ; 9=3 ; 11=2 ; 12=1 "$ ) \#GLUC $k$ Wisdom (Reversed)

WIS.PA\$GW7<-car: : recode(WIS.PA\$q15.3_7,"6=1;7=2;9=3;11=4;12=5") \#GLuck Wisdom

WIS.PA $\$$ GW8<-car: : recode(WIS.PA $\$$ q15.3_8, "6=1;7=2;9=3;11=4;12=5") \#GLuck Wisdom

WIS.PA\$GW9<-car : : recode(WIS.PA\$q15.3_9,"6=1;7=2;9=3;11=4;12=5") \#GLuck Wisdom

WIS.PA $\$$ GW10<-car: : recode(WIS.PA $\$$ q15.3_10, "6=1;7=2;9=3;11=4;12=5") \#GLu ck Wisdom

WIS.PA\$GW11<-car: :recode(WIS.PA $\$$ q15.3_11, "6=1;7=2;9=3;11=4;12=5") \#GLu ck Wisdom

WIS.PA $\$$ GW12<-car: : recode(WIS.PA $\$$ q15.3_12, "6=1;7=2;9=3;11=4;12=5") \#GLu ck Wisdom

WIS.PA\$GW13<-car: : recode(WIS.PA $\$$ q15.3_13, "6=1;7=2;9=3;11=4;12=5") \#GLu ck Wisdom

WIS.PA\$GW14r<-car: : recode(WIS.PA\$q15.3_14,"6=5;7=4;9=3;11=2;12=1") \#GL uck Wisdom (Reversed)

WIS.PA\$GW15<-car: : recode(WIS.PA $\$$ q15.3_15, "6=1;7=2;9=3;11=4;12=5") \#GLu ck Wisdom

WIS.PA $\$$ GW16<-car : :recode(WIS.PA $\$$ q15.3_16, "6=1;7=2;9=3;11=4;12=5") \#GLu ck Wisdom

WIS.PA\$GW17<-car: : recode(WIS.PA $\$$ q15.3_17, "6=1;7=2;9=3;11=4;12=5") \#GLu ck Wisdom

WIS.PA $\$$ GW18<-car : :recode(WIS.PA $\$$ q15.3_18, "6=1;7=2;9=3;11=4;12=5") \#GLu ck Wisdom

WIS.PA\$GW19<-car: : recode(WIS.PA $\$$ q15.3_19, "6=1;7=2;9=3;11=4;12=5") \#GLu ck Wisdom

WIS.PA\$GW20r<-car: : recode(WIS.PA\$q15.3_20,"6=5;7=4;9=3;11=2;12=1") \#GL 
uck Wisdom (Reversed)

WIS.PA\$GW21<-car: : recode(WIS.PA\$q15.3_21, "6=1;7=2;9=3;11=4;12=5") \#GLu ck Wisdom

Demographics Recoding

names (WIS.PA) [names (WIS.PA) $==$ 'q1.1']<-'Age' \#Age

names (WIS.PA) [names(WIS.PA) =='q1.2']<-'Country' \#Country

WIS.PA\$Region<-car: : recode(WIS.PA\$Country, " 'Australia' = 'Europe \& Other $S^{\prime}$;

'Canada' = 'North America';

'England'= 'Europe \& Others';

'Germany'='Europe \& Others';

'Hong Kong ' = 'Europe \& Others';

'Ireland'='Europe \& Others';

'israel'='Europe \& Others';

'Mexico' = 'North America';

'Scotland'= 'Europe \& Others';

'Spain'='Europe \& Others';

'Sweden' = 'Europe \& Others' ;

'The Netherlands' = 'Europe \& Others';

'Netherlands' = 'Europe \& Others';

'UK' = 'Europe \& Others' ; 'US'= 'North America'

")

WIS.PA\$Language<-car: : recode(WIS.PA\$q1.3, "1='English';2='0ther'") \#Fir st Language

WIS.PA\$Gender<-car: : recode(WIS.PA\$q1.4, "1= 'Male ';2= 'Female ' ; 3= 'Other ' " ) \#Gender

WIS.PA\$Ethnicity<-car: : recode(WIS.PA\$q16.3, "1= 'East Asian '; $2=$ 'African ' ;3='White/European'; 4='Latino'; 5='Other'; 7='South Asian';8='Middle Eas tern';6='Other'") \#Ethnicity

describe(WIS.PA\$Ethnicity)

\#\# WIS.PA\$Ethnicity

\#\# $\quad n$ missing distinct

$\begin{array}{llll}\# \# & 300 & 0 & 7\end{array}$

\#\#

\#\# Value African

ern

\#\# Frequency

10

East Asian

Latino Middle East

2
\#\# Proportion
0.033
0.043
0.047
$\theta$.

007 


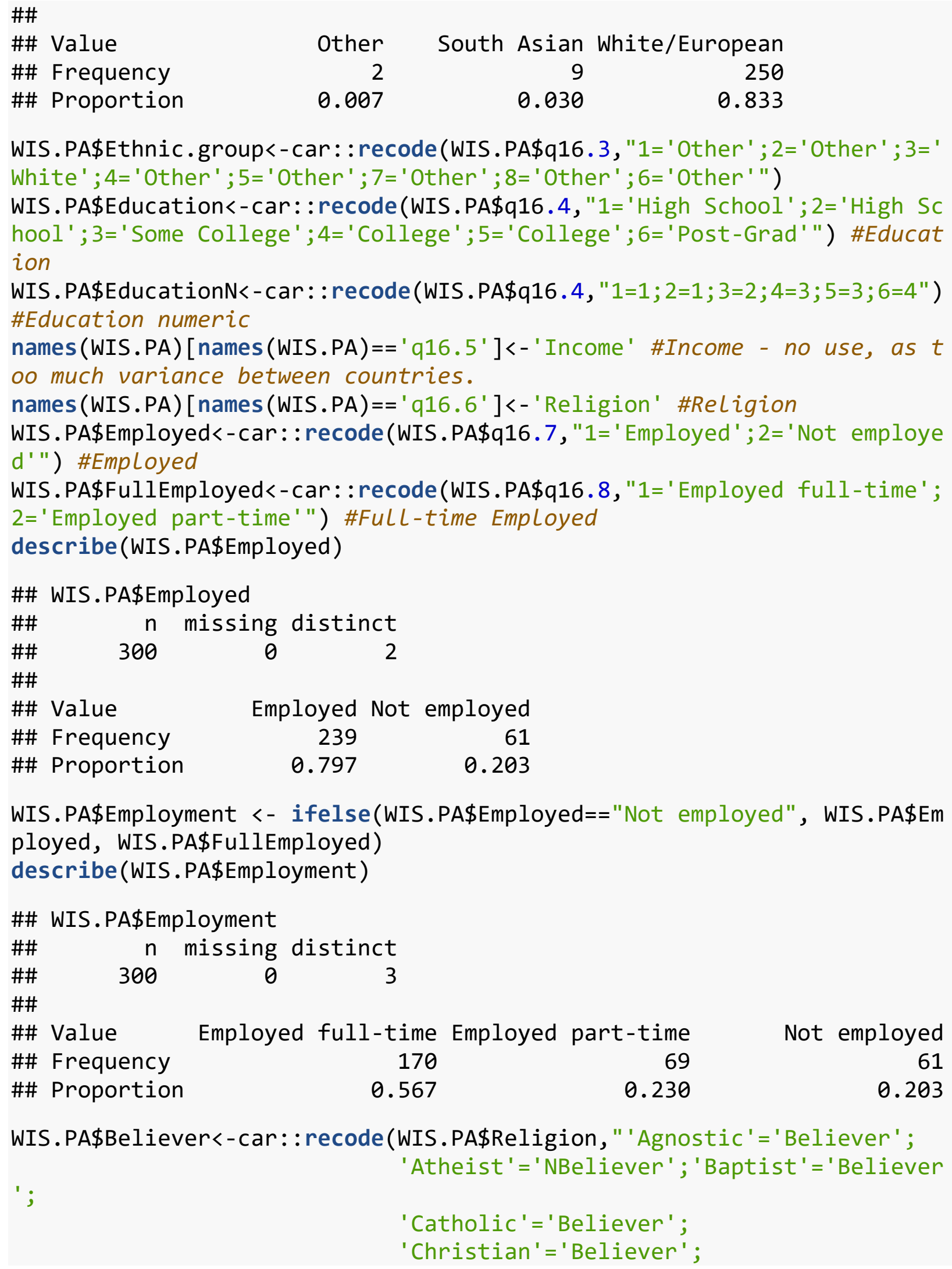




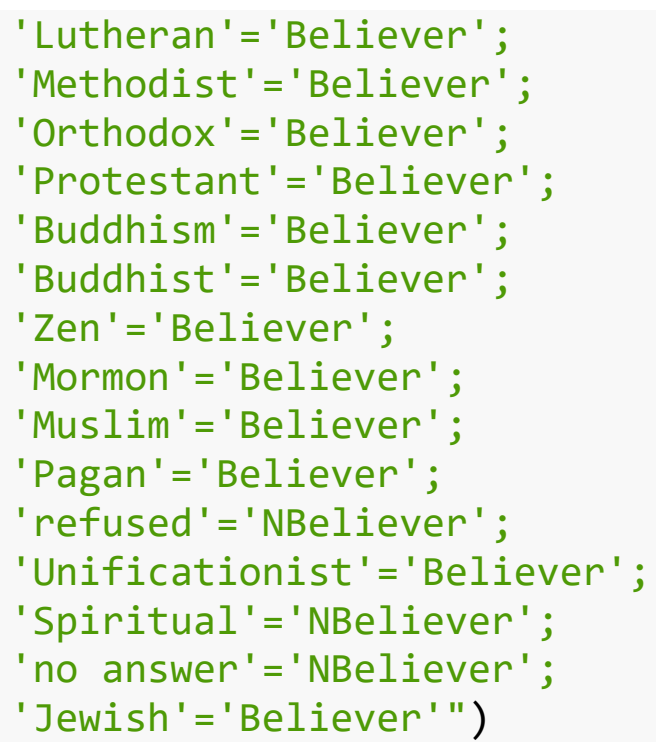

WISE REASONING COMPOSITES (Average of individual dimensions and overall scale)

WIS.PA\$C1.Perspective.ave<-apply(WIS.PA[, c( 'C1.W1' , 'C1.W2 ' , 'C1.W3 ', 'C1 .W4')], 1, mean, na.rm=TRUE) \#Conflict 1: Wise reasoning - Perspective Seeking Aspect

WIS.PA\$C1.ChangeOutcomes . ave<-apply(WIS.PA[ , c( 'C1.W5 ', 'C1.W6 ', 'C1.W7', 'C1.W8')], 1, mean, na.rm=TRUE) \#Conflict 1: Wise reasoning - Change a nd Multiple Outcomes Aspect

WIS.PA\$C1.Limits .ave<-apply(WIS.PA[ , C( 'C1.W9 ', 'C1.W10', 'C1.W11' , 'C1.W1 $\left.2^{\prime}\right)$ ], 1, mean, na.rm=TRUE) \#Conflict 1: Wise reasoning - Limits of Kno wledge Aspect

WIS.PA\$C1.CompResolve.ave<-apply(WIS.PA[ , c( 'C1.W13 ' , 'C1.W14 ', 'C1.W15 ', 'C1.W16', 'C1.W17')], 1, mean, na.rm=TRUE) \#Conflict 1: Wise reasoning

- Compromise and Resolution Aspect

WIS.PA\$C1.Outsider.ave<-apply(WIS.PA[, c( 'C1.W18' , 'C1.W19' , 'C1.W20' , 'C1 .W21')], 1, mean, na.rm=TRUE) \#Conflict 1: Wise reasoning - Outsider $P$ erspective Aspect

WIS.PA\$C1.wisdom. ave<-apply(WIS.PA[, c( 'C1.Perspective.ave ' , 'C1. Change0 utcomes.ave', 'C1.Limits.ave', 'C1.CompResolve.ave', 'C1.Outsider.ave' )], 1, mean, na.rm=TRUE) \#Conflict 1: Wise reasoning - Overall

WIS.PA\$C2.Perspective.ave<-apply(WIS.PA[, c ('C2.W1' , 'C2.W2 ' , 'C2.W3 ' , 'C2 .W4')], 1, mean, na.rm=TRUE) \#Conflict 2: Wise reasoning - Perspective Seeking Aspect

WIS.PA\$C2.ChangeOutcomes .ave<-apply(WIS.PA[, c ( 'C2.W5 ', 'C2.W6' , 'C2.W7' , 'C2.W8')], 1, mean, na.rm=TRUE) \#Conflict 2: Wise reasoning - Change a nd Multiple Outcomes Aspect

WIS.PA\$C2.Limits . ave<-apply(WIS.PA[ , C( 'C2.W9 ', 'C2.W10', 'C2.W11', 'C2.W1 $\left.2^{\prime}\right)$ ], 1, mean, na.rm=TRUE) \#Conflict 2: Wise reasoning - Limits of Kno wledge Aspect 
WIS.PA\$C2 . CompResolve.ave<-apply(WIS.PA[ , c( 'C2.W13 ', 'C2.W14 ', 'C2.W15 ', 'C2.W16','(C2.W17')], 1, mean, na.rm=TRUE) \#Conflict 2: Wise reasoning - Compromise and Resolution Aspect WIS.PA\$C2. Outsider . ave<-apply(WIS. PA[ , c( 'C2.W18 ' , 'C2.W19 ' , 'C2. W20 ' , 'C2 .W21')], 1, mean, na.rm=TRUE) \#Conflict 2: Wise reasoning - Outsider $P$ erspective Aspect

WIS.PA\$C2.wisdom. ave<-apply(WIS.PA[, c ( 'C2.Perspective.ave ', ' C2. ChangeO utcomes.ave ', 'C2.Limits.ave ', 'C2.CompResolve.ave ', 'C2.Outsider.ave ')], 1, mean, na.rm=TRUE) \#Conflict 2: Wise reasoning - Overall

create subsets of data for comparison of reliability in wisdom in North America vs.

Europe/Others.

wisdom.dataNA=subset(WIS.PA, Region=='North America') \#create subsets of North America (as in prior studies)

wisdom.dataEU=subset(WIS.PA, Region=='Europe \& Others') \#create a subs et of Europe + a few other countries.

wisdom. dataUK=subset (WIS.PA, Country== 'UK ' |Country== ' Scotland ' | Country $==$ 'Ireland '|Country=='England') \#create a subset of UK participants.

WISE REASONING RELIABILITY

psych: :alpha(WIS.PA[, c('C1.W1', 'C1.W2', 'C1.W3 ', 'C1.W4')], check.keys=F ALSE)[1] \#Conflict 1: Wise reasoning - Perspective Seeking Aspect

\#\# \$total

\#\# raw_alpha std.alpha G6(smc) average_r S/N ase mean

$\begin{array}{lllllllll}\text { \#\# } & 0.8 \overline{1} 72851 & 0.8180979 & 0.7875921 & 0.52927 \overline{1} 2 & 4.497463 & 0.01736213 & 2.8525\end{array}$

\#\# $\quad$ sd

\#\# 0.8929501

psych: :alpha(WIS.PA[, c('C1.W5 ', 'C1.W6', 'C1.W7', 'C1.W8')], check. keys=F ALSE)[1] \#Conflict 1: Wise reasoning - Change and Multiple Outcomes $A$ spect

\#\# \$total

\#\# raw_alpha std.alpha $\mathrm{G6}(\mathrm{smc})$ average_r $\mathrm{S} / \mathrm{N}$ ase mean

sd

$\begin{array}{lllllllllll}\text { \#\# } & 0.8164724 & 0.8168445 & 0.863677 & 0.5271779 & 4.459841 & 0.01864352 & 3.18 & 0 .\end{array}$ 9372539

psych: :alpha(WIS.PA[, c('C1.W9', 'C1.W10', 'C1.W11', 'C1.W12' )], check.key s=FALSE)[1] \#Conflict 1: Wise reasoning - Intellectual Humility Aspect

\#\# \$total

\#\# raw_alpha std.alpha G6(smc) average_r $\mathrm{S} / \mathrm{N}$ ase mea n 


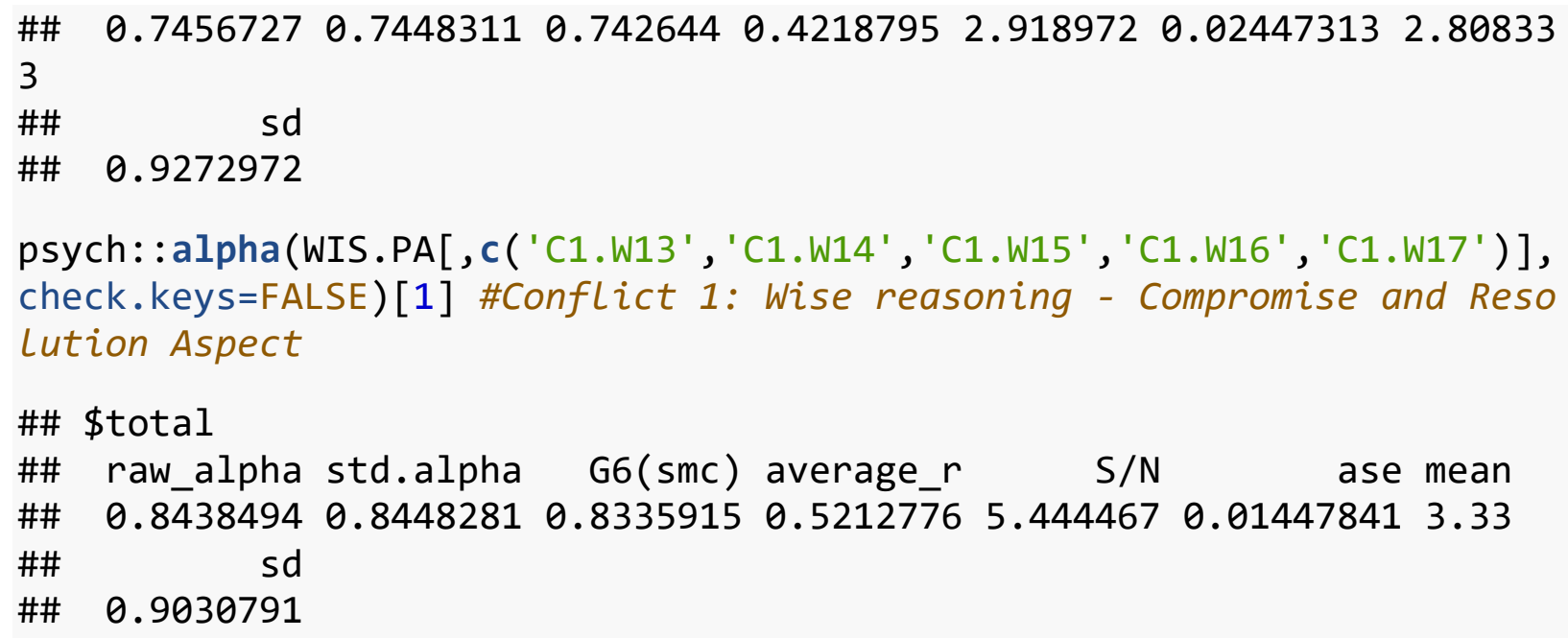

psych: :alpha(WIS.PA[, c('C1.W18', 'C1.W19', 'C1.W20', 'C1.W21')], check.ke ys=FALSE)[1] \#Conflict 1: Wise reasoning - Outsider Perspective Aspect

\#\# \$total

\#\# raw_alpha std.alpha G6(smc) average_r $\quad$ S/N ean

$\begin{array}{lllllllll}\text { \#\# } & 0.9141507 & 0.9142103 & 0.8918907 & 0.7270818 & 10.65641 & 0.008113494 & 2.541\end{array}$ 667

\#\# sd

\#\# 1.188862

psych: :alpha(WIS.PA[, c('C1.W1 ', 'C1.W2' , 'C1.W3 ', 'C1.W4 ' , 'C1.W5 ', 'C1.W6' , 'C1.W7' , 'C1.W8 ', 'C1.W9 ', 'C1.W10 ', 'C1.W11 ', 'C1.W12 ', 'C1.W13 ', 'C1.W14 ', 'C1.W15 ', 'C1.W16', 'C1.W17', 'C1.W18', 'C1.W19', 'C1.W20', 'C1.W21')], chec k.keys=FALSE)[1] \#Conflict 1: Wise reasoning - Overall

\#\# \$total

\#\# raw_alpha std.alpha G6(smc) average_r $\mathrm{S} / \mathrm{N}$ ase me

an

$\begin{array}{llllllll}\text { \#\# } & 0.920708 & 0.9215593 & 0.9500573 & 0.358749 & 11.74849 & 0.00669827 & 2.9609\end{array}$

52

\#\# $\quad$ sd

\#\# $\quad 0.7455558$

psych: :alpha(wisdom.dataNA[, c( 'C1.W1', 'C1.W2 ' , 'C1.W3 ', 'C1.W4 ' , 'C1.W5 ', 'C1.W6', 'C1.W7' , 'C1.W8 ', 'C1.W9' , 'C1.W10', 'C1.W11' , 'C1.W12 ', 'C1.W13 ', 'C 1.W14 ', 'C1.W15 ', 'C1.W16 ', 'C1.W17', 'C1.W18 ', 'C1.W19 ', 'C1.W20 ', 'C1.W21 ' ) ], check.keys=FALSE)[1] \#Conflict 1: Wise reasoning - North America Overall

\#\# \$total

\#\# raw_alpha std.alpha G6(smc) average_r $\mathrm{S} / \mathrm{N}$ ase mean 


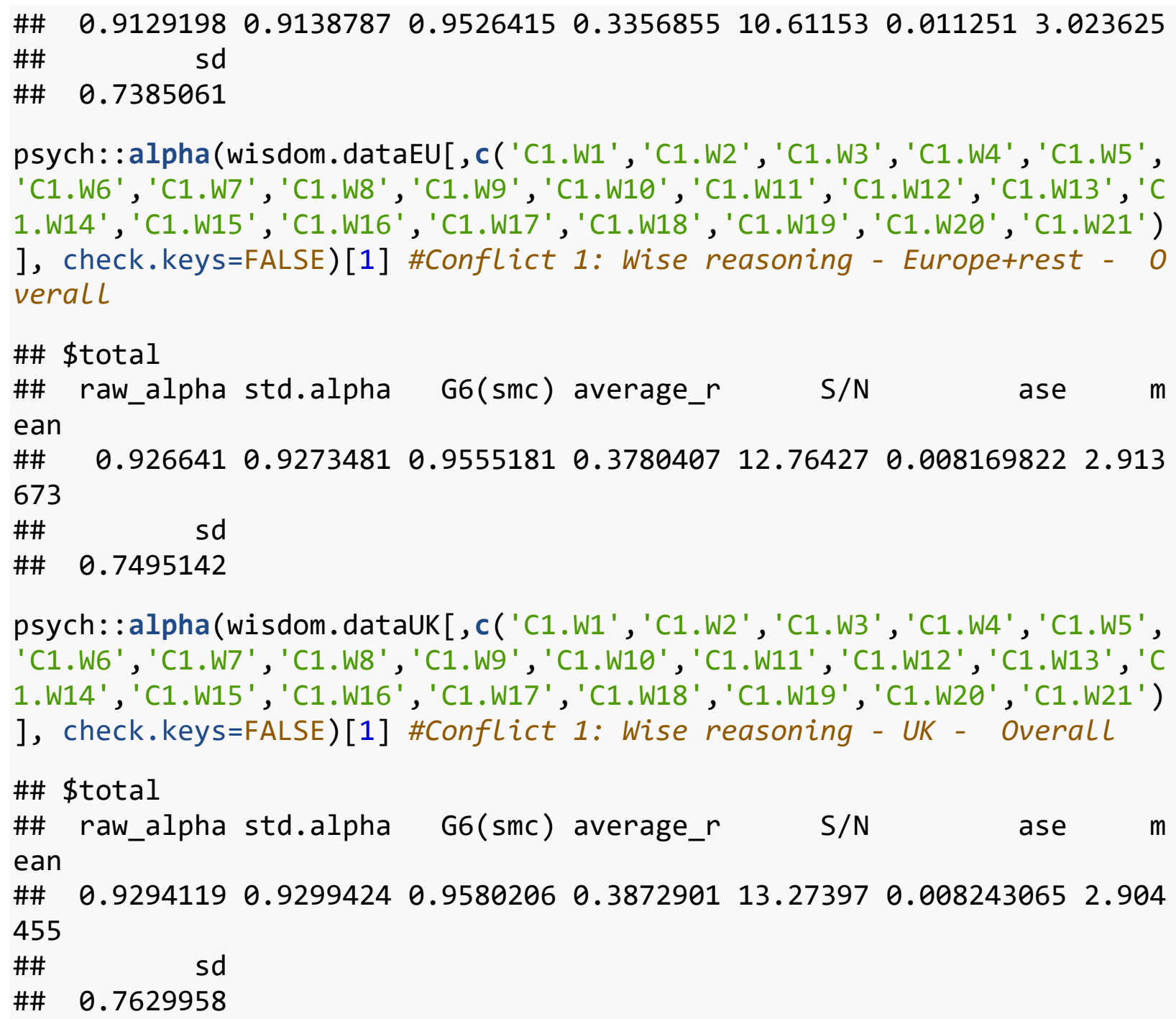

\#\# raw_alpha std.alpha G6(smc) average_r S/N ase me an 


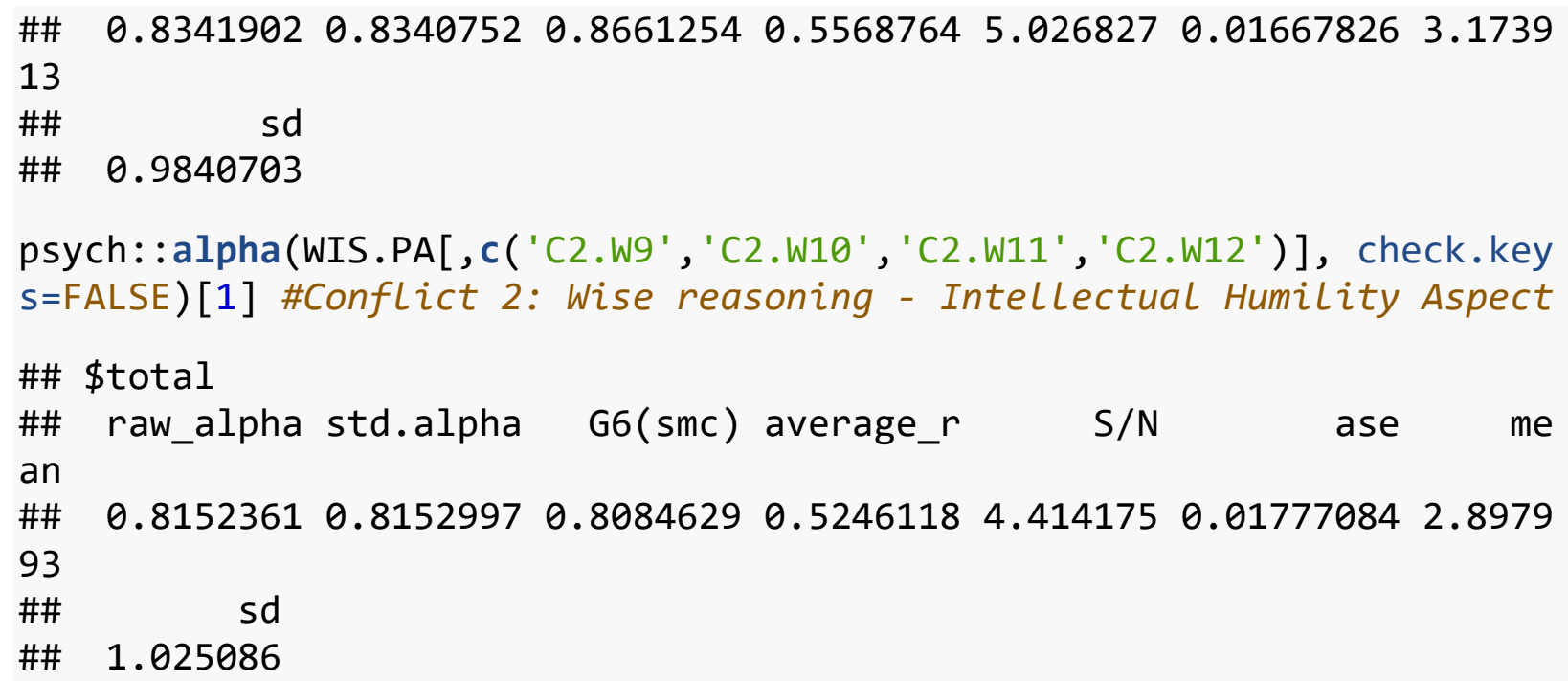
$\mathrm{s}=\mathrm{FALSE}$ [1] \#Conflict 2: Wise reasoning - Intellectual Humility Aspect

psych: :alpha(WIS.PA[, c('C2.W18', 'C2.W19', 'C2.W20', 'C2.W21')], check.ke ys=FALSE)[1] \#Conflict 2: Wise reasoning - Outsider Perspective Aspect \#\# \$total

\#\# raw_alpha std.alpha $\mathrm{G6}(\mathrm{smc})$ average_r $\mathrm{S} / \mathrm{N}$ ase $\mathrm{m}$ $\begin{array}{llllllll}\text { ean } & 0.9313952 & 0.9315459 & 0.9167608 & 0.7728348 & 13.60833 & 0.006560585 & 2.642\end{array}$ 977

\#\# $\quad$ sd

\#\# 1.25295

psych: :alpha(WIS.PA[, c( 'C2.W1', 'C2.W2', 'C2.W3 ', 'C2.W4' ', 'C2.W5 ' , 'C2.W6' , 'C2.W7' , 'C2.W8', 'C2.W9' , 'C2.W10', 'C2.W11', 'C2.W12' , 'C2.W13' , 'C2.W14' , 'C2.W15', 'C2.W16', 'C2.W17' , 'C2.W18', 'C2.W19', 'C2.W20', 'C2.W21' )], chec k.keys=FALSE)[1] \#Conflict 2: Wise reasoning - Overall

\#\# \$total

\#\# raw_alpha std.alpha G6(smc) average_r S/N ase me

an

$\begin{array}{lllllllll}\# \# & 0.9277329 & 0.9287871 & 0.9600787 & 0.3831223 & 13.0424 & 0.006167389 & 3.0242\end{array}$

08 


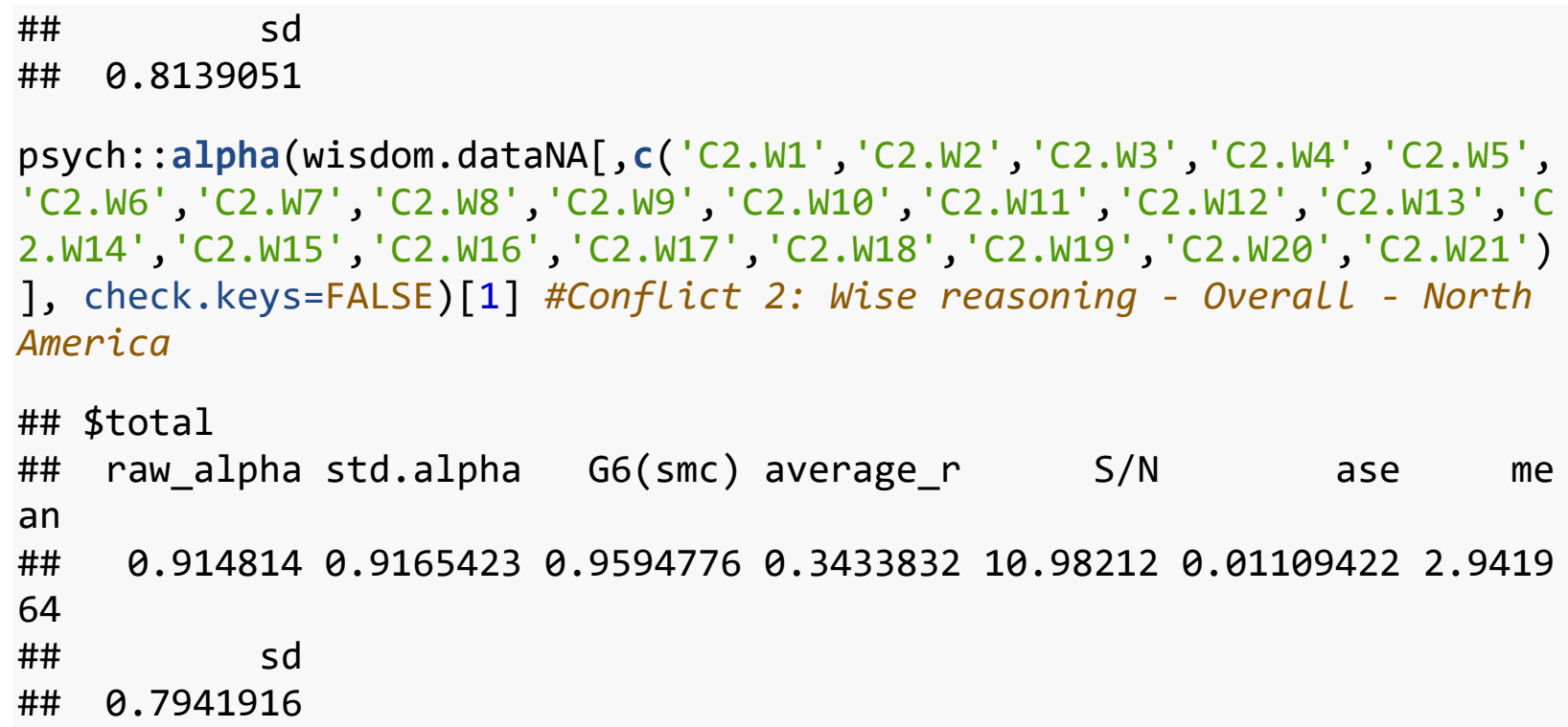

psych: :alpha(wisdom.dataEU[, c( 'C2.W1 ', 'C2.W2 ' , 'C2.W3 ', 'C2.W4 ', 'C2.W5 ' , 'C2.W6', 'C2.W7', 'C2.W8 ', 'C2.W9' , 'C2.W10', 'C2.W11', 'C2.W12 ', 'C2.W13 ', 'C 2.W14 ', 'C2.W15 ', 'C2.W16 ', 'C2.W17', 'C2.W18 ', 'C2.W19 ' , 'C2.W20 ' , 'C2.W21 ' ) ], check.keys=FALSE)[1] \#Conflict 2: Wise reasoning - Overall - Europe

\#\# \$total

\#\# raw_alpha std.alpha G6(smc) average_r $\mathrm{S} / \mathrm{N}$ ase me

an

$\begin{array}{lllllllll}\text { \#\# } & 0.9360334 & 0.9368452 & 0.9662492 & 0.4139661 & 14.83411 & 0.007233428 & 3.085\end{array}$

77

\#\# $\quad$ sd

\#\# 0.8252973

psych: :alpha(wisdom.dataUK [, c( 'C2.W1 ', 'C2.W2 ', 'C2.W3 ', 'C2.W4 ', 'C2.W5 ', 'C2.W6 ', 'C2.W7' , 'C2.W8 ', 'C2.W9' , 'C2.W10', 'C2.W11', 'C2.W12 ', 'C2.W13 ', 'C 2.W14 ', 'C2.W15 ', 'C2.W16 ', 'C2.W17', 'C2.W18 ', 'C2.W19 ', 'C2.W20 ', 'C2.W21 ' ) ], check.keys=FALSE)[1] \#Conflict 2: Wise reasoning - OveralL - UK

\#\# \$total

\#\# raw_alpha std.alpha G6(smc) average_r S/N ase me

an

$\begin{array}{lllllllll}\text { \#\# } & 0.9391699 & 0.9399247 & 0.968854 & 0.4269463 & 15.64578 & 0.007229403 & 3.0734\end{array}$

25

\#\# sd

\#\# 0.8517013

WISE REASONING PCA

principal(WIS.PA[, c("C1.Perspective.ave", "C1.ChangeOutcomes .ave", "C1.L imits.ave", "C1.CompResolve.ave", "C1.Outsider.ave")], nfactors=1, residua 
Is=TRUE, rotate="oblimin", missing=TRUE, impute="median") \#Conflict 1: Ge tting PCA scores (2nd order factor across means of 5 dimensions) /61\% var explained

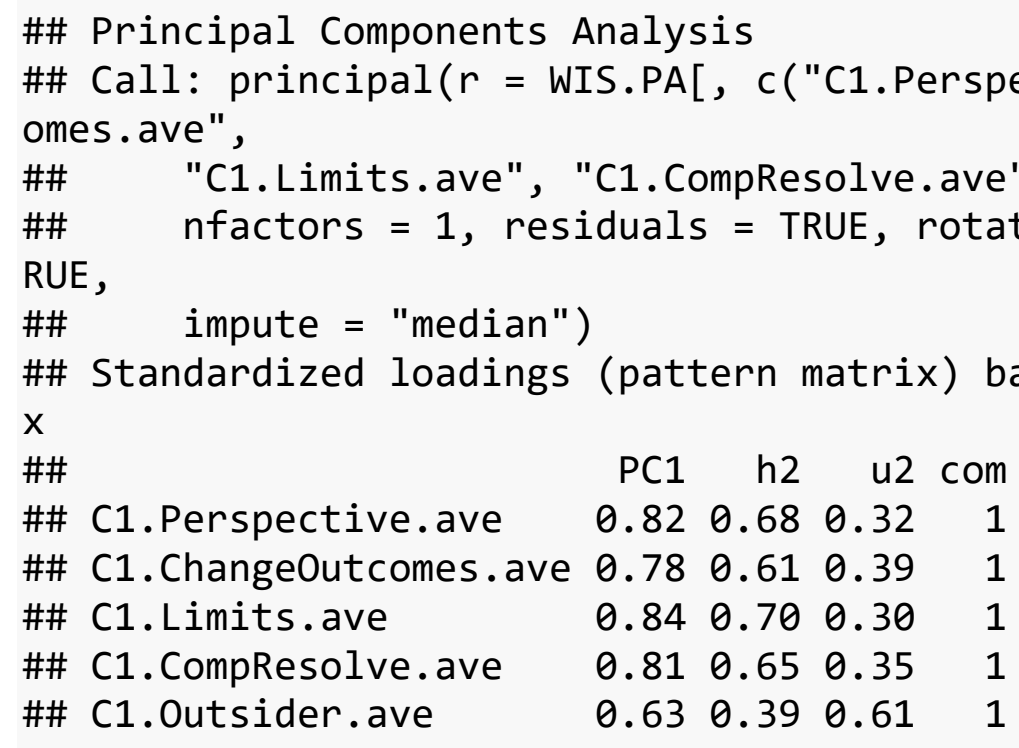
imits.ave", "C2.CompResolve.ave", "C2.Outsider.ave") ], nfactors=1, residua ls=TRUE, rotate="oblimin", missing=TRUE, impute="median") \#Conflict 2: Ge tting PCA scores (2nd order factor across means of 5 dimensions) / 59\% variance explained.

\#\# Principal Components Analysis

\#\# Call: principal( $r=$ WIS.PA[, c("C2.Perspective.ave", "C2.ChangeOutc omes.ave",

\#\# "C2.Limits.ave", "C2.CompResolve.ave", "C2.Outsider.ave")],

\#\# nfactors $=1$, residuals $=$ TRUE, rotate $=$ "oblimin", missing $=\mathrm{T}$ RUE, 


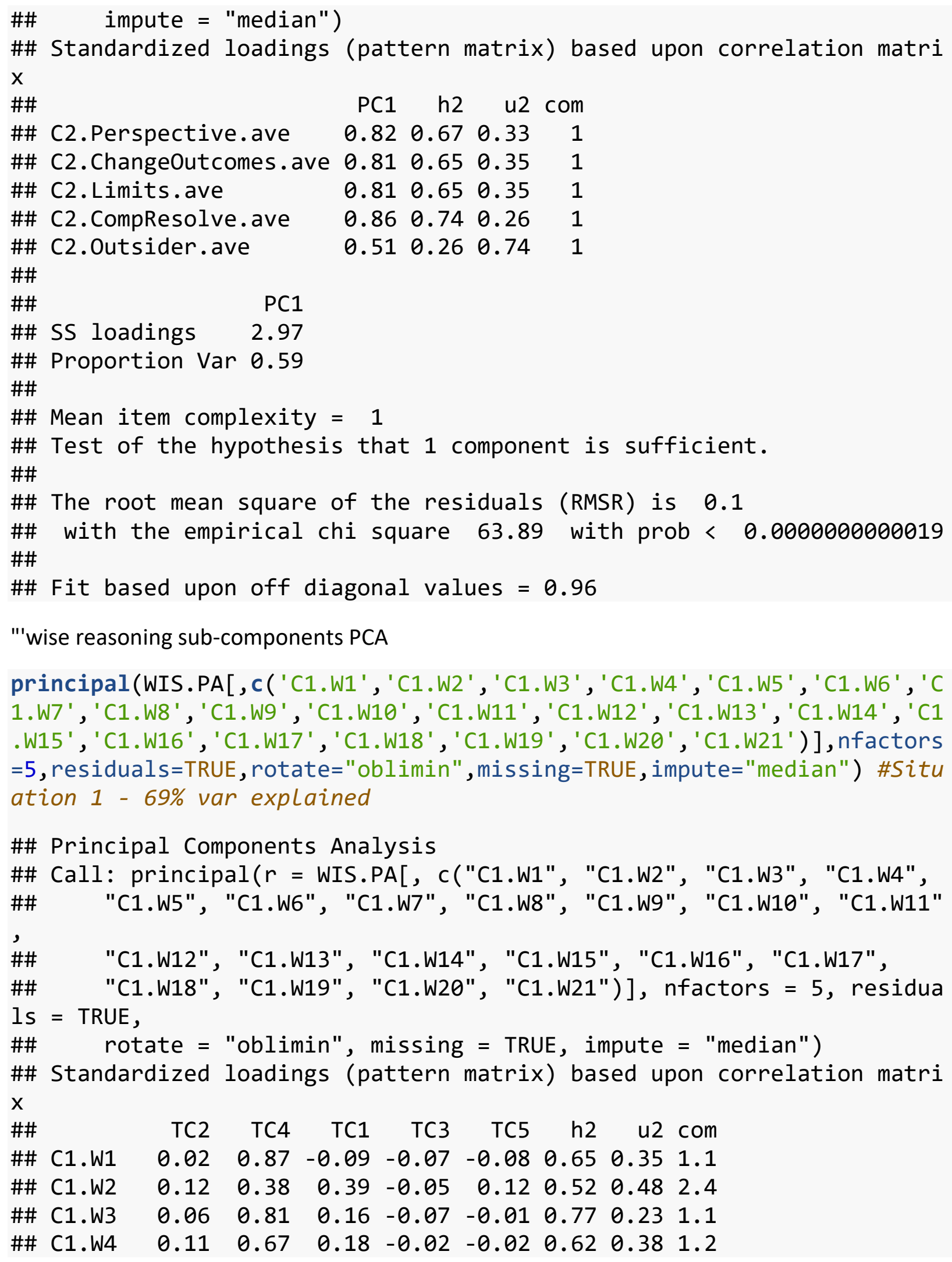




\begin{tabular}{|c|c|c|c|c|c|c|c|c|c|}
\hline \#\# & C1.W5 & 0.08 & & & & & & & \\
\hline \#\# & C1.W6 & 0.00 & 0.27 & 0.33 & 0.39 & 0.04 & .58 & .42 & \\
\hline \#\# & C1.W7 & 0.02 & 0.00 & 0.06 & 0.90 & -0.09 & 83 & .17 & 1.0 \\
\hline \#\# & C1.W8 & 0.04 & -0.11 & 0.02 & 0.92 & 0.01 & 83 & 0.17 & 1.0 \\
\hline \# & C1.W9 & .00 & 0.42 & -0.19 & 0.33 & & & 32 & 3.2 \\
\hline \#\# & C1.W10 & 0.00 & 0.57 & -0.08 & 0.33 & 0.19 & 0.65 & 0.35 & 1.9 \\
\hline$\#$ & C1.W11 & 0.19 & 0.09 & 0.05 & 0. & & & 0.37 & 1.3 \\
\hline \#\# & C1.W12 & 0.10 & -0.12 & 0.06 & -0.13 & 0.82 & 0.66 & 0.34 & 1.1 \\
\hline \# & C1.W13 & -0.05 & 0.08 & 0.55 & 0.00 & 0. & & 0.38 & 1.9 \\
\hline \# & C1.W14 & -0.05 & 0.10 & 0.56 & 0.11 & & & 0.35 & 1.8 \\
\hline \# & C1.W15 & -0.06 & 0.19 & 0.54 & 0.07 & 0.29 & 0. & 0.36 & 1.9 \\
\hline & C1.W16 & 0.03 & -0.06 & 0.83 & 0.04 & -0 . & & 0.35 & 1.1 \\
\hline & C1.W17 & 0.13 & 0.07 & 0.69 & 0.12 & -0.04 & 0.64 & 0.36 & 1.2 \\
\hline & C1.W18 & & 0.05 & -0.05 & -0.01 & -0 & & 0.23 & 1.0 \\
\hline & C1.W19 & 0.88 & 0.04 & -0.01 & 0.02 & 0.06 & 0. & 0.15 & 1.0 \\
\hline & $1 . W 20$ & 0.95 & -0.08 & 0.06 & -0.02 & -0.06 & & 0.15 & 1.0 \\
\hline \# & 1 . W21 & 0.78 & 0.06 & -0.04 & 0.06 & 0.15 & 0.75 & 0.25 & 1.1 \\
\hline
\end{tabular}

\#\#

\#\#

TC2 TC4 TC1 TC3 TC5

\#\# SS loadings $\quad \begin{array}{lllllll}3.38 & 3.32 & 3.11 & 2.48 & 2.10\end{array}$

$\begin{array}{llllllll}\text { \#\# Proportion Var } & 0.16 & 0.16 & 0.15 & 0.12 & 0.10\end{array}$

\#\# Cumulative Var $\quad 0.160 .320 .47 \quad 0.590 .69$

$\begin{array}{lllllll}\text { \# Proportion Explained } & 0.23 & 0.23 & 0.22 & 0.17 & 0.15\end{array}$

\#\# Cumulative Proportion $0.23 \quad 0.47 \quad 0.68 \quad 0.85 \quad 1.00$

\#\#

\#\# With component correlations of

\#\# $\quad$ TC2 TC4 TC1 TC3

$\begin{array}{lllllll}\text { \#\# TC2 } & 1.00 & 0.33 & 0.24 & 0.25 & 0.29\end{array}$

$\begin{array}{lllllll}\text { \#\# TC4 } & 0.33 & 1.00 & 0.35 & 0.32 & 0.39\end{array}$

$\begin{array}{lllllll}\text { \#\# TC1 } & 0.24 & 0.35 & 1.00 & 0.33 & 0.29\end{array}$

$\begin{array}{lllllll}\text { \#\# TC3 } & 0.25 & 0.32 & 0.33 & 1.00 & 0.22\end{array}$

$\begin{array}{lllllll}\text { \#\# TC5 } & 0.29 & 0.39 & 0.29 & 0.22 & 1.00\end{array}$

\#\#

\#\# Mean item complexity $=1.6$

\#\# Test of the hypothesis that 5 components are sufficient.

\#\#

\#\# The root mean square of the residuals (RMSR) is 0.06

\#\# with the empirical chi square 396.81 with prob $<0.000000000000$ 000000000000000000012

\#\#

\#\# Fit based upon off diagonal values $=0.98$

\#component 1: 13-17 -all Compromise-resolution items! 15\% var unique $v$ ar

\#component 2: 18-21 - all outsider perspective items! $16 \%$ var unique $v$ 
ar

\#Component 3: 6-8 - all outcomes/change [5 seems distributed between $t$ his component, component 1, and component 4]

\#items 5 is "Looked for different solutions as the situation evolved" - makes sense to be between outcomes, compromise, and perspective-taki ng | $12 \%$ unique var

\#component 4: 1, 3, 4, 10 - most of the perspective-taking, plus one Limits [2 seems distributed between this component and component 1; 5 between this component 1, and 3; item 9 - between this component and component 5]

\#item 10 "Double-checked whether the other person's opinions might be correct" makes sense to be between perspective-taking and Limits / 16\% unique var

\#component 5: 9, 11, 12 - most of the limits / 10\% unique var

\#\#\#overall -very good convergence and mapping on the original distribu tion when presented with 5 components.

principal(WIS.PA[, c( 'C2.W1', 'C2.W2', 'C2.W3 ', 'C2.W4 ', 'C2.W5 ', 'C2.W6 ', 'C 2.W7' , 'C2.W8', 'C2.W9' , 'C2.W10 ', 'C2.W11 ', 'C2.W12 ' , 'C2.W13 ' , 'C2.W14 ' , 'C2 .W15 ', 'C2.W16', 'C2.W17' , 'C2.W18 ', 'C2.W19 ', 'C2.W20 ', 'C2.W21 ') ], nfactors =5, residuals=TRUE, rotate="oblimin", missing=TRUE, impute="median") \#Situ ation 2 - total 73\% var explained

\#\# Principal Components Analysis

\#\# Call: principal(r = WIS.PA[, c("C2.W1", "C2.W2", "C2.W3", "C2.W4", \#\# "C2.W5", "C2.W6", "C2.W7", "C2.W8", "C2.W9", "C2.W10", "C2.W11"

\#\# "C2.W12", "C2.W13", "C2.W14", "C2.W15", "C2.W16", "C2.W17", \#\# "C2.W18", "C2.W19", "C2.W20", "C2.W21")], nfactors = 5, residua Is = TRUE,

\#\# $\quad$ rotate = "oblimin", missing = TRUE, impute = "median")

\#\# Standardized loadings (pattern matrix) based upon correlation matri $\mathrm{x}$

$\begin{array}{lrrrrrrrr}\text { \#\# } & \text { TC2 } & \text { TC1 } & \text { TC5 } & \text { TC4 } & \text { TC3 } & \text { h2 } & \text { u2 } & \text { Com } \\ \text { \#\# C2.W1 } & 0.02 & -0.10 & 0.93 & -0.01 & 0.00 & 0.77 & 0.23 & 1.0 \\ \text { \#\# C2.W2 } & -0.02 & 0.19 & 0.75 & -0.08 & -0.04 & 0.64 & 0.36 & 1.2 \\ \text { \#\# C2.W3 } & 0.01 & -0.02 & 0.90 & 0.02 & 0.02 & 0.83 & 0.17 & 1.0 \\ \text { \#\# C2.W4 } & 0.05 & 0.16 & 0.67 & 0.10 & 0.06 & 0.75 & 0.25 & 1.2 \\ \text { \#\# C2.W5 } & -0.10 & 0.51 & 0.10 & 0.23 & 0.18 & 0.62 & 0.38 & 1.8 \\ \text { \#\# C2.W6 } & -0.12 & 0.43 & 0.09 & 0.17 & 0.36 & 0.63 & 0.37 & 2.6 \\ \text { \#\# C2.W7 } & 0.02 & -0.02 & 0.05 & -0.02 & 0.93 & 0.88 & 0.12 & 1.0 \\ \text { \#\# C2.W8 } & 0.02 & 0.00 & -0.05 & 0.03 & 0.94 & 0.88 & 0.12 & 1.0 \\ \text { \#\# C2.W9 } & 0.06 & 0.13 & 0.00 & 0.75 & 0.03 & 0.71 & 0.29 & 1.1 \\ \text { \#\# C2.W10 } & 0.00 & 0.10 & 0.04 & 0.74 & 0.05 & 0.70 & 0.30 & 1.0 \\ \text { \#\# C2.W11 } & 0.17 & -0.07 & 0.20 & 0.61 & 0.02 & 0.60 & 0.40 & 1.4\end{array}$




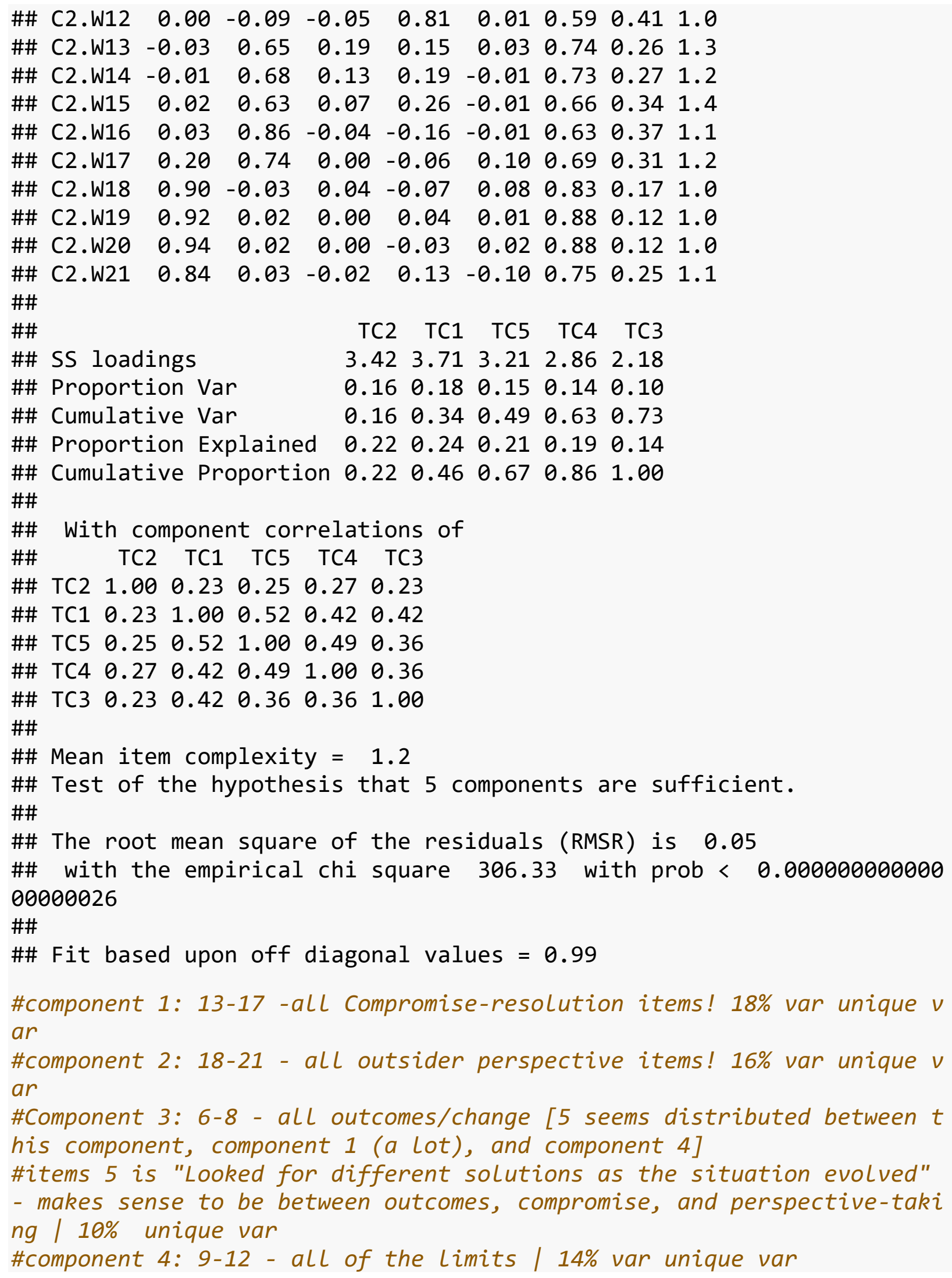
his component, component 1 (a Lot), and component 4] \#items 5 is "Looked for different solutions as the situation evolved" - makes sense to be between outcomes, compromise, and perspective-taki ng | $10 \%$ unique var

\#component 4:9-12 - all of the Limits / 14\% var unique var 
\#component 5: 1-4, 10 - all perspective-taking! /15\% var unique var \#\#\#overall -Excellent convergence and mapping on the original distribu tion when presented with 5 components!

examine PCAs for Europe and North America separately North America

principal(wisdom.dataNA[, c("C1. Perspective.ave", "C1. ChangeOutcomes .ave ", "C1.Limits.ave", "C1.CompResolve.ave", "C1.Outsider.ave")], nfactors=1, residuals=TRUE, rotate="oblimin", missing=TRUE, impute="median") \#Conflic $t$ 1: Getting PCA scores (2nd order factor across means of 5 dimensions ) $159 \%$ var explained

\#\# Principal Components Analysis

\#\# Call: principal( $r=$ wisdom.dataNA[, c("C1.Perspective.ave", "C1.Cha ngeOutcomes.ave",

\#\# "C1.Limits.ave", "C1.CompResolve.ave", "C1.Outsider.ave")],

\#\# $\quad$ nfactors $=1$, residuals $=$ TRUE, rotate $=$ "oblimin", missing $=\mathrm{T}$

RUE,

\#\# $\quad$ impute $=$ "median")

\#\# Standardized loadings (pattern matrix) based upon correlation matri $x$

\#\# $\quad$ PC1 1 h2 u2 com

$\begin{array}{lllllll}\text { \#\# C1.Perspective.ave } \quad 0.81 & 0.66 & 0.34 & 1\end{array}$

\#\# C1.ChangeOutcomes.ave $0.78 \quad 0.61 \quad 0.39 \quad 1$

$\begin{array}{lllllll}\text { \#\# C1.Limits.ave } \quad 0.86 & 0.74 & 0.26 & 1\end{array}$

$\begin{array}{llllll}\text { \#\# C1.CompResolve.ave } \quad 0.76 & 0.58 & 0.42 & 1\end{array}$

$\begin{array}{llllll}\text { \#\# C1.Outsider.ave } & 0.61 & 0.37 & 0.63 & 1\end{array}$

\#\#

\#\# PC1

\#\# SS loadings 2.95

\#\# Proportion Var 0.59

\#\#

\#\# Mean item complexity $=1$

\#\# Test of the hypothesis that 1 component is sufficient.

\#\#

\#\# The root mean square of the residuals (RMSR) is 0.12

\#\# with the empirical chi square 34.29 with prob $<0.0000021$

\#\#

\#\# Fit based upon off diagonal values $=0.95$

principal(wisdom.dataNA[, c("C2. Perspective.ave", "C2. ChangeOutcomes .ave ", "C2.Limits.ave", "C2.CompResolve.ave", "C2.Outsider.ave")], nfactors=1, residuals=TRUE, rotate="oblimin", missing=TRUE, impute="median") \#Conflic $t$ 2: Getting PCA scores (2nd order factor across means of 5 dimensions ) / 55\% variance explained (outsider a bit weak but overall OK). 
\#\# Principal Components Analysis

\#\# Call: principal( $r=$ wisdom.dataNA[, c("C2.Perspective.ave", "C2.Cha ngeOutcomes.ave",

\#\# "C2.Limits.ave", "C2.CompResolve.ave", "C2.Outsider.ave")],

\#\# $\quad$ nfactors $=1$, residuals $=$ TRUE, rotate $=$ "oblimin", missing $=\mathrm{T}$

RUE,

\#\# $\quad$ impute $=$ "median")

\#\# Standardized loadings (pattern matrix) based upon correlation matri $x$

\#\# $\quad$ PC1 1 h2 u2 com

$\begin{array}{llllll}\text { \#\# C2.Perspective.ave } \quad 0.82 & 0.67 & 0.33 & 1\end{array}$

$\begin{array}{lllllll}\text { \#\# C2.ChangeOutcomes.ave } & 0.77 & 0.60 & 0.40 & 1\end{array}$

\#\# C2.Limits.ave $\quad 0.740 .55 \quad 0.45 \quad 1$

\#\# C2.CompResolve.ave $\quad \begin{array}{lllll}0.84 & 0.70 & 0.30 & 1\end{array}$

$\begin{array}{llllll}\text { \#\# C2.Outsider.ave } & 0.47 & 0.22 & 0.78 & 1\end{array}$

\#\#

\#\# PC1

\#\# SS loadings 2.73

\#\# Proportion Var 0.55

\#\#

\#\# Mean item complexity $=1$

\#\# Test of the hypothesis that 1 component is sufficient.

\#\#

\#\# The root mean square of the residuals (RMSR) is 0.12

\#\# with the empirical chi square 37.64 with prob $<0.00000044$

\#\#

\#\# Fit based upon off diagonal values $=0.93$

"'wise reasoning sub-components PCA in North America

principal(wisdom.dataNA[, C('C1.W1', 'C1.W2' , 'C1.W3 ', 'C1.W4 ' , 'C1.W5 ', 'C1 .W6 ', 'C1.W7' ', 'C1.W8', 'C1.W9', 'C1.W10', 'C1.W11', 'C1.W12', 'C1.W13' , 'C1.W 14' , 'C1.W15 ', 'C1.W16', 'C1.W17', 'C1.W18' , 'C1.W19' , 'C1.W20' , 'C1.W21' ) ], n factors $=5$, residuals=TRUE, rotate="oblimin", missing=TRUE, impute="median" ) \#Situation 1 - 69\% var explained

\#\# Principal Components Analysis

\#\# Call: principal( $r=$ wisdom.dataNA[, c("C1.W1", "C1.W2", "C1.W3", "C 1.W4",

\#\# "C1.W5", "C1.W6", "C1.W7", "C1.W8", "C1.W9", "C1.W10", "C1.W11"

,

\#\# "C1.W12", "C1.W13", "C1.W14", "C1.W15", "C1.W16", "C1.W17",

\#\# "C1.W18", "C1.W19", "C1.W20", "C1.W21")], nfactors = 5, residua

$1 \mathrm{~s}=$ TRUE,

\#\# $\quad$ rotate $=$ "oblimin", missing $=$ TRUE, impute $=$ "median") 
\#\# Standardized loadings (pattern matrix) based upon correlation matri $\mathrm{x}$

$\begin{array}{lrrrrrrrr}\text { \#\# } & \text { TC2 } & \text { TC3 } & \text { TC5 } & \text { TC4 } & \text { TC1 } & \text { h2 } & \text { U2 } & \text { Com } \\ \text { \#\# C1.W1 } & -0.06 & -0.15 & 0.93 & 0.03 & 0.07 & 0.78 & 0.22 & 1.1 \\ \text { \#\# C1.W2 } & 0.14 & 0.22 & 0.57 & -0.03 & -0.20 & 0.55 & 0.45 & 1.7 \\ \text { \#\# C1.W3 } & 0.03 & 0.02 & 0.86 & -0.03 & 0.05 & 0.77 & 0.23 & 1.0 \\ \text { \#\# C1.W4 } & 0.17 & 0.08 & 0.69 & -0.03 & -0.07 & 0.62 & 0.38 & 1.2 \\ \text { \#\# C1.W5 } & 0.09 & 0.47 & 0.19 & 0.04 & 0.49 & 0.67 & 0.33 & 2.4 \\ \text { \#\# C1.W6 } & -0.04 & 0.52 & 0.14 & 0.17 & 0.41 & 0.65 & 0.35 & 2.3 \\ \text { \#\# C1.W7 } & 0.08 & -0.07 & 0.02 & 0.90 & 0.02 & 0.82 & 0.18 & 1.0 \\ \text { \#\# C1.W8 } & 0.08 & 0.04 & -0.06 & 0.89 & 0.03 & 0.83 & 0.17 & 1.0 \\ \text { \#\# C1.W9 } & 0.14 & 0.21 & 0.33 & 0.13 & 0.46 & 0.63 & 0.37 & 2.7 \\ \text { \#\# C1.W10 } & 0.06 & 0.13 & 0.41 & 0.18 & 0.52 & 0.71 & 0.29 & 2.4 \\ \text { \#\# C1.W11 } & 0.33 & 0.36 & 0.24 & -0.02 & -0.10 & 0.47 & 0.53 & 2.9 \\ \text { \#\# C1.W12 } & 0.20 & 0.55 & 0.10 & -0.36 & -0.31 & 0.51 & 0.49 & 2.8 \\ \text { \#\# C1.W13 } & 0.02 & 0.77 & 0.02 & 0.01 & -0.12 & 0.63 & 0.37 & 1.1 \\ \text { \#\# C1.W14 } & 0.01 & 0.88 & -0.13 & 0.05 & 0.18 & 0.76 & 0.24 & 1.1 \\ \text { \#\# C1.W15 } & -0.03 & 0.73 & 0.13 & 0.13 & -0.05 & 0.71 & 0.29 & 1.1 \\ \text { \#\# C1.W16 } & -0.09 & 0.36 & 0.11 & 0.48 & -0.46 & 0.67 & 0.33 & 3.1 \\ \text { \#\# C1.W17 } & -0.04 & 0.33 & 0.29 & 0.41 & -0.27 & 0.61 & 0.39 & 3.6 \\ \text { \#\# C1.W18 } & 0.89 & -0.08 & 0.03 & 0.01 & -0.05 & 0.78 & 0.22 & 1.0 \\ \text { \#\# C1.W19 } & 0.89 & -0.06 & 0.07 & 0.06 & 0.01 & 0.84 & 0.16 & 1.0 \\ \text { \#\# C1.W20 } & 0.93 & 0.01 & -0.09 & 0.00 & -0.01 & 0.82 & 0.18 & 1.0 \\ \text { \#\# C1.W21 } & 0.84 & 0.07 & -0.01 & 0.04 & 0.09 & 0.77 & 0.23 & 1.0\end{array}$

\#\#

\#\#

\#\# SS loadings

TC2 TC3 TC5 TC4 TC1

\#\# Proportion Var

$\begin{array}{lllll}3.62 & 3.64 & 3.41 & 2.48 & 1.44\end{array}$

\#\# Cumulative Var

$\begin{array}{llllllll}0.17 & 0.17 & 0.16 & 0.12 & 0.07\end{array}$

\#\# Proportion Explained

$\begin{array}{llllll}0.17 & 0.35 & 0.51 & 0.63 & 0.69\end{array}$

$\begin{array}{lllllll}\text { \#\# Cumulative Proportion } 0.25 & 0.50 & 0.73 & 0.90 & 1.00\end{array}$

\#\#

\#\# With component correlations of

\#\# $\quad$ TC2 TC3 TC5 TC4 TC1

$\begin{array}{lllllll}\text { \#\# TC2 } & 1.00 & 0.24 & 0.36 & 0.18 & 0.06\end{array}$

$\begin{array}{llllllll}\text { \#\# TC3 } & 0.24 & 1.00 & 0.41 & 0.35 & 0.01\end{array}$

$\begin{array}{lllllll}\text { \#\# TC5 } & 0.36 & 0.41 & 1.00 & 0.32 & 0.14\end{array}$

$\begin{array}{lllllll}\text { \#\# TC4 } & 0.18 & 0.35 & 0.32 & 1.00 & 0.13\end{array}$

$\begin{array}{lllllllllll}\text { \#\# TC1 } & 0.06 & 0.01 & 0.14 & 0.13 & 1.00\end{array}$

\#\#

\#\# Mean item complexity $=1.7$

\#\# Test of the hypothesis that 5 components are sufficient.

\#\#

\#\# The root mean square of the residuals (RMSR) is 0.06 
\#\# with the empirical chi square 171.39 with prob $<0.00051$

\#\#

\#\# Fit based upon off diagonal values $=0.98$

principal(wisdom.dataNA[, c('C2.W1' , 'C2.W2' , 'C2.W3 ', 'C2.W4 ', 'C2.W5 ', 'C2 .W6' , 'C2.W7' , 'C2.W8' , 'C2.W9' , 'C2.W10', 'C2.W11', 'C2.W12', 'C2.W13' , 'C2.W 14' , 'C2.W15 ', 'C2.W16' , 'C2.W17' , 'C2.W18' , 'C2.W19' , 'C2.W20' , 'C2.W21' ) ], n factor $s=5$, residuals=TRUE, rotate="oblimin", missing=TRUE, impute="median" ) \#Situation 2 - total 73\% var explained

\#\# Principal Components Analysis

\#\# Call: principal( $r=$ wisdom.dataNA[, c("C2.W1", "C2.W2", "C2.W3", "C 2.W4",

\#\# "C2.W5", "C2.W6", "C2.W7", "C2.W8", "C2.W9", "C2.W10", "C2.W11"

\#\# "C2.W12", "C2.W13", "C2.W14", "C2.W15", "C2.W16", "C2.W17",

\#\# "C2.W18", "C2.W19", "C2.W20", "C2.W21")], nfactors = 5, residua $1 \mathrm{~s}=\mathrm{TRUE}$,

\#\# $\quad$ rotate $=$ "oblimin", missing = TRUE, impute = "median")

\#\# Standardized loadings (pattern matrix) based upon correlation matri $x$

$\begin{array}{lllllllll}\# \# & \text { TC1 } & \text { TC2 } & \text { TC5 } & \text { TC3 } & \text { TC4 } & \text { h2 } & \text { u2 com }\end{array}$

$\begin{array}{lllllllll}\text { \#\# C2.W1 } & -0.01 & 0.02 & 0.84 & 0.02 & 0.04 & 0.75 & 0.253 & 1.0\end{array}$

$\begin{array}{llllllllll}\text { \#\# C2.W2 } & 0.13 & 0.01 & 0.59 & 0.00 & 0.08 & 0.49 & 0.511 & 1.1\end{array}$

$\begin{array}{llllllllll}\text { \#\# C2.W3 } & -0.07 & 0.00 & 0.93 & 0.03 & 0.03 & 0.84 & 0.159 & 1.0\end{array}$

$\begin{array}{lllllllll}\text { \#\# C2.W4 } & 0.24 & 0.00 & 0.72 & 0.02 & 0.03 & 0.78 & 0.222 & 1.2\end{array}$

$\begin{array}{lllllllll}\text { \#\# C2.W5 } & 0.49 & -0.13 & 0.11 & 0.23 & 0.31 & 0.68 & 0.318 & 2.5\end{array}$

$\begin{array}{lllllllll}\text { \#\# C2.W6 } & 0.42 & -0.16 & 0.03 & 0.24 & 0.46 & 0.70 & 0.295 & 2.8\end{array}$

$\begin{array}{lllllllll}\text { \#\# C2.W7 } & -0.04 & 0.02 & 0.08 & -0.02 & 0.91 & 0.86 & 0.145 & 1.0\end{array}$

$\begin{array}{lllllllll}\text { \#\# C2.W8 } & 0.03 & 0.07 & 0.01 & -0.04 & 0.90 & 0.84 & 0.162 & 1.0\end{array}$

$\begin{array}{lllllllll}\text { \#\# C2.W9 } & 0.09 & 0.07 & 0.23 & 0.67 & -0.06 & 0.68 & 0.323 & 1.3\end{array}$

$\begin{array}{lllllllll}\text { \#\# C2.W10 } & 0.16 & 0.00 & 0.23 & 0.66 & -0.07 & 0.68 & 0.320 & 1.4\end{array}$

$\begin{array}{lllllllll}\text { \#\# C2.W11 } & -0.12 & 0.18 & 0.22 & 0.59 & 0.09 & 0.54 & 0.462 & 1.6\end{array}$

$\begin{array}{lllllllll}\text { \#\# C2.W12 } & 0.00 & 0.06 & -0.20 & 0.76 & 0.04 & 0.53 & 0.468 & 1.2\end{array}$

$\begin{array}{lllllllll}\text { \#\# C2.W13 } & 0.77 & -0.04 & 0.10 & 0.10 & 0.01 & 0.74 & 0.259 & 1.1\end{array}$

$\begin{array}{lllllllll}\text { \#\# C2.W14 } & 0.80 & -0.01 & 0.06 & 0.08 & 0.05 & 0.77 & 0.233 & 1.0\end{array}$

$\begin{array}{lllllllll}\text { \#\# C2.W15 } & 0.80 & 0.04 & -0.13 & 0.20 & 0.08 & 0.76 & 0.242 & 1.2\end{array}$

$\begin{array}{lllllllll}\text { \#\# C2.W16 } & 0.79 & 0.06 & 0.10 & -0.25 & -0.06 & 0.62 & 0.384 & 1.3\end{array}$

$\begin{array}{lllllllll}\text { \#\# C2.W17 } & 0.73 & 0.24 & 0.09 & -0.12 & -0.01 & 0.69 & 0.313 & 1.3\end{array}$

$\begin{array}{lllllllll}\text { \#\# C2.W18 } & 0.02 & 0.92 & -0.04 & 0.00 & 0.09 & 0.86 & 0.140 & 1.0\end{array}$

$\begin{array}{lllllllll}\text { \#\# C2.W19 } & -0.01 & 0.95 & -0.02 & 0.02 & 0.06 & 0.90 & 0.096 & 1.0\end{array}$

$\begin{array}{lllllllll}\text { \#\# C2.W20 } & 0.02 & 0.94 & 0.02 & -0.04 & 0.02 & 0.89 & 0.109 & 1.0\end{array}$

$\begin{array}{lllllllll}\text { \#\# C2.W21 } & 0.05 & 0.77 & 0.07 & 0.16 & -0.20 & 0.72 & 0.283 & 1.3\end{array}$

\#\#

\#\#

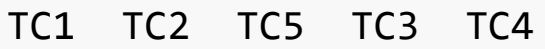




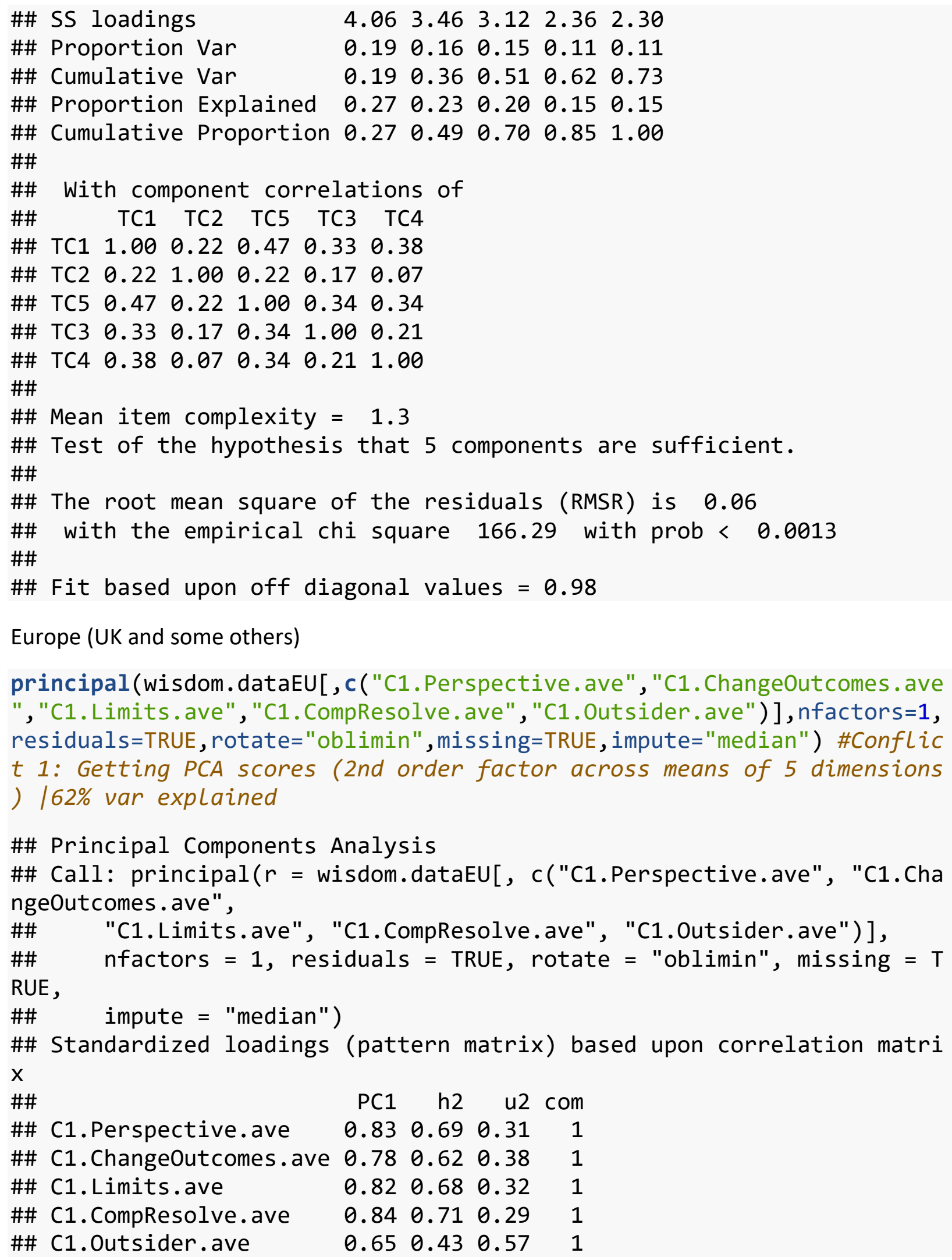




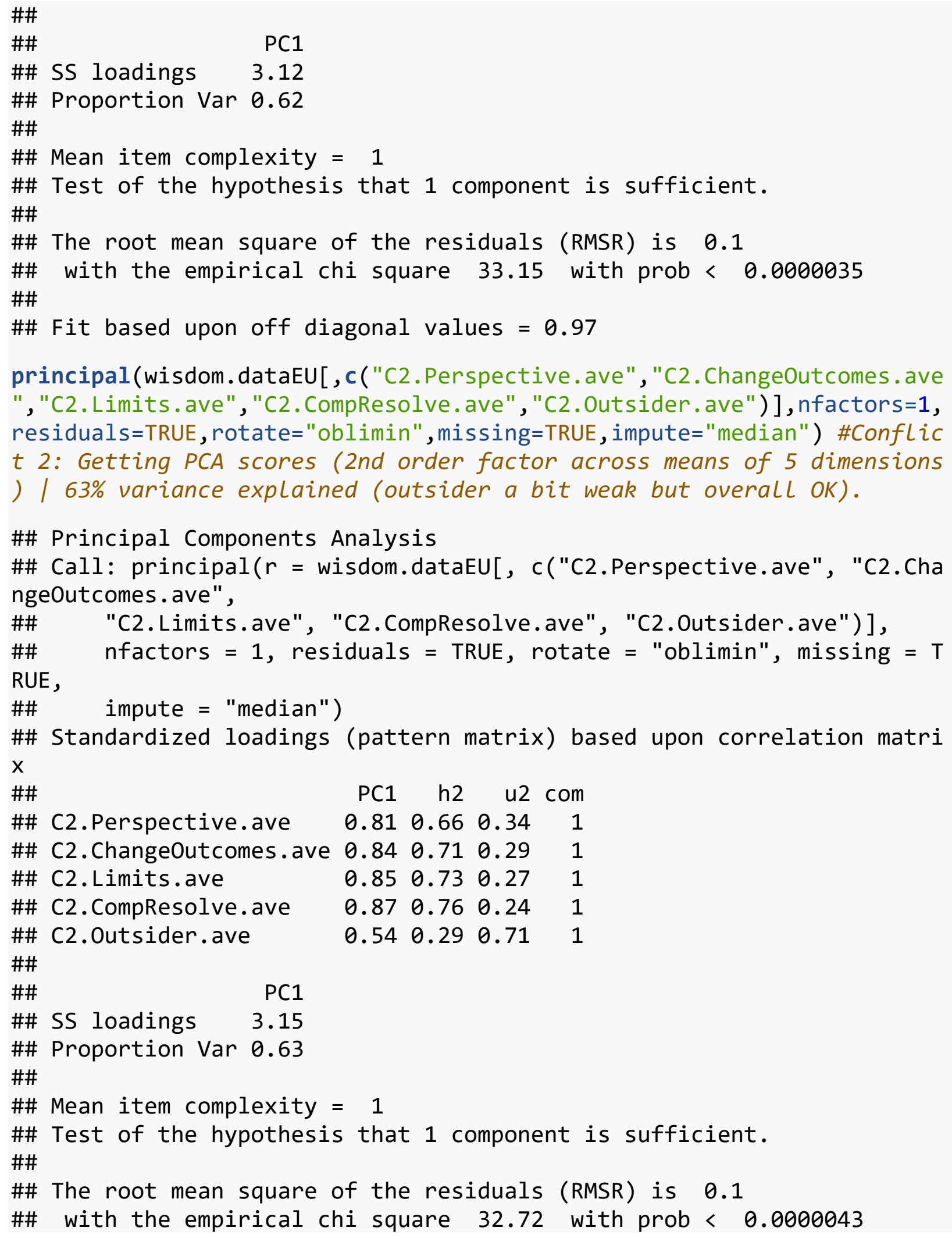


\#\#

\#\# Fit based upon off diagonal values $=0.97$

principal(wisdom.dataEU[, c( 'C1.W1' , 'C1.W2' , 'C1.W3 ' , 'C1.W4 ' , 'C1.W5 ', 'C1 .W6 ', 'C1.W7' , 'C1.W8', 'C1.W9', 'C1.W10', 'C1.W11' , 'C1.W12 ', 'C1.W13 ', 'C1.W 14', 'C1.W15 ', 'C1.W16', 'C1.W17', 'C1.W18 ', 'C1.W19' , 'C1.W20 ', 'C1.W21' )] , n factors=5, residuals=TRUE, rotate="oblimin", missing=TRUE, impute="median" ) \#Situation 1 - 70\% var explained

\#\# Principal Components Analysis

\#\# Call: principal( $r$ = wisdom.dataEU[, c("C1.W1", "C1.W2", "C1.W3", "C 1.W4",

\#\# "C1.W5", "C1.W6", "C1.W7", "C1.W8", "C1.W9", "C1.W10", "C1.W11"

\#\# "C1.W12", "C1.W13", "C1.W14", "C1.W15", "C1.W16", "C1.W17",

\#\# "C1.W18", "C1.W19", "C1.W20", "C1.W21")], nfactors = 5, residua ls = TRUE,

\#\# $\quad$ rotate = "oblimin", missing = TRUE, impute = "median")

\#\# Standardized loadings (pattern matrix) based upon correlation matri

$\mathrm{x}$

$\begin{array}{llllllll}\text { \#\# } & \text { TC1 } & \text { TC2 } & \text { TC4 } & \text { TC3 } & \text { TC5 } & \text { h2 } & \text { u2 com }\end{array}$

$\begin{array}{lllllllll}\text { \#\# C1.W1 } & -0.18 & 0.08 & 0.00 & -0.02 & 0.90 & 0.75 & 0.25 & 1.1\end{array}$

$\begin{array}{lllllllll}\text { \#\# C1.W2 } & 0.54 & 0.07 & 0.05 & 0.00 & 0.31 & 0.58 & 0.42 & 1.6\end{array}$

$\begin{array}{lllllllll}\text { \#\# C1.W3 } & 0.25 & 0.04 & 0.09 & 0.02 & 0.69 & 0.78 & 0.22 & 1.3\end{array}$

$\begin{array}{lllllllll}\text { \#\# C1.W4 } & 0.31 & 0.03 & -0.01 & 0.11 & 0.59 & 0.65 & 0.35 & 1.6\end{array}$

$\begin{array}{lllllllll}\text { \#\# C1.W5 } & 0.62 & 0.08 & 0.09 & 0.23 & 0.02 & 0.65 & 0.35 & 1.4\end{array}$

$\begin{array}{lllllllll}\text { \#\# C1.W6 } & 0.48 & 0.05 & 0.13 & 0.36 & 0.03 & 0.63 & 0.37 & 2.1\end{array}$

$\begin{array}{lllllllll}\text { \#\# C1.W7 } & 0.04 & -0.02 & -0.05 & 0.93 & 0.05 & 0.87 & 0.13 & 1.0\end{array}$

$\begin{array}{llllllllll}\text { \#\# C1.W8 } & -0.07 & 0.05 & 0.03 & 0.93 & -0.07 & 0.85 & 0.15 & 1.0\end{array}$

$\begin{array}{lllllllll}\text { \#\# C1.W9 } & 0.02 & -0.08 & 0.75 & 0.17 & 0.09 & 0.70 & 0.30 & 1.1\end{array}$

$\begin{array}{lllllllll}\text { \#\# C1.W10 } & 0.20 & -0.03 & 0.47 & 0.17 & 0.21 & 0.60 & 0.40 & 2.1\end{array}$

$\begin{array}{lllllllll}\text { \#\# C1.W11 } & 0.05 & 0.11 & 0.80 & -0.01 & -0.02 & 0.73 & 0.27 & 1.0\end{array}$

$\begin{array}{llllllllll}\text { \#\# C1.W12 } & -0.07 & 0.07 & 0.83 & -0.10 & -0.06 & 0.62 & 0.38 & 1.1\end{array}$

$\begin{array}{lllllllll}\text { \#\# C1.W13 } & 0.51 & -0.03 & 0.25 & -0.02 & 0.23 & 0.58 & 0.42 & 1.9\end{array}$

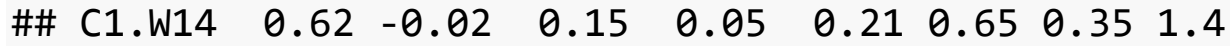

$\begin{array}{lllllllll}\text { \#\# C1.W15 } & 0.52 & -0.05 & 0.24 & 0.00 & 0.24 & 0.59 & 0.41 & 1.9\end{array}$

$\begin{array}{llllllllll}\text { \#\# C1.W16 } & 0.84 & 0.02 & -0.09 & -0.07 & -0.12 & 0.59 & 0.41 & 1.1\end{array}$

$\begin{array}{llllllllll}\text { \#\# C1.W17 } & 0.75 & 0.19 & 0.00 & 0.04 & -0.08 & 0.66 & 0.34 & 1.2\end{array}$

$\begin{array}{lllllllll}\text { \#\# C1.W18 } & -0.05 & 0.87 & -0.05 & 0.04 & 0.10 & 0.77 & 0.23 & 1.0\end{array}$

$\begin{array}{llllllllll}\text { \#\# C1.W19 } & 0.07 & 0.87 & 0.09 & 0.00 & -0.02 & 0.85 & 0.15 & 1.0\end{array}$

$\begin{array}{llllllllll}\text { \#\# C1.W20 } & 0.03 & 0.95 & -0.10 & 0.00 & -0.01 & 0.85 & 0.15 & 1.0\end{array}$

$\begin{array}{lllllllll}\text { \#\# C1.W21 } & 0.00 & 0.73 & 0.24 & 0.01 & 0.02 & 0.74 & 0.26 & 1.2\end{array}$

\#\#

\#\#

\#\# SS loadings

TC1 TC2 TC4 TC3 TC5

$\begin{array}{lllll}3.96 & 3.24 & 2.85 & 2.23 & 2.40\end{array}$ 


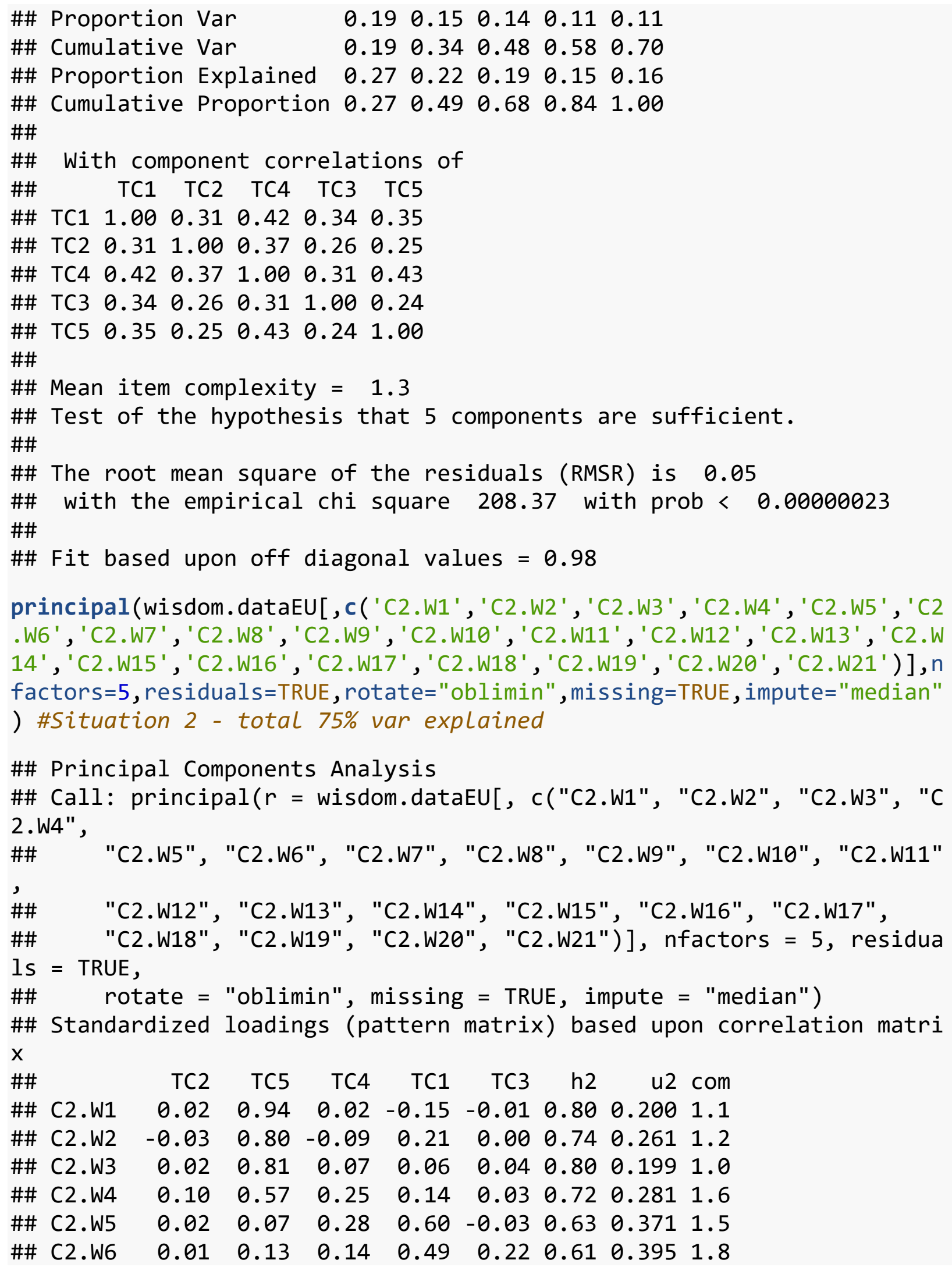




\begin{tabular}{|c|c|c|c|c|c|c|c|c|c|}
\hline \# & C2.W7 & 0.04 & 0.07 & -0.05 & 0.01 & 0.94 & & 0.084 & 1.0 \\
\hline \#\# & C2.W8 & -0.02 & -0.10 & 0.10 & 0.01 & 0.92 & 0.89 & 0.110 & 1.0 \\
\hline$\#$ & C2.W9 & 0.06 & -0.05 & 0.77 & 0.18 & 0.04 & 0.77 & 0.234 & 1.1 \\
\hline & C2.W10 & 0.02 & -0.01 & 0.77 & 0.08 & 0.07 & 0.73 & 0.274 & 1.0 \\
\hline & C2.W11 & 0.19 & 0.17 & 0.64 & -0.03 & -0.01 & 0.66 & 0.344 & 1.3 \\
\hline \# & C2.W12 & -0.04 & 0.08 & 0.80 & -0.17 & 0.10 & 0.67 & 0.331 & 1.1 \\
\hline & C2.W13 & -0.04 & 0.34 & 0.13 & 0.52 & 0.12 & 0.75 & 0.251 & 2.0 \\
\hline \#\# & C2.W14 & 0.00 & 0.23 & 0.29 & 0.49 & 0.03 & 0.70 & 0.298 & 2.1 \\
\hline \# & C2.W15 & 0.01 & 0.32 & 0.27 & 0.37 & 0.02 & 0.61 & 0.393 & 2.8 \\
\hline \# & C2.W16 & -0.01 & -0.05 & -0.07 & 0.85 & 0.01 & 0.65 & 0.350 & 1.0 \\
\hline \# & C2.W17 & 0.16 & 0.06 & -0.04 & 0.69 & 0.19 & 0.71 & 0.291 & 1.3 \\
\hline & C2.W18 & 0.88 & 0.09 & -0.13 & -0.08 & 0.11 & 0.81 & 0.188 & 1.1 \\
\hline & C2.W19 & 0.91 & 0.01 & 0.08 & 0.05 & -0.04 & 0.87 & 0.127 & 1.0 \\
\hline & C2.W20 & 0.94 & -0.04 & -0.01 & 0.00 & 0.01 & 0.86 & 0.138 & 1.0 \\
\hline$\pi$ & C2.W21 & 0.89 & -0.06 & 0.08 & 0.03 & -0.05 & 0.80 & 0.204 & 1.0 \\
\hline
\end{tabular}

\#\#

\#\#

\#\# SS loadings

TC2 TC5 TC4 TC1 TC3

\#\# Proportion Var

$\begin{array}{lllll}3.49 & 3.45 & 3.29 & 3.28 & 2.17\end{array}$

\#\# Cumulative Var

$\begin{array}{lllllll}0.17 & 0.16 & 0.16 & 0.16 & 0.10\end{array}$

\#\# Proportion Explained

\#\# Cumulative Proportion $0.220 .44 \quad 0.650 .861 .00$

\#\#

\#\# With component correlations of

\#\# $\quad$ TC2 TC5 TC4 TC1 TC3

$\begin{array}{llllll}\text { \#\# TC2 } & 1.00 & 0.25 & 0.31 & 0.19 & 0.35\end{array}$

$\begin{array}{lllllll}\text { \#\# TC5 } & 0.25 & 1.00 & 0.49 & 0.45 & 0.29\end{array}$

$\begin{array}{lllllll}\text { \#\# TC4 } & 0.31 & 0.49 & 1.00 & 0.44 & 0.44\end{array}$

$\begin{array}{lllllll}\text { \#\# TC1 } & 0.19 & 0.45 & 0.44 & 1.00 & 0.40\end{array}$

$\begin{array}{lllllll}\text { \#\# TC3 } & 0.35 & 0.29 & 0.44 & 0.40 & 1.00\end{array}$

\#\#

\#\# Mean item complexity $=1.3$

\#\# Test of the hypothesis that 5 components are sufficient.

\#\#

\#\# The root mean square of the residuals (RMSR) is 0.05

\#\# with the empirical chi square 155.21 with prob $<0.0074$

\#\#

\#\# Fit based upon off diagonal values $=0.99$

Only UK

principal(wisdom.dataUK [, c("C1.Perspective.ave", "C1. ChangeOutcomes.ave ", "C1. Limits.ave", "C1. CompResolve.ave", "C1. Outsider.ave") ] , nfactors=1, residuals=TRUE, rotate="oblimin", missing=TRUE, impute="median") \#Conflic 
t 1: Getting PCA scores (2nd order factor across means of 5 dimensions ) $163 \%$ var explained

\#\# Principal Components Analysis

\#\# Call: principal( $r=$ wisdom.dataUK [, c("C1.Perspective.ave", "C1.Cha ngeOutcomes.ave",

\#\# "C1.Limits.ave", "C1.CompResolve.ave", "C1.Outsider.ave")],

\#\# $\quad$ nfactors $=1$, residuals $=$ TRUE, rotate $=$ "oblimin", missing $=\mathrm{T}$

RUE,

\#\# $\quad$ impute $=$ "median")

\#\# Standardized loadings (pattern matrix) based upon correlation matri $x$

$\begin{array}{lrrrr}\text { \#\# } & \text { PC1 } & \text { h2 } & \text { u2 } & \text { com } \\ \text { \#\# C1.Perspective.ave } & 0.83 & 0.68 & 0.32 & 1 \\ \text { \#\# C1.ChangeOutcomes.ave } & 0.81 & 0.65 & 0.35 & 1 \\ \text { \#\# C1.Limits.ave } & 0.83 & 0.69 & 0.31 & 1 \\ \text { \#\# C1.CompResolve.ave } & 0.84 & 0.70 & 0.30 & 1 \\ \text { \#\# C1.Outsider.ave } & 0.66 & 0.44 & 0.56 & 1\end{array}$

$\# \#$

\#\# PC1

\#\# SS loadings 3.16

\#\# Proportion Var 0.63

\#\#

\#\# Mean item complexity $=1$

\#\# Test of the hypothesis that 1 component is sufficient.

\#\#

\#\# The root mean square of the residuals (RMSR) is 0.1

\#\# with the empirical chi square 29.21 with prob $<0.000021$

\#\#

$\# \#$ Fit based upon off diagonal values $=0.97$

principal(wisdom.dataUK [ , c("C2.Perspective.ave", "C2. ChangeOutcomes .ave ", "C2. Limits.ave", "C2. CompResolve.ave", "C2. Outsider.ave") ], nfactors=1, residuals=TRUE, rotate="oblimin", missing=TRUE, impute="median") \#Conflic $t$ 2: Getting PCA scores (2nd order factor across means of 5 dimensions ) / 64\% variance explained (outsider a bit weak but overall OK).

\#\# Principal Components Analysis

\#\# Call: principal( $r=$ wisdom.dataUK [, c("C2.Perspective.ave", "C2.Cha ngeOutcomes.ave",

\#\# "C2.Limits.ave", "C2.CompResolve.ave", "C2.Outsider.ave")],

\#\# $\quad$ nfactors $=1$, residuals $=$ TRUE, rotate $=$ "oblimin", missing $=\mathrm{T}$

RUE,

\#\# $\quad$ impute $=$ "median")

\#\# Standardized loadings (pattern matrix) based upon correlation matri 


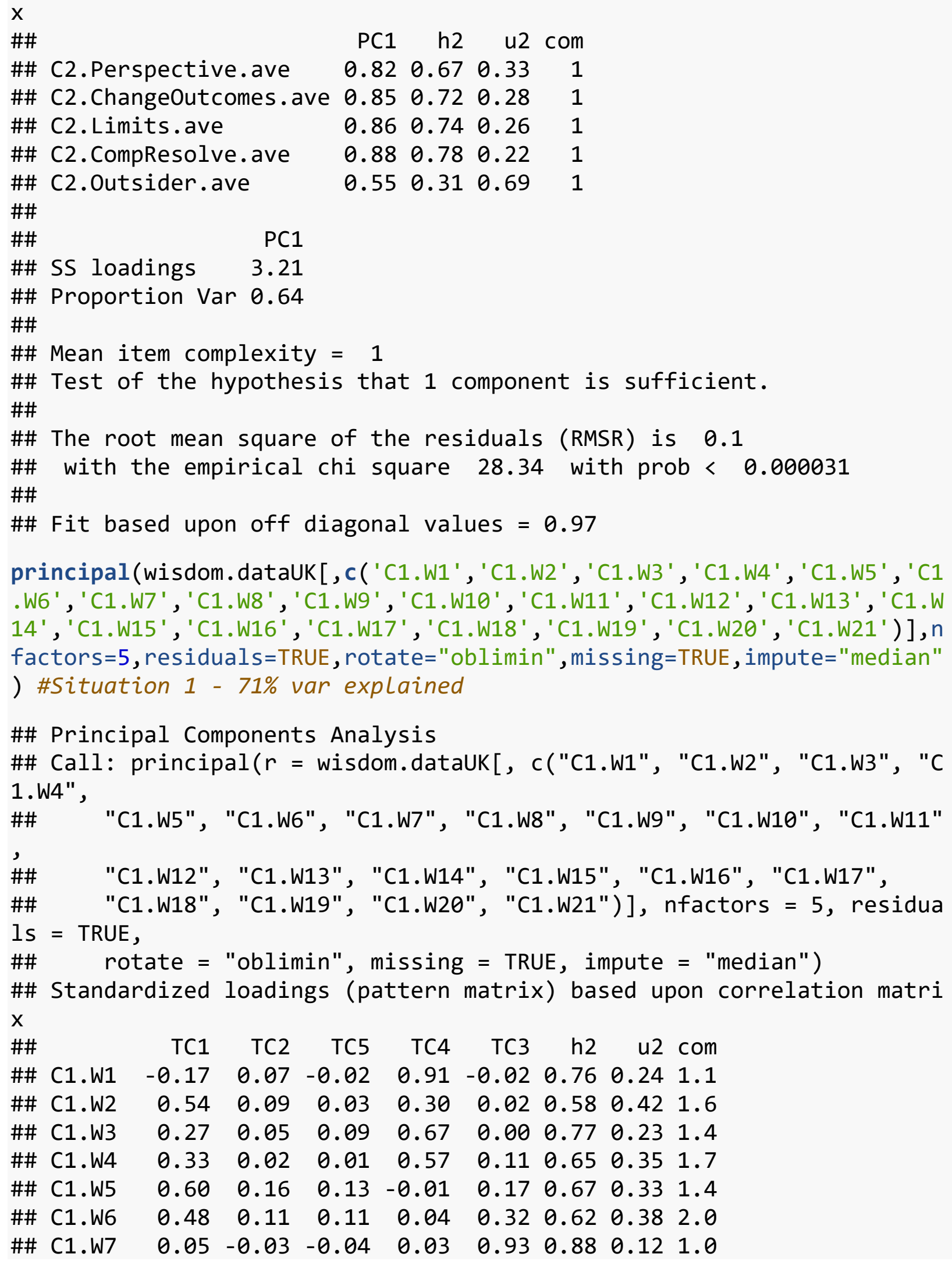




$\begin{array}{lrrrrrrrr}\text { \#\# C1.W8 } & -0.09 & 0.04 & 0.02 & -0.06 & 0.95 & 0.85 & 0.15 & 1.0 \\ \text { \#\# C1.W9 } & 0.04 & -0.06 & 0.67 & 0.15 & 0.19 & 0.68 & 0.32 & 1.3 \\ \text { \#\# C1.W10 } & 0.22 & 0.02 & 0.36 & 0.31 & 0.19 & 0.63 & 0.37 & 3.2 \\ \text { \#\# C1.W11 } & 0.07 & 0.07 & 0.81 & -0.01 & 0.00 & 0.75 & 0.25 & 1.0 \\ \text { \#\# C1.W12 } & -0.07 & 0.05 & 0.88 & -0.09 & -0.07 & 0.68 & 0.32 & 1.1 \\ \text { \#\# C1.W13 } & 0.54 & -0.04 & 0.19 & 0.24 & 0.01 & 0.59 & 0.41 & 1.7 \\ \text { \#\# C1.W14 } & 0.66 & -0.02 & 0.15 & 0.16 & 0.04 & 0.66 & 0.34 & 1.2 \\ \text { \#\# C1.W15 } & 0.56 & -0.04 & 0.17 & 0.27 & 0.02 & 0.64 & 0.36 & 1.7 \\ \text { \#\# C1.W16 } & 0.86 & -0.01 & -0.10 & -0.14 & -0.04 & 0.60 & 0.40 & 1.1 \\ \text { \#\# C1.W17 } & 0.77 & 0.16 & 0.01 & -0.09 & 0.01 & 0.66 & 0.34 & 1.1 \\ \text { \#\# C1.W18 } & -0.08 & 0.89 & -0.01 & 0.09 & 0.00 & 0.78 & 0.22 & 1.0 \\ \text { \#\# C1.W19 } & 0.07 & 0.86 & 0.09 & -0.03 & 0.00 & 0.84 & 0.16 & 1.0 \\ \text { \#\# C1.W20 } & 0.04 & 0.94 & -0.12 & -0.03 & 0.02 & 0.83 & 0.17 & 1.0 \\ \text { \#\# C1.W21 } & 0.00 & 0.72 & 0.22 & 0.05 & 0.00 & 0.72 & 0.28 & 1.2 \\ \text { \#\# } & & & & & & & & \end{array}$

\#\#

TC1 TC2 TC5 TC4 TC3

\#\# SS loadings $\quad 4.19 \quad 3.26 \quad 2.70 \quad 2.47 \quad 2.23$

$\begin{array}{lllllll}\text { \#\# Proportion Var } & 0.20 & 0.16 & 0.13 & 0.12 & 0.11\end{array}$

$\begin{array}{llllllll}\text { \# Cumulative Var } & 0.20 & 0.35 & 0.48 & 0.60 & 0.71\end{array}$

$\begin{array}{lllllll}\text { \# Proportion Explained } & 0.28 & 0.22 & 0.18 & 0.17 & 0.15\end{array}$

\#\# Cumulative Proportion $0.28 \quad 0.50 \quad 0.68 \quad 0.85 \quad 1.00$

\#\#

\#\# With component correlations of

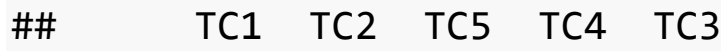

$\begin{array}{lllllll}\text { \#\# TC1 } & 1.00 & 0.33 & 0.42 & 0.35 & 0.34\end{array}$

$\begin{array}{lllllll}\text { \#\# TC2 } & 0.33 & 1.00 & 0.37 & 0.24 & 0.28\end{array}$

$\begin{array}{lllllll}\text { \#\# TC5 } & 0.42 & 0.37 & 1.00 & 0.40 & 0.32\end{array}$

$\begin{array}{lllllll}\text { \#\# TC4 } & 0.35 & 0.24 & 0.40 & 1.00 & 0.25\end{array}$

$\begin{array}{llllllll}\text { \#\# TC3 } & 0.34 & 0.28 & 0.32 & 0.25 & 1.00\end{array}$

\#\#

\#\# Mean item complexity = 1.4

\#\# Test of the hypothesis that 5 components are sufficient.

\#\#

\#\# The root mean square of the residuals (RMSR) is 0.05

\#\# with the empirical chi square 190.51 with prob $<0.000012$

\#\#

\#\# Fit based upon off diagonal values $=0.98$

principal(wisdom.dataUK [ , c('C2.W1' , 'C2.W2' , 'C2.W3 ', 'C2.W4 ' , 'C2.W5 ' , 'C2 .W6 ', 'C2.W7' , 'C2.W8 ', 'C2.W9' , 'C2.W10', 'C2.W11 ' , 'C2.W12 ' , 'C2.W13 ', 'C2.W 14 ', 'C2.W15 ', 'C2.W16' , 'C2.W17' , 'C2.W18 ', 'C2.W19 ', 'C2.W20 ', 'C2.W21 ' ) ], n factors =5, residuals=TRUE, rotate="oblimin", missing=TRUE, impute="median" ) \#Situation 2 - total 73\% var explained 
\#\# Principal Components Analysis

\#\# Call: principal( $r=$ wisdom.dataUK [, c("C2.W1", "C2.W2", "C2.W3", "C 2.W4",

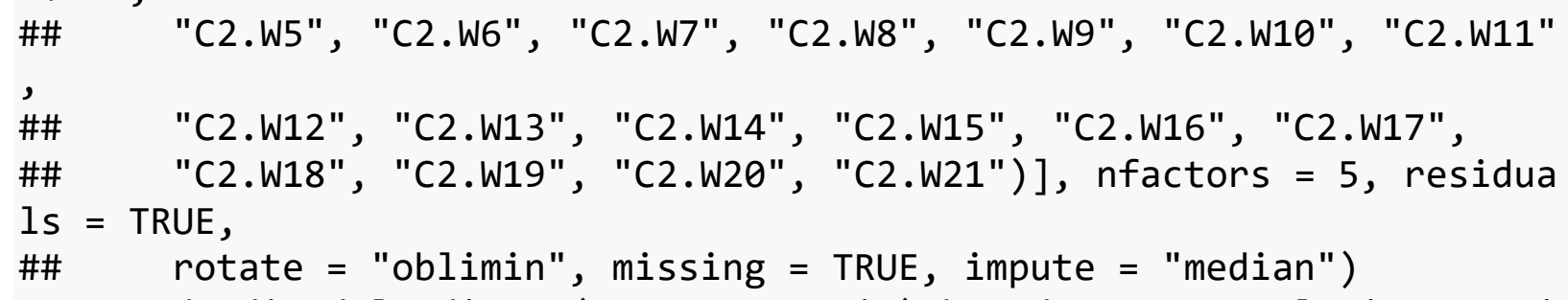




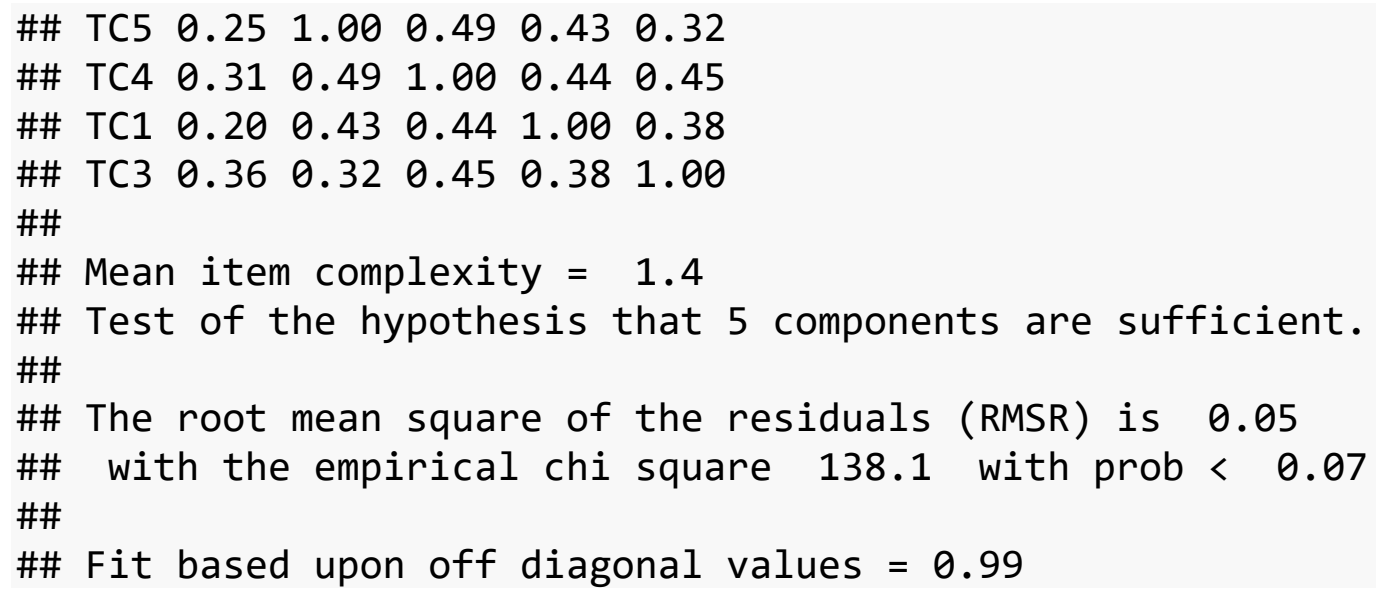

CONCLUSION: if anything - the effect is even stronger in Europe (UK being the main driver) than in the US - Factor structure holds well.

\#create trait-wisdom score

WIS.PA\$trait.wisdom. ave<-apply(WIS.PA[, c ('C1.wisdom.ave ', 'C2.wisdom.av $\left.\left.\mathrm{e}^{\prime}\right)\right], 1$, mean, na.rm=TRUE)

WIS.PA\$state.wisdom.1<- WIS.PA\$C1.wisdom.ave - WIS.PA $\$$ trait.wisdom.ave WIS.PA\$state.wisdom.2<- WIS.PA\$C2.wisdom.ave - WIS.PA\$trait.wisdom.av e

\section{INFLUENCE/ADJUSTMENT RELIABILITY}

psych: :alpha(WIS.PA[, c('C1.IA1', 'C1.IA2', 'C1.IA3', 'C1.IA4')], check.ke ys=FALSE)[1] \#Conflict 1: Influence

\#\# \$total

\#\# raw_alpha std.alpha G6(smc) average_r S/N ase me

an

$\begin{array}{lllllllll}\text { \#\# } & 0.8173736 & 0.8207841 & 0.7906919 & 0.533792 & 4.579861 & 0.01743765 & 4.1288\end{array}$

89

\#\# sd

\#\# 1.157958

psych: :alpha(WIS.PA[, c( 'C1.IA5 ', 'C1.IA6', 'C1.IA7' , 'C1.IA8 ', 'C1.IA9 ')], check.keys=FALSE)[1] \#Conflict 1: Adjustment

\#\# \$total

\#\# raw_alpha std.alpha G6(smc) average_r S/N ase me

an

$\begin{array}{lllllllll}\text { \#\# } & 0.783212 & 0.7867184 & 0.7998211 & 0.4245357 & 3.688637 & 0.02025972 & 2.3046\end{array}$

67

\#\# sd

\#\# 1.040739 
psych: :alpha(WIS.PA[, c('C2.IA1', 'C2.IA2', 'C2.IA3', 'C2.IA4')], check.ke ys=FALSE)[1] \#Conflict 2: Influence

\#\# \$total

\#\# raw_alpha std.alpha $\mathrm{G6}(\mathrm{smc})$ average_r $\mathrm{S} / \mathrm{N}$ ase me

an

$\begin{array}{lllllllll}\text { \#\# } & 0.8177406 & 0.8187137 & 0.7972015 & 0.5303034 & 4.516135 & 0.01716425 & 4.0245\end{array}$

26

\#\# sd

\#\# 1.216996

psych: :alpha(WIS.PA[, c('C2.IA5', 'C2.IA6', 'C2.IA7', 'C2.IA8', 'C2.IA9' )], check.keys=FALSE)[1] \#Conflict 2: Adjustment

\#\# \$total

\#\# raw_alpha std.alpha G6(smc) average_r S/N ase mean

$\begin{array}{lllllllll}\text { \#\# } & 0.8522402 & 0.8531893 & 0.87017 & 0.5375291 & 5.811491 & 0.01388888 & 2.334281\end{array}$

\#\# $\quad$ sd

\#\# 1.195196

\section{INFLUENCE/ADJUSTMENT COMPOSITE}

WIS. PA\$C1. Influence<-apply(WIS.PA[, c ('C1.IA1' , 'C1.IA2' , 'C1.IA3 ', 'C1. IA $\left.4^{\prime}\right)$ ], 1, mean, na.rm=TRUE) \#Conflict 1: Influence

WIS.PA\$C1.Adjustment<-apply(WIS.PA[, c( 'C1.IA5 ', 'C1.IA6', 'C1.IA7' , 'C1. I A8', 'C1.IA9')], 1, mean, na.rm=TRUE) \#Conflict 1: Adjustment

WIS. PA\$C2. Influence<-apply(WIS.PA [, c( 'C2. IA1' , 'C2. IA2' , 'C2. IA3 ' , 'C2. IA $\left.4^{\prime}\right)$ ], 1, mean, na.rm=TRUE) \#Conflict 2: Influence

WIS.PA\$C2.Adjustment<-apply(WIS.PA [, c( 'C2.IA5 ', 'C2.IA6 ' , 'C2. IA7' , 'C2. I A8', 'C2.IA9')], 1, mean, na.rm=TRUE) \#Conflict 2: Adjustment

\section{GLUCK WISDOM PCA}

GW<-na.omit(WIS.PA[, c( 'GW1', 'GW2 ', 'GW3' ', 'GW4' , 'GW5 ' , 'GW6r' , 'GW7 ' , 'GW8 ' , 'GW9' , 'GW10', 'GW11 ', 'GW12', 'GW13' , 'GW14r' , 'GW15 ' , 'GW16 ', 'GW17 ' , 'GW18 ' , 'GW19' , 'GW20r', ' GW21' ) ])

ev <- eigen(cor(GW)) \#Getting eigenvalues - 5 eigen values greater tha n 1

ap <- parallel (subject=nrow(GW), $\operatorname{var}=n \operatorname{col}(\mathrm{GW}), \mathrm{rep}=100$, cent=.05)

nS <- nScree(x=ev\$values, aparallel=ap\$eigen\$qevpea)

plotnscree(nS) \#Scree plot 


\section{Non Graphical Solutions to Scree Test}

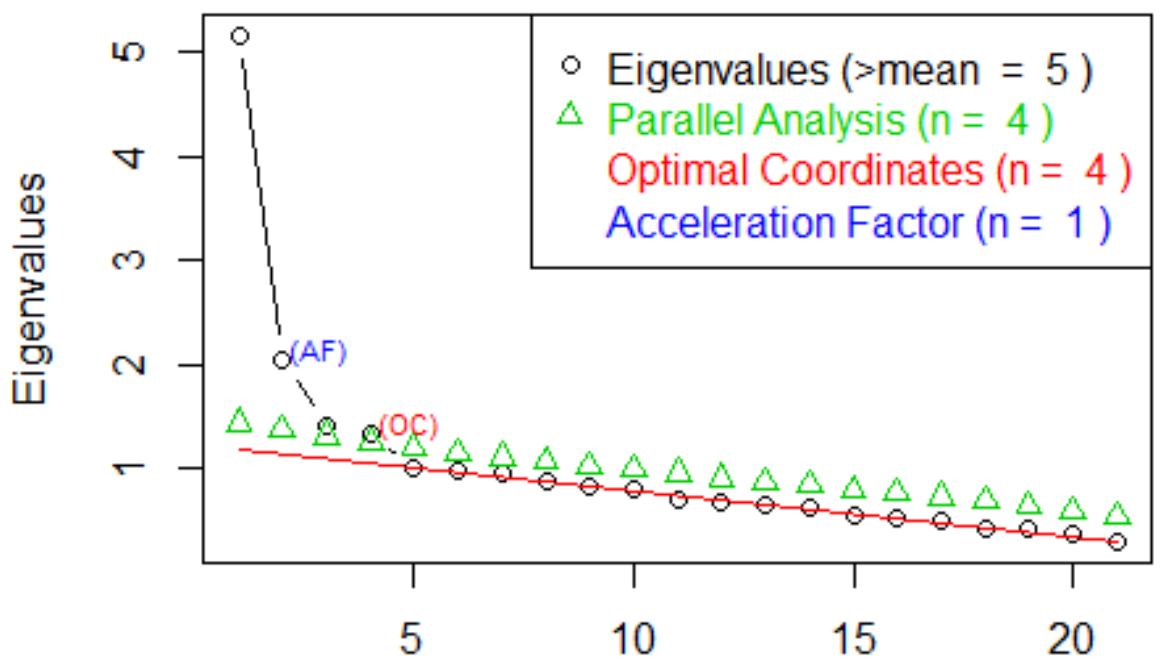

Components

principal(WIS.PA[, c( 'GW1 ', 'GW2 ', 'GW3 ' , 'GW4 ' , 'GW5 ' , 'GW6r ' , 'GW7 ' , 'GW8 ' , GW9 ' , 'GW10 ', 'GW11 ', 'GW12 ' , 'GW13 ' , 'GW14r ', 'GW15 ' , 'GW16 ' , 'GW17 ' , 'GW18 ' , GW19 ', 'GW20r' , 'GW21 ')], nfactors=4, residuals=TRUE, rotate="oblimin", miss ing=TRUE, impute="median") \#GLuck Wisdom PCA with 5 factors

\#\# Principal Components Analysis

\#\# Call: principal(r = WIS.PA[, c("GW1", "GW2", "GW3", "GW4", "GW5", " GW6r",

\#\# "GW15", "GW16", "GW17", "GW18", "GW19", "GW20r", "GW21")],

\#\# nfactors $=4$, residuals $=$ TRUE, rotate $=$ "oblimin", missing $=\mathrm{T}$

RUE,

\#\# $\quad$ impute $=$ "median")

\#\# Standardized loadings (pattern matrix) based upon correlation matri $\mathrm{x}$

$\begin{array}{lllllll}\# \# & \text { TC1 } & \text { TC2 } & \text { TC4 } & \text { TC3 } & \text { h2 } & \text { u2 com }\end{array}$

$\begin{array}{llllllll}\text { \#\# GW1 } & 0.53 & 0.34 & 0.05 & -0.22 & 0.55 & 0.45 & 2.1\end{array}$

$\begin{array}{llllllll}\text { \#\# GW2 } & 0.70 & -0.13 & -0.11 & 0.15 & 0.50 & 0.50 & 1.2\end{array}$

$\begin{array}{lllllllll}\text { \#\# GW3 } & 0.42 & 0.27 & 0.01 & -0.01 & 0.32 & 0.68 & 1.7\end{array}$

$\begin{array}{llllllll}\text { \#\# GW4 } & 0.62 & 0.06 & 0.31 & -0.23 & 0.57 & 0.43 & 1.8\end{array}$

$\begin{array}{llllllll}\text { \#\# GW5 } & 0.19 & 0.46 & 0.08 & 0.14 & 0.36 & 0.64 & 1.6\end{array}$

$\begin{array}{lllllllll}\text { \#\# GW6r } & 0.21 & 0.10 & 0.72 & -0.16 & 0.66 & 0.34 & 1.3\end{array}$

$\begin{array}{lllllllll}\text { \#\# GW7 } & 0.46 & 0.21 & -0.13 & 0.17 & 0.36 & 0.64 & 1.9\end{array}$ 


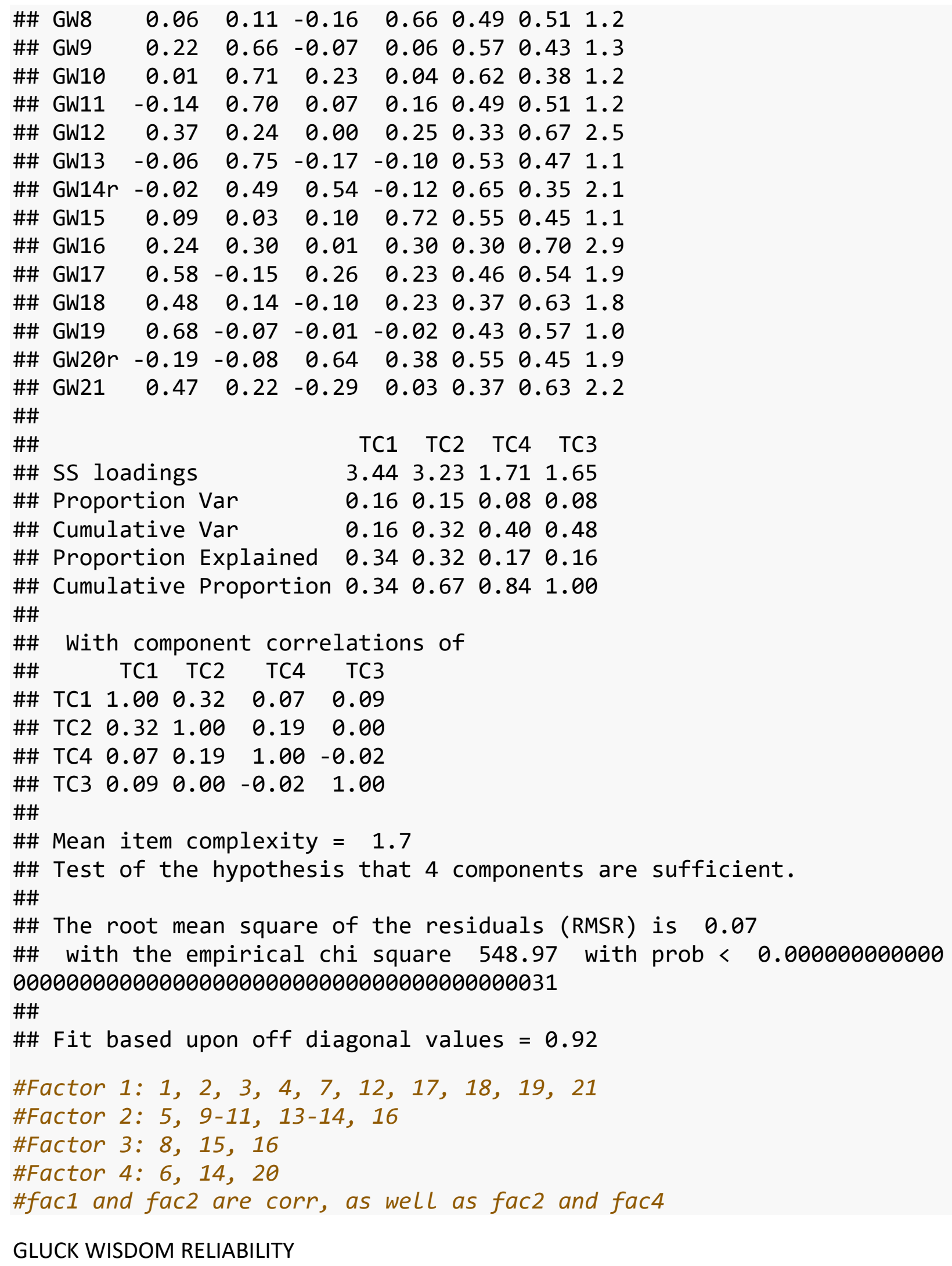


psych: :alpha(WIS.PA[ , c( 'GW1' , 'GW2' , 'GW3' , 'GW4' ', 'GW5' , 'GW6r' , 'GW7' ', 'GW8 ', 'GW9 ', 'GW10' , 'GW11', 'GW12' , 'GW13' , 'GW14r' , 'GW15 ', 'GW16 ' , 'GW17' , 'GW18 ', 'GW19', 'GW20r', 'GW21')], check.keys=FALSE) [1] \#GLuck Wisdom

\#\# \$total

\#\# raw_alpha std.alpha G6(smc) average_r S/N ase me

an

$\begin{array}{lllllllll}\text { \#\# } & 0.8285165 & 0.8378854 & 0.8638427 & 0.1975077 & 5.168476 & 0.01424199 & 3.4790\end{array}$

59

\#\# sd

\#\# $\quad 0.4862852$

psych: : alpha(wisdom.dataNA[ , c('GW1', 'GW2' , 'GW3' , 'GW4' , 'GW5' , 'GW6r' , 'GW 7', 'GW8' , 'GW9' , 'GW10', 'GW11', 'GW12' , 'GW13' , 'GW14r' , 'GW15 ' , 'GW16' , ' GW17 ', 'GW18', 'GW19', 'GW20r' , 'GW21')], check.keys=FALSE)[1] \#GLuck Wisdom N orth America

\#\# \$total

\#\# raw_alpha std.alpha G6(smc) average_r S/N ase mean

$\begin{array}{lllllllll}\text { \#\# } & 0.8531681 & 0.8573196 & 0.89566 & 0.2224719 & 6.008669 & 0.01833093 & 3.541839\end{array}$

\#\# sd

\#\# $\quad 0.5272197$

psych: :alpha(wisdom.dataEU[, c( 'GW1', 'GW2 ', 'GW3 ', 'GW4 ', 'GW5 ', 'GW6r ' , 'GW 7', 'GW8 ', 'GW9' , 'GW10 ', 'GW11' , 'GW12 ', 'GW13 ' , 'GW14r ', 'GW15 ' , 'GW16 ', ' GW17 ', 'GW18', 'GW19', 'GW20r', 'GW21')], check.keys=FALSE)[1] \#GLuck Wisdom E urope - slightly Lower than in North America, but both are alpha >.80

\#\# \$total

\#\# raw_alpha std.alpha G6(smc) average_r S/N ase me

an

$\begin{array}{lllllllll}\text { \#\# } & 0.8002876 & 0.8154609 & 0.8535537 & 0.1738433 & 4.418906 & 0.02214016 & 3.4320\end{array}$

66

\#\# sd

\#\# 0.4490902

psych: :alpha(wisdom.dataUK[ , c( 'GW1', 'GW2' , 'GW3 ', 'GW4 ', 'GW5 ', 'GW6r ' , 'GW 7' , 'GW8 ', 'GW9 ', 'GW10 ', 'GW11' , 'GW12 ', 'GW13 ' , 'GW14r ' , 'GW15 ' , 'GW16 ', 'GW17 ', 'GW18', 'GW19', 'GW20r' ', 'GW21')], check.keys=FALSE)[1] \#GLuck Wisdom U $K$ - slightly Lower than in North America, but both are alpha > .80

\#\# \$total

\#\# raw_alpha std.alpha G6(smc) average_r S/N ase mea $\mathrm{n}$ $\begin{array}{lllllllll}\text { \#\# } & 0.8040482 & 0.8190992 & 0.8583076 & 0.1773703 & 4.52789 & 0.02282354 & 3.44655\end{array}$ 9 


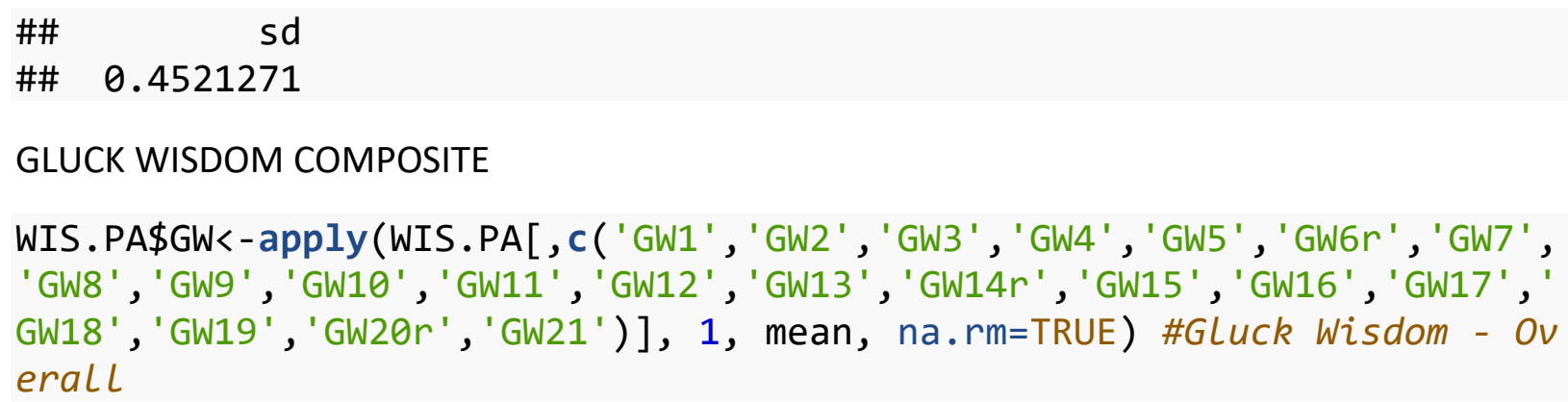




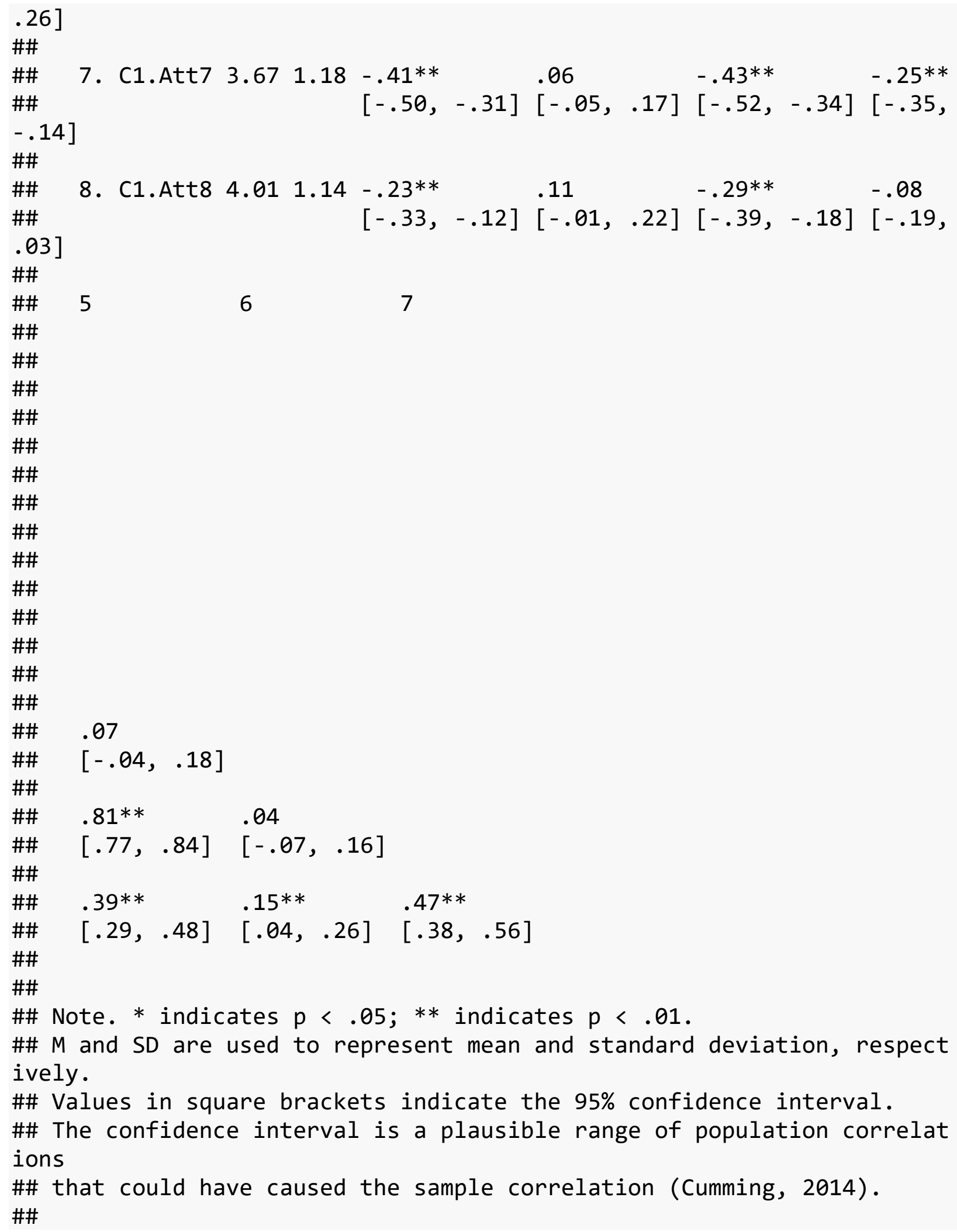


apa.cor.table(as.matrix(attrib.matrix2)) \#regret - "could have been $m$ ore wise" - well correlated with respective self vs. other-focused att ributions

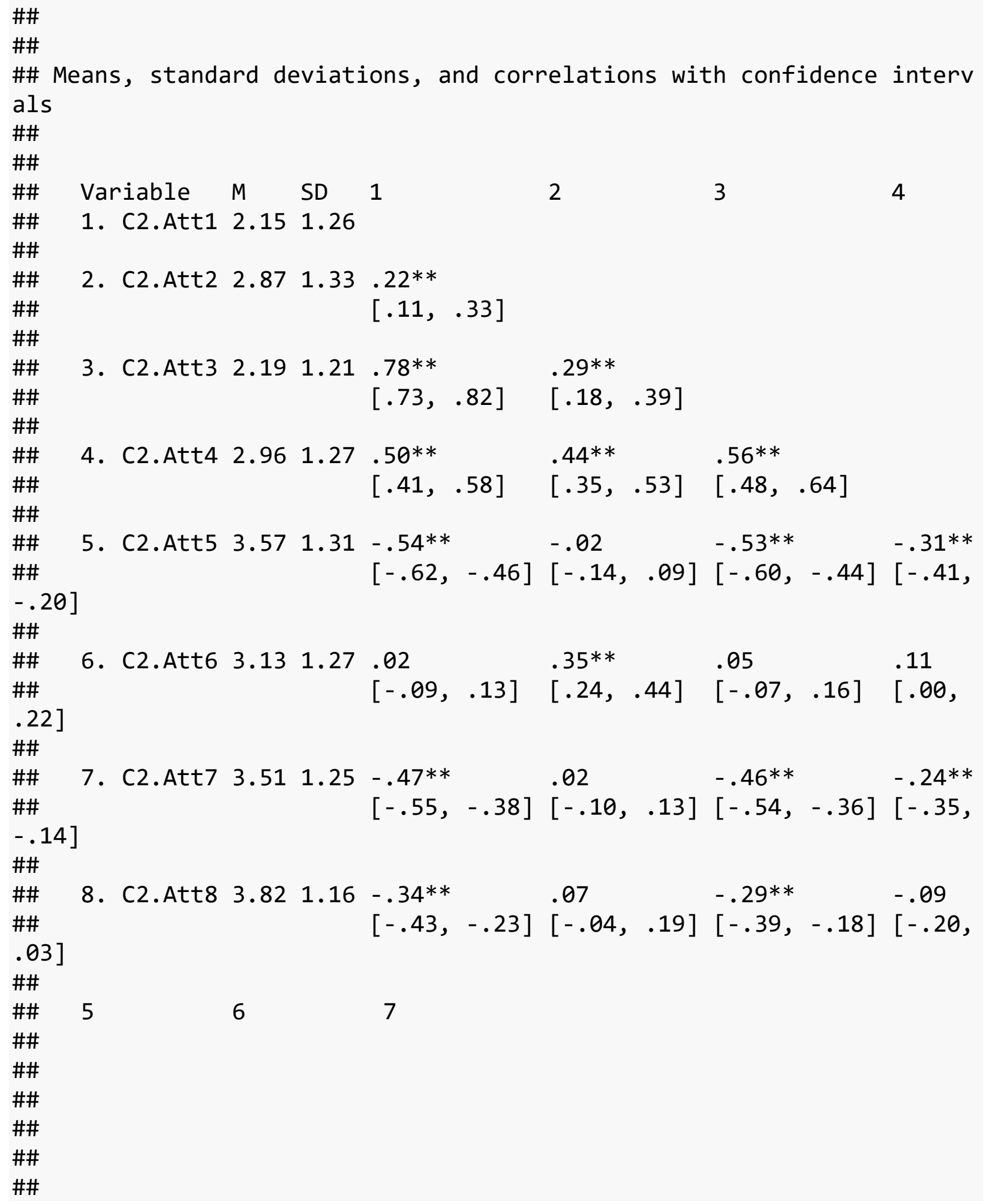




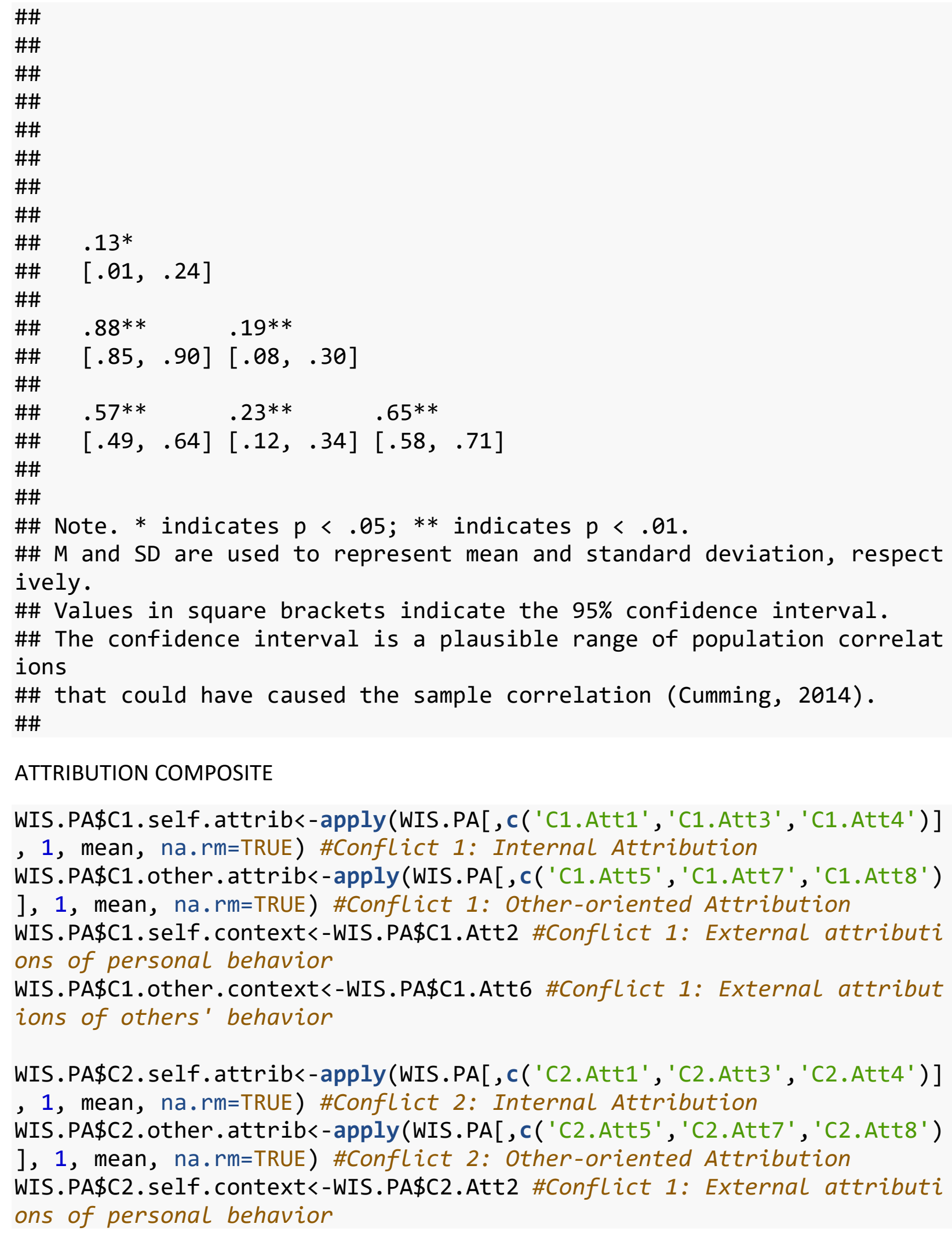


WIS.PA\$C2. other. context<-WIS.PA\$C2. Att6 \#Conflict 1: External attribut ions of others' behavior

Create balance scores using the Thompson, Griffin, and Zanna attitude ambivalence formula $(\mathrm{P}+\mathrm{N}) / 2-|\mathrm{P}-\mathrm{N}|$

WIS.PA\$C1. internal. attrib.balance $=$ (WIS.PA\$C1. self. attrib+WIS.PA $\$ C 1.0$ ther.attrib)/2 - abs(WIS.PA\$C1.self.attrib-WIS.PA\$C1.other.attrib) WIS.PA\$C2. internal. attrib.balance $=$ (WIS. PA\$C2. self. attrib+WIS. PA\$C2.0 ther.attrib)/2 - abs(WIS.PA\$C2.self.attrib-WIS.PA\$C2.other.attrib)

WIS.PA\$C1. goals. balance $=($ WIS.PA $\$ C 1$. Influence $+W I S \cdot P A \$ C 1$. Adjustment $) / 2$ - abs(WIS.PA\$C1. Influence-WIS.PA\$C1.Adjustment)

WIS. $P A \$ C 2$. goals . balance $=($ WIS. $P A \$ C 2$. Influence $+W I S . P A \$ C 2$. Adjustment $) / 2$ - abs(WIS.PA\$C2. Influence-WIS.PA\$C2. Adjustment)

descriptives of relevant scores goals

apa.cor.table(as.matrix(WIS.PA[, c ('C1.Influence ', 'C1.Adjustment' , 'C1.g oals.balance',

oals.balance')]))

'C2. Influence' , 'C2. Adjustment' , 'C2.g

\#\#

\#\#

\#\# Means, standard deviations, and correlations with confidence interv als

\#\#

\#\#

\#\#

Variable M SD 1 2 3

\#\#
1. C1.Influence
4.131 .16

\#\#

\#\#

\#\#

2. C1.Adjustment

$2.301 .04-.04$

\#\#

\#\#

\#\#

\#\#

\#\#

\#\#

3]

\#\#

\#\#

\#\#

\#\#

3. C1.goals balance

$1.181 .43-.22 * *$

$[-.33,-.11][.85, .90]$

4. C2.Influence

$4.02 \quad 1.22 \quad .50^{* *}$

$$
[.41, .58]
$$

$-.00$

$-.15 *$

5. C2.Adjustment

$2.331 .20 .12 *$

$[.01, .23]$

$.37 * *$

\#\#

6. C2.goals.balance

1.241 .57 .00

$.42 * *$

$.42 * *$ 


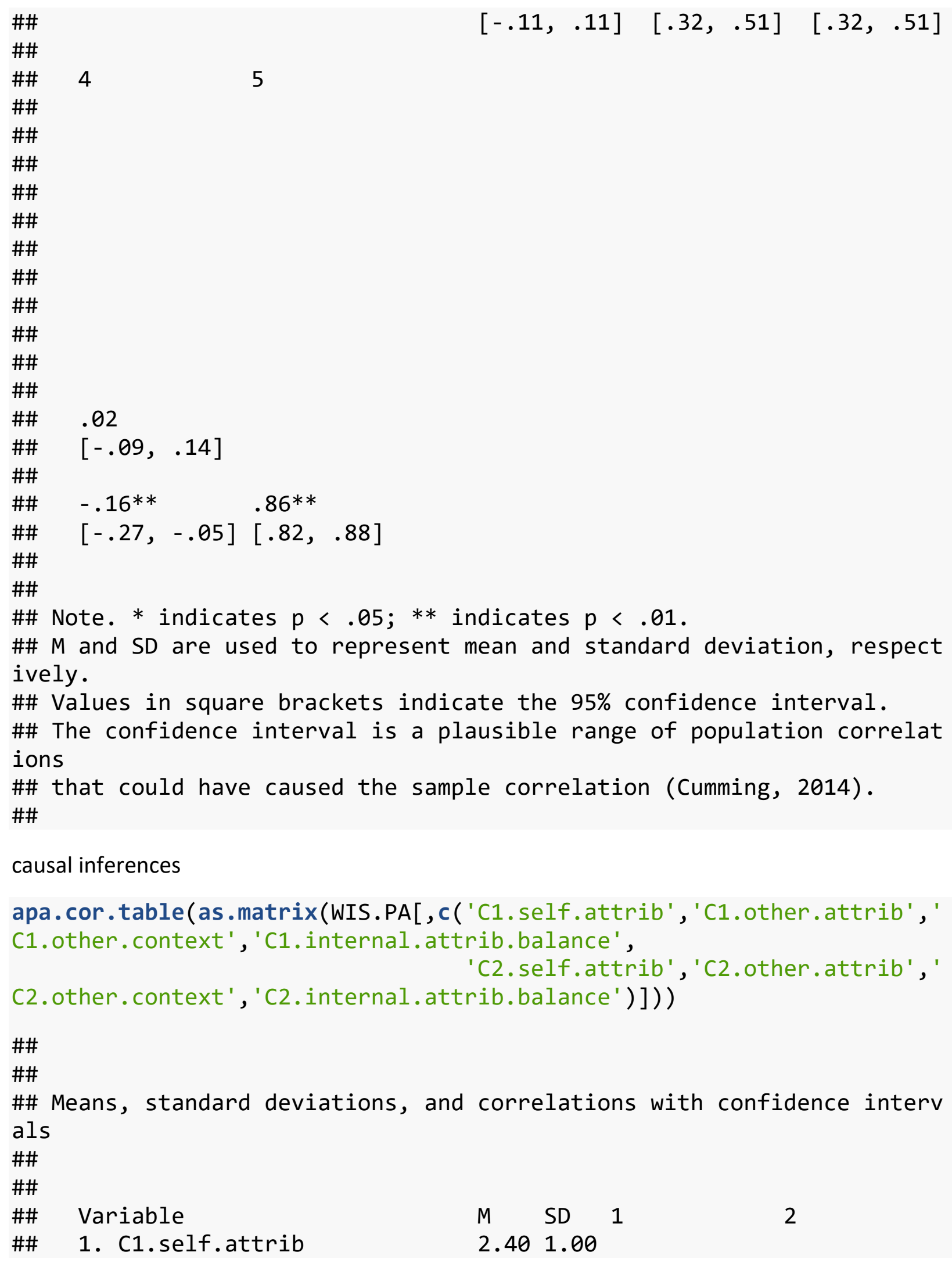




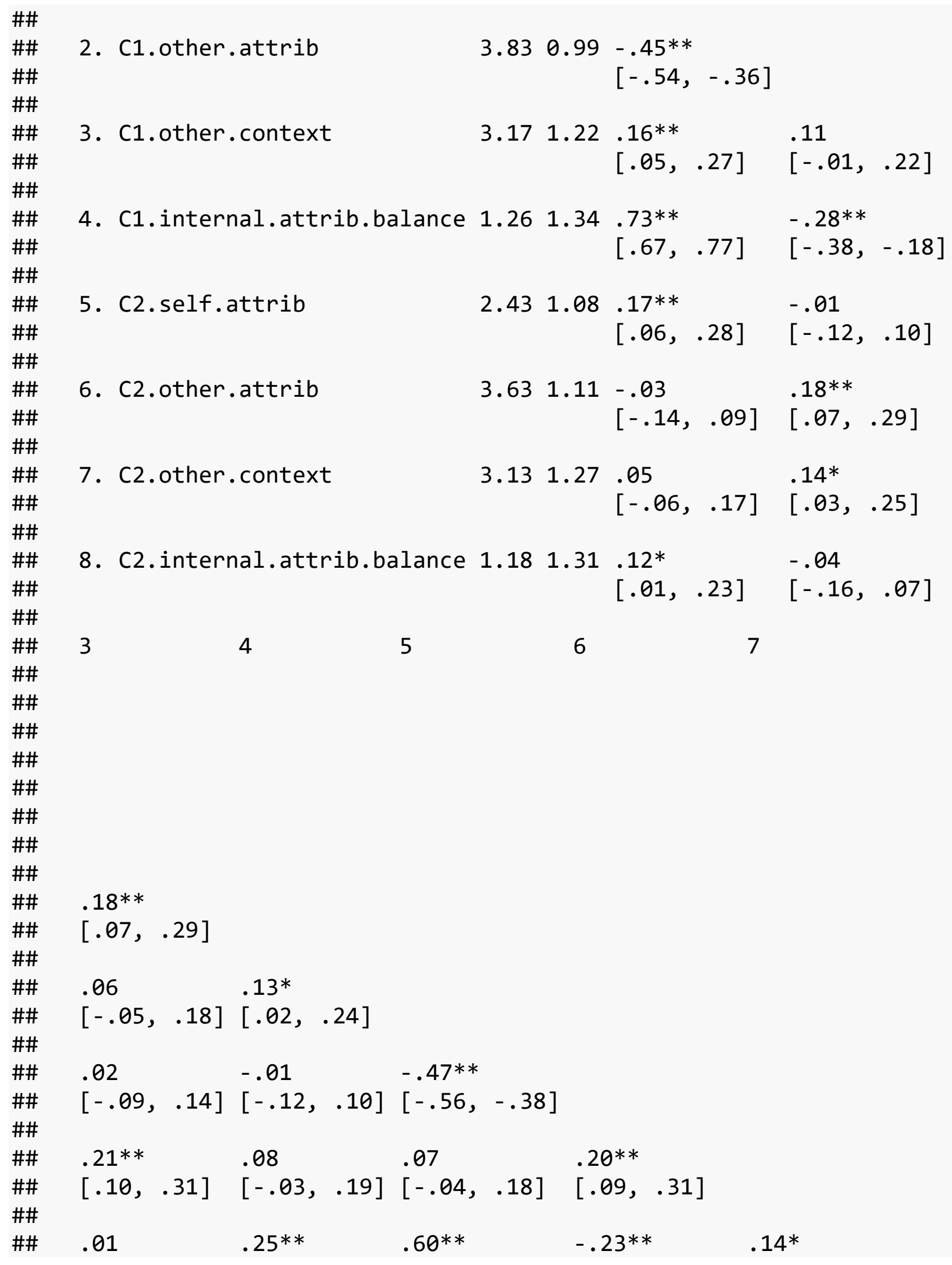




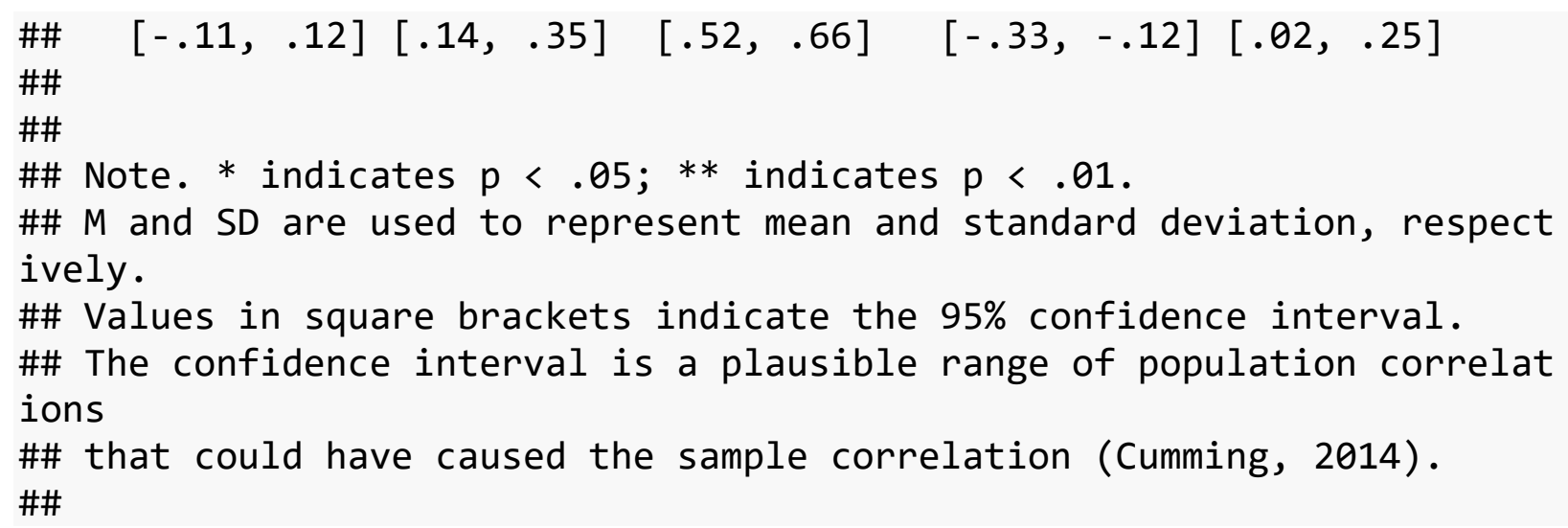

Prepare data for multi-level analyses prepare matrixes and create long-format datafile for MLM analyses

wisdom.matrix=WIS.PA[ , c( 'GW', 'trait.wisdom.ave ', 'C1.wisdom.ave ', 'C2.wi sdom.ave')]

state1.matrix=WIS.PA[, c( 'GW', 'C1.wisdom.ave ', 'C1.goals.balance ', 'C1. In fluence', 'C1.Adjustment ', 'C1.internal.attrib.balance ', 'C1.self.attrib' , 'C1.other.attrib', 'C1.other.context ')]

state2.matrix=WIS.PA[, c( 'GW', 'C2.wisdom.ave ', 'C2.goals.balance ', 'C2. In fluence ', 'C2.Adjustment ', 'C2.internal.attrib.balance ', 'C2.self.attrib' , 'C2.other.attrib', 'C2.other.context')]

tolong.data = WIS.PA[, c('V1', 'GW', 'trait.wisdom.ave', 'C1.wisdom.ave ', ' C1. Influence', 'C2. Influence', 'C1.Adjustment ', 'C2.Adjustment ', 'C1.goals .balance ', 'C1.self.attrib', 'C2.self.attrib', 'C1.other.attrib', 'C2.othe r.attrib', 'C1.internal.attrib.balance ', 'C1.other.context ', 'C2.wisdom.a ve', 'c2.goals.balance ', 'C2.internal.attrib.balance ', 'c2.other.context ' )]

\#convert to long format for within-subject analyses goal.balance.long = gather(tolong.data, type, goal.balance, C1.goals.b alance, C2.goals.balance, factor_key=TRUE) attribution.balance.long = gather(tolong.data, type, attr.balance, C1. internal.attrib.balance, C2.internal.attrib.balance, factor_key=TRUE) goal.influence.long = gather(tolong.data, type, goal.influence, C1.Inf luence, C2. Influence, factor_key=TRUE)

goal.adjustment.long = gather(tolong.data, type, goal.adjustment, C1.A djustment, C2. Adjustment, factor_key=TRUE)

attribution.self.long = gather(tolong.data, type, attr.self, C1.self.a ttrib,c2.self.attrib, factor_key=TRUE)

attribution.other.long = gather(tolong.data, type, attr.other, $\mathrm{C} 1$.othe r.attrib, C2.other.attrib, factor_key=TRUE)

attribution.othercontext.long = gather(tolong.data, type, attr.otherco ntext, C1.other. context, C2.other. context) 
\#calculate state-level variations in wisdom (from the aggregate trait) goal.balance. long\$state. wisdom <- ifelse (goal.balance. long \$type=="C1.g oals.balance", goal.balance.long\$C1.wisdom.ave - goal.balance.long\$tra it.wisdom.ave, goal.balance.long $\$ C 2$. wisdom.ave - goal.balance.long $\$$ tra it.wisdom.ave)

attribution.balance.long\$state.wisdom <- ifelse(attribution.balance. 10 ng\$type $==" C 1$.internal.attrib.balance", attribution.balance.long $\$ C 1$.wis dom.ave - attribution.balance.long \$trait.wisdom.ave, attribution.balan ce.long $\$ C 2$.wisdom.ave - attribution.balance.long $\$$ trait.wisdom.ave) attribution.othercontext.long\$state.wisdom <- ifelse(attribution.other context. long $\$$ type $==" \mathrm{C} 1$.other . context", attribution.othercontext.long $\$ C$ 1.wisdom.ave - attribution.othercontext.long \$trait.wisdom.ave, attribu tion.othercontext.long $\$ C 2$.wisdom.ave - attribution.othercontext.long $\$ t$ rait.wisdom.ave)

goal.influence. long\$state. wisdom <- ifelse (goal.influence. long $\$$ type==" C1.Influence", goal.influence.long $\$ C 1$.wisdom.ave - goal.influence.long \$trait.wisdom.ave, goal.influence.long $\$ C 2$.wisdom.ave - goal.influence. long\$trait.wisdom.ave)

goal.adjustment. long\$state. wisdom <- ifelse (goal.adjustment.long $\$$ type= ="C1.Adjustment", goal.adjustment.long $\$ C 1$.wisdom.ave - goal.adjustment .long\$trait.wisdom.ave, goal.adjustment.long $\$ C 2$.wisdom.ave - goal.adju stment. long\$trait.wisdom.ave)

attribution.self.long\$state.wisdom <- ifelse(attribution.self.long $\$$ typ $\mathrm{e}==" C 1$.self.attrib", attribution.self.long $\$ C 1$.wisdom.ave - attribution .self.long\$trait.wisdom.ave, attribution.self.long $\$ C 2$.wisdom.ave - att ribution.self.long\$trait.wisdom.ave)

attribution.other. long\$state.wisdom <- ifelse(attribution.other. long $\$ t$ ype=="C1.other.attrib", attribution.other.long $\$ C 1$.wisdom.ave - attribu tion.other.long $\$$ trait.wisdom.ave, attribution.other. long $\$ C 2$. wisdom.ave - attribution.other.long\$trait.wisdom.ave)

\#calcualate degree of closeness in situation

WIS.PA\$C1.CloseGroup<-WIS. PA\$C1.Person

WIS.PA\$C2.CloseGroup<-NA

WIS. PA\$C2. CloseGroup<-ifelse (is . na (WIS. PA\$C2. CloseGroup)\&WIS. PA\$C2. Con flictPartner $==1$, "romantic partner",WIS.PA\$C2.CloseGroup)

WIS. PA\$C2. CloseGroup<-ifelse (is . na (WIS. PA\$C2 . CloseGroup)\&WIS. PA\$C2. Con flictFamily==1, "family member", WIS.PA\$C2.CloseGroup)

WIS. PA\$C2. CloseGroup<-ifelse (is . na (WIS. PA\$C2. CloseGroup)\&WIS. PA\$C2. Con flictFriend==1, "friend", WIS.PA\$C2. CloseGroup) 
WIS. PA\$C2.CloseGroup<-ifelse (is . na (WIS. PA\$C2. CloseGroup)\&WIS. PA $\$ C 2$. Con flictCoworker ==1, "co-worker", WIS.PA\$C2.CloseGroup)

\section{DEMOGRAPHICS DESCRIPTIVES}

describe(WIS.PA[, c( 'Age' , 'Gender', 'Religion' , 'Country', 'Language ', 'Eth nicity', 'Education', 'Employment')])

\#\# WIS.PA[, c("Age", "Gender", "Religion", "Country", "Language", "Eth nicity", "Education", "Employment")]

\#\#

\#\# 8 Variables $\quad 300$ Observations

\#\# - - - - -

\#\# Age

\#\# $\mathrm{n}$ missing distinct Info Mean Gmd

.10

\#\#

300

$0 \quad 50$

0.999

37.6

12.51

23.95

2

5.00

$\begin{array}{lrrrrr}\# \# & .25 & .50 & .75 & .90 & .95\end{array}$

$\begin{array}{llllll}\text { \#\# } & 29.00 & 35.00 & 45.00 & 54.00 & 60.00\end{array}$

\#\#

\#\# lowest : 18192021 22, highest: 6466686970

\#\#

\#\# Gender

$\begin{array}{rrrr}\# \# & \mathrm{n} & \text { missing } & \text { distinct } \\ \# \# & 300 & 0 & 3\end{array}$

\#\#

\#\# Value Female Male Other

$\begin{array}{llll}\text { \#\# Frequency } & 166 & 132 & 2\end{array}$

$\begin{array}{llll}\text { \#\# Proportion } 0.553 & 0.440 & 0.007\end{array}$

\#\# ---- -

\#\# Religion

\#\# $\quad n$ missing distinct

\#\# $299 \quad 1 \quad 20$

\#\#

\#\# Agnostic (15, 0.050), Atheist (139, 0.465), Baptist (4, 0.013), Bud dhism

\#\# (1,0.003), Buddhist (3,0.010), Catholic (19,0.064), Christian (6 7,

\#\# 0.224$)$, Jewish $(2,0.007)$, Lutheran $(3,0.010)$, Methodist $(3,0.010$ ) ,

\#\# Mormon (2, 0.007), Muslim (10,0.033), no answer $(14,0.047)$, Ortho 
$\operatorname{dox}(2$, \#\# 0.007), Pagan $(4,0.013)$, Protestant $(4,0.013)$, refused $(2,0.007)$ ,

\#\# Spiritual (3, 0.010$)$, Unificationist $(1,0.003)$, Zen $(1,0.003)$

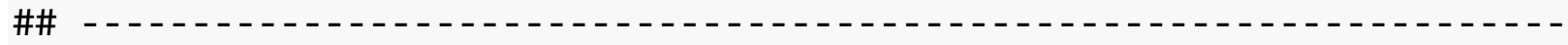
$--1---$

\#\# Country

\#\# $\quad n$ missing distinct

$\begin{array}{llll}\# \# & 300 & 0 & 14\end{array}$

\#\#

\#\# Australia (5, 0.017), Canada (13, 0.043), England (53, 0.177), Germ any $(3$, \#\# 0.010$)$, Hong Kong $(1,0.003)$, Ireland $(2,0.007)$, israel $(1,0.003)$ \#\# Mexico (2, 0.007), Scotland $(11,0.037)$, Spain $(4,0.013)$, Sweden ( 1 ,

\#\# 0.003$)$, The Netherlands $(1,0.003)$, UK $(89,0.297)$, US $(114,0.380)$

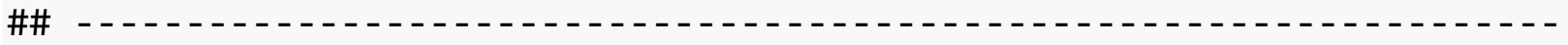

\#\# Language

\#\# $\quad \mathrm{n}$ missing distinct

\#\# $300 \quad 0 \quad 2$

\#\#

\#\# Value English Other

\#\# Frequency $\quad 269 \quad 31$

$\begin{array}{lll}\text { \#\# Proportion } 0.897 & 0.103\end{array}$

\#\# -

\#\# Ethnicity

$\begin{array}{rrr}\# \# & \mathrm{n} & \text { missing distinct } \\ \# \# & 300 & 0\end{array}$

\#\#

\#\# Value

ern

\#\# Frequency

2

$\begin{array}{lllll}\text { \#\# Proportion } & 0.033 & 0.043 & 0.047 & 0 .\end{array}$

007

\#\#

\#\# Value

\#\# Frequency

\#\# Proportion

Other South Asian White/European

\#\#

$\begin{array}{rrr}2 & 9 & 250 \\ 0.007 & 0.030 & 0.833\end{array}$

(n) 


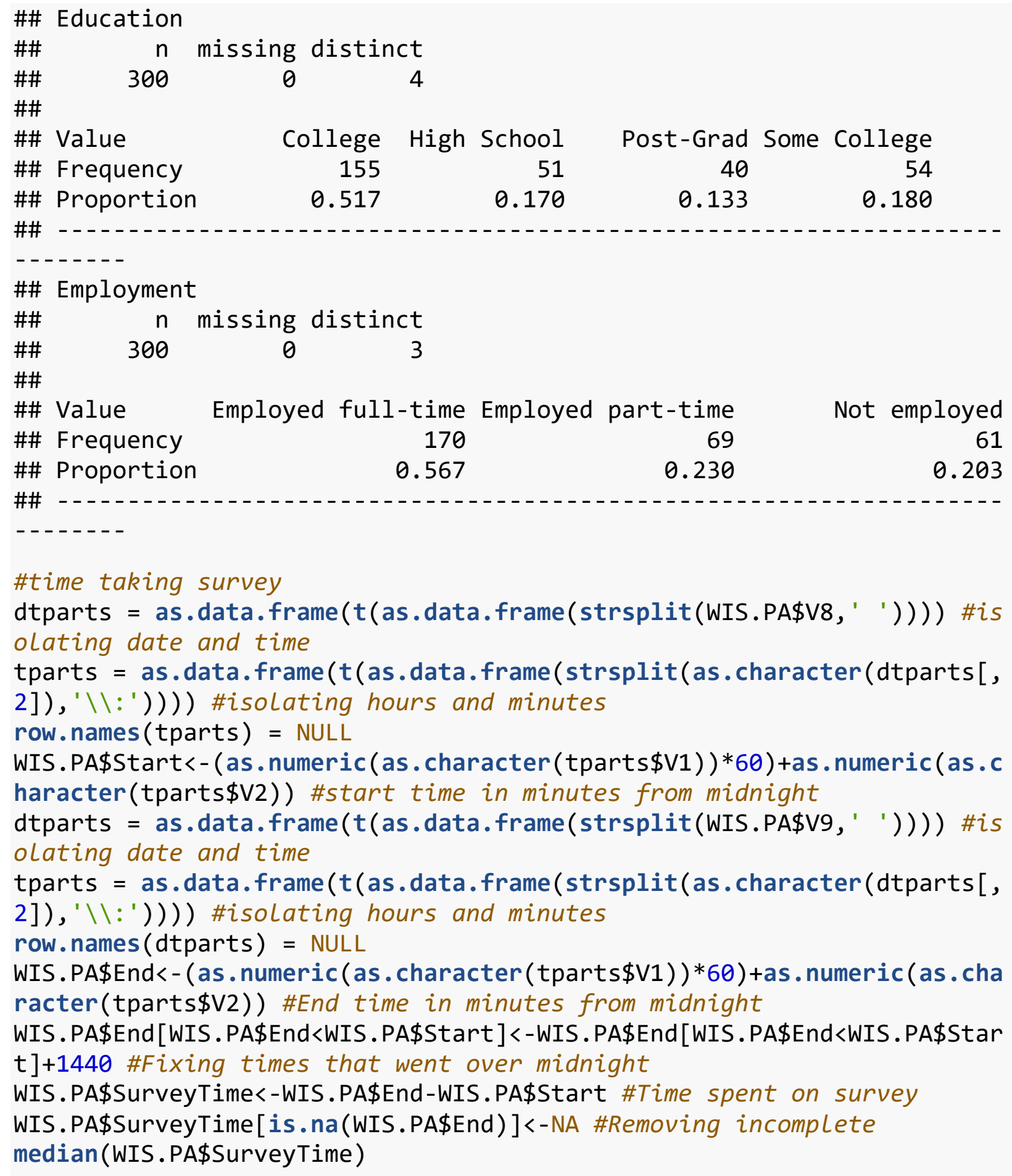




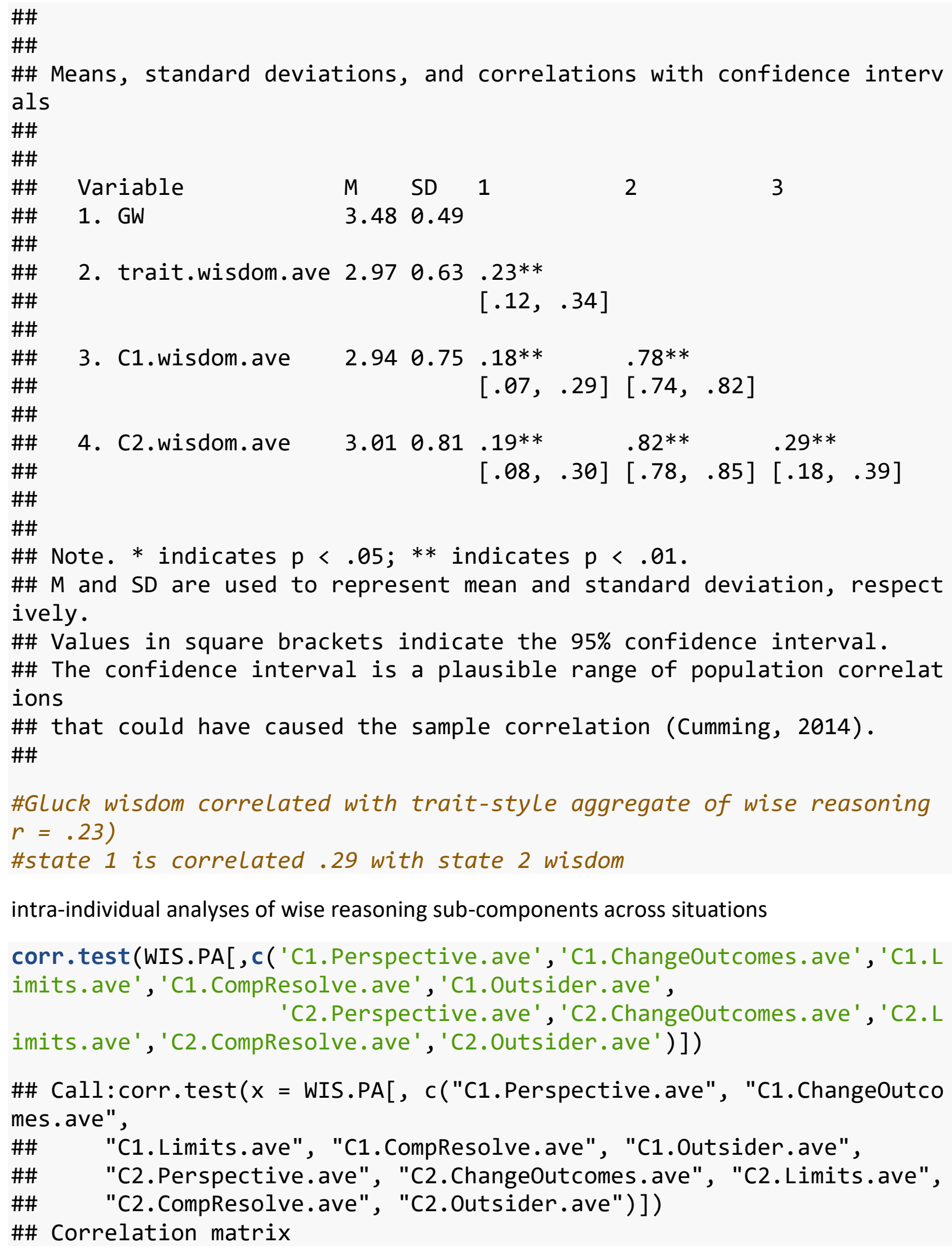




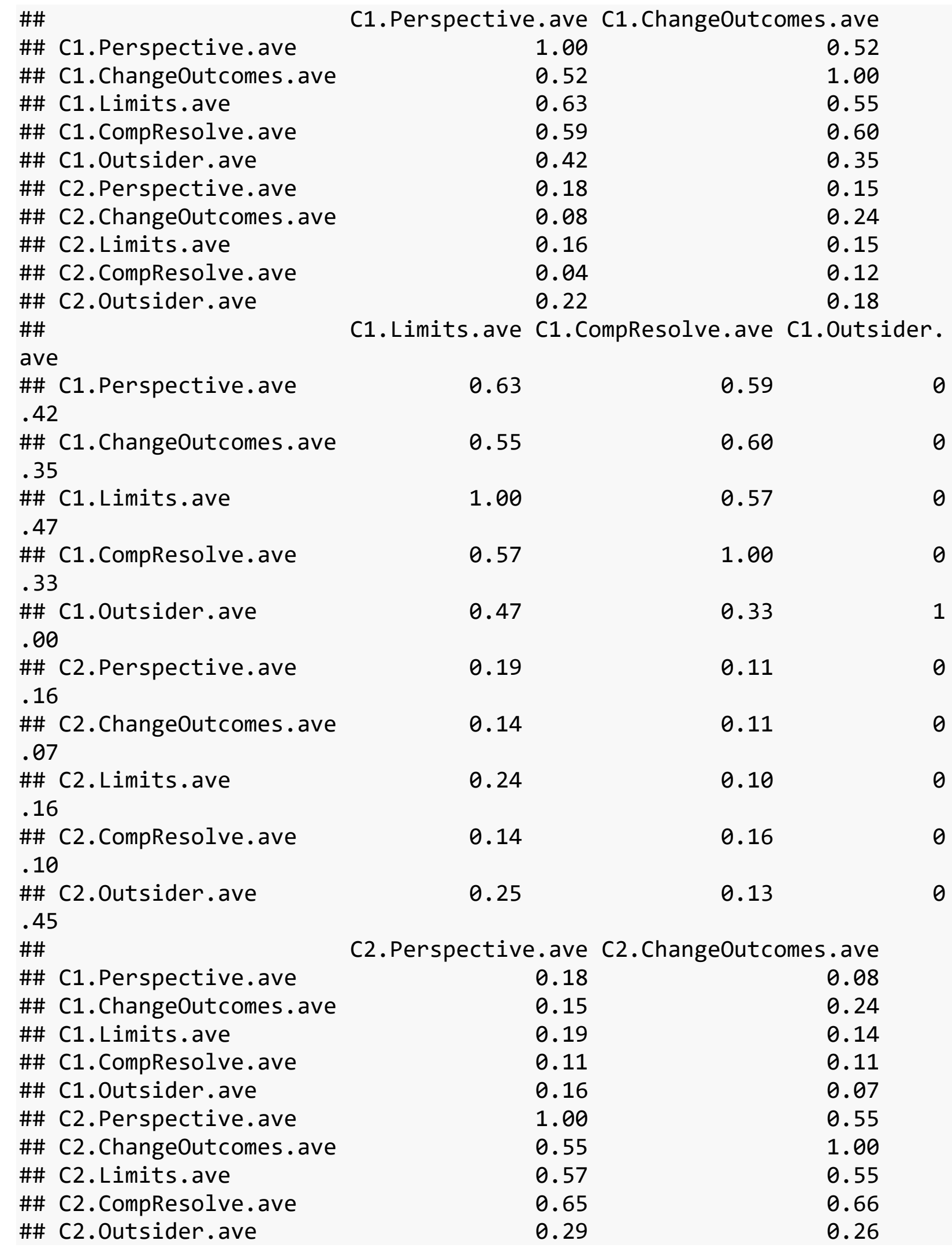




\begin{tabular}{|c|c|c|c|}
\hline \multirow{2}{*}{\multicolumn{4}{|c|}{ C2.Limits.ave C2.CompResolve.ave C2.Outsider. }} \\
\hline & & & \\
\hline $\begin{array}{l}\# \# \text { C1.Perspective.ave } \\
.22\end{array}$ & 0.16 & 0.04 & 0 \\
\hline $\begin{array}{l}\# \# \text { C1.ChangeOutcomes.ave } \\
.18\end{array}$ & 0.15 & 0.12 & $\theta$ \\
\hline \#\# C1.Limits.ave & 0.24 & 0.14 & 0 \\
\hline .25 & & & \\
\hline $\begin{array}{l}\text { \#\# C1.CompResolve.ave } \\
.13\end{array}$ & 0.10 & 0.16 & $\theta$ \\
\hline $\begin{array}{l}\# \# \text { C1.Outsider.ave } \\
.45\end{array}$ & 0.16 & 0.10 & 0 \\
\hline $\begin{array}{l}\# \# \text { C2. Perspective.ave } \\
.29\end{array}$ & 0.57 & 0.65 & 0 \\
\hline $\begin{array}{l}\text { \#\# C2. ChangeOutcomes.ave } \\
.26\end{array}$ & 0.55 & 0.66 & 0 \\
\hline $\begin{array}{l}\# \# \text { C2. Limits.ave } \\
.36\end{array}$ & 1.00 & 0.58 & \\
\hline $\begin{array}{l}\# \# \text { C2.CompResolve.ave } \\
.31\end{array}$ & 0.58 & 1.00 & $\theta$ \\
\hline $\begin{array}{l}\# \# \text { C2.Outsider.ave } \\
.00 \\
\# \# \text { Sample Size }\end{array}$ & 0.36 & 0.31 & 1 \\
\hline 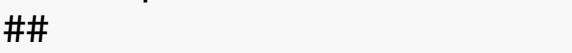 & C1.Perspective & .ave C1.ChangeOutc & :omes.ave \\
\hline \#\# C1.Perspective.ave & & 300 & 300 \\
\hline \#\# C1.ChangeOutcomes.ave & & 300 & 300 \\
\hline \#\# C1.Limits.ave & & 300 & 300 \\
\hline \#\# C1.CompResolve.ave & & 300 & 300 \\
\hline \#\# C1.Outsider.ave & & 300 & 300 \\
\hline \#\# C2.Perspective.ave & & 299 & 299 \\
\hline \#\# C2.ChangeOutcomes.ave & & 299 & 299 \\
\hline \#\# C2.Limits.ave & & 299 & 299 \\
\hline \#\# C2.CompResolve.ave & & 299 & 299 \\
\hline \#\# C2.Outsider.ave & & 299 & 299 \\
\hline $\begin{array}{l}\# \# \\
\text { ave }\end{array}$ & C1.Limits.ave & C1.CompResolve.ave & C1.Outsider. \\
\hline $\begin{array}{l}\# \# \text { C1.Perspective.ave } \\
300\end{array}$ & 300 & 300 & \\
\hline $\begin{array}{l}\# \# \text { C1. ChangeOutcomes . ave } \\
300\end{array}$ & 300 & 300 & \\
\hline $\begin{array}{l}\# \# \text { C1.Limits.ave } \\
300\end{array}$ & 300 & 300 & \\
\hline $\begin{array}{l}\text { \#\# C1.CompResolve.ave } \\
300\end{array}$ & 300 & 300 & \\
\hline
\end{tabular}




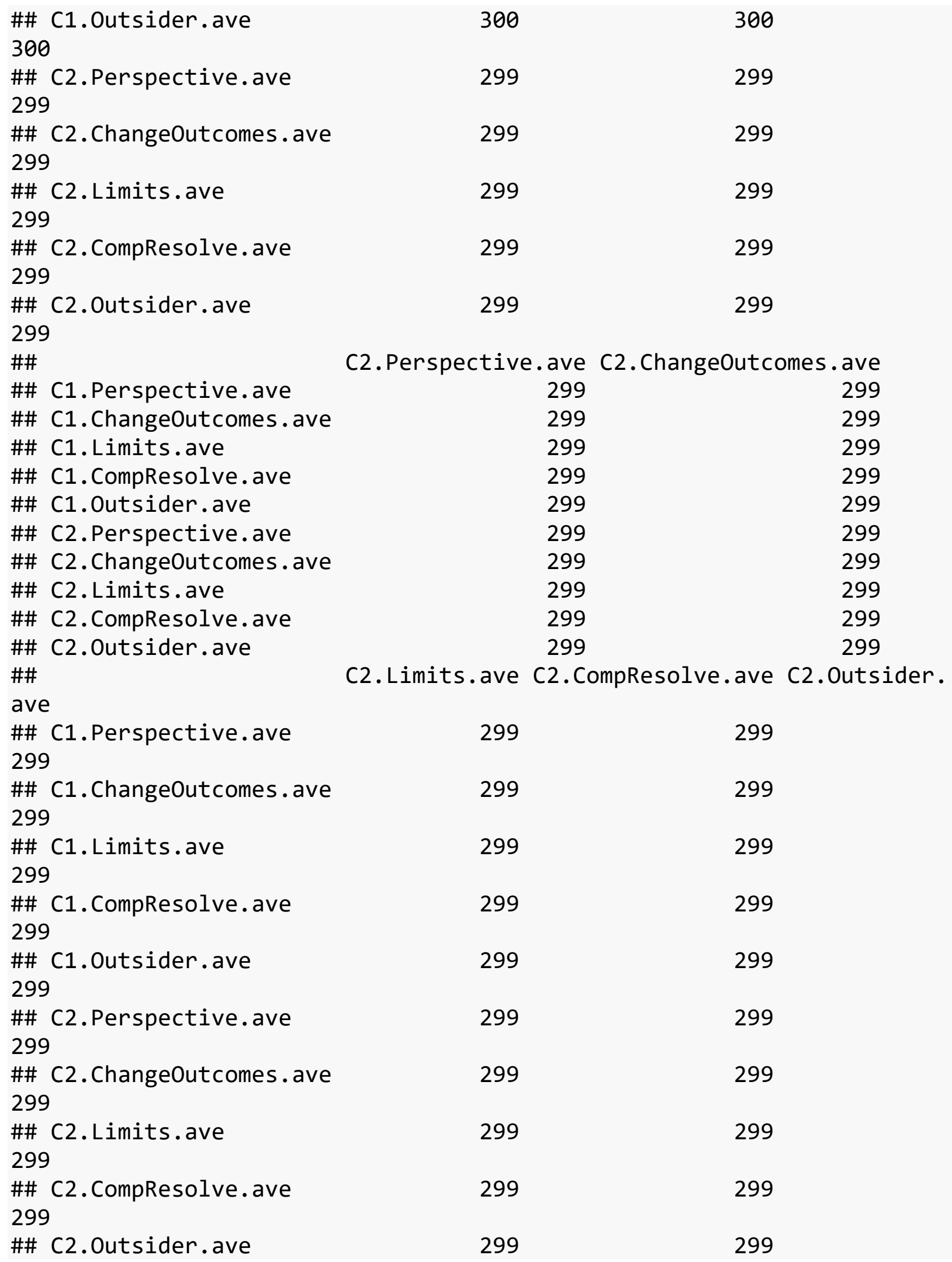


299

\#\# Probability values (Entries above the diagonal are adjusted for mul tiple tests.)

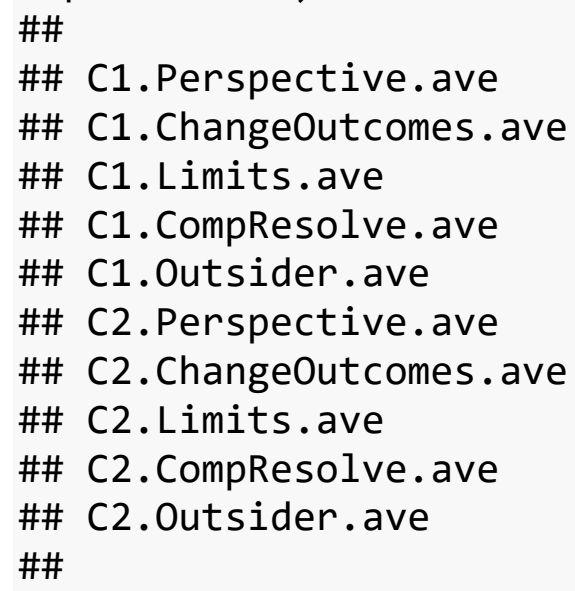




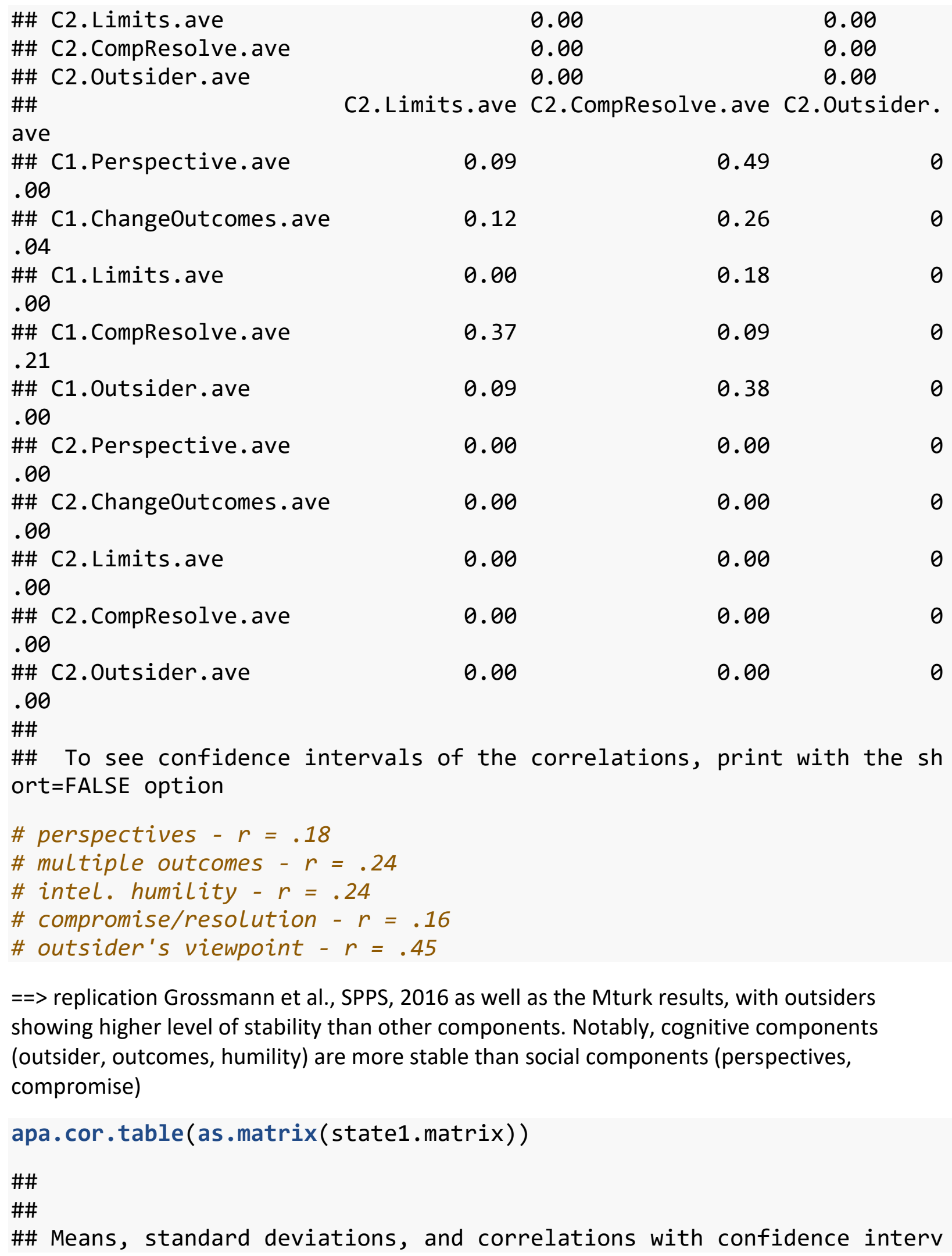

0.00

\#\#

C2.Limits.ave C2.CompResolve.ave C2.Outsider.

ave

\#\# C1.Perspective.ave

0.09

0.49

0

.00

\#\# C1. ChangeOutcomes.ave

0.12

0.26

0

.04

\#\# C1.Limits.ave

0.00

0.18

0

.00

\#\# C1.CompResolve.ave

0.37

0.09

0

.21

\#\# C1.Outsider.ave

0.09

0.38

0

.00

\#\# C2.Perspective.ave

0.00

0.00

0

.00

\#\# C2.ChangeOutcomes.ave

0.00

0.00

0

.00

\#\# C2. Limits.ave

0.00

0.00

0

.00

\#\# C2.CompResolve.ave

0.00

0.00

0

.00

\#\# C2. Outsider.ave

0.00

0.00

0 .00

\#\#

\#\# To see confidence intervals of the correlations, print with the sh ort=FALSE option

\# perspectives $-r=.18$

\# multiple outcomes $-r=.24$

\# intel. humility $-r=.24$

\# compromise/resolution - $r=.16$

\# outsider's viewpoint $-r=.45$

$==>$ replication Grossmann et al., SPPS, 2016 as well as the Mturk results, with outsiders showing higher level of stability than other components. Notably, cognitive components (outsider, outcomes, humility) are more stable than social components (perspectives, compromise)

apa.cor.table(as.matrix (state1.matrix))

\#\#

\#\#

\#\# Means, standard deviations, and correlations with confidence interv 


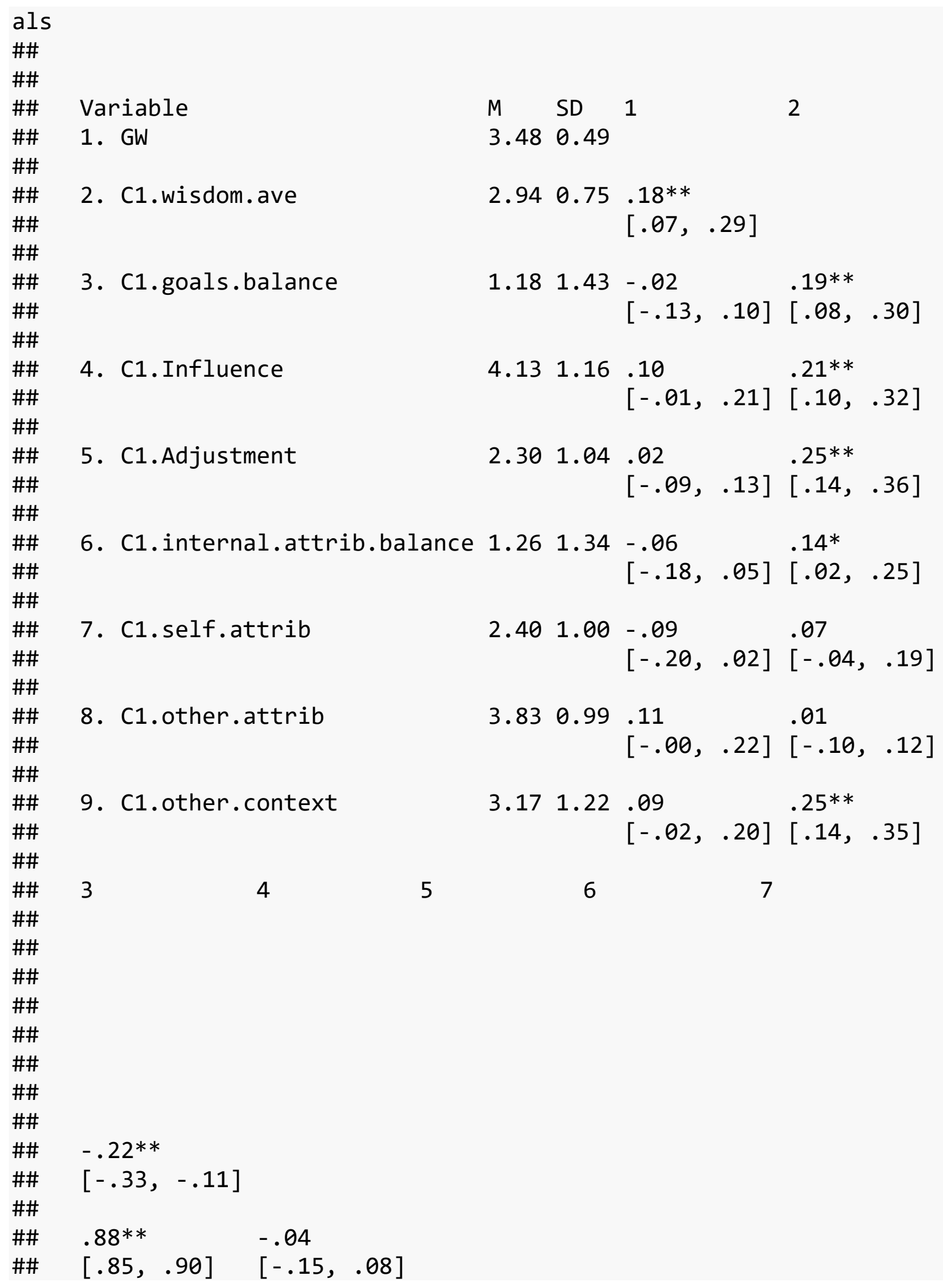




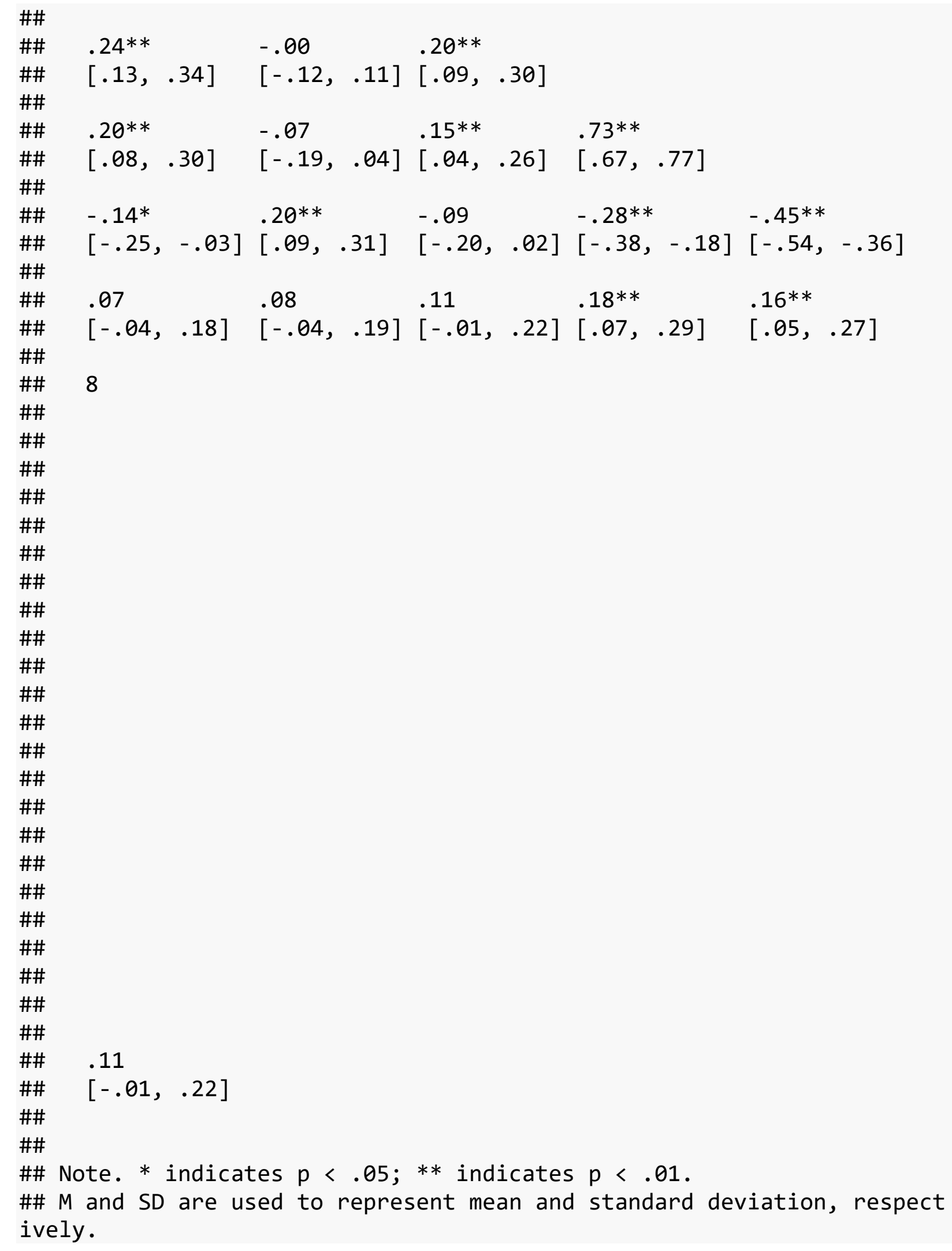


\#\# Values in square brackets indicate the $95 \%$ confidence interval. \#\# The confidence interval is a plausible range of population correlat ions

\#\# that could have caused the sample correlation (Cumming, 2014). \#\#

\#GLueck's measure is not related to anything balance-related... \#in contrast, state wise reasoning is linked to goal and causal infere nce balance and consideration of how context influenced others' behavi or apa.cor.table(as .matrix(state2.matrix))

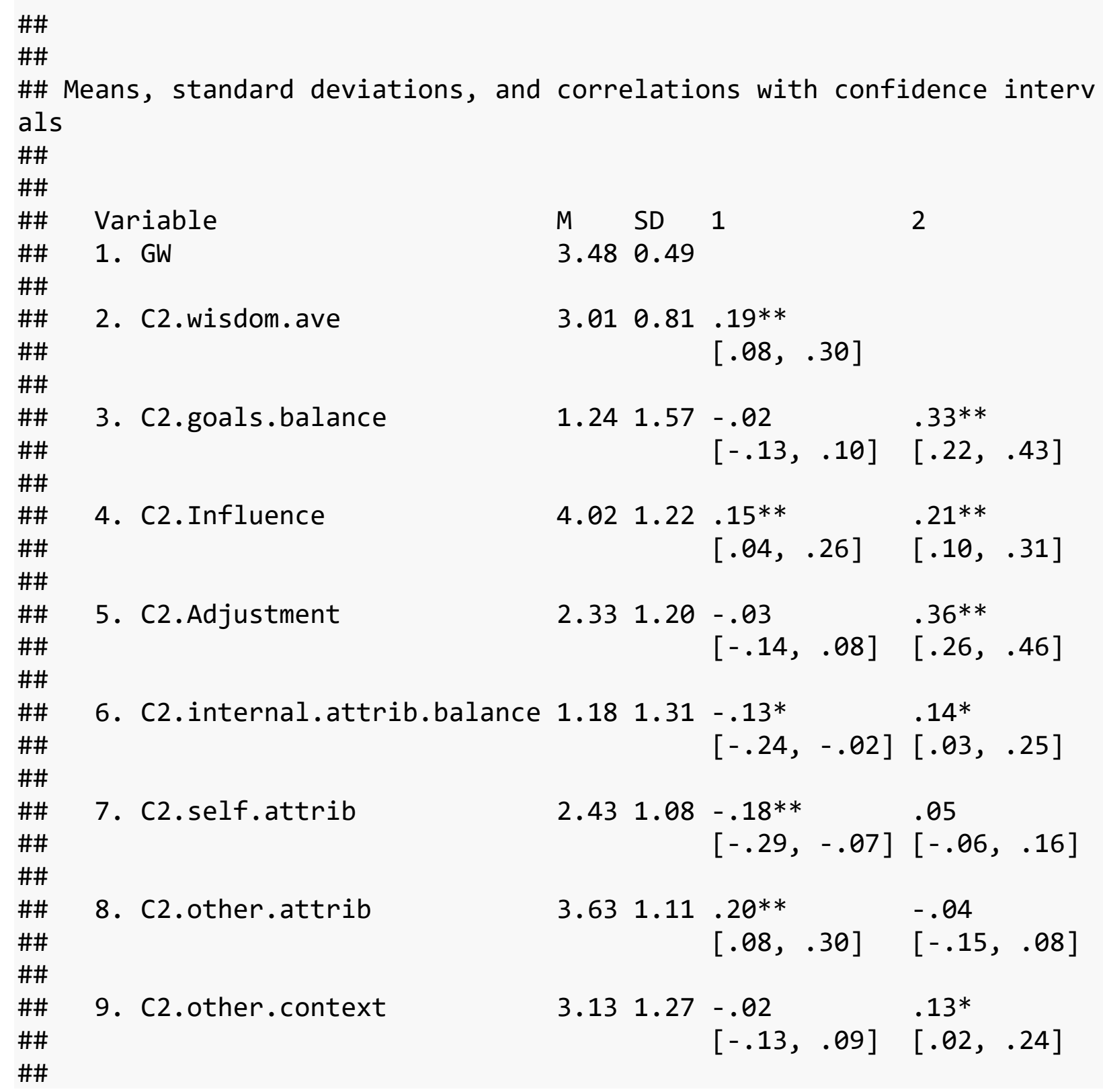




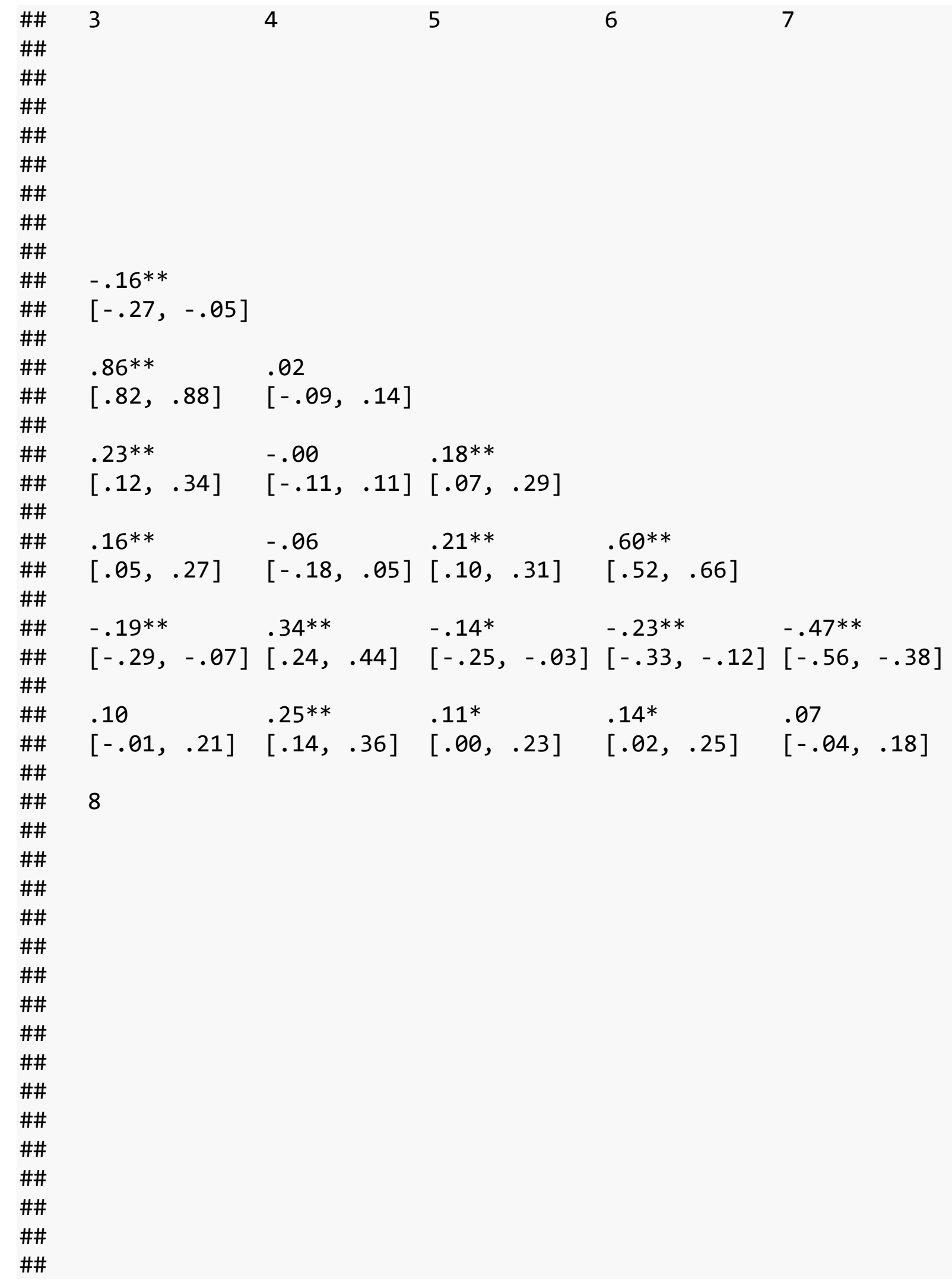




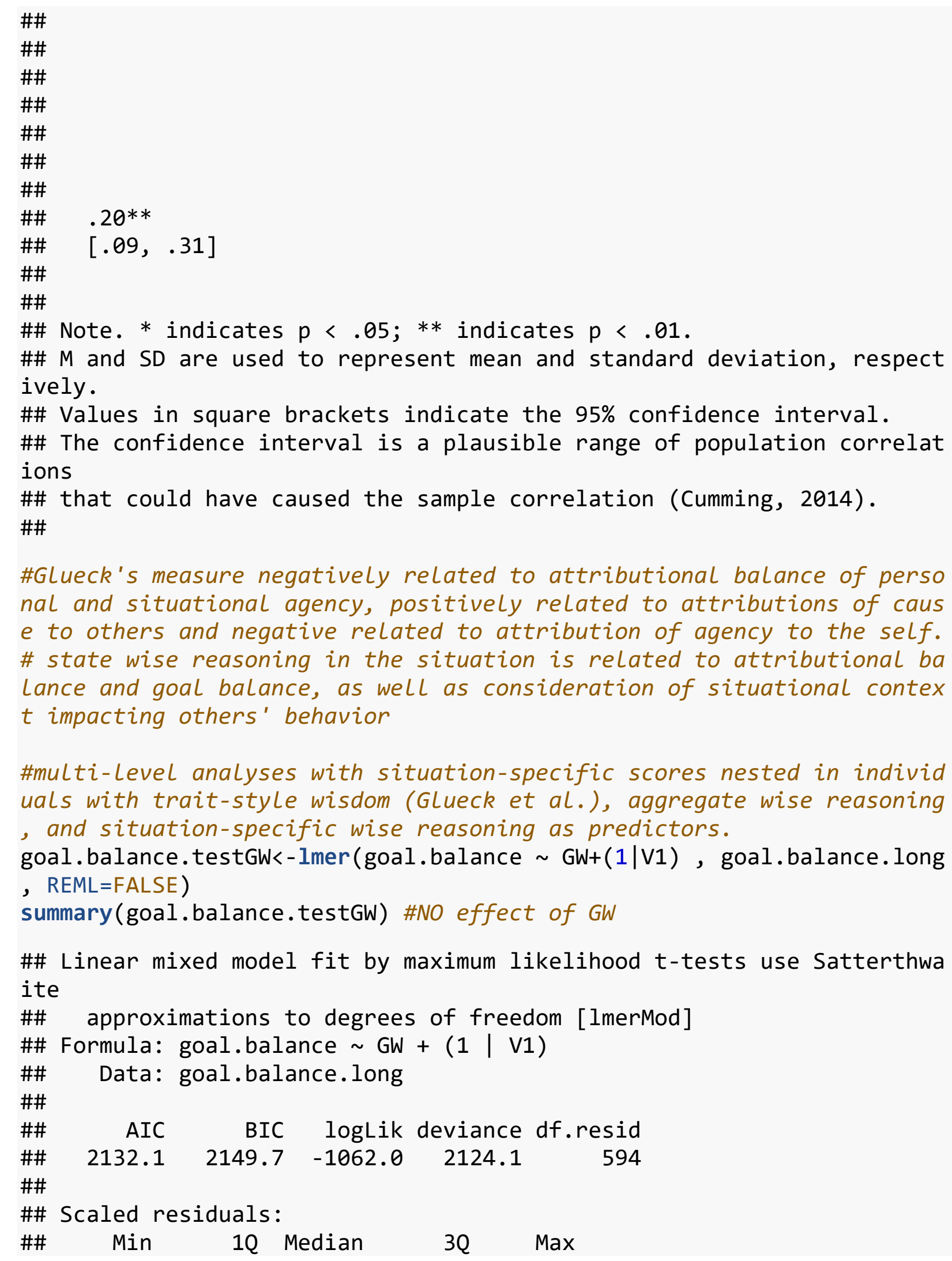




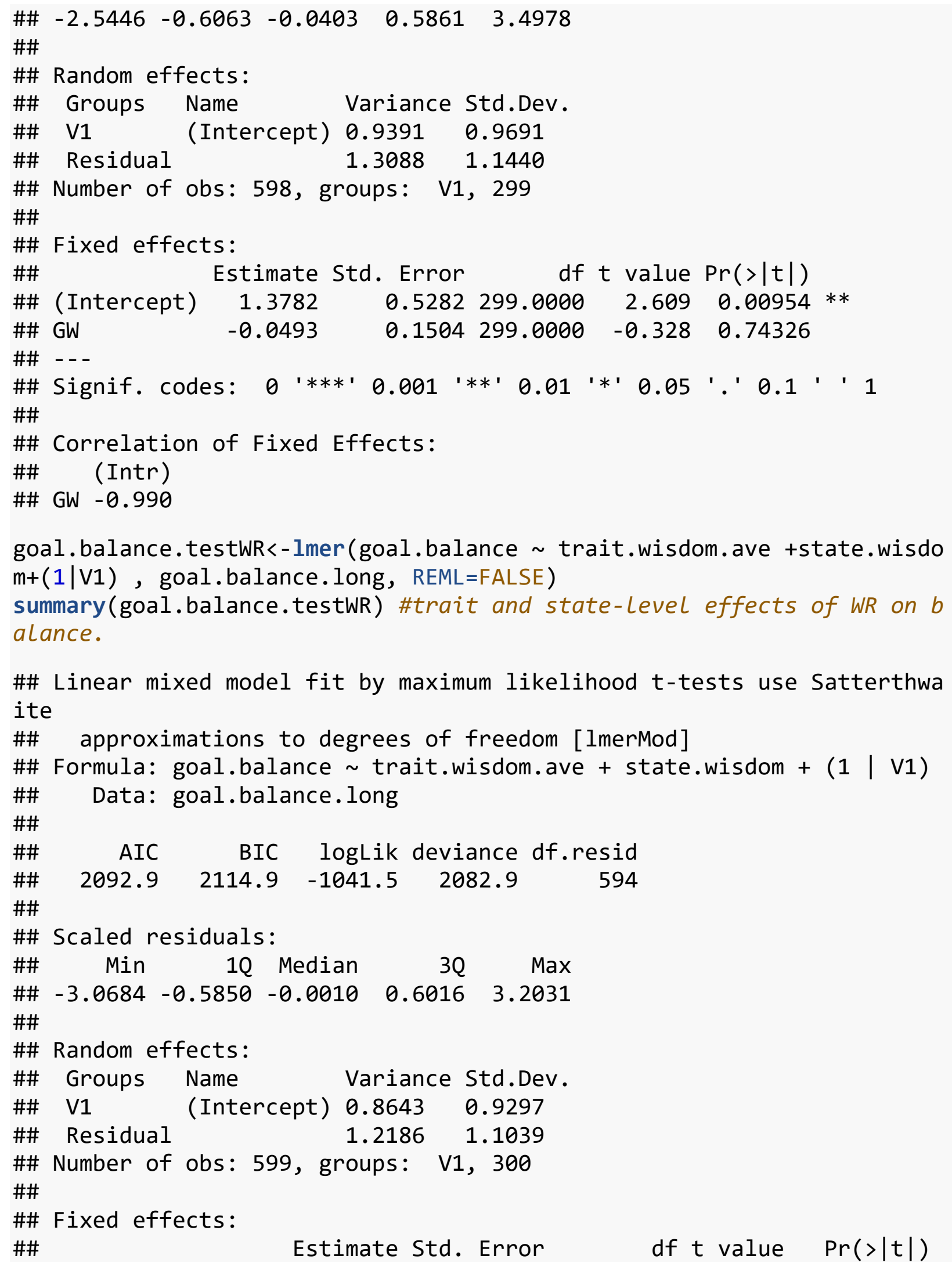




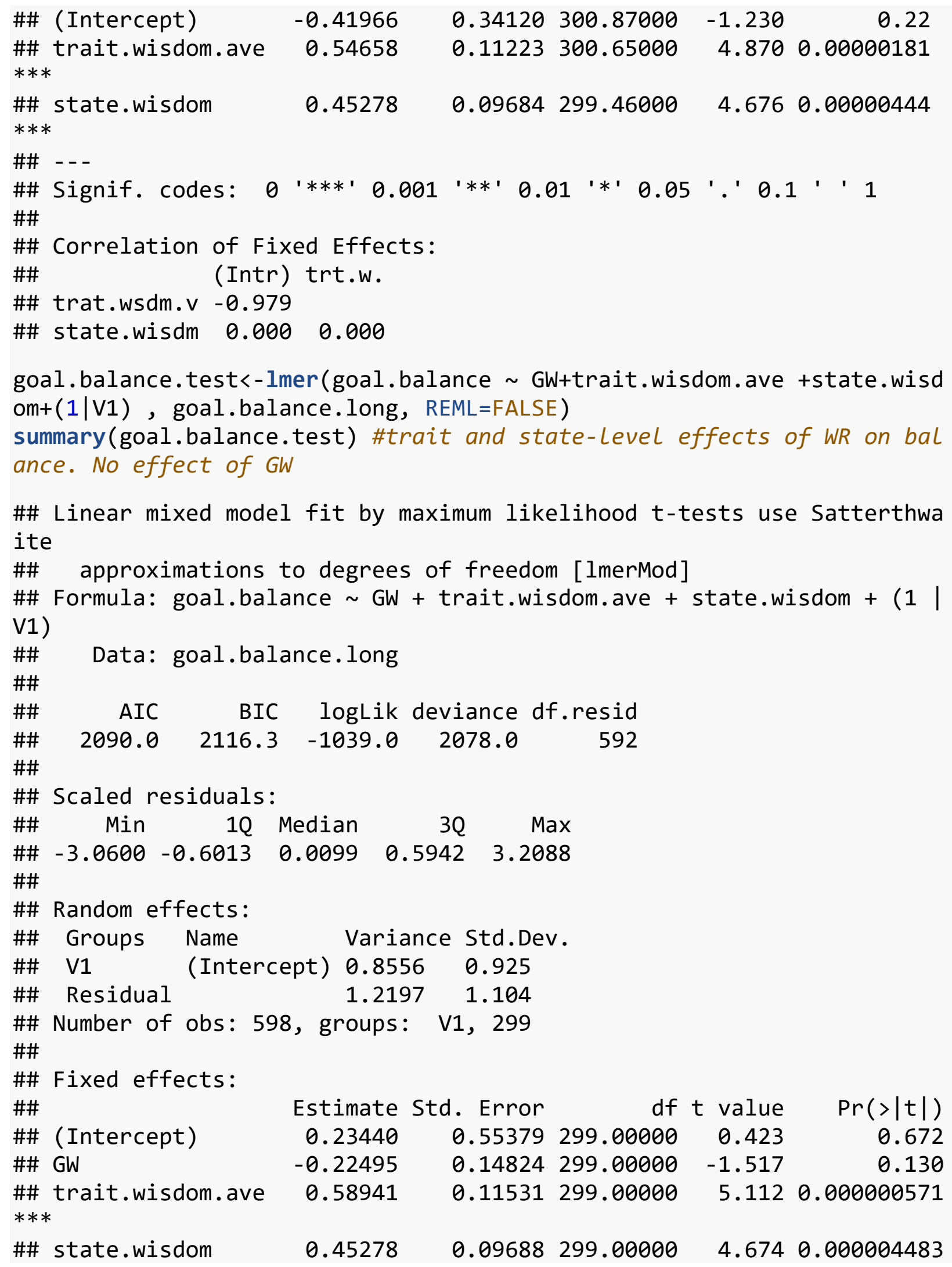




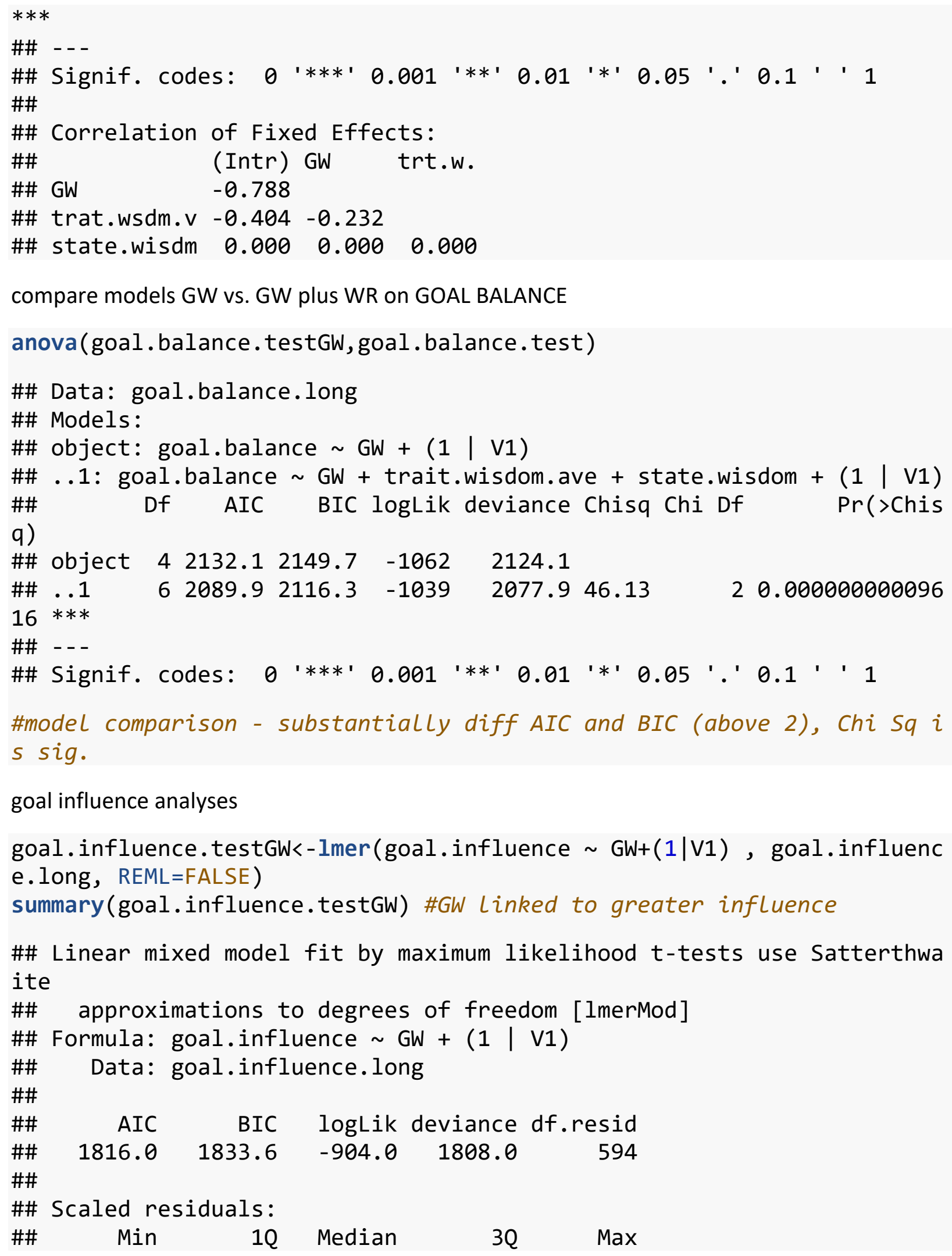




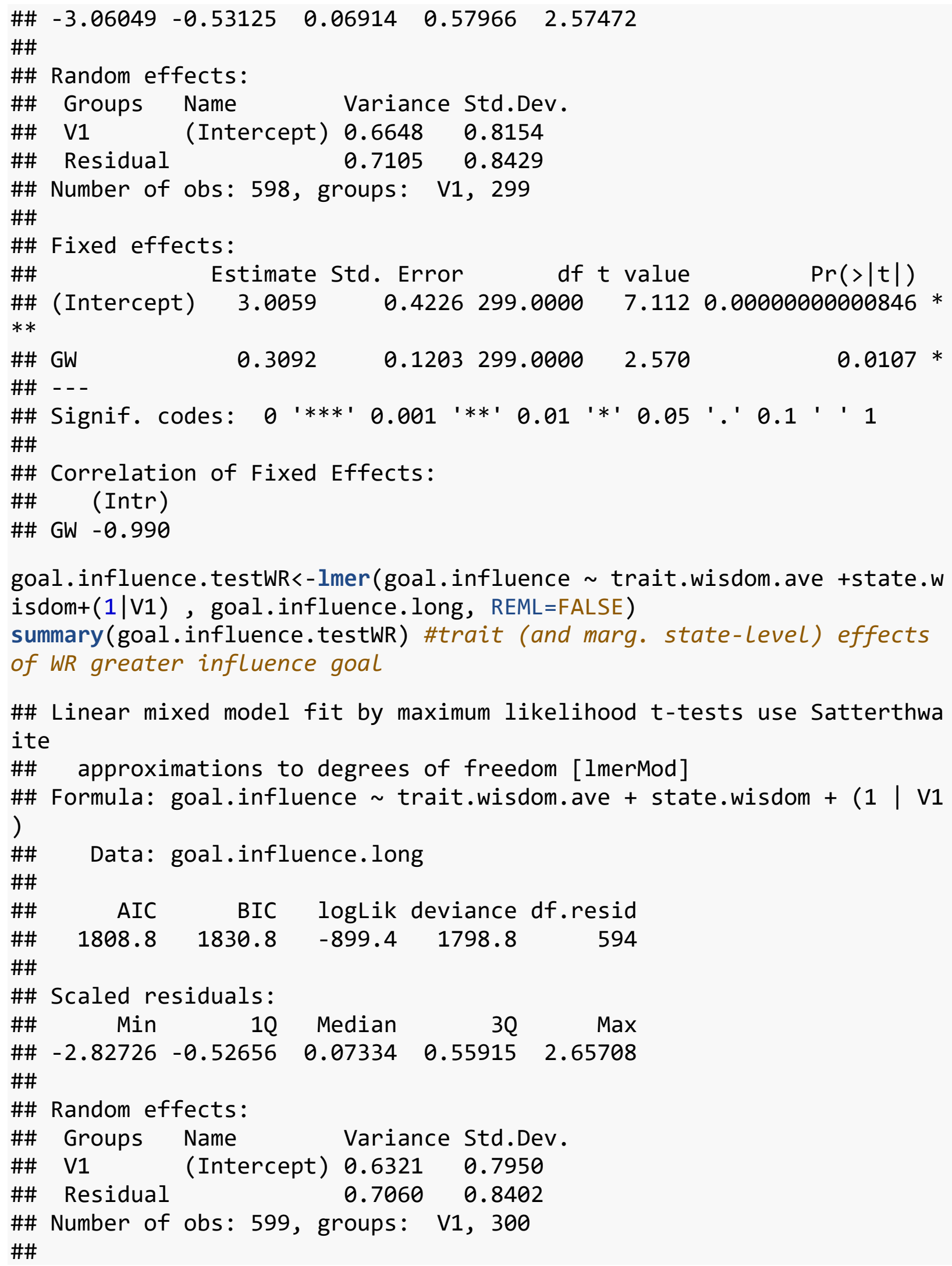




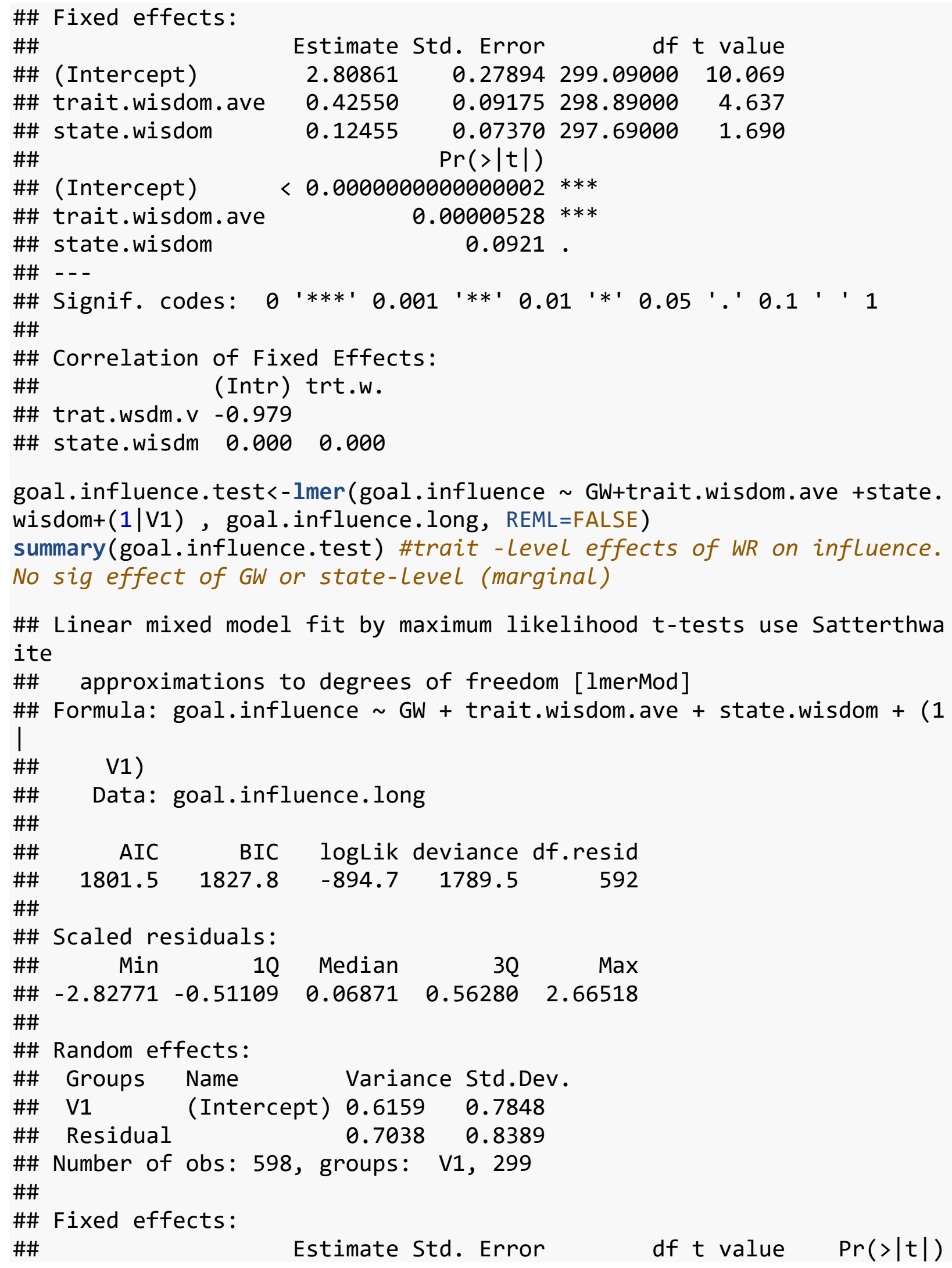




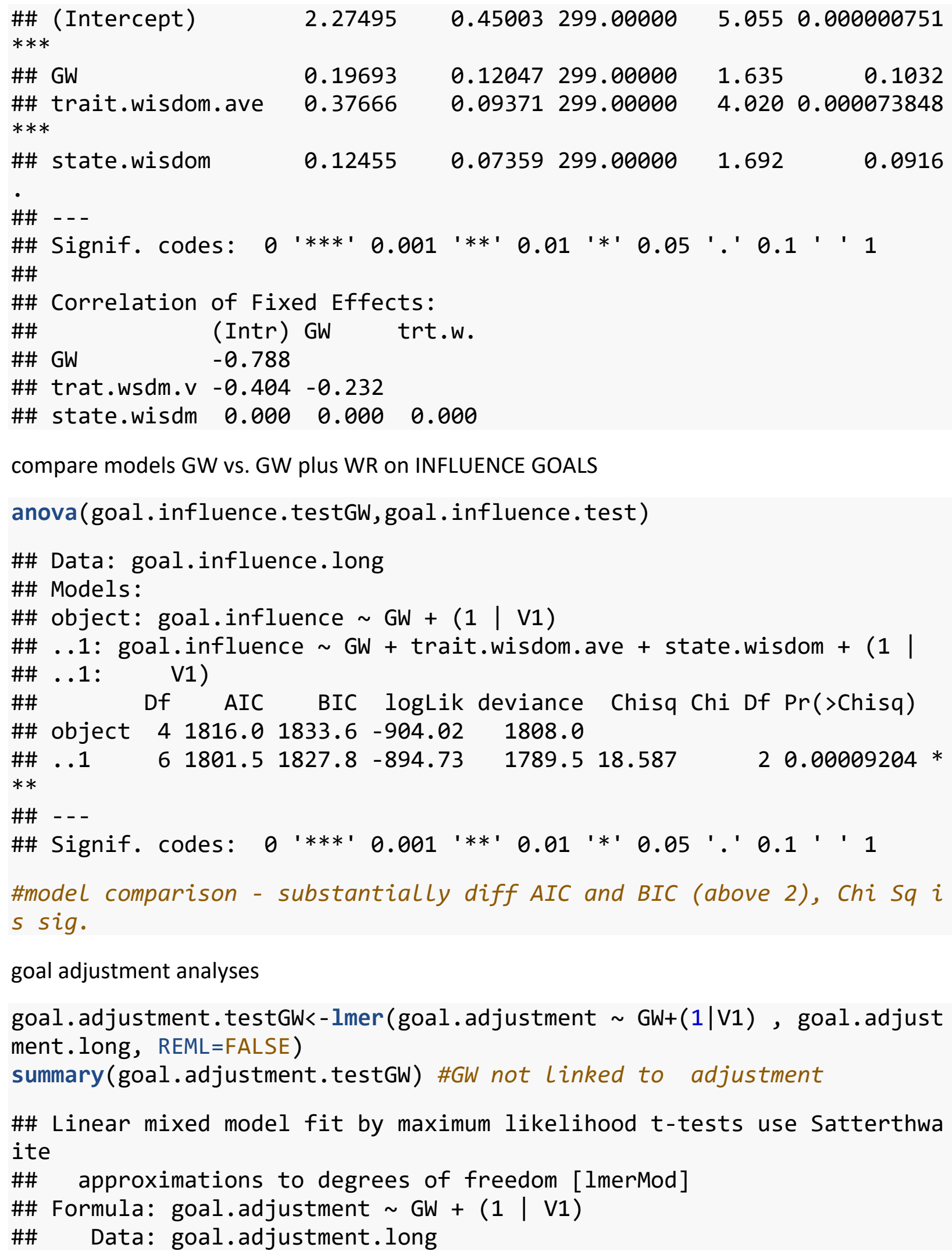




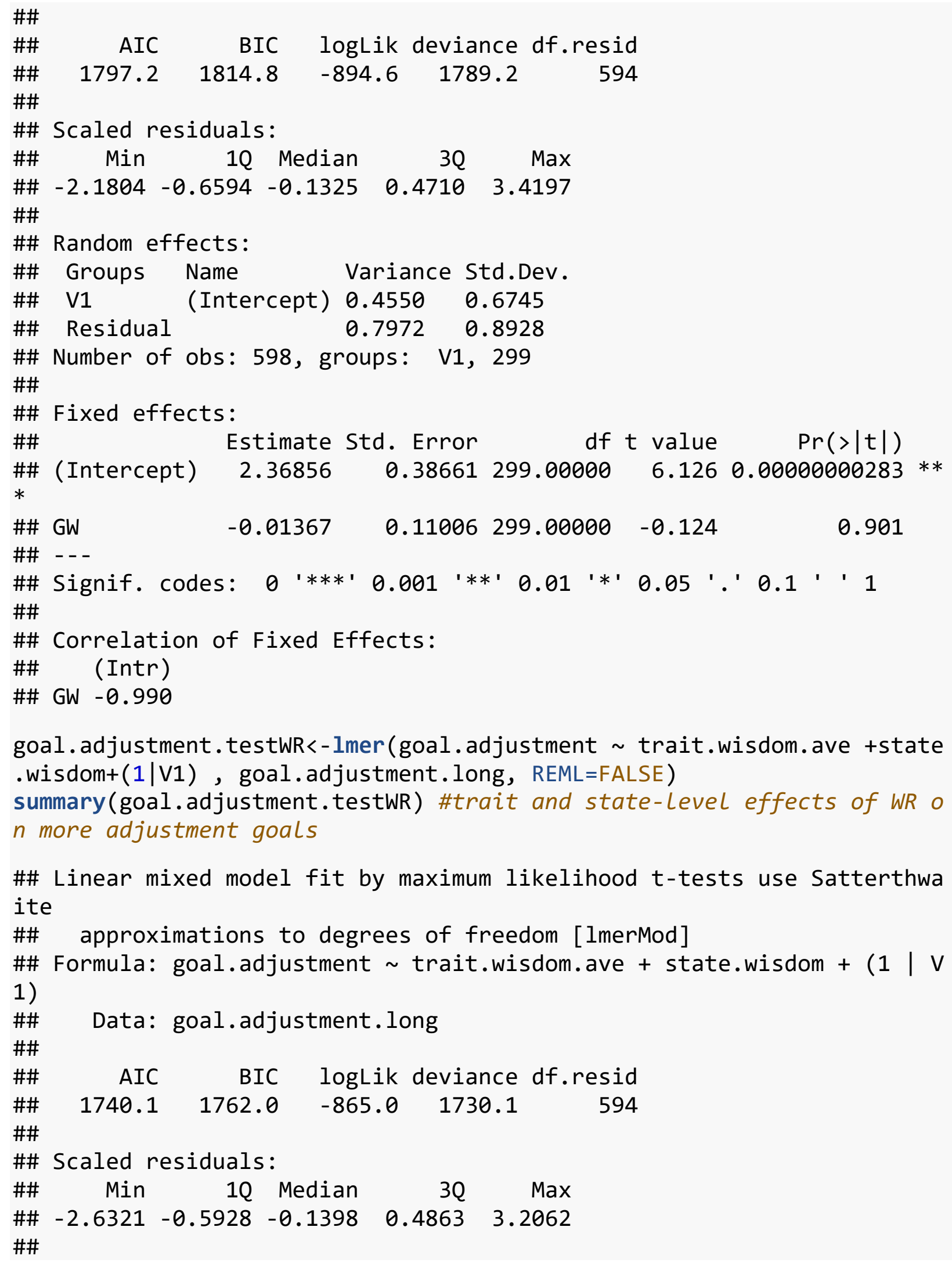




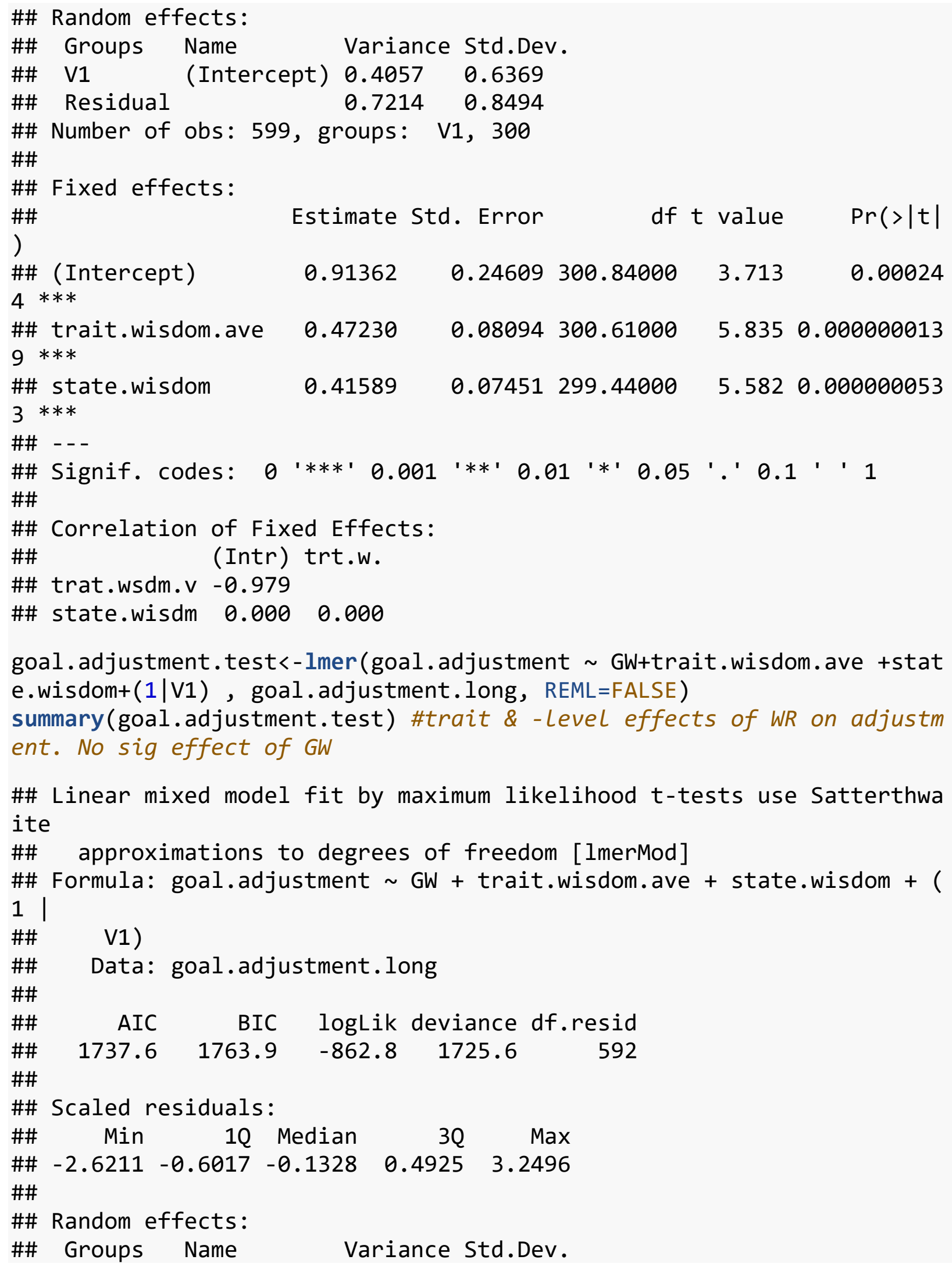




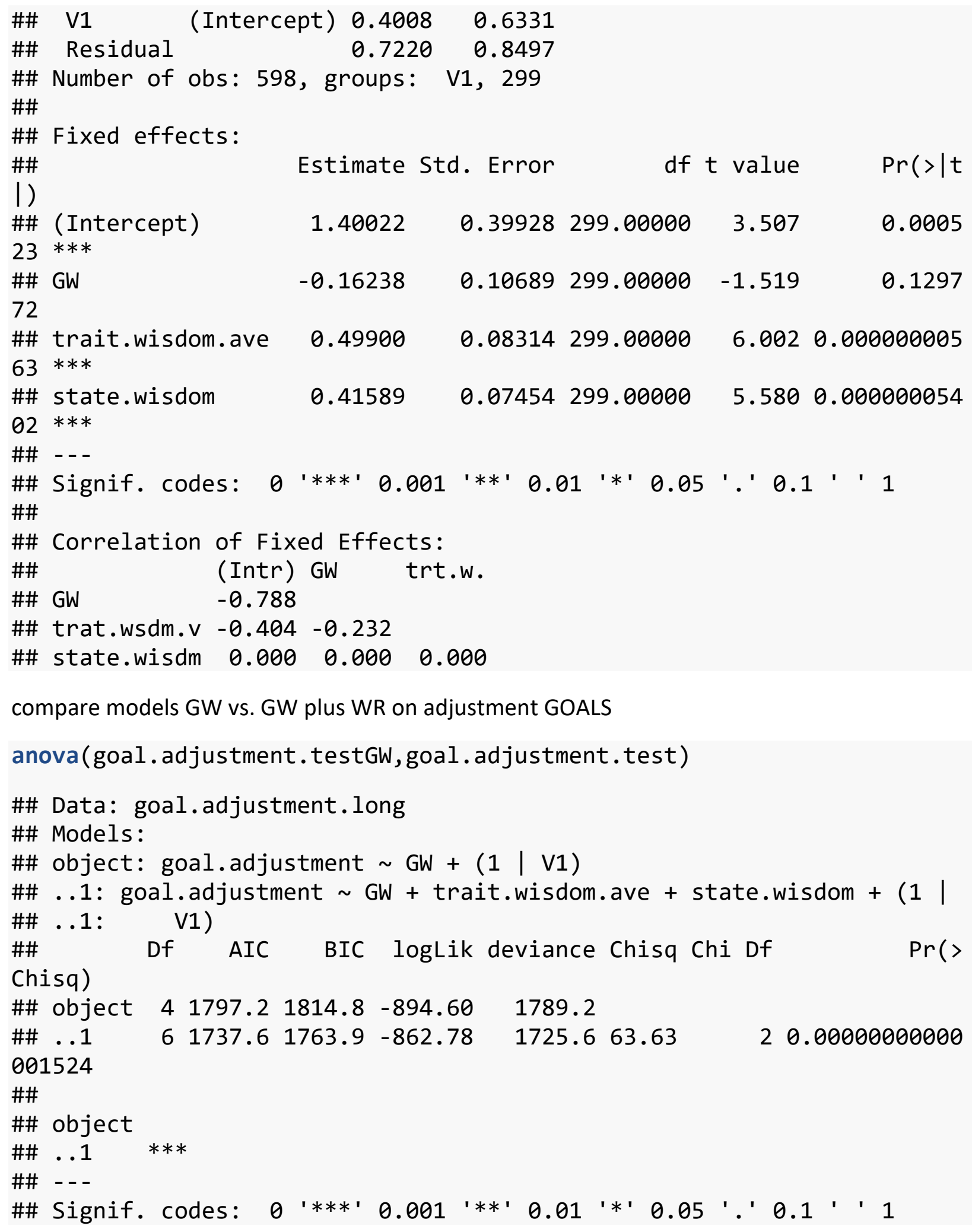


\#model comparison - substantially diff AIC and BIC (above 2), Chi Sq i s sig. HUGE EFFECT

CAUSAL INFERENCE Analyses balance

attribution.balance.testGW<-Imer(attr.balance $\sim \mathrm{GW}+(1 \mid \mathrm{V} 1)$, attributio n.balance.long, REML=FALSE)

summary(attribution.balance.testGW) \#marg NEG effect of GW on balance in attributions

\#\# Linear mixed model fit by maximum likelihood t-tests use Satterthwa ite

\#\# approximations to degrees of freedom [lmerMod]

\#\# Formula: attr.balance GW + (1 | V1)

\#\# Data: attribution.balance.long

\#\#

\#\# AIC BIC logLik deviance df.resid

$\begin{array}{llllll}\text { \#\# } & 2016.9 & 2034.4 & -1004.4 & 2008.9 & 594\end{array}$

\#\#

\#\# Scaled residuals:

\#\# Min $1 Q$ Median $3 Q \quad$ Max

$\begin{array}{llllll}\text { \#\# } & -2.09423 & -0.73851 & -0.00776 & 0.71257 & 2.34329\end{array}$

\#\#

\#\# Random effects:

\#\# Groups Name Variance Std.Dev.

$\begin{array}{llll}\text { \#\# V1 } & \text { (Intercept) } & 0.4124 & 0.6422\end{array}$

\#\# Residual $1.3218 \quad 1.1497$

\#\# Number of obs: 598, groups: V1, 299

\#\#

\#\# Fixed effects:

\#\# Estimate Std. Error df $t$ value $\operatorname{Pr}(>|t|)$

\#\# (Intercept) $\quad 2.1565 \quad 0.4335299 .0000 \quad 4.974 \quad 0.00000111$ ***

$\begin{array}{llllll}\text { \#\# GW } & -0.2685 & 0.1234 & 299.0000 & -2.176 & 0.0304\end{array}$

\#\# - - -

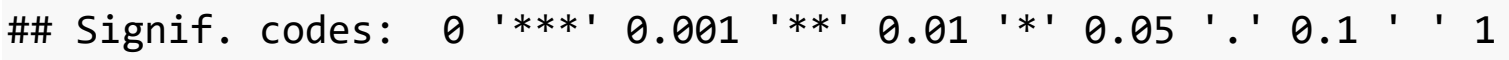

\#\#

\#\# Correlation of Fixed Effects:

\#\# (Intr)

\#\# GW -0.990

attribution.balance. testWR<-Imer (attr. balance $\sim$ trait. wisdom. ave +stat e.wisdom+(1|V1), attribution.balance.long, REML=FALSE)

summary(attribution.balance.testWR) \#trait (and marg. state-Level) eff ects of WR on balance. 
\#\# Linear mixed model fit by maximum likelihood t-tests use Satterthwa ite

\#\# approximations to degrees of freedom [lmerMod]

\#\# Formula: attr.balance trait.wisdom.ave + state.wisdom + (1 | V1)

\#\# Data: attribution.balance.long

\#\#

\#\#

\#\#

$\begin{array}{rrrrr}\text { AIC } & \text { BIC } & \text { logLik } & \text { deviance df.resid } \\ 2017.5 & 2039.5 & -1003.7 & 2007.5 & 594\end{array}$

\#\#

\#\# Scaled residuals:

\#\# $\quad$ Min $\quad 1 Q$ Median $3 Q \quad$ Max

$\begin{array}{llllll}\# \# & -1.99635 & -0.76629 & 0.01397 & 0.68695 & 2.30561\end{array}$

\#\#

\#\# Random effects:

\#\# Groups Name Variance Std.Dev.

$\begin{array}{llll}\text { \#\# V1 } & \text { (Intercept) } & 0.4117 & 0.6416\end{array}$

\#\# Residual $\quad 1.3094 \quad 1.1443$

\#\# Number of obs: 599, groups: V1, 300

\#\#

\#\# Fixed effects:

\#\#

\#\# (Intercept)

Estimate Std. Error df $t$ value $\operatorname{Pr}(>|t|)$

\#\# trait.wisdom.ave

$0.4381 \quad 0.2903 \quad 300.2200$

1.5090 .13236

\#\# state.wisdom

0.2623

0.0955299 .9800

2.746

$0.00639 * *$

\#\# ---

\#\# Signif. codes:

0.1757

0.1004298 .8600

1.751

0.08103 .

\#\#

\#\# Correlation of Fixed Effects:

\#\# (Intr) trt.w.

\#\# trat.wsdm.v -0.979

\#\# state.wisdm $0.000 \quad 0.000$

attribution.balance.test<-Imer(attr.balance $\sim$ GW+trait.wisdom.ave +sta te.wisdom+(1|V1), attribution.balance.long, REML=FALSE)

summary(attribution.balance.test) \#trait (and marg state-level) effect $s$ of WR on balance. NEG effect of GW !

\#\# Linear mixed model fit by maximum likelihood t-tests use Satterthwa ite

\#\# approximations to degrees of freedom [lmerMod]

\#\# Formula: attr.balance $\sim \mathrm{GW}+$ trait.wisdom.ave + state.wisdom + (1 I V1)

\#\# Data: attribution.balance.long

\#\#

\#\#

AIC BIC logLik deviance df.resid 


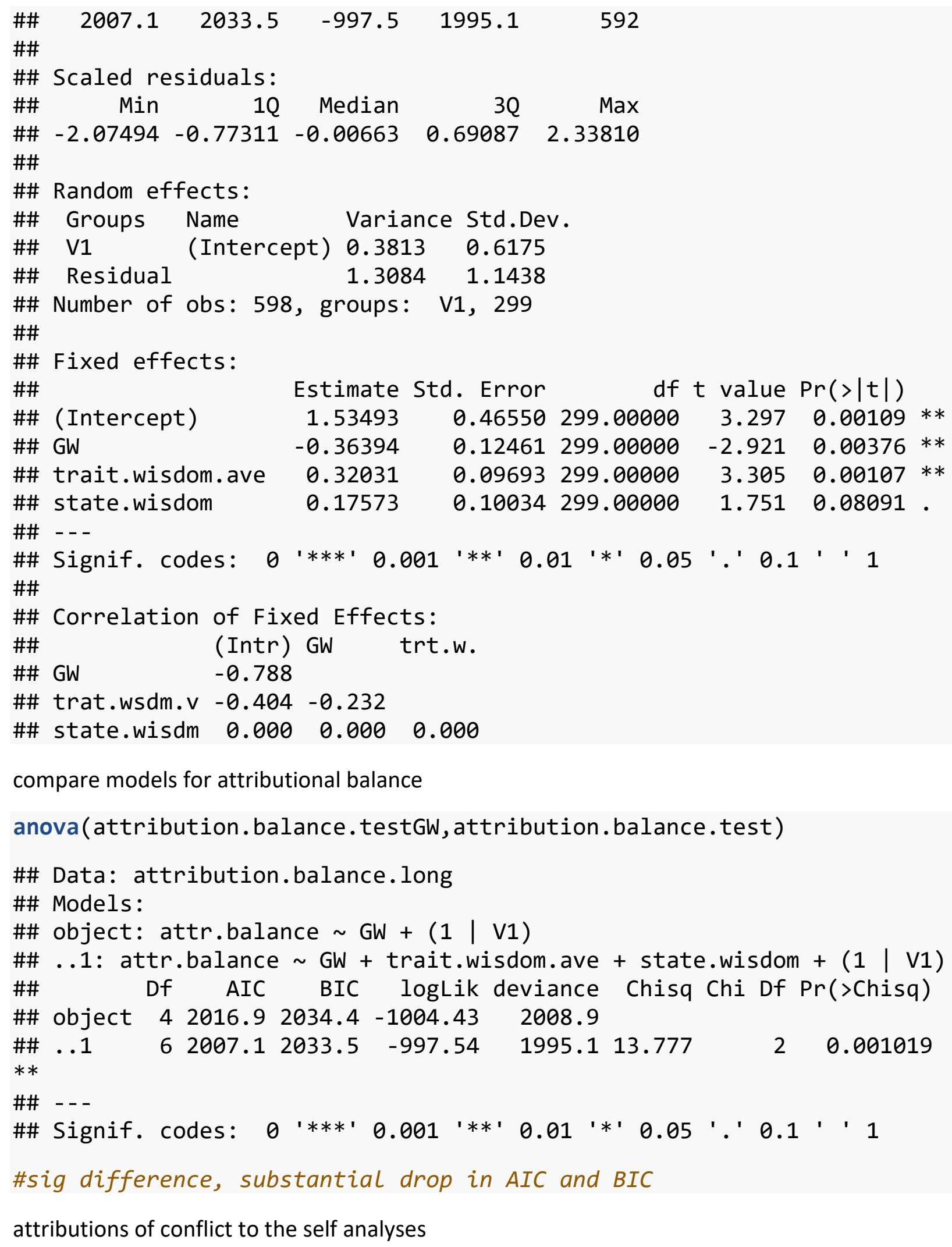


attribution.self.testGW<-lmer(attr.self GW+(1|V1), attribution.self .long, REML=FALSE)

summary(attribution.self.testGW) \#marg NEG effect of GW on self-focuse $d$ attributions

\#\# Linear mixed model fit by maximum likelihood t-tests use Satterthwa ite

\#\# approximations to degrees of freedom [1merMod]

\#\# Formula: attr.self GW + (1 | V1)

\#\# Data: attribution.self.long

\#\#

\#\# AIC BIC logLik deviance df.resid

$\begin{array}{llllll}\# \# & 1729.2 & 1746.8 & -860.6 & 1721.2 & 594\end{array}$

\#\#

\#\# Scaled residuals:

\#\# Min 1 Q Median $3 Q \quad$ Max

$\begin{array}{llllll}\# \# & -1.75124 & -0.76104 & -0.09754 & 0.59591 & 2.52525\end{array}$

\#\#

\#\# Random effects:

\#\# Groups Name Variance Std.Dev.

$\begin{array}{llll}\text { \#\# V1 } & \text { (Intercept) } & 0.1608 & 0.4010\end{array}$

\#\# Residual $\quad 0.89280 .9449$

\#\# Number of obs: 598, groups: V1, 299

\#\#

\#\# Fixed effects:

\#\#

$|t|)$

\#\# (Intercept) $\quad 3.43115 \quad 0.32607299 .00000 \quad 10.523<0.000000000000$

0002

\#\# GW

$-0.29104$

$0.09282 \quad 299.00000-3.135$

0.0

0189

\#\#

\#\# (Intercept) $* * *$

\#\# GW

\#\# ---

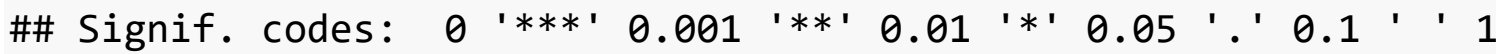

\#\#

\#\# Correlation of Fixed Effects:

\#\# (Intr)

\#\# GW -0.990

attribution.self.testWR<-lmer(attr.self $\sim$ trait.wisdom. ave +state.wisd $\mathrm{om}+(1 \mid \mathrm{V} 1)$, attribution.self.long, REML=FALSE)

summary(attribution.self.testWR) \#WR unrelated to self attributions. 


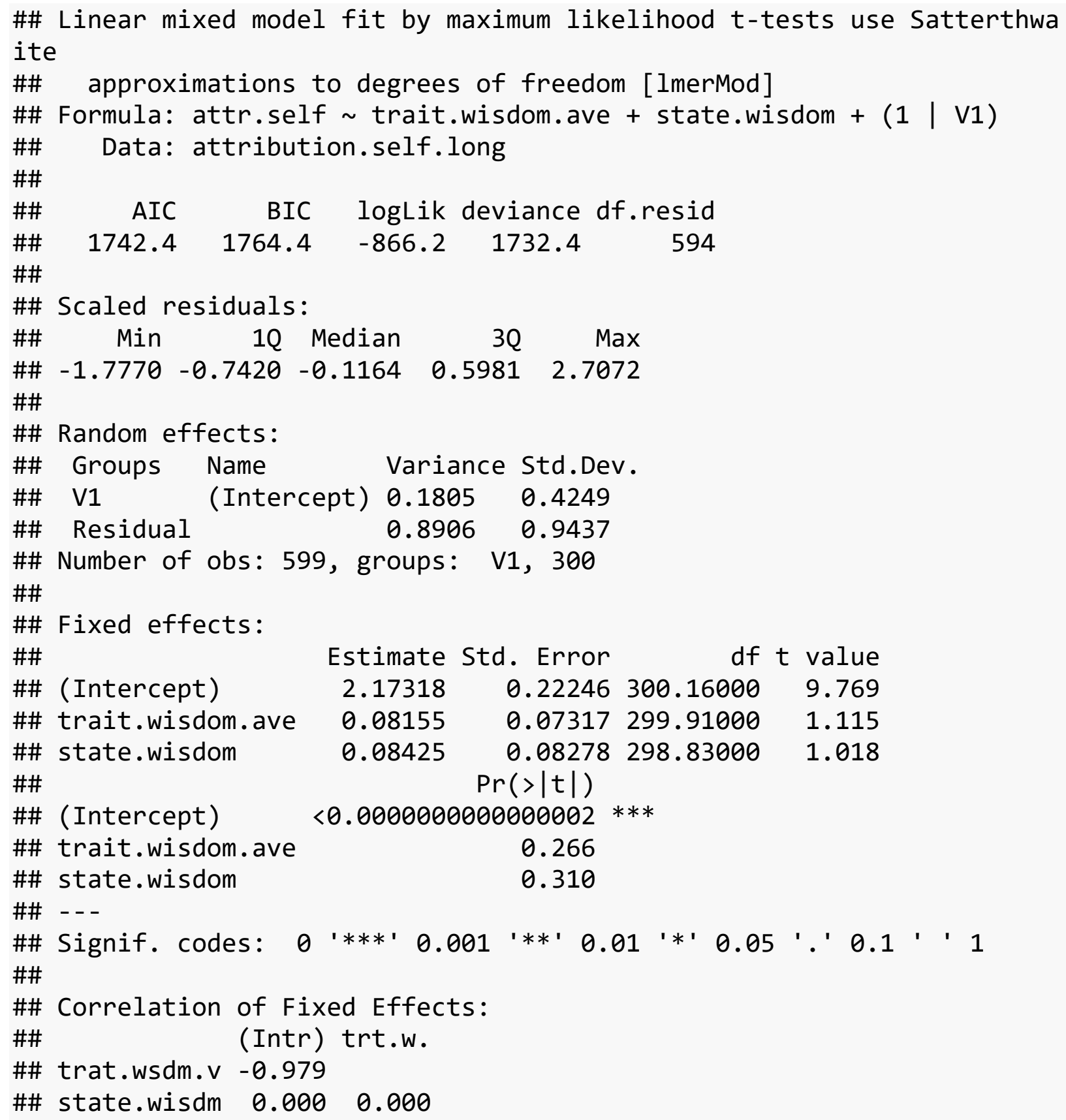




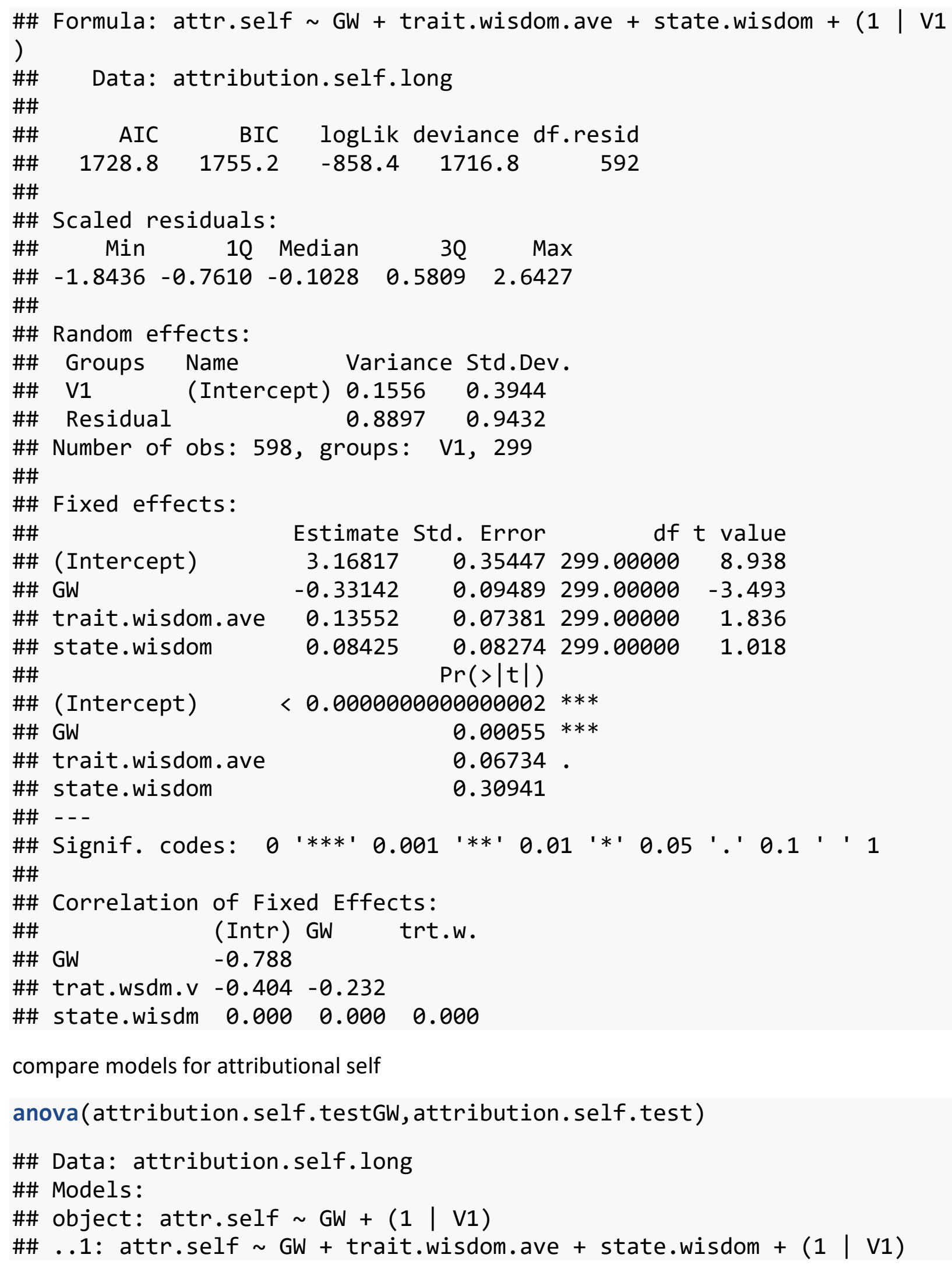




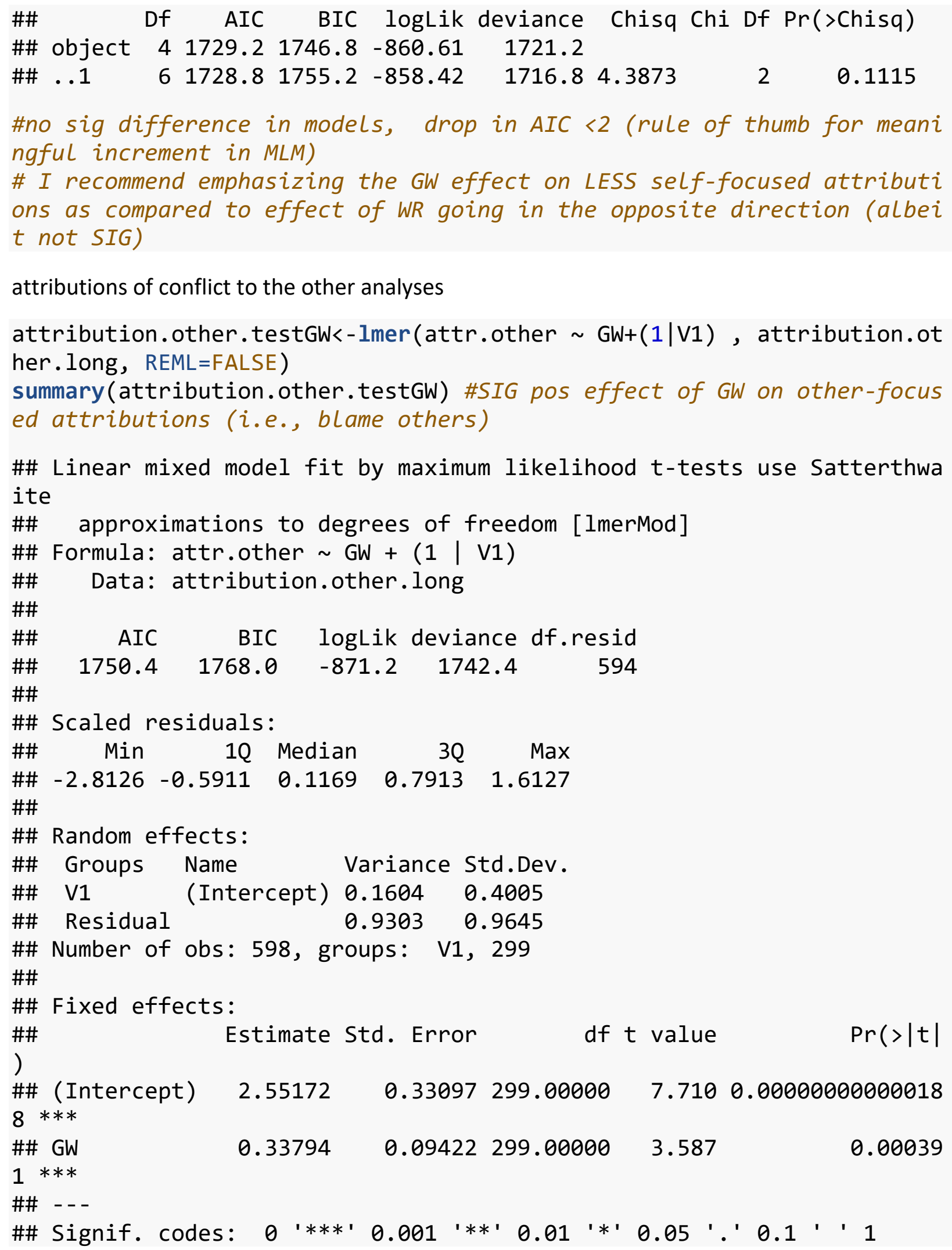




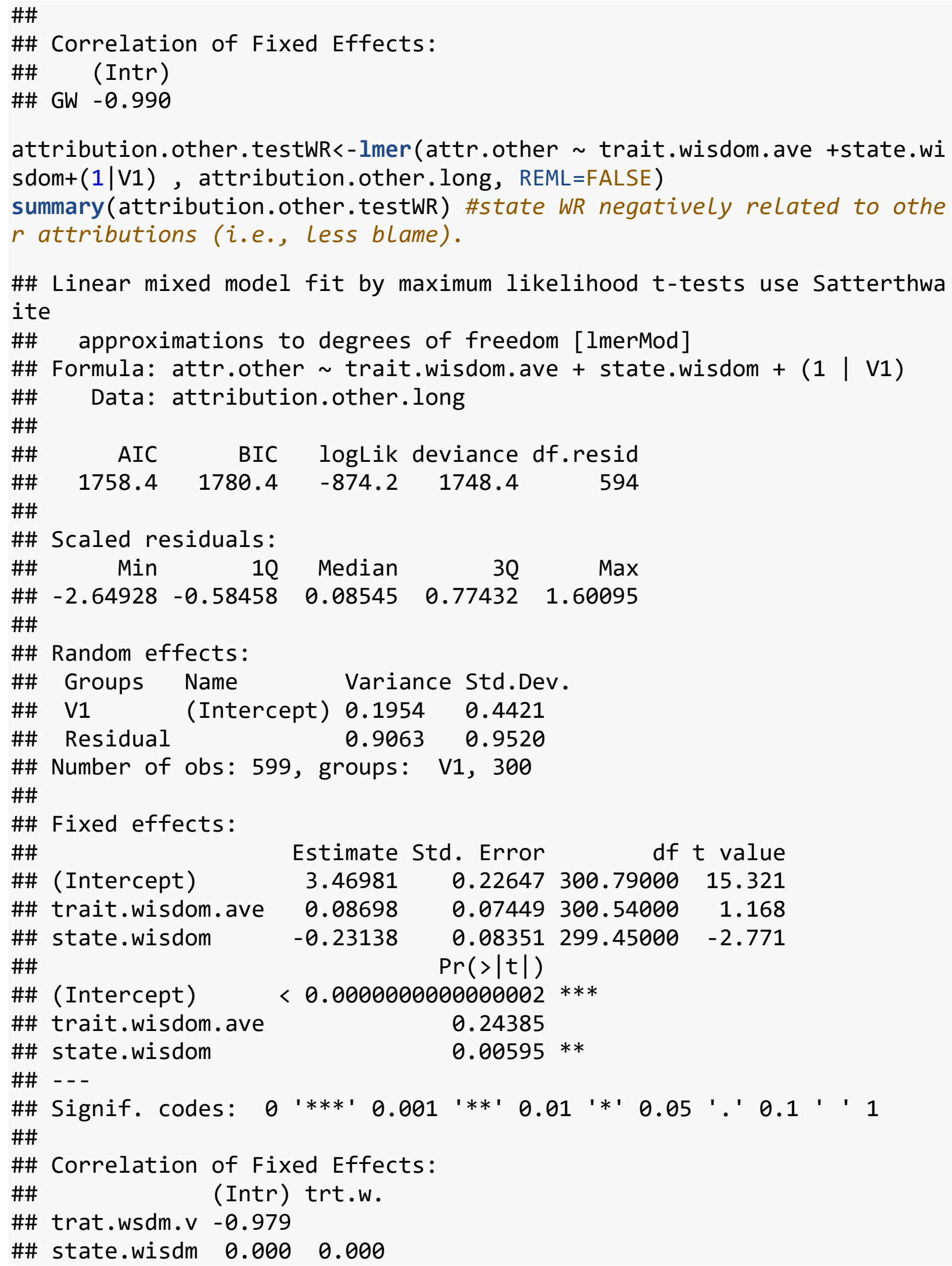


attribution.other.test<-1mer(attr.other GW+trait.wisdom.ave +state.w isdom+(1|V1), attribution.other.long, REML=FALSE)

summary(attribution.other.test) \#\#sig state-level effects of WR on low er attribution to the other party. GW POS linked to attribution to the other party!

\#\# Linear mixed model fit by maximum likelihood t-tests use Satterthwa ite

\#\# approximations to degrees of freedom [lmerMod]

\#\# Formula: attr.other GW + trait.wisdom.ave + state.wisdom + (1 | V

1)

\#\# Data: attribution.other.long

\#\#

\#\#

AIC BIC logLik deviance df.resid

\#\#

1746.7

1773.1

$-867.4 \quad 1734.7$

592

\#\#

\#\# Scaled residuals:

\#\# $\quad$ Min $1 Q$ Median $3 Q \quad$ Max

$\begin{array}{llllll}\text { \#\# } & -2.75171 & -0.53445 & 0.09451 & 0.76037 & 1.67025\end{array}$

\#\#

\#\# Random effects:

\#\# Groups Name Variance Std.Dev.

$\begin{array}{llll}\text { \#\# } & \text { V1 } \quad \text { (Intercept) } & 0.1717 & 0.4144\end{array}$

\#\# Residual 0.90710 .9524

\#\# Number of obs: 598, groups: V1, 299

\#\#

\#\# Fixed effects:

\#\#

$\mathrm{t} \mid)$

$\begin{array}{lllll}\text { \#\# (Intercept) } \quad 2.49227 & 0.36172 & 299.00000 & 6.890 & 0.000000000\end{array}$

$033 * * *$

$\begin{array}{llllll}\text { \#\# GW } & 0.32881 & 0.09683 & 299.00000 & 3.396 & 0.000\end{array}$

$777 * * *$

$\begin{array}{llllll}\text { \#\# trait.wisdom.ave } & 0.03063 & 0.07532 & 299.00000 & 0.407 & 0.684\end{array}$

490

$\begin{array}{llllll}\text { \#\# state.wisdom } & -0.23138 & 0.08355 & 299.00000 & -2.769 & 0.005\end{array}$

$966 * *$

\#\# - - -

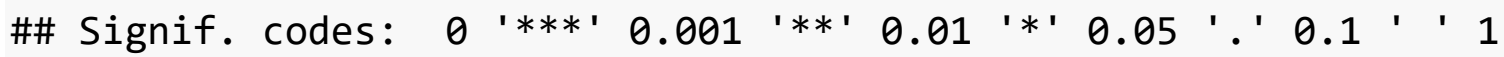

\#\#

\#\# Correlation of Fixed Effects:

\#\# (Intr) GW trt.w.

\#\# GW $\quad-0.788$

\#\# trat.wsdm.v $-0.404-0.232$

$\begin{array}{lllll}\text { \#\# state.wisdm } & 0.000 & 0.000 & 0.000\end{array}$ 
compare models for attributional other

anova(attribution.other.testGW, attribution.other.test) \#sig difference $s$ in model fit delta AIC >2, chi-sq is SIG

\#\# Data: attribution.other.long

\#\# Models:

\#\# object: attr.other GW + (1 | V1)

\#\# ..1: attr.other GW + trait.wisdom.ave + state.wisdom + (1 | V1)

\#\# Df AIC BIC logLik deviance Chisq Chi Df $\operatorname{Pr}(>C h i s q)$

\#\# object $4 \begin{array}{llll}1750.4 & 1768.0 & -871.22 & 1742.4\end{array}$

$\begin{array}{llllllllll}\# \# & . .1 & 6 & 1746.7 & 1773.1 & -867.35 & 1734.7 & 7.7387 & 2 & 0.02087\end{array}$ *

\#\# - - -

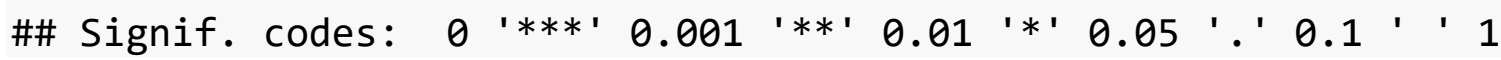

attributions of other's behavior to contextual factors (i.e. situational attributions)

attribution. context. testGW<-1mer(attr.othercontext GW+(1|V1), attri bution. othercontext. long, REML=FALSE)

summary(attribution. context.testGW) \#no effect of GW on context-focuse d attributions

\#\# Linear mixed model fit by maximum likelihood t-tests use Satterthwa ite

\#\# approximations to degrees of freedom [1merMod]

\#\# Formula: attr.othercontext $~ \mathrm{GW}+(1 \mid \mathrm{V} 1)$

\#\# Data: attribution.othercontext.long

\#\#

\#\# AIC BIC logLik deviance df.resid

$\begin{array}{llllll}\text { \#\# } & 1952.4 & 1970.0 & -972.2 & 1944.4 & 594\end{array}$

\#\#

\#\# Scaled residuals:

\#\# Min $1 Q$ Median $30 \quad$ Max

$\begin{array}{llllll}\# \# & -1.95047 & -0.64089 & -0.05869 & 0.65528 & 1.72923\end{array}$

\#\#

\#\# Random effects:

\#\# Groups Name Variance Std.Dev.

$\begin{array}{llll}\text { \#\# V1 } & \text { (Intercept) } & 0.318 & 0.5639\end{array}$

\#\# Residual $\quad 1.227 \quad 1.1079$

\#\# Number of obs: 598, groups: V1, 299

\#\#

\#\# Fixed effects:

\#\# $\quad$ Estimate Std. Error $d f t$ value $\operatorname{Pr}(>|t|)$

$\begin{array}{llllll}\text { \#\# (Intercept) } & 2.85332 & 0.40391 & 299.00000 & 7.064 & 0.0000000000114\end{array}$ $* * *$

\#\# GW

0.08734

0.11498299 .00000

0.760

0.448 


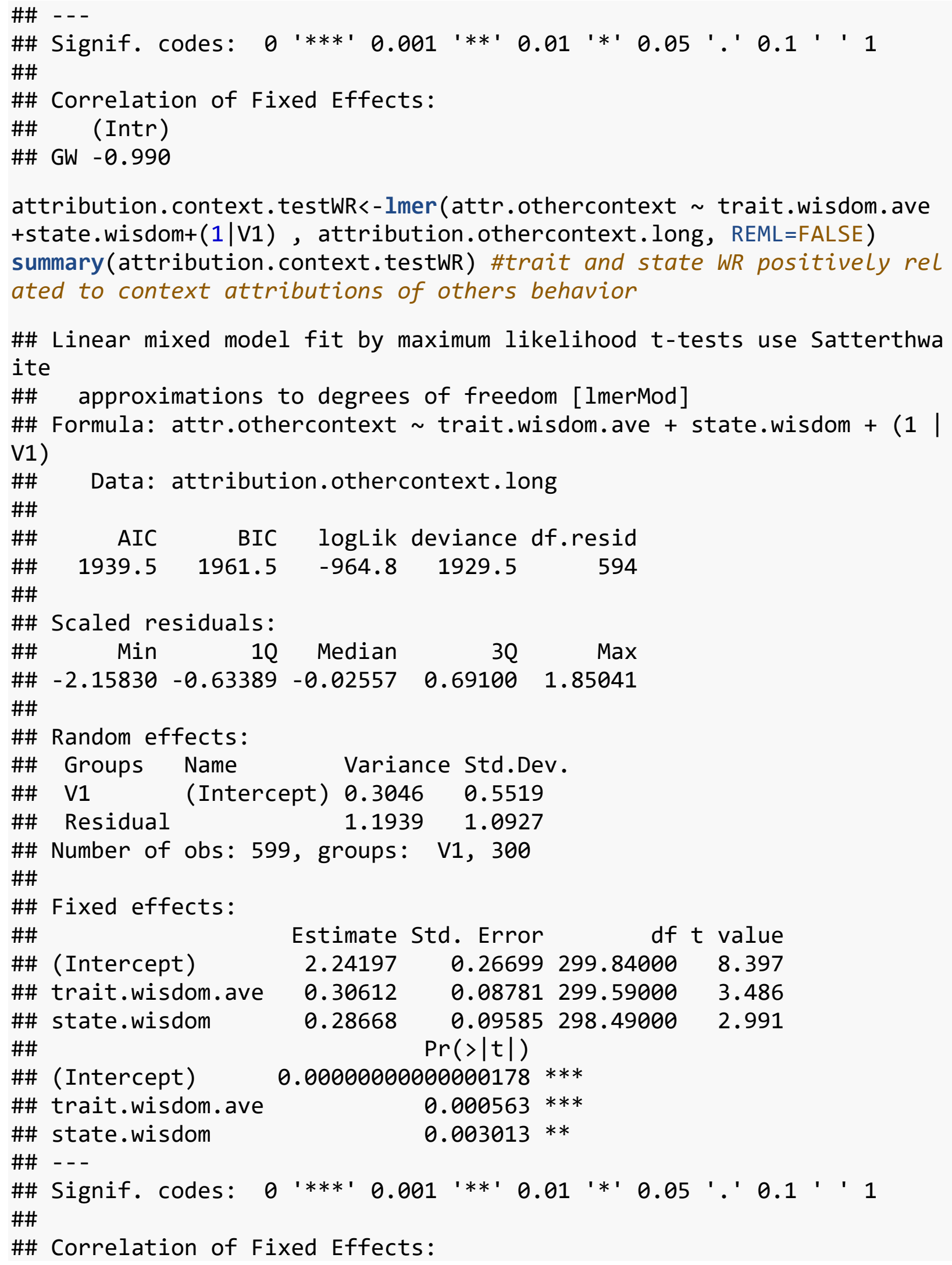




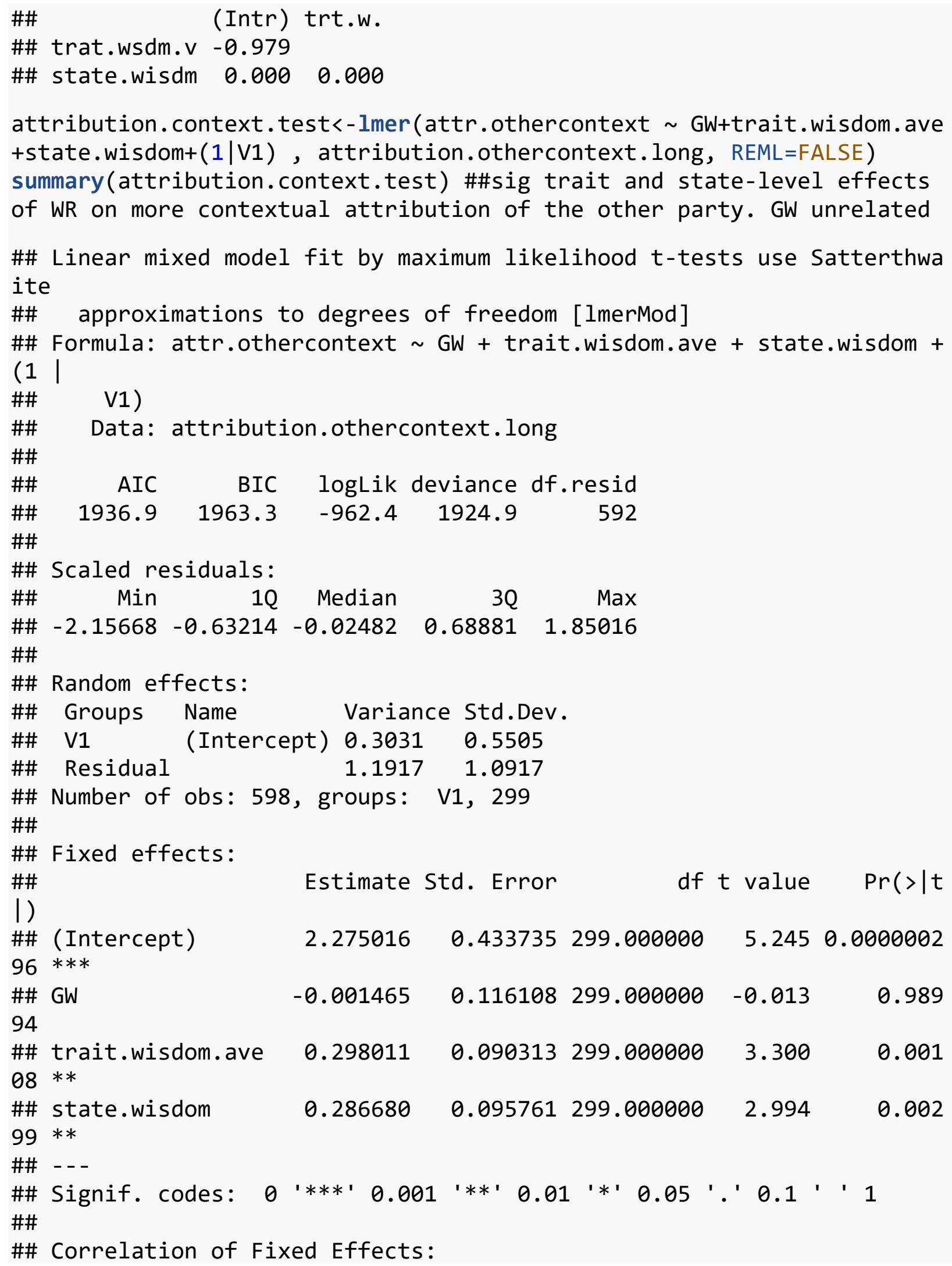




$\begin{array}{lrrr}\text { \#\# } & (\text { Intr) } & \text { GW } & \text { trt.w. } \\ \text { \#\# GW } & -0.788 & & \\ \text { \#\# trat.wsdm.v } & -0.404 & -0.232 & \\ \text { \#\# state.wisdm } & 0.000 & 0.000 & 0.000\end{array}$

compare models for attributional context

anova (attribution. context. testGW, attribution. context. test)

\#\# Data: attribution.othercontext.long

\#\# Models:

\#\# object: attr.othercontext $\sim \mathrm{GW}+(1 \mid \mathrm{V} 1)$

\#\# ..1: attr.othercontext $\sim \mathrm{GW}+$ trait.wisdom.ave + state.wisdom + (1

1

\#\# ...1: V1)

\#\# Df AIC BIC logLik deviance Chisq Chi Df $\operatorname{Pr}$ (>Chisq)

\#\# object 4 1952.4 $1970.0-972.21 \quad 1944.4$

$\begin{array}{lllllllll}\text { \#\# ..1 } & 6 & 1936.9 & 1963.2 & -962.45 & 1924.9 & 19.525 & 2 & 0.00005756\end{array}$ *

**

\#\# -- -

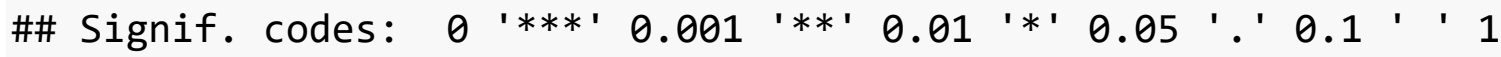

\#sig differences in model fit delta AIC >2, chi-sq is SIG

\#\#\#\# S8: STUDY 8 \#\#\#\#

LOADING PACKAGES

require(psych)

require(car)

require(nFactors)

library (Hmisc)

library(tidyr)

library (dplyr)

library(lme4)

library(lmerTest)

library (apaTables)

library (yarrr)

library (QuantPsyc)

library (ggplot2)

LOADING AND PREPARING FILE

WIS.DEM. RAW<-WIS. RAW

WIS. DEM<-WIS.DEM. RAW[WIS.DEM. RAW\$DupFlag==0, ]

WIS.DEM[WIS.DEM=-99]<-NA \# Removing missing values 
WIS.DEM[WIS.DEM=='NA']<-NA \# Removing NA string

WIS.DEM[WIS.DEM==' ']<-NA \# Removing blank string

ANALYSES FOR DEMOGRAPHICS first, just age and gender, as many vars are missing for other groups.

WIS.DEM\$Age. $\mathrm{Sq}=\mathrm{scale}(\text { WIS.DEM } \$ \text { Age, } \mathrm{scale}=\mathrm{F})^{\wedge} 2$

summary (lm (Normalize (wisdom. ave) scale(Age, scale=F)+Age. sq+gender, data $=$ WIS.DEM))

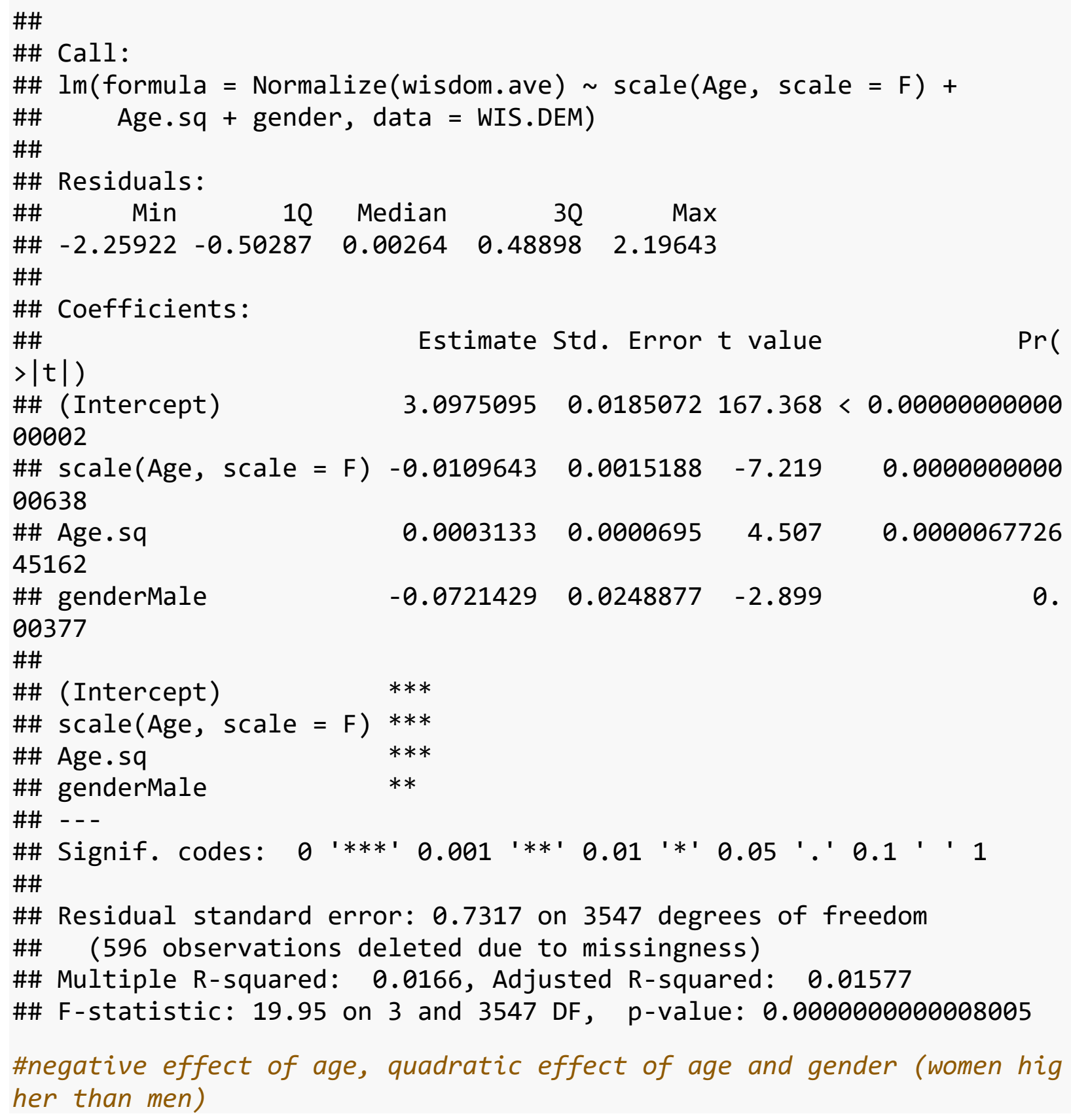


ggplot(WIS.DEM, aes(x=Age, $y=$ wisdom.ave)) +

geom_point (shape=1) + \# Use hollow circles

geom_smooth ()$+$ theme_bw ()$+\operatorname{labs}(y=$ "Wise reasoning: Average across 5 Components") \# Add a loess smoothed fit curve with confidence region $g$ eom_smooth()' using method = 'Loess'

\#\#'geom_smooth()' using method = 'gam'

\#\# Warning: Removed 589 rows containing non-finite values (stat_smooth ) .

\#\# Warning: Removed 589 rows containing missing values (geom_point).

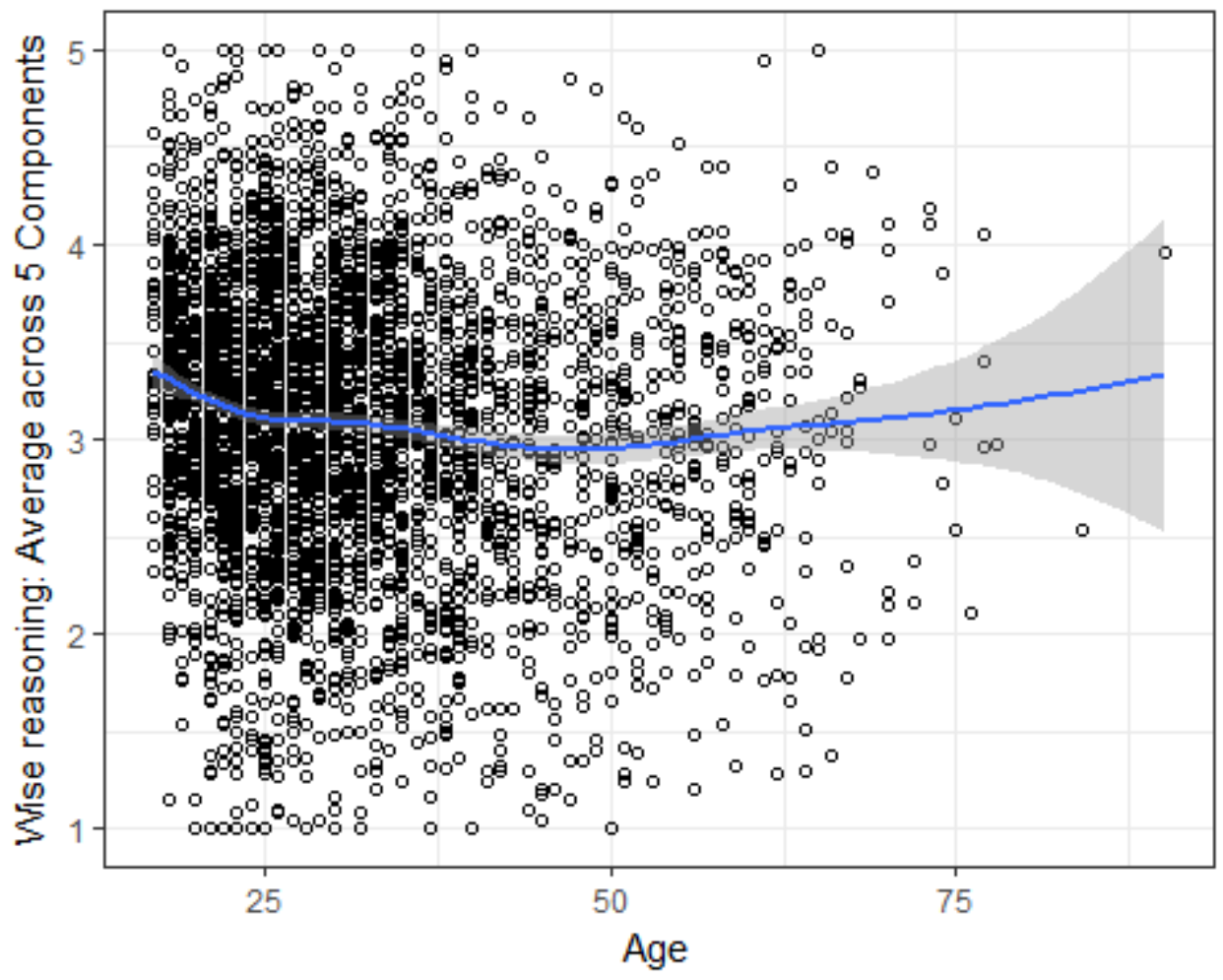

\#neg effect of age on wisdom until middle-aged (until 45) and pos effe ct thereafter

WIS.DEM.old=subset(WIS.DEM, Age > 44)

WIS.DEM.young=subset (WIS.DEM, Age < 45)

WIS.DEM. young $\$$ Age. $s q=$ scale $($ WIS.DEM. young $\$$ Age, $s c a l e=F) \wedge 2$

summary (lm(Normalize (wisdom.ave) Age, data=WIS.DEM.young)) 


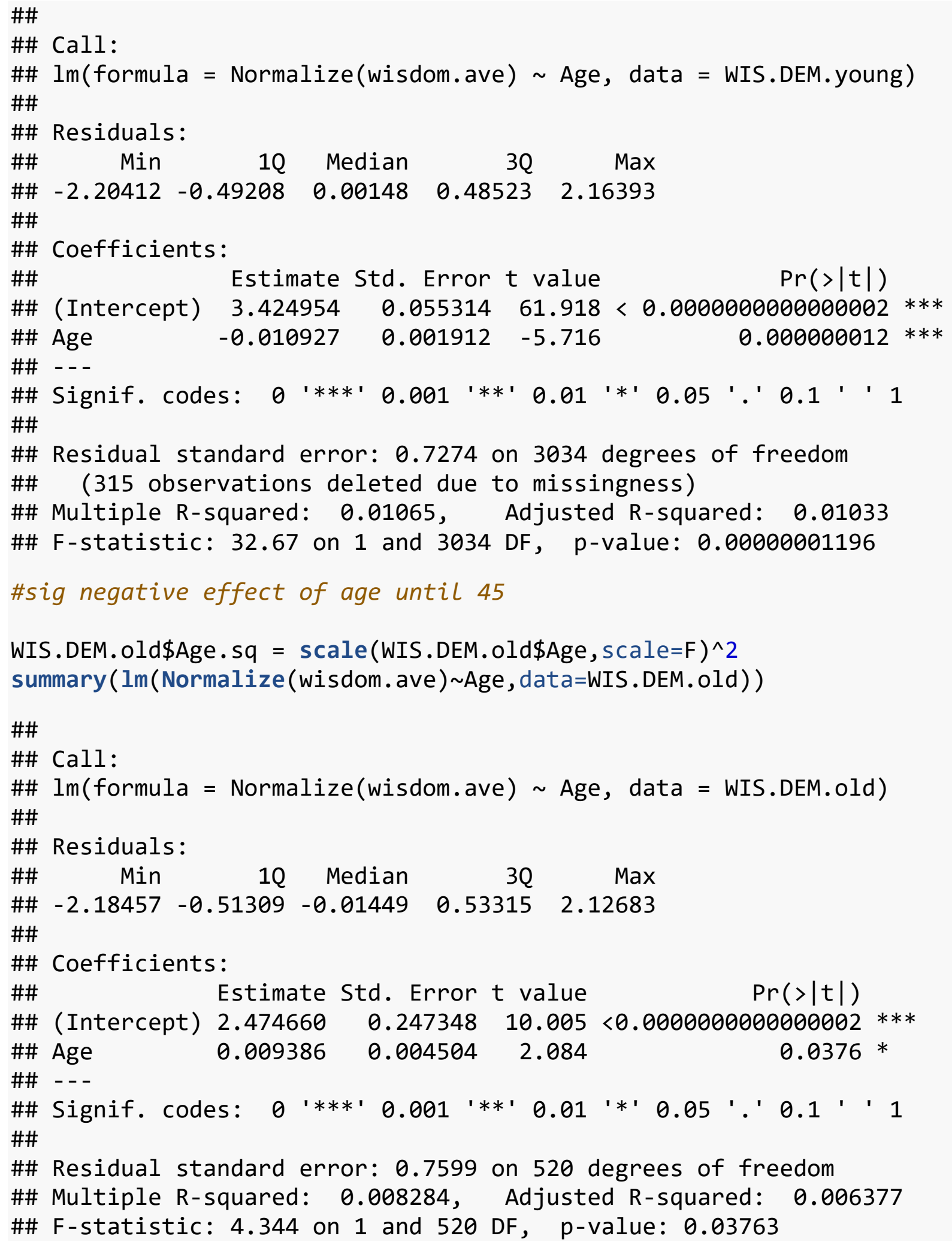


\#sig positive effect of age after 45

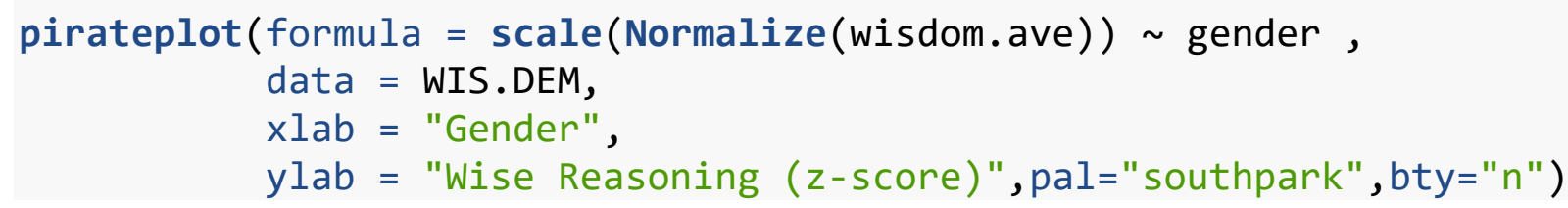

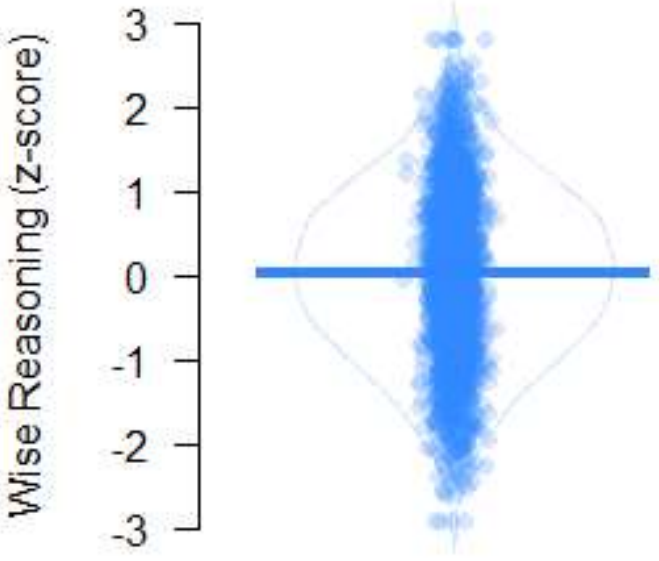

Female

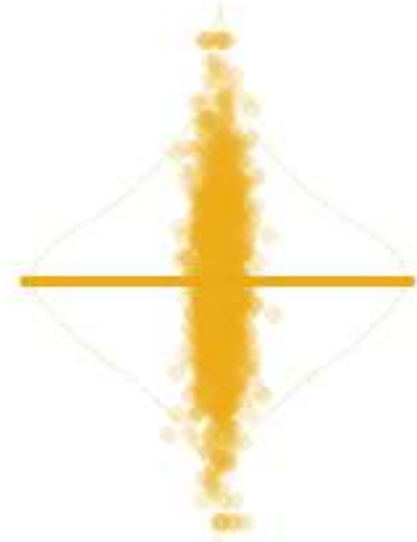

Male

Gender

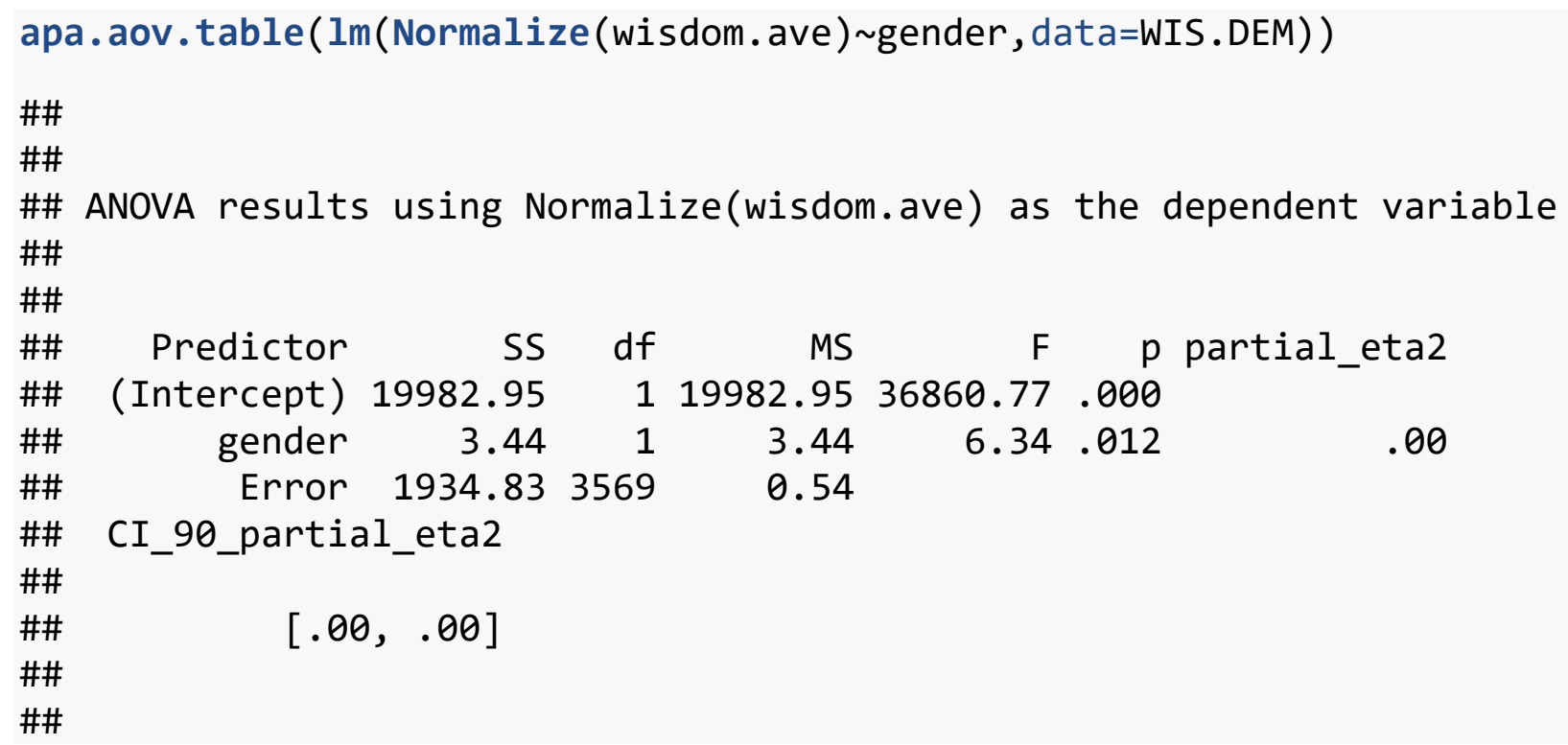

\#\#$$
[.00, .00]
$$

\#\#

\#\# 
\#\# Note: Values in square brackets indicate the bounds of the $90 \%$ conf idence interval for partial eta-squared

WIS.DEM. men=subset (WIS.DEM, gender $=={ }^{\prime M a l e '}$ )

WIS.DEM. women=subset $\left(\right.$ WIS.DEM, gender $=={ }^{\prime}$ Female' )

Stderr <- function $(x) \operatorname{sqrt}(\operatorname{var}(x$, na.rm=TRUE $) /$ length $($ na.omit $(x)))$

psych: : describe(WIS.DEM. men\$wisdom. ave)

\#\# vars $\mathrm{n}$ mean sd median trimmed mad min max range skew kurt osis

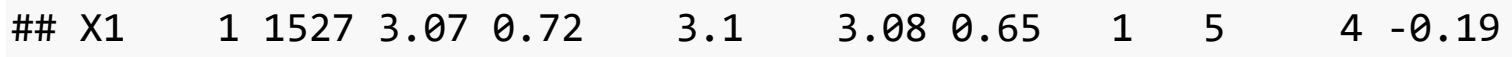

0.27

\#\# se

\#\# X1 0.02

Stderr (WIS.DEM.men\$wisdom.ave)

\#\# [1] 0.01840702

psych: : describe(WIS.DEM. women\$wisdom.ave)

\#\# vars $\mathrm{n}$ mean sd median trimmed mad min max range skew kurt osis

$\begin{array}{lllllllllllll}\text { \#\# X1 } & 1 & 2044 & 3.12 & 0.75 & 3.17 & 3.15 & 0.74 & 1 & 5 & 4 & -0.27 & -\end{array}$ 0.14

\#\# se

\#\# X1 0.02

Stderr (WIS.DEM.women\$wisdom.ave)

\#\#[1] 0.0166868

next, analyses of other demographic variables

\#Ethnicity

apa.aov.table(lm(Normalize(wisdom.ave) Ethnicity, data=WIS.DEM))

\#\#

\#\#

\#\# ANOVA results using Normalize(wisdom.ave) as the dependent variable \#\#

\#\#

\#\# Predictor SS df MS F p partial_eta2

\#\# (Intercept) 2286.16 $12286.16 \quad 4136.44 \quad .000$ 

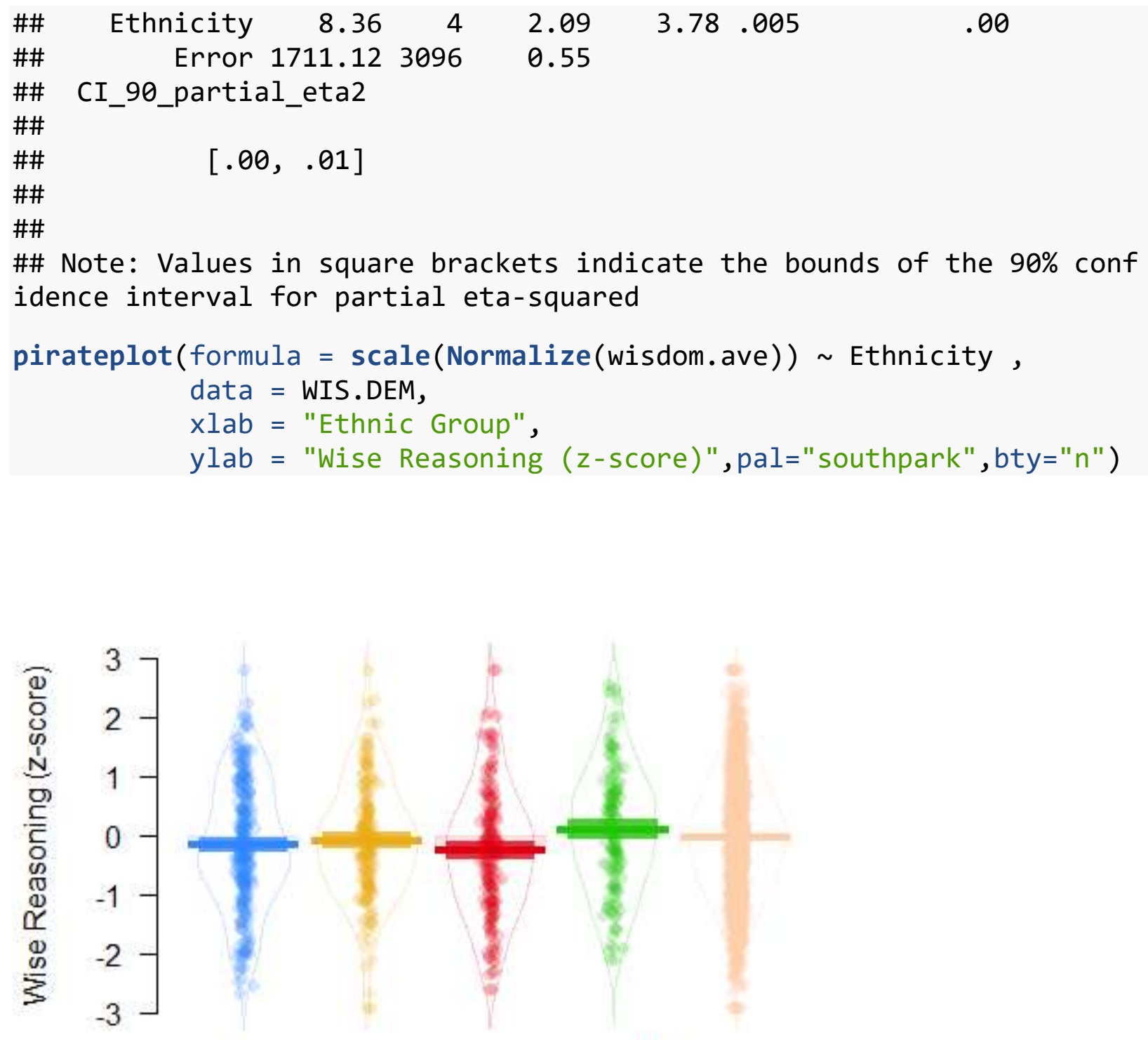

African-AraterigałAmericalnatino Othe/Nhite/European

\section{Ethnic Group}

apa.d.table(Ethnicity, wisdom.ave, WIS.DEM)

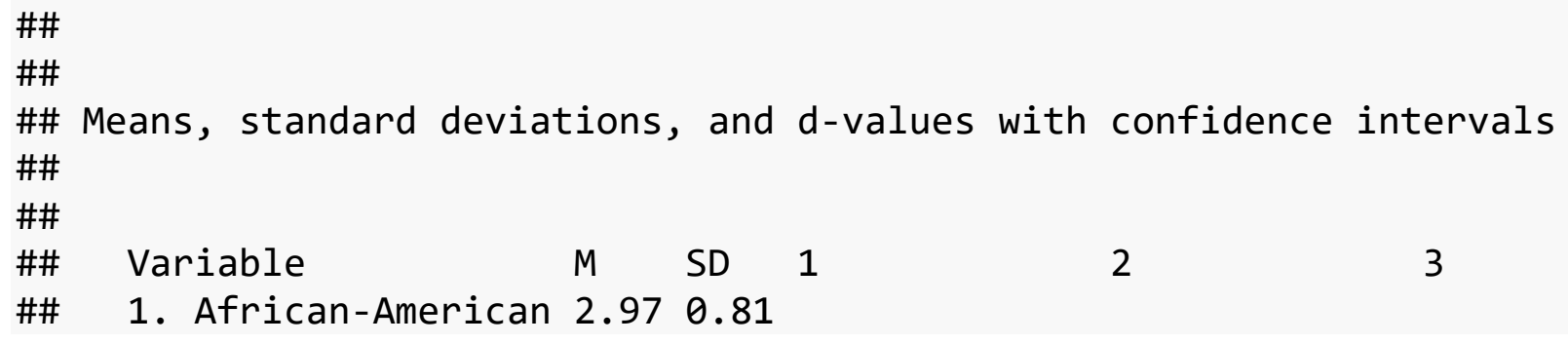




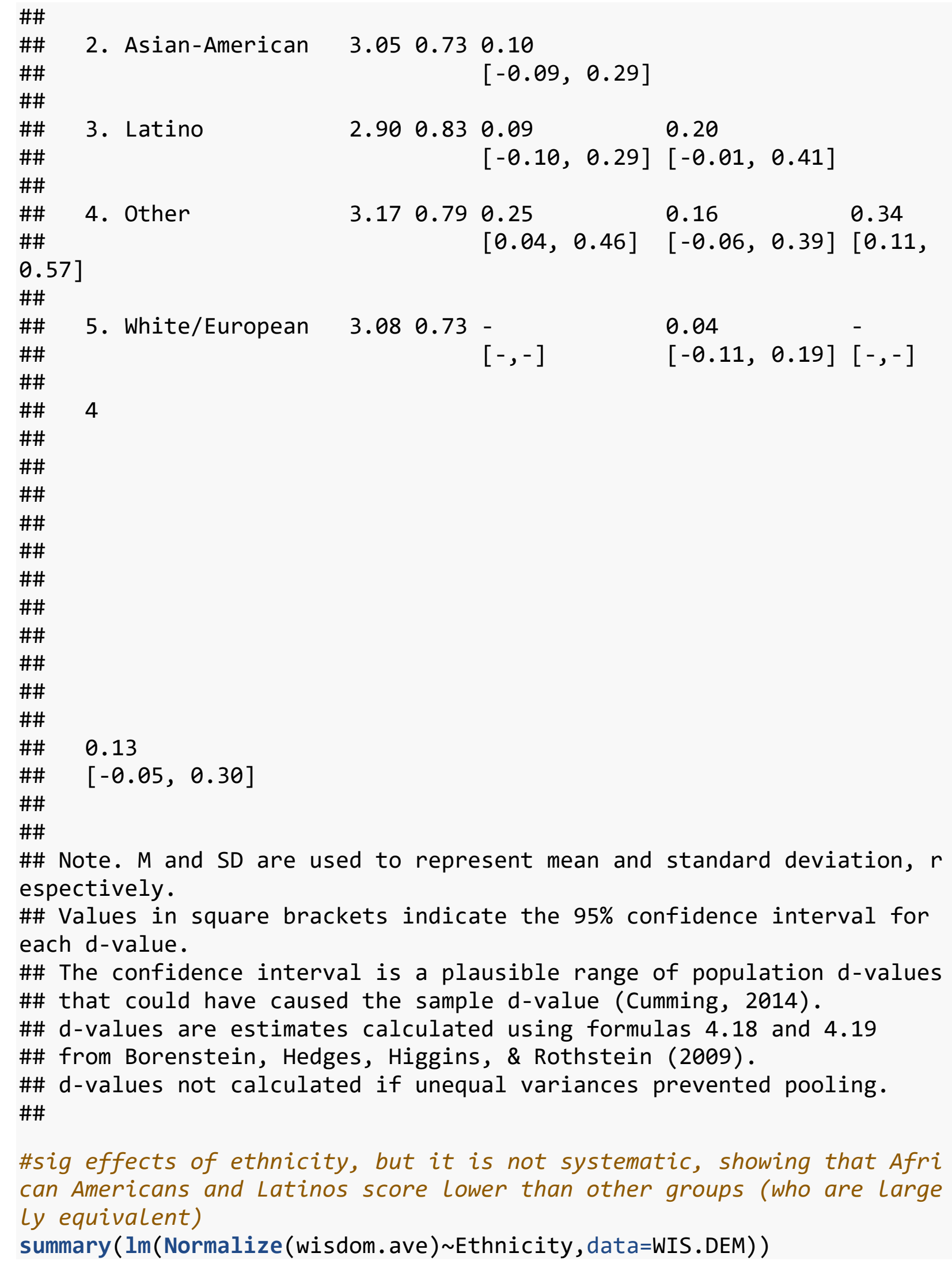




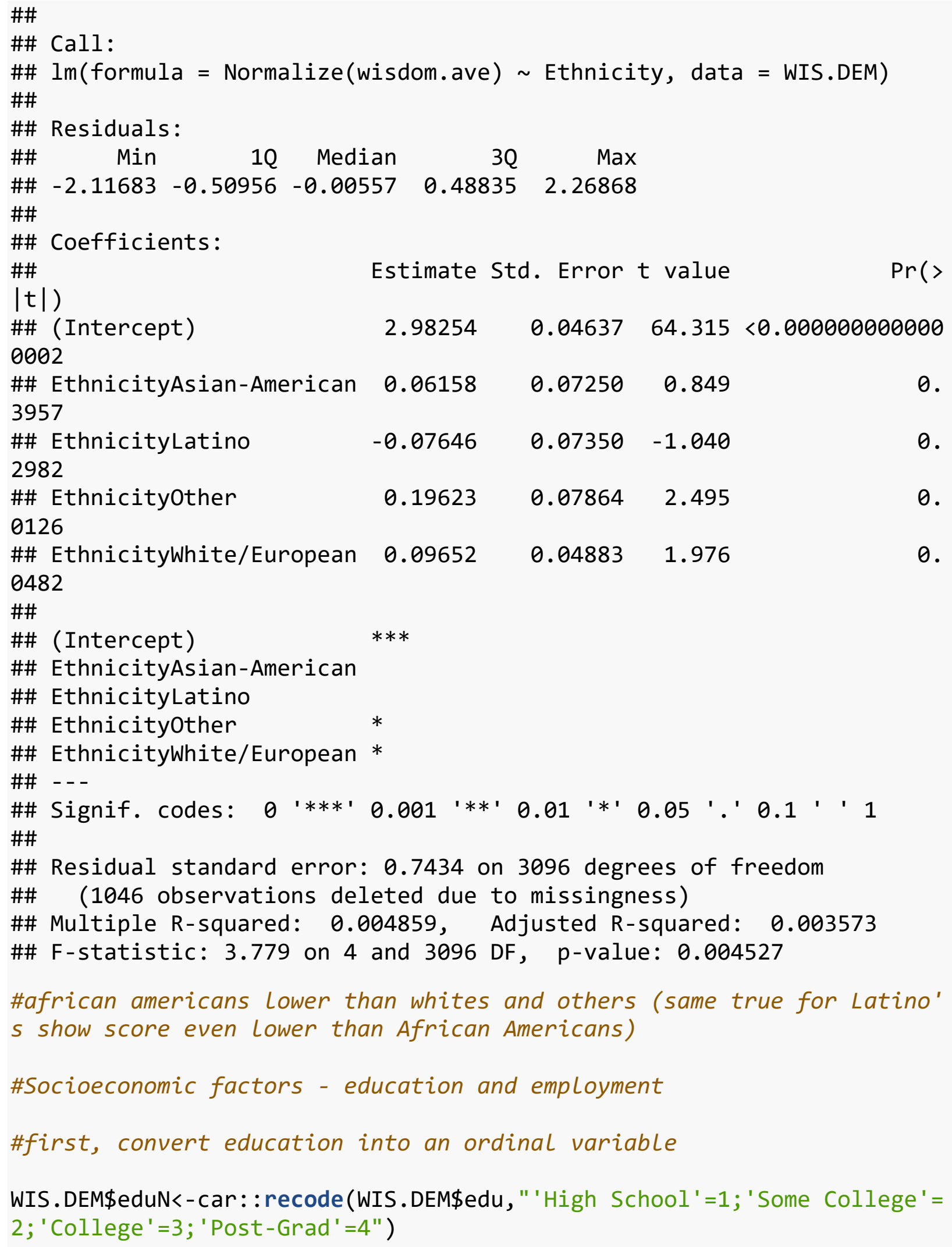




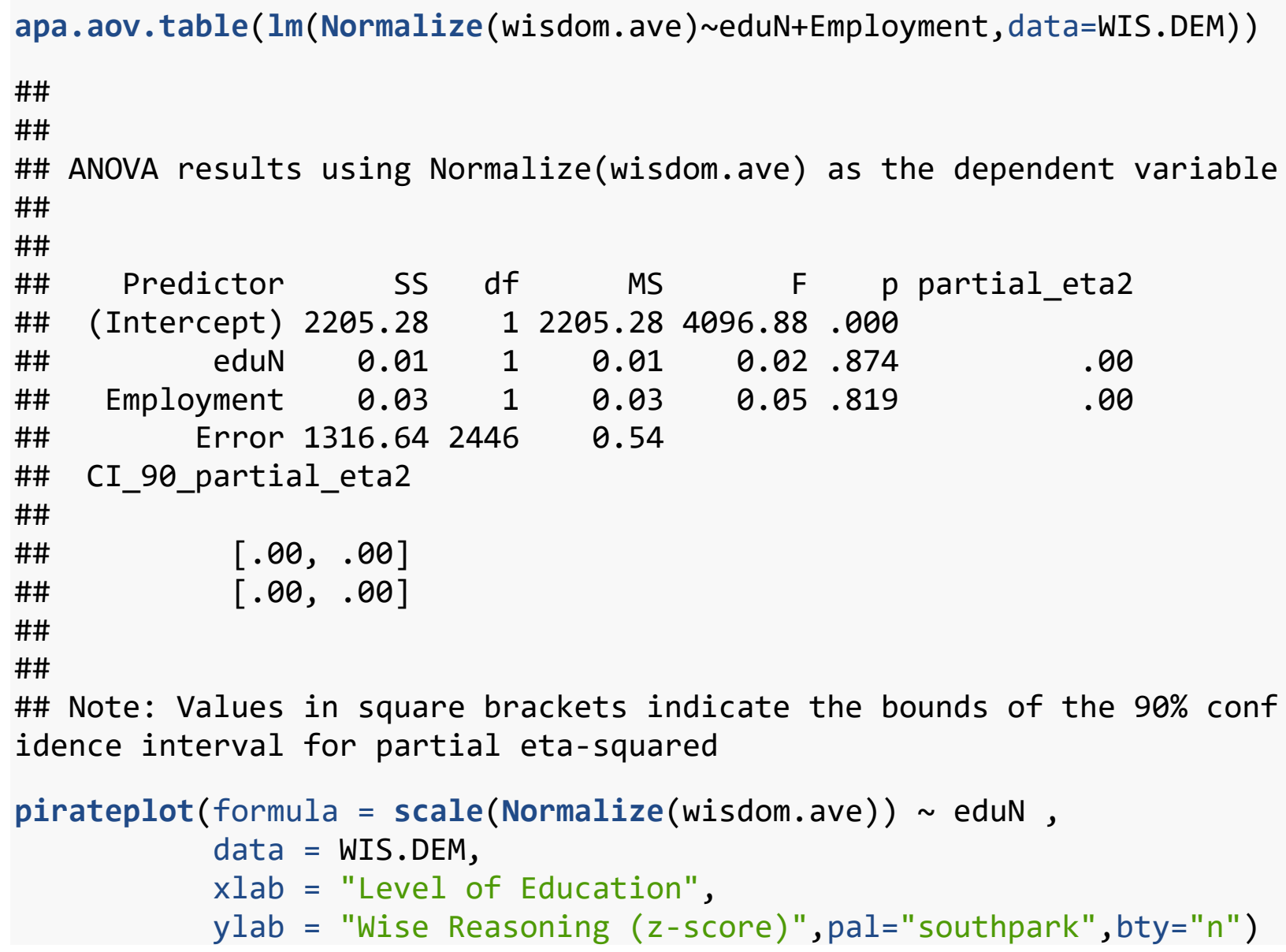




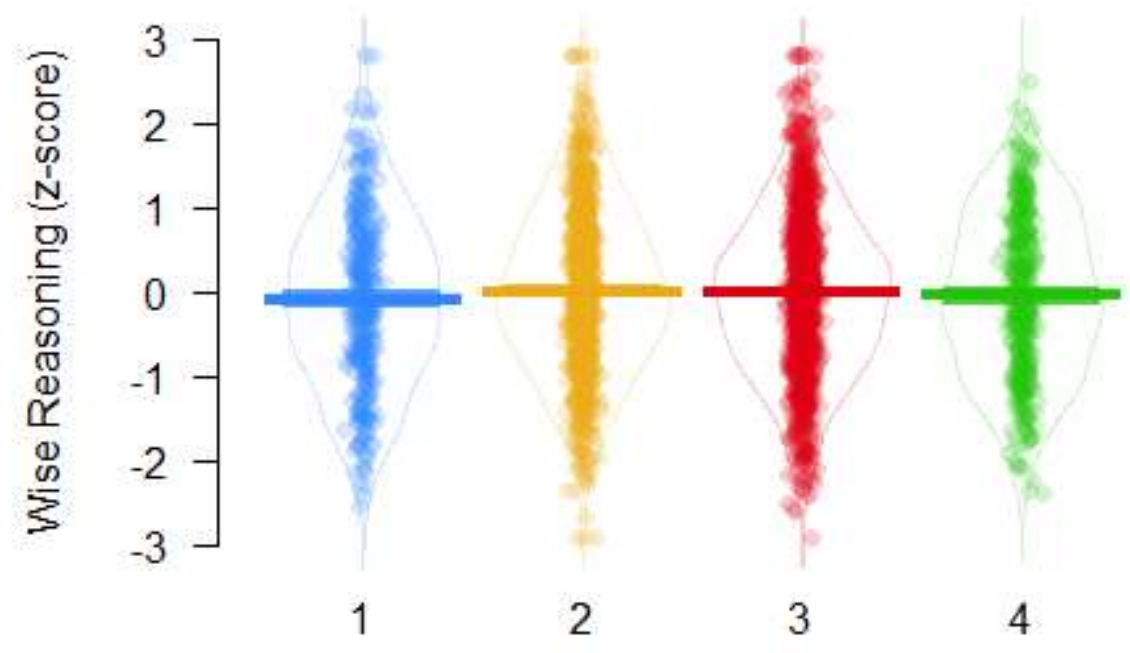

Level of Education

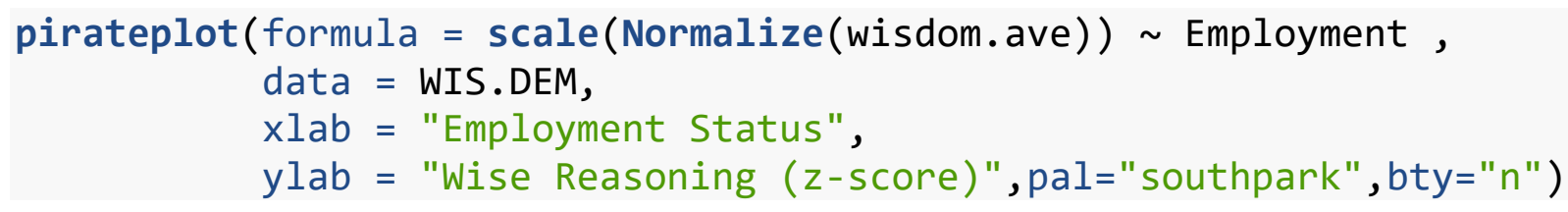




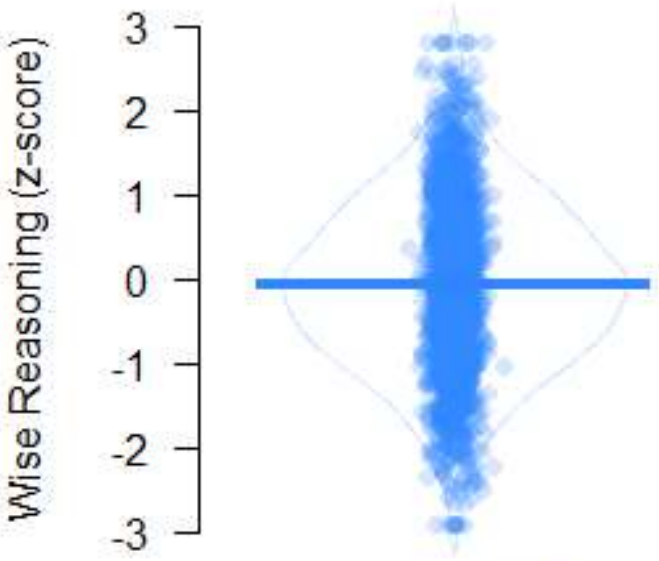

Employed

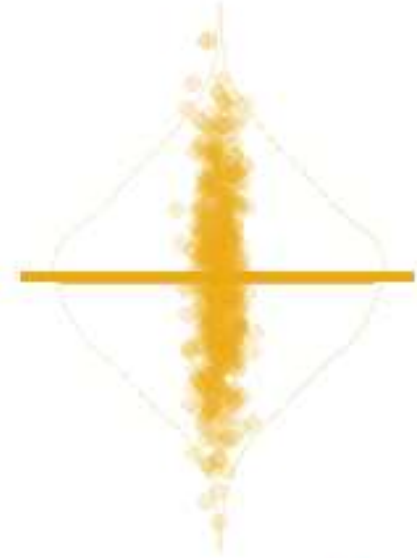

Unemployed

\section{Employment Status}

\#no clear effect of level of education or employment (though, unemploy ed see a tiny bit wiser)

ANALYSES OF CONTEXT FACTORS: Seriousness and Gender of the other person psych: :describe(WIS.DEM[, c('Serious ', 'OtherGender' ')])

\#\# $\quad$ vars $n$ mean sd median trimmed mad min max range

skew

\#\# Serious

$\begin{array}{llll}1 & 2177 & 2.87 & 1.13\end{array}$

3

$2.821 .48 \quad 1 \quad 5$

4

0.37

\#\# OtherGender*

NaN

$228353.00 \quad 0.00$

3

$3.000 .00 \quad 3 \quad 3$

0

\#\#

\#\# Serious

\#\# OtherGender* kurtosis se

$\begin{array}{lll}-0.7 & 0.02\end{array}$

NaN 0.00

WIS.DEM\$OtherGenderX<-car: : recode (WIS.DEM\$OtherGender, " 'Male ' = 'Male ' ; " Female'='Female';3=NA") \#Other person's gender recode (eliminate 3 for the same of analyses)

WIS.DEM. men\$OtherGenderX<-car: : recode (WIS.DEM. men\$OtherGender, " 'Male ' = 'Male'; 'Female' = 'Female';3=NA") \#Other person's gender recode (elimina te 3 for the same of analyses)

WIS.DEM. women\$OtherGenderX<-car : : recode(WIS.DEM. women\$OtherGender, " "Ma 
le'='Male' ; 'Female' = 'Female'; 3=NA") \#Other person's gender recode (eli minate 3 for the same of analyses)

WIS.DEM\$OtherGenderN<-car: : recode(WIS. DEM\$OtherGender, " 'Male ' =0; ' Femal $e^{\prime}=1 ; 3=N A "$ ) \#recode to numeric

WIS.DEM\$genderN<-car: : recode(WIS.DEM\$gender, "'Male'=0; 'Female'=1") \#re code to numeric

summary ( $\mathrm{lm}$ (Normalize(wisdom. ave) scale(OtherGenderN, scale=F)*scale (gen derN, scale=F)+Serious, data=WIS.DEM))

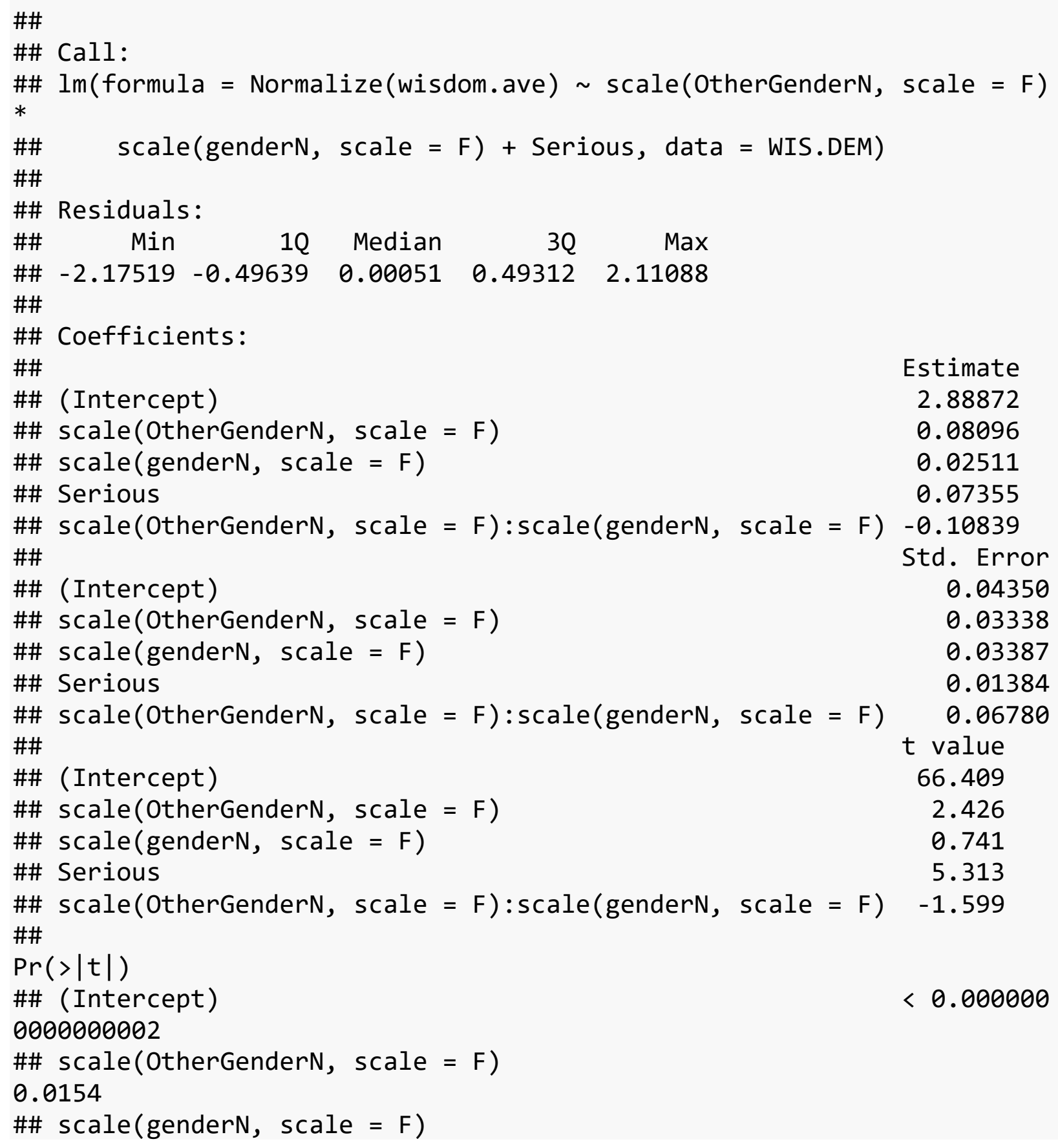




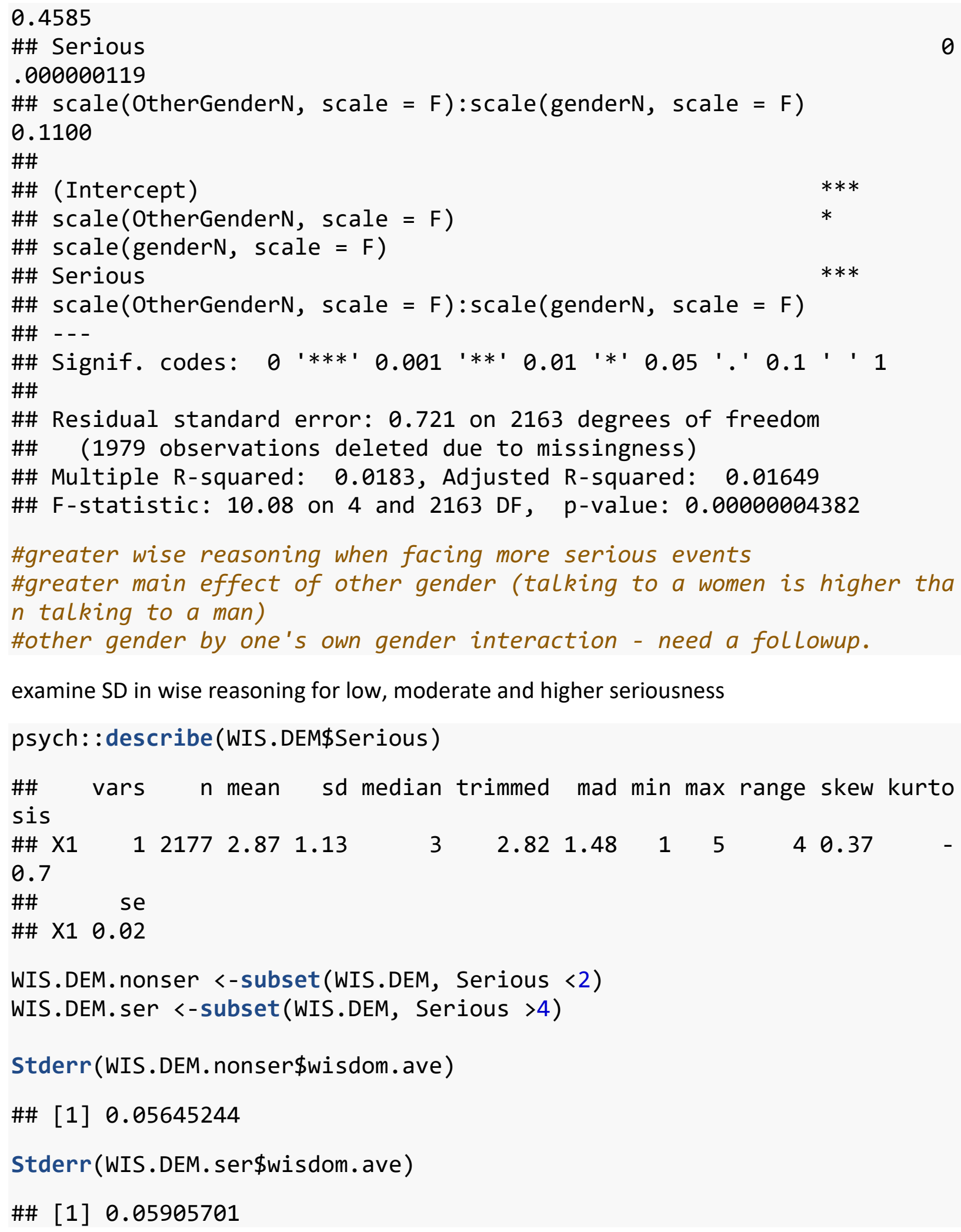


pirateplot $($ formula $=\operatorname{scale}($ Normalize $($ wisdom. ave $)) \sim$ OtherGenderX+gende $r$,

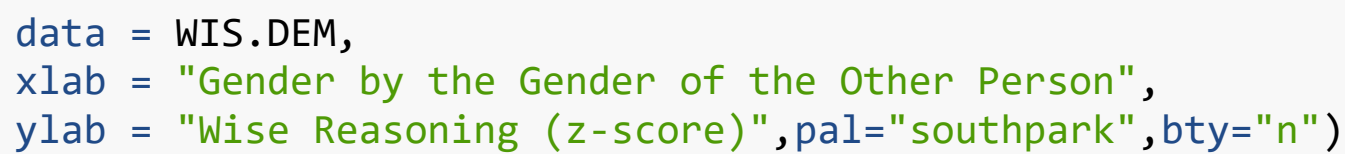

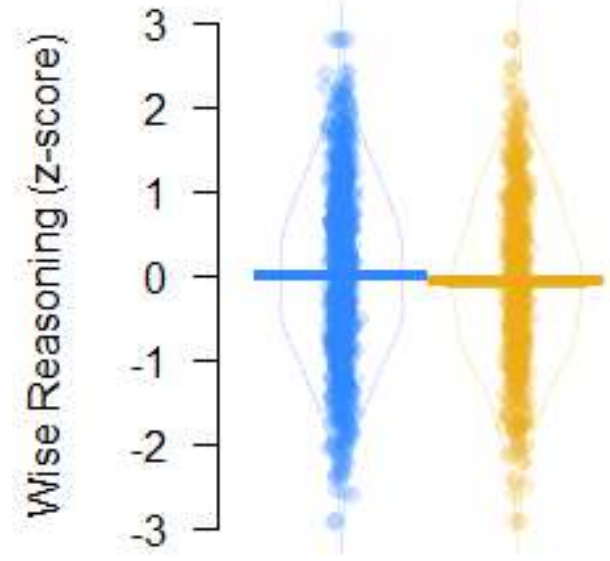

gender

jenderX
Female

Female Male

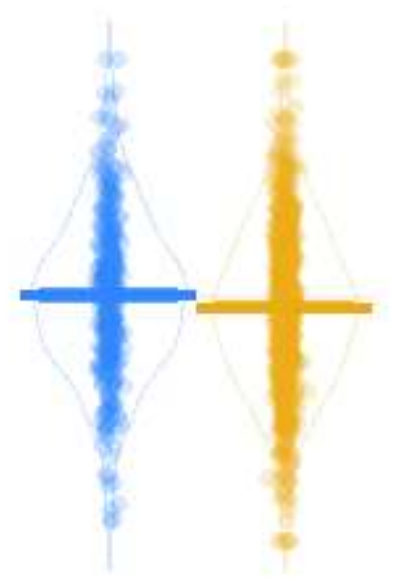

Male

Female Male

\#\#though effect in the same direction, it seems like for women, there is little diff whom they talk to whereas men are wiser when talking to women vs. men (cultural norm?)

\#examine this interaction in more detail by splitting up the sample by gender

summary ( $\operatorname{lm}($ Normalize (wisdom. ave) 0therGenderX, data=WIS.DEM.men)) \#effe ct present for men

\#\#

\#\# Call:

\#\# $\operatorname{lm}($ formula $=$ Normalize(wisdom.ave) $\sim$ OtherGenderX, data = WIS.DEM.m en)

\#\#

\#\# Residuals:

\#\# $\quad$ Min $1 Q$ Median $3 Q \quad$ Max

$\begin{array}{llllll}\# \# & -1.91411 & -0.48107 & -0.00599 & 0.48905 & 1.99979\end{array}$ 


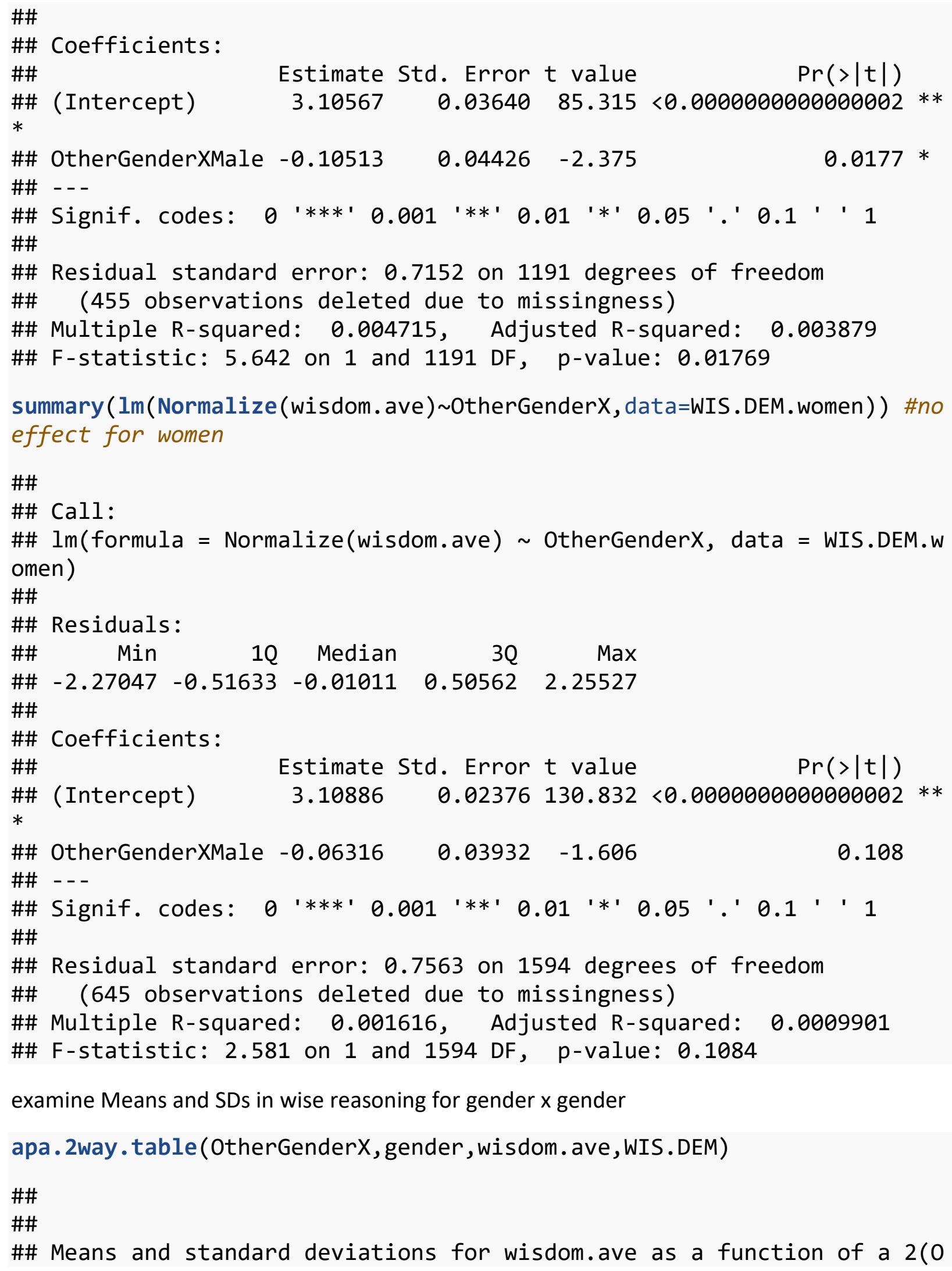




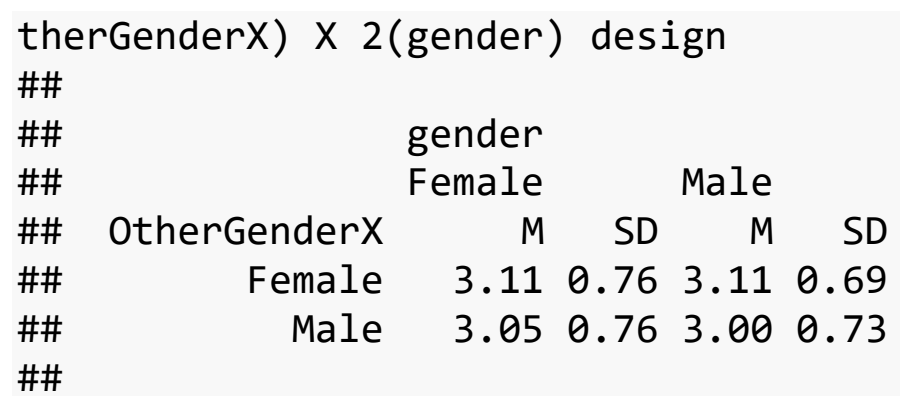

\#\# Note. M and SD represent mean and standard deviation, respectively. describe.by (WIS.DEM\$wisdom. ave, list(WIS.DEM\$gender, WIS.DEM\$OtherGender $\mathrm{X})$ )

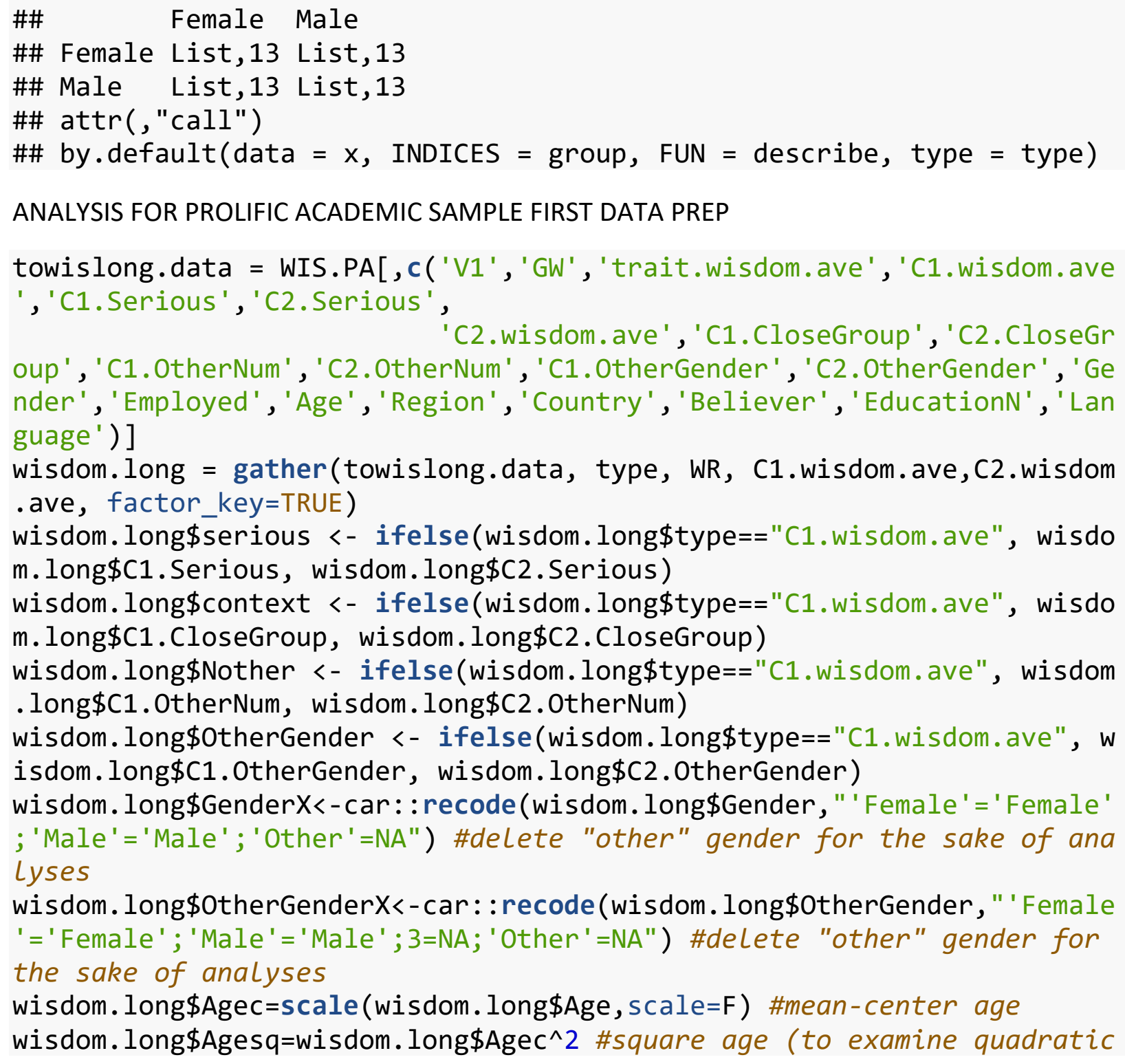




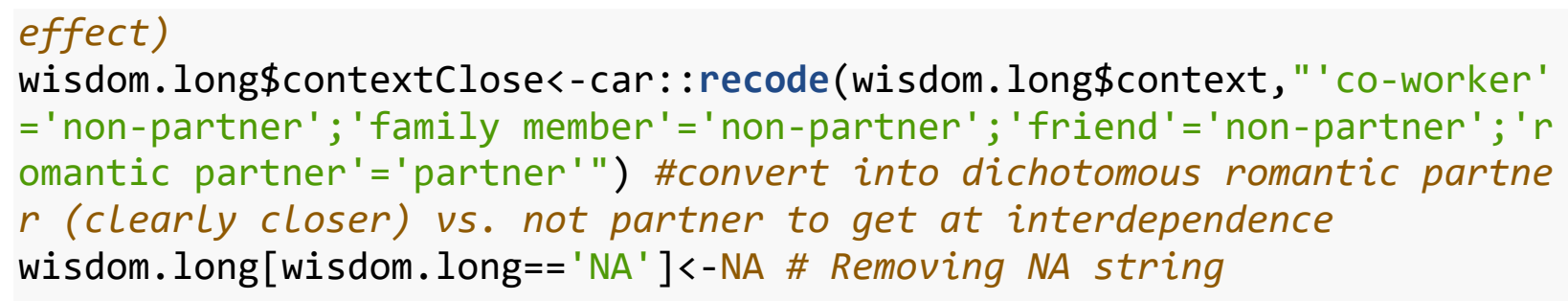

wisdom. long $\$$ EmployedN<-car: : recode (wisdom.long\$Employed, " ' Not employed '=0; 'Employed'=1") \#recode to numeric describe (wisdom. long $\$$ EmployedN)

\#\# wisdom. long\$EmployedN

\#\# $\mathrm{n}$ missing distinct Info Sum Mean Gmd

$\begin{array}{llllllll}\# \# & 600 & 0 & 2 & 0.486 & 478 & 0.7967 & 0.3245\end{array}$

wisdom. long\$OtherGenderN<-car: : recode (wisdom. long\$OtherGenderX, " 'Male ' $=0$; Female'=1") \#recode to numeric

wisdom. long $\$$ Gender $<$-car : : recode (wisdom. long $\$$ GenderX, " 'Male ' =0; 'Female "=1") \#recode to numeric 
wisdom.longUS=subset(wisdom.long, Region=='North America') \#create sub sets of North America (as in prior studies)

wisdom. longEU=subset (wisdom.long, Region=='Europe \& Others') \#create a subset of Europe + a few other countries. wisdom. longUK=subset (wisdom. long, Country== ' UK ' | Country== 'Scotland ' |Co untry==' Ireland '|Country==' England' ) \#create a subset of UK.

examine effects of demographics and social context

summary (Imer (WR +Agec*scale (RegionN, scale=F)+scale(serious, scale=F)* scale (RegionN, scale $=F$ ) +Agesq*scale (RegionN, scale=F)+scale (EducationN, scale $=F) * s c a l e($ RegionN, scale $=F)+$ scale (EmployedN, scale=F)*scale (RegionN , scale $=F$ )+scale (GenderN, scale $=F) * s c a l e($ OtherGenderN, scale $=F) * s c a l e(R e$ gionN, scale $=F)+(1 \mid V 1)$, wisdom.long))

\#\# Linear mixed model fit by REML t-tests use Satterthwaite approximat ions

\#\# to degrees of freedom [1merMod]

\#\# Formula:

\#\# WR +Agec * scale $($ RegionN, scale $=F)+$ scale $($ serious, scale $=F)$

$*$

\#\# $\quad$ scale $($ RegionN, scale $=F)+$ Agesq $* \operatorname{scale}($ RegionN, scale $=F)+$

\#\# $\quad$ scale(EducationN, scale $=\mathrm{F}) * \operatorname{scale}($ RegionN, scale $=\mathrm{F})+$

\#\# $\quad$ scale $($ EmployedN, scale $=F) * \operatorname{scale}($ RegionN, scale $=F)+$

\#\# $\quad$ scale $($ GenderN, scale $=\mathrm{F}) * \operatorname{scale}($ OtherGenderN, scale $=\mathrm{F}) *$

\#\# $\quad$ scale(RegionN, scale = F ) + (1 | V1)

\#\# Data: wisdom.long

\#\#

\#\# REML criterion at convergence: 1408.1

\#\#

\#\# Scaled residuals:

\#\# Min 1 Q Median $3 Q \quad$ Max

$\begin{array}{llllll}\# \# & -2.61083 & -0.61689 & 0.05433 & 0.57105 & 2.99212\end{array}$

\#\#

\#\# Random effects:

\#\# Groups Name Variance Std.Dev.

$\begin{array}{llll}\text { \#\# V1 } & \text { (Intercept) } & 0.1531 & 0.3913\end{array}$

\#\# Residual $\quad 0.43250 .6576$

\#\# Number of obs: 589, groups: V1, 298

\#\#

\#\# Fixed effects:

\#\#

Estimate \#\# (Intercept) 


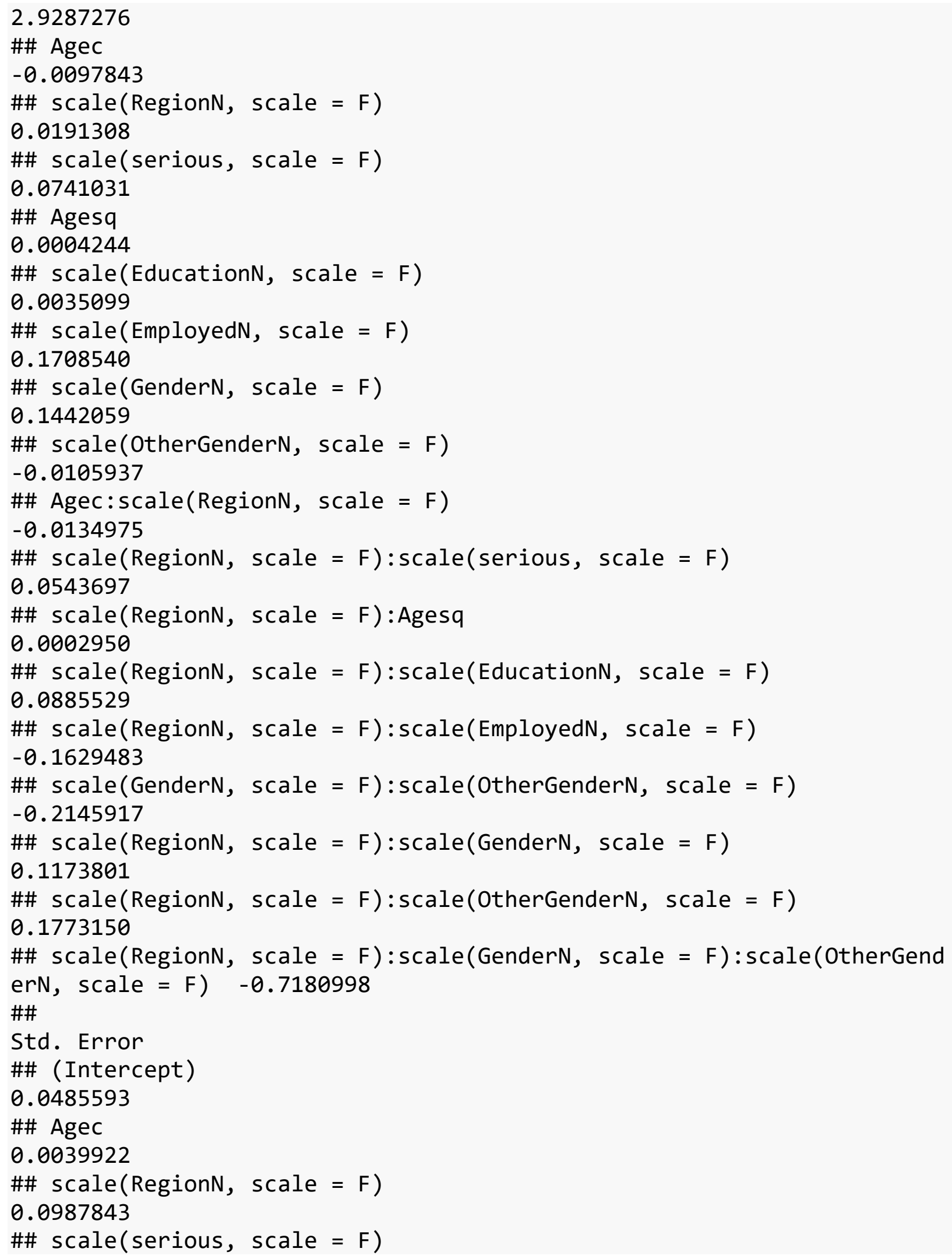




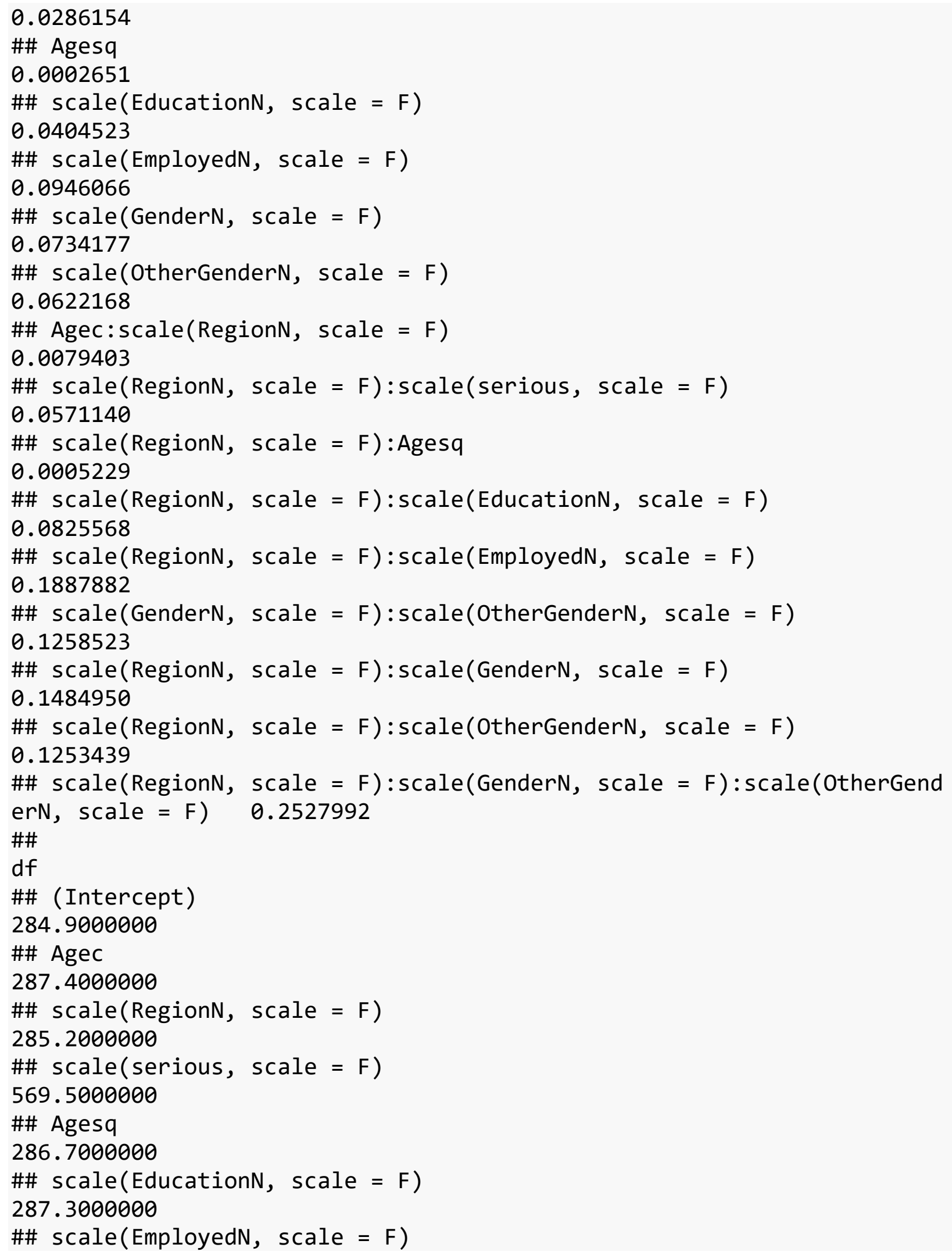




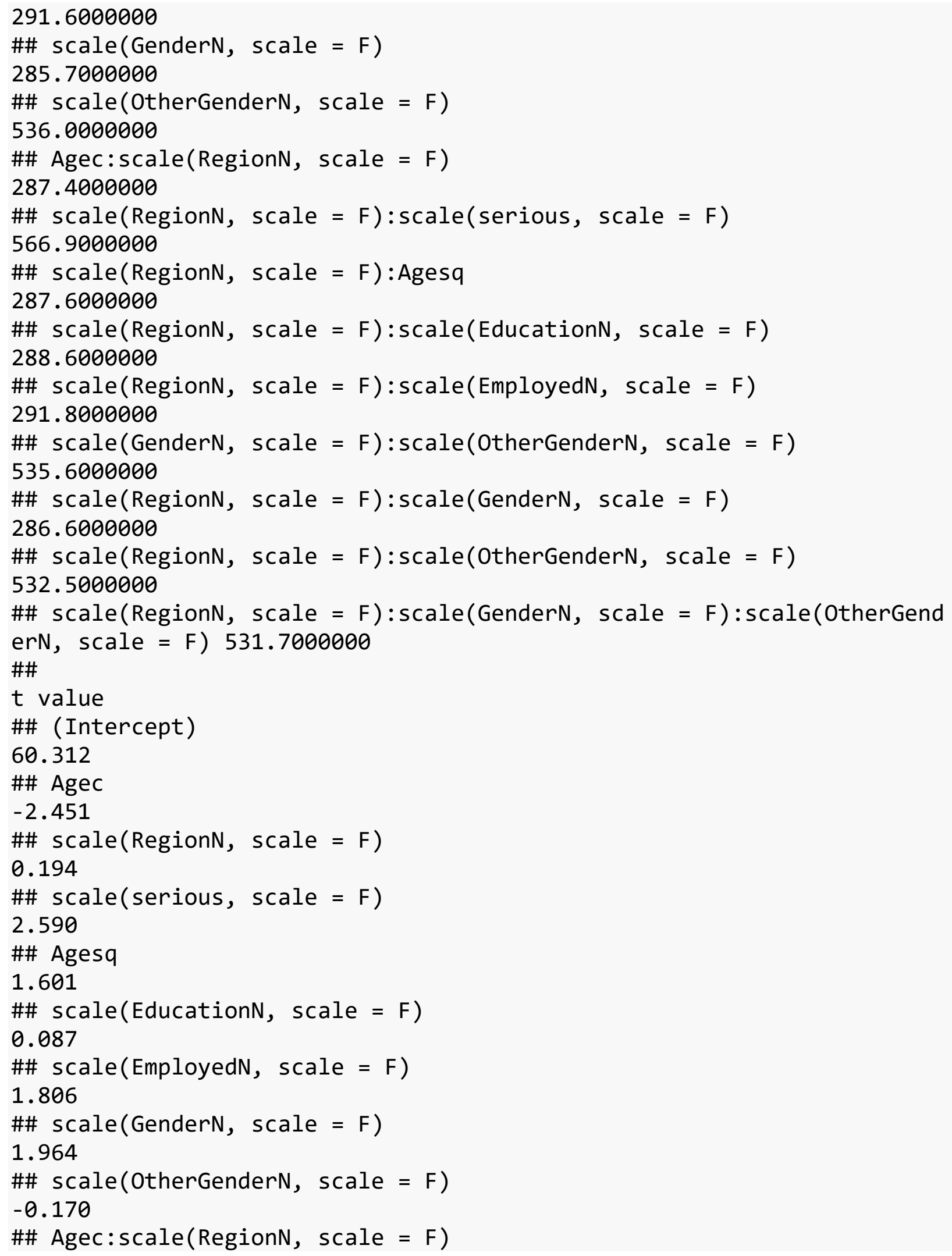


$-1.700$

\#\# scale(RegionN, scale $=F)$ : scale (serious, scale $=F$ )

0.952

\#\# scale(RegionN, scale = F): Agesq

0.564

\#\# scale(RegionN, scale = F): scale (EducationN, scale $=F)$

1.073

\#\# scale (RegionN, scale = F): scale $($ EmployedN, scale $=F)$

$-0.863$

\#\# scale (GenderN, scale = F): scale (OtherGenderN, scale = F)

$-1.705$

\#\# scale(RegionN, scale = F): scale $($ GenderN, scale $=F)$

0.790

\#\# scale (RegionN, scale = F): scale (OtherGenderN, scale $=F$ )

1.415

\#\# scale(RegionN, scale = F): scale (GenderN, scale = F): scale(OtherGend erN, scale = F) -2.841

\#\#

$\operatorname{Pr}(>|t|)$

\#\# (Intercept)

$<0.0000000000000002$

\#\# Agec

0.01485

\#\# scale(RegionN, scale = F)

0.84658

\#\# scale(serious, scale = F)

0.00985

\#\# Agesq

0.11055

\#\# scale (EducationN, scale = F)

0.93092

\#\# scale (EmployedN, scale = F)

0.07196

\#\# scale (GenderN, scale = F)

0.05048

\#\# scale(OtherGenderN, scale = F)

0.86486

\#\# Agec:scale(RegionN, scale = F)

0.09024

\#\# scale(RegionN, scale = F): scale (serious, scale $=F$ )

0.34153

\#\# scale(RegionN, scale = F): Agesq

0.57310

\#\# scale(RegionN, scale = F): scale (EducationN, scale $=F$ ) 


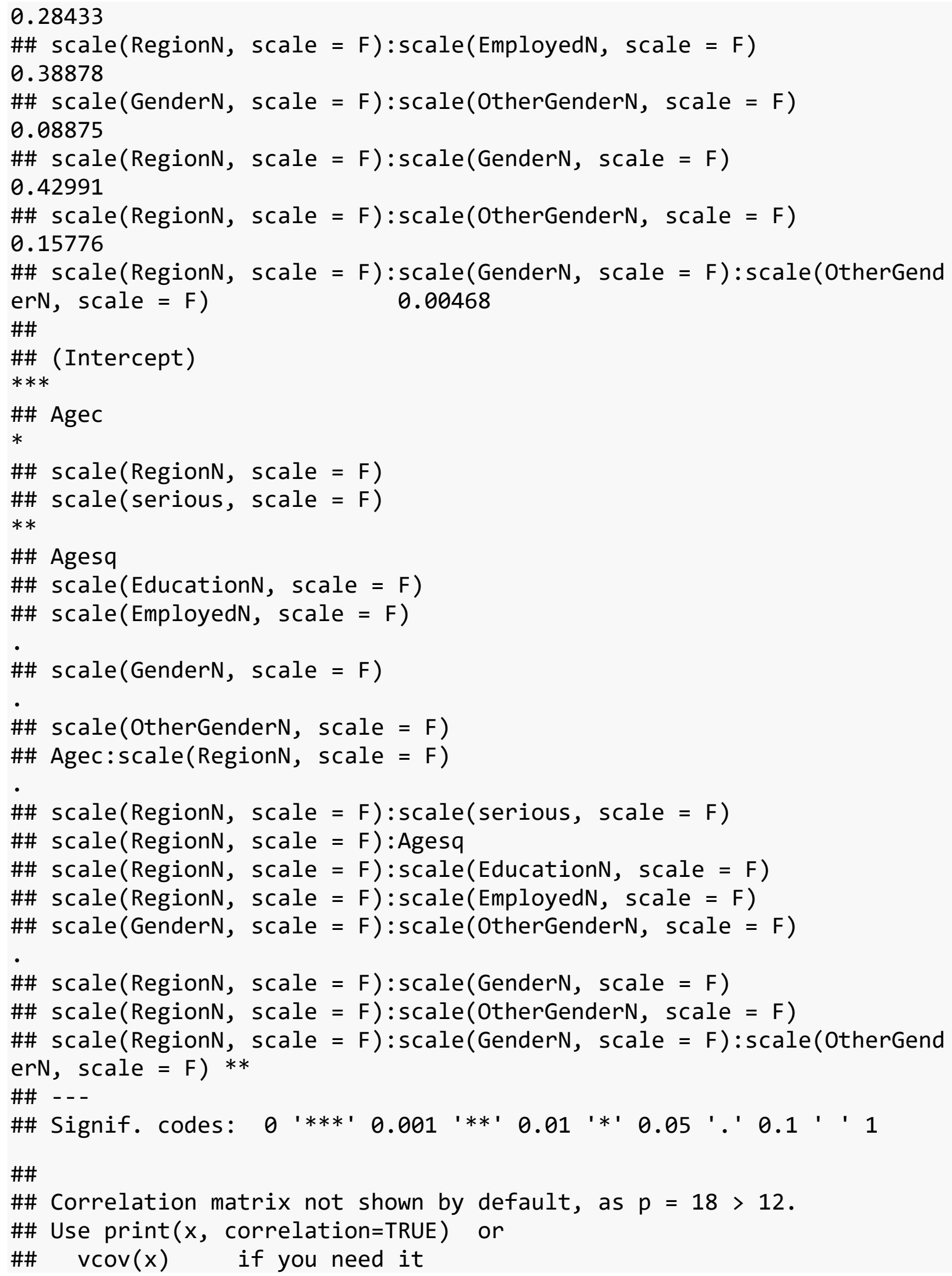


\#\# fit warnings:

\#\# Some predictor variables are on very different scales: consider res caling

str (wisdom. long\$RegionN)

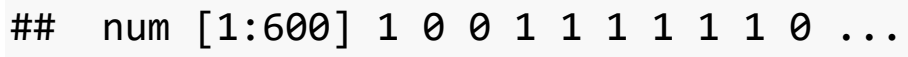

\#effect of seriousness -wiser if more serious

\#negative effect of age, but moderated by region

\#marginal curvilinear effect of age

\#effect of employment - employed score somewhat wiser than unemployed \#effect of gender $x$ gender of the other person, moderated by region

examine trait vs. state effects of seriousness Hypothesis 1: Wiser reasoners are more likely to view events as serious compared to less wise reasoners $==>$ trait $\mathrm{W}$ on seriousness

serious.long = gather(towislong.data, type, serious, C1.Serious, C2.Ser ious, factor_key=TRUE)

serious.long $\$$ wisdom <- ifelse (serious.long $\$$ type=="C1.Serious", serious .long $\$ C 1$. wisdom.ave, serious.long $\$ C 2$.wisdom.ave)

serious. long\$wisdom.trait<-apply(serious. long [, c( 'C1.wisdom.ave ', ' C2.w isdom.ave')], 1, mean)

serious. long\$wisdom. state<-serious. long\$wisdom - serious. long\$wisdom.t rait

serious.longs<-subset( serious.long, wisdom.state!='NA' )

summary(lmer(serious wisdom.trait+wisdom.state+(1|V1), serious.longS ))

\#\# Linear mixed model fit by REML t-tests use Satterthwaite approximat ions

\#\# to degrees of freedom [lmerMod]

\#\# Formula: serious wisdom.trait + wisdom.state + (1 | V1)

\#\# Data: serious.longs

\#\#

\#\# REML criterion at convergence: 1817.5

\#\#

\#\# Scaled residuals:

\#\# Min $1 Q$ Median $3 Q \quad$ Max

$\begin{array}{llllll}\# \# & -1.9513 & -0.5745 & -0.2288 & 0.4997 & 2.3931\end{array}$

\#\#

\#\# Random effects:

\#\# Groups Name Variance Std.Dev.

$\begin{array}{llll}\text { \#\# V1 } \quad \text { (Intercept) } & 0.2588 & 0.5087\end{array}$

$\begin{array}{lll}\text { \#\# Residual } & 0.9888 & 0.9944\end{array}$ 
\#\# Number of obs: 596, groups: V1, 299

\#\#

\#\# Fixed effects:

\#\#

$|t|)$

Estimate Std. Error

$d f$ value

$\operatorname{Pr}(>$

\#\# (Intercept)

2.27619

0.24471296 .74000

$9.302<0.000000000000$

0002

\#\# wisdom.trait

0.09717

0.08047296 .94000

1.208

$\theta$.

2282

\#\# wisdom.state

0.18930

0.08726297 .22000

2.169

$\theta$.

0308

\#\#

\#\# (Intercept)

\#\# wisdom.trait

\#\# wisdom.state *

\#\# ---

\#\# Signif. codes: $0{ }^{\prime * * *}$ ' $0.0011^{\prime * *}, 0.011^{\prime *} 0.05$ '.' 0.1 ' 1

\#\#

\#\# Correlation of Fixed Effects:

\#\# (Intr) wsdm.t

\#\# wisdom.trat -0.979

\#\# wisdom.stat $-0.001 \quad 0.001$

anova(lmer(serious wisdom.state+(1|V1), serious.longs), lmer(serious $\sim$ wisdom.trait+wisdom.state+(1|V1), serious.longS))

\#\# refitting model(s) with ML (instead of REML)

\#\# Data: serious.longs

\#\# Models:

\#\# object: serious wisdom.state $+(1 \mid \mathrm{V} 1)$

\#\# ..1: serious wisdom.trait + wisdom.state + (1 | V1)

\#\# Df AIC BIC logLik deviance Chisq Chi Df $\operatorname{Pr}$ (>Chisq)

\#\# object $4 \begin{array}{llll}1816.6 & 1834.2 & -904.30 & 1808.6\end{array}$

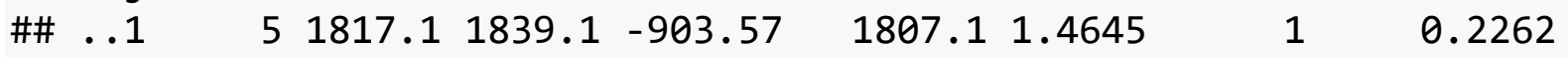

\#only effect of state wisdom, suggesting that trait wisdom does not un iquely contribute to seriousness

\#rather, the effect is situational.

hypothesis 2: Serious situations call for greater wisdom $==>$ state seriousness on WR

wisdom. long\$serious.trait<-apply (wisdom. long [ , c('C1.Serious ' , 'C1. Serio us') ], 1, mean)

wisdom. long\$serious.state<-wisdom. long\$serious - wisdom.long\$serious.t rait 


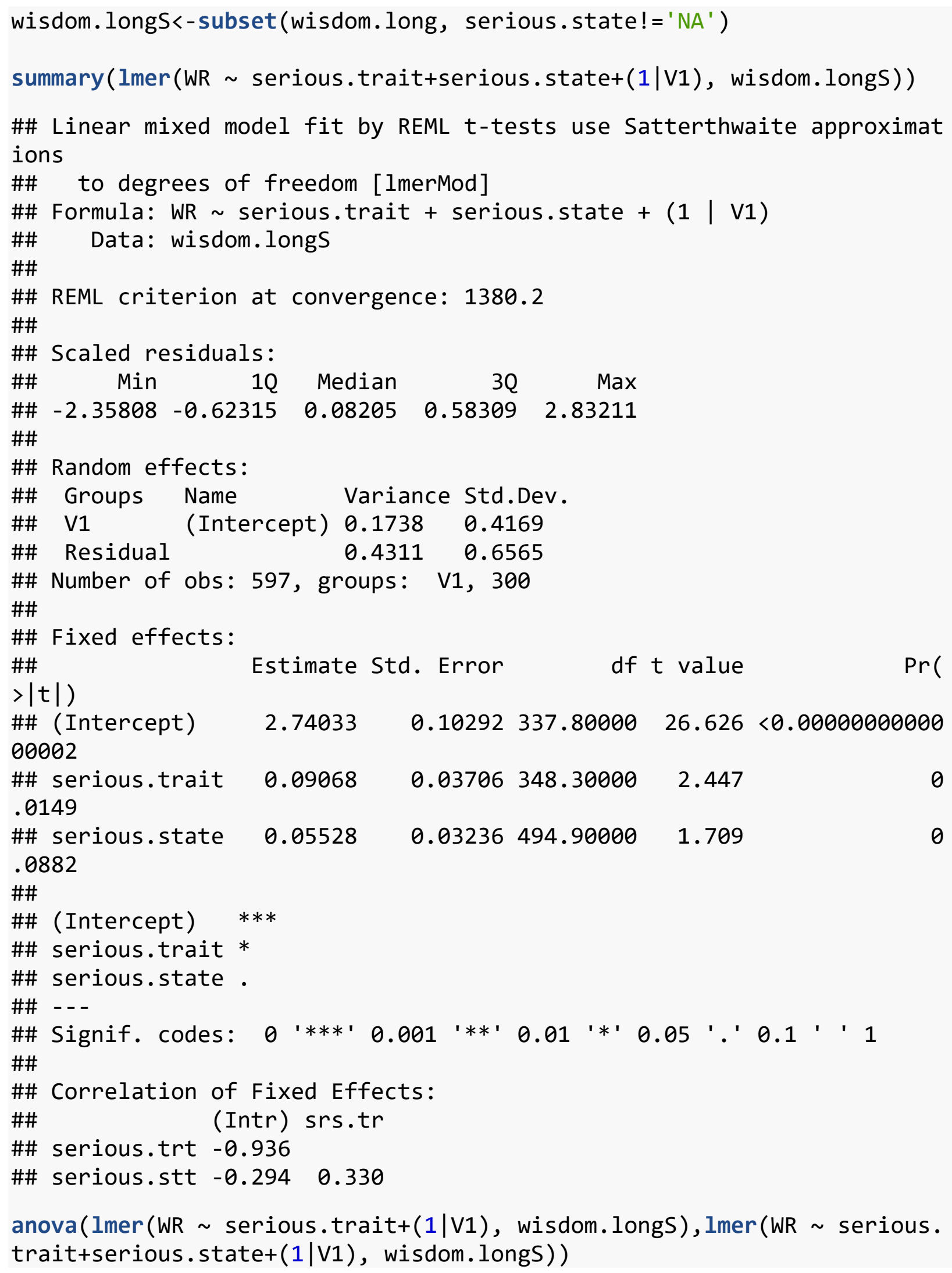


\#\# refitting model(s) with ML (instead of REML)

\#\# Data: wisdom.longs

\#\# Models:

\#\# object: WR serious.trait + (1 | V1)

\#\# ..1: WR serious.trait + serious.state + (1 | V1)

\#\# Df AIC BIC logLik deviance Chisq Chi Df Pr(>Chisq)

\#\# object 4 1376.4 1393.9 -684.18 1368.4

$\begin{array}{llllllllll}\text { \#\# } & \ldots 1 & 5 & 1375.5 & 1397.4 & -682.74 & 1365.5 & 2.8859 & 1 & 0.08936\end{array}$.

\#\# - - -

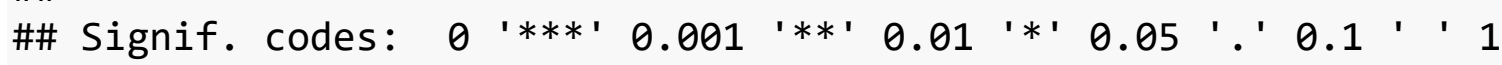

\#both trait and state components of seriousness for WR - trait compone nt stronger than state

\#adding state seriousness marginally contributes to WR

Age Analyses

ggplot (wisdom.long, aes $(x=$ Age, $y=W R))+$ geom_point (shape=1) + \# Use hollow circles geom_smooth ()$+$ theme_bw( $)+$ labs $(y=$ "Wise reasoning: Average across

5 Components") \# Add a loess smoothed fit curve with confiden ce region geom_smooth()' using method = 'Loess'

\#\#'geom_smooth()' using method = 'loess' 


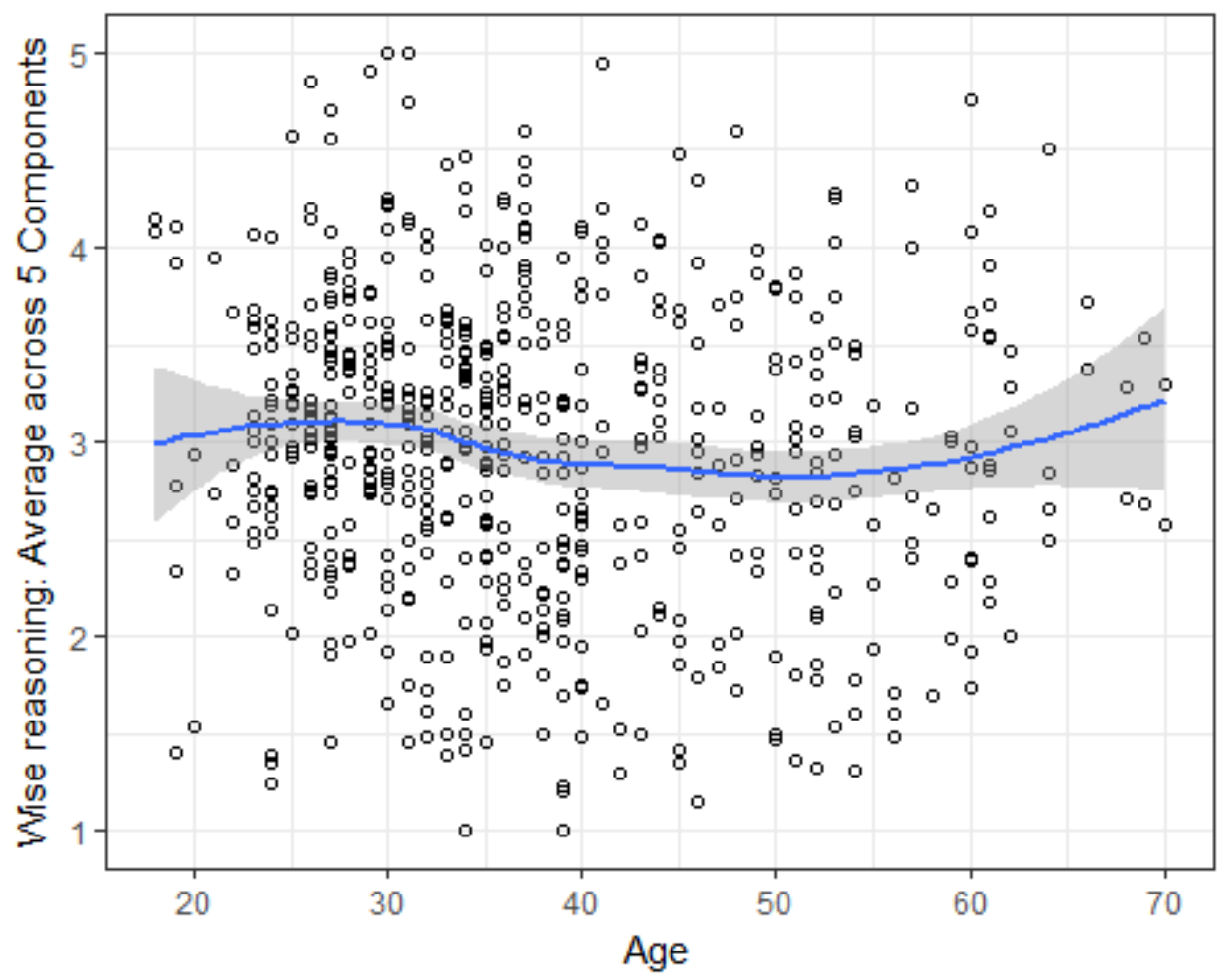

\#age neg related til 45 and than goes up, Like in Mturk sample! wisdom. long. young=subset (wisdom. long, Age < 45) wisdom. long.old=subset ( wisdom.long, Age $>44$ )

summary (Imer (WR +Agec*scale (RegionN, scale=F)+scale(serious, scale=F)* scale (RegionN, scale=F)+scale (EducationN, scale=F)*scale(RegionN, scale= F)+scale (EmployedN, scale $=F) * s c a l e($ RegionN, scale $=F)+$ scale (GenderN, scal e=F)*scale (OtherGenderN, scale $=F) *$ scale (RegionN, scale=F)+(1|V1), wisdom .long.young))

\#\# Linear mixed model fit by REML t-tests use Satterthwaite approximat ions

\#\# to degrees of freedom [lmerMod]

\#\# Formula:

\#\# WR $\sim$ +Agec $*$ scale $($ RegionN, scale $=F)+$ scale $($ serious, scale $=F)$ *

\#\# $\quad$ scale $($ RegionN, scale $=F)+\operatorname{scale}($ EducationN, scale $=F) *$

\#\# $\quad$ scale $($ RegionN, scale $=\mathrm{F})+\operatorname{scale}($ EmployedN, scale $=\mathrm{F}) *$

\#\# $\quad$ scale $($ RegionN, scale $=F)+\operatorname{scale}($ GenderN, scale $=F) * \operatorname{scale}(0$ therGenderN,

\#\# $\quad$ scale $=\mathrm{F}) * \operatorname{scale}($ RegionN, scale $=\mathrm{F})+(1 \mid \mathrm{V} 1)$

\#\# Data: wisdom.long.young

\#\# 


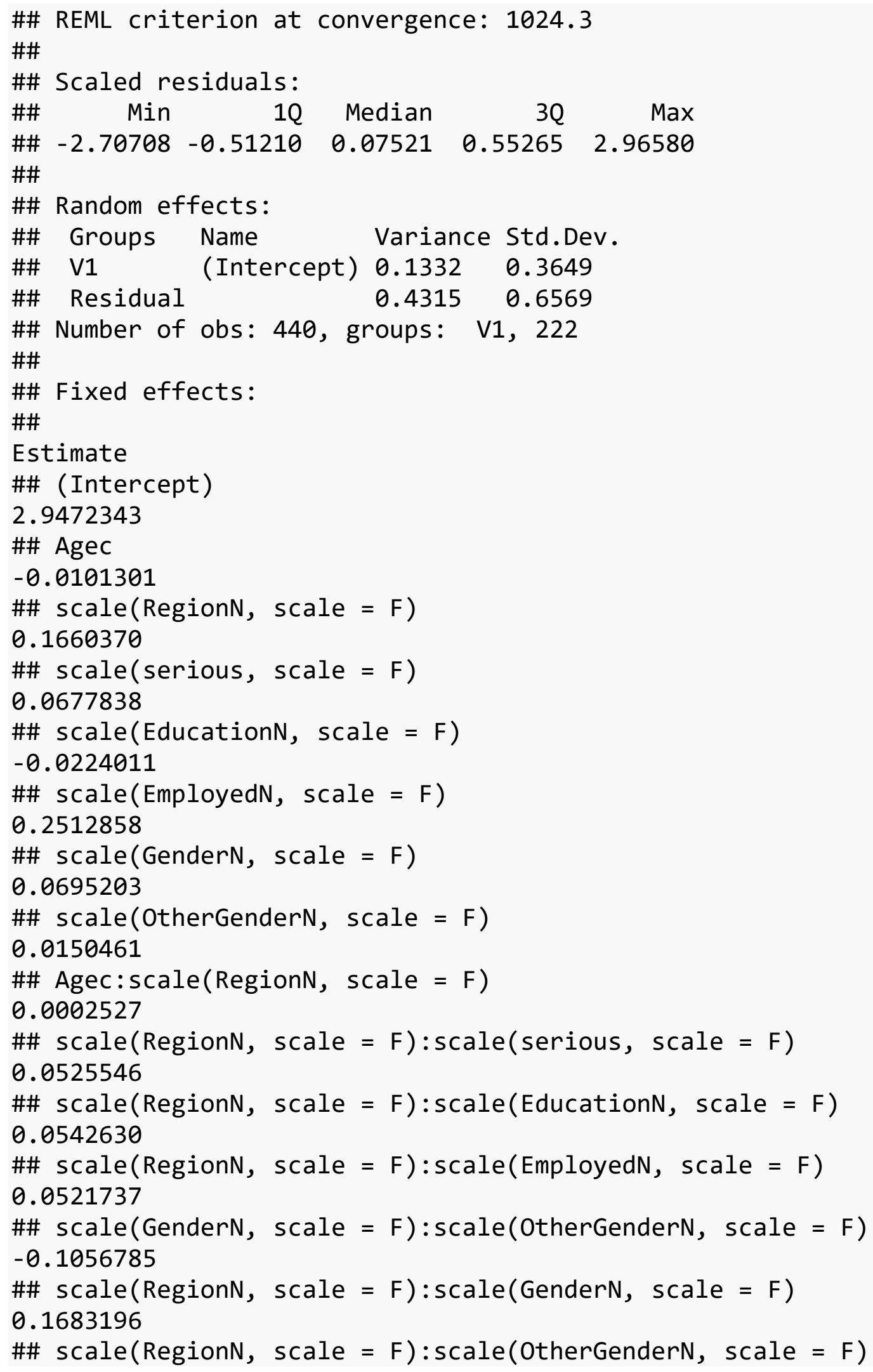




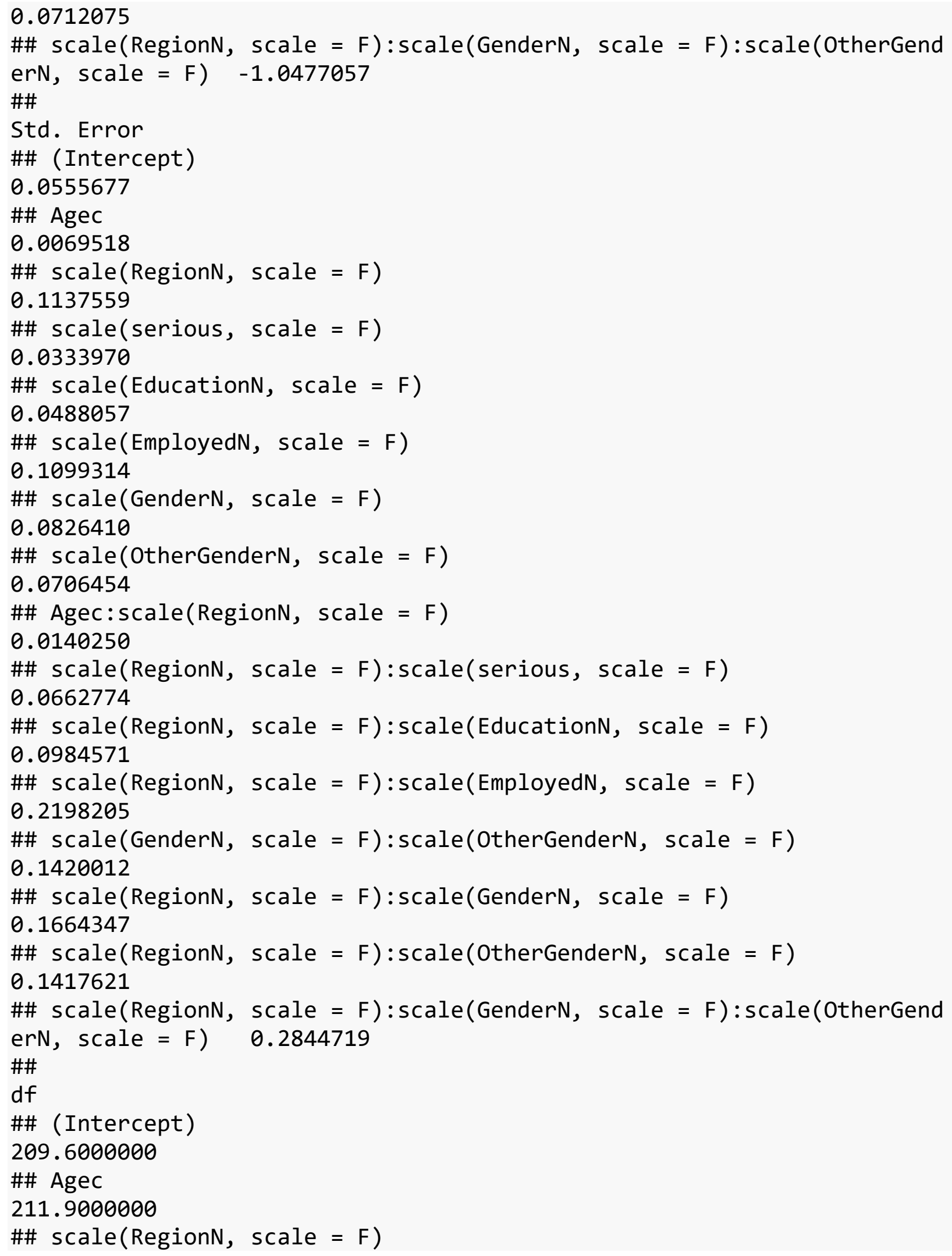




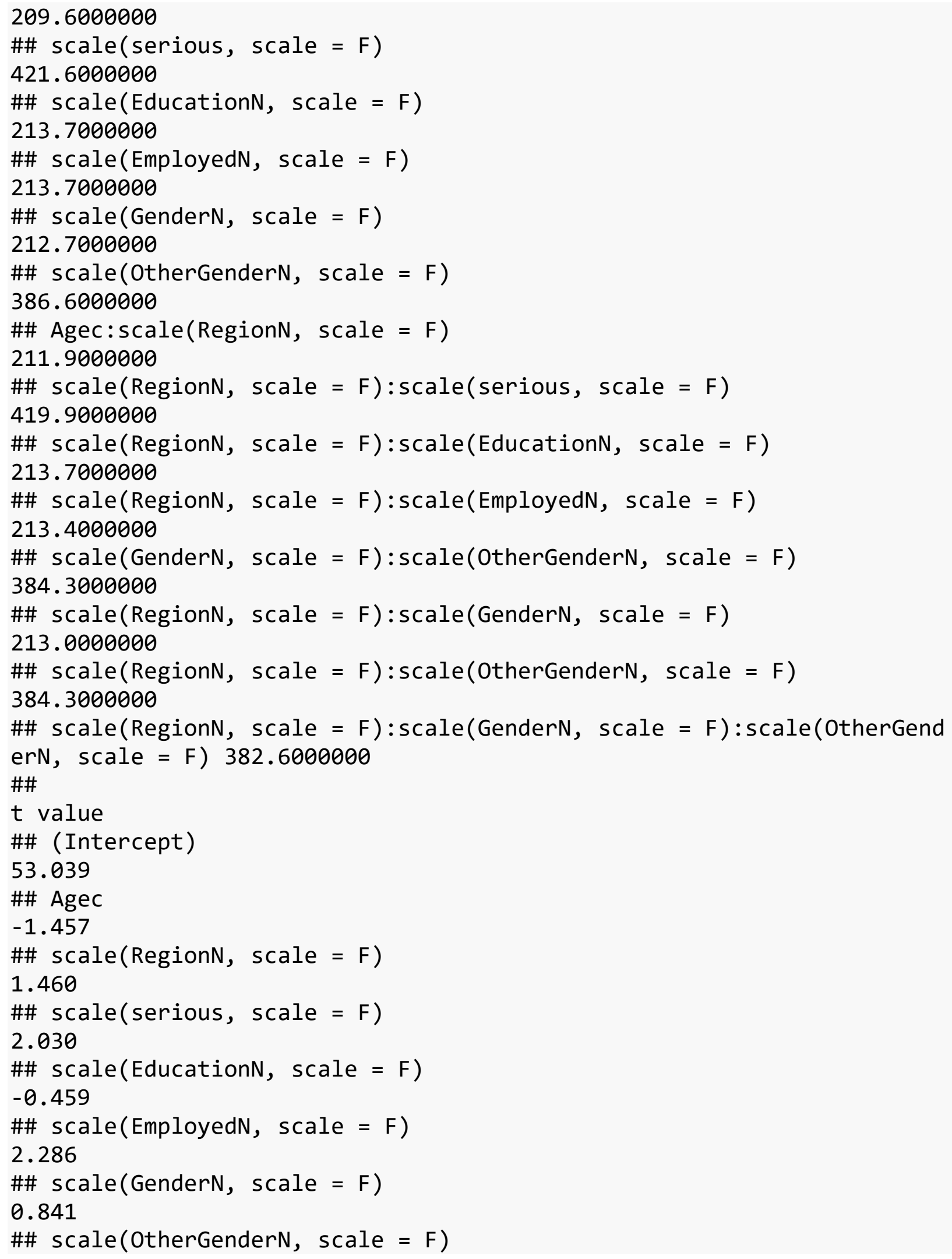




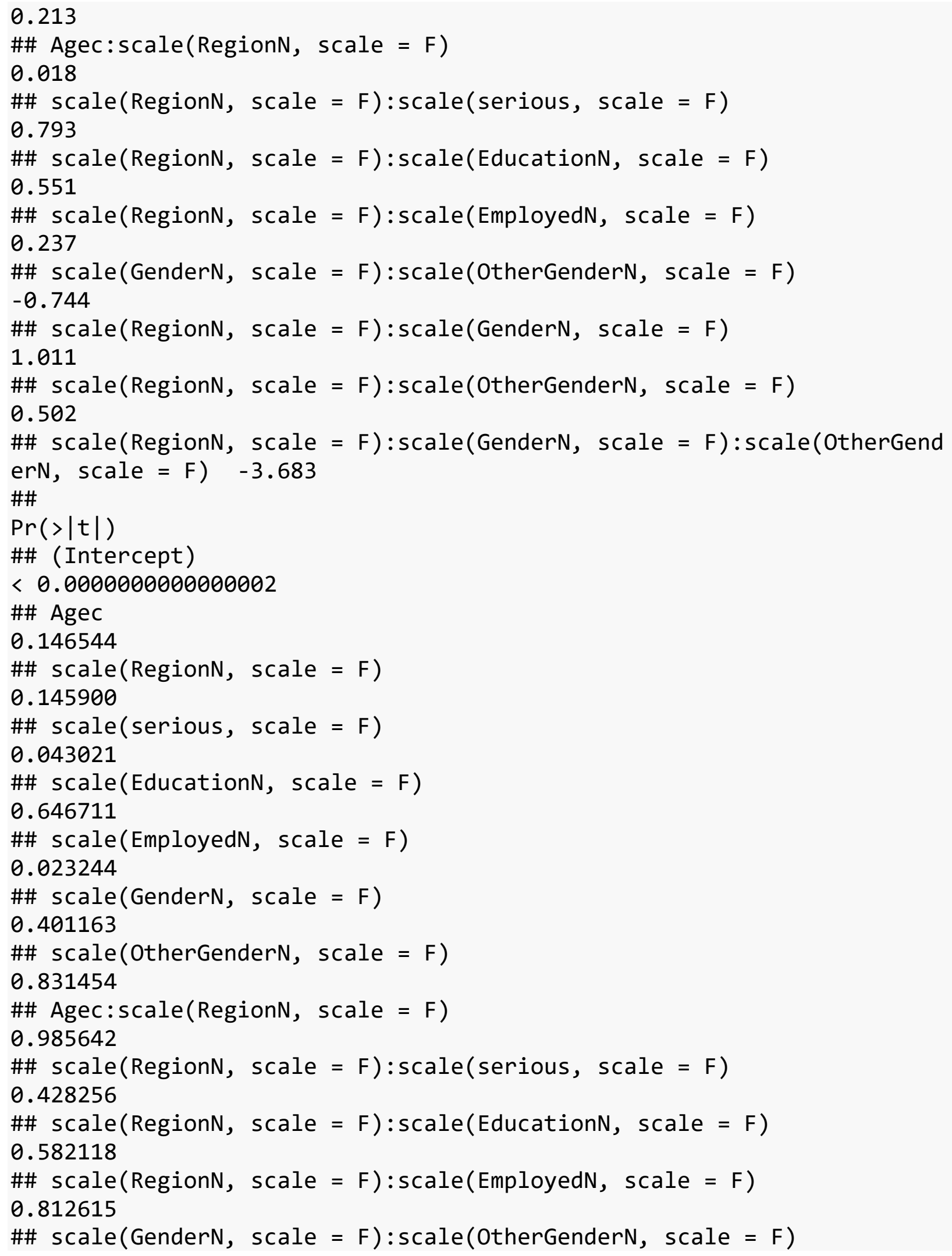




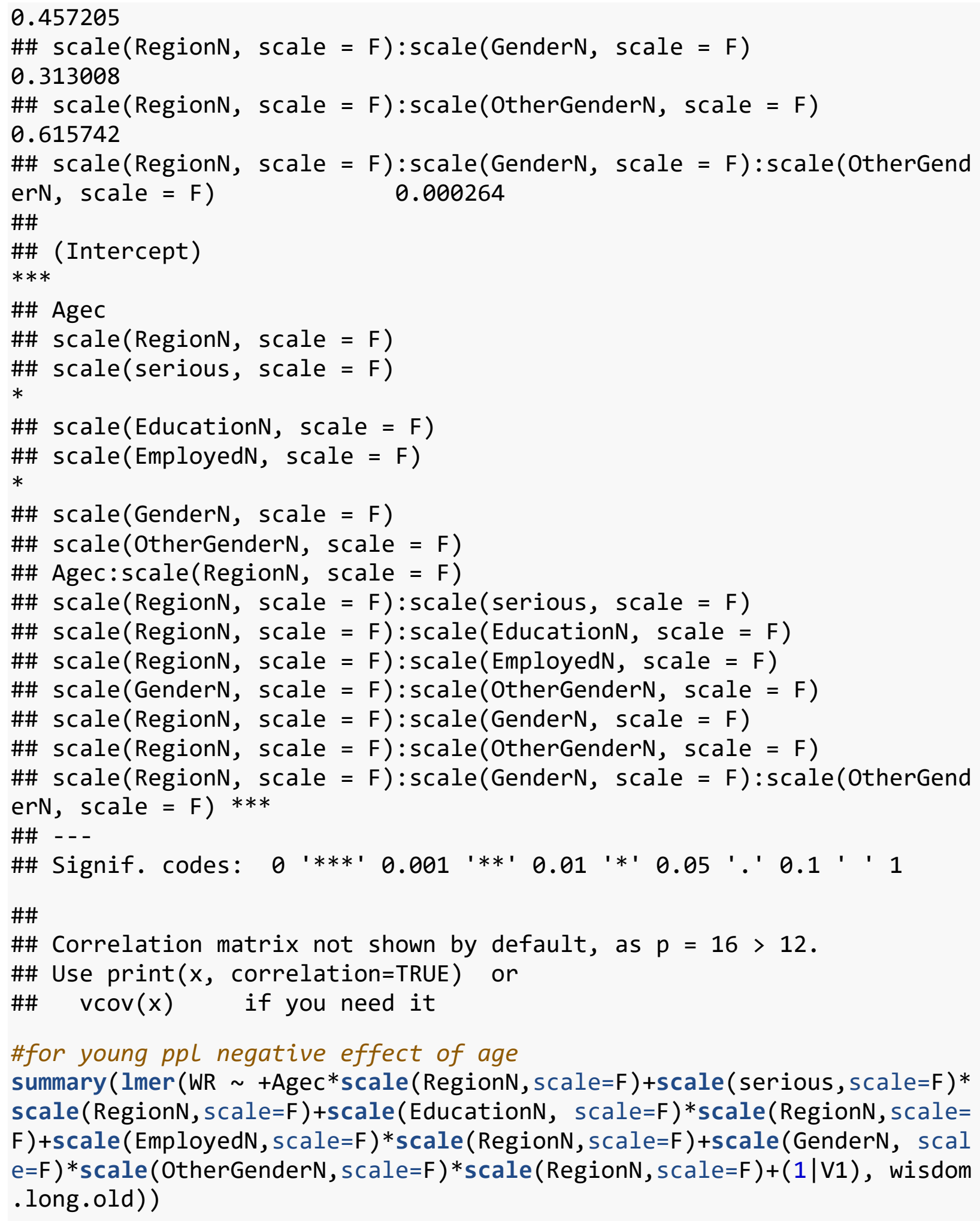

\#\# Linear mixed model fit by REML t-tests use Satterthwaite approximat ions 


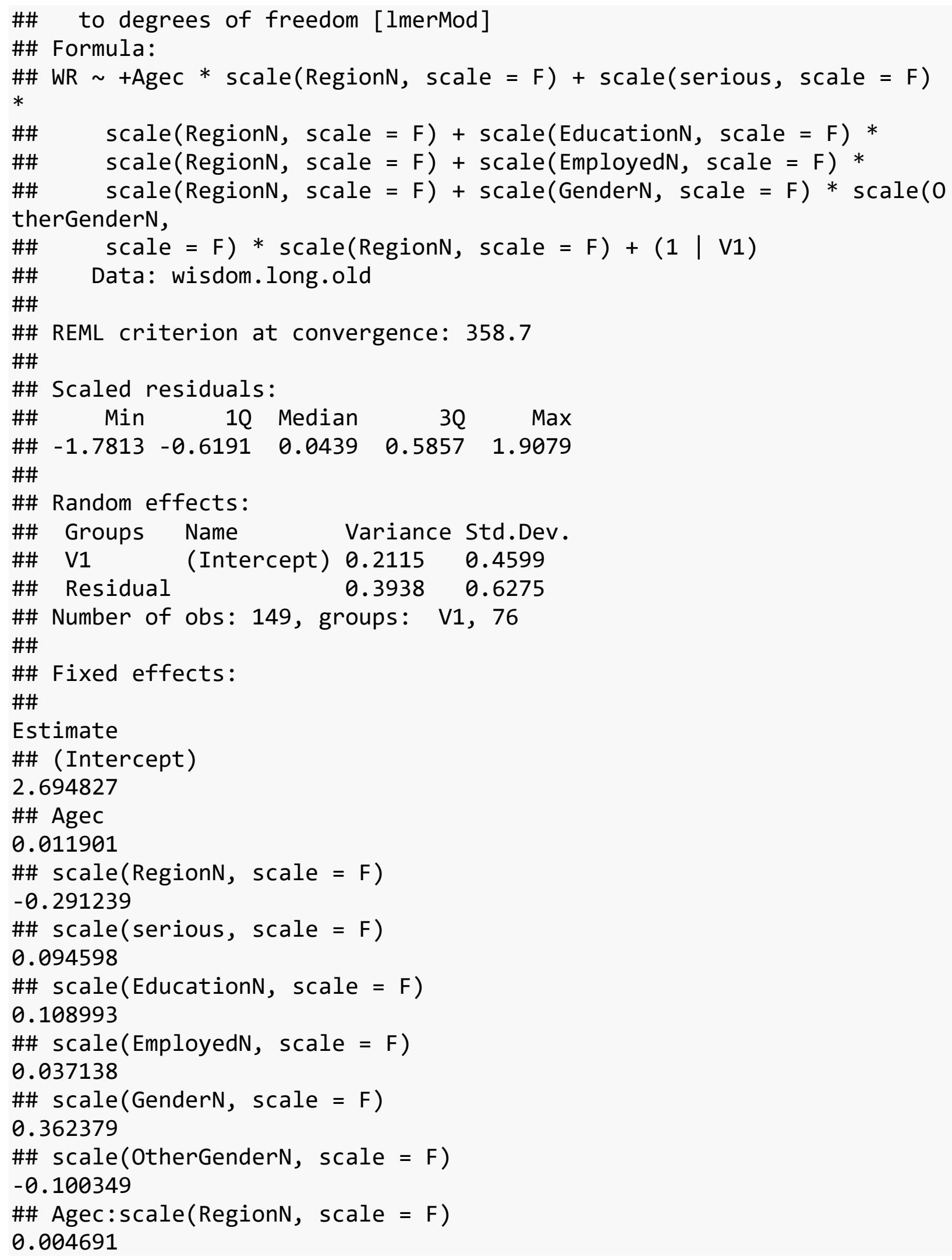




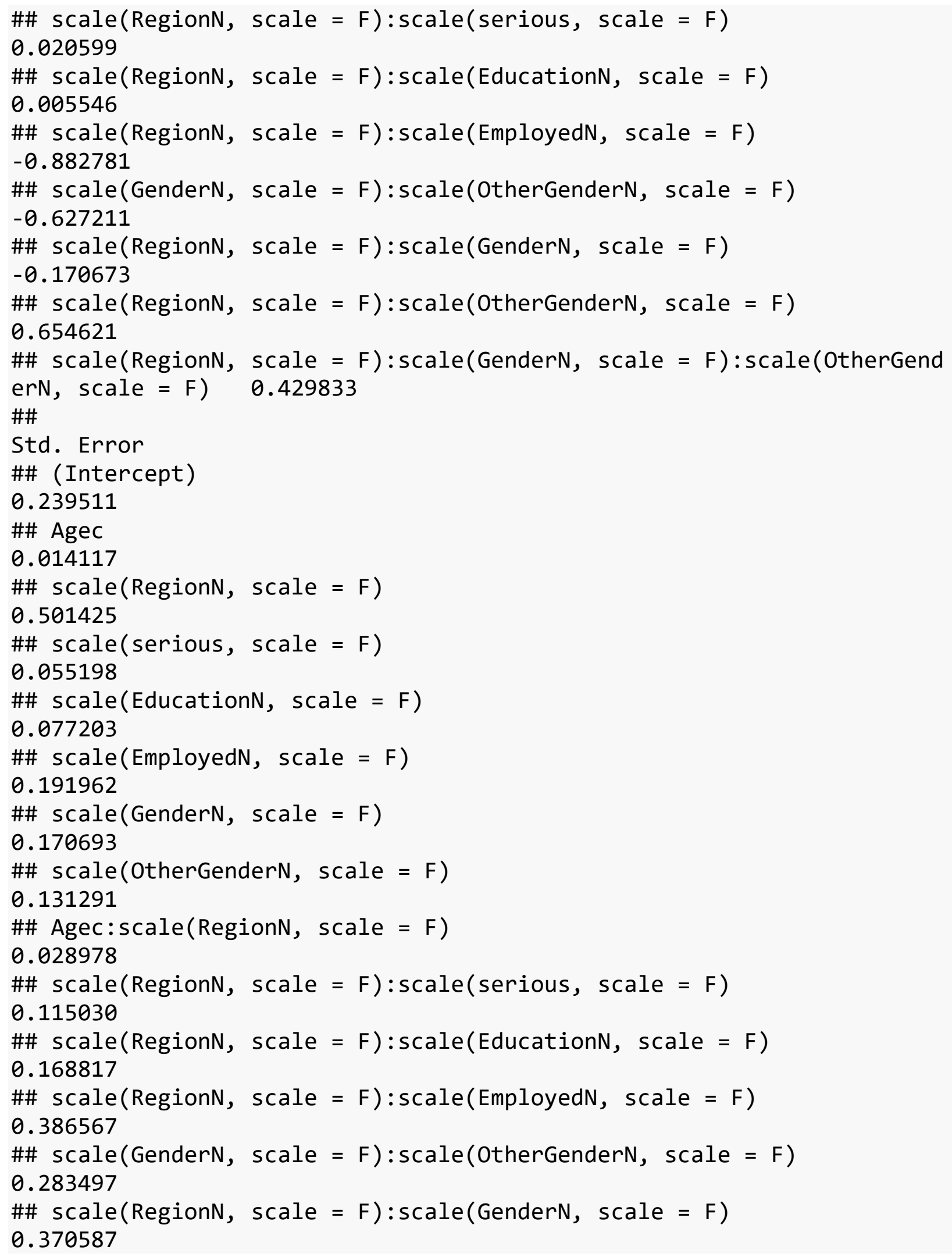




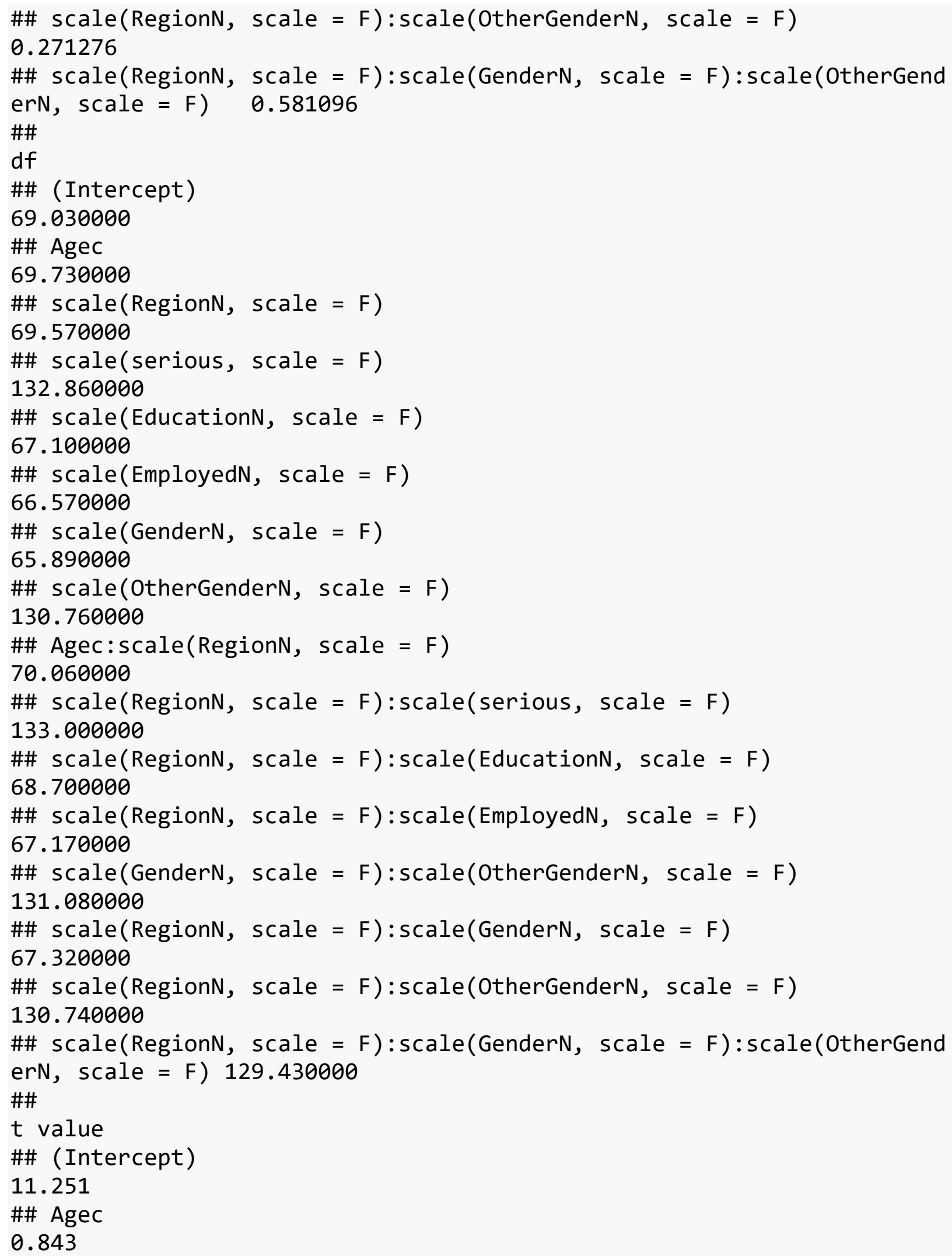




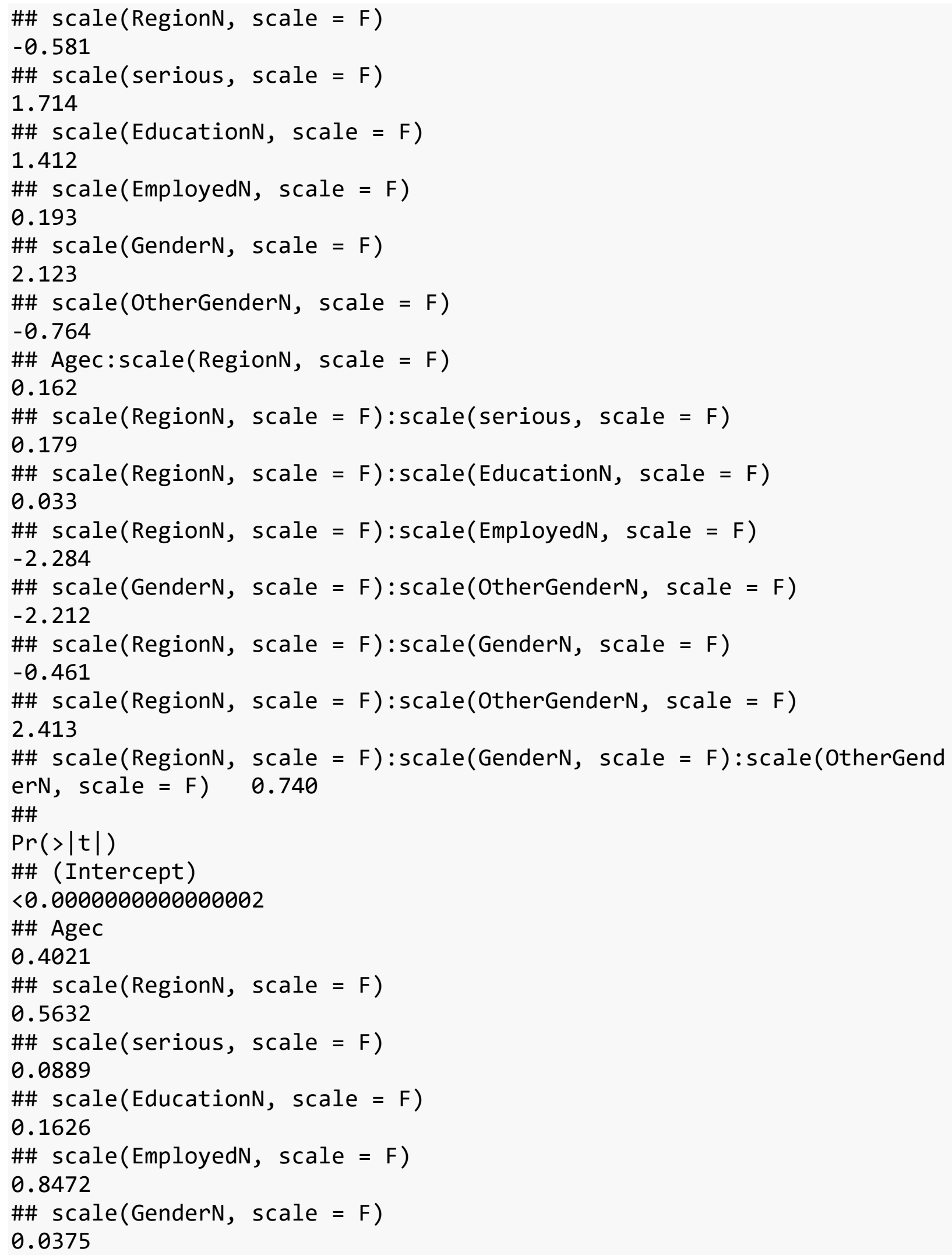




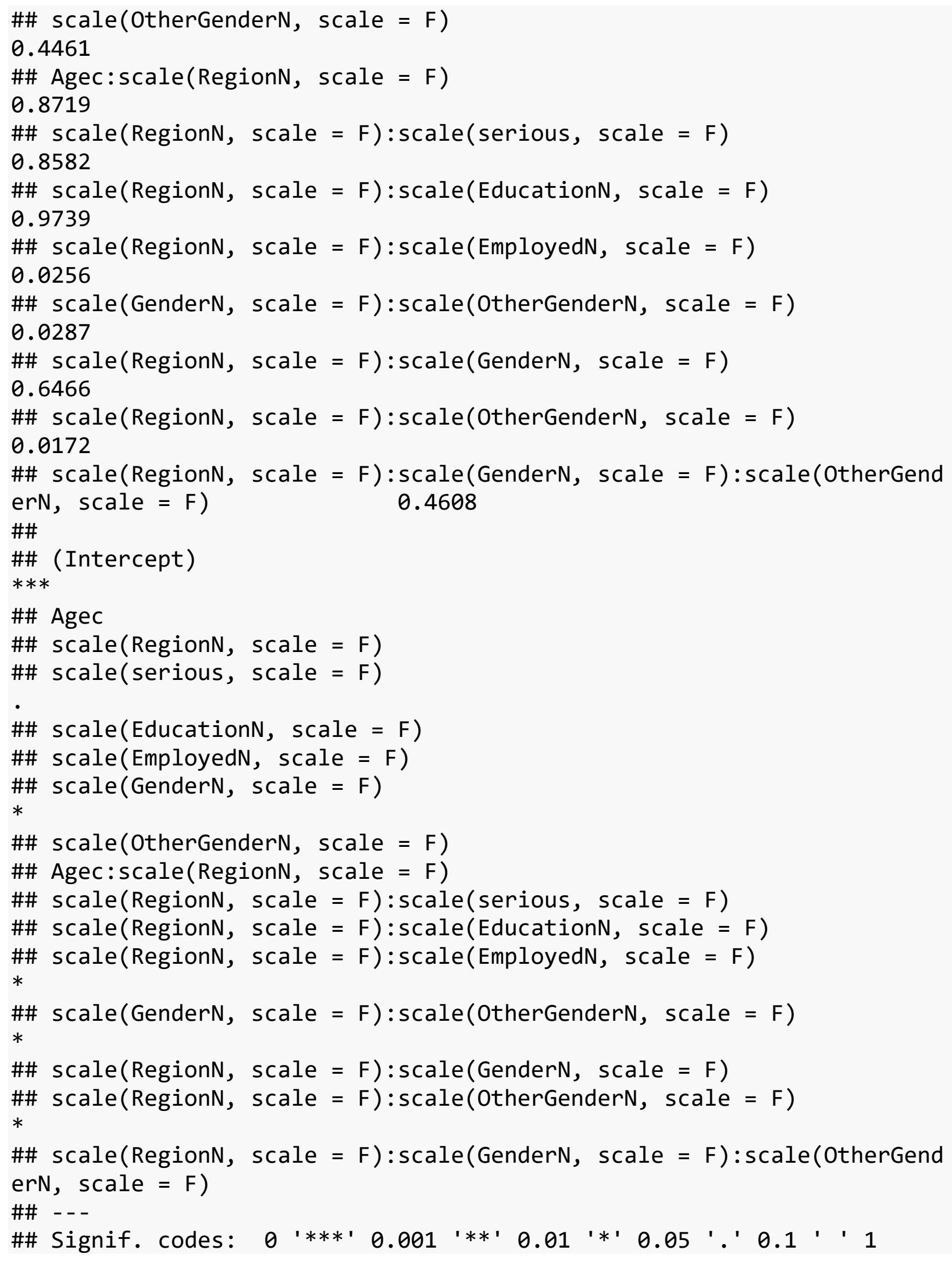




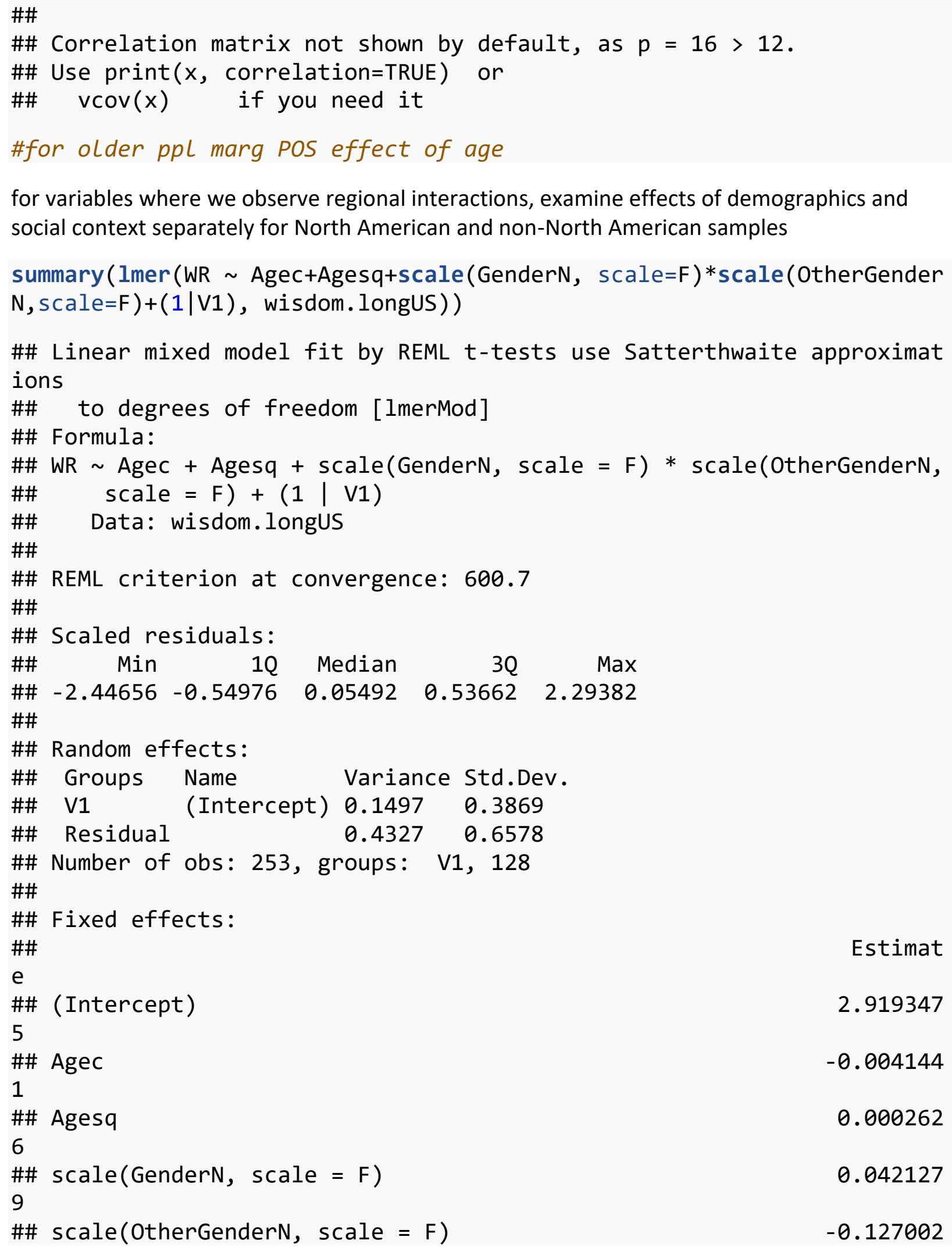




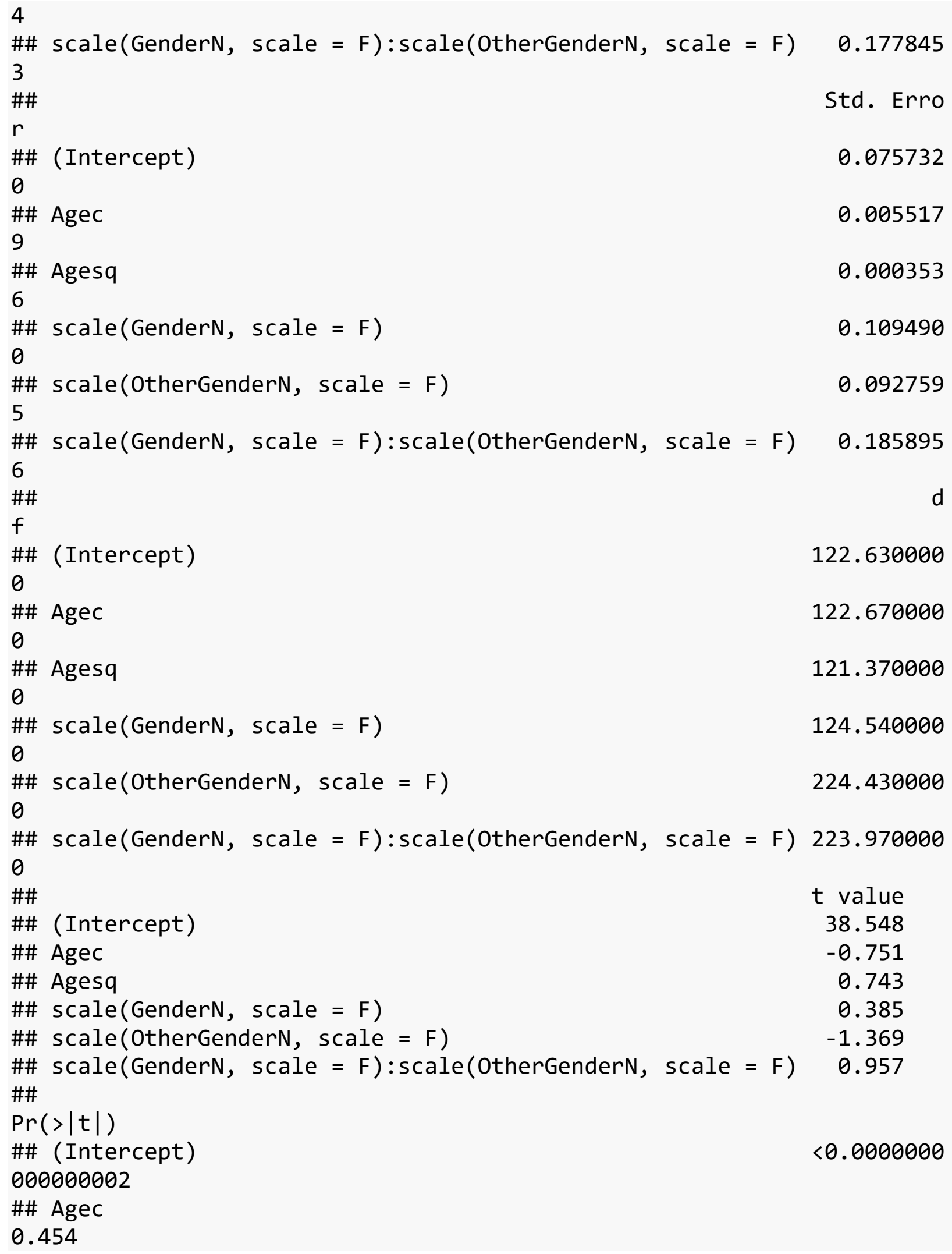




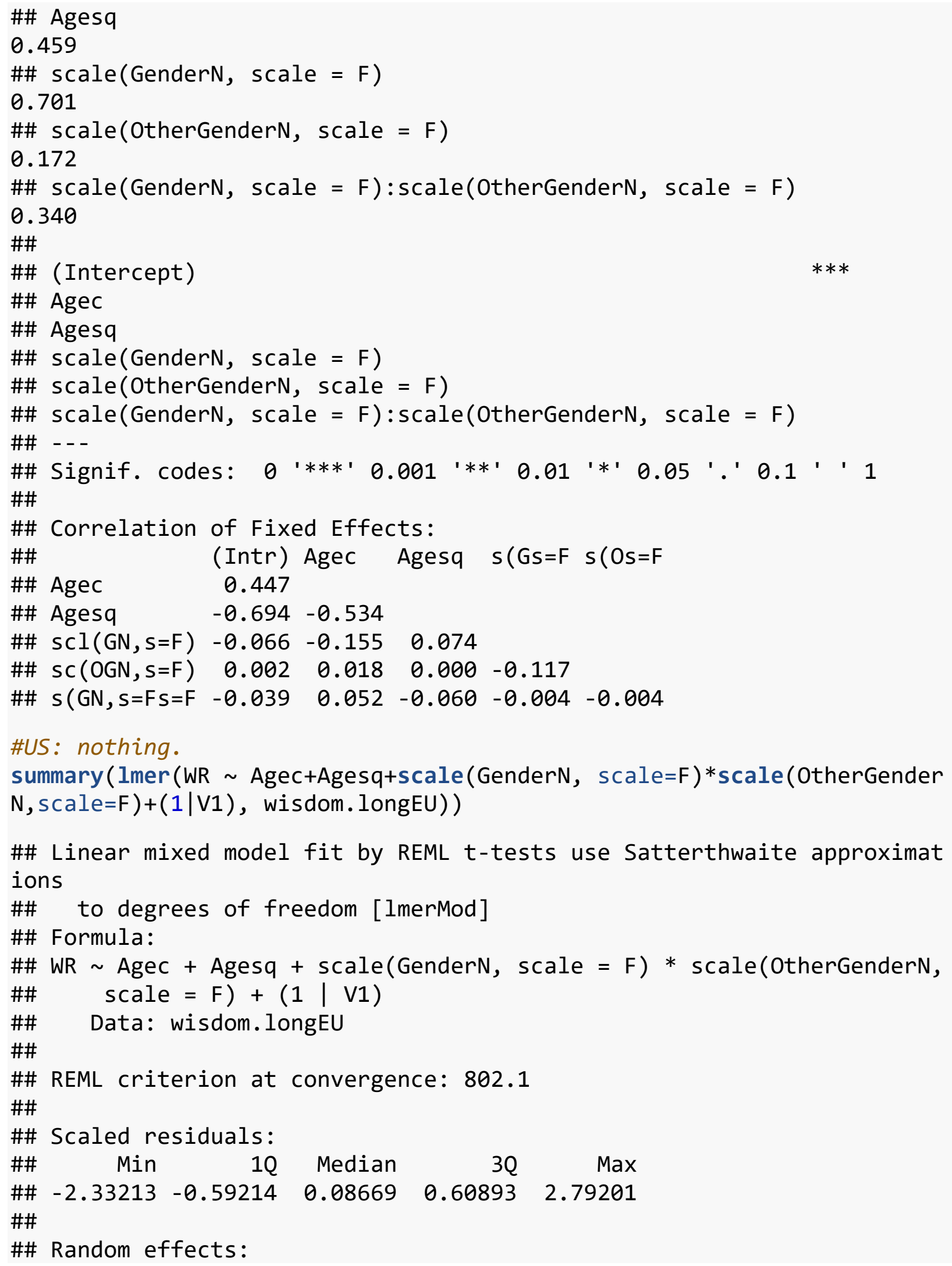




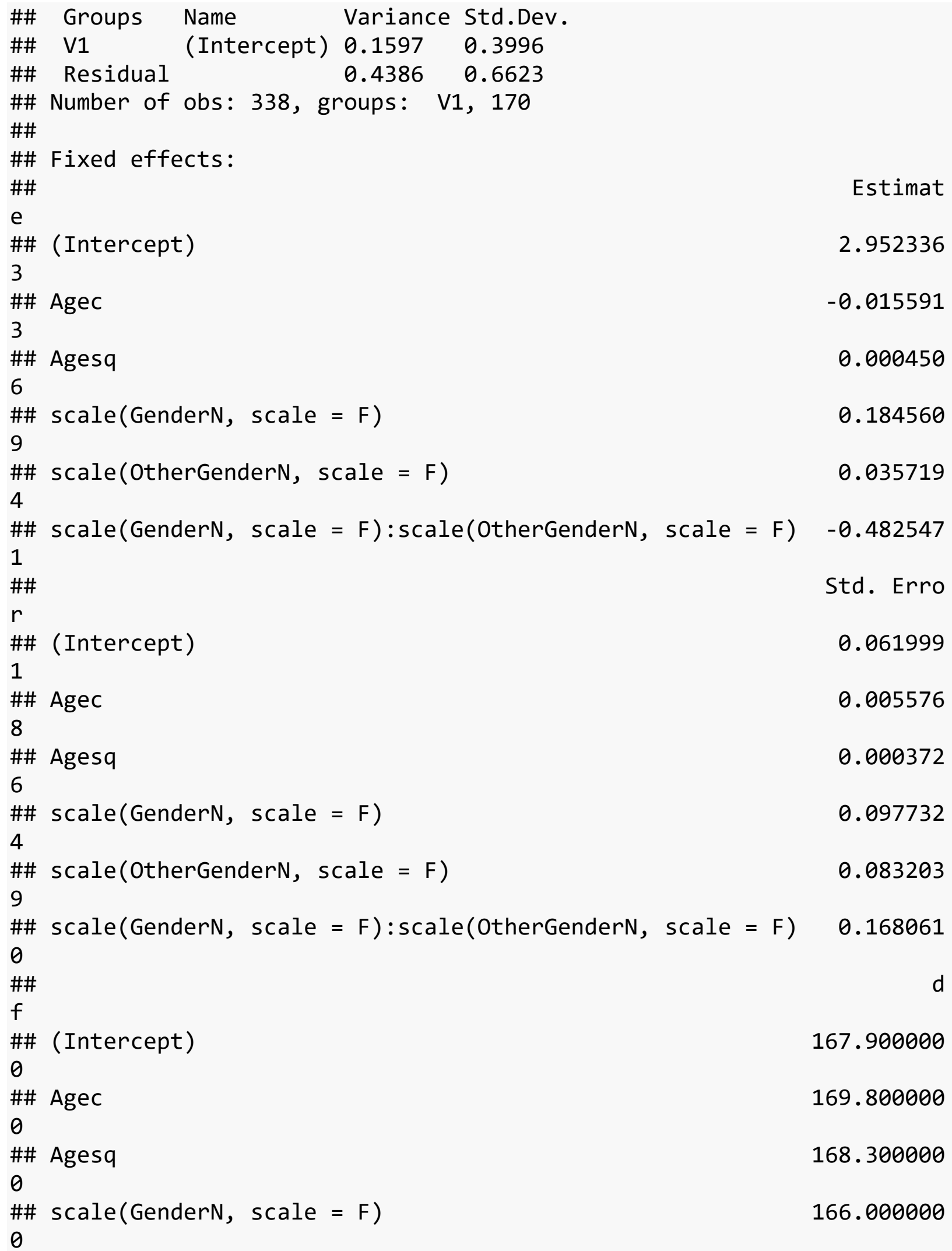




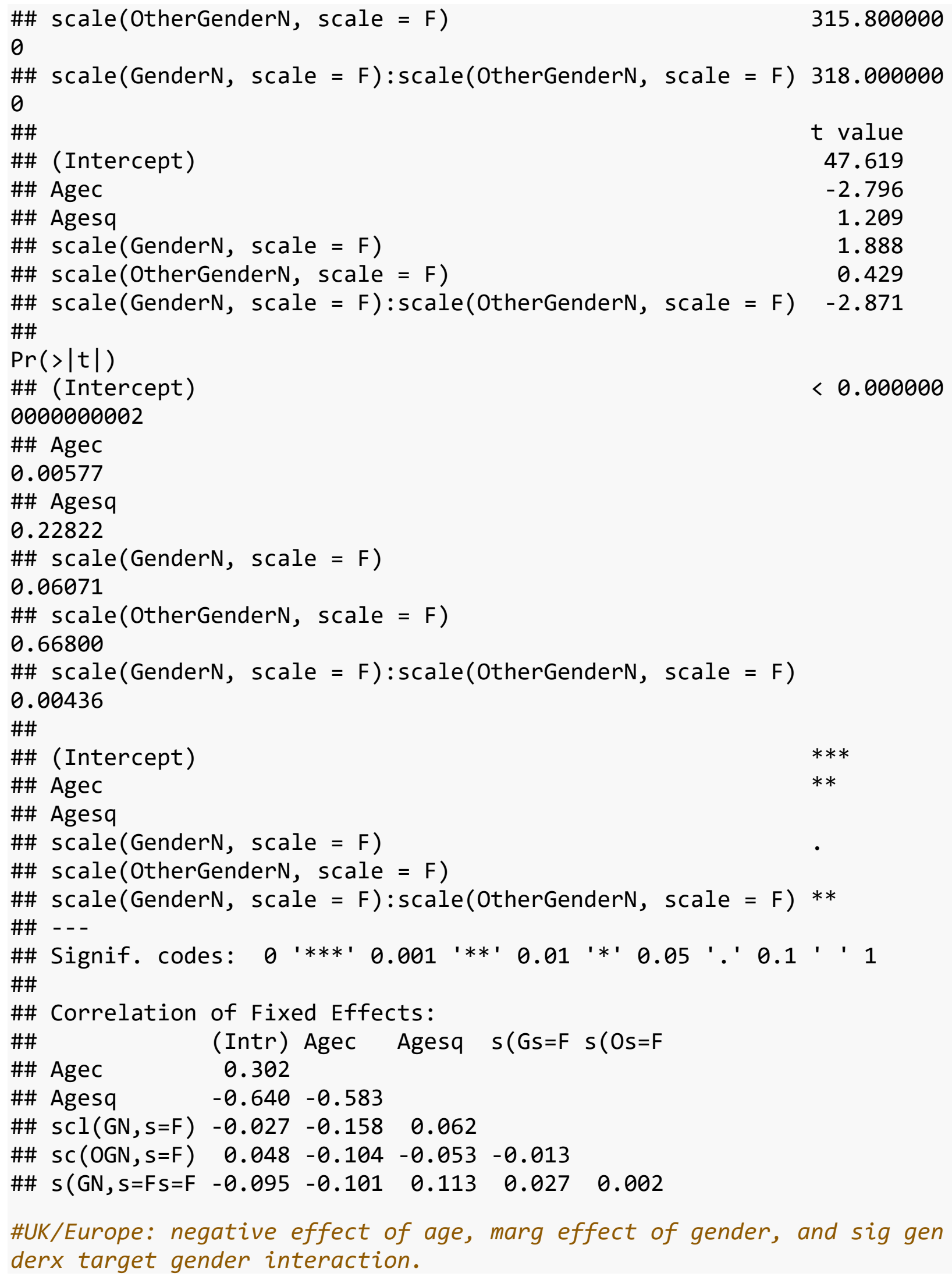


summary (Imer (WR Agec+Agesq+scale(GenderN, scale=F)*scale(OtherGender $\mathrm{N}$, scale $=\mathrm{F})+(1 \mid \mathrm{V} 1)$, wisdom. longUK $))$

\#\# Linear mixed model fit by REML t-tests use Satterthwaite approximat ions

\#\# to degrees of freedom [1merMod]

\#\# Formula:

\#\# WR Agec + Agesq + scale (GenderN, scale = F) * scale (OtherGenderN, $\# \quad$ scale $=F)+(1 \mid V 1)$

\#\# Data: wisdom.longUK

\#\#

\#\# REML criterion at convergence: 743.2

\#\#

\#\# Scaled residuals:

\#\# Min $1 Q$ Median $30 \quad$ Max

$\begin{array}{llllll}\# \# & -2.27388 & -0.61099 & 0.08953 & 0.59385 & 2.72833\end{array}$

\#\#

\#\# Random effects:

\#\# Groups Name Variance Std.Dev.

$\begin{array}{llll}\text { \#\# V1 } & \text { (Intercept) } & 0.1726 & 0.4155\end{array}$

\#\# Residual $\quad 0.45760 .6765$

\#\# Number of obs: 306, groups: V1, 154

\#\#

\#\# Fixed effects:

\#\#

Estimat

e

\#\# (Intercept)

2.948677

1

\#\# Agec

$-0.016266$

2

$\begin{array}{ll}\text { \#\# Agesq } & 0.000487\end{array}$

0

\#\# scale(GenderN, scale = F) $\quad 0.168604$

3

\#\# scale(OtherGenderN, scale = F) $\quad 0.029929$

3

\#\# scale(GenderN, scale = F): scale(OtherGenderN, scale = F) $\quad-0.485697$ 6

\#\#

Std. Erro

$r$

\#\# (Intercept)

0.066043

5

\#\# Agec

0.006172

6

\#\# Agesq

0.000402 


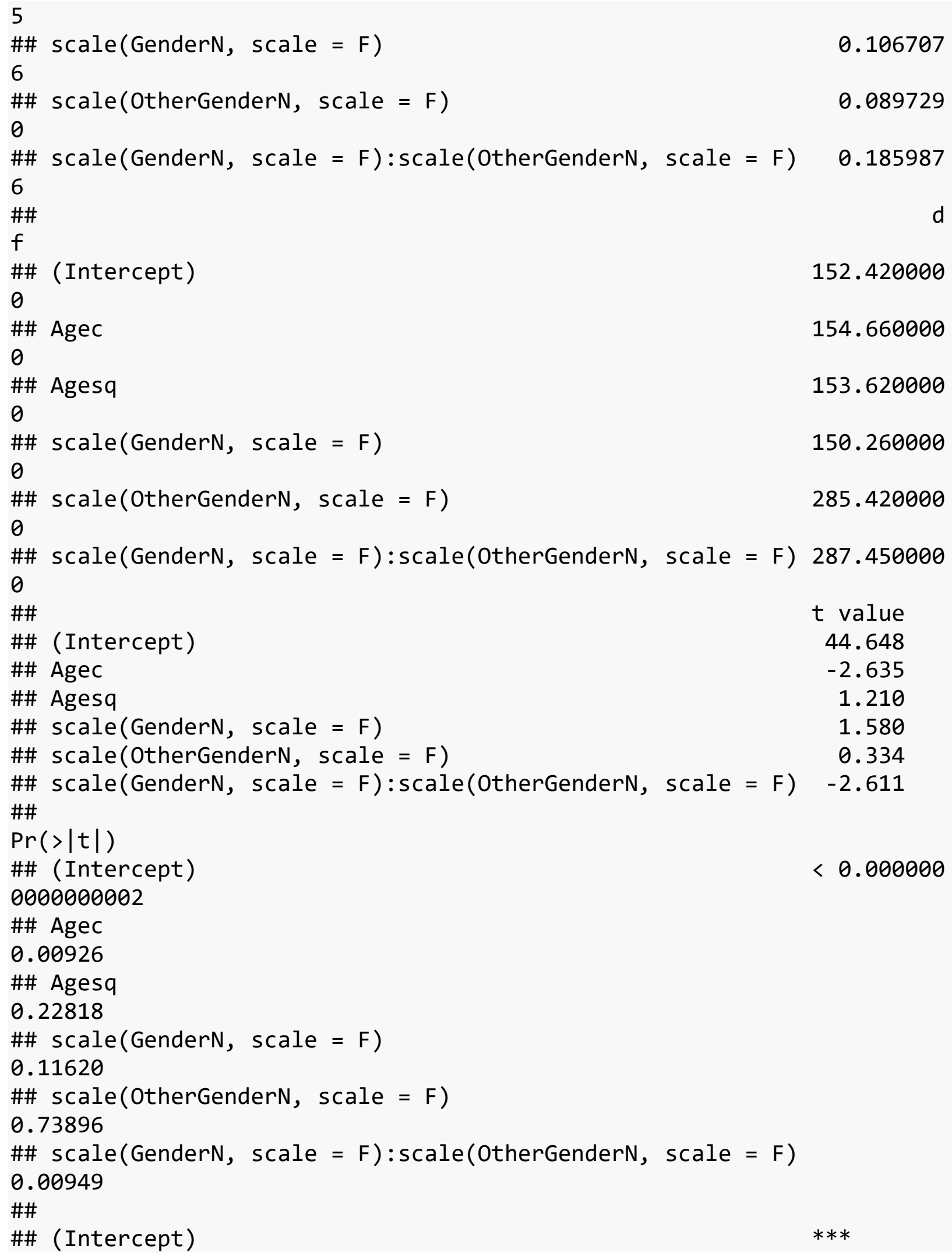




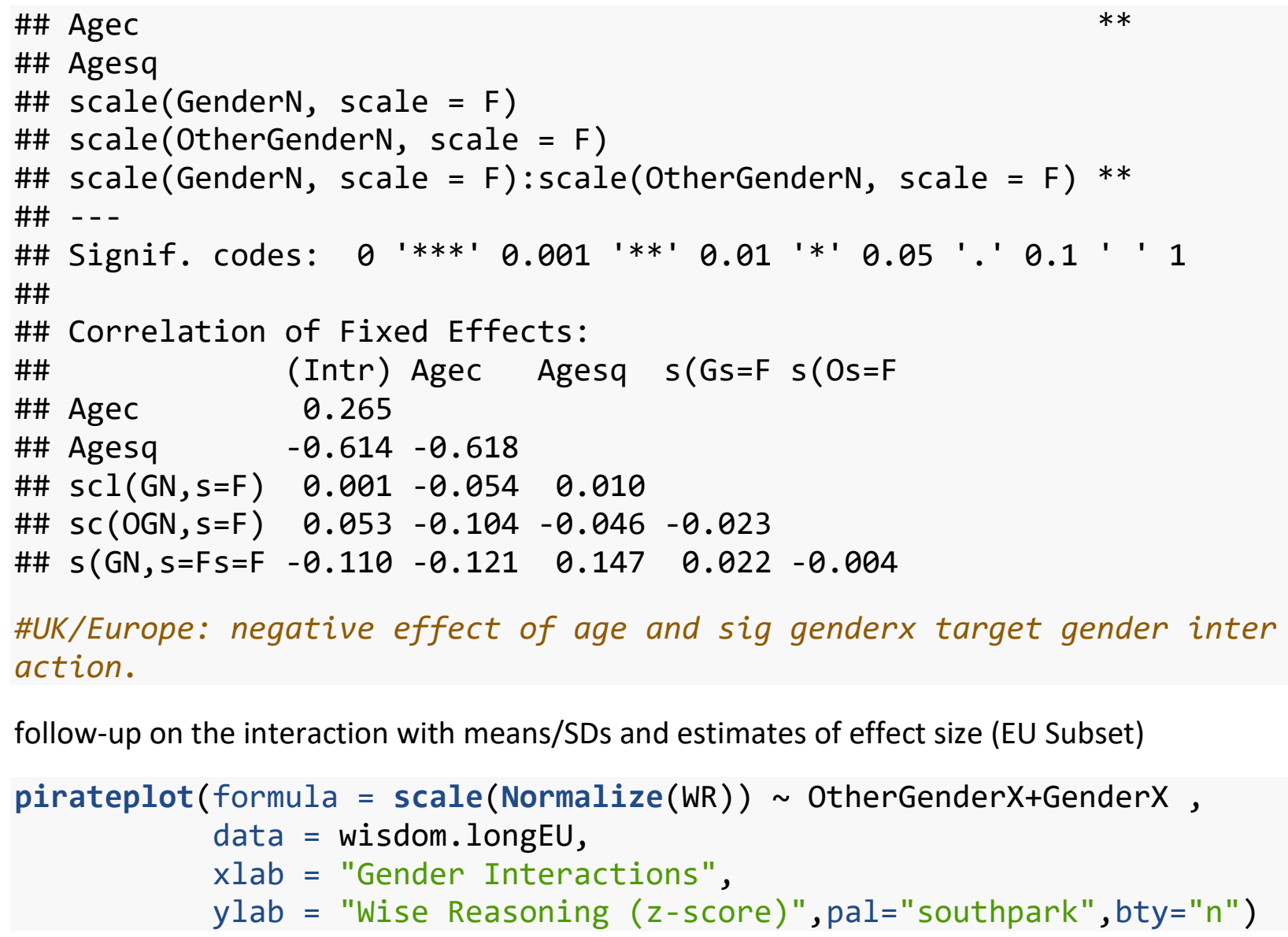




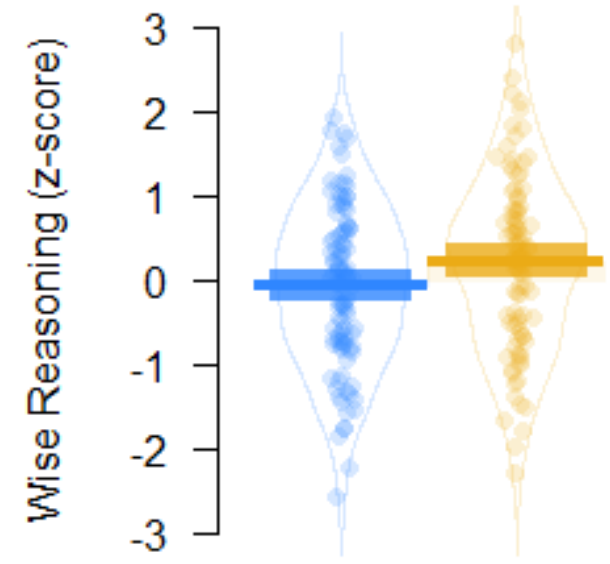

jenderX jender $X$
Female

Female Male

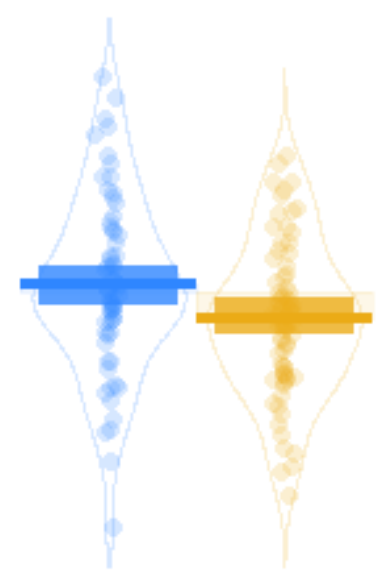

Male

Female Male

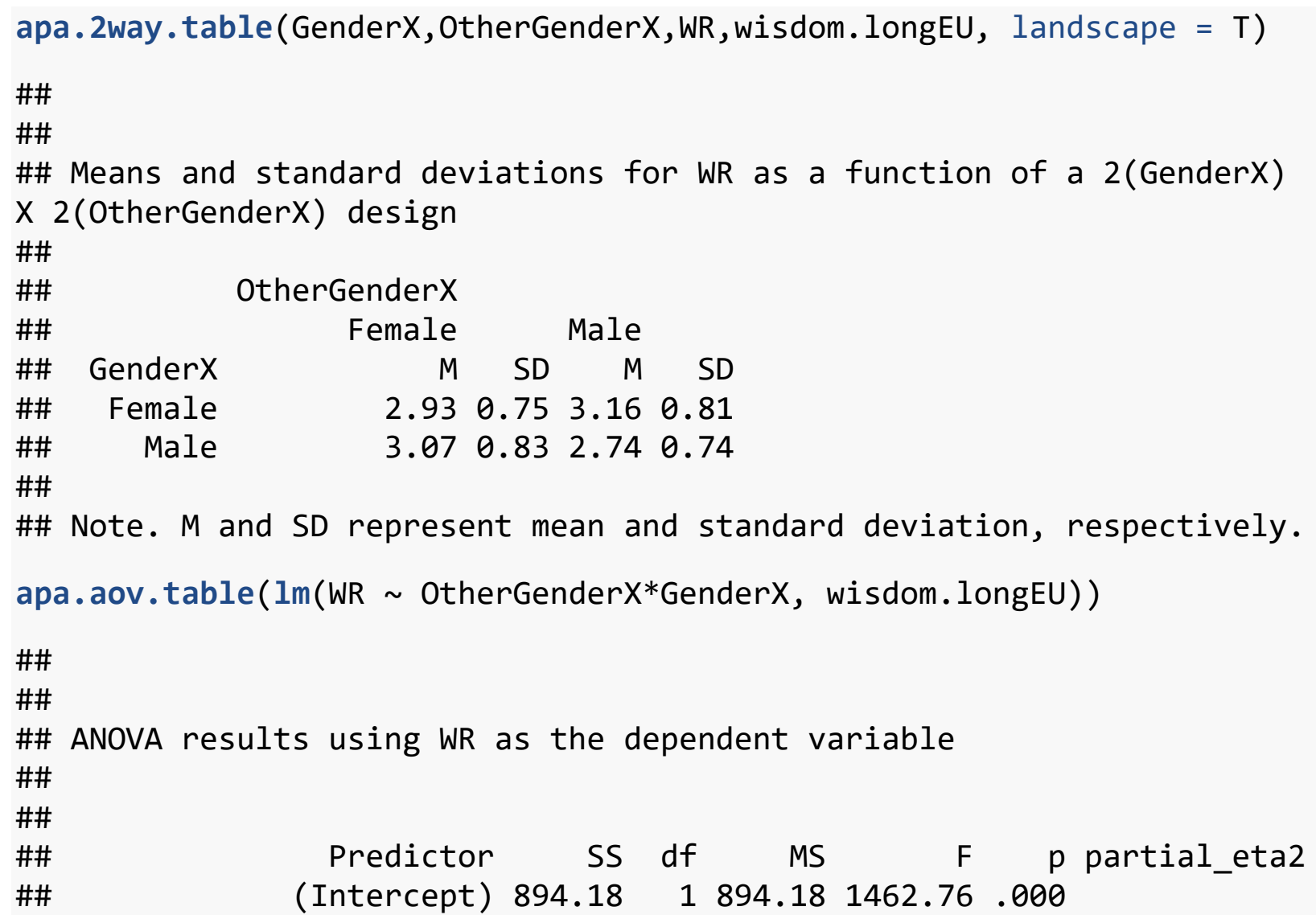




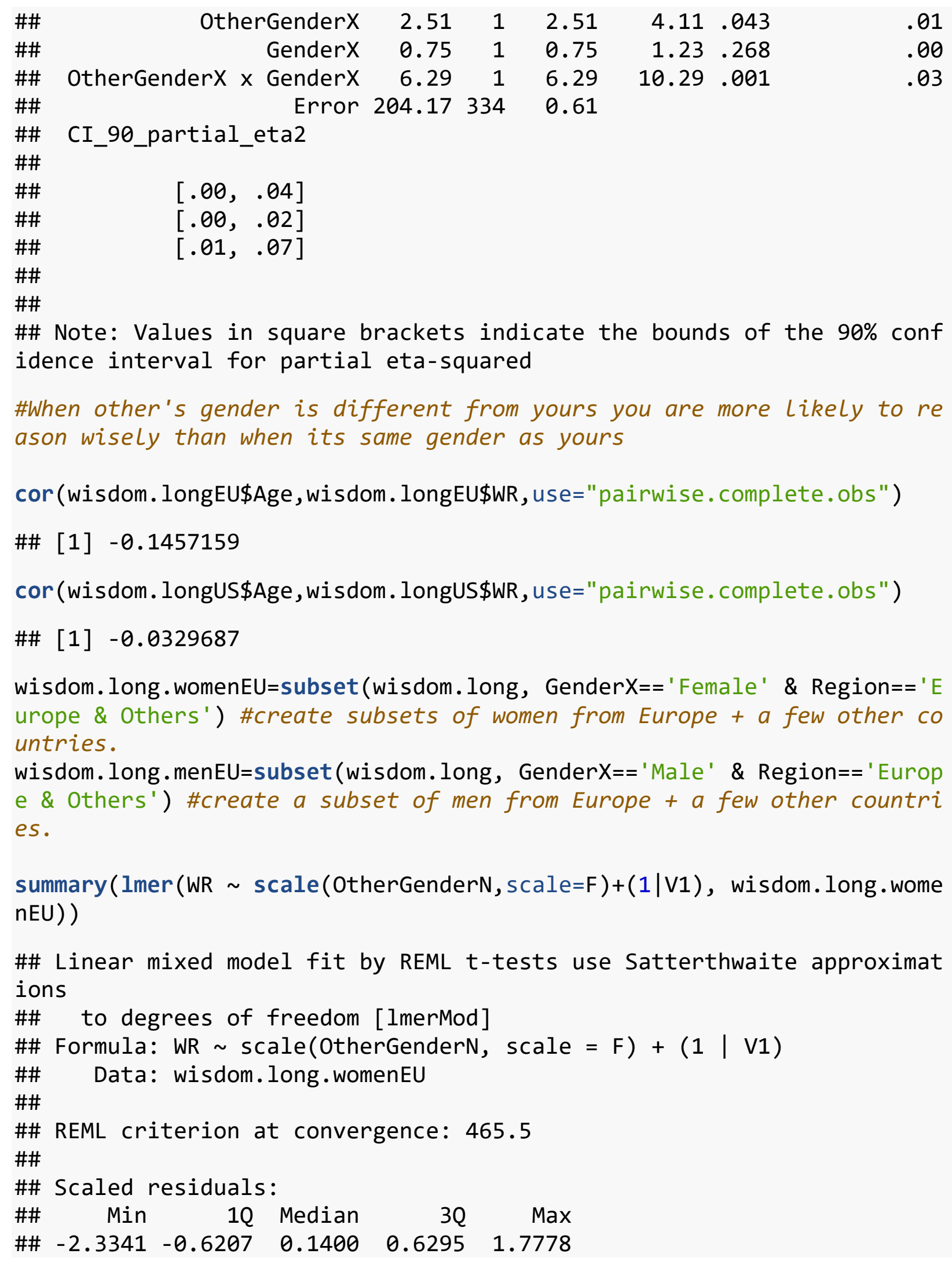




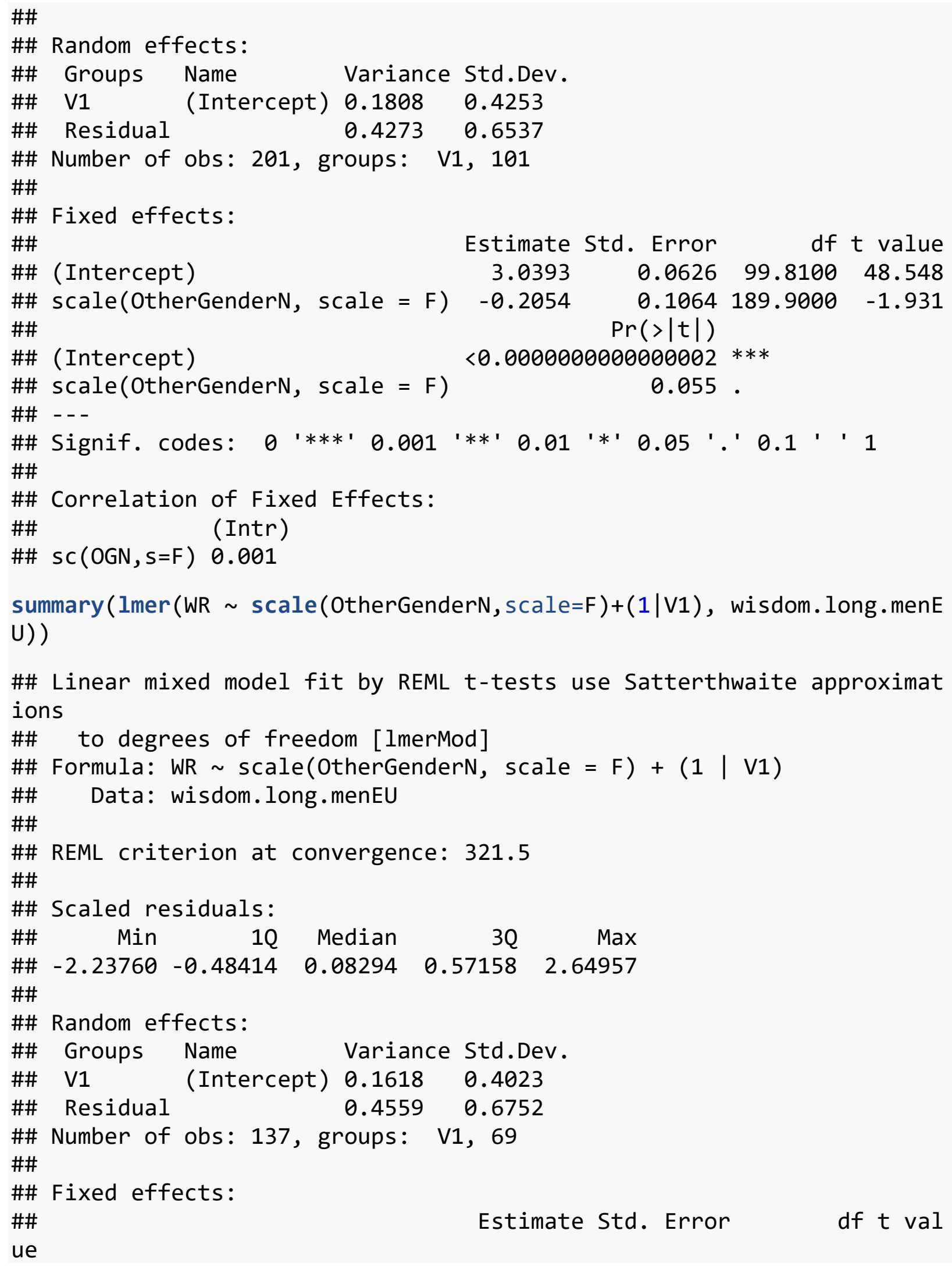




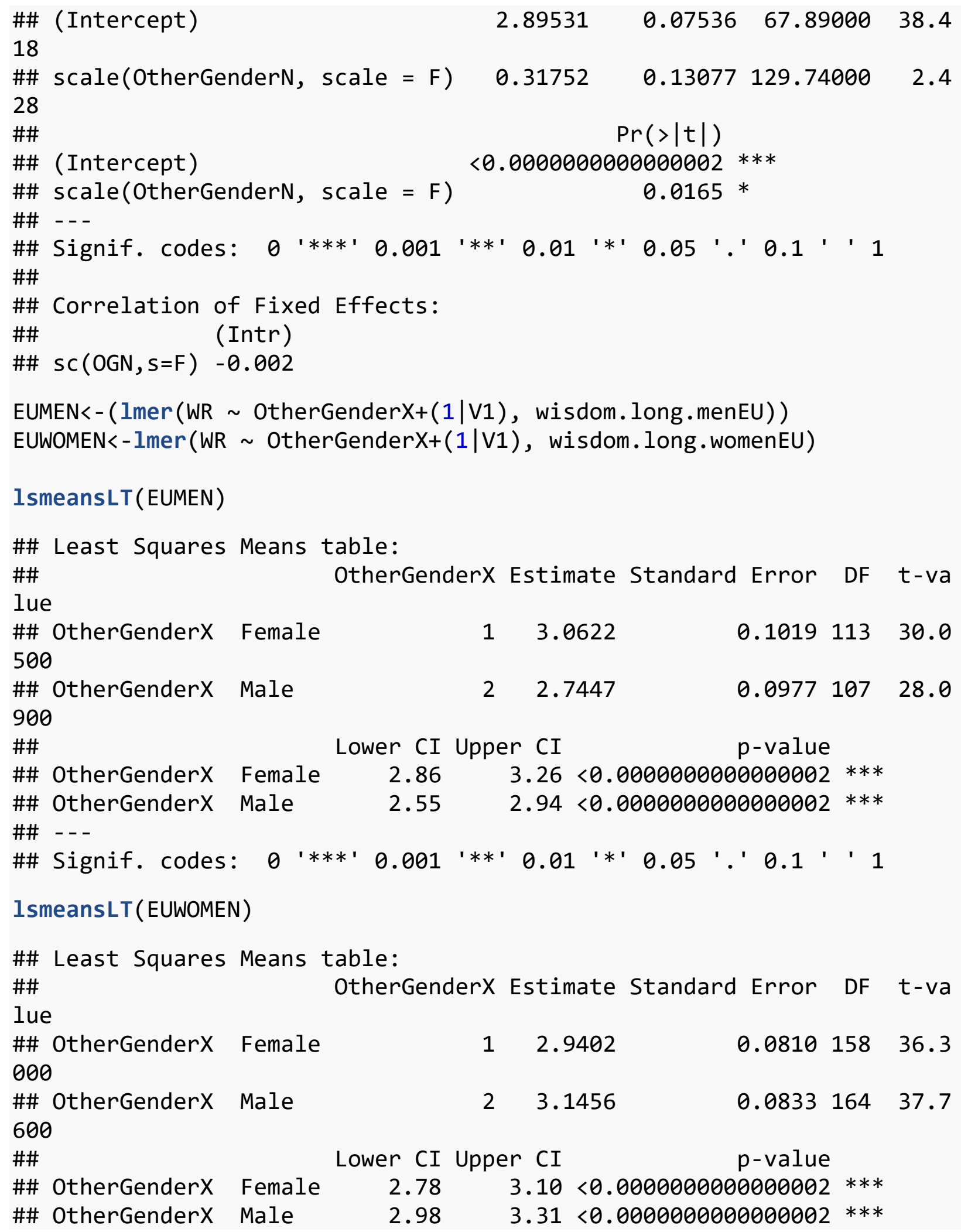


\#\# - - -

\#\# Signif. codes: $0{ }^{\prime * * *}$ ' $0.0011^{\prime * *}{ }^{\prime} 0.011^{\prime *}{ }^{\prime} 0.05$ '.' 0.1 ' 1

follow-up on the interaction with means/SDs and estimates of effect size (UK Subset)

pirateplot $($ formula $=\operatorname{scale}(\operatorname{Normalize}($ WR $)) \sim 0$ therGenderX+GenderX , data $=$ wisdom.longUK,

$\mathrm{xlab}=$ "Gender Interactions",

$\mathrm{ylab}=$ "Wise Reasoning (z-score)",pal="southpark", bty="n")

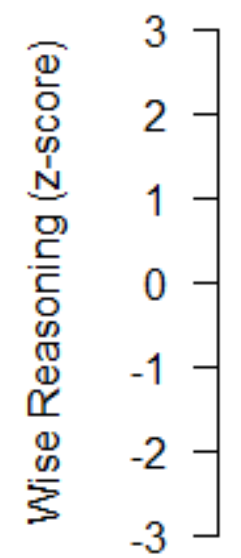

jenderX

jenderX
Female

Female Male

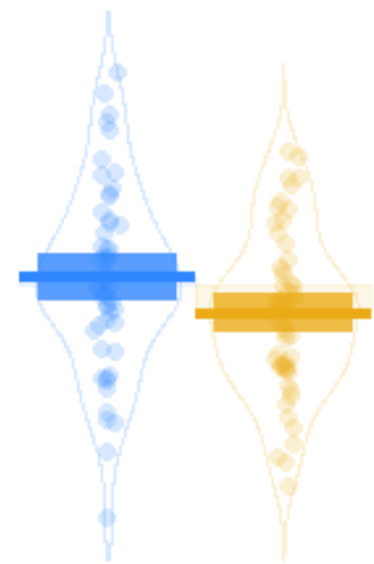

Male

apa.2way.table(GenderX, OtherGenderX, WR, wisdom.longUK, landscape $=\mathrm{T}$ )

\#\#

\#\#

\#\# Means and standard deviations for WR as a function of a 2(GenderX) X 2(OtherGenderX) design

\#\#

\#\#

\#\#

\#\# GenderX

OtherGenderX

\#\# Female

Female

Female Male

\#\#

Male

$M \quad S D \quad M \quad S D$

$2.93 \quad 0.75 \quad 3.14 \quad 0.82$

$\begin{array}{llll}3.05 & 0.88 & 2.70 & 0.77\end{array}$

\#\#

\#\# Note. M and SD represent mean and standard deviation, respectively. 


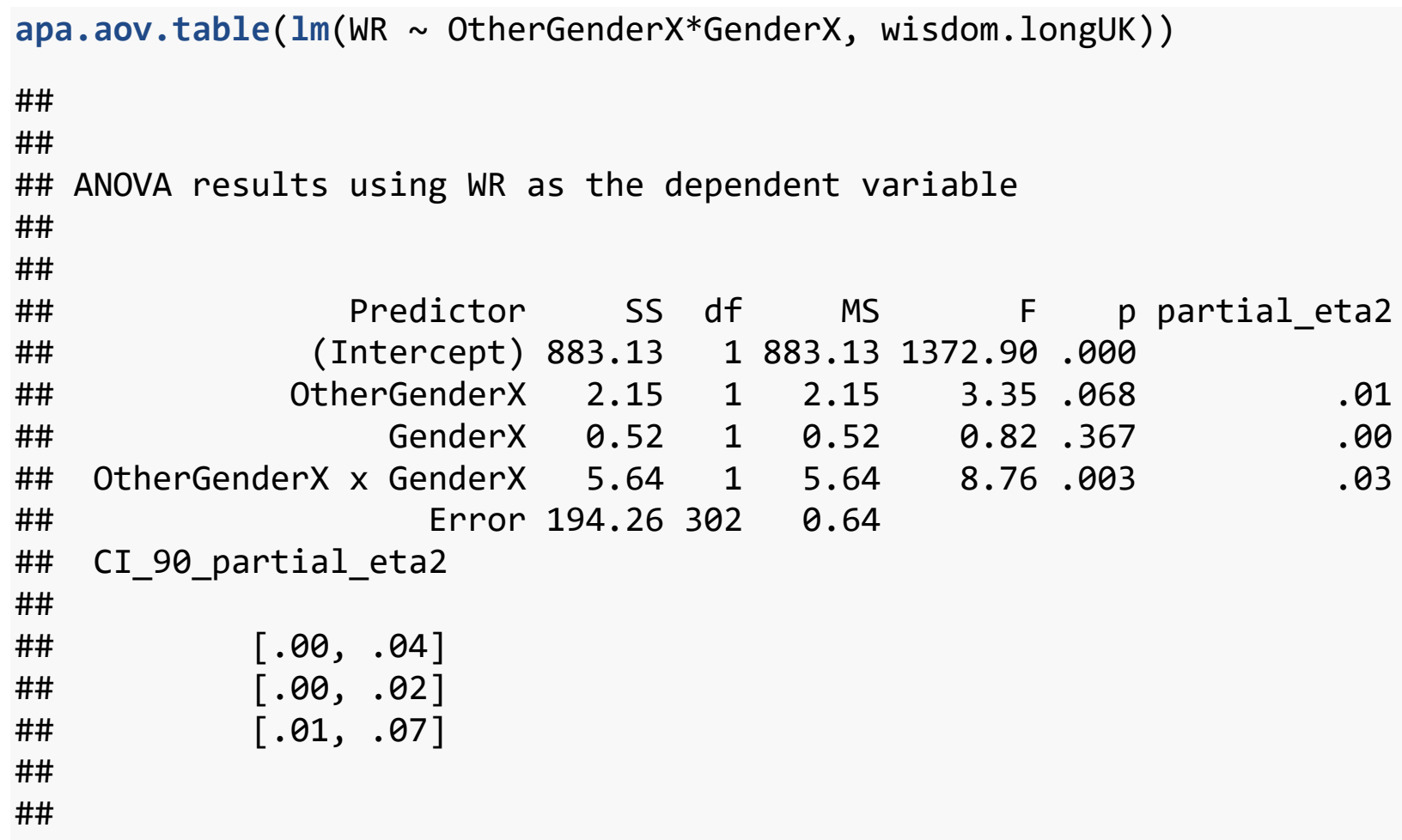

\#\# Note: Values in square brackets indicate the bounds of the $90 \%$ conf idence interval for partial eta-squared

\#When other's gender is different from yours you are more likely to re ason wisely than when its same gender as yours

cor (wisdom. longUK\$Age, wisdom. longUK\$WR, use="pairwise . complete.obs")

\#\# [1] -0.1527542

cor (wisdom. longUS\$Age, wi sdom. longUS\$WR, use="pairwise. complete.obs")

\#\# [1] -0.0329687

wisdom. long. womenUK=subset ( wisdom. long, GenderX=='Female' \& (Country== 'UK ' |Country==' 'Scotland ' |Country==' Ireland ' Country==' England' )) \#crea te subsets of women from UK.

wisdom. long. menUK=subset ( wisdom. long, GenderX=='Male' \& (Country==' 'UK' |Country==' Scotland '|Country==' Ireland '|Country==' England')) \#create a subset of men from UK.

summary (Imer (WR scale(OtherGenderN, scale=F)+(1|V1), wisdom.long. wome nUK))

\#\# Linear mixed model fit by REML t-tests use Satterthwaite approximat ions 


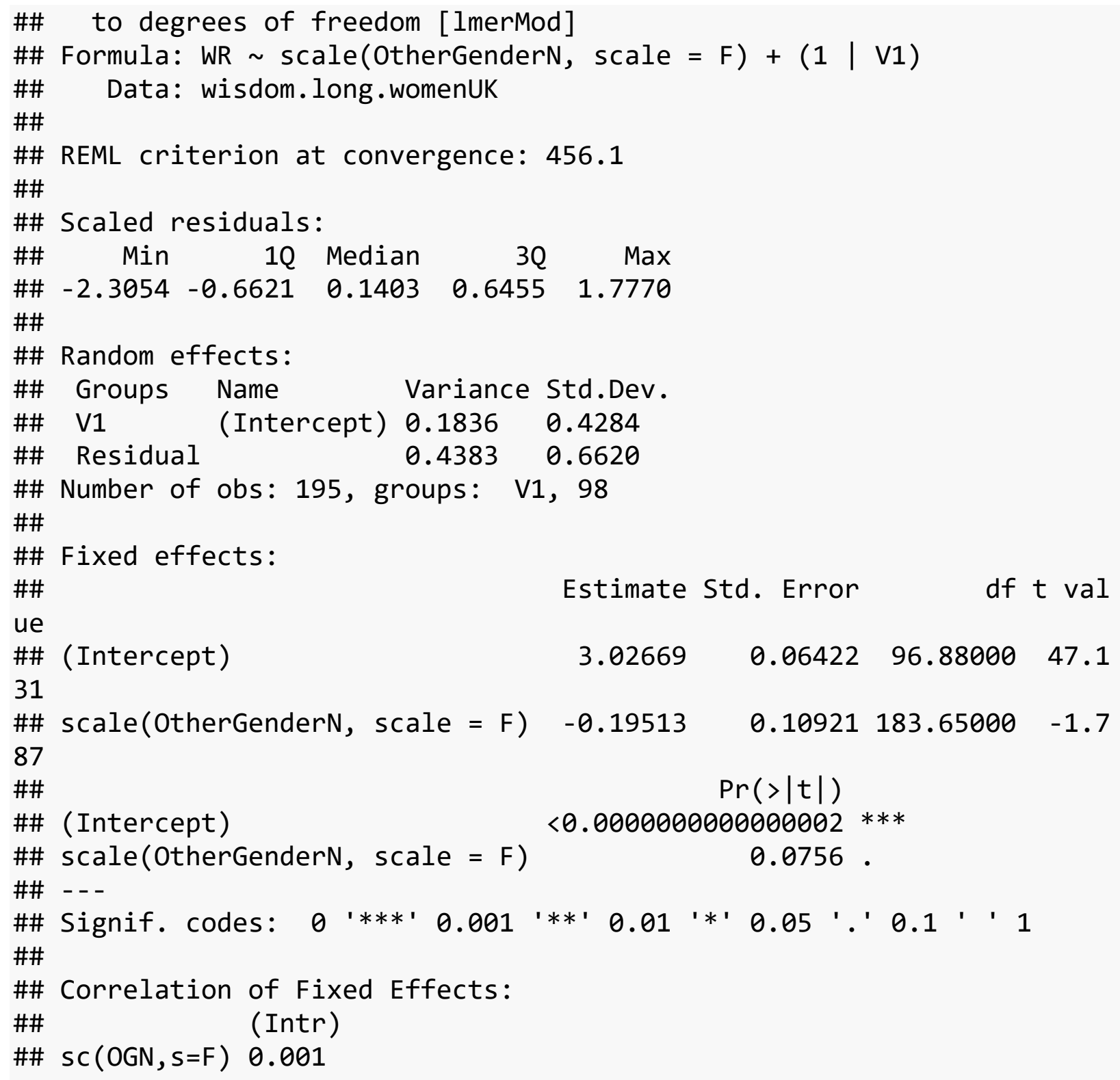
K))

\#\# Linear mixed model fit by REML t-tests use Satterthwaite approximat ions

\#\# to degrees of freedom [lmerMod]

\#\# Formula: WR scale(OtherGenderN, scale = F) + (1 V1)

\#\# Data: wisdom.long.menUK

\#\#

\#\# REML criterion at convergence: 271.2

\#\#

\#\# Scaled residuals: 


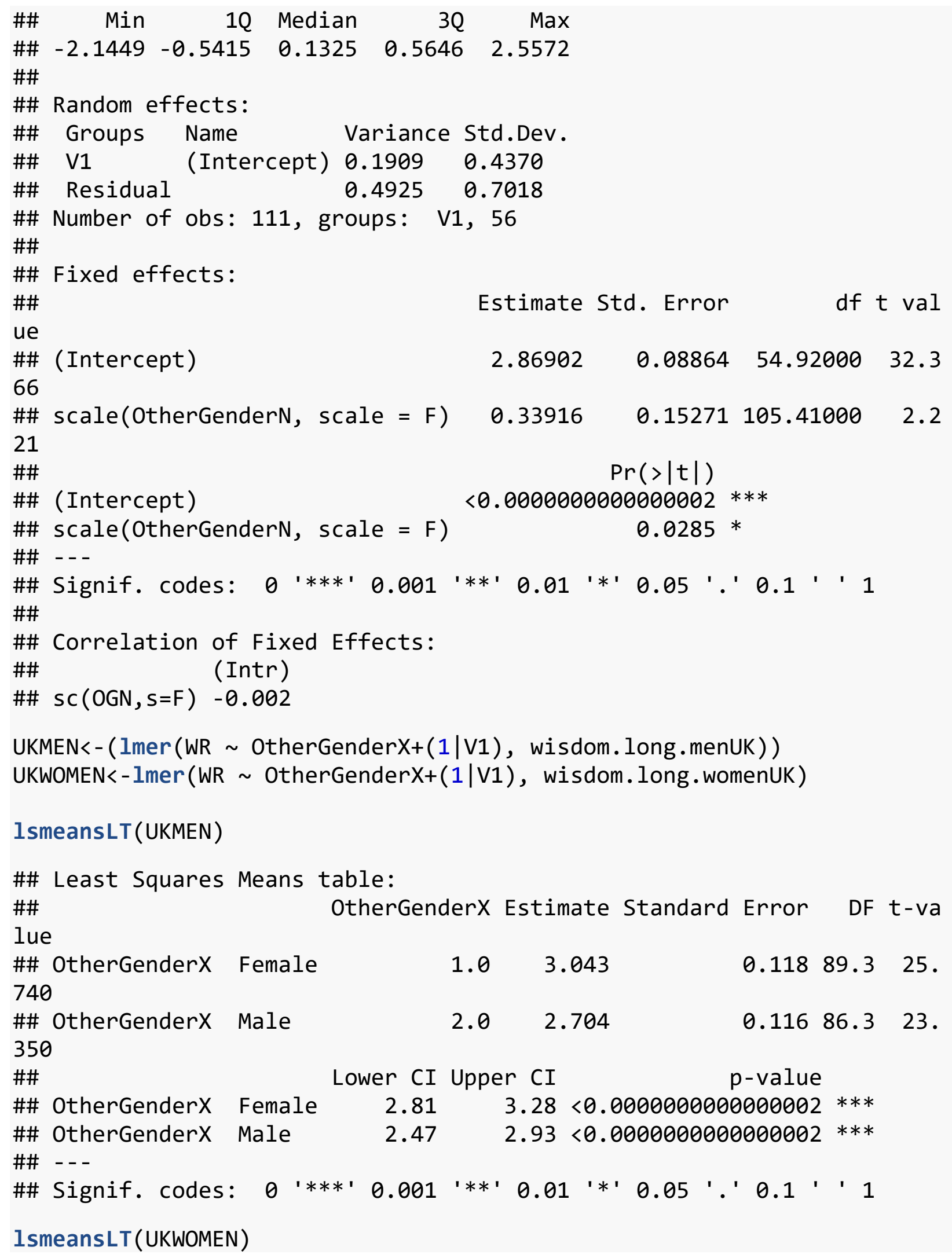




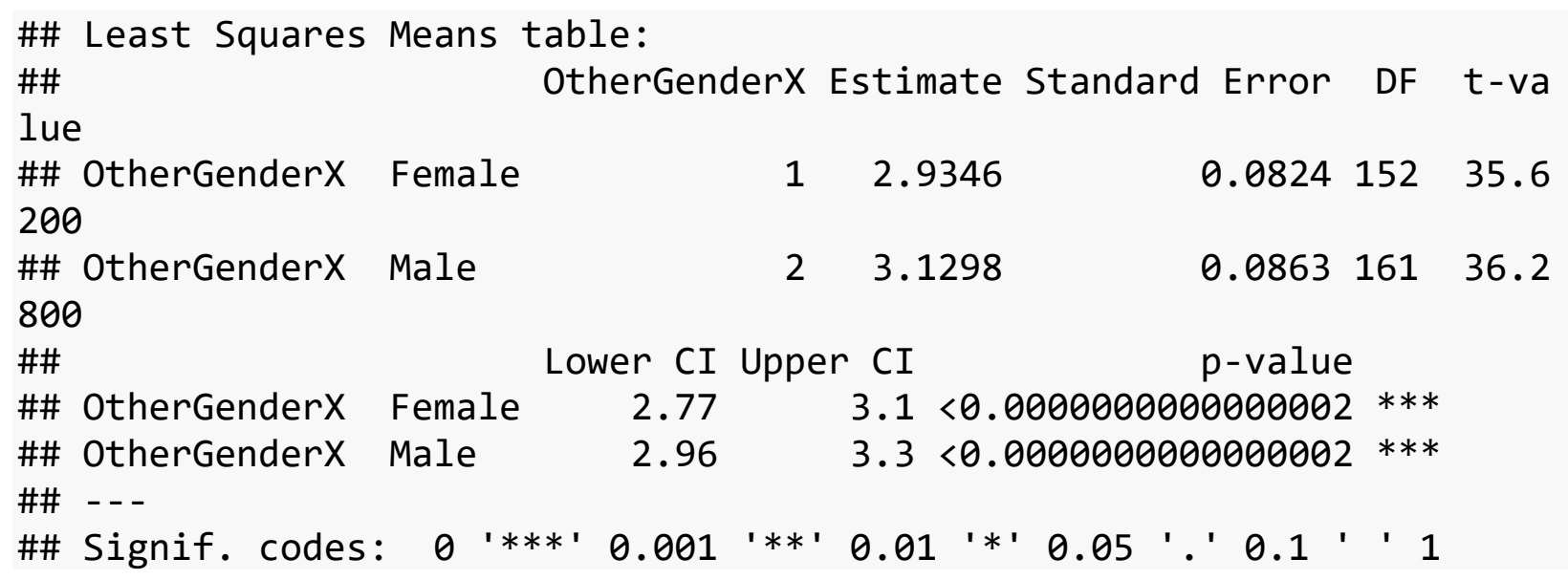




\section{Additional Materials}

Table S14.

Event reconstruction and final wise reasoning items.

In this section we would like you to think about a difficult situation that has happened to you with another person, specifically in your workplace (e.g., a disagreement, conflict) / specifically with a close friend. This should be a situation that you yourself were involved in, whether or not you were the person who initiated the situation. We would like you to take a moment to recall the situation and visualize the events in your mind's eye; consider who was involved and what happened, what you thought and how you felt. After doing so, please respond to the following questions:

1. When did this situation first begin?

i. This week

ii. Within the last month

iii. Within the last 6 months

iv. Within the last year

v. Over a year ago

2. What day of the week was it?

i. $\mathrm{M}$

ii. $\mathrm{T}$

iii. $\mathrm{W}$

iv. $\mathrm{T}$

v. $\mathrm{F}$

vi. $S$

vii.S

viii. Don't remember

3. What time of day was it?

i. Morning

ii. Afternoon

iii. Evening

iv. Don't remember

4. What were you doing when it happened? This only needs to be a sentence or two.

i. $\{$ text box $\}$

5. Where were you?

i. $\{$ text box $\}$

6. Who was involved in this situation? Check any/all that apply - you may select more than one for any person: a coworker may also be a friend. (This question is omitted when assessing conflicts with a close friend)

i. Boss, supervisor, or manager

ii. Mentor

iii. Trainer 
iv. Colleague or Coworker

v. Subordinate

vi. Mentee

vii.Trainee or Apprentice

viii. Customer or Client

ix. Supplier

x. Friend

xi. Family

7. Was the person the same gender as you?

i. Yes

ii. No

8. As you were thinking about this situation, what thoughts came to your mind? Please write your thoughts in the space provided.

i. $\{$ text box $\}$

Please continue to think about the situation you called to mind in the previous section and recall the extent to which you engaged in the following thoughts and behaviors - what you actually did as the situation unfolded. None of the statements listed below are supposed to be "good" or "bad". We are simply interested in how people approach difficult situations. Therefore, it is very important to us that you answer as accurately as possible - your honesty is appreciated, and your replies are, of course, anonymous.

"While this situation was unfolding, I did the following..." (from 1 - not at all, to 5 - very much)

1. Put myself in the other person's shoes

2. Tried to communicate with the other person what we might have in common

3. Made an effort to take the other person's perspective

4. Took time to get the other person's opinions on the matter before coming to a conclusion

5. Looked for different solutions as the situation evolved

6. Considered alternative solutions as the situation evolved

7. Believed the situation could lead to a number of different outcomes

8. Thought the situation could unfold in many different ways

9. Double-checked whether my opinion on the situation might be incorrect

10. Double-checked whether the other person's opinions might be correct

11. Looked for any extraordinary circumstances before forming my opinion

12. Behaved as if there may be some information to which I did not have access

13. Tried my best to find a way to accommodate both of us

14. Though it may not have been possible, I searched for a solution that could result in both of us being satisfied

15. Considered first whether a compromise was possible in resolving the situation

16. Viewed it as very important that we resolve the situation

17. Tried to anticipate how the conflict might be resolved

18. Wondered what I would think if I was somebody else watching the situation

19. Tried to see the conflict from the point of view of an uninvolved person 
20. Asked myself what other people might think or feel if they were watching the conflict 21. Thought about whether an outside person might have a different opinion from mine about the situation

\section{Legend}

Items 1-4: others' perspectives; items 5-8: consideration of change and multiple ways situation may unfold; items 9-12: intellectual humility/recognition of limits of knowledge; items 13-17: search for a compromise / conflict resolution; items 18-21: view of the event through the vantage point of an outsider 
Table S15.

\section{Crimea Case Scenario}

Please read the following article carefully. You will later be asked about your opinion on this issue.

Ukraine shares history and cultural heritage with many of its different neighbors. People in Eastern Ukraine identify with Russia, and many Russians see Ukraine as part of their motherland. In contrast, people in Western Ukraine identify with Western Europe. In the last few years Ukraine became a battleground for political and economic influence from Russia and the West. The country is in an economic recession and suffers a huge deficit: it requires more goods from abroad than it produces and sells. Many Ukrainians wish to join with Russia to avoid significant economic hardship. Many others wish to cut ties with Russia and seek deals with the European Union, where they see opportunities for more jobs.

Last year, pro-Western Ukrainians hoped that a trade agreement with European Union would help the economy. However, at the last moment, Ukraine's President Yanukovych turned away a European deal in favor of a $\$ 15$ billion bailout from Russia. Some say it was a corrupt decision made under pressure from the Kremlin. Following this, hundreds of thousands of people took the protest to the streets. In the weeks and months that followed, the protests turned into a general outcry against governmental corruption and police violence. Eventually, after much destruction and violence protesters took control of Kiev's city center, and parliament voted to remove Mr. Yanukovych from office, who in turn fled the country. Some say the protesters have been financially backed up by the Western powers, interested in the natural resources of the country.

Recently in Crimea, a southern peninsula of Ukraine with a predominantly Russian ethnic majority $(58 \%)$ and a large Russian military presence, an internationally-disputed election took place in which the majority voted in favor of independence from Ukraine and to join Russia. Some fear that that the events in Crimea are a sign of things to come and that Ukraine will be split as a result of the current crisis. 
Table S16.

Intergroup Wise Reasoning Measure

As you reflected on the conflict, to what extent did you engage in the following thoughts and behaviors? Note, none of the statements listed below are supposed to be "good" or "bad". We are simply interested in how people approach difficult situations. Please select the extent to which you engaged in the following thoughts and behaviors:

"While I was contemplating and writing about the previous scenario, I did the following..." (from $\underline{1-\text { not at all, to } 5 \text { - very much) }}$

1. Put myself in both parties' shoes

2. Thought about the things both parties might have in common

3. Made an effort to take both parties' perspective

4. Took time to consider both parties' opinions on the matter before coming to a conclusion

5. Looked for different solutions to the evolving conflict

6. Considered alternative solutions as I learned about the conflict

7. Believed the situation could lead to a number of different outcomes

8. Thought the situation could unfold in many different ways

9. Double-checked whether my opinion on the situation might be incorrect

10. Double-checked whether the either party's opinions might be correct

11. Looked for any extraordinary circumstances before forming my opinion

12. Behaved as if there may be some information to which I do not have access

13. Tried my best to find a way to accommodate both parties' perspectives

14. Though it may not have been possible, I searched for solutions that could result in both parties being satisfied

15. Considered first whether a compromise was possible in resolving the situation

16. Viewed it as very important that the parties resolve the situation

17. Tried to anticipate how the conflict might be resolved

18. Wondered what I would think if I were somebody else considering the situation

19. Tried to see the conflict from the point of view of an uninvolved person

20. Asked myself what other people might think or feel if they were considering the conflict

21. Thought about whether an outside person might have a different opinion from mine about the situation 


\section{Table S17.}

\section{Global Wise Reasoning Scale}

We would like you to think about how you handle difficult situations involving others, specifically with a close friend (e.g., a disagreement, or a conflict). We ask you to answer these questions as accurately and honestly as possible.

To what extent do you agree with the statements below? $(1$ - strongly disagree to 6 - strongly agree)

1. I am good at seeing things from other people's point of view

2. It comes naturally to me to understand other people's thoughts and emotions

3. I know that most situations will have an element of unpredictability to them

4. I keep an eye out for ways things might change over time

5. I often consider multiple ways how social events may unfold

6. In social events, I keep an eye out for how the situation may change direction

7. I think that the best way to solve difficult situations is to ignore the distracting details (discarded item)

8. I am skilled at looking out for the little "unknowns" in social situations

9. In most difficult situations I tend to compromise with other people

10. During conflicts I am very much the kind of person who will look for ways to benefit both myself and the other people involved in the conflict.

11. In social conflict situations I tend to be the "problem-solver" - looking for solutions how to optimally resolve the conflict

12. In social situations, I focus a lot on how to resolve the issue

13. I try to "step outside of myself" to see how I might appear when I am engaged in difficult social situations

14. In difficult situations, I try to "step out of my own shoes" and see myself from a thirdperson/observer perspective 
Table S18.

Attribution Vignettes

\section{Vignette 1}

Steve Jensen is the president of a large construction company in New York. Last year, local government fined the company, as unstable scaffolding caused problems resulting in injuries to several people.

Recently, Steve Jensen started a special discount house building program for large families. Also, he decided to donate a large sum of money to a local orphanage.

\section{Vignette 2}

Sara Martin is a top executive of a company "XinK Int." "XinK Int." is one of the leading pharmaceutical companies in the US. However, the company has experienced a decline in their public image which has led to a decline in sales in the last half a year. Recently, the company started several activities, which were focused on the stabilization of their leading position in the pharmaceutical market.

Not too long ago, "XinK Int." developed a new drug for treating malaria. Shortly after that several African countries experienced an outbreak of malaria. As soon as Sara Martin found out about this event, she decided to donate a lot of medicine to the regions in Africa that needed assistance. Local mass media showed different reactions to this news.

\section{Vignette 3}

Since his childhood, David Conner wanted to become a doctor. Now, he is a young surgeon at a local hospital in the Baltimore area. During his first year he has had a wonderful track record. However, due to a recent argument with the head physician, any little mistake would mean that he would be fired.

Last week, a patient died during a surgery performed by David Conner because another doctor had given her an incorrect diagnosis. However, David decided to hide this fact and told the woman's the other doctor's incorrect diagnosis - that the weak heart of the patient was the reason for her death, and therefore the doctors could not save her.

\section{Vignette 4}

Emma Peterson is a banker at a large bank in Cincinnati, OH. Several major pension funds are heavily invested in the bank. In the last couple of months, the bank lost a large amount of money on the stock market. The current financial difficulties of the bank may devalue the bank's shares.

However, Emma Peterson did not reveal the loss to the company's shareholders in order to avoid causing panic. Instead, Emma Peterson reported a sizeable profit at the annual meeting of the shareholders, hoping that the annual balance of the company would still be positive in comparison to the last year. 
Table S19.

Influence and Accommodation

Here are questions regarding the incident you recalled and the other person involved in the situation.

How important was it for you to... $(1-$ Not at all important to 5 - Extremely important)

1. Appear confident

2. Assert yourself

3. Have an impact on the other person

4. Have the other person listen to what you have to say

5. Keep your thoughts or feelings to yourself

6. Not reveal what you are really like

7. Go along with what the other person wants

8. Do what the other person wanted you to do

9. Make sure that the other person does not see you as getting in his/her way 
Table S20.

Causal Inferences

Here are questions regarding the incident you recalled and the other person involved in the situation. (1 - Not at all, to 5 - Very much)

1. Do you think that you were primarily responsible for the incident?

2. Do you think the situation may have influenced you to say or do things that you otherwise would not have done?

3. To what extent were you to blame?

4. Could you have been more "wise" in the situation?

5. Do you think that the other person was primarily responsible for the incident?

6. Do you think the situation may have influenced this other person to say or do things that they otherwise would not have done?

7. To what extent was this other person to blame?

8. Could this other person have been more "wise" in the situation? 